\title{
What Dreams May Come
}

Ambient Suite for Jazz Orchestra

by

Louisa Beth Williamson

\section{An exegesis}

submitted to the Victoria University of Wellington in fulfilment of the requirements for the degree of Master of Music in Composition

Victoria University of Wellington 31 May 2020 


\section{Abstract}

What Dreams May Come is a five-movement suite for jazz orchestra, intended to create a calm and relaxing listening experience. The project is inspired by the mystery of dreaming, and it attempts to communicate musical ideas which reflect the relaxed state one is in when sleeping. The aims of What Dreams May Come are to highlight the timbral combinations available in a jazz orchestra and to draw on characteristics of ambient music to give the listener a relaxing atmosphere.

This exegesis explores timbre both in music that served as inspiration for this composition and in the composition itself, and it describes how emphasising timbre in my compositional process affected other musical elements of the piece. Chapter 1 explores Brian Eno's ambient album Ambient 1: Music for Airports, specifically looking at the role of timbre and texture in the album, and at the overall structuring techniques used by Eno on the album to create coherency. Chapter 2 analyses two compositions for jazz orchestra by Maria Schneider, "Nocturne" and "Sea of Tranquility", examining the role of timbre in the compositions, as well as the ways Schneider uses soft dynamics and harmonic techniques to structure the pieces. These two chapters look into how Eno and Schneider, in different ways, both highlight timbre in their compositional approaches and processes. Each chapter dives deep into timbral and textural analysis, with additional analysis of form and harmony. Chapter 3 reflects on the ways these two composers informed What Dreams May Come, focussing on how I used techniques from Eno and Schneider to challenge myself in composing for jazz orchestra.

In the course of the project, I strove to tap into music's therapeutic qualities, putting this idea at the forefront of my intentions as a composer. Using dreaming as aesthetic and conceptual influence, Brian Eno's ambient music as inspiration, and Maria Schneider's compositions as a musical guide, I have been able to produce a work which not only challenges traditional jazz orchestra techniques but also relaxes listeners by complementing their environments. 


\section{Acknowledgements}

Ironically, composing music intended to relax to was not always a relaxing experience in itself. There were many times I embraced ambient music in order to reduce stress caused by diving into the exact music I was listening to. I am grateful for Brian Eno and his ambient works for helping to keep me sane throughout the course of this project, and of course to Maria Schneider for her fearless, beautiful compositions.

Firstly I would like to give a huge thank you to my supervisor, Dave Wilson. Without his persistently incredible guidance I would definitely not have finished this. Thank you Dave, for being so inspiring in the way you have supported me to complete this. I would also like to thank my other supervisor, Dave Lisik, who has played a large role in my formation as a musician and gave great advice for these compositions. Thank you for giving me such excellent mentoring and guidance from day one. I am deeply grateful to Victoria University for allowing me to pursue this work by scholarship. I am also extremely thankful for the professionalism of Daniel Hurley, who was paramount in the final few weeks of proofreading and formatting.

To my parents, Jana, Amy and Fleur, thank you for how much you believe in me. Making you all proud is always my motivation to succeed.

I'm grateful to have been locked down in a flat with such amazing friends and musicians Keanu, Katelin, Niamh and Zane, who were nothing but supportive and encouraging. I am deeply grateful for all of my musical comrades in New Zealand and abroad who were so invested in this project and its progress. Thank you Dylan for always being willing to help me, you are a gift to the world.

Finally, I would like to thank Keanu for bearing the brunt of my stress, setting up a workspace for me to focus on this work, making so many sacrifices for me to complete this to a high level and encouraging me every day. 
Chapter 2 A Figureless Landscape: Timbre and Texture in Brian Eno's Ambient 1: Music for

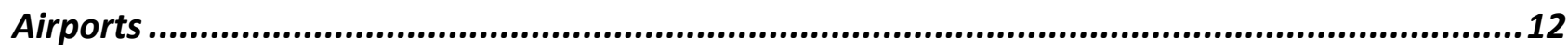

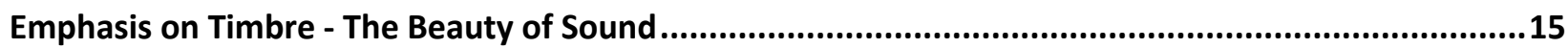

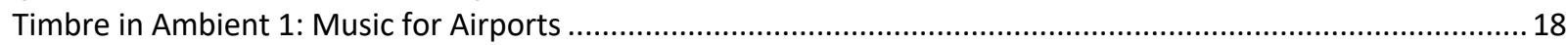

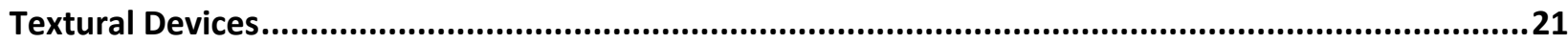

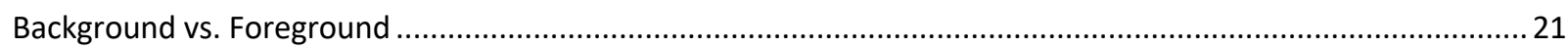

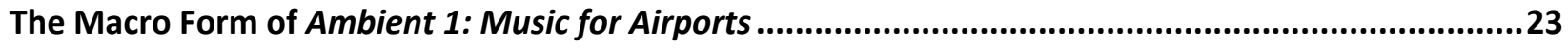

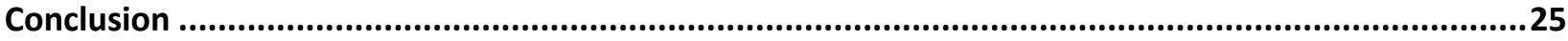

Chapter 3 Timbre, Dynamics and Harmony in "Nocturne" and "Sea of Tranquility" by Maria

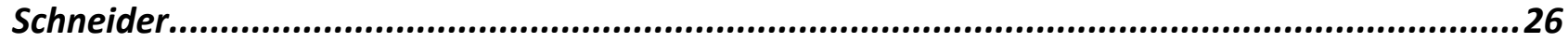

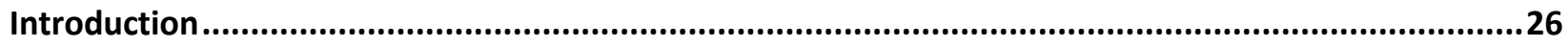

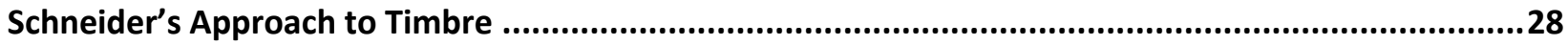

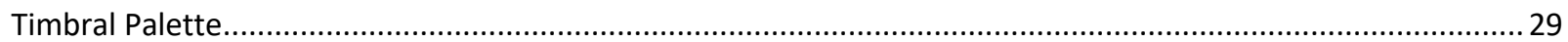

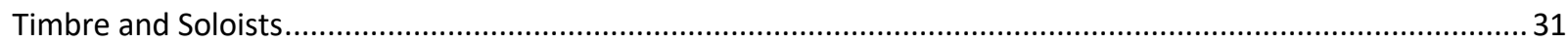

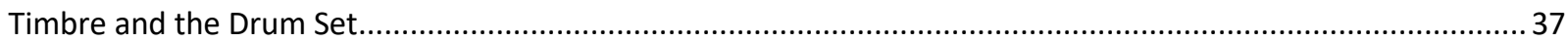

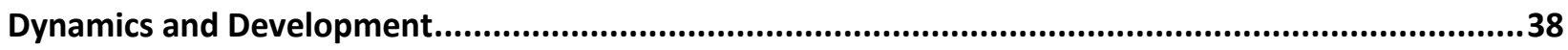

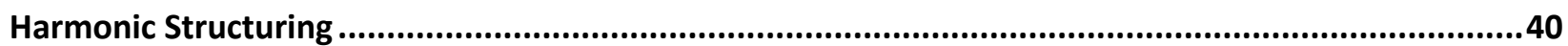

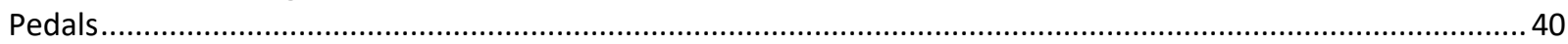

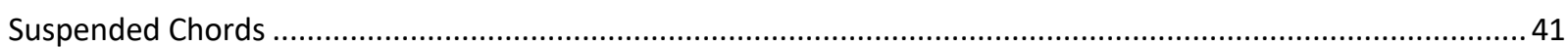

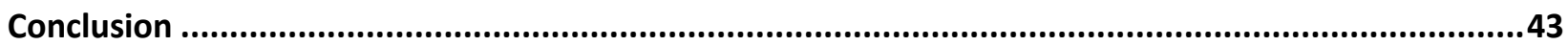

Chapter 4 Analysis and Reflections on What Dreams May Come ............................................44

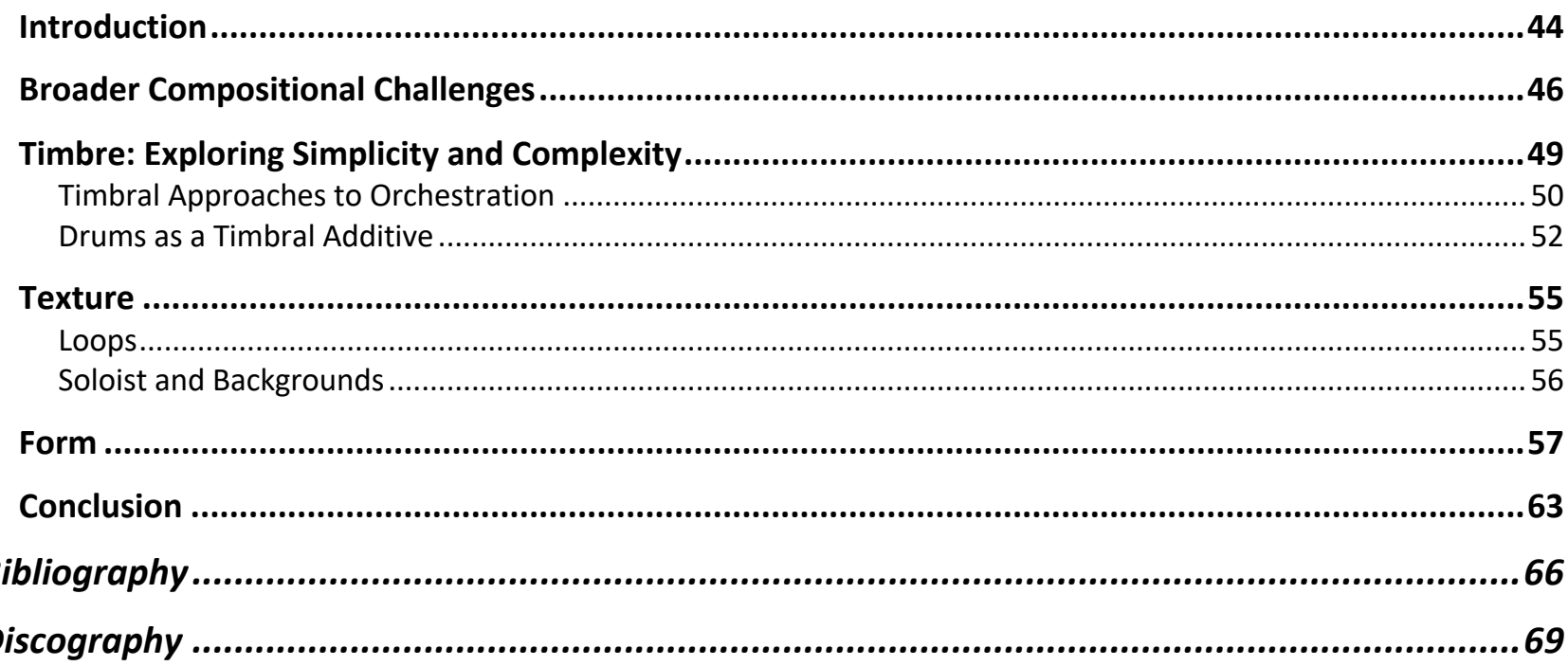




\section{Table of Figures}

Figure 2. 1 Graphic Score of Ambient 1: Music for Airports by Brian Eno 17

Figure 3. 1 Thinning of orchestra weight at bar 19 of "Nocturne"

Figure 3. 2 Backgrounds behind piano solo in "Nocturne", bars 41-48 33

Figure 3. 3 Melodies from backgrounds written into the soloist's (piano) at Bar 41 of "Nocturne" 36

Figure 3. 4 Piano and bass parts from bar 37-40 of "Nocturne" 36 41

Figure 4. 1 The combination of mutes in the trumpets at Bar 70 of movement five 52

Figure 4. 2 A drum roll on the floor tom accompanying a bass pedal in Bar 1 of the third movement 54

Figure 4. 3 Guitar and piano at bar 55-56 of movement one

Figure 4. 4 The five motifs/loops used in fifth movement

Figure 4. 5 Main motif in movement two played by Alto 1 and 2 (bar 32 of movement two)___ 56

Figure 4. 6 Motif 1 in piano and bass (bar 37 of movement 2) __ 58

Figure 4. 7 Motif 1 in piano (bar 1 of movement 3)

Figure 4. 8 Motif 2 in guitar and piano (bar 97 of movement 3)

Figure 4. 9 Motif 2 carried on over new, unfamiliar bass line (Bars 121 to 125 in movement three) __ 60

Figure 4. 10 Time intervals between entrances in each part begin to decrease in bars 71-77 of movement five __ 62 


\section{Chapter 1}

\section{Introduction}

What Dreams May Come is an original, multi-movement suite for jazz orchestra, inspired by the mysteries and anomalies of dreaming. This project is informed by ambient music, specifically the album Ambient 1: Music for Airports (1978) ${ }^{1}$ by Brian Eno, and by modern big band jazz, specifically two compositions from the album Allegresse $(2000)^{2}$ by Maria Schneider, "Nocturne" and "Sea of Tranquility". This extended composition comprises a suite of five movements: "1.", "2.", "2.3.2. (Dream within a Dream)", "4. (The White Room)" and " 5 ." with a total duration of 47 minutes. The aim of this project is to intentionally create music to induce comfort.

This project is inspired by and named after the movie What Dreams May Come (1998), ${ }^{3}$ directed by Vincent Ward and adapted by Ronald Bass from the 1978 novel of the same name by Richard Matheson. ${ }^{4}$ The movie follows the life and afterlife of a man who travels from heaven to hell to save his wife who had committed suicide. This visually stunning movie beautifully depicts and interprets the afterlife, as if it is one big dream, bringing a sense of comfort and hope to whoever is watching. The inspiration behind this movie and novel originates from the story of Shakespeare's Hamlet. 'What dreams may come' is a well-known phrase spoken by Hamlet in his famed 'To be or not to be?' soliloquy:

To die, to sleep--

To sleep--perchance to dream: ay, there's the rub,

For in that sleep of death what dreams may come ${ }^{5}$ This excerpt equates death with sleeping, pondering 'what dreams may come' when one dies. This contemplation of death is a major theme throughout Hamlet, as well as in the film What Dreams May Come. This movie is a stunning visual interpretation of the goingson inside the human brain when the unconsciousness has more freedom. My focus is on

\footnotetext{
${ }^{1}$ Brian Eno, “Ambient 1: Music for Airports" (Polydor Records, 1978).

${ }^{2}$ Maria Schneider, "Allegresse." (Enja Records, 2000).

${ }^{3}$ Vincent Ward, What Dreams May Come (United States: PolyGram Filmed Entertainment, 1998).

${ }^{4}$ Richard Matheson, What Dreams May Come (Putnam, 1978).

${ }^{5}$ Wiliam Shakespeare, "Hamlet: A Monologue," Monologue Archive, accessed November 13, 2019, http://www.monologuearchive.com/s/shakespeare_001.html.
} 
dreams specifically, but I use this movie as inspiration to help interpret the mysteries of the unconscious mind.

A certain level of mystery, wonder and enigma is always present with the discussion of the afterlife or dreaming. Everybody dreams, but many people go through life accepting that dreaming is a mystery. So, what is the purpose of dreaming? Dreaming could be considered to be the most relaxed state in which the brain and body can exist. Dreaming is total freedom for the subconscious to process anything it needs to, with almost total stillness of the body. Whether remembered or not, dreams are an important part in the workings of the phenomena of the brain.

Many theories of the purposes behind dreaming exist, with no general consensus. One theory is that people dream to process information and emotions the conscious brain is not capable of processing. ${ }^{6}$ Dreams can often be connected to issues or concern currently on a person's mind whilst awake. ${ }^{7}$ Similarly, to me it seems daydreaming is a more conscious form of dreaming, where people can play out potential scenarios or events to process information. During a daydream, one is usually in a relatively relaxed state. Of the many mental processes the brain undergoes every day, it seems as though dreaming could be one way that the mind resolves suppressed or complex emotions.

For this project, I wanted to musically portray the comforting and therapeutic elements of dreaming. I determined that ambient music would be the best inspiration, as it also possesses therapeutic qualities through its soothing, minimal soundscapes. Ambient music is a genre of popular music that emphasises space, texture and timbre over other musical conventions such as melody, moving harmonies and rhythm, typically using electronic instruments such as synthesisers. It is slow-moving in tempo, and specifically designed to not be listened to directly but rather to be played in the background to accompany other activities. This genre has a similar intent to dreaming in the sense it can be therapeutic, serving to relax and ease the listener. In this sense, ambient music is like the dreamscape of the awake world.

\footnotetext{
${ }^{6}$ Clara E Hill, Working with Dreams in Psychotherapy (Guilford Press, 1996).

${ }^{7}$ Morton F. Reiser, "Revising Dream Theory," in Memory in Mind and Brain (Yale University Press, 1994$), 177$.
} 
In conceiving this suite of compositions, my general desires were to create music that provides a calming listening experience, by focussing on timbre and exploring softer dynamics. In my analysis of the work of Eno and Schneider, the focus on timbre lies at the centre of my uncovering of how calmness is achieved in their composition. I also explore how putting emphasis on timbre throughout a compositional process affects other musical elements such as dynamics, rhythm, harmony, melody, and form. In my own suite of compositions, I model some of my strategies on compositional techniques I observe in my analysis of the work of Eno and Schneider.

The genre of ambient music was invented by Brian Eno, a musician, composer, and producer. Eno has composed and produced music in an abundance of different styles, and has also worked as a visual artist, sculptor and digital artist. Eno is fiercely creative, and has helped and inspired an enormous amount of people worldwide with his work, including me and my work on What Dreams May Come. Eno developed the concept of ambient music inspired by earlier styles of 'furniture music', invented by Erik Satie in the 1920s, and 'muzak'. ${ }^{8}$ In my analysis of his Ambient 1: Music for Airports in Chapter 2, I focus mostly on describing timbre on the album and on Eno's overall approach to timbre, and I look into how he uses a small number of timbres effectively. I also analyse his approach to texture in terms of the hierarchy of sounds, and his overall structuring of the album with regard to its coherency, the placement of silence between tracks, the use of drones, and tuning. I have adopted many of Eno's compositional techniques for What Dreams May Come, applying them to the context of jazz orchestra instrumentation primarily in a conceptual manner.

I decided to also study two works of Maria Schneider in order to apply or interpret more practical and tangible compositional techniques. Maria Schneider utilises timbre and texture in a way that embraces the potential of a jazz orchestra, which is why I chose to study two of her works to inform my own writing. The two pieces "Nocturne" and "Sea of Tranquility" feature as two of the slower-paced, "ballad' components of Allegresse. One element I analyse and draw inspiration from in Schneider's two works are her use of instrumentation to achieve variety in timbre and texture, through the use of doubling instruments, mutes, and the use of the drum kit as a timbral additive. I also analyse her

\footnotetext{
${ }^{8}$ Herve Vanel, “John Cage's Muzak-Plus: The Fu(Rni)Ture of Music,” Representations 102, no. 1 (2008): 94.
} 
use of dynamics to shape a piece, and her harmonic approach and techniques, such as application of dissonance.

Eno and Schneider have very different compositional approaches and processes, and they work in two very different realms of musical recording and performance. But they share a similar appreciation for timbre, aiming to bring attention to the beauty of sound. Eno works only in the digitally performed realm and does the bulk of his composing in-studio, whilst Schneider works with both live performance and studio recording, for primarily acoustic instruments. Taking elements of both Eno and Schneider's compositional approach, with dreaming as my overarching inspiration, has resulted in a suite of music which suits the aesthetics of how the inner workings of our relaxed imagination, be it in the afterlife or in our dreams.

This exegesis comprises a chapter each analysing Brian Eno's Ambient 1: Music for Airports and Maria Schneider's two pieces, "Nocturne" and "Sea of Tranquility", respectively. These chapters delve into timbral and textural analysis, with some additional analysis on harmony, form and dynamics. Following these two chapters is a chapter describing, analysing and reflecting on my own compositions in relation to the analysis of Eno's and Schneider's work.

Using a big band as a sonic vessel for a composition informed by ambient music comes with several obstacles, such as issues around stamina, endurance, and engagement for the performer, but also several opportunities for innovation and creativity, like having the jazz orchestra play at a quiet volume for a prolonged period of time, instruments of the rhythm section acting as timbral additives instead of providing the foundations of pulse and rhythmic foundations, and the inclusion of many timbral and textural possibilities. This is a contrast to the big band music I usually hear performed in my community, which can often be raucous and powerfully loud. This project acknowledges the wide range of dynamic possibilities for a jazz orchestra, exploring the ensemble's softer, more delicate side through the lens of ambient music. My other goal was to produce music where many different levels of attentiveness are available to the listener, giving listeners discretion with regard to how they want the music to affect them, whether they seek to be fully immersed 
in the musical experience, or to allow the music to play in the background of their thoughts, much like Eno's intentions behind his ambient music. ${ }^{9}$

As ambient composition and jazz orchestra composition have a considerable number of contradictory musical characteristics, the main challenge in this project is finding where the two genres can live harmoniously. I wanted to challenge myself with studying a style of music I did not understand well (ambient music) and applying it to a type of ensemble I already knew well (jazz orchestra). In doing this I hoped to confront some of the traditional conventions of big band jazz such as the inclusion of improvisation, musical conceptions of narrative and the role of the drum kit. This project interprets dreaming by combining ambient music with big band jazz in an innovative and captivating way. What Dreams May Come is an experimental venture where I challenged and stretched my compositional ability and uncovered more about the capabilities of a jazz orchestra, in the process hopefully creating a relaxing and reflective experience for whoever is listening. Among the project's goals are to ease stress for the listeners, where they can still be present with their thoughts, and have a meaningful and reflective experience, similar to daydreaming.

\footnotetext{
${ }^{9}$ Brian Eno, "Music for Airports; Liner Notes," accessed February 20, 2020, http://music.hyperreal.org/artists/brian_eno/MFA-txt.html.
} 


\section{Chapter 2}

\section{A Figureless Landscape: Timbre and Texture in Brian Eno's Ambient 1: Music for Airports}

Brian Eno has produced an enormous body of influential work in both audio and visual art. A composer, producer, and multi-instrumentalist, Eno's public career began playing synthesisers in the progressive rock group Roxy Music in the 1970s. Since then he has released 20 solo albums, in genres spanning ambient to punk. ${ }^{10}$ Whilst he began his career as a performer, he is now held in high regard as a studio musician and producer. Eno considers the studio an instrument and 'compositional tool,'11 and has produced or coproduced over 100 albums for artists such as David Bowie, Coldplay, U2 and Grace Jones ${ }^{12}$. Eno is famously known for considering himself a 'non-musician'13 and does not rely on a wealth of knowledge of music theory or technical abilities on instruments. Because of this he has honed and developed innovative approaches to creating music inside the studio. He coined the term 'ambient music' in the liner notes to the album Ambient 1: Music for Airports (1978), ${ }^{14}$ which is the first of a series of four ambient albums he released between 1978 and 1982. ${ }^{15}$ Ambient 1: Music for Airports consists of four tracks ("1/1", "2/1", "1/2", "2/2"), each with a duration between 9 and 17 minutes long.

Ambient music is a genre of popular music that emphasises space, texture and timbre over other musical elements such as melody, harmonic motion, and active, engaging rhythms. In its emphasis on texture and timbre, ambient music typically features electronic instruments such as synthesisers. Ambient music typically unfolds slowly over a long period of time, and is specifically designed to not be listened to directly but rather to be played in the background to accompany other activities. A significant moment in the

\footnotetext{
${ }^{10}$ Eric Tamm, Brian Eno - His Music and the Vertical Color of Sound (New York: Da Capo Press, 1995).

${ }^{11}$ Brian Eno, "The Studio as a Compositional Tool," in Audio Culture: Readings in Modern Music (Bloomsbury Academic, 2004), 127-30.

12 “Brian Eno,” Discogs, accessed May 27, 2020, https://www.discogs.com/artist/634-Brian-

Eno?filter_anv=0\&subtype=Albums\&type=Releases.

${ }^{13}$ Lester Bangs, "Lester Bangs Interviews Eno," Musician, n.d.,

http://music.hyperreal.org/artists/brian_eno/interviews/musn79.html (accessed 14 December 2019).

${ }^{14}$ Eno, "Music for Airports; Liner Notes."

15 The other albums in this series are: Ambient 2: The Plateaux of Mirror (1980) (By Eno and Harold Budd), Ambient

3: Day of Radiance (1980) (By Laraaji / Produced by Eno), Ambient 4: On Land (1982)
} 
development of Eno's concept of ambient music was in 1975 when he spent several months in a body cast lying in a hospital bed after being hit by a car, forced to listen to eighteenth-century harp music that was slightly too quiet. He discovered that the harp music was effectively merging into his environment, creating 'many levels of listening attention without enforcing one in particular'. ${ }^{16}$ This listening experience inspired his mission, for Music for Airports and subsequent ambient projects, to create music where the listener is able to think freely, whilst 'enhancing and incorporating'17 other outside sounds. In creating ambient music, Eno aimed to create music that could swallow up any environmental, atmospheric or exterior sounds and turn them into part of the composition. Eno also marks another particular experience as profoundly inspiring his conception of Music for Airports. He was sitting in an airport with stunning architectural design in Cologne, Germany, and he had a realisation that the 'awful' radio-style music playing in the airport did not make sense in relation to the airport's design, especially considering how much money and effort had clearly been put into the architecture and design of the rest of the ambiance. ${ }^{18}$

Airports could be considered by many to be an extremely stress-inducing environment. The stress of airport travel also inspired Eno to write Ambient 1: Music for Airports, with one of his stated aims behind the album being to bring a sense of calm to travellers, although due to the widespread popularity of this album it is listened to in many environments other than airports. ${ }^{19}$ Eno believes ambient music, and most art, causes people to 'surrender'. ${ }^{20}$ Perhaps what Eno means by 'surrender', is that engaging with art can cause a lost sense of control, whilst letting something else take over. Since releasing Music for Airports in 1978, Eno has worked on several projects that develop this idea, such as producing soothing soundscapes and light installations for hospitals. ${ }^{21}$

\footnotetext{
${ }^{16}$ Brian Eno quoted in: Robert Fink, "FOUR 'A POX MANFREDINI': The Long-Playing Record, the Baroque Revival, and the Birth of Ambient Music," in Repeating Ourselves: American Minimal Music as Cultural Practice (University of California Press, 2005), 200.

${ }^{17}$ Tamm, Brian Eno - His Music and the Vertical Color of Sound.

${ }^{18}$ Nathanidiothend, "Brian Eno Interview," 2007,

https://www.youtube.com/results?search_query=brian+eno+music+for+airports+interview.

${ }^{19}$ Eno, "Music for Airports; Liner Notes."

${ }^{20}$ Simon Hattenstone, "Brian Eno: 'We've Been in Decline for 40 Years - Trump Is a Chance to Rethink," The Guardian, 2017, https:/www.theguardian.com/music/2017/jan/23/brian-eno-not-interested-in-talking-about-mereflection\#maincontent.

${ }^{21}$ Jenn Pelly, "Brian Eno Creates Healing Music and Light Installations for Hospitals," Pitchfork, accessed February 21, 2020, https://pitchfork.com/news/50402-brian-eno-creates-healing-music-and-light-installations-for-hospitals/.
} 
My analysis in this chapter brings to the forefront the compositional strategies I drew on to inform my suite of ambient big band music. I chose to study Ambient 1: Music for Airports because it is an excellent example of the genre of ambient music. Not incidentally, it is the first album explicitly named 'ambient' by Eno. ${ }^{22}$ Underlying my analysis is the question of how ambient music is distinct from other styles of music that can be played quietly in the background to add ambiance. One distinctive element of ambient music is that it is designed and born out of an intention for it to be played in the background, to be 'ignorable as it is interesting'. ${ }^{23}$ My musical analysis explores what Eno implemented to intentionally make the music 'ambient', especially considering that the album was intended for people who are experienced heightened levels of stress, specifically at an airport, and not, for example, someone enjoying a sunny afternoon.

With my own background of studying in a university jazz programme for four years, I noticed that, in my initial listening to this album, my attention was drawn to certain musical elements that were almost opposite to those prioritised by ambient music. Working towards mastering facility and technique on the saxophone, assimilating vocabulary to navigate complex choral structures, and developing a strong sense of keeping in time were all highlighted in my education as being important in the development of becoming a jazz musician. I was attracted to ambient music perhaps out of resistance or defiance to the value for virtuosity of some jazz music, and the differences or similarities in the intentions of an ambient composer versus those of a jazz composer. Relating back to the idea of dreaming being a sort of therapeutic aid for the brain, this album was conceived out of Eno's desire to reduce stress in an airport. I argue, through this study of ambient music and through my composition, that the therapeutic aid and service that music gives cannot be taken for granted.

The first section of my analysis focuses on how Eno highlights timbre and tone within his compositions and how it is a commonly overlooked musical element. I then analyse the structure and design of the album in its entirety, as Eno intended for it to be played, start to

\footnotetext{
${ }^{22}$ Mark Eyles and Roger Eglin, “Ambient Games, Revealing a Route to a World Where Work Is Play?,” ed. Kok Wai Wong, International Journal of Computer Games Technology 2008 (2008): 176056, https://doi.org/10.1155/2008/176056.

${ }^{23}$ Eno, "Music for Airports; Liner Notes."
} 
finish. I adopt these techniques within my own compositions, which I discuss and analyse in Chapter 4.

\section{Emphasis on Timbre - The Beauty of Sound}

One element that is often overlooked in analyses of jazz and other musical genres is timbre. My personal experience of jazz education (performance and composition) involved little to no emphasis on the importance of timbre. While I have been told on several occasions that 'sound is the first thing people hear', which suggests the importance of a musician developing a personal timbre on an instrument, this concept of 'having a good sound' is also subjective and defines a narrow timbre range for each instrument. This shows that timbral variety is not a principal focus, whereas harmonic, rhythmic, and melodic variety and complexity typically receive much time and attention in musical training.

In his introduction to the collection The Relentless Pursuit of Tone: Timbre in Popular Music, ${ }^{24}$ Robert Fink discusses that experts have not developed sufficient analytical tools to talk about timbre, in contrast with the many tools available to interpret and analyse harmony and melody. This has much to do with the existence of notation systems, and a focus on composition over performance, especially in European classical music.

Compared to all other musical elements, timbre ends up almost becoming an afterthought. ${ }^{25}$ Timbre refers to the colour of the sound itself, and, in its most straightforward form, differentiates instruments from one another. ${ }^{26}$ Like dynamics or melody, for example, timbre is a vehicle for musical expression wherein performers' 'sound' acts a part in expressing what they play, and can be altered to fit a certain mood.27

\footnotetext{
${ }^{24}$ Robert Fink, Melinda Latour, and Zachary Wallmark, eds., The Relentless Pursuit of Tone: Timbre in Popular Music (New York: Oxford University Press, 2018).

${ }^{25}$ Caroline Traube, "Instrumental and Vocal Timbre Perception" (Quebec, Canada: Musicology Department University of Graz), accessed September 3, 2020,

https://www.researchgate.net/profile/Caroline_Traube/publication/267939679_Instrumental_and_vocal_timbre_percept ion_Vocal_timbre_perception/links/56854c8908aele63f1f3308e/Instrumental-and-vocal-timbre-perception-Vocaltimbre-perception.pdf.

${ }^{26}$ Tamm, Brian Eno - His Music and the Vertical Color of Sound.

${ }^{27}$ Pamela J. Goad and Douglas H. Keefe, "Timbre Discrimination of Musical Instruments in a Concert Hall," Music Perception 10, no. 1 (1992): 43.
} 
The 'sonic envelope' of a note is a significant element in the discussion of timbre. This describes how the sound changes over a period of time. Put simply, an envelope breaks a note into four sections: attack, decay, sustain and release (ADSR). ${ }^{28}$ These four defining aspects are easy to distinguish when looking at the waveform of a note, a visual representation of how the note sounds. In electronic music, where Brian Eno thrives, digital alteration of the envelope and other elements of notes/sound waves by a composer is typical. It is a purposeful aesthetic choice by the composer to take away acoustic or live sonic elements of a sound and create something more digital, perhaps sounding robotic. This can either be achieved through a synthesiser, creating a timbre from scratch, or like Eno has done in this case, recording an acoustic instrument and then using digital alteration to create a desired effect. When working electronically, the composer has control over how a note sounds, with the help of digital alteration. In jazz, where there is more freedom for a performer's own interpretation and expression, the composer has far less control over timbre. Jazz composers engage with timbre to varying degrees. For example, Maria Schneider, as I explore in the next chapter, pays particular attention to combinations of specific timbres in her compositions, and perhaps exercises greater control over timbre than other jazz composers. Eno's compositional process involves him setting up machines to play automated continuous loops with notes he has sonically designed. These notes are played by synthesisers, piano, vibraphone and the human voice, which he has recorded and then digitally altered, spliced up, and rearranged. Here I explore how Eno modifies individual timbres and the effect these timbres have on the broader composition.

Within the idiom of ambient music, including on the album Music for Airports, timbre is emphasised through the use of sustained notes and chords, and through the relentless repetition of a small collection of pitches. The composer does not want the music to interfere too much, or at all, with the listener's thoughts and environment. Rather, the music is meant to complement and add ambiance to the overall aural experience. When the pace of a track is slow-moving with many sustained pitches, it requires less conscious attention from the listener, and therefore blends more easily into an atmosphere. The variety of timbres of Ambient 1: Music for Airports could be described as angelic and joyous in some cases, but as deep, dark and ominous in others. Eno combines acoustic

\footnotetext{
${ }^{28}$ Karl Kristoffer, "Timbre Models of Musical Sounds Kristoffer Jensen Datalogisk Institut, Københavns Universitet Department of Computer Science , University of Copenhagen” (University of Copenhagen, 1999).
} 
and electronic sounds to create a soundscape that evokes sensations of humanness amongst electronic, more robotic musical material.

The graphic score of Ambient 1: Music for Airports by Eno (Fig. 2.1) shows his personal visual representation of the timbres, shapes and forms of each of the album's four tracks. Having a visual representation is helpful for reinforcing my ideas throughout the analysis, as it provides, for example, a visual depiction of the relentless repetition of motifs, timbres, and textures. The graphic score is also helpful in understanding the 'loops' Eno uses to structure his compositions. The loops are clearly seen as consistently spaced symbols on each horizontal 'line' of each track.
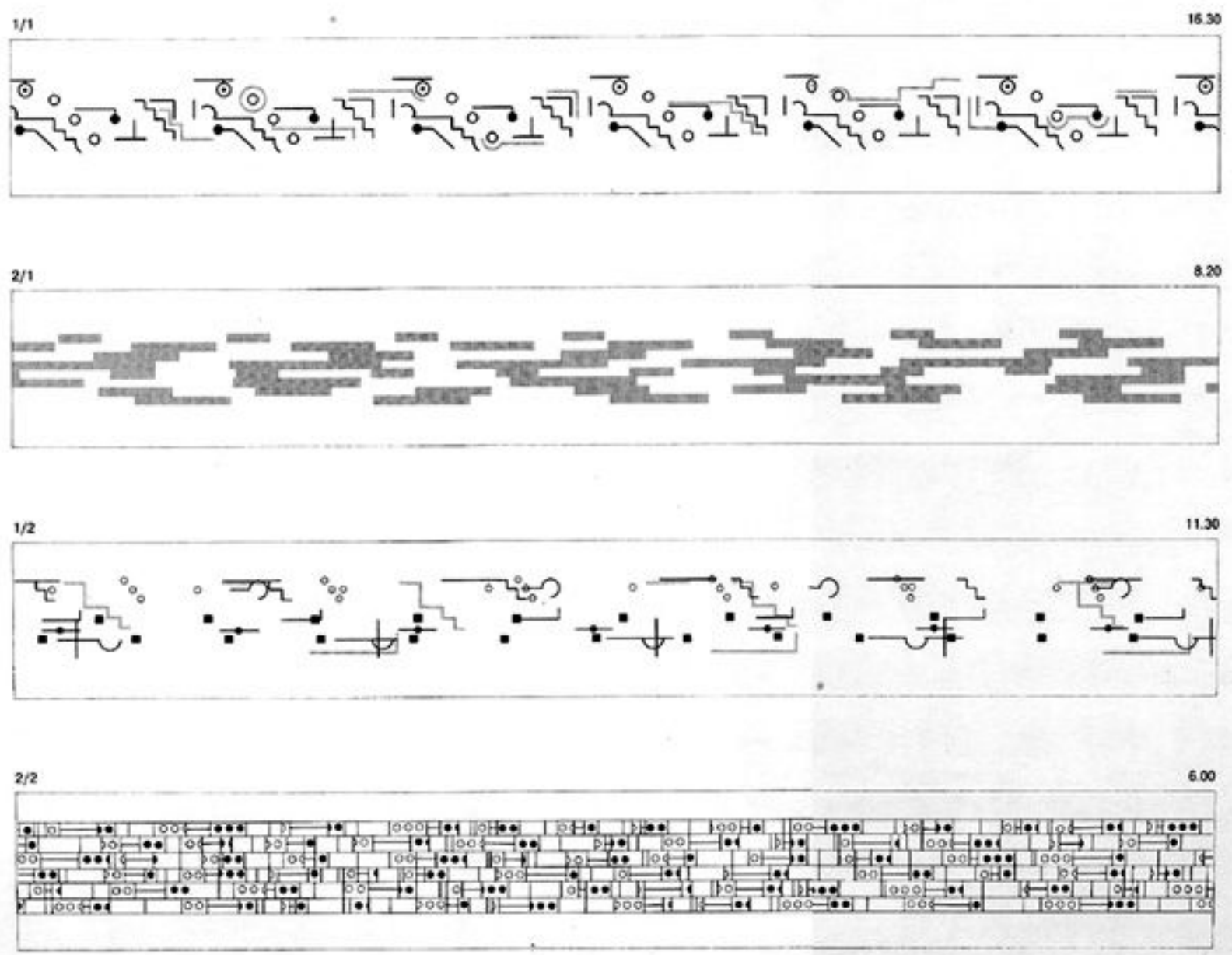

Figure 2.1 Graphic Score of Ambient 1: Music for Airports by Brian Eno ${ }^{29}$

\footnotetext{
${ }^{29}$ Jimmy Stamp, "5 1/2 Examples of Experimental Music Notation,” smithsonianmag.com, accessed February 25, 2020, https://www.smithsonianmag.com/arts-culture/5-12-examples-of-experimental-music-notation-92223646/.
} 


\section{Timbre in Ambient 1: Music for Airports}

The first track, "1/1," starts out with piano and vibraphone playing a combination of either single pitches or two- or three-pitch chords. Melodies are sometimes played in heterophony, where the same melody is being played by two instruments at generally, but not exactly, the same time. This relationship between melodies creates a sense of tranquillity and calmness; both instruments seem to be intentionally carefree, each one only loosely connected to the other. Both instruments evenly utilise their wide pitch range. Because the piano and vibraphone are percussive instruments, the sustain and decay time depends on the weight of the attack of a given note. The envelope of these notes and chords on "1/1" have a sharp attack, and a long, slow decay, due to the sustain pedals of the piano and vibraphone being held down to let the notes 'ring out'. This is unlike for wind or bowed instruments, where musicians can purposefully control the sound of the sustain and decay of any given note. The sustain and decay on the piano and vibraphone are slow enough and long enough for chords to be created and continuously overlap with one another. The resulting harmony, then, is like a stream made up of many pitches that sound both individually and simultaneously in various combinations, like a sonic realisation of raindrops falling into a stream of water. The piano and vibraphone form the backbone of the composition, following a steady pulse for the most part, whilst chords from synthesisers grow in and out of the melodies, contributing to the overall dynamic shape of the piece as the intensity level of the backbone stays the same. The sporadic chords from the synthesisers are much brighter, shinier and brassier than the softer, fluffier timbre of the vibraphone, and the familiar, docile, acoustic sound of the piano. These synthesiser chords become much more consistent, introducing new harmonies throughout the second half of the track. The only other sound heard in this piece is a subtle, breathy tone which quietly appears briefly in between notes struck by the piano and vibraphone, adding a faint layer to the soundscape. All timbres in "1/1" have varying intensities of vibrato. Vibrato is a prominent feature of sustained or decaying pitches in "1/1", which is arguably a feature of timbre according to Bregman. ${ }^{30}$

\footnotetext{
${ }^{30}$ Albert. S Bregman, Auditory Scene Analysis: The Perceptual Organization of Sound (Cambridge, Massachusetts: MIT Press, 1990).
} 
Track two, "2/1," consists exclusively of electronically modified human voice. Seven single sustained pitches sung by what sounds like a woman are layered on top of one another to produce harmonies and rhythmic movement. The voice has been electronically modified to be given a sparkling, angelic tone. There is a glassy quality to the sound, making it seem almost transparent. Introducing this human-made timbre after the first track is refreshing, especially considering that this second track consists of one timbre, with moments of silence scattered throughout. Since the music has more space in the form of long drones and silences, external sound can blend with the track more easily, and the track, as ambient music, can blend into any given atmosphere. Especially because this album concept was conceived in an airport, it was intended for there to be enough space to not impose on flight announcements or other messages over the airport speakers. ${ }^{31}$ Eno's choice of the electronically modified human voice also lends itself to blending in with real life human interaction and speaking in the airport itself, or in other settings where humans are speaking and interacting.

The third track "1/2" also includes piano, but with a slightly liver, plainer acoustic timbre than the piano in the first track. The only other timbre included in this track is the same electronically modified voices from the previous track, "2/1". The fact that Eno utilises more than one piano timbre in the same album speaks to his fascination with the instrument. In 1985 Eno was quoted in an interview calling piano one of his two favourite instruments, along with the tambura. ${ }^{32}$ Piano is often used in ambient and background music, as it is such a familiar and versatile instrument which is heard in many styles of music, ranging from classical to pop. In his words:

But now I think what makes a piano so interesting is that it's generating so much complex information. Like... if you play an $\mathrm{A}$ at $44 \mathrm{OHz}$, it generates harmonics at $880 \mathrm{~Hz}, 1320 \mathrm{~Hz}, 1660 \mathrm{~Hz}, 2220 \mathrm{~Hz}$... and so on. These harmonics are very important to your appreciation of the feeling of a piano note. If you subtract these and just leave the fundamental, it won't sound like a piano...The nature of acoustic instrumentsnot synthesisers, I might add-is that they have these complex harmonics. Not only

\footnotetext{
${ }^{31}$ Nathanidiothend, "Brian Eno Interview."

${ }^{32}$ Phil South, "The Sound of Silence: A Thursday Afternoon with Brian Eno," More Dark Than Shark, 1985, https://www.moredarkthanshark.org/eno_int_eamm-dec85.html (accessed 8 May 2019).
} 
do these notes generate their whole harmonic series, but the harmonics interact. You get both the product and the difference of them. ${ }^{33}$

In addition to the piano playing single line melodies and chords, Eno incorporates the same choral effect of the looped voice from the previous track. With exactly the same timbre as in the previous track, the voices flow in and out of interacting with the piano. The effect of having the same timbre in the form of the electronically modified voice over two tracks gives a strong sense of continuity and familiarity, whilst the incorporation of the new piano timbre establishes a subtle development in the album.

The final track "2/2" consists of one instrument, a synthesiser triggering a particular sound. The timbre of this sound is clean and bright, with the attack of each note seeming fuzzy, then leading into almost a clean, rounded sine wave. All notes have a prominent wavering vibrato. Each pitch lasts no longer than seven seconds, which means that as pitches roll over one another, there is a layering effect, and because everything is the same timbre it all blends into one big soundscape. The pace of the pitches rolling over one another feels a lot faster than that of the same effect in previous tracks on the album. To compare this to my earlier metaphor of rain dropping into a stream, there is simply more rain falling in this track.

Timbre plays a significant role in the ability for ambient music to serve as accompaniment. Firstly, timbres must be soothing and round with the right combination of frequencies for them to be comfortably heard at a low volume, as ambient music is intended to be played. Secondly, timbres must be reasonably familiar to the listener. The human voice is obviously familiar, and the piano is a common instrument across many styles. The vibraphone is distinguishable as being within the same family as the piano, or keyboard, which helps with its familiarity.

Through analysing the timbres on this album, I have developed a strong appreciation for meticulous attention to detail when it comes to composing music with a palette of sounds. Within a jazz orchestra there are many timbres available to me as a composer, and many potential combinations of sounds. Applying a stronger awareness of timbre to jazz 
orchestra has resulted in a multitude of new sounds, and has forced me to take a different approach to other compositional elements such as rhythm, melody, pace, and harmony.

\section{Textural Devices}

Much like the consistency of timbre, dynamics and pace, the consistency of texture is an important element of ambient music. Here I look into how various timbres are situated as background or foreground of the soundscape of the album's tracks, and how the addition and subtraction of timbres creates differences in texture.

\section{Background vs. Foreground}

A focus of Eno's compositional technique in Music for Airports is blurring the lines between background and foreground. Eno utilises similar timbres that blend well with one another, and uses a similar dynamic level for each of the various elements of a given track throughout. The ear is drawn to melody in most forms of popular music, jazz, and European classical music, as melody is usually the 'voice' of a composition which is often viewed as 'telling the story'. In Music for Airports, by contrast, the piano, vibraphone and synthesiser play melodies in "1/1", "1/2" and "2/2", but because they are interacting either with silence or closely related timbres, there is not a strong sense of hierarchy. They are too rarely at the foreground to be considered 'lead voices'. Because of this destabilised hierarchy between foreground and background, any sense of linear narrative takes a back seat, since there is no voice to 'tell a story', so to speak. Looking to other genres for comparison in texture, popular music and European classical music typically features a hierarchy of voices, where the melody is most prominent. It seems as though Eno is challenging traditional European constructs of music having a linear narrative. In a 2017 interview with Pitchfork Eno states his ambient music is based on 'abstract expressionism', which was a modernist movement in visual art in the 1940s. The abstract approach challenged earlier conventions in visual art of narrative or linearity. ${ }^{34}$ Eno also describes his ambient music a field which can be sonically wandered over, 'deliberately devoid of personalities...So there's not somebody in that field leading you around; you find your own

\footnotetext{
${ }^{34}$ Vytautas Kavolis, “Abstract Expressionism and Puritanism,” The Journal of Aesthetics and Art Criticism 21, no. 3 (1963): 315-19, https://doi.org/10.2307/427440.
} 
way.' ${ }^{\prime} 5$ Of course this is the whole intention of ambient music, to accompany and leave enough room for other sounds.

The concept of minimising focus on melody in ambient music presents a challenge to me as a composer as I have weighed out whether and how to include improvised solos in my own compositions. Ambient music is intentionally devoid of featured soloists, and any sense of narrative. ${ }^{36} \mathrm{Jazz}$, by contrast, typically features improvisation, including Maria Schneider's music which usually has a clear story and theme. ${ }^{37}$ This is one of the many differences between Eno and Schneider's music, which I have had to navigate in my own compositions that seek to blend the two approaches. In the liner notes to Eno's famous ambient album Ambient 4: On Land, ${ }^{38}$ he describes how he made an effort to blur the lines between a listener's sense of foreground and background. He also addresses the idea that once a figure is painted inside a landscape, the painting then becomes about the figure instead of the landscape. A soloist can be compared to being a figure in such a painting. To me, jazz orchestra music is a painting full of figures and faces, and ambient music is a landscape painting.

In track "1/2" on two occasions, the voices disappear, leaving only the piano, the first time for about 30 seconds (01:48-02:15) and the second time for about one minute (03:5104:55). This simplification of texture through subtraction of timbre resets the listener's ear and creates a sense of calm and cadence. In " $2 / 1$ ", the same electronically modified voices are occasionally unaccompanied by piano for brief periods (6:20-6:27 is one example). This gives the listener less to listen to, and therefore more space to relax with the soothing, angelic collection of voices. This concept of taking musical aspects away from something already so musically simplistic is effective, because it gives the listener even more space to be left with their thoughts.

\footnotetext{
${ }^{35}$ Philip Sherburne, “A Conversation with Brian Eno About Ambient Music,” Pitchfork, accessed November 13, 2019, https://pitchfork.com/features/interview/10023-a-conversation-with-brian-eno-about-ambient-music/.

${ }^{36}$ Sherburne.

${ }^{37}$ Alex Stewart, Making the Scene (Contemporary New York City Big Band Jazz) (University of California Press, 2007), https://www.jstor.org/stable/10.1525/j.ctt1pnr6g.1.

${ }^{38}$ Brian Eno, “Ambient 4: On Land, Liner Notes,” accessed September 11, 2019, music.hyperreal.org/artists/brian_eno/onland-txt.html.
} 


\section{The Macro Form of Ambient 1: Music for Airports}

The form of Music for Airports is structured by a number of factors. Narrow dynamic range, silence, and drones structure the album in a way that intentionally does not produce too much conscious engagement for the listener. Eno also creates a sense of cohesion through the album by incorporating the same musical material across various tracks. The album is also shaped in a way to be reminiscent of the experience of airport travel, which in itself can be stressful, yet Eno manages to translate the experience elegantly into his album, as I explore through the analysis of drones and tuning.

Perhaps the most effective concept in the third track, "1/2", is how it takes material from the album so far and combines it to create something new, whilst maintaining a sense of familiarity and security. This puts listeners in a position where they hear the same timbral and textural material again in a different context. Perhaps Eno took inspiration from one of his famous Oblique Strategies cards, 39 'Repetition is a form of change'. Taking these two pieces and overlapping and combining the musical material creates something completely new and something greater than the sum of its parts.

In all four tracks, the dynamic range is narrow because of the nature of the structure being formed by automated loops which have all been set to a similar volume. Again, with regard to the concept of narrative, dynamics usually play an important role in shaping a 'story' in popular music and can have a huge effect on the listening experience. Eno intended for each piece to be a similar volume, individually and together as an album. This lack of 'shape' maintains a constant level of volume, which successfully blends into an environment. This album is designed to be played at a low volume, or just loud enough to add a desired level of ambiance. Because of this, it does not have extreme spikes and drops in volume, as that could attract too much attention to the music.

The lower the dynamic, the closer the sound is to reaching silence. Silence is usually more noticeable when preceded or followed by sounds with extremely high volumes. When sounds are low in volume, silence is not so significant. Eno explores the relationship between sound and silence by intentionally placing sound and silence side by side to

\footnotetext{
${ }^{39}$ Peter Eno, Brian. Schmidt, Oblique Strategies (Peter Notron, 1996).
} 
complement one another. An average of 30 seconds of silence separates the end of each track from the beginning of the next. Silence can feel uneasy to a listener who would like to be distracted, but because of the low volume and relaxing nature of the album the silence is welcomed and prepares the ear for the next piece. This may seem like a significant amount of time, but in relation to the durations of the tracks these gaps are not especially long. The first and last track contain no silence throughout, due to a constant underlying drone in each one. The middle two tracks, however, have silence scattered throughout. The first 8 seconds of the last track, "2/2", consist of only a clean, brassy drone, which then continues throughout the entirety of the track. This drone is an almost unnoticeably quiet, slightly flat $\mathrm{Bb} 2(115 \mathrm{~Hz})$ that subtly rings out underneath the rest of the track. Only when I listen to the piece with headphones can I properly hear the drone. What is the point in having something so low and quiet if it is almost inaudible in the intended listening environment (e.g. an airport)? Perhaps there is more to it than the sound itself; perhaps it is more to do with the calming vibrations of this frequency. Perhaps Eno is trying to subtly emulate the experience of the airport travel experience, the first and last tracks representing being grounded by the use of the consistent low drone acting as the ground, then the lack of grounding in the middle tracks represents being up in the air.

Eno also seems to use tuning create this same concept of emulating the airport travel experience. The first and last tracks use equal-temperament tuning, but their tonal centres are pitches whose frequencies fall in between pitches common in popular music and European classical music. For example, the tonal centre of " $2 / 2$ " is approximately $115 \mathrm{~Hz}$, which is somewhere between A and Ab. By contrast, the middle tracks " $2 / 1$ " and "1/2" are both in the key of $A b(207 \mathrm{~Hz})$, their tonal centre based on a common key in popular and classical music. Eno's approach to tuning may reflect his view of the timeline of a stressed airport traveller. The first and last tracks could represent the mental state of a traveller in the airports on either side of the flight, feeling slightly uneasy (for Eno, centred on a pitch that is outside of commonly used key centres), slightly stressed to catch a flight on time, or slightly stressed hoping that the correct luggage will appear on the carousel. The sterile white lights, security lines and crowds can make travellers feel anxious and slightly off balance. The middle tracks could represent the long flight, based on a familiar tonal centre, with voices almost sounding like they are floating through the air, just like the listener. The two middle tracks already almost blend into one because of the identical vocal timbres in both, and their tonal centres within the equal-tempered system familiar from popular music 
could reflect a traveller being more at ease when sitting on the plane, powerless to do anything but sit and relax.

Whilst some of this analysis, especially the first and last tracks, depicts the music as reflecting a stressful experience, the music remains soothing to the listener. The drones and tuning are very unnoticeable until close analytical inspection. If anything, these elements only very subconsciously represent a sense of what the airport experience really is, framed in a way to calm and ground the listener.

\section{Conclusion}

I have discovered through this analysis that although timbre is only beginning to receive attention in music research and education, its effect on a composition is paramount in informing other musical elements, such as melody, harmony, dynamics and pace. Timbre has a significant effect on the psychological response to a composition, for example the effect of using a familiar timbre such as piano, or a soothing timbre such as a fuzzy synthesiser. Timbre is key in producing a soothing, relaxing experience. Textural devices such as a destabilisation of a background/foreground hierarchy and the role a featured voice can also be effective to this end, and adding and subtracting to the textural palette can create variety for a listener in having enough space to be present with conscious thoughts, and being engaged in the music. It is at the listener's discretion as to how they wish to listen to the music. The overall shape and structure of the album is particularly effective through features such as maintaining a low dynamic throughout the album, utilising silence to incorporate breaks in the music, and structuring the album through drones and tuning to emulate airport travel. Ambient 1: Music for Airports has had a significant influence on my own compositions, in both a conceptual and practical sense. I turn now to the work of Maria Schneider, whose work also has influenced mine with regard to timbre and texture, as well as a number of other musical elements. 


\title{
Chapter 3
}

\section{Timbre, Dynamics and Harmony in "Nocturne" and "Sea of Tranquility" by Maria Schneider}

\begin{abstract}
All of a sudden I find myself daydreaming about something that happened in my life which that musical thing conjures up. It's as if it wants to come out and be manifested. I think a lot of times it's an internal healing process for me. I think that l've used music. I think a lot of people use music, and they don't realise it. I realise it afterwards. ${ }^{40}$

- Maria Schneider
\end{abstract}

\section{Introduction}

This chapter explores compositional techniques used by Maria Schneider in two of her pieces, "Nocturne" and "Sea of Tranquility", both from her album Allegresse. ${ }^{41}$ I chose these pieces because they both demonstrate how Schneider creates calmness and serenity in her music, and because they represent a more reserved, relaxed side of the repertoire for jazz orchestra. In the broader context of Allegresse, these two tracks are particularly restrained and simple in comparison to the rest of the tracks. They are both ballads, slower tempo tracks which complement the album's more hastened and energetic tracks such as "Hang Gliding" and "Allegresse". My main focus throughout this entire project is timbre, so throughout this analysis of Schneider I primarily look at ways Schneider highlights timbre as she creates a calming and relaxing listening experience. I also wanted to look specifically at Schneider's music not only because of my personal fascination with it, but because she is an active, modern-day jazz orchestra composer.

Allegresse was recorded by the Maria Schneider Orchestra, an ensemble that Schneider formed in 1992 and still leads today. The ensemble has performed all around the world and has won two Grammy awards. ${ }^{42}$ Her orchestra generally has the same instrumentation as a traditional jazz orchestra or big band from the Swing Era (1930s and 1940s), when big bands were most popular (e.g., the Count Basie Orchestra, the Glen Miller Orchestra,

\footnotetext{
40 Jennifer Kelly and Maria Schneider, "Maria Schneider," in In Her Own Words: Conversations with Composers in the United States (University of Illinois Press, 2013), 240.

${ }^{41}$ Schneider, "Allegresse."

42 "Maria Schneider Grammy Awards," Recording Academy, accessed April 13, 2020, https:/www.grammy.com/grammys/artists/maria-schneider.
} 
and the Duke Ellington Orchestra). Schneider's main influences are composers Gil Evans and Bob Brookmeyer, both of whom mentored her at various stages of her early career. ${ }^{43}$ Schneider is known for building on teachings from Evans and Brookmeyer to create new styles of writing. She composes for and conducts her orchestra, but unlike many jazz composers and big band leaders (though similar to her mentor Evans), she does not perform with the group, despite being a proficient pianist.

One of the distinguishing elements of Schneider's compositions is her approach to orchestration. Often, when I listen to Schneider's music, the ensemble reminds me of a classical chamber orchestra. Perhaps this is because she uses doubles such as oboe, flute and English horn that are common in European classical music, and rare in the jazz idiom. Schneider also has training in classical composition and has continued to receive commissions for classical ensembles. ${ }^{44}$

Both pieces are through-composed, which means Schneider completely maps out the entire composition without repeat signs even around solo sections, therefore having full control over how the music develops. Schneider acknowledges that she struggled to free herself from the 'confining expectations and conservatism of jazz musicians and teachers' 45 in terms of form, and acknowledges that her mentor Brookmeyer was a pioneer in developing freer styles of form in his compositions. Though Allegresse (2000) does not have liner notes, her first album, Evanescence (1994), includes comprehensive liner notes including comments from Brookmeyer and from Schneider herself. ${ }^{46}$ Brookmeyer comments on what he perceives as a lack of excellent big bands in New York at the time, observing that 'they have been fading since the 1970s, because no composers can also organise, conduct and perform convincingly in public'. ${ }^{47}$ He speaks highly of Schneider, and mentions that she quickly gained respect as a successful bandleader in New York and Europe.

\footnotetext{
${ }^{43}$ Maria Schneider, "Evanescence" (Enja Records, 1994) Liner notes.

${ }^{44}$ Kelly and Schneider, "Maria Schneider."

${ }^{45}$ Stewart, Making the Scene (Contemporary New York City Big Band Jazz).

${ }^{46}$ Schneider, "Evanescence" Liner notes.

${ }^{47}$ Kelly and Schneider, "Maria Schneider."
} 
Using the published scores for "Nocturne"48 and "Sea of Tranquility"49, I first examine how Schneider uses instrumentation techniques such as doubling and mutes to expand the amount of available timbres in a jazz orchestra. I then look at how Schneider uses the drum kit more as a timbral additive than as a rhythmic time- or groove-keeper, and I discuss the role timbre plays in the solo sections of each piece. Next, I explore Schneider's subtle use of dynamics, and how she explores the quieter dynamic of a jazz orchestra. In the final section of the chapter I look at some of Schneider's approaches to harmony, such as the use of pedals, suspended chords, dissonance, and differences in harmonic rhythm to create structure and momentum.

\section{Schneider's Approach to Timbre}

So far, I have discussed the importance of timbre in ambient music and the role it plays in creating a soothing musical experience, and also the effect a compositional focus on timbre has on other musical elements. In my analysis of timbre in "Nocturne" and "Sea of Tranquility" here, I look at the use of doubling, the effect of mutes in the brass, some examples of different timbre combinations, the relationship between timbre and a soloist, and the use of drums as a timbral additive. Whilst changes in dynamics still exist, Schneider mostly varies the overall intensity of the piece by varying orchestral weight, in terms of thickening or thinning textures. In doing so she keeps the listener engaged by utilising the plethora of instrument combinations and sounds available in a big band. She heavily uses doubles, (e.g., saxophone players on other woodwinds, trumpet players on flugelhorns) to draw on an even wider range of timbres. Her emphasis on timbre is similar to Eno's approach, but she uses live acoustic instruments instead of electronic synthesised sounds. I am inspired not only by Schneider's timbre combinations, but also by how she continuously develops contrasting sounds with a careful, thoughtful, and restrained approach.

Schneider explores timbre combinations to a higher extent than is common in big bands I hear in my own community, but she is not the only jazz composer to explore timbre in this way. For example, the works of two of her most direct mentors, Bob Brookmeyer and Gil

\footnotetext{
${ }^{48}$ Maria Schneider, "Nocturne" (MSF Music, 1999).

${ }^{49}$ Maria Schneider, "Sea of Tranquility" (MSF Music, 1999).
} 
Evans, demonstrate similar approaches to timbre, including extensive use of woodwind doubles and of a variety of mutes in the brass sections.

\section{Timbral Palette}

Traditionally, reflecting earlier jazz orchestra styles, the recipe for jazz orchestra arrangement largely involves section playing. This means an arrangement is typically broken up into four sections: rhythm section, saxophone section, trombone section, and trumpet section. Trumpet 1, Trombone 1, and Alto 1 are often the main 'voices', tying the three wind sections together, with rhythm section as accompaniment. The rest of the trumpet, trombone and saxophone players play harmonised lines underneath their respective section leaders. In contrast to this style of jazz arrangement, Schneider's composition largely destabilise the section-based hierarchy of instruments in melody and harmony. In terms of timbre, the difference between having instruments stay with their sections and the more destabilised structure is considerable, as, in Schneider's approach, all instruments frequently intertwine across sections to create a much larger range of timbre combinations and continuous changing of shades and colour. As well as this destabilisation of section playing, Schneider utilises doubling to further broaden the timbral possibilities.

Doubling is a technique used throughout the history of jazz orchestra composition. Big band composers typically expand on the timbres of the five saxophones, four trombones and four or five trumpets typical of a big band by including sections in their compositions where individual players play additional instruments, or 'doubles'. Schneider composes using doubles throughout both "Nocturne" and "Sea of Tranquility", and this has a strong effect on the music, since, especially to the untrained ear, timbral variety is very noticeable. Doubling broadens the timbral palette of the jazz orchestra, because the number of instruments in that palette is greater than it would be otherwise. In "Nocturne" twelve different instruments are played: alto flute, C flute, oboe, English horn, tenor saxophone, soprano saxophone, bass clarinet, clarinet, trumpet, flugelhorn, piccolo trumpet and trombone. The four trombone parts do not contain any doubles. Each of the four trumpets changes to flugelhorn at some point in the composition, with Trumpet 1 and 2 also both switching to piccolo trumpet for only four bars. Tenor 1 never changes to another instrument. Throughout what would normally be the Alto 1 part, alto flute and $C$ 
flute are both played, while the alto saxophone is not played. In what would normally be Alto 2, oboe and English horn are both played, while, again, the alto saxophone is not played at all. Baritone saxophone is also replaced completely with bass clarinet, which also changes to clarinet, and Tenor 2 changes to soprano saxophone for part of the piece. In "Sea of Tranquility", out of the fourteen woodwind or brass players, only one changes instrument, which happens when the Tenor Saxophone 1 part switches to flute in bar 81. Also, the musicians who would typically play alto saxophone are instructed to play flute (for Alto Saxophone 1) and clarinet (for Alto Saxophone 2) throughout the whole piece. Excluding the timbre of the alto saxophone distances both pieces from the typical sound of a traditional jazz orchestra.

Schneider also creates many possible timbre combinations by adding mutes in many of the brass parts, which result in possibilities for softer dynamics and for further timbral variety. Throughout both pieces, Schneider utilises bucket, cup and harmon mutes in both the trumpets and the trombones. Cup mutes produce a softer muffled sound, harmon mutes produce a more metallic and buzzed sound, and bucket mutes produce a smoother, deadened sound. Within a brass section in a jazz orchestra, mutes can help blend the section sound because they are not only lowering the volume, but also creating a more consistent sound across individual players by only allowing certain frequencies of a player's full sound to be heard. This also helps blend the section into the rest of the ensemble. Schneider does not always have every member in the section playing the same mute, whereas, if adhering to the traditional style of jazz orchestra arranging where each section functions as an individual unit and therefore has a consistent timbre, all members of a brass section would typically use the same mute at the same time. Schneider also at times directs players to play 'in stand,' which provides a more muffled timbre and dynamic, a similar but less drastic effect than using a bucket mute. Schneider is meticulous with her instructions for using mutes. For example, in "Nocturne", Trumpet 2 is instructed at bar 45 to use a cup mute for only three bars, then switches to play flugelhorn from bar 53 to 69 , and later to use a harmon mute from bar 73 to 76 , and then to play piccolo trumpet from bar 79 to 83, before switching back to trumpet with cup mute from bar 85 to the end (bar 101).

The beginning of "Nocturne" provides a clear example of how Schneider emphasises a broad timbral palette. The piece begins with 19 bars of muted, soft trumpets and 
trombones in bucket and cup mutes, with emphasis being put on the woodwind instruments such as oboe and alto flute which are not often heard in a jazz orchestra. Bars 1-4 consist of the breathy timbre of the alto flute, the solid, sharp sound of the oboe, the bass clarinet played in its upper-middle register, Trumpet 1 in a bucket mute, three trumpets in cup mutes, fourth trombone in a bucket mute, guitar, piano and double bass. Alto flute, oboe and Trumpet 1 (with a bucket mute) play the lead melody in unison during bb. 1-4, after which the oboe plays the melody through bar 19. All instruments have a piano dynamic instruction which also produces a softer timbre. During bb. 1-8, the double bass plucks in its high register, creating a sense of uneasiness, since the bass is more commonly played in the lower register, which has a more rounded, dark, richer timbre. A sustained pitch in the low bass register provides a feeling of stability and cadential reinforcement. The piano plays chords to accompany the woodwinds and brass from bar 1 to 18 , after which it is featured as a melody instrument and soloist for the rest of the piece. In order for the piano to be clearly audible, all brass and woodwinds play at a quiet volume throughout the majority of the piece (where the piano is being featured as the soloist), which can create a softer, fluffy, breathy timbre. These combinations of timbres are relaxing because they are not abrasive, due to the woodwinds providing a gentler, smoother timbre compared to what would usually be the brassier, sharper timbre of the saxophone, and the mutes contributing a much softer, more mellow timbre compared to the typically cutting, vibrant sound of the unmuted trumpet. The instrumentation creates a softer overall timbre, like a soft blanket which is comfortable and soothing.

\section{Timbre and Soloists}

Timbre also plays a role in how a solo instrument impacts compositional strategies. Soloists play an important role in being a 'voice' of a composition, with the timbre of the soloist being featured. As is typical for ballads in jazz orchestra composition, "Nocturne" features a single soloist, the piano. "Sea of Tranquility" primarily features a baritone saxophone soloist, but also includes a secondary flugelhorn solo. Schneider weaves the soloists into each through-composed piece, and says she uses the soloist to help the arrival of a contrasting section in the piece feel inevitable. ${ }^{50}$ This is like Schneider using the

\footnotetext{
${ }^{50}$ Alex Stewart, "Contemporary New York City Big Bands: Composition, Arranging and Individuality in Orchestral Jazz," Ethnomusicology 48, no. 2 (2004): 178.
} 
soloist as a voice in the narrative of the piece, to help transition one part of the story to the next.

I discussed the familiarity of the timbre of piano in the previous chapter, and it is also relevant here. This familiarity has a huge impact on the listener's experience of "Nocturne", compared to if the soloist was, say, baritone saxophone, which is a less commonly heard solo instrument. Schneider uses various techniques throughout "Nocturne" to highlight the timbre of the piano. Sometimes she composes melodies in the piano part in unison with another instrument, such as from bb. 85-92, where the piano is playing a melody in unison with the flute, clarinet and Trumpet 1 with cup mute. The absence of drums helps in lowering the overall volume of the piece to feature the acoustic volume limitations of the piano. When the piano is first introduced as being the featured soloist at bar 19, the orchestration thins out to include only bass clarinet, Trumpet 1 and Trombone 3 for one bar, who are joined by alto flute and oboe in playing a melodic answer to the piano (see Fig. 3.1). This change in orchestral weight provides more space for the timbre of the piano to be featured. 

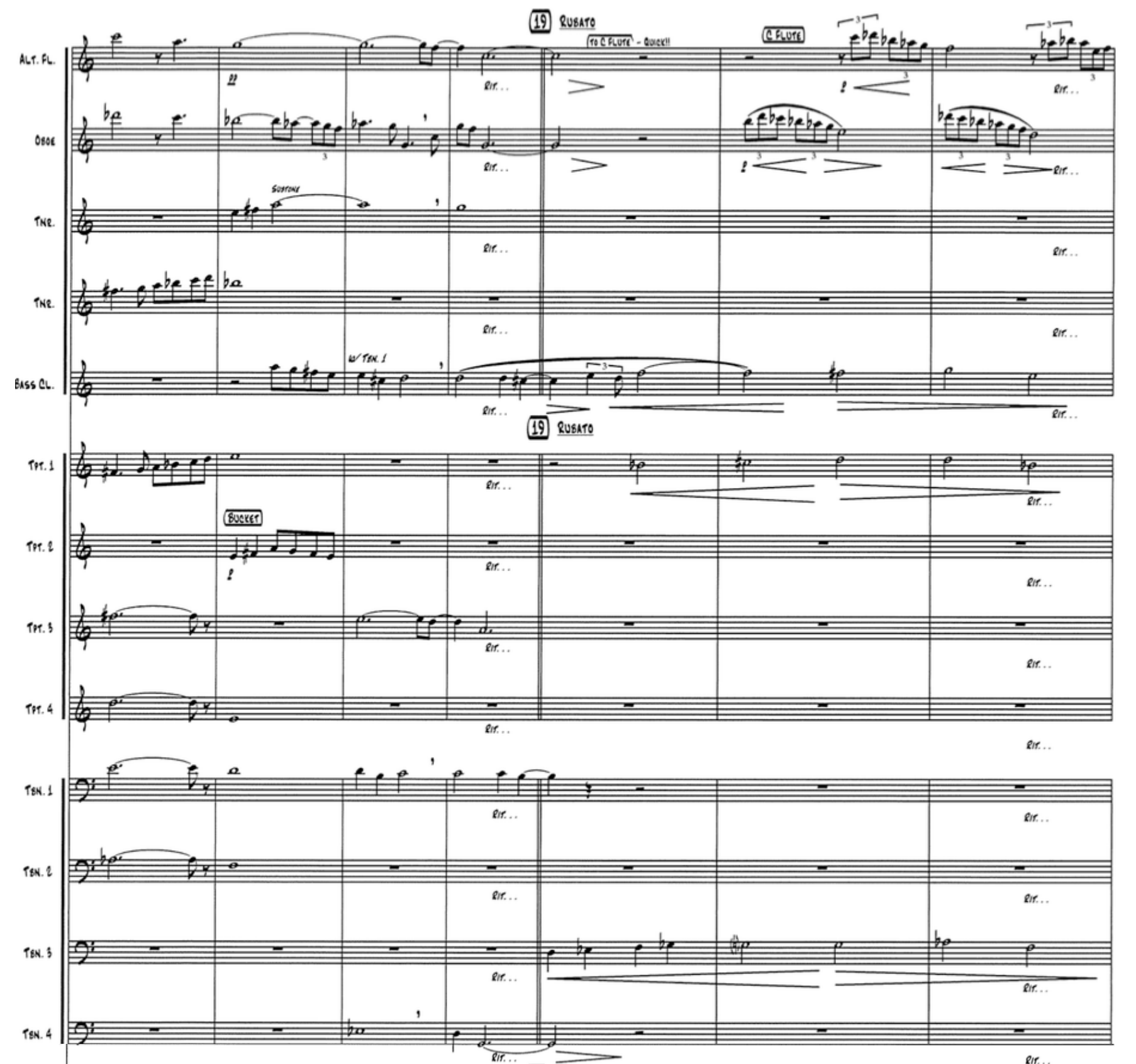

(19. Ruskro

are.

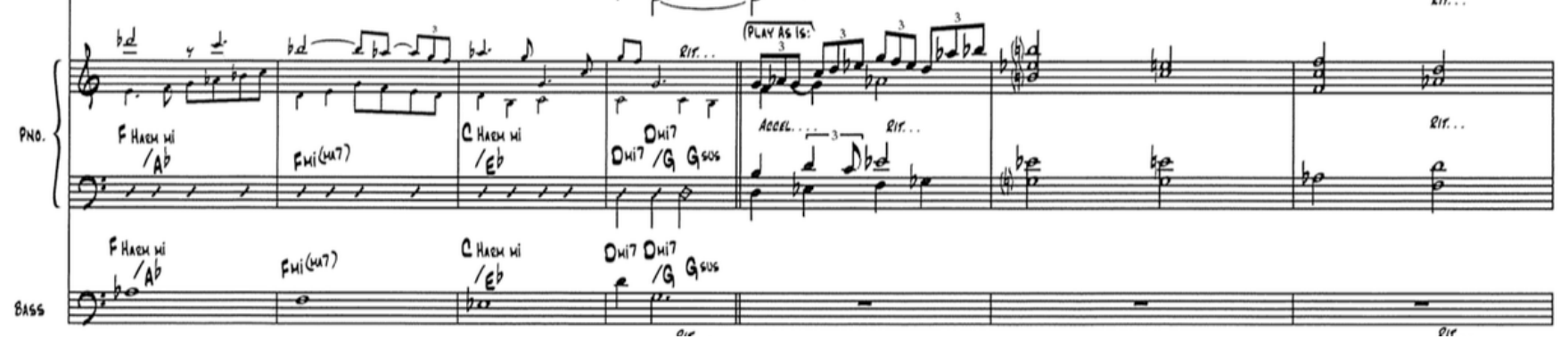

Figure 3.1 Thinning of orchestra weight at bar 19 of "Nocturne"51

${ }^{51}$ Schneider, Nocturne. 
In "Sea of Tranquility" the main soloist is baritone saxophone, complemented by a secondary soloist on flugelhorn. The baritone saxophone has a history of being featured with a big band, though it is a less common solo instrument than tenor or alto saxophones. Well known examples of baritone saxophone solos include Ronnie Cuber on "Moanin"' by Charles Mingus (1993), ${ }^{52}$ and Harry Carney on "Sophisticated Lady" by Duke Ellington (1932). ${ }^{53}$ "Sea of Tranquility" begins with a pickup by the baritone saxophone, which leads into bar one when the piano and guitar begin accompanying the saxophone. The pickup in the baritone saxophone is a concert Ab, which ascends to a concert Eb (both in the higher octave of the saxophone) held for almost two bars at a tempo of 55bpm. Usually, in big band orchestration, it is commonplace for the baritone saxophone to play lines in unison with the upright bass and/or bass trombone, covering the lower frequencies of the big band. The baritone saxophone in "Sea of Tranquility" contributes a less frequently featured timbre to the ear of a listener familiar with big band music. The timbre of the baritone saxophone in the high register is grittier than that of the alto or tenor, and, in this example, sounds rich with an almost human voice quality. Long notes in the upper register of the baritone saxophone comprise much of the melody, providing a dustier, rougher timbre through an instrument which typically sounds fuller and rounder in its middle and lower registers. The simple melody of long, sustained notes in the baritone saxophone at the beginning of the piece is effective because the simple, slower rhythmic activity allows more room to start the improvised solo, where the rhythmic activity eventually develops to become faster and more complex. This simplicity, along with the slightly unstable timbre in the higher register of the saxophone, creates a sense of unpredictability.

The flugelhorn enters at 02:27, playing a written melody as the baritone saxophone begins improvising around this new second voice. Having a second soloist join the main voice helps develop and build the piece in a subtle way. Two voices or soloists mimic the idea of two people in conversation, which can be engaging to a listener. The timbre of the flugelhorn is mellow and velvety, contrasting the dusty and almost abrasive sound of the baritone saxophone. This pairing of instruments or sounds is uncommon but not without precedent in popular big band jazz. (For example, in Thad Jones' "Three and One" [1966], ${ }^{54}$ the two instruments, along with the double bass, play the melody together.)

\footnotetext{
52 Charles Mingus, “Nostalgia in Times Square” (Dreyfus Jazz, 1993).

${ }^{53}$ Duke Ellington, "Soul Call" (Verve Records, 1967).

54 Thad Jones and Mel Lewis, "Presenting Thad Jones/Mel Lewis and the Jazz Orchestra" (Solid State Records, 1966).
} 
The baritone saxophone is featured much more heavily than the flugelhorn in this piece. The flugelhorn disappears after its solo, then reappears at 06:50 playing the initial baritone saxophone melody from the beginning of the piece. When the baritone reappears with the melody, the trumpets (with bucket mutes) create soft, muted chords in the background, which creates a timbre reminiscent of, and similar to, the sound of the flugelhorn. This happens at bar 81 , where the three trumpets play in harmony in bucket mutes, bringing their timbre closer to the softer sound of a flugelhorn. This ties the secondary soloist (the flugelhorn) and the rest of the piece together. The baritone gently improvises from bar 89 until the end of the piece.

Timbre combinations are almost always constantly changing throughout the backgrounds behind soloists in "Nocturne" (and in "Sea of Tranquility"). A prominent technique used by Schneider to achieve this is by passing around small melodic phrases from instrument to instrument. Because of this movement and the melodies in the backgrounds of soloists, Schneider weakens the hierarchy of the soloist being in the foreground, and the 'backgrounds' being in the background. Usually in jazz orchestra compositions, a melody is played slightly louder and more prominently than the backgrounds, therefore making its timbre dominant to the other accompanying instruments. The cumulative timbre of a group of instruments playing together is defined strongly by the melodic timbre at the forefront of the sound. Examples of how Schneider weakens the foregrounding of the soloist through this passing around of a melody behind the piano solo is seen four times between bb. 4148 of "Nocturne" (Fig. 3.2). Prominent melodies are passed around through the English horn, flugelhorn, trumpet with cup mute, and Tenor 1 . These melodies are also indicated in the piano part (Fig. 3.3). This is so the soloist can play improvised melodies around these written melodies, tying the soloist and the backgrounds together. 


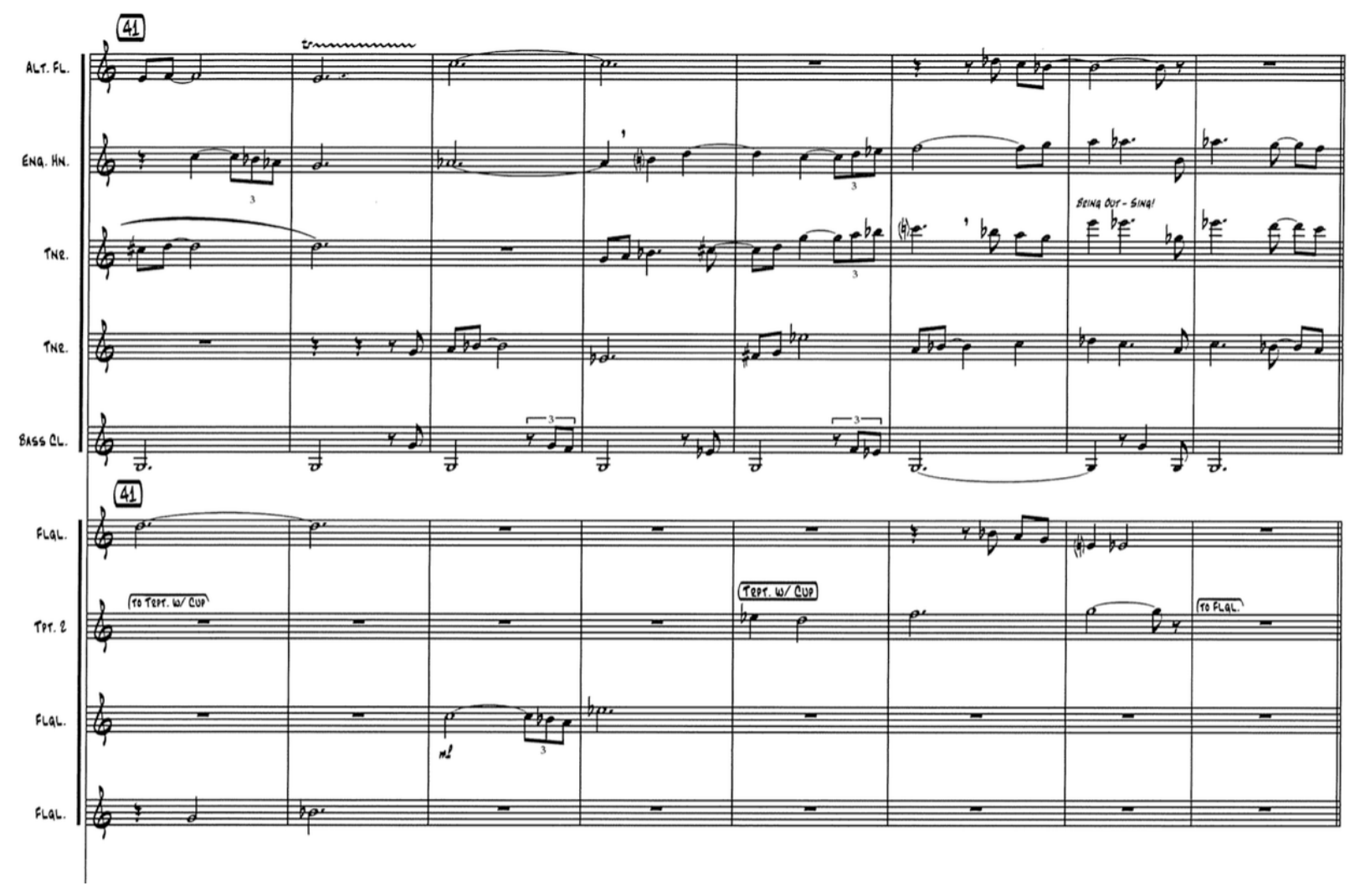

Figure 3.2 Backgrounds behind piano solo in "Nocturne", bars $41-48^{55}$

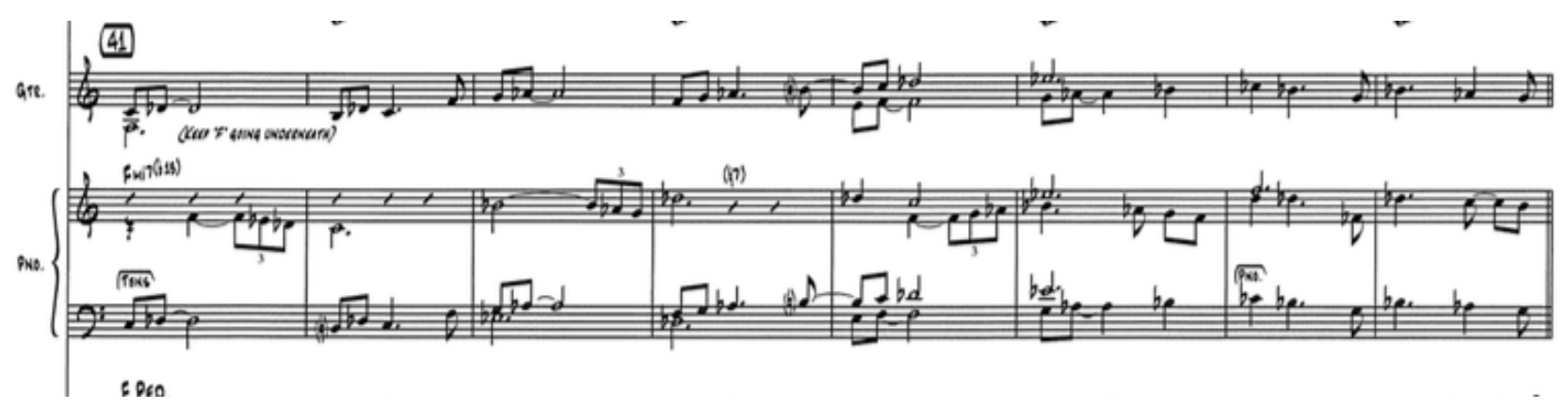

Figure 3.3 Melodies from backgrounds written into the soloist's (piano) at Bar 41 of "Nocturne" 56

This variation of timbre changes the overall timbral quality of the band every two bars, and it is significant whilst a solo is happening because it pulls some attention away from the soloist and draws the attention to the backgrounds. The attention put into timbral variation of the solo backgrounds helps to destabilise a hierarchy of a foreground versus a

\footnotetext{
${ }^{55}$ Schneider, Nocturne.

${ }^{56}$ Schneider.
} 
background, which gives the music a closer relationship to the same concept from Eno's Music for Airports.

\section{Timbre and the Drum Set}

"Nocturne" is completely free of drums and "Sea of Tranquility" only uses drums as a timbral additive-mostly just colours on the cymbals - from the midpoint of the piece to the end. Schneider shows restraint in her compositions through the absence of drums. This absence of drum kit helps the pieces stay at a low intensity of volume for longer, and draws more of the listeners' attention to timbre, melody and harmony rather than to rhythmic elements and pulse. The pulse is not outlined clearly in any of the parts so the individual rhythms, which also lack a strong sense of pulse, are left with a sense that they are floating in the air. In "Nocturne", because of the complete absence of drums, the upright bass appears to take on the role of the main 'time-keeper', primarily outlining quarter notes and half notes from bb. 1-8, while other instruments often play with a freer and more expressive feel. In "Sea of Tranquility", the drum set adds timbral effects only, contributing to subtle builds and drops in intensity.

As "Sea of Tranquility" and "Nocturne" are both ballads, they are slow in tempo and they feature a soloist who can lead the rubato tempo, stretching and pulling the pulse as the ensemble follows. Strongly tied to vocalists, ballads are known for being a strong vehicle for expression and emotion in jazz orchestra music. Usually the drummer uses brushes or sometimes mallets, instead of sticks, to be able to play at a much softer volume. Utilising sticks, brushes and mallets brings timbral exploration and difference to the drum part. If a jazz orchestra is playing above a mezzo forte, sticks will probably be required for the drummer to be heard properly. Brushes and mallets are used for softer dynamics, and respectively bring different timbres out of the drum kit. Brushes, when played on the snare drum, emulate the sound of rain, and a diffused, metallic timbre on the cymbals. Mallets offer a muffled, round timbre. As Schneider demonstrates in "Sea of Tranquility", the drum kit can be used for much more than providing a strong sense of rhythm and pulse. Throughout "Sea of Tranquility", Schneider instructs the drummer to use sticks, mallets and brushes at various times, with the overall effect from the drums and cymbals featuring timbral augmentation more heavily than maintaining a pulse. 
The first instruction for the drummer is at bar 33, where Schneider requests 'colours' to be played on the cymbals with mallets. The drummer switches to sticks at bar 45 , until bar 81 . Whilst the drummer is using the sticks, the drum part has very specific rhythms to play on the cymbals that line up with other parts. This insinuates the drummer is to play the notation precisely, though the performer may enhance what is already there with colour from the cymbals. In bar 73, the drummer plays a cymbal roll with crescendo (with sticks), joining all other parts which also have a one-bar crescendo. Sometimes in other styles of jazz arranging a bar that includes a dramatic dynamic build involves the drum kit rhythmically filling the space with a 'drum fill', but in this case a cymbal roll only adds a timbral effect to the overall sound. Bar 81 instructs brushes to be used (for the rest of the composition), with only slashes and no specific rhythms given for instruction. The significance of having sticks, mallets and brushes all within the same drum part again indicates the attention to detail Schneider puts into timbral exploration.

Schneider's approach to timbre is intricate and thorough, with instruments sometimes being doubled twice within the space of 16 bars, and mutes also being used for very specific amounts of time. Schneider also highlights the timbre of the soloist whilst also sometimes bringing attention to the backgrounds. The timbral approach Schneider takes to the drum kit results in a subtle, polite and complementary addition to the music.

\section{Dynamics and Development}

These two compositions both demonstrate how a jazz orchestra can perform effectively at a softer dynamic in a captivating manner. Schneider builds intensity through adding and taking away instruments through different timbre combinations, which has a more subtle effect than indicating dramatic rises and drops in dynamics in the score. This concept of a quiet, unobtrusive big band is the basis of what I am exploring for my compositions for this project.

Dynamically, both pieces span a reasonably narrow range, located on the quieter side. Similar to ambient music, they offer a feeling of calmness and stillness, with a softer overall volume over an extended period of time. One reason "Nocturne" maintains a low volume is that, as I mention above, the big band must play underneath the acoustic piano 
soloist. Towards the end of the piece, beginning at bar 79, a dramatic increase in volume and intensity occurs, with all instruments crescendoing over a full bar from mezzo forte, and decrescendoing slightly over the last two beats of the next bar. This higher intensity only lasts for around 20 seconds.

Schneider heavily controls subtle developments in intensity through dynamics. She takes into consideration the difference of natural loudness and power of each instrument. For example, in bar 5 of "Nocturne" the two tenor saxophones enter at pianissimo, joining the rest of the woodwinds and brass (in bucket or cup mutes), which are all at a dynamic marking of piano. This creates an extremely delicate and subtle entry, helping all of the instruments blend together since pianissimo on tenor saxophone has a similar loudness to a flute, oboe, or muted trumpet playing piano. A similar example is at bar 78 , where the woodwinds play mezzo forte and the brass play mezzo piano. This technique is seen throughout Schneider's other compositions, as she uses it to control and effectively balance her desired blend of the band.

Throughout both pieces, Schneider avoids silence. The space is always filled up with something, whether it be long sustained notes played by multiple woodwinds and brass, a texture of soloist and rhythm section, the piano playing completely solo, or one instrument sustaining a single pitch. Because of the lack of silence, the ear is given no complete break. This has a strong impact on how the listener feels when it comes to the silence at the very end of the piece, similar to the ambient works of Eno. To many listeners and musicians, the silence in between the end of a piece of music and the beginning of the applause can be the most gratifying part of the performance. By the same token, the silence which immediately precedes a piece can also seem more effective. Perhaps this is why Eno chose to have 30 seconds of silence between his pieces in Ambient 1: Music for Airports.

Dynamics play a vital role in the development of a piece of music and the level of engagement from the listener. In the case of these two compositions, the journey and narrative the dynamics bring is smooth and gradual, as opposed to jolting. For the majority of both pieces the dynamic range is narrow and on the quiet side (common in jazz ballads), but both pieces do reach a climax in intensity and dynamics (none higher than forte). This brings the listener into a slower, more relaxed narrative, gradually developing 
the dynamics in waves to reach the climax. The dynamic climax only lasts for 4 bars in "Nocturne" and 8 bars in "Sea of Tranquility", proportionally very short in the greater scheme of the composition.

To compare these two pieces of Schneider's to Eno's ambient works, Eno's music is effective at not engaging the listener partly thanks to the lack of dynamic range, compared to Schneider's pieces where the dynamic range builds in a subtle manner but still engages the listener in a sense of narrative.

\section{Harmonic Structuring}

In both compositions, Schneider uses pedals and chords with upper extensions and altered extensions extensively. Here I analyse these chords and their effects, and the way she navigates through these complicated and often unexpected chords, and then look at how she uses harmonic rhythm to create momentum.

\section{Pedals}

Pedals are used throughout both compositions. A pedal provides a similar effect as that of a drone in ambient music, though while drones in ambient music often last the entirety of a composition, pedals are commonly used as harmonic tools to build or reduce intensity. Pedals can be any pitch, but pedals on the root or the fifth of a key centre are most often seen in jazz composition. Pedals can also be played by any instrument. In "Nocturne" all pedals appear in the upright bass, which is joined by Trombone 4 and the left hand of the piano in the last 16 bars of the composition. "Nocturne" has a pedal of $F$ through the last 16 bars, while in the first eight bars, a pedal is played by upright bass on a middle $C$. The octave placement of the $\mathrm{C}$ pedal is also worth noting, as it is played an octave higher than written, locating the pedal higher than the typical range of the upright bass, which also creates a sense that a resolution is coming, when the bass returns to its typical lower register. This $\mathrm{C}$ pedal acts as the fifth of the key centre, $\mathrm{F}$ minor. This introduces the piece with ambiguity and uncertainty and creates tension before resolving to the root, $F$. 
The rhythm of the $\mathrm{C}$ pedal in the first seven bars is displaced from the start of each bar by two beats, creating a 'floating' sensation from the bass. The chords change every bar for the first eight bars, and every chord is dissonant with half step and whole step intervals. After this pedal from bb. 1-8, an Ebmaj7(\#5) in bar 9 leads to an Abmaj7(\#11) in bar 10. These altered extensions create a further sense of ambiguity about the harmonic centre, creating a sense of anticipation and perhaps even uneasiness. Schneider embraces dissonance to the point that she often includes chords which transcend functional harmony. Sometimes Schneider specifies a scale for the soloist to play if the chord is unspecified, like at bar 40, where the chord symbol is ?/G for one bar (see Fig. 3.4), and the pitches for improvisation are specified, leading into 8 bars of $F$ minor.

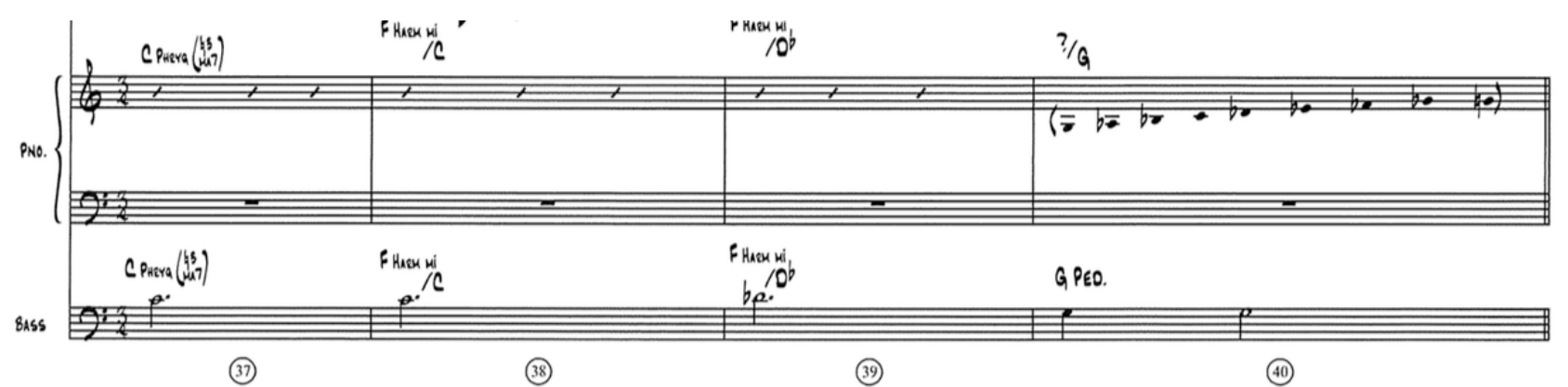

(37)

(38)

(39)

(40)

Figure 3.4 Piano and bass parts from bar 37-40 of "Nocturne"57

\section{Suspended Chords}

The harmony of "Sea of Tranquility" never reaches an obvious-sounding resolution because of the regular occurrence of altered and suspended chords. The beginning and end sections of the piece are built on a pedal of Eb. From bar 1 to bar 29 the piano and guitar both sustain pedals of Eb that underlie the other pitches and harmonies. This entire section is built upon Eb suspended chords, which is indicated in the piano part as the first two notes of each phrase is Eb to Ab, the root and the fourth of the chord, with the chords changing alterations every bar. Since suspended chords are neither clearly major nor obviously minor, this provides a feeling of floating whilst still being grounded by a bass note, and creates a sense of tranquillity whilst still producing a small feeling of being unsure, comparable to travelling on the sea. In addition to the sustained $\mathrm{Eb}$ and $\mathrm{Ab}$ pitches, other extensions such as E-natural (flattened ninth in an Eb chord), Db (flattened seventh), and $C$ (natural thirteenth) create a further sense of mystery. The baritone

\footnotetext{
${ }^{57}$ Schneider.
} 
saxophone melody outlines basic elements of the harmony, either holding the root, fourth, fifth, sixth or flat seventh of the chord, and avoiding the third. This outlines the harmony based on the Ebsus7 chord.

Generally the harmony moves in unexpected ways. For example, in bar 41 an F\#mi7(b5) chord leads to a B7sus(b9) chord, which seems to be a variation of a ii-V-i in E minor. But instead of the expected $\mathrm{E}$ minor chord resolution, the harmony moves to an Ebmi13. A few bars later, in bar 47, an Amin13 to Dsus7 follows the same pattern. It seems that it would lead to a $\mathrm{G}$ major chord in a ii-V-I pattern, but it instead leads into a $\mathrm{C}$ pedal with a suspended tonality. By not bringing the listener to the expected cadence of the tonic after a dominant $\mathrm{V}$ chord, it propels the harmony back up into the air of ambiguity.

"Nocturne" and "Sea of Tranquility" both frequently feature chords which contain close intervals within them, like major or minor seconds. These narrow intervals can easily clash and sound dissonant if not blended correctly or placed in the right context by a composer. Compositionally, Schneider manages to make even the most dissonant intervals sound beautiful. The balance between tension and release seems to lean heavier on the side of tension, perhaps even with a sense of continually rolling tension where the minor sonorities add a feeling of edginess. Chords with internal dissonance are much more ambiguous and mysterious because of the tension they are creating to eventually or potentially be released in unexpected ways. To me, this could be compared to night-time, where it is easier for the corrupt to be concealed in darkness, in contrast with daytime, where everything is much clearer, obvious and reassuring. Sounding haunting and slightly scary, "Nocturne" definitely captures the essence of the night-time as it brings the listener into a world of darkness. This may have been Schneider's intention considering the title of the piece, and I draw inspiration from these elements in my project, which gestures towards dreaming and its connections with the night. The title of "Sea of Tranquility" also has connotations of the night, and of a specific place, as the Sea of Tranquility is the name of the landing site of Apollo 11, on the moon. ${ }^{58}$

\footnotetext{
58 John Carl Villanueva, "Sea of Tranquility," Universe Today, accessed October 20, 2019, https:/www.universetoday.com/50525/sea-of-tranquility/.
} 
The harmonic rhythm throughout both pieces varies, with extended sections of one pedal and sections where the chords change every bar or every two beats, all of which help create lifts and drops of intensity. In "Nocturne," a C pedal in the bass plays for the first eight bars, with the chords over the top changing every bar, then in bar 9 to 19 the chords change every bar, until the piano is featured at bar 20 , when the harmonic rhythm changes every half a bar for 5 bars, eventually reaching a chord held for 2 bars. In bb. 25-37 the chords change every two bars. At bar 37 a 3/4 meter begins, and the chords change every bar for 4 bars (37-40), followed by another pedal on $\mathrm{F}$ for 8 bars. More pedals appear later on in the piece, all surrounded by sections where the harmony changes either every two bars or every bar. The faster the harmonic rhythm, the more the intensity level increases. The harmonic rhythm always speeds up to one chord per bar before a section with a pedal note in the bass. This is tension and release at work, the release being the pedal, which grounds the music in a key centre. Many pedals appear throughout this piece, the majority of which are on F. All other chords play a part in resolving back to the home key of $F$ minor. This is similar to "Sea of Tranquility", where the pedals of Eb contrast to faster moving chords and harmonic rhythms, creating sections with diverse paces in movement.

\section{Conclusion}

"Nocturne" and "Sea of Tranquility" by Maria Schneider contain many elements which are fitting to interpret into my own project and creating a calm, relaxing musical experience. Because timbre is my main focus, I adopt many of these timbral techniques Schneider uses, along with techniques like maintaining a low dynamic level and pedals. Subtleties in dynamic shifts and extended pedals also complement the focus put on timbral combination, simultaneously relating to my analysis on Brian Eno, whose music has a much narrower timbral palette but uses other similar musical devices to those of Schneider's music. Timbre seems to be at the forefront of Schneider's music, as she intentionally highlights the beauty of tone she finds in each of the instruments. In doing this she creates music for jazz orchestra which is calm, soothing and relaxing. 


\section{Chapter 4}

\section{Analysis and Reflections on What Dreams May Come}

\section{Introduction}

This chapter is a reflection on and discussion of my composition, What Dreams May Come, a suite for jazz orchestra in five movements. This project's aim was to challenge both the traditional role of the jazz orchestra and the techniques that are typically applied to jazz orchestra composition. To write this suite of music I drew on my analysis of Brian Eno and Maria Schneider, uncovering the differences and similarities between techniques and approaches of the two composers. Taking inspiration from two composers with such different musical styles and compositional processes meant that I often had to favour some musical concepts at the expense of others. Ambient music has always been the main inspiration for this project, but throughout the process its inspiration has become more conceptual and less obvious in terms of musical style. In this piece I initially aimed to maintain a 'balance' between the aesthetics of jazz and ambient music, but the product has resulted in this balance tipping more to the side of modern jazz, mostly because of the instrumentation of the jazz orchestra. The fear of the music not being 'interesting' or 'impressive' enough at times hindered my endeavours in musical exploration, but the desire to create a work which pushed the limitations or boundaries of a jazz orchestra helped me overcome this uncomfortable feeling.

Each movement of What Dreams May Come is unique and showcases concepts taken from Eno and Schneider in different ways. The first movement, "1.", is structured fully around two main motifs. To begin the suite, movement one takes the most direct musical inspiration from Brian Eno through timbral simplicity and repeated motifs. The second movement, "2.", features melodies which unfold as each instrument independently articulates a different sustained pitch. The third movement, "2.3.2.", heavily features a motif from movement two, along with a second motif. "2.3.2." is conceived around the concept of a dream within a dream, structured into three parts, where the first and final parts represent one dream and the second part represents the dream within the dream. Towards the end of the solo section, in the final part, the two main motifs combine, 
representing a blending of the two dreams into one, which creates ambiguity until the first motif (the first dream) is outlined once again to conclude the piece. Like Schneider's "Sea of Tranquility", the fourth movement, "4. (The White Room)", was inspired by a physical space or place and follows the clearest structure out of any movement. In the case of "4.", I draw on the physical space of "The White Room," a room in a London nightclub in the 1990s, Heaven, designed as a 'chill-out' recluse for ravers. ${ }^{59}$ This penultimate movement contains the most momentum and movement, bringing the suite to a climax before the more reserved "5.". The final movement, like the first, takes inspiration mostly from ambient music and Brian Eno's approach, and also does not include drums or bass.

The title of a piece of music is influential in a listening experience. Composers choose titles for tracks that reflect or that have been inspired by the music, or vice versa. In the case of Ambient 1: Music for Airports, Eno has purposefully given very non-imposing titles, based on a basic numbering system, to reflect the non-imposing music. I also adopted this concept and slightly altered it to involve some words in the titles of movements three and four.

In my introductory chapter I compared ambient music to being like the 'dreamscape' of the awake world. Ambient music, in my view, greatly assists in helping people relax and attain a sense of peace with their thoughts. Because I took such strong inspiration from ambient music for this project, I hope that this composition achieves a similar goal. The jazz orchestra is an unlikely ensemble to perform in the style of ambient music, and for good reason. The historical nature of the jazz orchestra is to be loud, live and rhythmic, which is basically the opposite of ambient music. Throughout history, jazz orchestra composers have gradually shifted these assumptions about the sound of a jazz orchestra. Maria Schneider is one of many composers who has refashioned the fabric of jazz orchestra composition and arrangement through her use of many techniques, including doubling and challenging of traditional roles of the drum kit. A main goal of this project was not only to challenge myself compositionally, but to challenge the musical terrain jazz orchestras are capable of exploring. Creating an ambient big band album has turned out to be a great challenge, and I have had to completely abandon certain ideas, settle on a middle ground in some areas, and push the conventions of a traditional jazz orchestra in other areas.

\footnotetext{
${ }^{59}$ Ciro Scotto, Kenneth M. Smith, and John Brackett, eds., The Routledge Companion to Popular Music Analysis: Expanding Approaches (New York: Routledge, 2019).
} 
In this chapter, I first discuss some of the broader challenges I worked through on this project such as issues around subjectivity, disparate ways of listening for ambient and jazz orchestra music, and techniques for translating ambient music aesthetics to a jazz orchestra setting. I then discuss the composition following three themes: limitations and combinations of timbre, texture, and form/structure. For timbre, the main focus of this project, I discuss my various approaches to timbre in orchestration, including a careful approach to doubling and mutes. For texture, I examine the use of heterophony, orchestral weight and the concept from Eno of the destabilisation of background and foreground. Finally, with regard to structure, I look at the use of through-composed form, improvisation and the issue of narrative, harmonic rhythm, dynamics, and the overall 'macro' form of the suite. All figures in this chapter are taken from the score of What Dreams May Come, submitted along with this exegesis.

\section{Broader Compositional Challenges}

Through consideration of issues of subjectivity, environments of listening and endurance, I found myself needing to make adjustments to prior expectations I had of my project. One of the broader issues that I faced in this project was that of subjectivity. In truth, any music can be defined as calm and relaxing if a person experiences it as such. I am not claiming that everyone will enjoy listening to my compositions, but I hope that some people can find some tranquillity in my work. I can only incorporate into my compositions my own experience of ambient music being relaxing, with the hope other people will feel the same. As I have mentioned, the intention of Brian Eno was always to create background ambiance to complement human experience in a given environment. Perhaps Schneider also wanted to make relaxing music, but I cannot be sure whether she had intended for it to be played in the background to accompany the activities of everyday life.

Though my aim for this suite of music was to make it relaxing for listeners - an aim I believe I achieved -in my compositional process I had to overcome the mental challenge that some people may find my compositions boring. Through this process I adjusted some musical aspects to find a middle ground so that the music could be played live, holding a balance of being ignorable and engaging. I have also come to realise that some aspects of 
my composition, like its lack of pulse-driven rhythmic activity in the drums, may be more captivating to listen to in a jazz orchestra setting, because they depart from typical expectations of a jazz orchestra composition. I explore this perspective further in the section dedicated to timbre in this chapter.

A major difference between Eno and Schneider is the environment in which their music is typically heard, and how the sounds are produced. Brian Eno works only in the studio, altering sounds digitally, to be produced onto a recording which is intended to be played in the background, electronically through a speaker or headphones. The environment in which Schneider intends her music to be played is most likely live with mostly acoustic instruments, in the typical mode of a jazz orchestra performance. Of course I am also able to listen to Schneider's music through a speaker or headphones quietly in the background in an 'ambient' fashion, but my focus is on both composers' intentions for performance environment, as well as on the differences I see between the two. This was something I battled with throughout my own process. I began conceptualising that this suite of music I would be composing would be intended to be recorded and played as literal background music, much like Eno's intentions. I then came to realise the implications of performing this suite of music with a live big band in a sit-down full-attention live performance, much like the typical setting for jazz orchestra music performance today. Would I be able to maintain enough interest for the listeners, and even the performers themselves? This leads to the fact that I wanted my compositions to be engaging enough to be played or recorded in the first place. The 'as ignorable as it is interesting' concept from Eno sums up what I am trying to achieve with this project. But what defines something to be ignorable, or interesting? Is it the prioritisation of timbre over elements of rhythm, melody and harmony which makes music less 'interesting'? Ambient music may be considered by many to be boring, whilst others may find ambient music to be interesting and jazz to be extremely boring. I believe my 'interest' in ambient music lies in its intentions for relaxing the listener and complementing an environment, whereas other styles of music are not necessarily composed with these reasons in mind. The fact that composers of ambient music do not necessarily want the listener to be engaged with it fascinated me to the point I found myself wanting to explore this concept in other styles of music, in this case the jazz orchestra. 
Writing What Dreams May Come has been a challenge when thinking about the difference between it being played live to a captive audience, and it being played in the background to complement the ambience of an environment. When conceptualising the album I originally thought of it being created to become recorded music that required little attention to be enjoyed, but ended up adjusting my approach so that I can craft a live performance too. I have not discarded the idea that most of the listening experience of modern jazz compositions is through recording technology. The approach to creating a recording of a jazz orchestra is again very different to producing ambient music. Generally speaking, the goal for most jazz recordings is to imitate what an ideal live performance would sound like. I came to realise that I not only wanted to use a jazz orchestra because of its timbral qualities and capacity for timbral exploration, I also wanted to use ambient music as a concept to challenge a jazz orchestra in any setting, which includes a live performance with an attentive audience.

One of the main issues that has been raised throughout this process was that, through emulating some elements of ambient music, much consideration had to be put into endurance and stamina, especially of brass players, within the jazz orchestra. I have come to realise ambient music and other styles of background music utilise instruments that do not struggle with physical endurance compared to brass players, such as piano, synthesisers, vibraphone, bass, guitar, and of course automated electronic loops which require no human interaction or stamina once they are set up. In movements one, three and four of What Dreams May Come the guitar and piano feature in playing consistent, repetitive motifs. I wanted to include this repetition, as well as passing the motif around from instrument to instrument, to have an element of consistency in timbre, balancing timbral exploration with timbral consistency. This was intentional as the guitar and piano do not have issues with endurance as, for example, a trumpet would when playing this same repetitive motif.

Throughout Eno's "2/2" from Music for Airports a quiet drone rings out for the entirety of the piece. A drone or pedal that lasts 10 minutes is undesirable for a musician to play on any instrument, though not impossible by any means. Circular breathing is one technique that I could have adopted to create drones in the winds, but it was not something I wanted to rely on. The beginning of movement three begins with the double bass using a bow to play an Eb for 17 bars, followed by a number of sustained notes for another 24 bars until 
returning to plucking. With this bowed double bass, I most closely imitate the effect of a bass drone, as is heard in "2/2". Also, to overcome this challenge of endurance whilst including repetition and consistency, I intentionally pass motifs and long notes around the brass and woodwind instruments to give the musicians breaks of varying lengths. I was also careful to not compose any notes that are longer than humanly possible on a given instrument, and included many specifically placed breath marks to instruct musicians where to breathe. These are often staggered in different places so musicians are not all breathing at the same time, in order to maintain continuity of sound. The musicians eventually playing my compositions will be jazz musicians, who are usually very versatile and used to many different styles of music underneath the broader umbrella of jazz. Much like when playing Schneider's music, the musicians playing my music will need patience and control over their instruments to play at a low volume for a prolonged amount of time.

\section{Timbre: Exploring Simplicity and Complexity}

Whilst timbre is quietly considered by some to be perhaps the most significant personal element of individual jazz performance, much more emphasis seems to be put on virtuosity, complicated harmony and rhythm. Musicians work hard to develop an individual sound. What has always enticed me is putting a jazz musician who has been working on a 'personal sound' into an environment of a jazz orchestra, where they now have to blend and work with three or four others to create one 'section' sound. Even more complicated are arrangements that explore more timbre combinations across sections, in which cases the musicians must blend into the composite of the full jazz orchestra.

Ambient 1: Music for Airports, "Nocturne" and "Sea of Tranquility" all feature intentional and effective uses of timbre. This concept serves as the foundation for What Dreams May Come, along with the exploration of concepts drawn from either composer and the similarities and differences in their approaches. Timbre has been at the forefront of the analysis I have done so far. I chose Eno and Schneider because although they have very different approaches and work in very different musical fields, they both have a strong relationship to and affiliation with timbre. The main difference in these composers' approaches to timbre is largely based on the environment and medium in which they compose. I have made it clear that Eno uses a small number of individual timbres, 
whereas Schneider draws on much broader timbral palette through the capabilities of the jazz orchestra.

Both composers noticeably appreciate the role of timbre within their composition in their own unique ways that have been inspiring to me. Composers of all genres consider timbre to some degree, but taking a more conscious approach to centring my analysis around timbre has resulted in a higher awareness of the impact timbre has within a composition. This in turn has affected other areas of the composition, forcing me to take a different approach to compositional elements such as rhythm, pace, melody and harmony. For example, throughout both composers' works are an abundance of long, sustained notes and chords which slow the pace of the music, fewer active rhythmic roles for the members of the rhythm section, and less harmonic deviation and exploration.

The timbral palette of the jazz orchestra is well established, but Schneider has played a significant role in expanding the types of instruments included in a jazz orchestra, such as oboe, English horn, and even accordion. The timbre palette of ambient music, on the other hand, is much more flexible and does not have a historical narrative that would strictly predetermine a particular timbral palette. Because the medium of my project is, of course, jazz orchestra, I chose to take Schneider's approach of timbral exploration as the basis and combine it, to an extent, with Eno's concept of timbral simplicity. Examples of both are intentionally placed side by side or combined throughout each of the movements of What Dreams May Come.

\section{Timbral Approaches to Orchestration}

Timbral exploration in jazz orchestra composition involves interaction between instruments across sections. I adopt this technique from Schneider's compositions, exploring many timbral combinations. I refrained from applying this technique until the second movement. The reason I did this was to use the first movement as an 'introduction' to the sections within the jazz orchestra. The trumpet section (all playing flugelhorns in cup mutes) features many times throughout this first movement (bb. 1-30, 86-91, 108-120 and 167175), being either completely unaccompanied, or accompanied with occasional notes from the double bass and drum kit. The muted flugelhorn offers a fluffier, smoother timbre than the trumpet, and having all four members of the trumpet section playing flugelhorn creates 
a richer more mellow overall timbre, compared to the usually piercing sound of a trumpet section, which usually cuts through the many layers of a jazz orchestra. The reason I chose to have timbral consistency (the only timbre being flugelhorns with cup mutes) to begin the suite was to make a clear distinction from the timbral exploration I was about to explore further in movement two. Because "1.1" is structured around the trumpet section on flugelhorn, I intentionally maintained timbral simplicity across the rest of the band. This movement paints a more traditional picture of the way jazz orchestras historically have been arranged in composition (three melodies interacting in three 'blocks': saxophone section, trombone section and trumpet section, accompanied by rhythm section). This is the most simplistic timbral approach to a big band, and one that is traditional in big bands such as Count Basie's band. I decided that having a simplistic timbral approach for the first movement would contrast the rest of the suite as exploring timbre to a much larger extent, making it more effective.

The first bar of movement two comes as a sudden divergence from timbral simplicity. The movement begins with Alto 1, Tenor 1, Trumpet 1 and Trombone 1 playing a melody together with individually attacked sustained notes, much like the flugelhorns at the beginning of movement one, but this time spread across sections and timbres. Movement five explores this concept of timbral exploration to the furthest extent, through the use of melodies being passed around members of the band, with differing intervals of time in between.

I have applied doubling to all movements of this suite as Schneider does in "Nocturne" and "Sea of Tranquility", in the saxophone and trumpet sections. I have augmented the traditional jazz orchestra instrumentation with the timbres of flute, clarinet, bass clarinet and flugelhorn. In many parts, I have utilised several instruments over the course of one movement. For example, in movement 3, the 'Alto 1' musician plays alto saxophone, soprano saxophone and flute. In movement 2, 'Tenor 1' plays tenor saxophone, clarinet, and flute. This variation in timbre through doubling occurs much more regularly in the saxophone section than the brass, where flugelhorns in the trumpet section are the only doubling instruments. The use of doubling has created a large amount of timbral exploration throughout the suite, adding to the destabilisation of the tendency for each section to play as a singular unit on the same or similar instruments. Examples of this include at bar 117 of movement three where flute changes to soprano saxophone four 
bars later, or movement five where both tenor saxophones swap to clarinet from 34 to bar 45 (then back to tenor). Considerations with using doubling instruments include allowing enough time for a player to change instruments (having at least 4 bars rest between changes), and allocating appropriate instruments to certain musicians.

When initially conceiving this composition, I first considered the inclusion of synthesisers and a range of pedals for the electric guitar to incorporate some element of digitalised timbre, like what makes up ambient music. I concluded I would only utilise acoustic timbres and attempt to imitate synthesised sound. The concept of altering sounds digitally as Eno does in the studio, such as the digitally-altered human voice, can be translated acoustically by the alteration of timbre of brass instruments by using mutes, as Schneider demonstrates in her compositions. Throughout my own compositions I use mutes extensively, not only to dampen or lower the volume and achieve a softer, less imposing dynamic, but to augment the overall timbral palette. I also use different combinations of mutes together to achieve an even wider timbral palette. An example of this is clearly seen in bar 70 of movement five, where each trumpet below Trumpet 1 uses a different mute (Trumpet 2 uses cup mute, Trumpet 3 uses bucket mute, Trumpet 4 uses harmon mute) (see Fig. 4.1). This results in a rich, fuzzy, soft timbre. Another clear example of using a mute is in bar 29 of movement three, where Trombone 1 is playing a sustained Eb in unison with piano, and an octave lower than the flute (with arco bass also playing Eb two octaves lower). The trombone uses a bucket mute, deadening the note slightly to aid in blending with the flute, piano and bass.

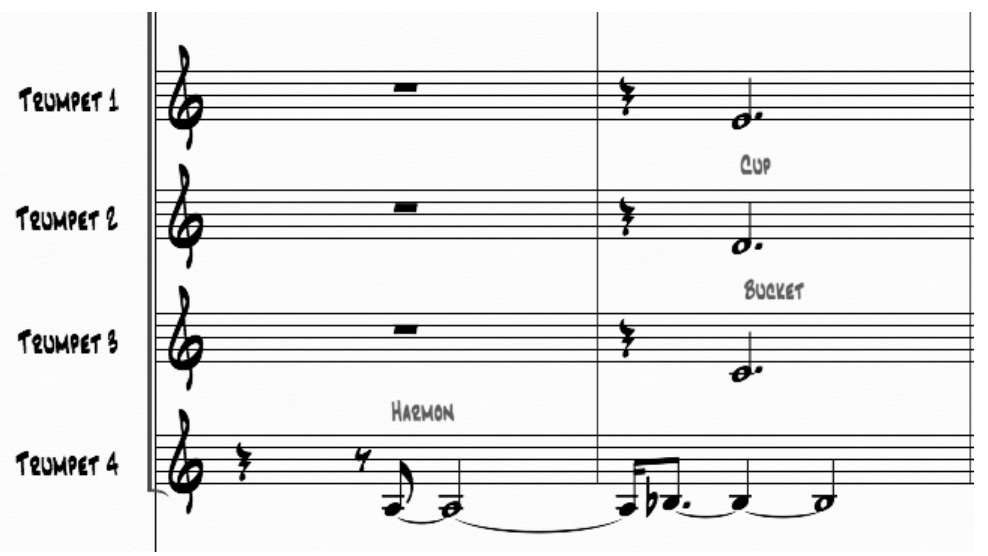

Figure 4.1 The combination of mutes in the trumpets at Bar 70 of movement five 
Focusing on timbre in What Dreams May Come has also had an effect on the techniques I apply in composing for drum kit. I adopted and interpreted several techniques from Eno and Schneider to apply to the drum kit in my compositions. The drum kit typically plays a significant role in supplying intensity, busy-ness, and rhythm to a piece of music for jazz orchestra. Remove the drum kit, like in "Nocturne", and this results in more emphasis put on timbre, texture, melody and harmony in the composition as a whole. This less rhythmic and more timbral approach to drums also helps with keeping the band at a quieter volume, especially when the drums are played with brushes or mallets.

Eno's ambient music does not have any drum kit, or any percussive instruments to provide a sense of rhythm. This contributes to the music sounding nearly nonmetrical. The lack of a solid sense of pulse, along with the presence of consistent sustained notes and chords, and scattered silence, results in a more emotive and expressive feeling. I had intended on having a few movements without drums (and even considered not including drums altogether) but then decided for most of the movements to explore the way Schneider uses the drum kit in a way that contributes to timbre, instead of providing the 'beat' or 'holding the band together'. The fifth movement of my suite is the only movement which is completely void of drums. I intentionally composed the last movement to be devoid of drums and upright bass, concluding the suite with relaxing melodies scattered across instruments, with no solid sense of pulse. The fourth movement is designed to include the climactic section and peak of the whole suite, and the fifth movement is intended on having a lower intensity of rhythm and harmony, like a 'warm-down'.

I use a number of other strategies in the drums in my timbral approach in the suite. In the second movement, I instruct the drummer to only play rhythms on the cymbals for the first 30 bars, in unison with each note being articulated from different brass and woodwind players. This adds a metallic, sharp timbre to the culminations of timbres being articulated. To accompany a 'drone' (pedal), I often utilise a long drum roll on the floor tom to last the entirety of the pedal. For example, at the beginning of the third movement, the upright bass bows a pedal on Eb and the drums play a roll on the floor tom (see fig. 4.2). This is designed to only complement the bowing of the upright bass note, adding richness and depth. 


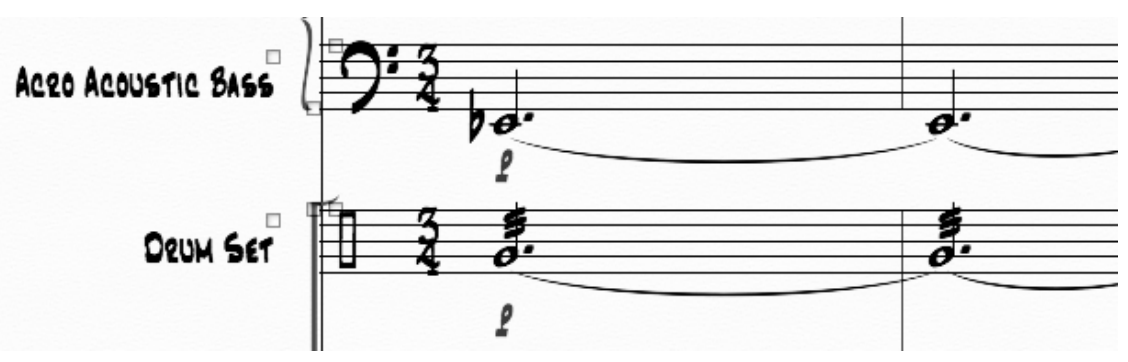

Figure 4.2 A drum roll on the floor tom accompanying a bass pedal in Bar 1 of the third movement

Subtleties or complete lack of drums largely contribute to the overall volume of the band. Using the drums more as a timbral additive also helps maintain a low volume, creating more opportunity for the music to be a more subtle environment enhancer, similar to ambient music. In addition to this role in the drums, I have indicated mainly soft dynamics for the drums' parts in the majority of the movements.

Throughout the suite's five movements, I feature a handful of timbres, whether it be through a melody or an improvised solo, an approach that follows both Schneider and Eno. Schneider features soloists in both "Sea of Tranquility" (baritone saxophone and flugelhorn) and "Nocturne" (piano), throughout the majority of the piece. Eno features certain timbres in a different way, like in track " $2 / 1$ ", where he features electronically modified human voice by not including any other timbre. Movement one features the timbre of the flugelhorn in cup mutes, and also a tenor saxophone solo. Movement two features the timbre of two alto saxophones. Movement three features a trombone solo and the timbre of piano and guitar together. Movement four features the timbre of alto saxophone in unison with guitar, and also a guitar solo. Movement five has no soloist or featured instruments.

I feature timbral uniformity several times in the suite. The opening of movement one ("1.”) features the trumpet section on flugelhorns all in cup mutes (specifically no vibrato), from bar 1 to 30, 108 to 120, and 167 to 175, and also (on trumpets, 'in-stand') at bb. 86-91. Bars 210-219 involve Trumpets 2 and 3 with Trombone 1, all 'in stand', which gives a timbre and weight which is close to four trumpets. Another area where I explored the idea of timbral uniformity is in movement two ("2."), where I feature two alto saxophones playing together in bb. $75-84$, and from bar 94 to the end. 
In my analysis of both Eno and Schneider's compositions I comment on how the timbre of the piano is versatile, covering many genres, therefore being a familiar and soothing sound. I altered the concept of featuring the familiar timbre of piano with featuring piano with guitar many times throughout the suite. Examples include movement one in bb. 55-65 (see Fig. 4.3), 90-95 and 226-240, movement two in bb. 9-20, movement three in bb. 75137, and movement four in bb. 33-45 and 197-215.

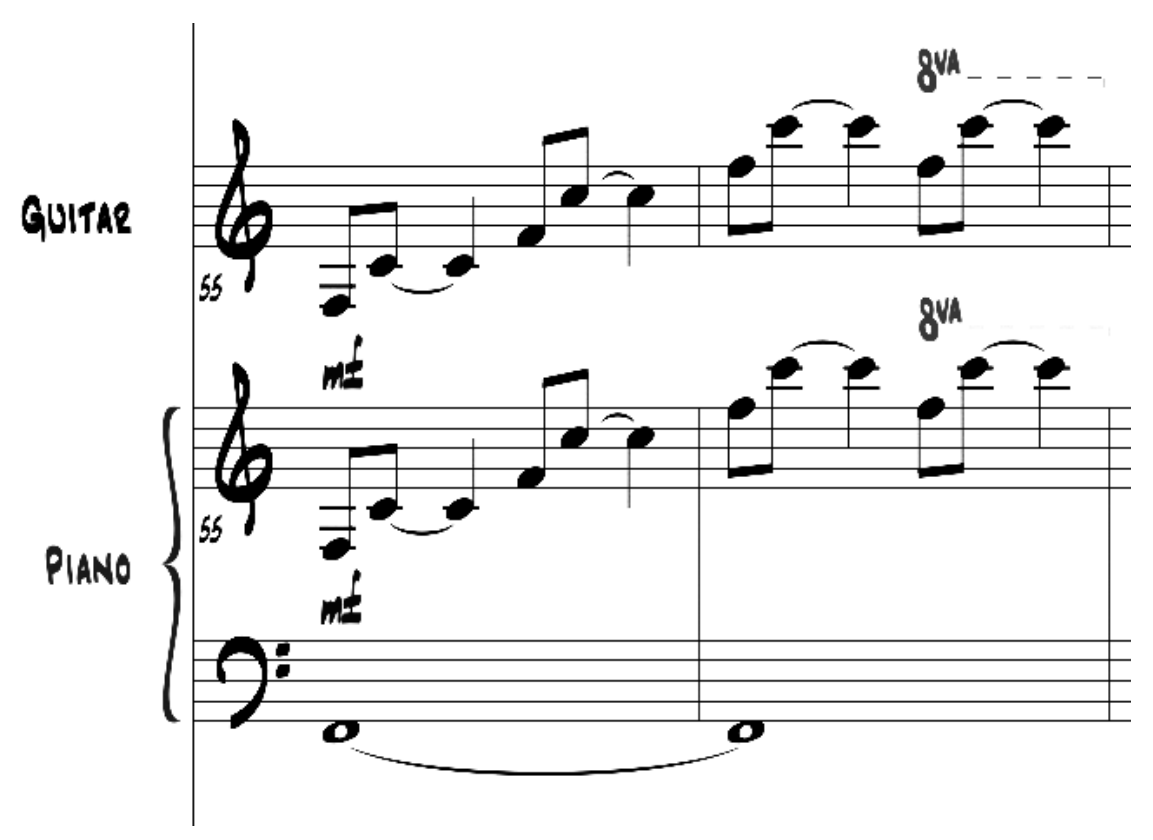

Figure 4.3 Guitar and piano, bb. 55-56 of movement one

\section{Texture}

Loops

The construction of Ambient 1: Music for Airports is made up of several automated loops of varying lengths, individually crafted by Eno, and set on repeat to overlap and interact with one another. I explore this concept in movement five, where I took five melodic motifs as 'loops' (see Fig. 4.4) and developed them over ten minutes by having them interact in different ways across instruments, whilst making an attempt to never stray too far from the original 'loops'. 


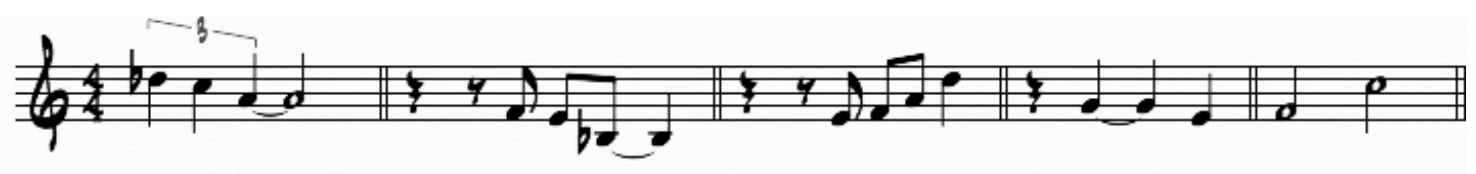

Figure 4.4 The five motifs/loops used in fifth movement

Throughout this process I realised the similarities between a 'loop' and a 'motif', and that the 'motivic development' in this instance was extremely restricted, to achieve the goal of not straying far from the original motif (or 'loop'). This is a significant departure from motivic development in the context of jazz improvisation, where the goal is often to begin with a motif and develop it through a 'story', changing and skewing the melody in its development. Motivic development and repetition occur many times over the course of the entire suite, as I carry motifs from one movement and incorporate them into another (this I explore in the section on macro-form).

Movement two contains a motif which is developed throughout the composition. The motif first appears in bar 9 and again in bar 10 in the piano and guitar part. The next time the motif appears is bar 32 in the Alto 1 and Alto 2 parts (see Fig. 4.5). In bar 70 and 72 the motif appears again (after a long development of the main motif of movement 2). Bar 125 sees a development of this motif, with the intervals being slightly different but with the same shape and essence. The motif appears again in bar 143, played by piano and guitar in unison.

A. $5 \times 1$

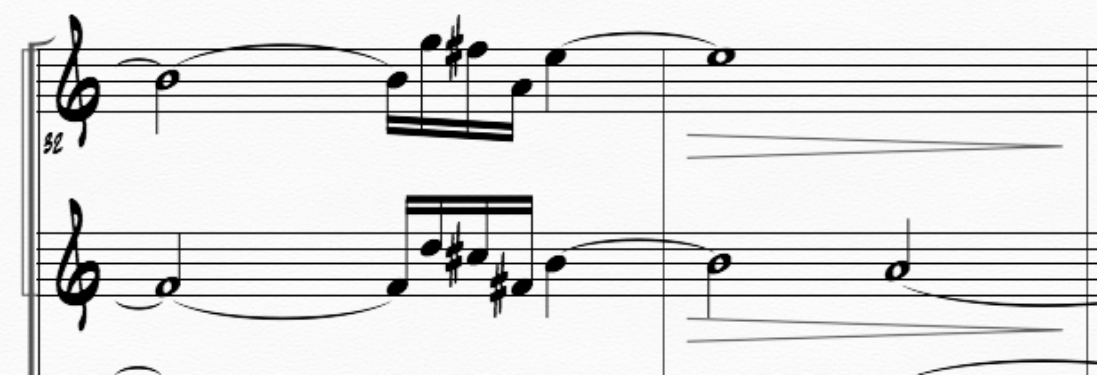

Figure 4.5 Main motif in movement two played by Alto 1 and 2 (bar 32 of movement two) 
Deciding whether and how to include soloists in this composition took much deliberation. All movements include an improvised solo except movements two and five. Because I am focussing more on timbre and texture in this composition, having a soloist for most of the composition (like in Schneider's compositions) may draw attention away from this. Whilst a soloist adds a voice and narrative to a composition, the inclusion of improvisation seems like it has to be inevitable when writing for jazz orchestra, especially since improvisation is such a strong feature of jazz. I found a balance between having 'voice-less' composition and incorporating a voice/improvised solo in a subtle way, where the 'backgrounds' of the solo were busy enough to bridge the gap in any sense of hierarchy between the soloist and the rest of the band. An example of busy solo backgrounds is in movement three. Here, separate melodies interact across instruments, developing phrases and different rhythms behind the trombone solo. This blurring between background and foreground also serves my inspiration of dreaming, in which the narratives I did end up embracing reflect the often confusing yet comfortable feel of dreaming. Having two movements without the expected featured improvised solos assists in intentionally disregarding narrative in the suite overall. The strong correlation between improvisation and storytelling has become apparent throughout the process of writing this suite. Therefore, involving improvisation within my compositions has resulted in a sense of narrative being unavoidable, which is something I have largely embraced.

In some cases, I included instructions for soloists to play sparsely, like at the beginning of the Tenor 1 solo in movement one, where I wrote 'Play Minimal - Long Notes'on the part. Although this takes away some of the improviser's creative agency, it still gives the player expressive freedom within restrictions.

\section{Form}

I have modelled my compositions after Schneider's two compositions in terms of duration, with lengths of between eight to ten minutes. This is also similar in duration to " $2 / 1$ " and "2/2" off Ambient 1: Music for Airports. One challenge was maintaining a subtle sense of development over this amount of time to engage the listener to a desired level.

I took inspiration from several elements of Brian Eno's Ambient 1: Music for Airports in structuring the movements into a suite as a whole to create coherence. Each movement 
can be played on its own, but to experience the full effect of the intricacies and subtle motivic connections of the suite, it is designed to be listened to in one sitting. Concepts taken from Eno related to the macro-form of the suite include development of musical material (such as motifs) across movements, and how the structure of the album may reflect a dreaming experience. Structurally, all of the movements are mostly throughcomposed, like Schneider's compositions. This means I avoid repeated sections, which are often common in jazz charts, especially for a solo section. Using through-composed form also creates a controlled environment to carry the soloist entirely through the solo, fully determining changes in volume and texture. Each movement has a different overall structural approach, with some having more obvious sections than others. Movement four has a more obvious form, structured generally around a melody section, a solo section, and a melody section repeated again in a slight departure from through-composition. Movement five has very little structure.

An example of musical material being carried over movements to create a sense of coherency is heard throughout movements two and three, which are connected through the use of two repeated motifs. The first motif consists of four notes, ascending by a half step, then a fifth, and then a major second (Figs. 4.6-4.7). The second main motif is repeated groups of three notes with the same shape (down a fourth or a sixth then up a half or whole step, Fig. 4.8).

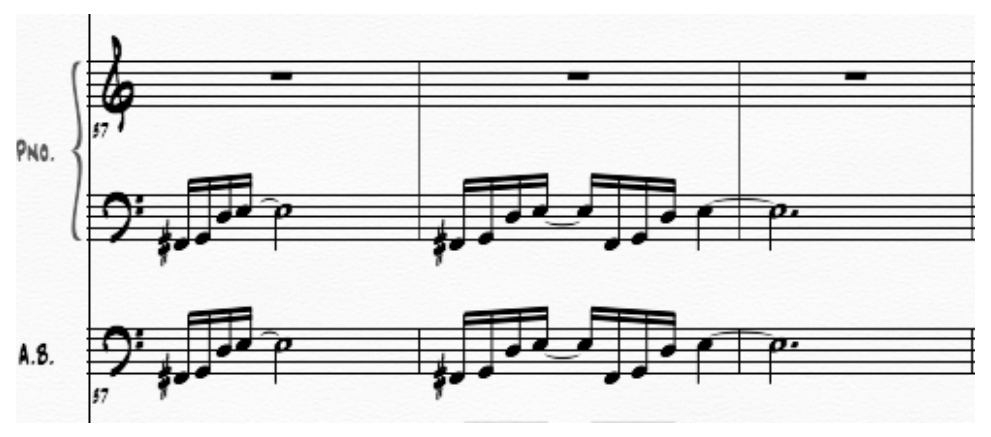

Figure 4.6 Motif 1 in piano and bass (bar 37 of movement 2) 


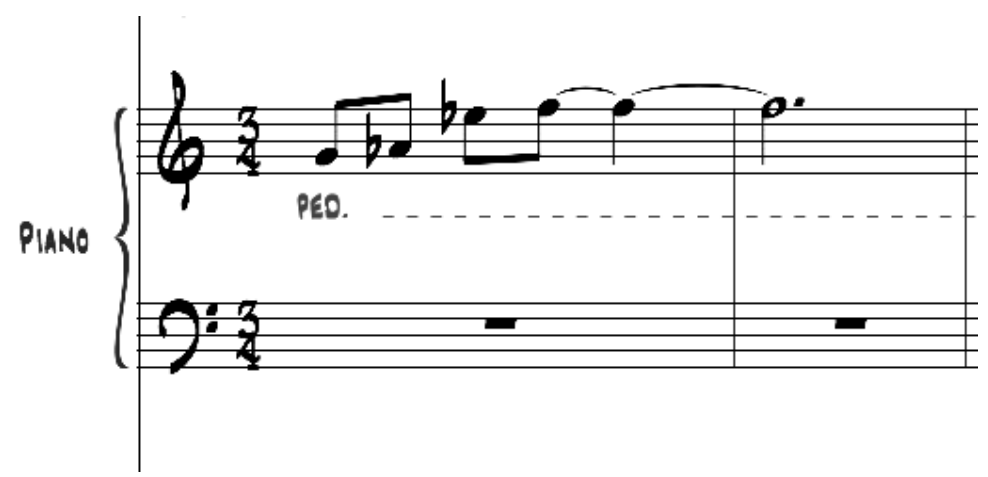

Figure 4.7 Motif 1 in piano (bar 1 of movement 3)

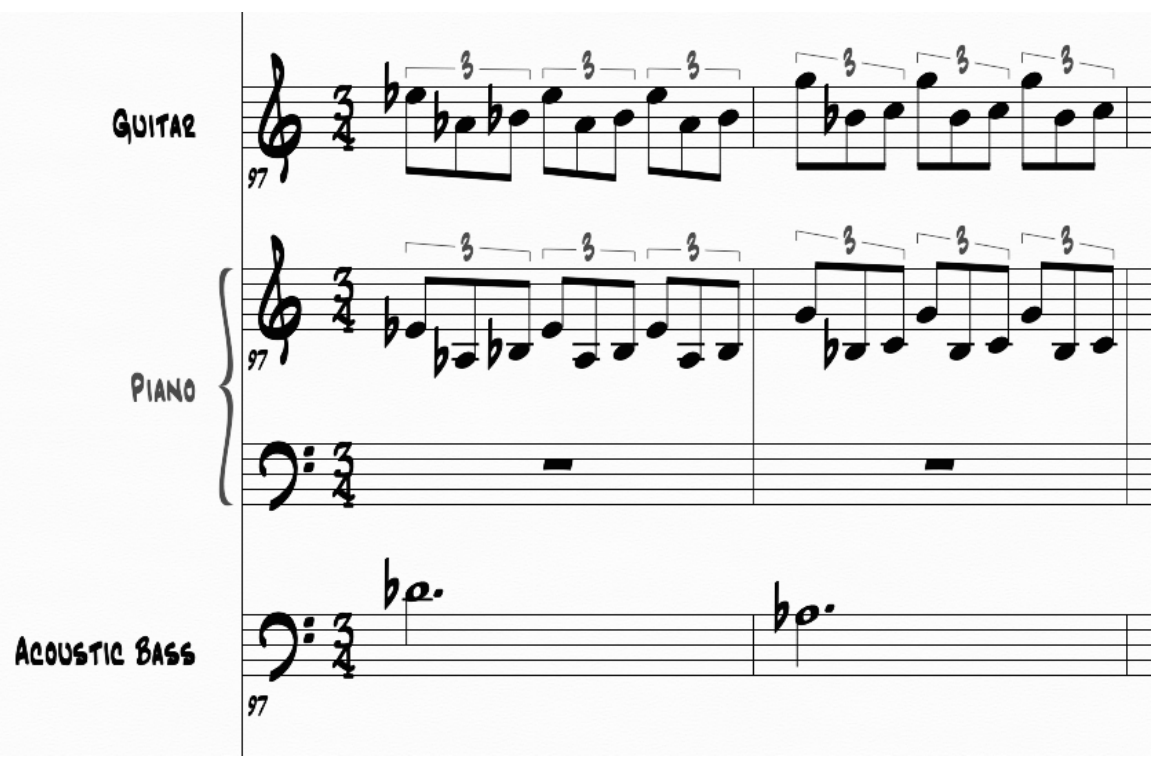

Figure 4.8 Motif 2 in guitar and piano (bar 97 of movement 3)

Dreams often do not make sense to our conscious mind, but usually feature familiar places and characters. I have reflected this in my own compositions, where one consistent motif carries through the entirety of the piece to give the listener familiarity to carry them through the composition. For example, in bar 121 of "2.3.2", I introduce a new, unfamiliar bass line, but I maintain the consistent triplet figure in the piano, to keep a sense of familiarity and tie the section into the rest of the piece (Fig. 4.9). 


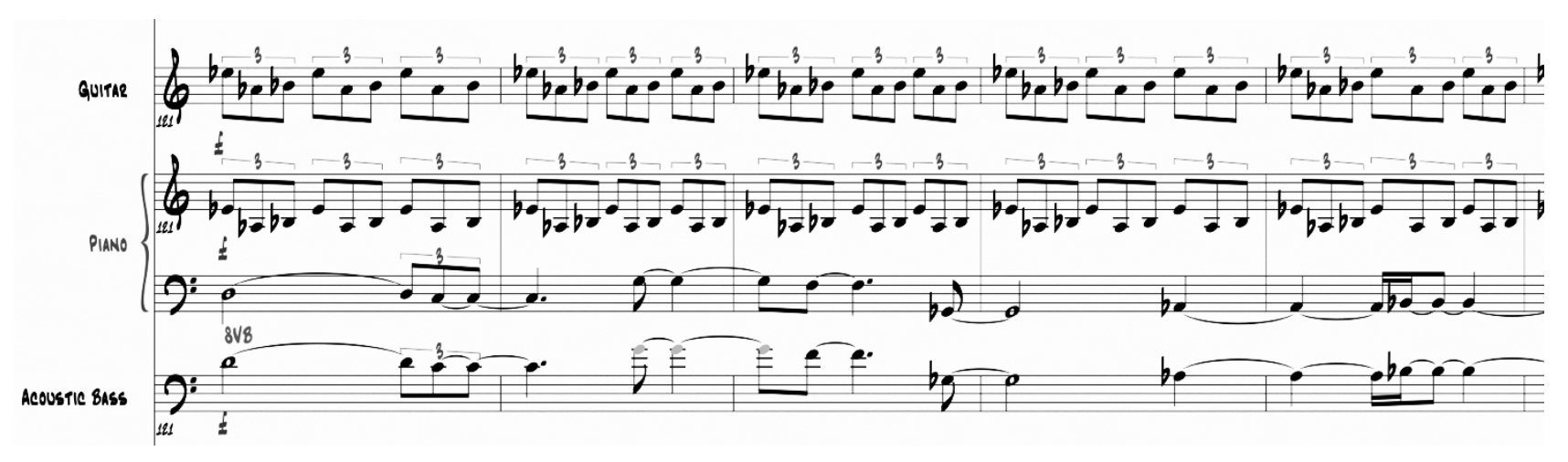

Figure 4.9 Motif 2 carried on over new, unfamiliar bass line (bb. 121-125 in movement three)

Harmony and harmonic rhythm both also have effects on structuring a composition. The harmony used within Ambient 1: Music for Airports is created by automated loops repeating a relatively small selection of pitches, normally no more than seven. This type of harmony is a step away from traditional, functional jazz harmony, which often involves chromatic movement and substituted chords to create moments of tension and release.

Schneider uses variation in harmonic rhythm to structure and develop her compositions. Throughout my compositions, the harmonic rhythm is constantly changing, to complement the style of through-composed form where repetition is minimal. The harmony in each movement is largely centred around one key, like Schneider's compositions, and contain several 'pedals' where the bass stays on the same note for an extended period of time between 4 and 16 bars. The harmony accompanying these pedals stay on a suspended chord, for example in movement two in bb. 57-66, and in movement four in bb. 146-153. Throughout my compositions, suspended chords are featured to create a sense of ambiguity and mystery, rather than tension. This contributes to helping the music sound 'dreamy' and also creates a sense of the music being unsettled, ungrounded, or 'up in the air', which gives the effect and feel of floating, which can be very relaxing for the listener.

The concept of embracing 'tension' with suspended chords is also reflected in a consistent use of close intervals and dissonance scattered throughout the arrangements. This is exhibited clearly in the alto saxophones feature in movement two, where they are interacting either in half or whole steps from bb. 98-114. This question of how and why 
dissonance and tension contribute to making music sound relaxing has continually stimulated me as a composer throughout the process of writing this suite.

Dynamics relate very closely to a narrative and shaping of a piece and its structure, and I intentionally did not include many dramatic changes in dynamic level and volume. As I have mentioned in previous chapters, changes in dynamics have a large effect on altering the level of engagement and attention from the listener. Dynamically Eno's music is very flat, and therefore does not engage the attention of the listener. An issue that arose for me while composing this piece is the question of whether applying a consistent, softer dynamic to jazz orchestra actually draws more of listeners' attention, since it may depart from listeners' expectations. I maintain a low dynamic throughout the entire suite, never reaching a dynamic level higher than forte, and when these fortes did appear they were never for very long, such as in bb. 47-49 of movement two (forte for two bars), or in bb. 193-198 of movement three (forte for six bars). This is much like the proportionately short climactic sections in Schneider's pieces that contrast the reserved, quiet nature of the rest of the composition.

In my analysis, I mention how the timbral role of the drums in Schneider's pieces has an effect on keeping the dynamic level low. I follow Schneider's model, altering intensity only subtly, and not through changes in dynamics but through changes in orchestral weight, which keeps individual dynamic levels mostly constant. Movement five is a perfect example of the dynamic of each part staying the same, but intensity subtly increasing and decreasing through increasing or decreasing the number of instruments playing at once. At about halfway through the movement (bar 71) the intervals of time between instruments' entries begin to get shorter and shorter until all instruments are playing, overlapping with one another (see Fig. 4.10). The individual dynamic level for each instrument stays the same throughout (piano).

Eno's conception of ambient music involves the music being played at a low volume, just loud enough to add to the ambiance of a person's environment. This concept applied to jazz is most relevant to ballad playing. Ballads are usually softer, quieter and intend to captivate the audience by bringing them into the musicians' emotive performance. This comparison brought my attention to the flaw in my perception that a low volume always equals less attention and focus from the audience. I have come to realise this does not 
always apply; sometimes the softest or quietest music is the most captivating, and loud and boisterous music can turn listeners' attention away. In some instances, a live ambient big band performance could have a listener completely captivated. My compositions contain a similar dynamic range to Schneider's compositions, maintaining a low dynamic until a short climactic section towards the end, then coming down to finish at a lower dynamic level. Bars 164-173 of movement four is a section which is quite loud, because all instruments are playing and the rhythm section is instructed to play forte. In bb. 81-84 of the first movement a sudden increase in dynamics occurs, with a rest on beat one of bar 81 before all instruments enter on beat two at forte.

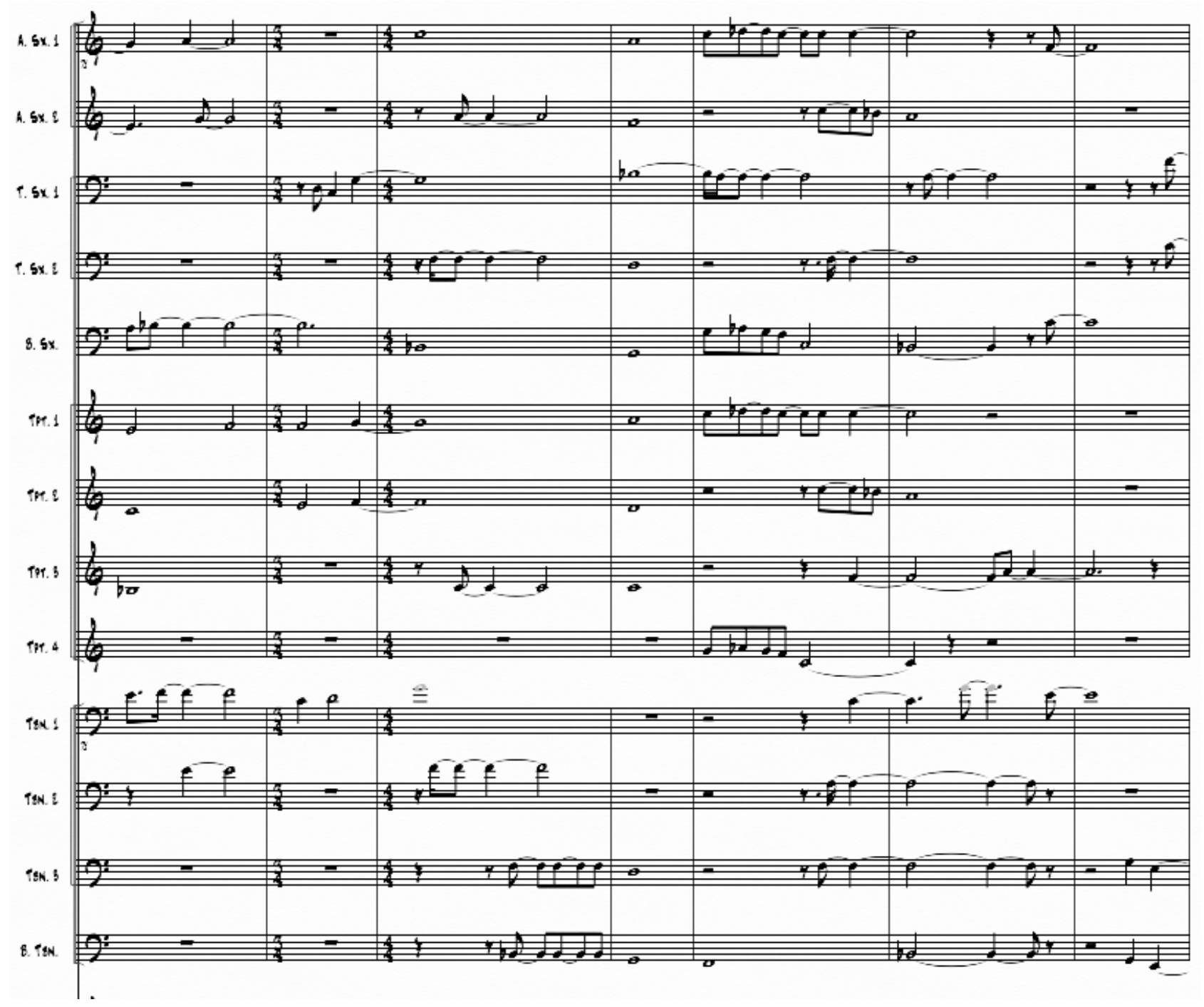

Figure 4.10 Time intervals between entrances in each part begin to decrease in bb. 71-77 of movement five

Dramatically decreasing dynamics feature much more than other dynamic changes. For example, during the final bars of movement four, the entire band builds up to a climax with 
a dramatic crescendo, after which the last chord is played only by the piano. Another example is the section in movement three, bb. 121-136, which is higher in volume, in comparison to a sudden drop immediately after to begin the trombone solo.

I also use silence throughout the composition to structure and shape the movements, much like how silence helps to shape Eno's compositions. A clear example of this is in bb. 50-52 of "2." Bar 50 is a bar of silence, bar 51 is a sustained chord in bar 51 from all instruments except baritone saxophone, trombone 4, piano and guitar, and bar 52 is another full bar of silence. In this particular case the silence also acts as a way to make thinning of orchestral weight more obvious. Each time these sustained chords appear after silence, fewer instruments are playing the chord, making the orchestral weight thinner and thinner.

\section{Conclusion}

What Dreams May Come explores innovative approaches to composition for jazz orchestra. Highlighting timbre gave me a different perspective on elements like motivic development (interpreting motifs more like loops), spreading musical material such as motifs across movements, maintaining a softer dynamic, and using simplified harmony. Through composing this music I felt as though I was making a conscious attempt to diverge from traditional jazz orchestra composition, and using Eno's compositions to inform my process was helpful in adding another perspective to my approach, especially considering my compositional priorities. Schneider's compositions influenced my composition a great deal, too, especially regarding solo backgrounds, the dynamic structure, and use of pedals. I found many similarities and relationships between elements and techniques by Eno and Schneider, which was fascinating to uncover as the two composers work in very different genres of music, two genres I once thought would be incompatible.

The combination of aesthetics of ambient music and jazz results in jazz orchestra music which allows much more room to think and breathe. What Dreams May Come, in my view, is less imposing and less forcefully engaging than other jazz compositions written today. I set my intentions very similarly to those of Eno, in that I don't mind if this music just plays 
in the background. Musical techniques I have adopted to achieve this include pedals and non-complicated harmony, incorporating melody in subtle ways and a timbral approach to the drum kit.

With my analysis informing my compositions, I was able to successfully write a suite of music which was as 'ignorable as it is interesting'. The concept of music being equally ignorable and listenable seems undesirable at first, especially when seeking out new music. Intentionally choosing to listen to ambient music appears to be a niche, but I would say most people enjoy the ambience of background music without realising it, whether it be in a movie, behind a motivational speaker, or even in an aquarium. Ambient music pops up as background music in many environments. Throughout this project I have sought to understand what makes ambient music different to any other style of music being played in the background. The most important differentiating feature to me lies in the early conceptual point in the process, where the composer sets her intentions on what she wants the music to achieve, and this permeates throughout the rest of the compositional process. Brian Eno intentionally creates music where he doesn't mind, or rather prefers, if the listener doesn't actively listen to it. He has no story to tell, no narratives to follow, and requires no emotional connection. Ambient music is really no different to the couch in your living room, in terms of the ambience they bring to the room. Applying this concept to jazz orchestra and combining the two aesthetics came with many issues, such as subjectivity and endurance. Because highlighting timbre was at the forefront of my analysis, and therefore of my composition as well, I explored the many beautiful timbres available in a jazz orchestra.

In addition to the concepts and techniques from Eno and Schneider, dreams have been at the forefront of my mind as I composed this suite. I took dreaming more as a conceptual and aesthetic inspiration, more so than trying to musically depict the mystery and wonder of dreams or a particular dream narrative. The relationship between dreaming and ambient music lies in the ability of both to create a space for people to process information. This project has stretched my compositional abilities in exercising restraint and experimenting with timbral possibilities. Through this process I now understand more about the significance of intent throughout a compositional process, and feel inspired by how Brian Eno and Maria Schneider push the boundaries of their respective musical fields, ultimately for the greater good and well-being of the listener. 


\section{Bibliography}

Bangs, Lester. "Lester Bangs Interviews Eno." Musician, n.d.

http://music.hyperreal.org/artists/brian_eno/interviews/musn79.html (accessed 14 December 2019).

Bregman, Albert. S. Auditory Scene Analysis: The Perceptual Organization of Sound. Cambridge, Massachusetts: MIT Press, 1990.

Discogs. "Brian Eno." Accessed May 27, 2020. https://www.discogs.com/artist/634-Brian-

Eno?filter_anv=0\&subtype=Albums\&type=Releases.

Eno, Brian. Schmidt, Peter. Oblique Strategies. Peter Notron, 1996.

Eno, Brian. “Ambient 4: On Land, Liner Notes.” Accessed September 11, 2019.

music.hyperreal.org/artists/brian_eno/onland-txt.html.

- - - "Music for Airports; Liner Notes." Accessed February 20, 2020.

http://music.hyperreal.org/artists/brian_eno/MFA-txt.html.

- - - "The Studio as a Compositional Tool." In Audio Culture: Readings in Modern Music, 12730. Bloomsbury Academic, 2004.

Eyles, Mark, and Roger Eglin. "Ambient Games, Revealing a Route to a World Where Work Is Play?" Edited by Kok Wai Wong. International Journal of Computer Games Technology 2008 (2008): 176056. https://doi.org/10.1155/2008/176056.

Fink, Robert. "FOUR 'A POX MANFREDINI': The Long-Playing Record, the Baroque Revival, and the Birth of Ambient Music." In Repeating Ourselves: American Minimal Music as Cultural Practice, 200. University of California Press, 2005.

Fink, Robert, Melinda Latour, and Zachary Wallmark, eds. The Relentless Pursuit of Tone: Timbre in Popular Music. New York: Oxford University Press, 2018.

Goad, Pamela J., and Douglas H. Keefe. "Timbre Discrimination of Musical Instruments in a Concert Hall." Music Perception 10, no. 1 (1992): 43.

Hattenstone, Simon. "Brian Eno: 'We've Been in Decline for 40 Years - Trump Is a Chance to Rethink." The Guardian, 2017. https://www.theguardian.com/music/2017/jan/23/brian-enonot-interested-in-talking-about-me-reflection\#maincontent.

Hill, Clara E. Working with Dreams in Psychotherapy. Guilford Press, 1996.

Kavolis, Vytautas. "Abstract Expressionism and Puritanism." The Journal of Aesthetics and Art Criticism 21, no. 3 (1963): 315-19. https://doi.org/10.2307/427440.

Kelly, Jennifer, and Maria Schneider. "Maria Schneider." In In Her Own Words: Conversations with Composers in the United States, 240. University of Illinois Press, 2013.

Kristoffer, Karl. "Timbre Models of Musical Sounds Kristoffer Jensen Datalogisk Institut, Københavns Universitet Department of Computer Science , University of Copenhagen.” University of Copenhagen, 1999. 
Recording Academy. "Maria Schneider Grammy Awards." Accessed April 13, 2020.

https://www.grammy.com/grammys/artists/maria-schneider.

Matheson, Richard. What Dreams May Come. Putnam, 1978.

Nathanidiothend. "Brian Eno Interview," 2007.

https://www.youtube.com/results?search_query=brian+eno+music+for+airports+interview.

Pelly, Jenn. "Brian Eno Creates Healing Music and Light Installations for Hospitals." Pitchfork.

Accessed February 21, 2020. https://pitchfork.com/news/50402-brian-eno-creates-healingmusic-and-light-installations-for-hospitals/.

Reiser, Morton F. "Revising Dream Theory." In Memory in Mind and Brain, 177. Yale University Press, 1994.

Schneider, Maria. Nocturne. MSF Music, 1999.

- - - Sea of Tranquility. MSF Music, 1999.

Scotto, Ciro, Kenneth M. Smith, and John Brackett, eds. The Routledge Companion to Popular Music Analysis: Expanding Approaches. New York: Routledge, 2019.

Shakespeare, Wiliam. "Hamlet: A Monologue." Monologue Archive. Accessed November 13, 2019. http://www.monologuearchive.com/s/shakespeare_001.html.

Sherburne, Philip. "A Conversation with Brian Eno About Ambient Music.” Pitchfork. Accessed November 13, 2019. https://pitchfork.com/features/interview/10023-a-conversation-with-brianeno-about-ambient-music/.

South, Phil. "The Sound of Silence: A Thursday Afternoon with Brian Eno." More Dark Than Shark, 1985. https://www.moredarkthanshark.org/eno_int_eamm-dec85.html (accessed 8 May 2019).

Stamp, Jimmy. "5 1/2 Examples of Experimental Music Notation." smithsonianmag.com. Accessed February 25, 2020. https://www.smithsonianmag.com/arts-culture/5-12-examples-ofexperimental-music-notation-92223646/.

Stewart, Alex. "Contemporary New York City Big Bands: Composition, Arranging and Individuality in Orchestral Jazz." Ethnomusicology 48, no. 2 (2004): 178.

- - - Making the Scene (Contemporary New York City Big Band Jazz). University of California Press, 2007. https://www.jstor.org/stable/10.1525/j.ctt1pnr6g.1.

Tamm, Eric. Brian Eno - His Music and the Vertical Color of Sound. New York: Da Capo Press, 1995.

Traube, Caroline. "Instrumental and Vocal Timbre Perception." Quebec, Canada: Musicology Department - University of Graz. Accessed September 3, 2020. https://www.researchgate.net/profile/Caroline_Traube/publication/267939679_Instrumental_a nd_vocal_timbre_perception_Vocal_timbre_perception/links/56854c8908ae1e63f1f3308e/Inst rumental-and-vocal-timbre-perception-Vocal-timbre-perception.pdf.

Vanel, Herve. “John Cage's Muzak-Plus: The Fu(Rni)Ture of Music.” Representations 102, no. 1 
(2008): 94.

Villanueva, John Carl. "Sea of Tranquility." Universe Today. Accessed October 20, 2019. https://www.universetoday.com/50525/sea-of-tranquility/.

Ward, Vincent. What Dreams May Come. United States: PolyGram Filmed Entertainment, 1998. 


\section{Discography}

Ellington, Duke. "Soul Call.” Verve Records, 1967.

Eno, Brian. “Ambient 1: Music for Airports.” Polydor Records, 1978.

Jones, Thad, and Mel Lewis. "Presenting Thad Jones/Mel Lewis and the Jazz Orchestra." Solid State Records, 1966.

Mingus, Charles. "Nostalgia in Times Square." Dreyfus Jazz, 1993.

Schneider, Maria. "Allegresse.” Enja Records, 2000.

Schneider, Maria. “Evanescence.” Enja Records, 1994. 
What Dreams May Come

Louisa Beth Williamson 



\section{Instrumentation}

Alto Saxophone 1 | Flute | Soprano

Alto Saxophone 2 | Clarinet

Tenor Saxophone 1 | Clarinet

Tenor Saxophone 2 | Clarinet

Baritone Saxophone | Bass Clarinet

Trumpet 1 | Flugelhorn

Trumpet 2 | Flugelhorn

Trumpet 3 | Flugelhorn

Trumpet 4 | Flugelhorn

Trombone 1

Trombone 2

Trombone 3

Bass Trombone

Guitar

Piano

Upright Bass

Drum Set 



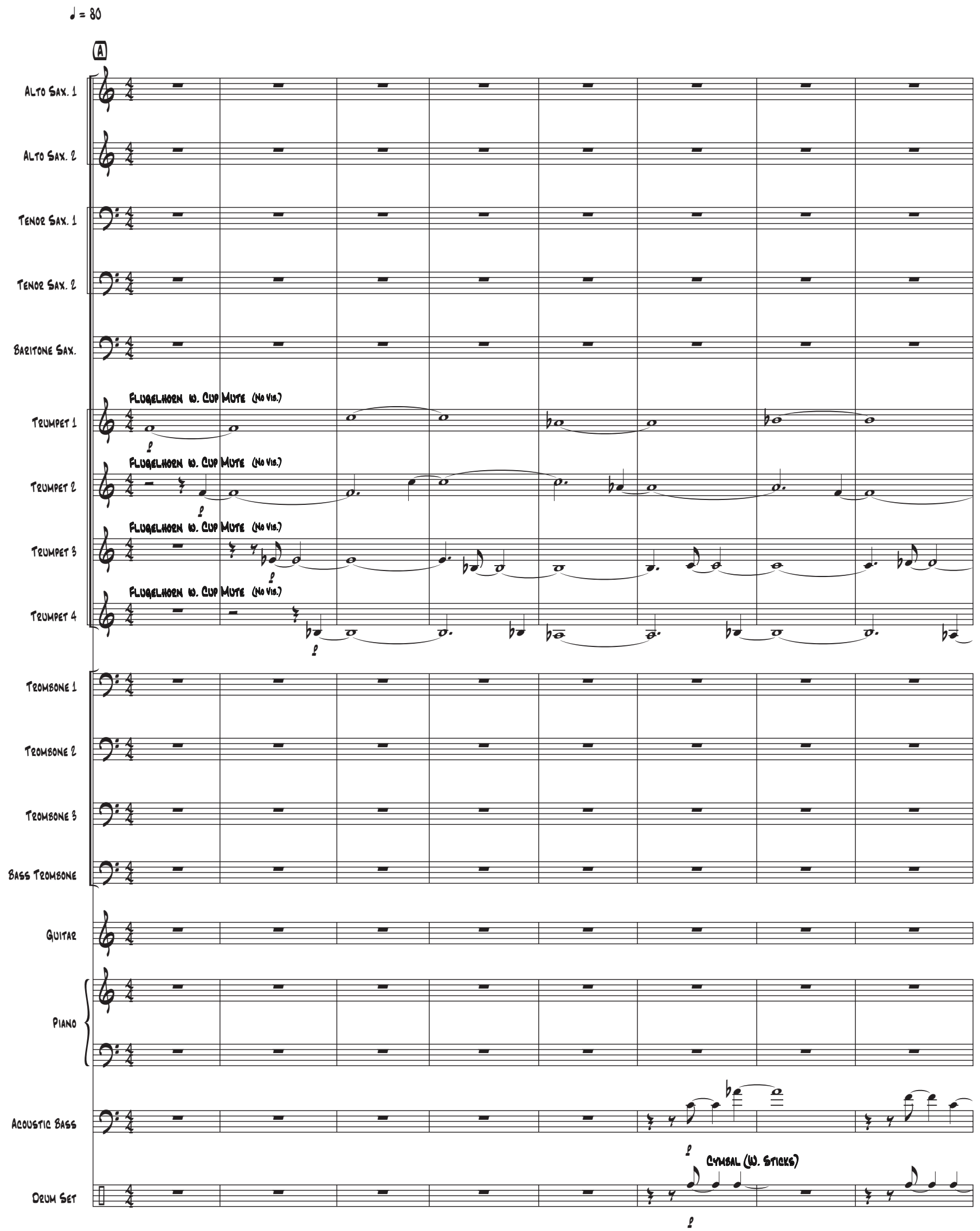



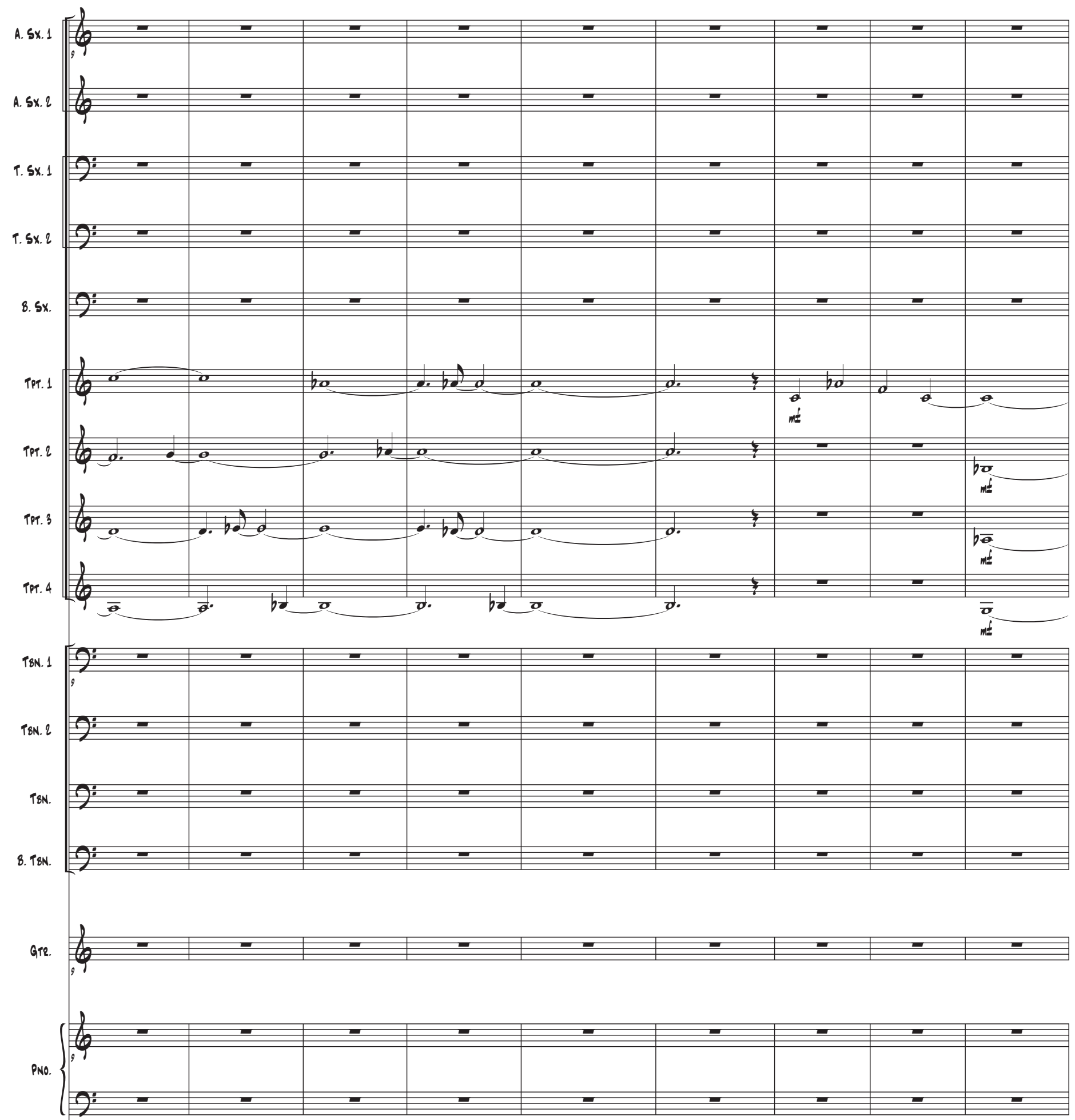

A.8.

$\int_{0}^{0}$

0.5.

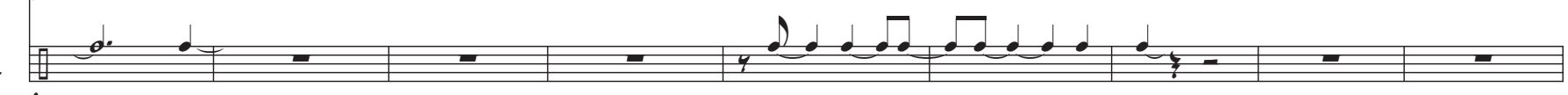



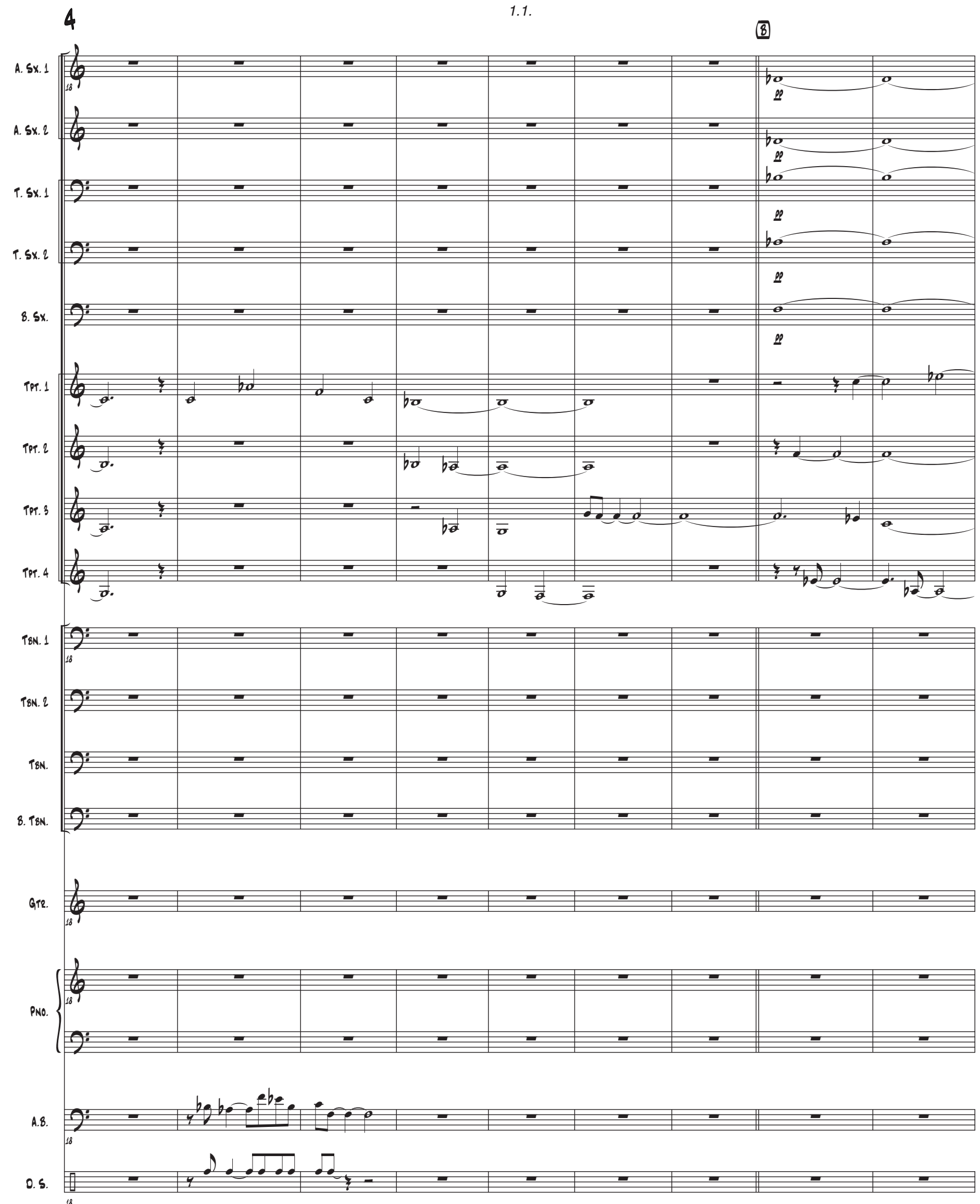

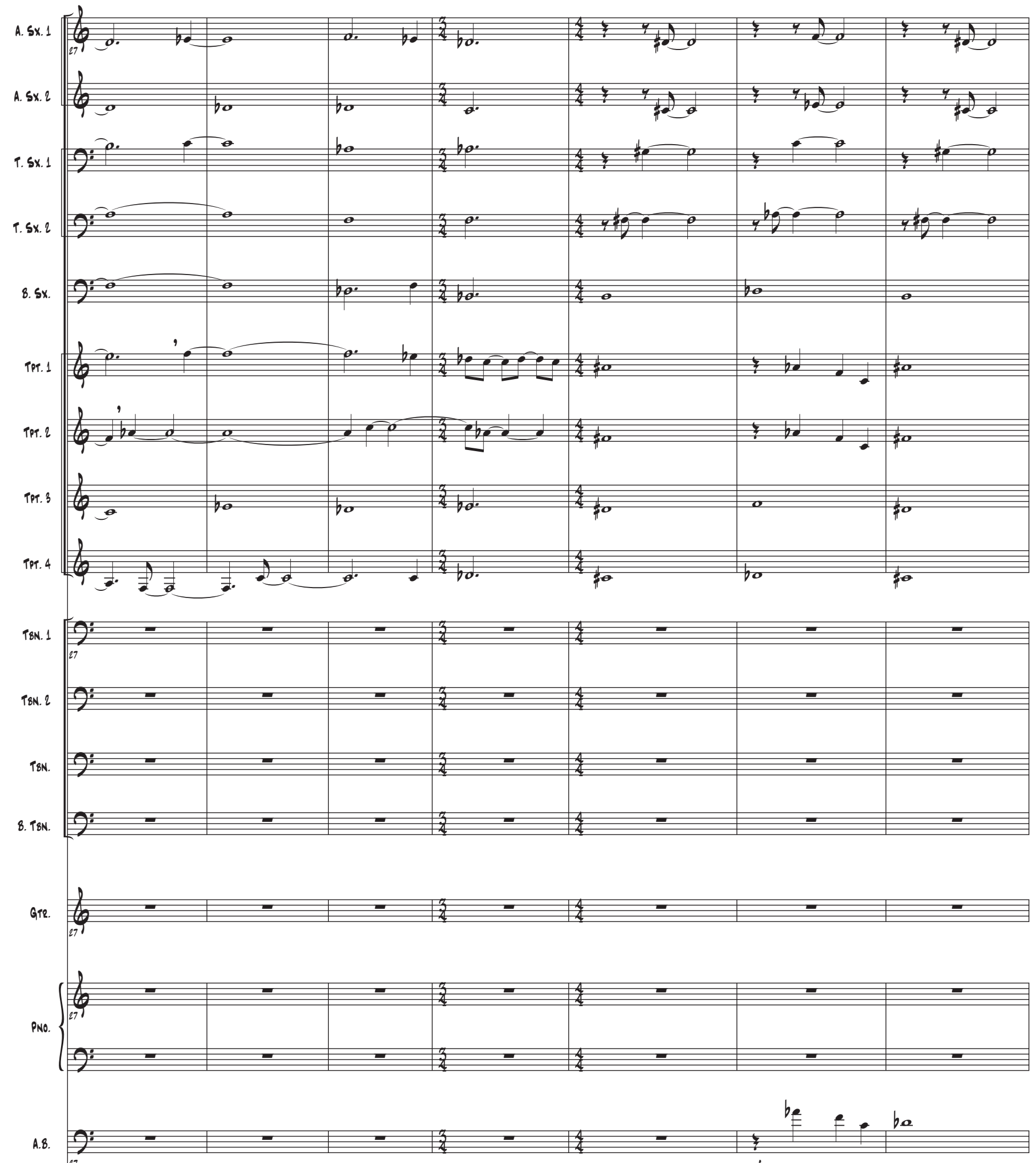

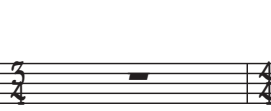

o.s.

27

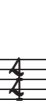

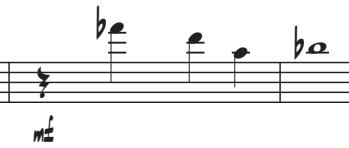



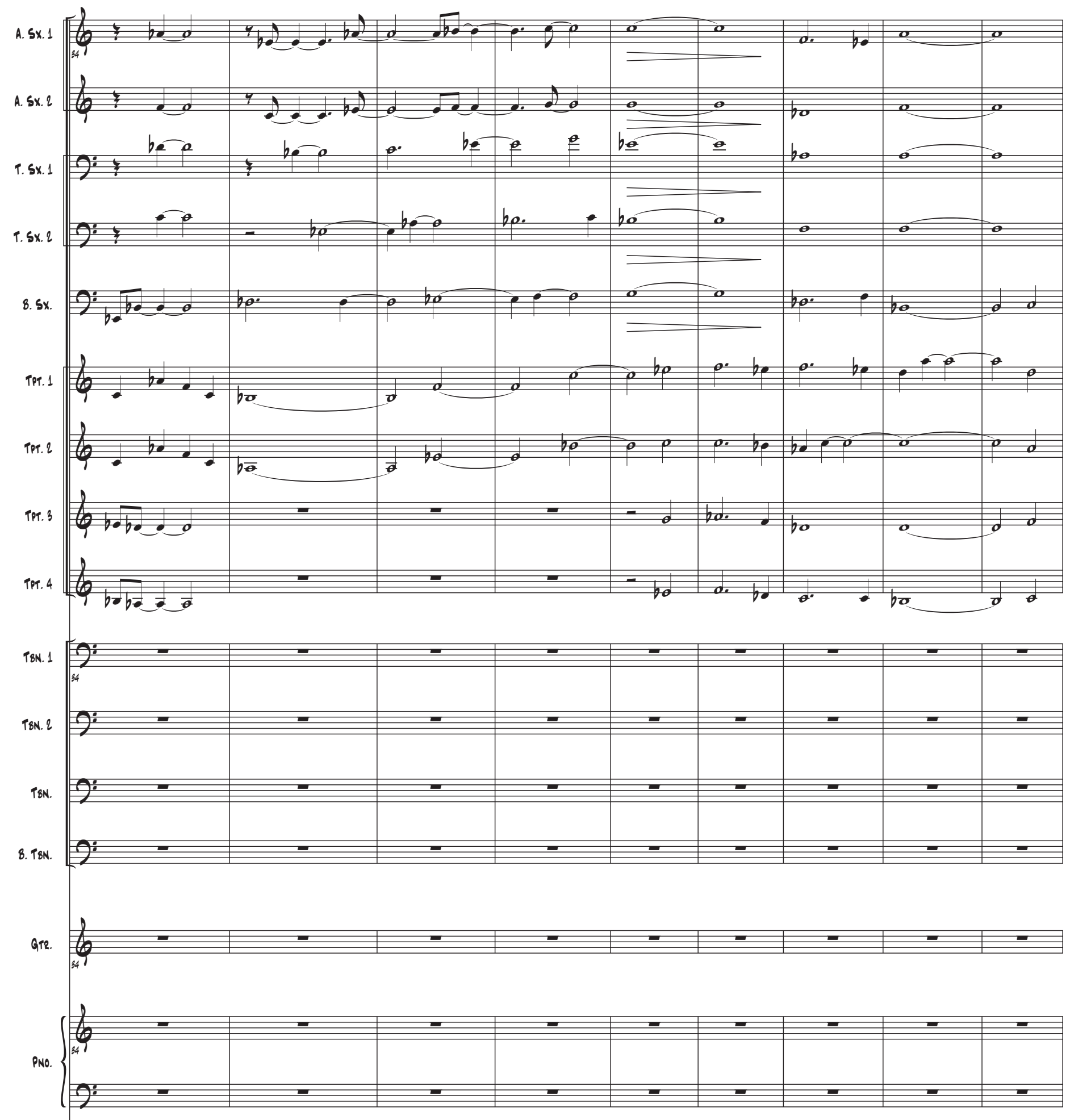

A.8.

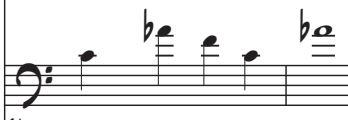

o. 5 .

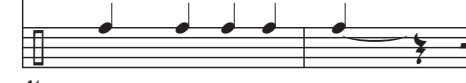



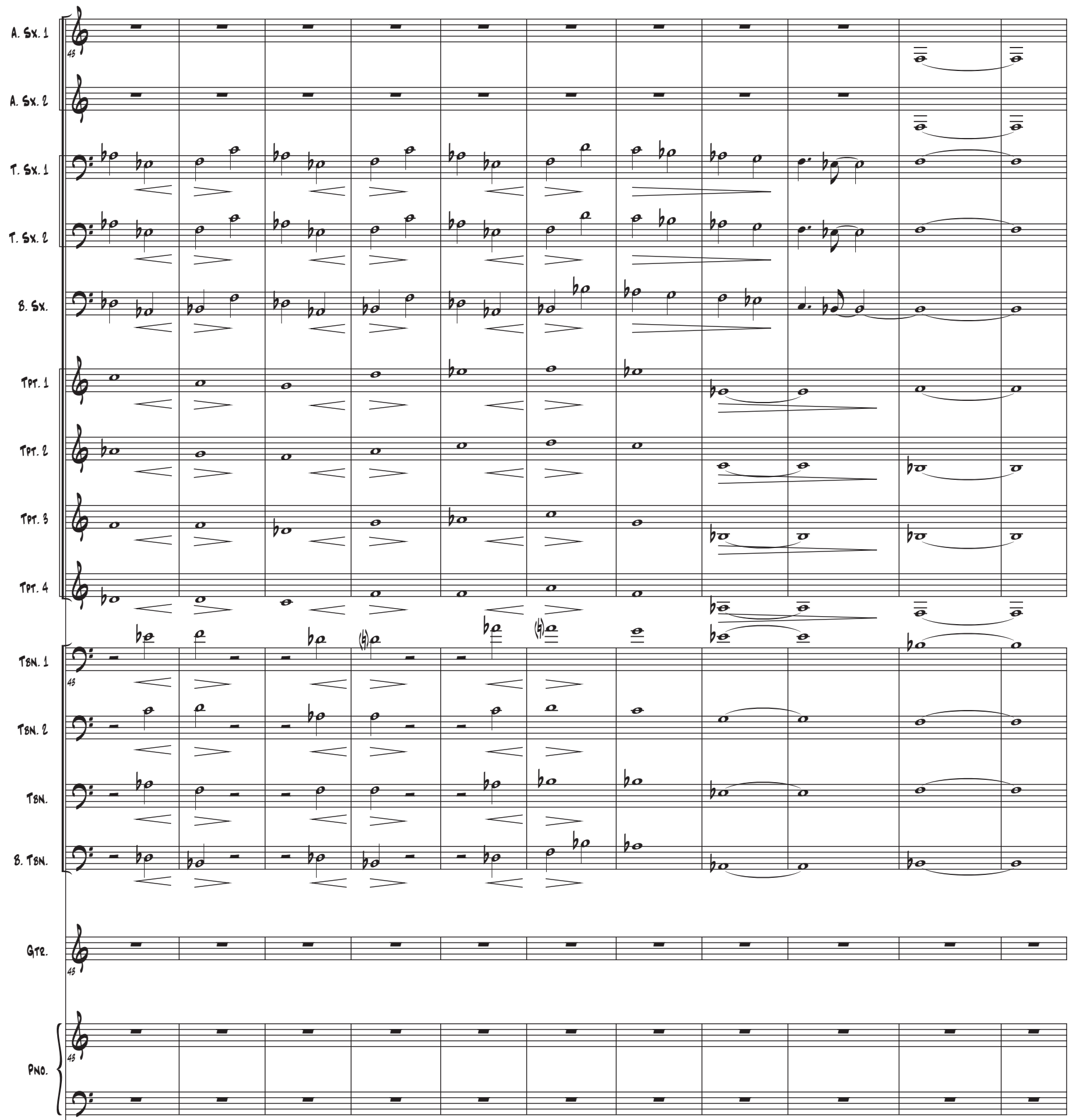

A.8.

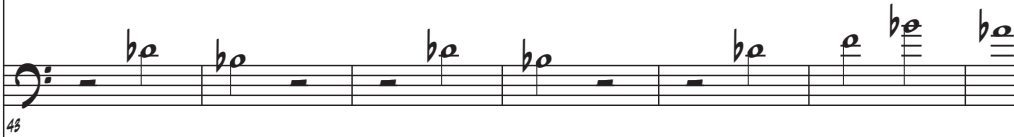

o. 5

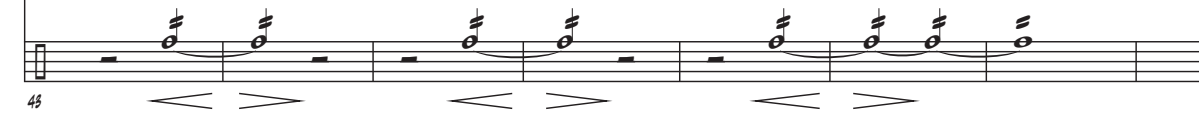



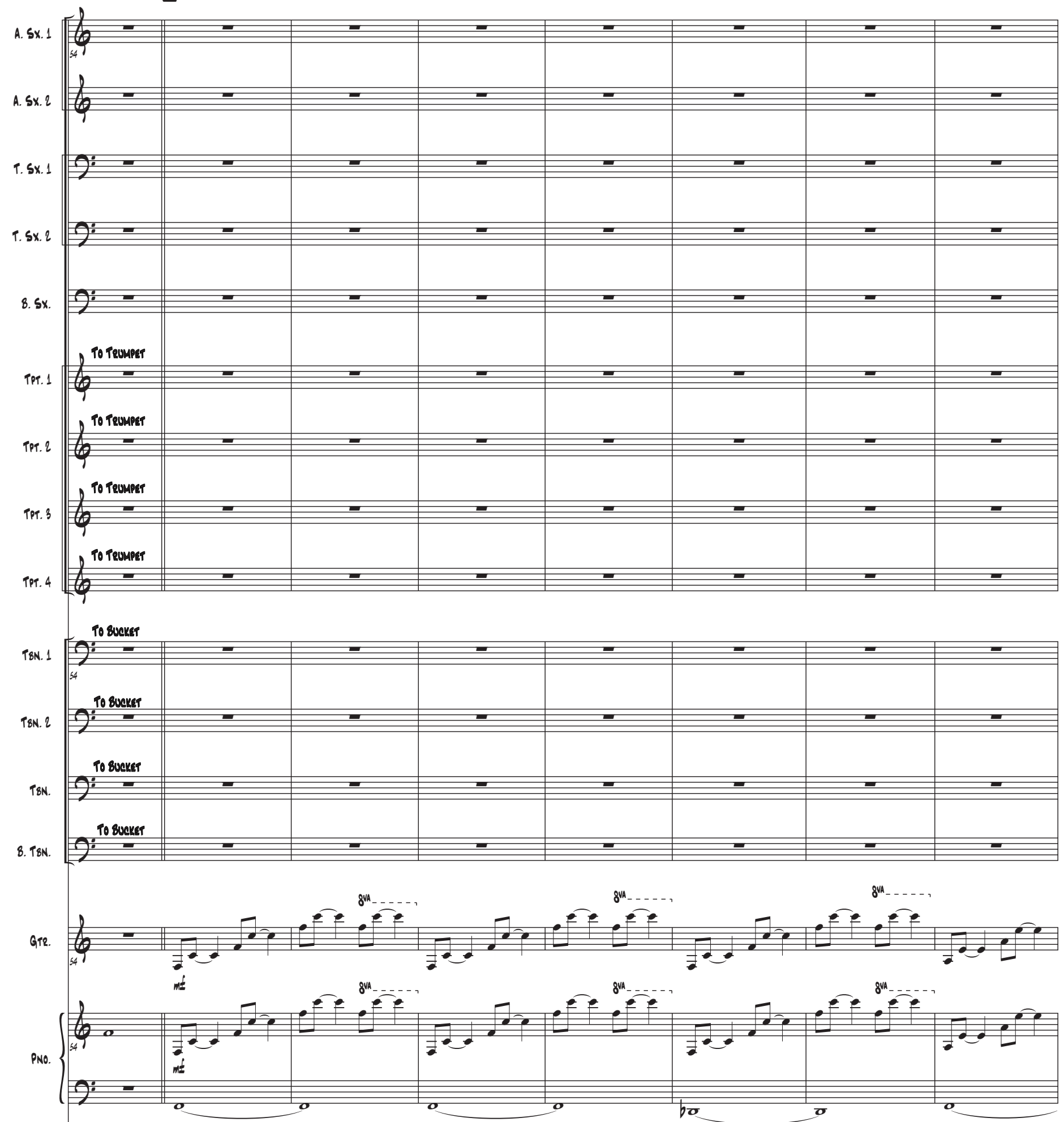

A.8.

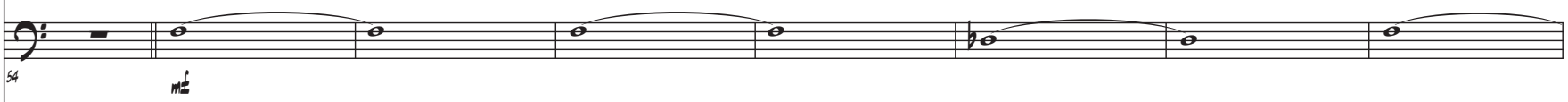

0.5

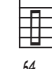



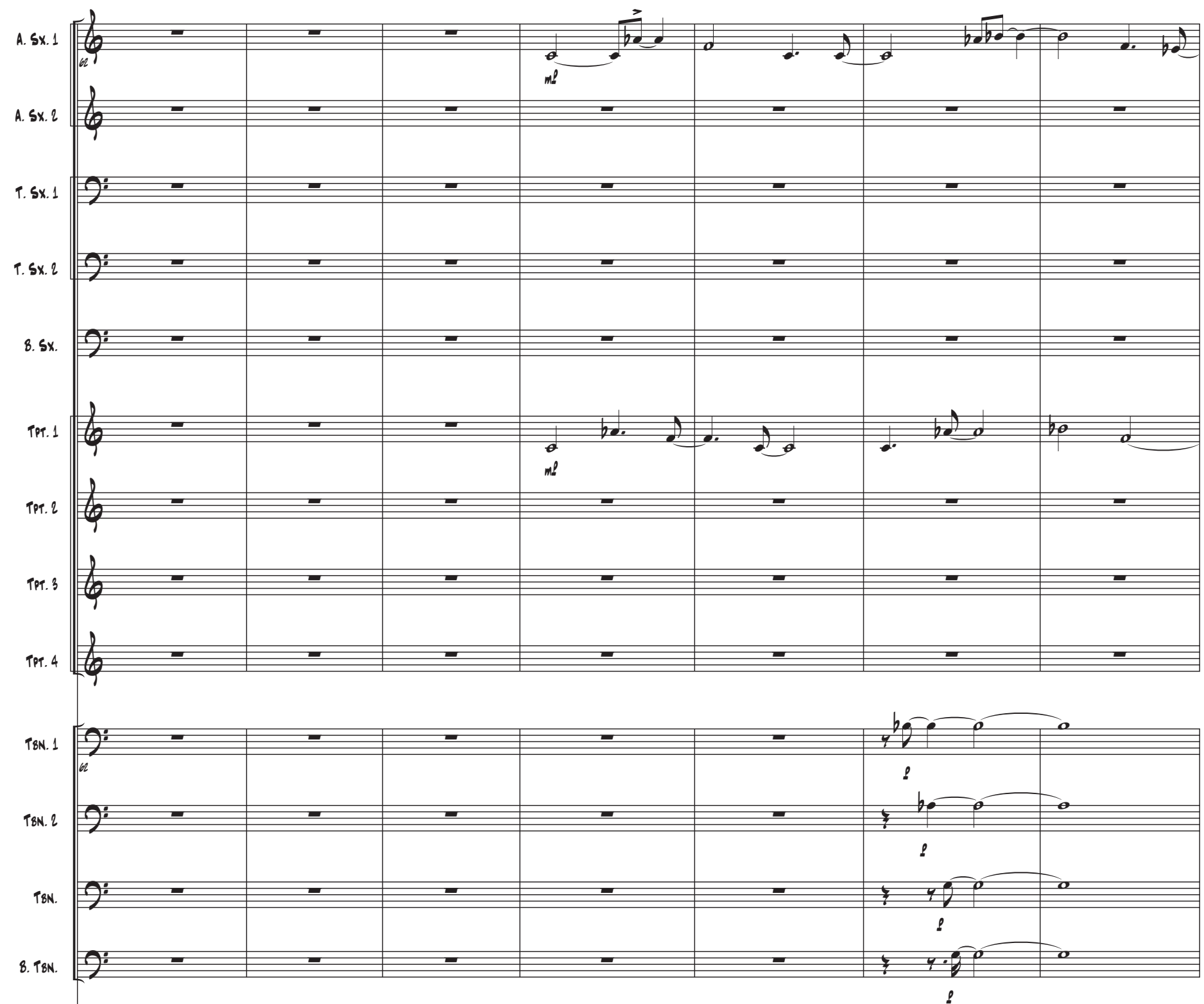
$8^{14}$.......

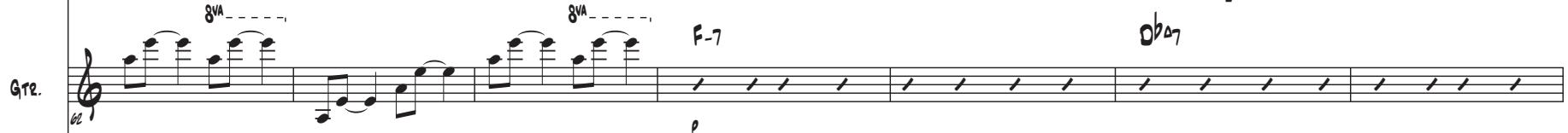

GT2.

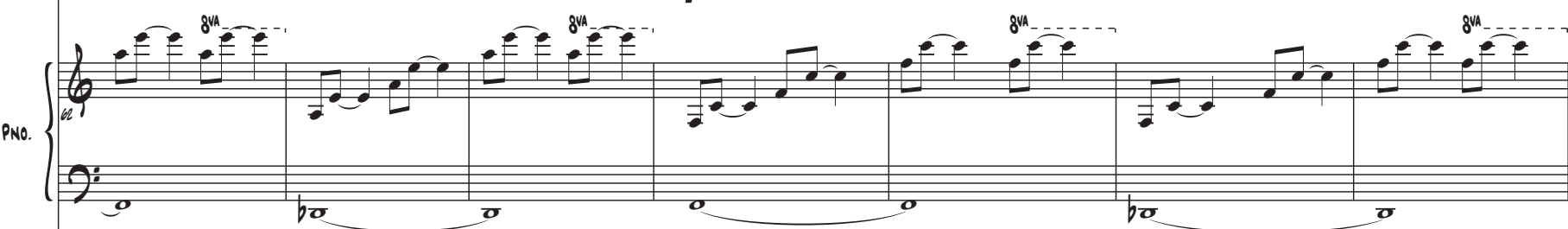

F-7

$b_{b 7}$

A.8.

T:०

0.5

62

ice

20 

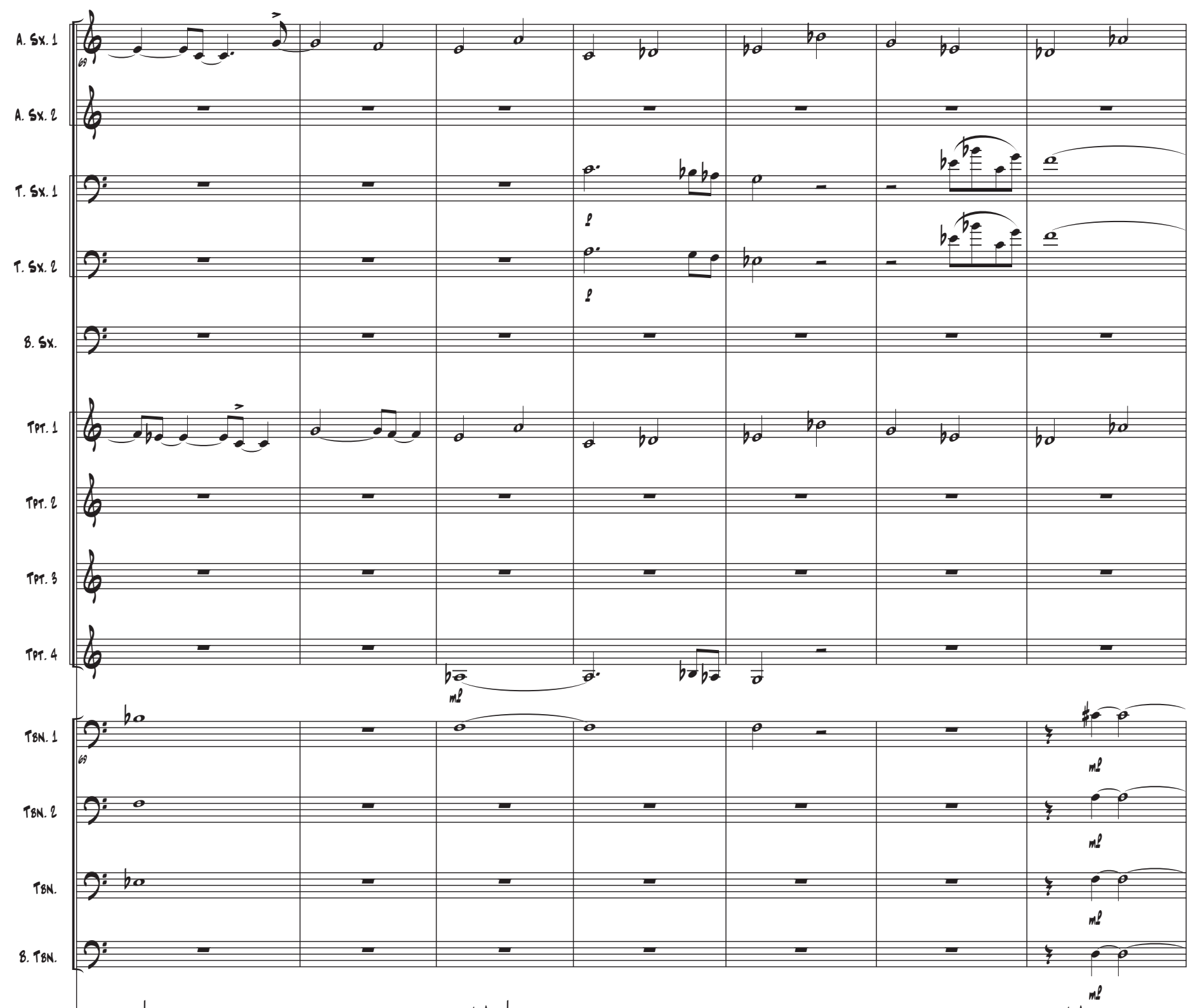

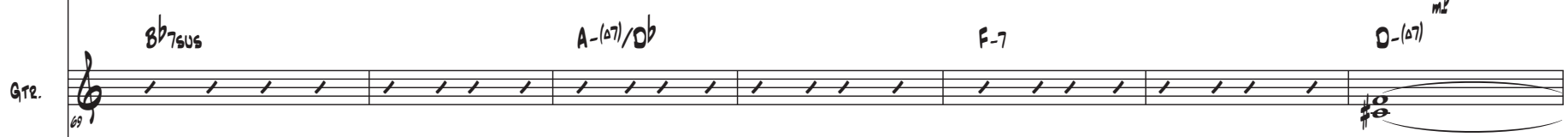

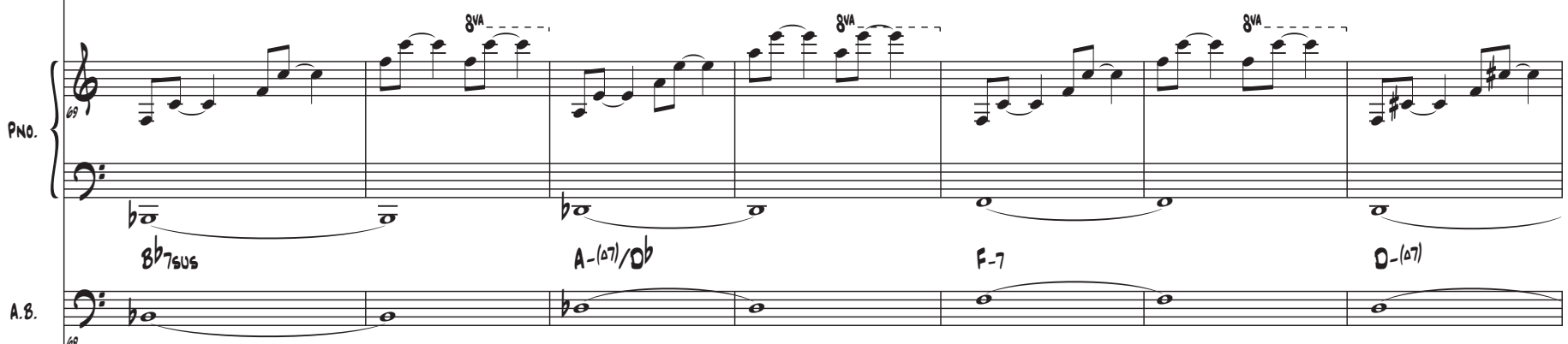

o. 5 .

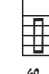



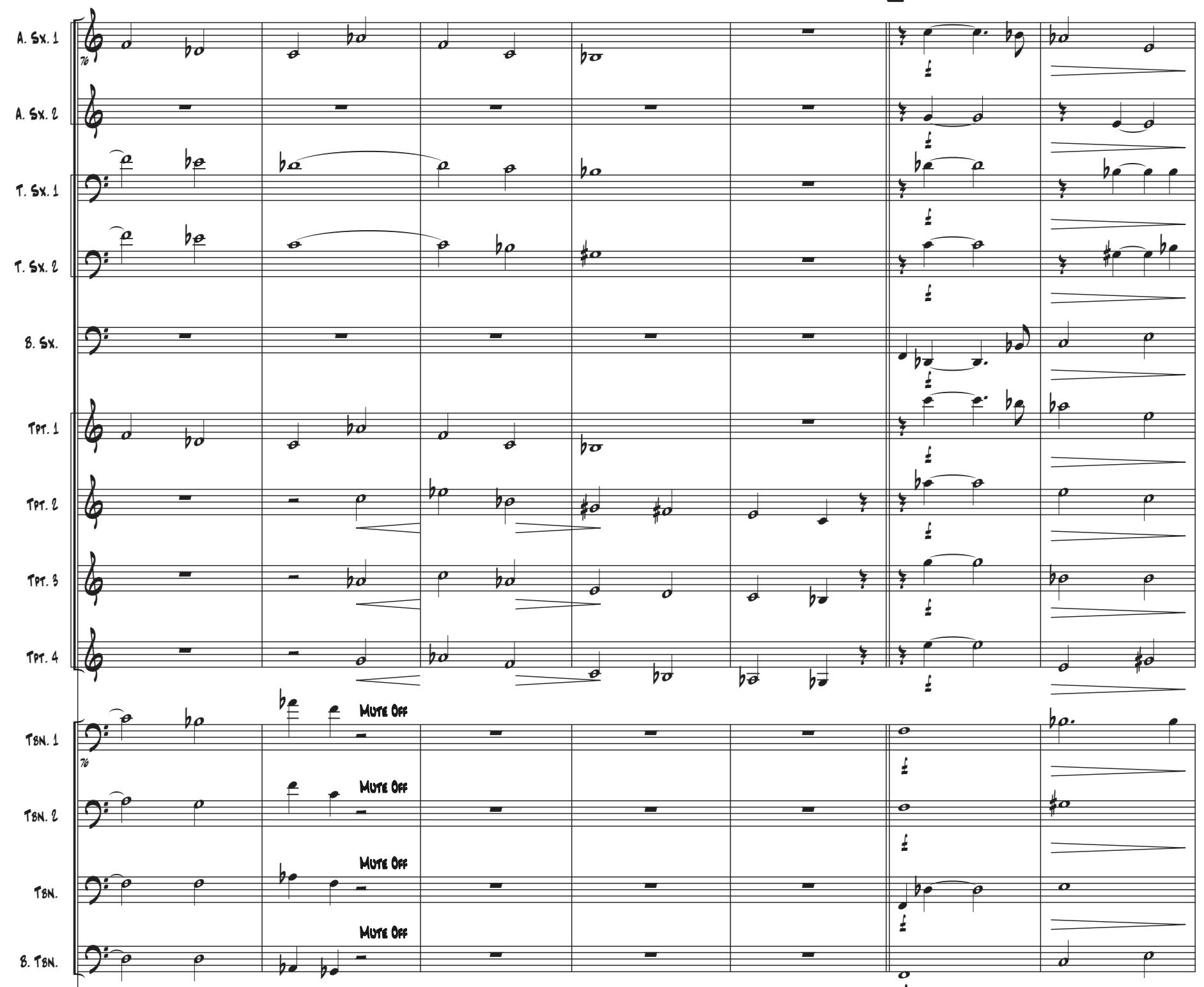

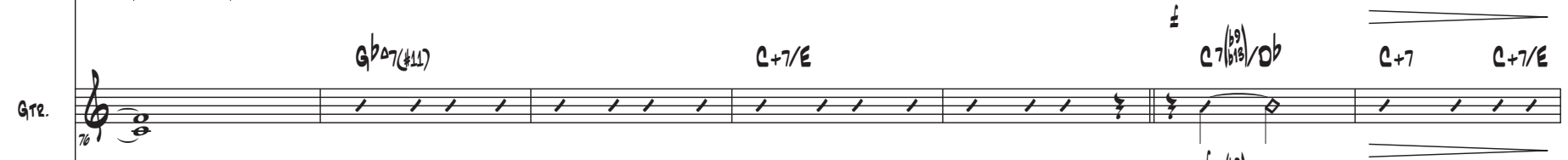

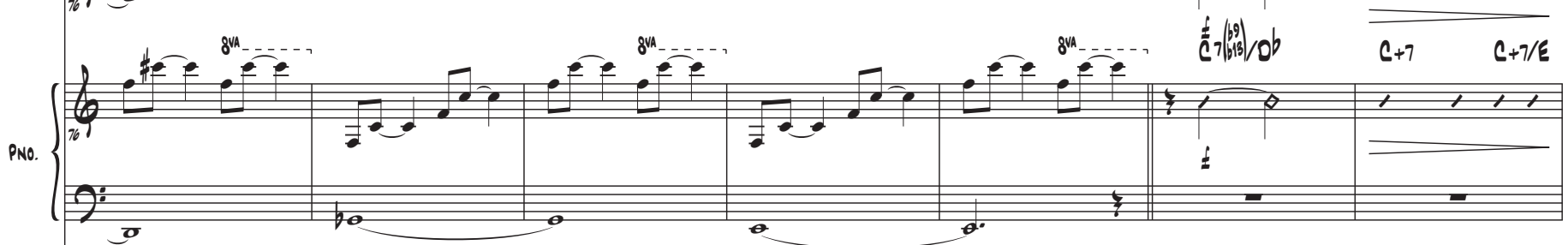

A.8.

$G^{b_{0}(41)}$

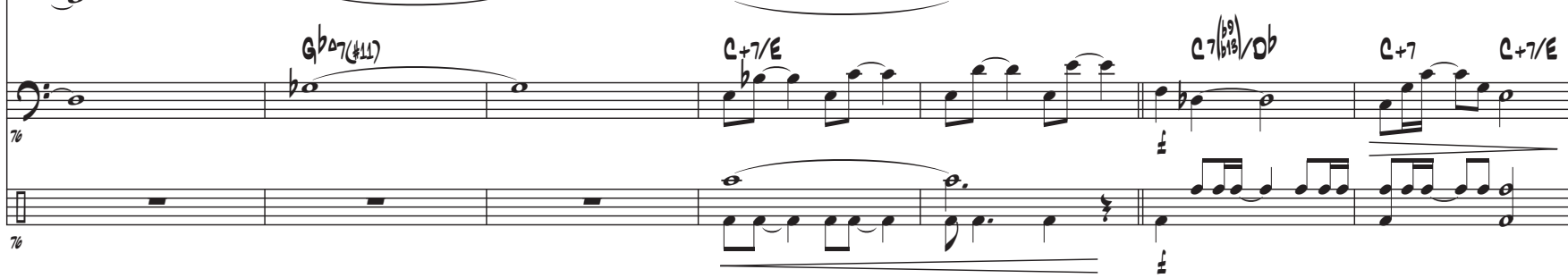



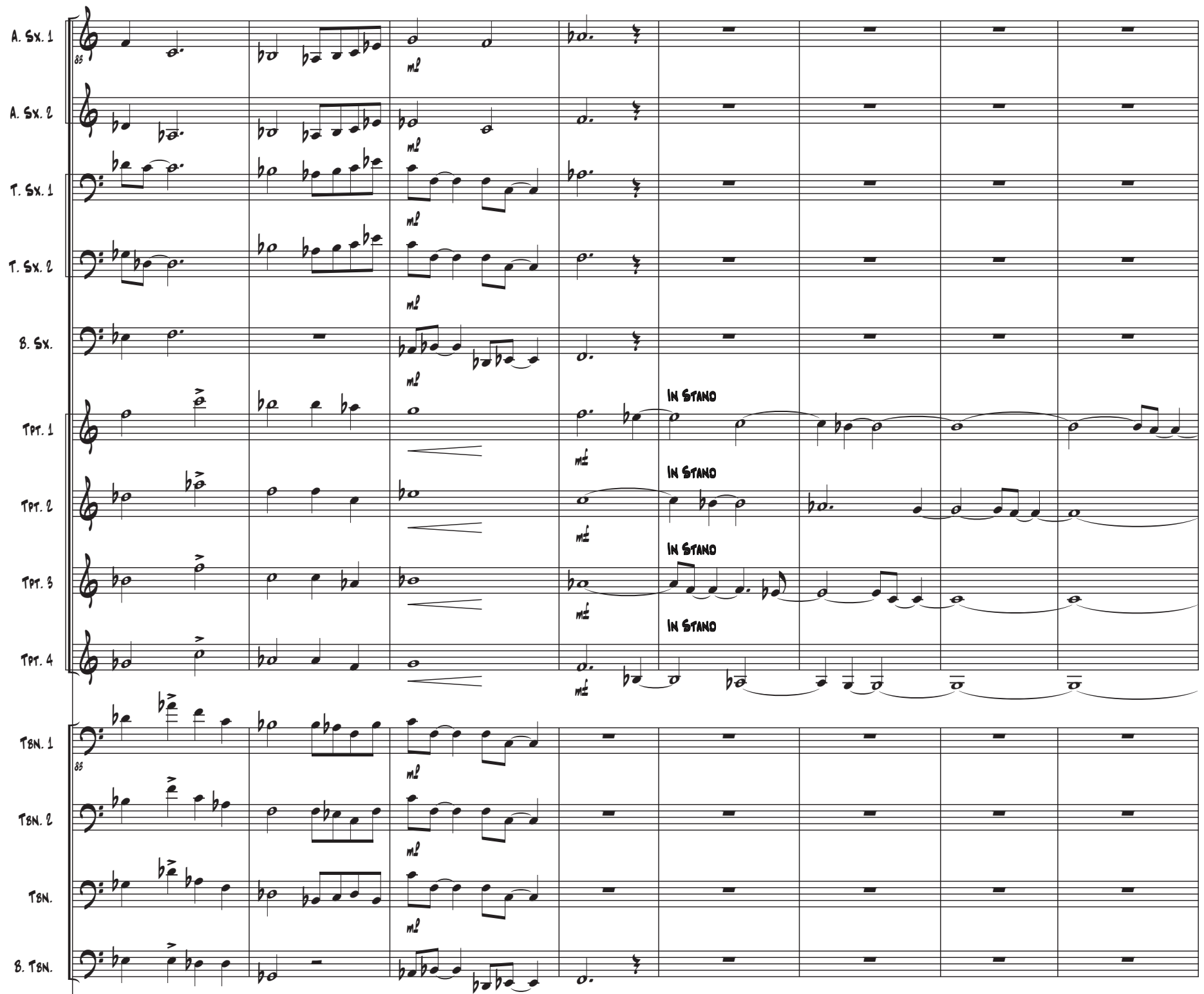

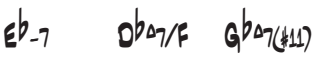
Ab ob
f-

Gie. $\int_{83}$
$E b_{-7} \quad O b_{\Delta 7 / F} \quad G b_{\Delta \rightarrow(411)}$
Ab ob

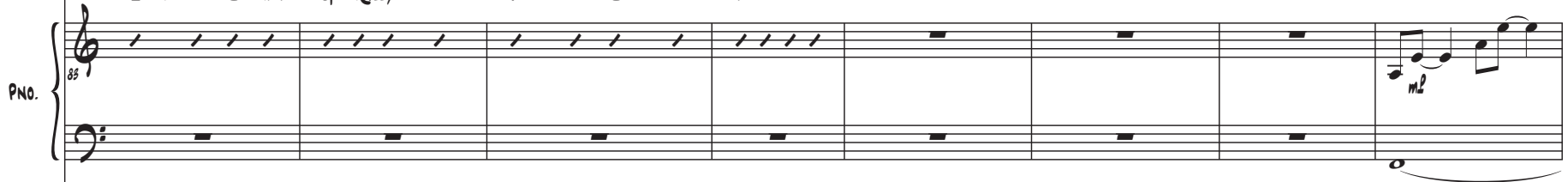

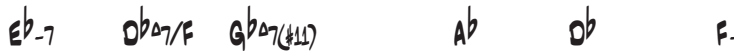

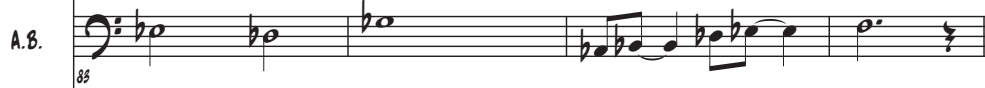

0.5.

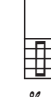


1.1.
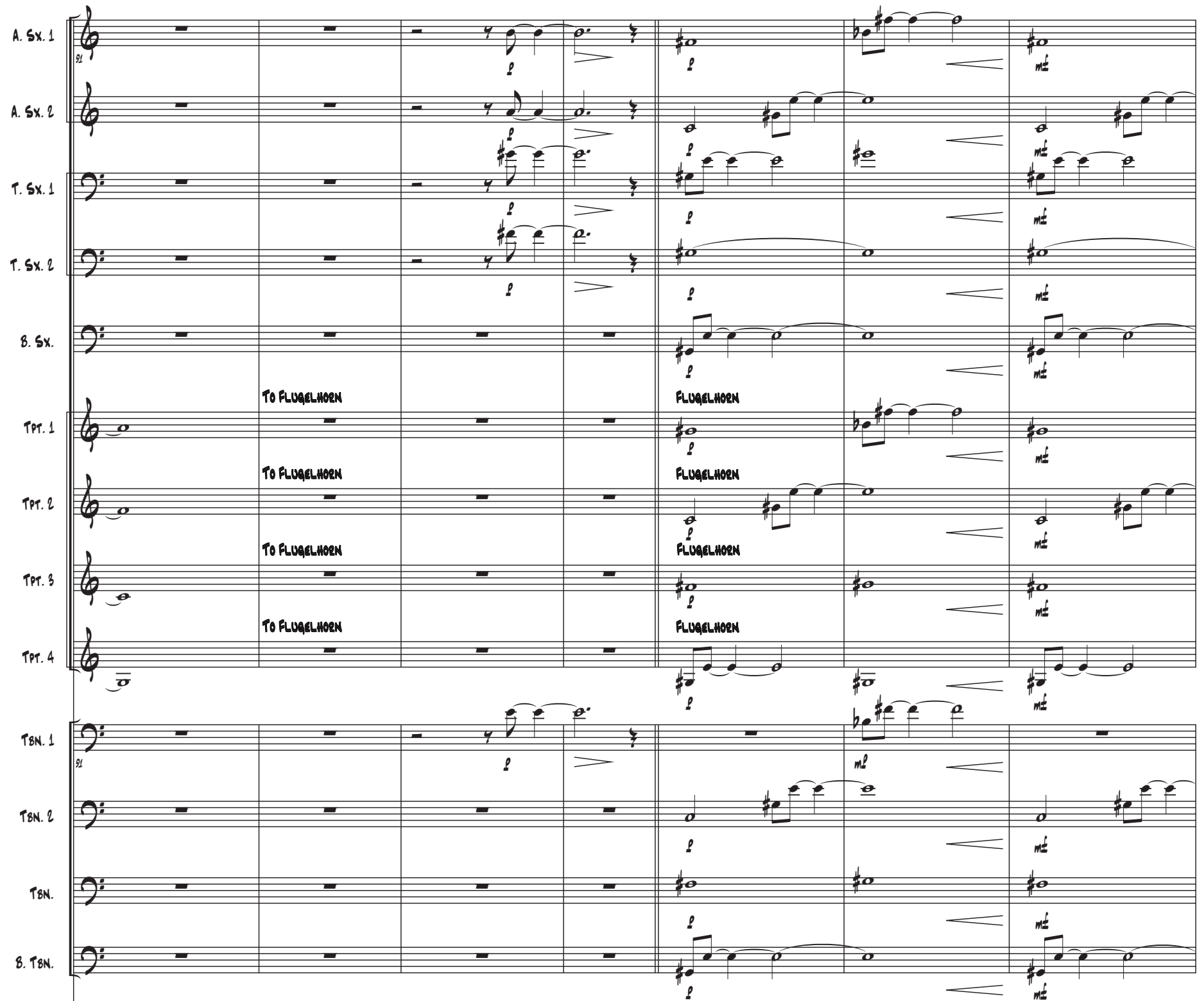
$8^{14}$

Gre.

Gre. $b_{1}=0$

Gre.

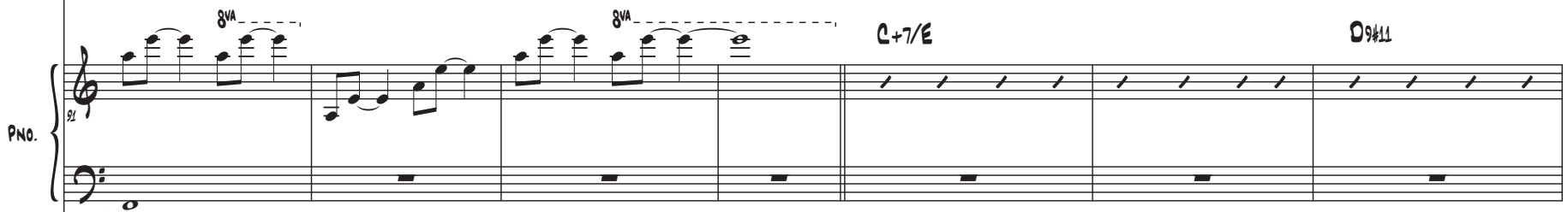

$C+7 / E$

O.41

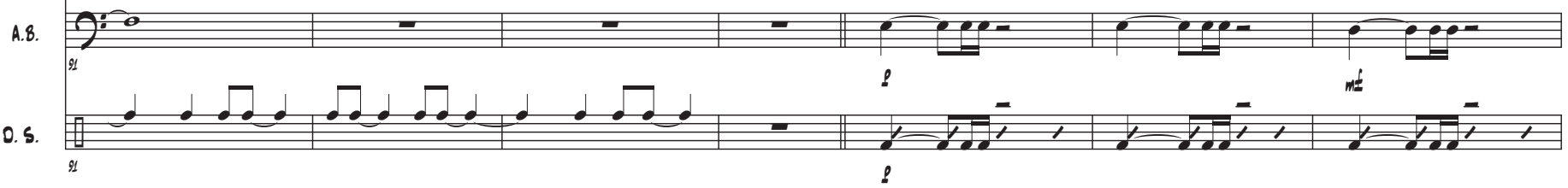



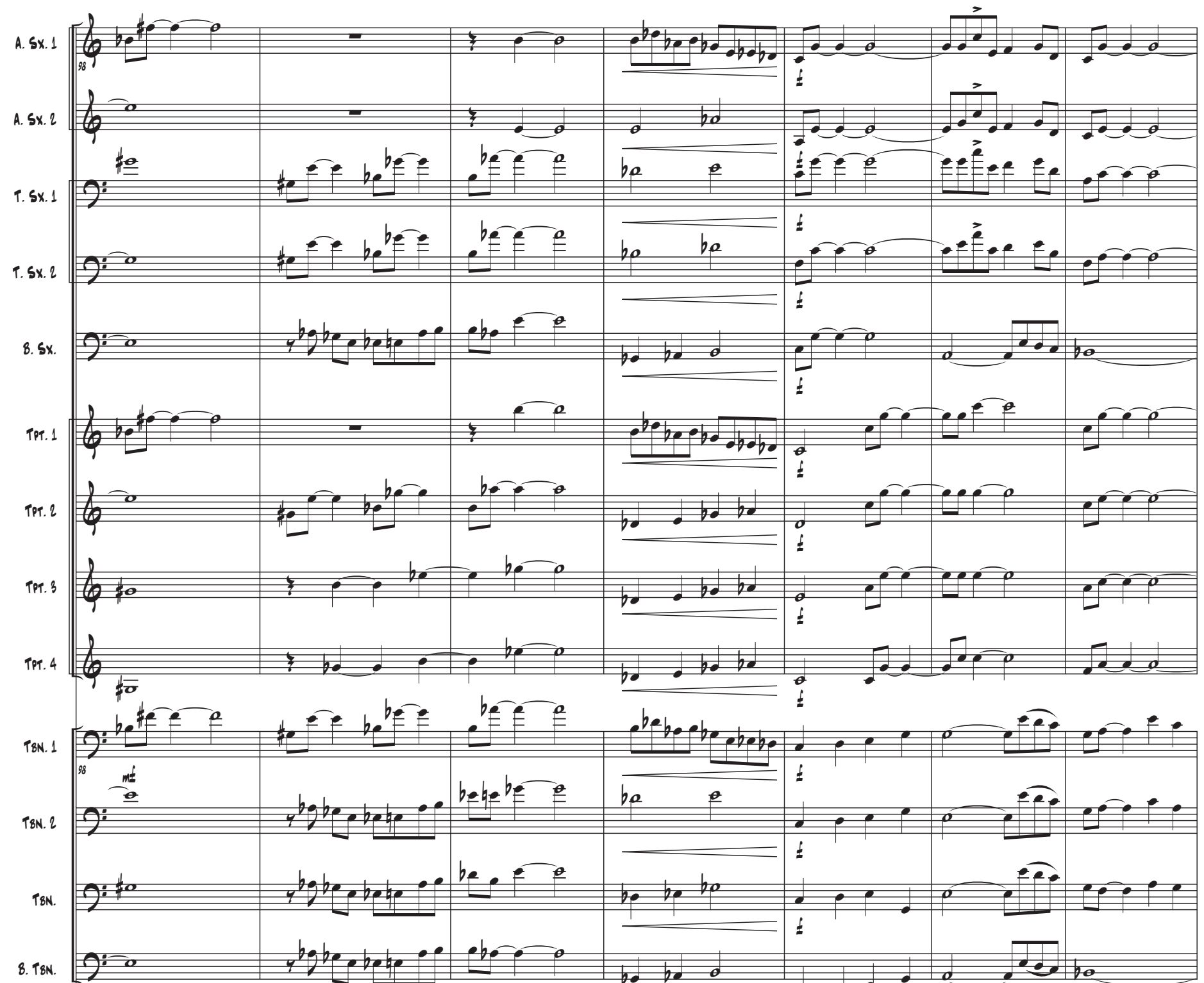

8. I8N.
$a b-11$

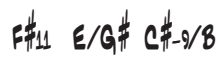
$\mathrm{CD}_{7}$
A-7
$8 b_{9}$

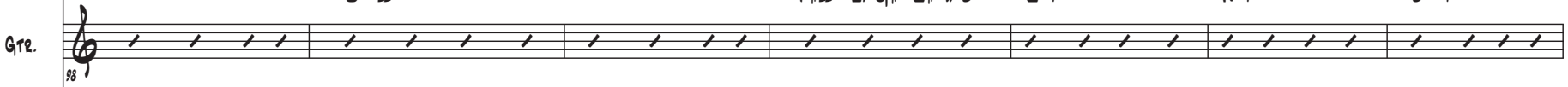

ob-11

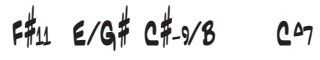

A-7

$B b \Delta$

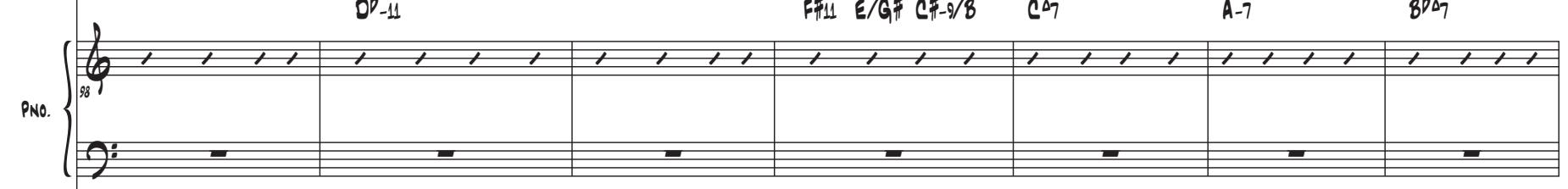

$0 b-11$

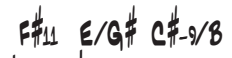

A.8.

A.8.

A.8.

0.5. 

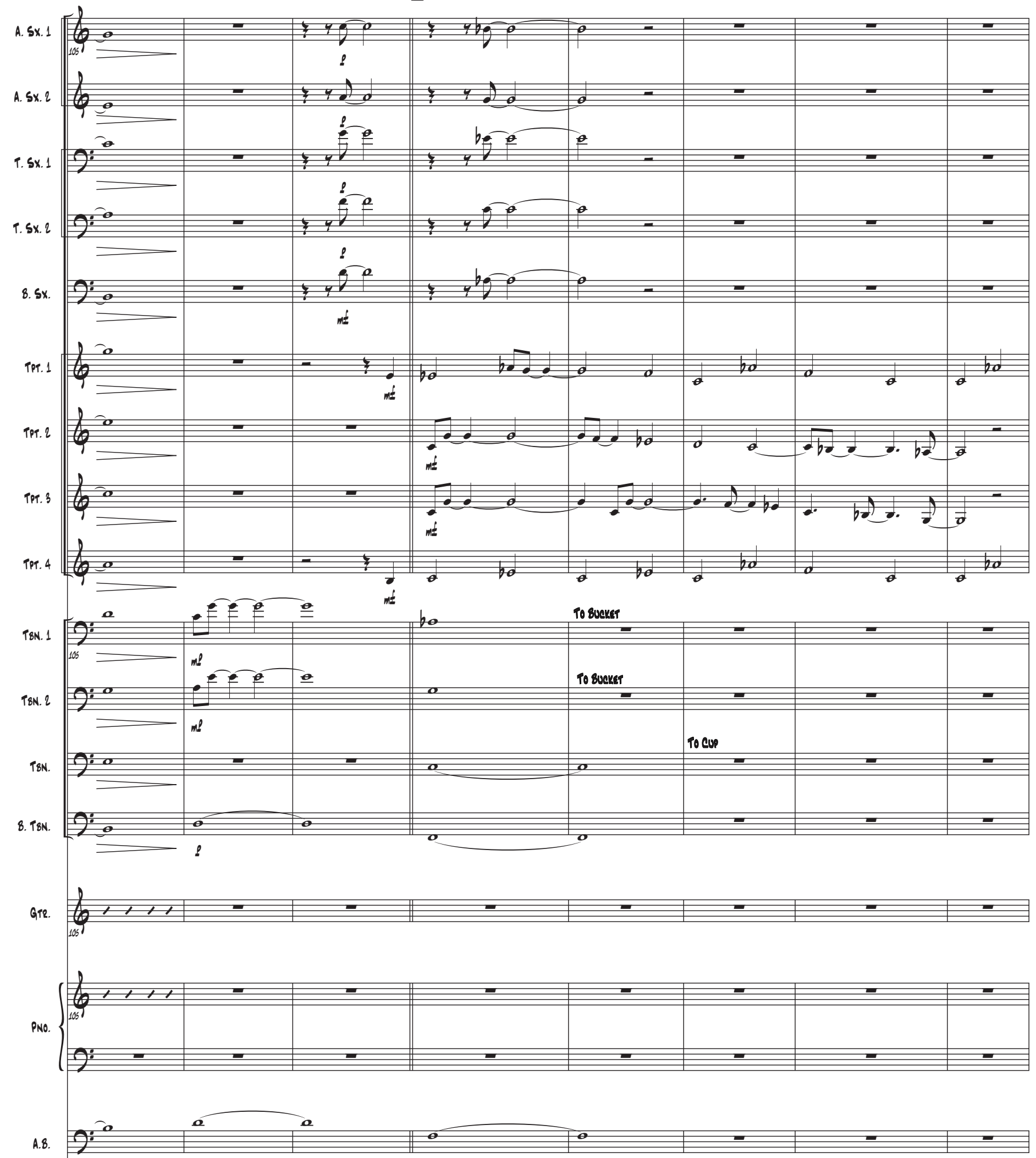
$105=\mathrm{m}$ ?

.5. 

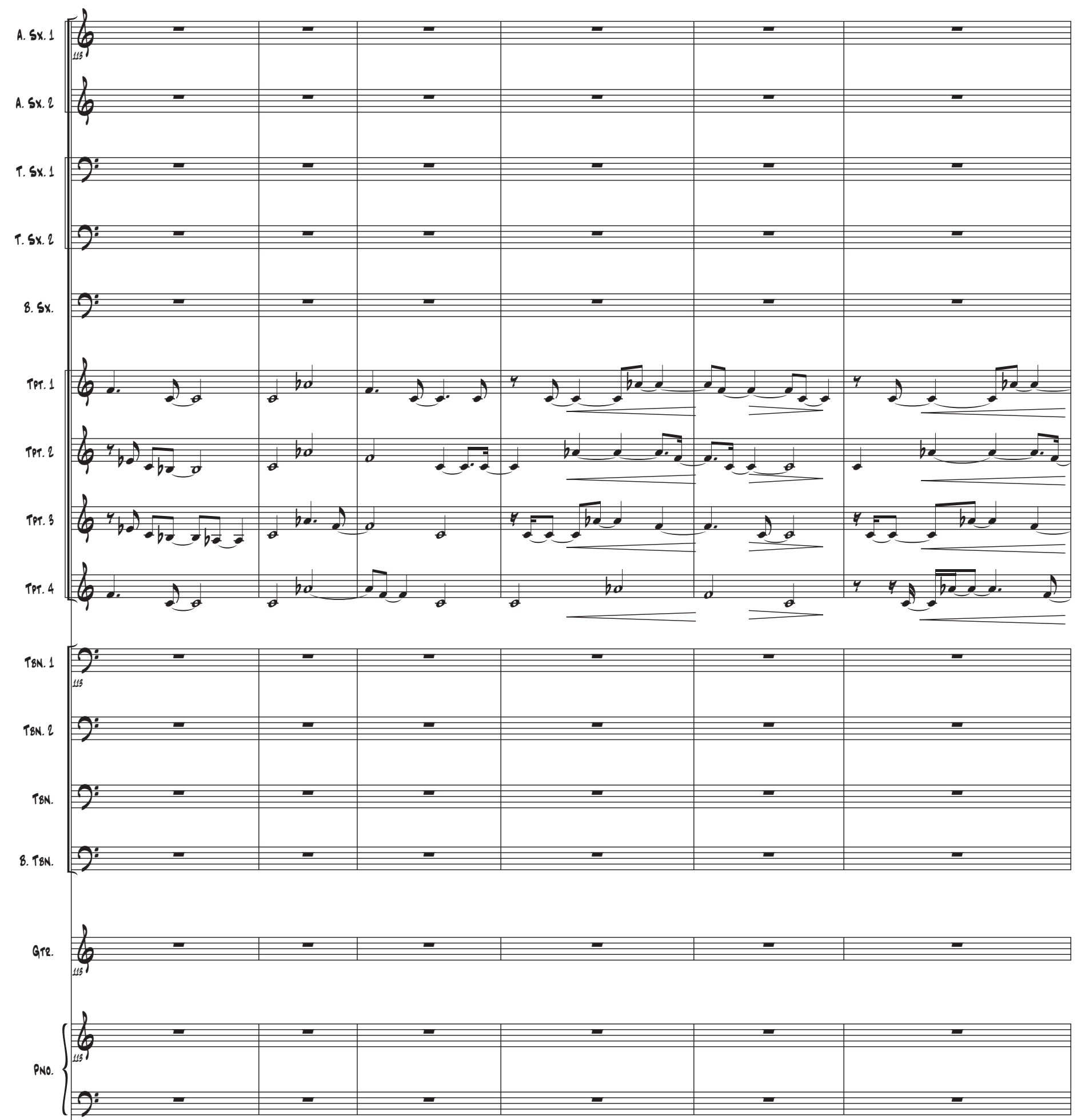

A.8.

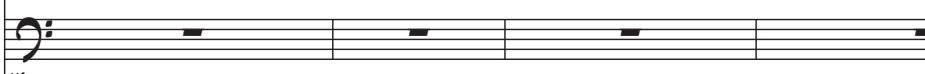

0.5 113

0.5. 

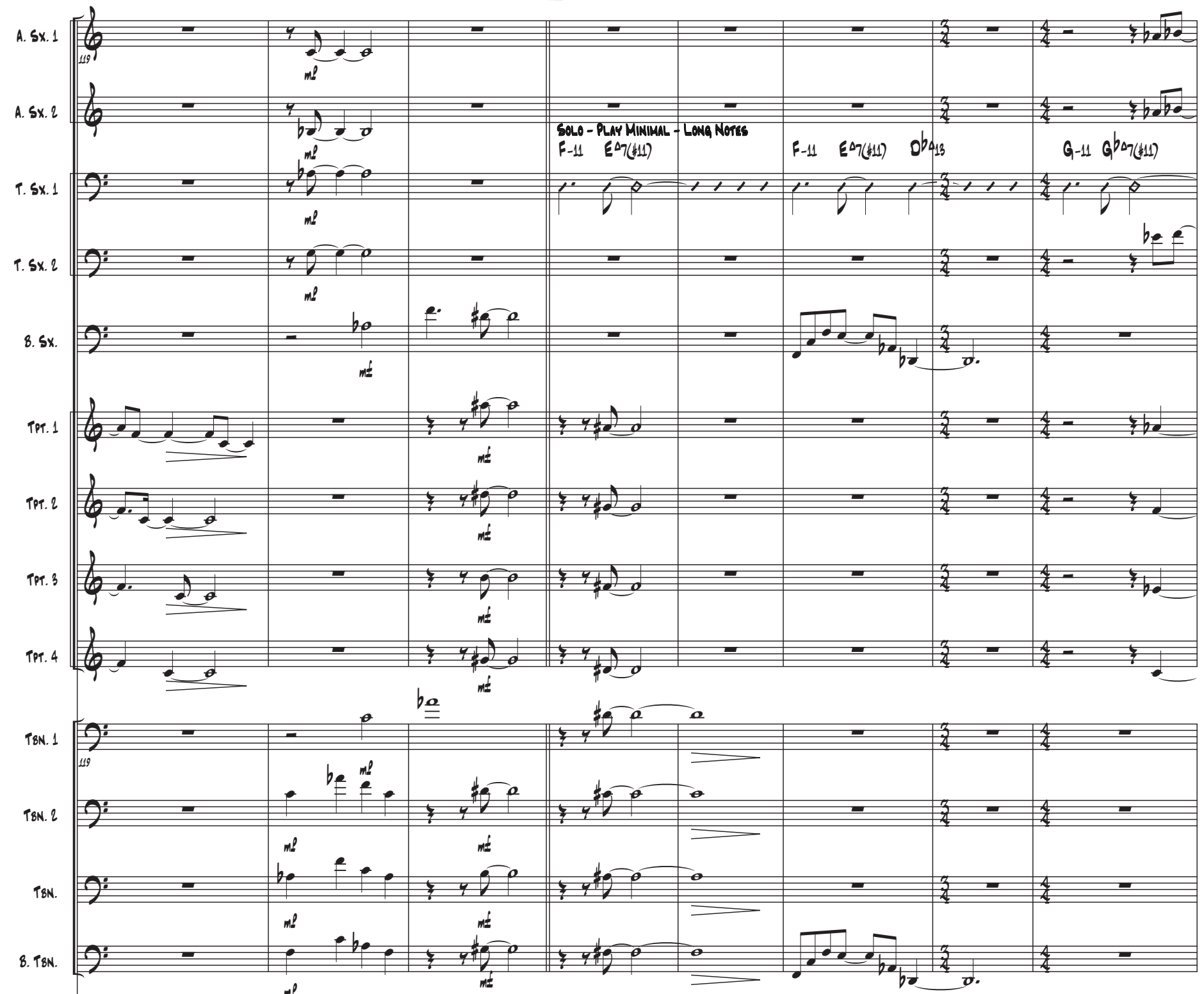

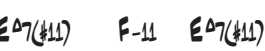
F-11 $\quad 507(411) \quad D b_{13}$
$G-11 G^{b} 07(411)$

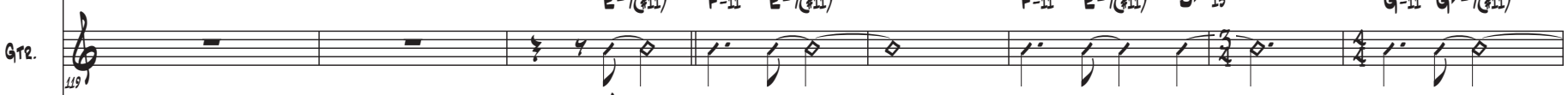

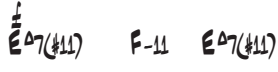
F-11 EAn(\$11) $\quad 0 b_{13}$
$G-11 G^{b} \rightarrow(411)$

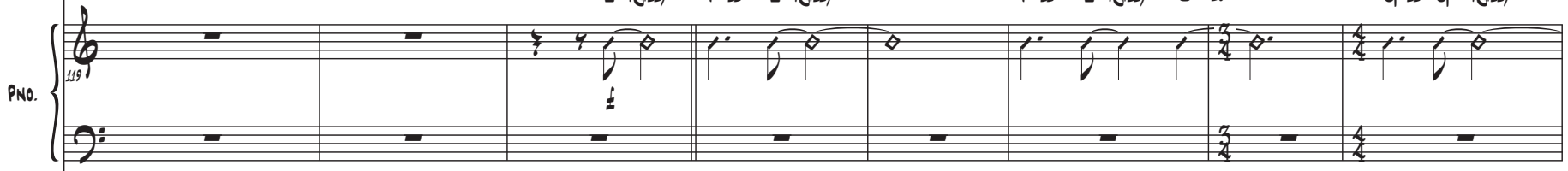

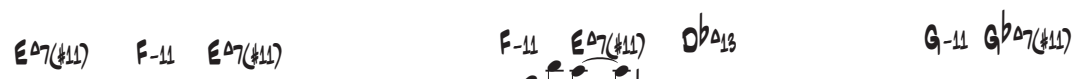

A.8.

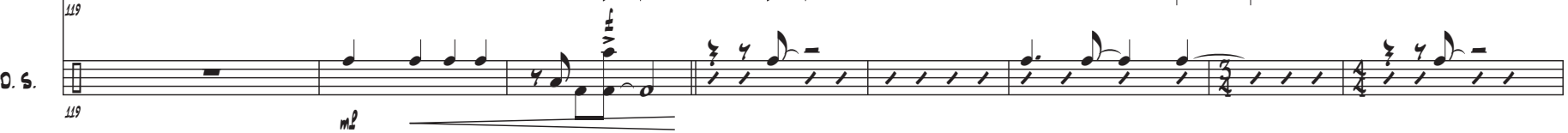




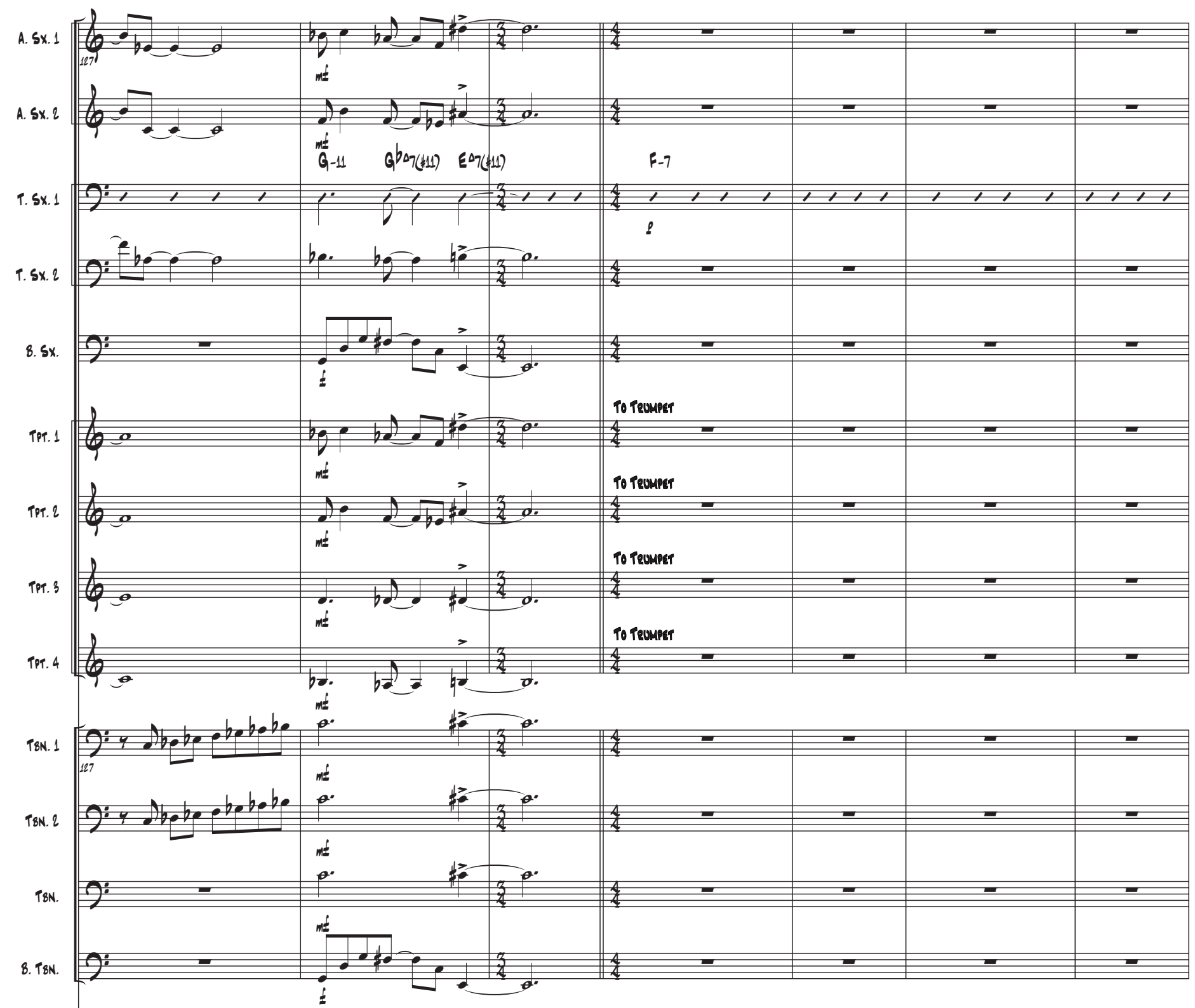
G-11 $\quad G_{\Delta \rightarrow(411)} E^{\Delta} \rightarrow(411)$
8-7

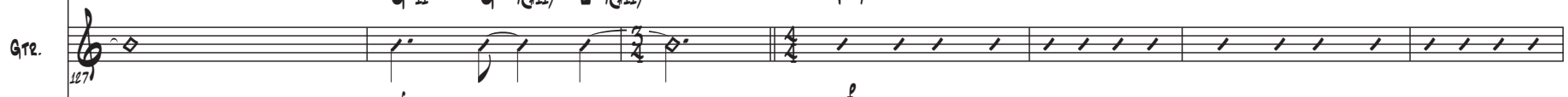

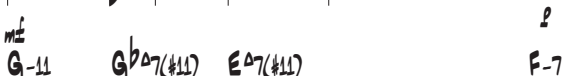

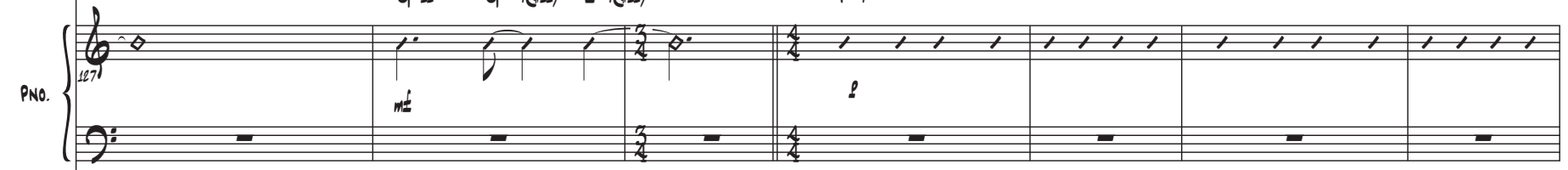

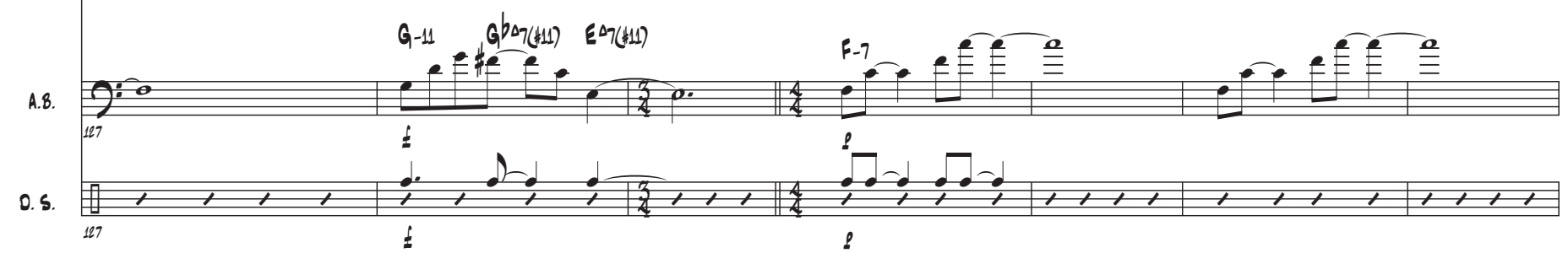




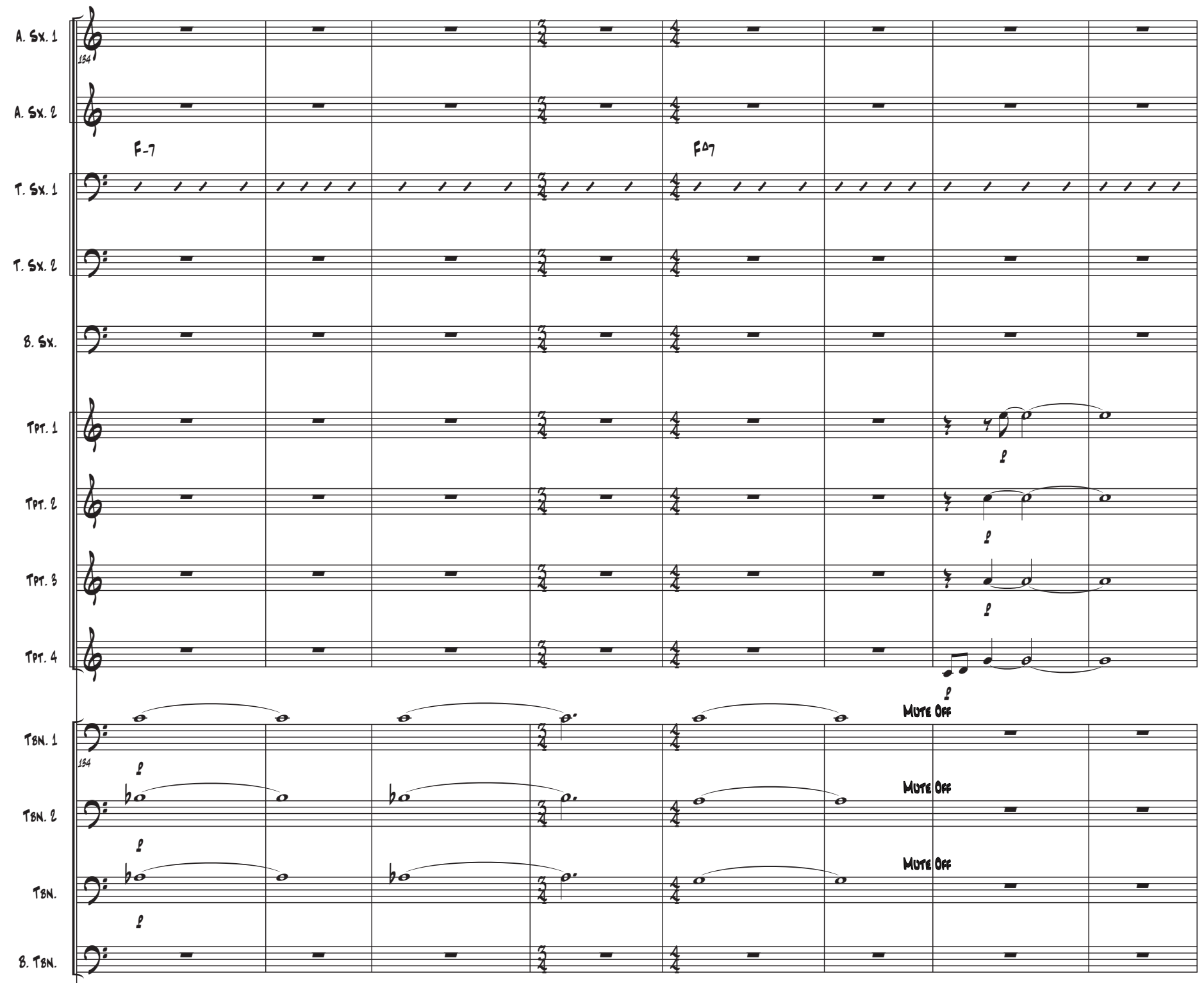

$\beta \Delta q$

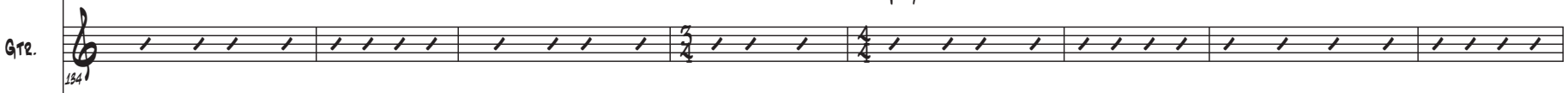
$\mathrm{FA}_{7}$

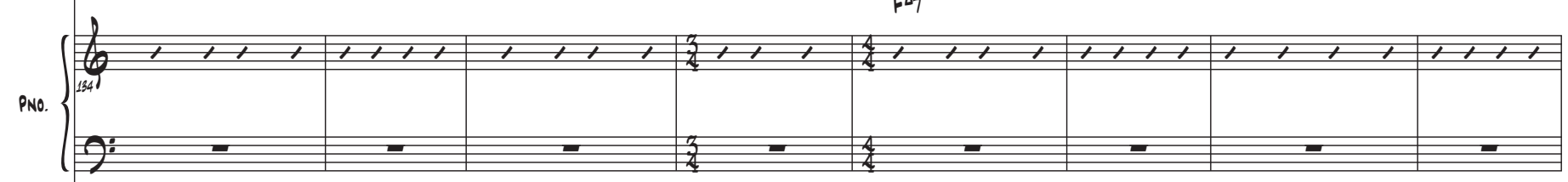

A.8.

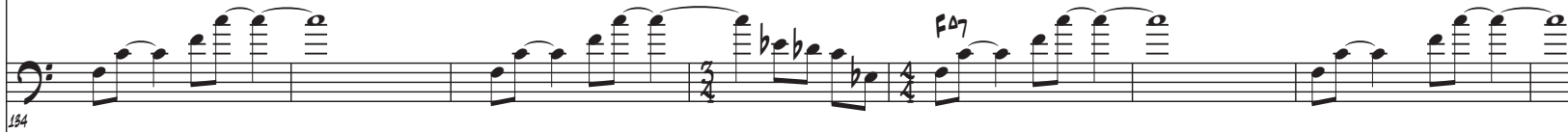

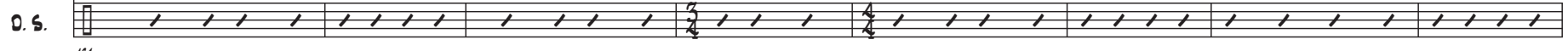




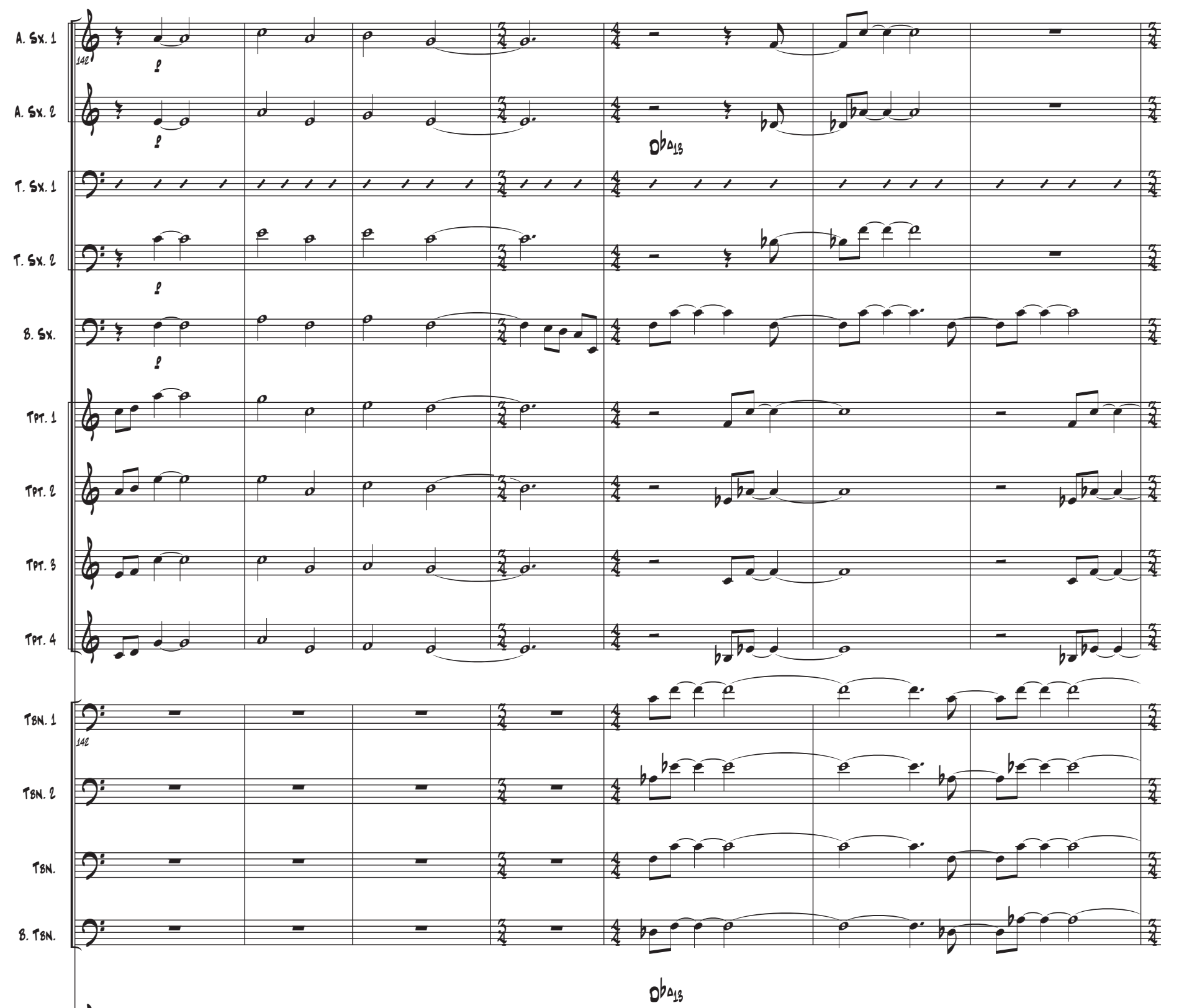

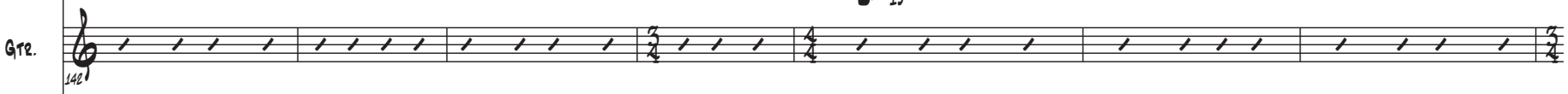
obda

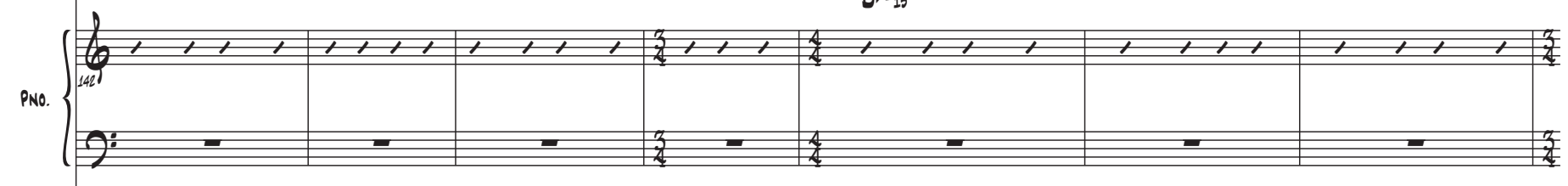

A.8.

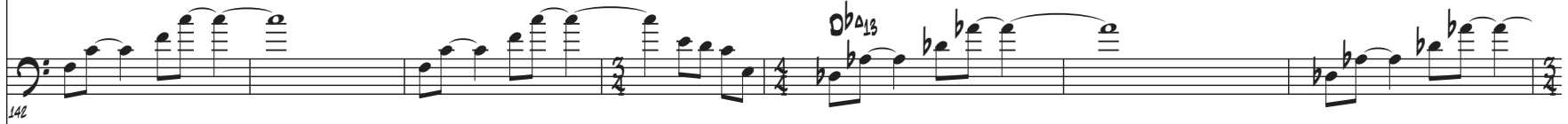

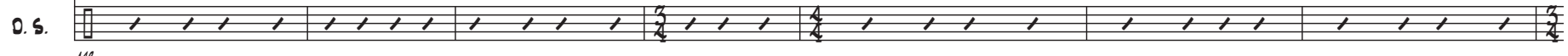



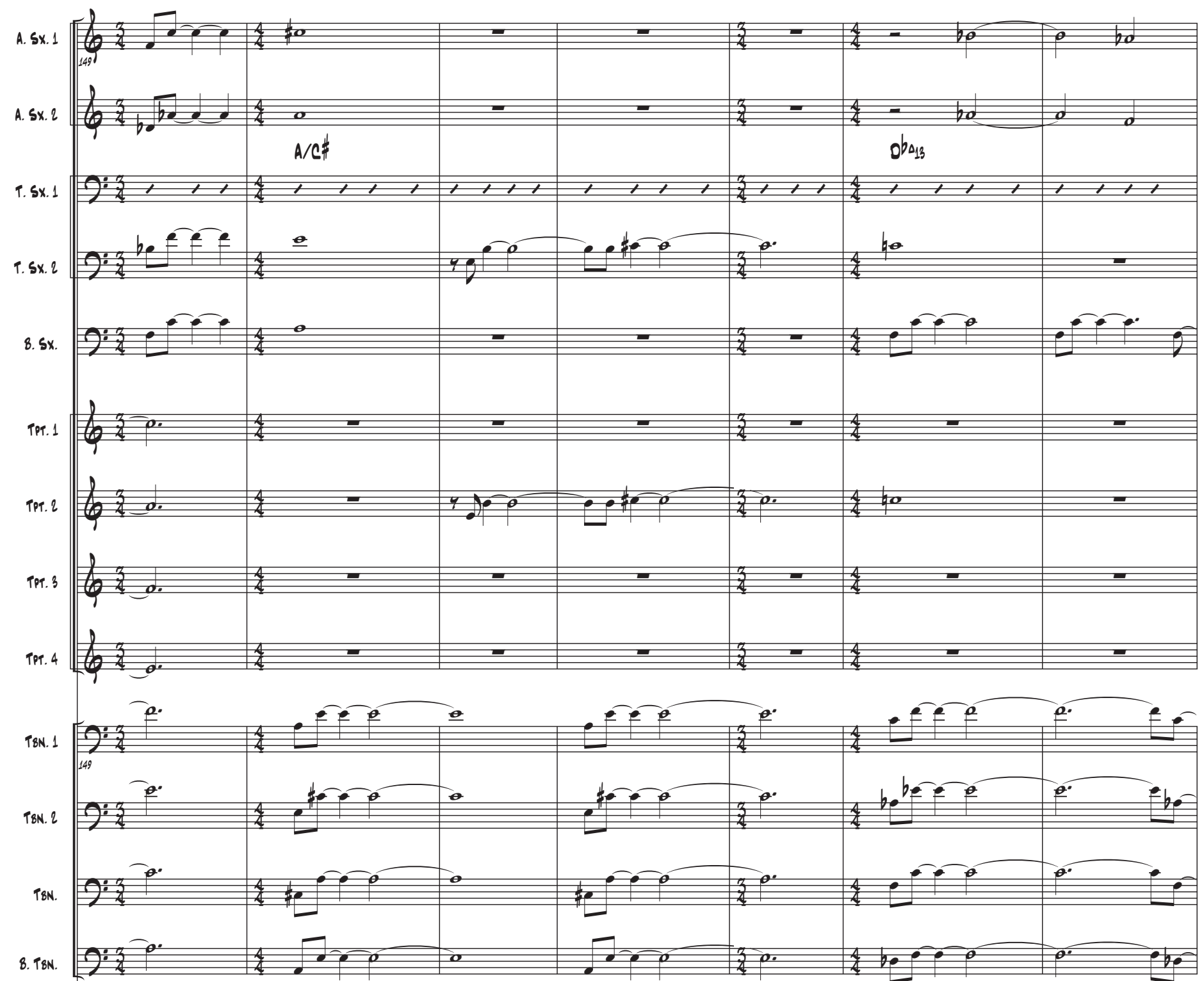
Gre.

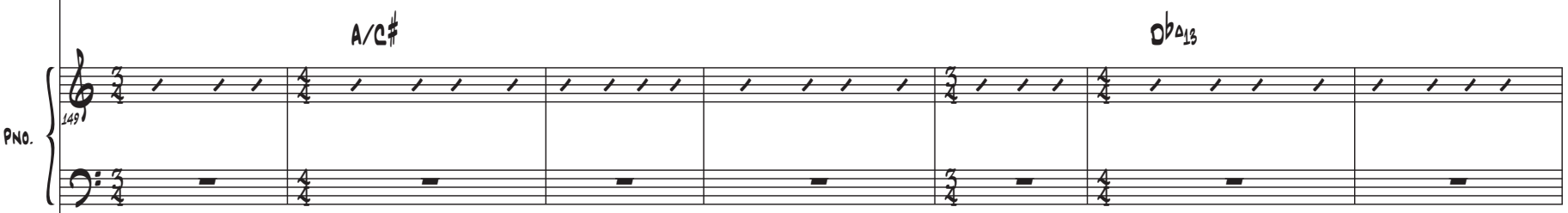
A.8.

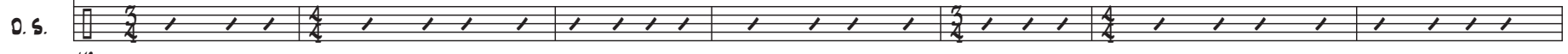



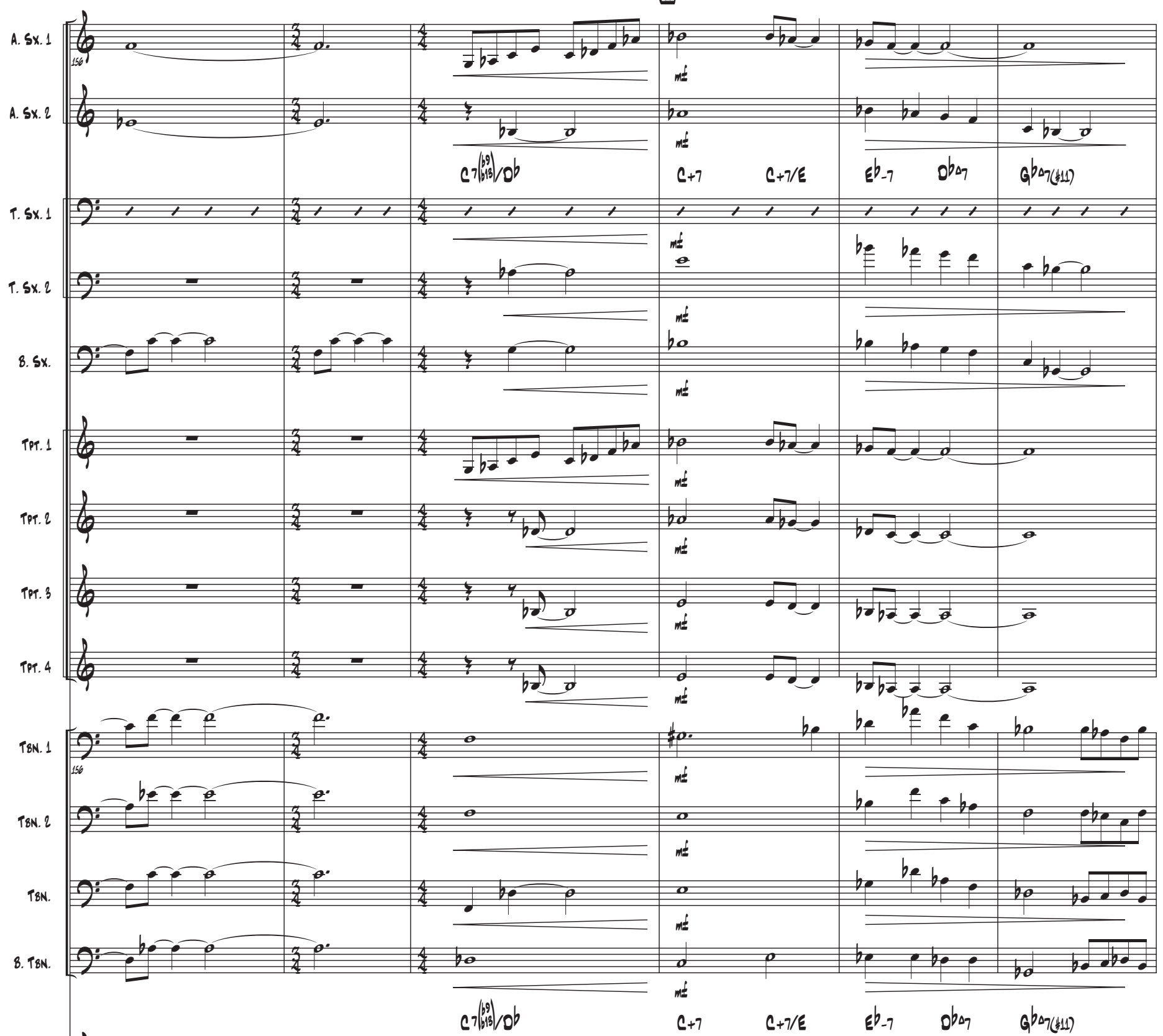

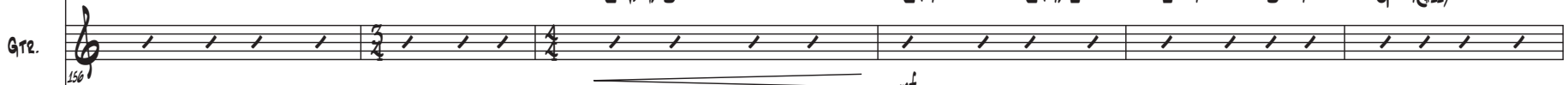

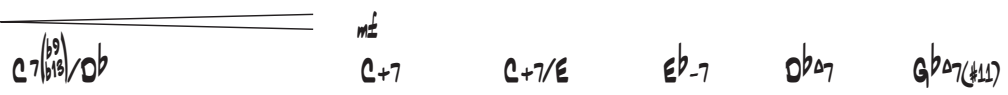

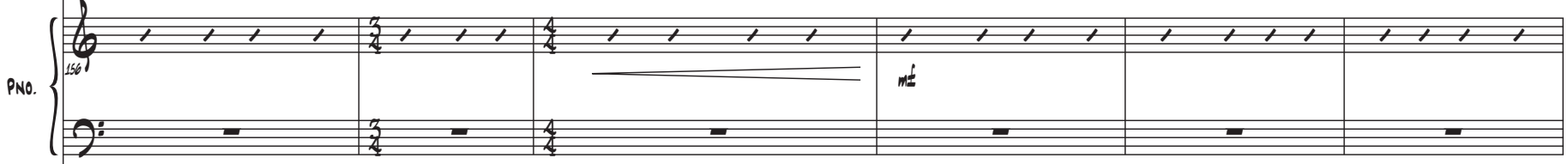

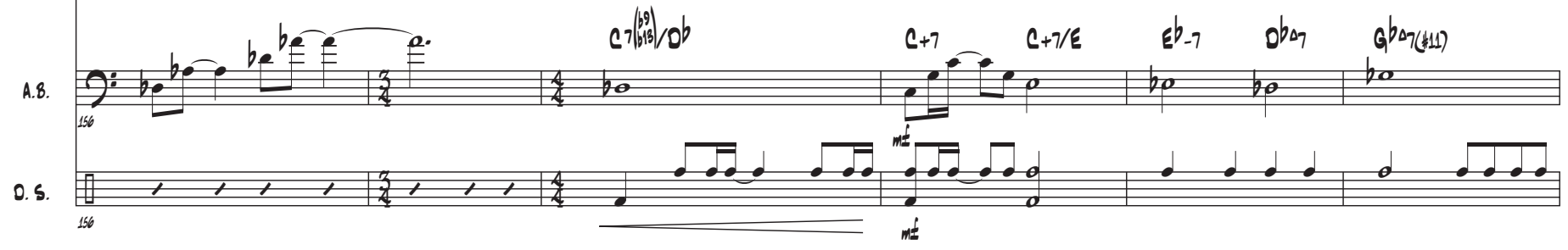



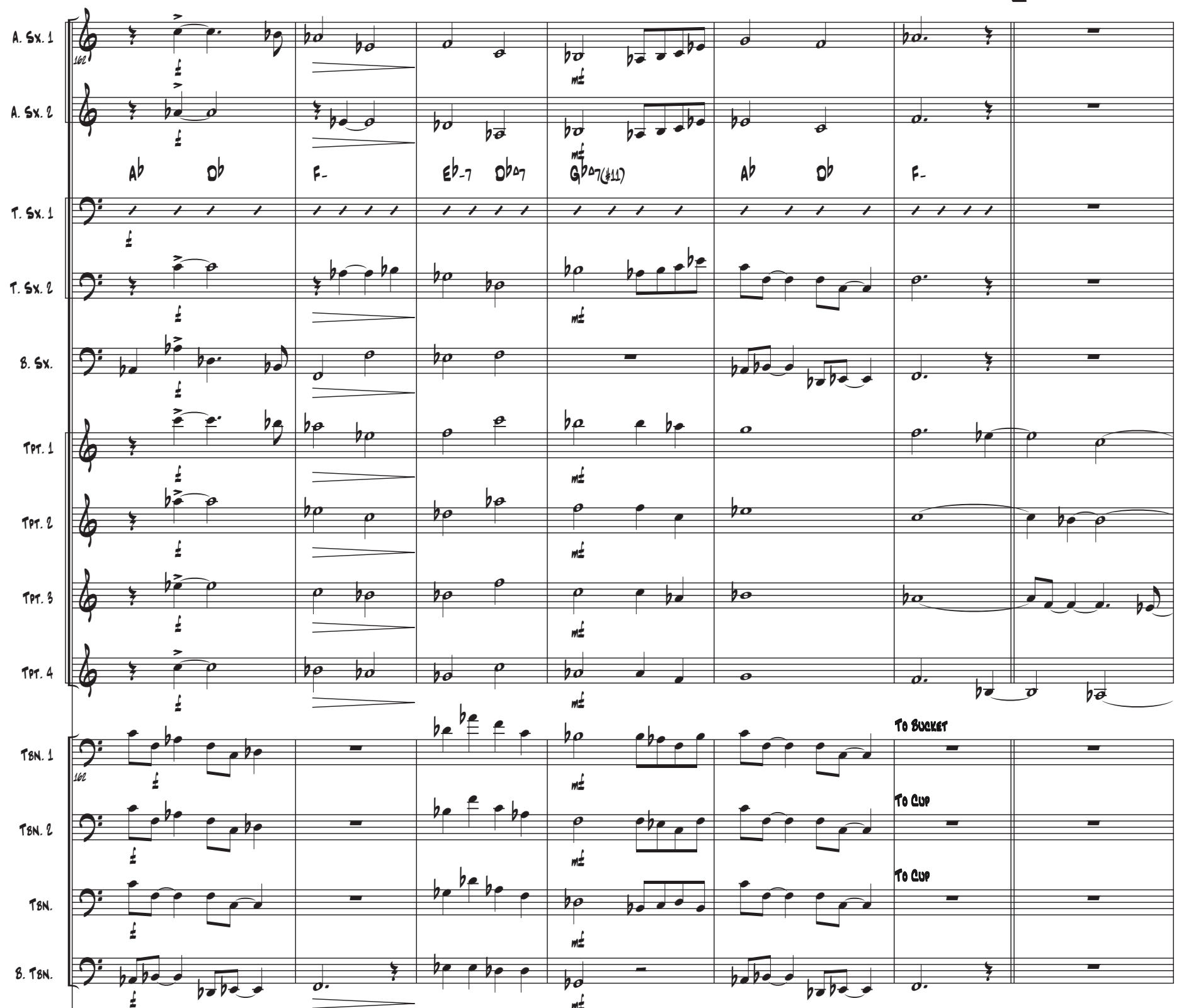

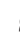
Ab ob

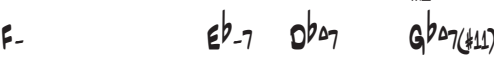
Ab ob
F-

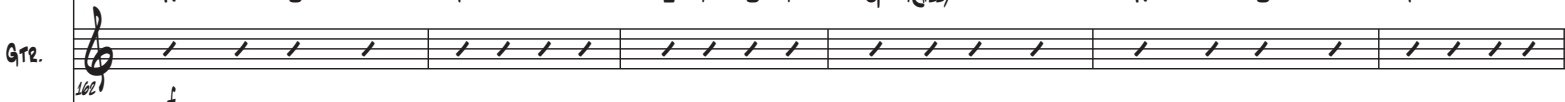

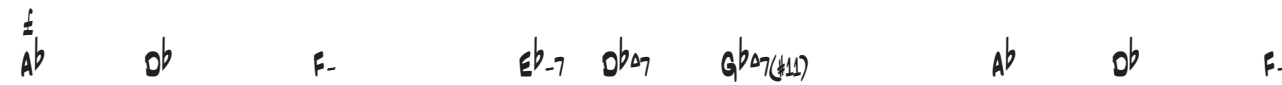

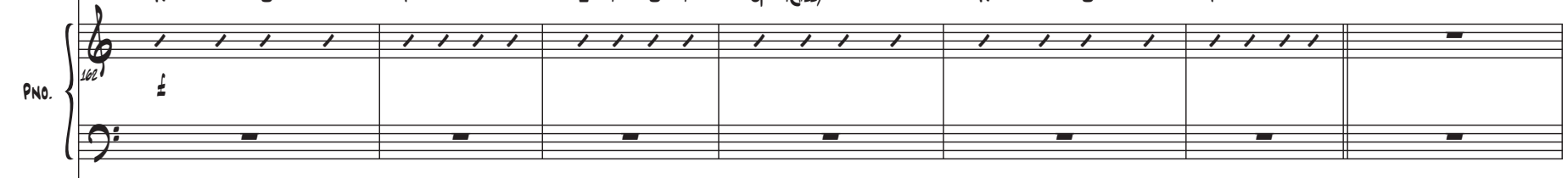
Ab ob
F-
Eb $b_{-7} \quad b_{\Delta \rightarrow} \quad G b_{\Delta 7(411)}$
Ab ob
F-

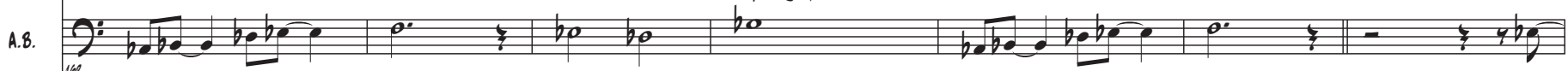

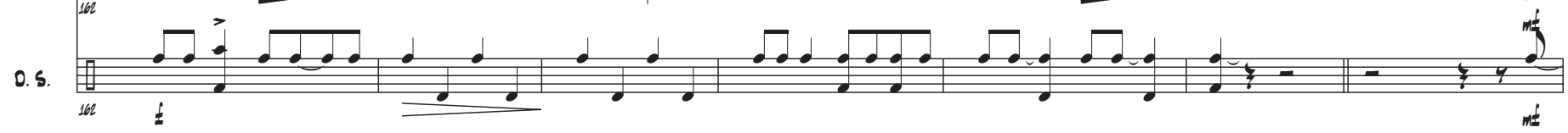



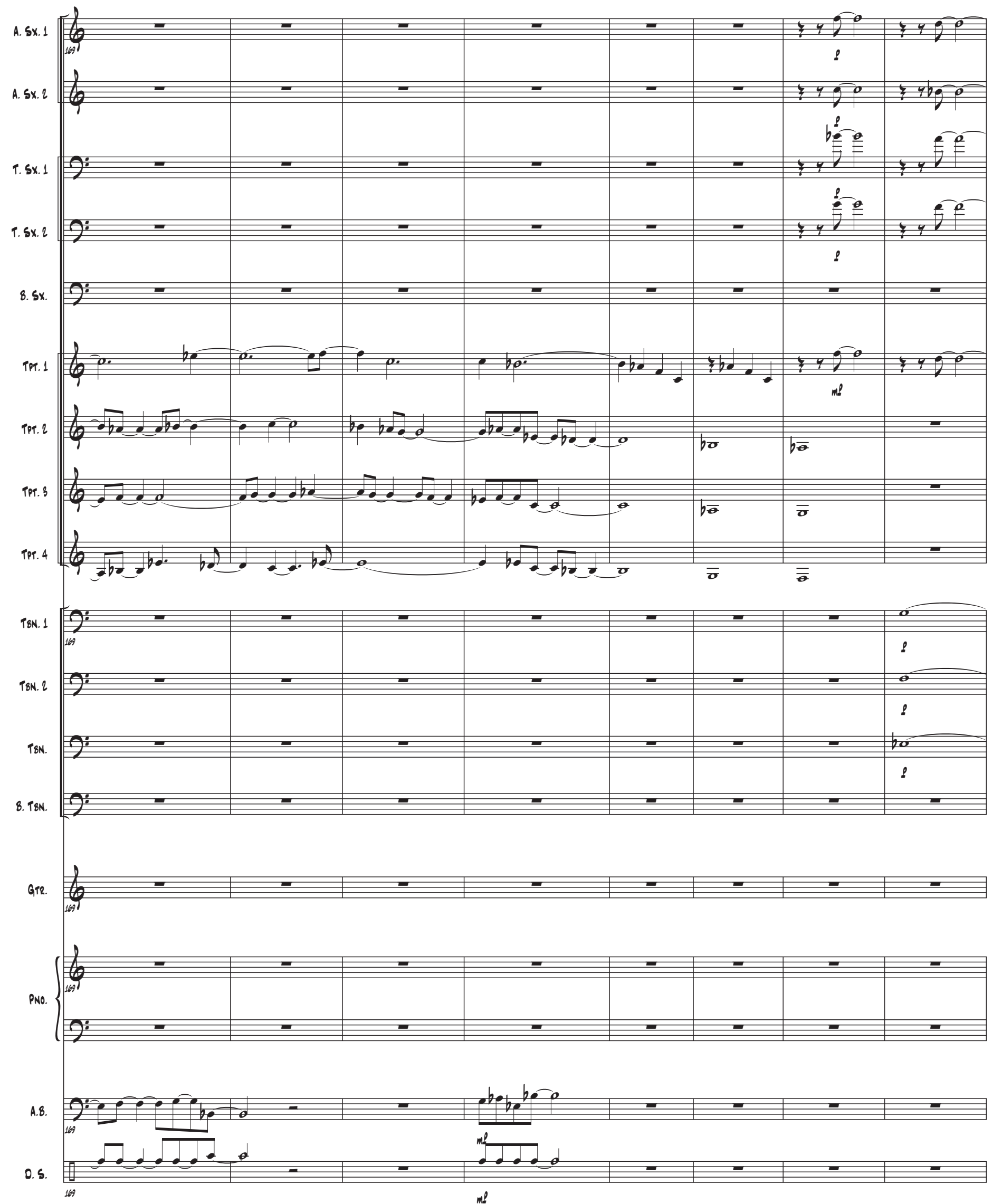

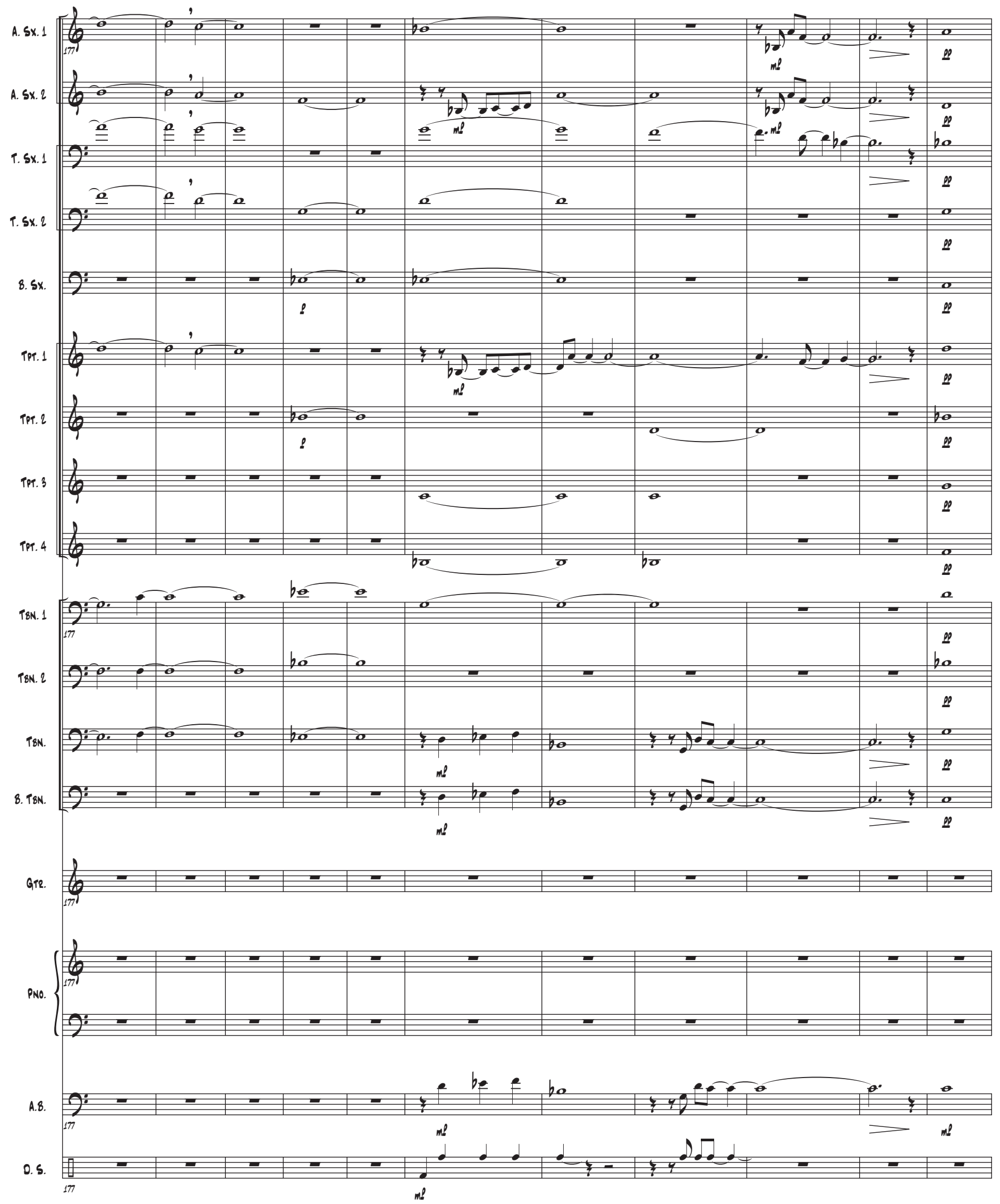


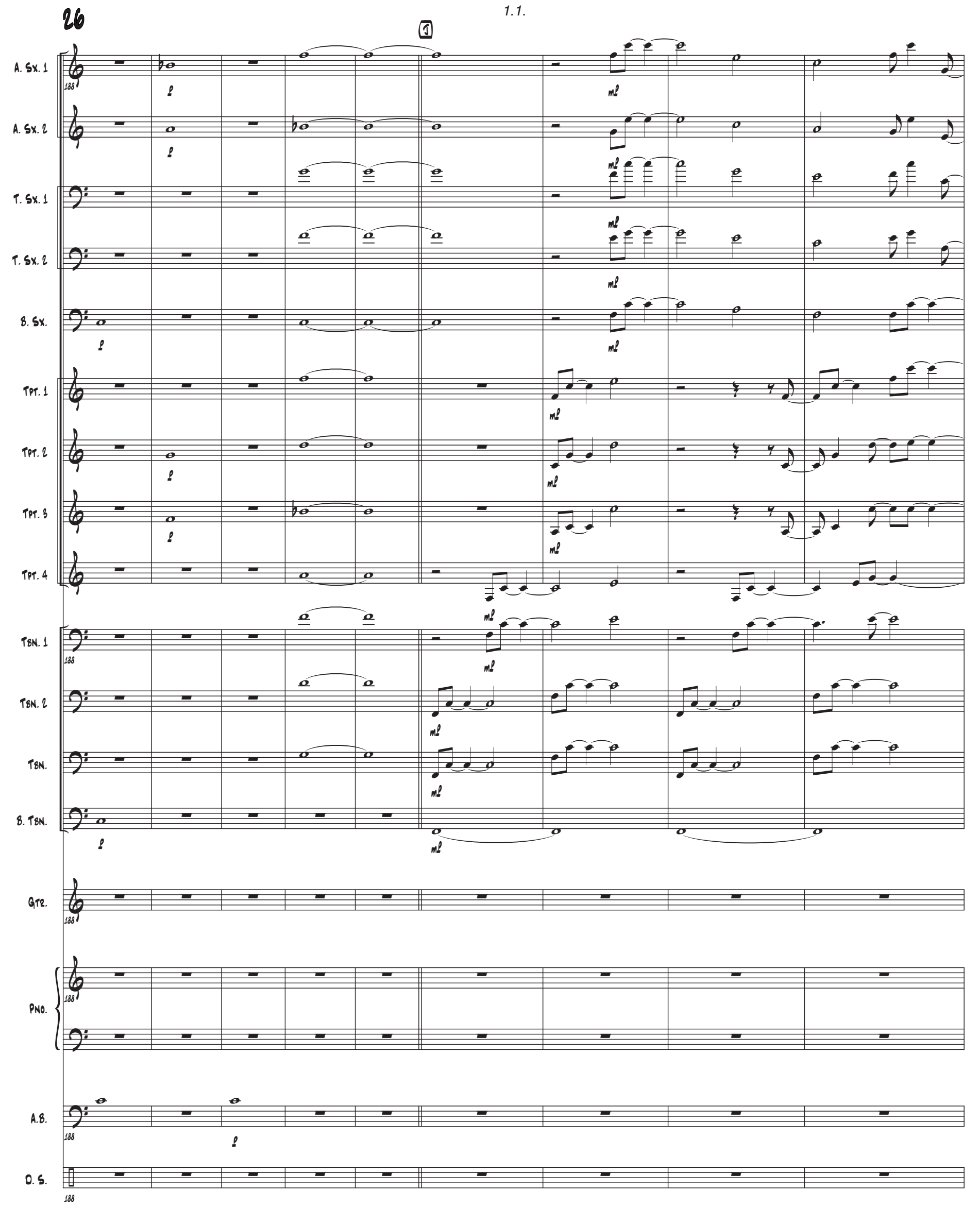



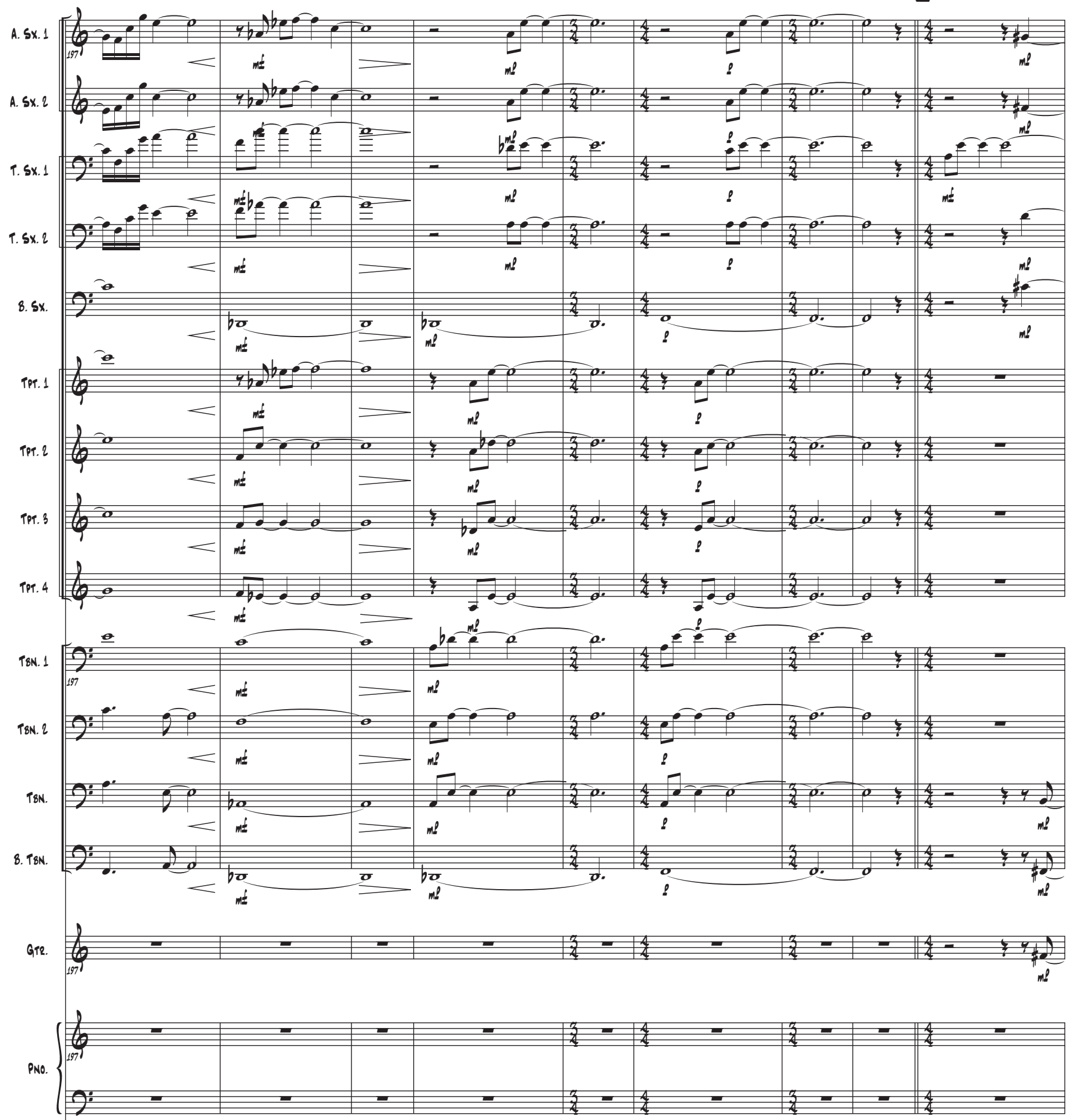

A.8.

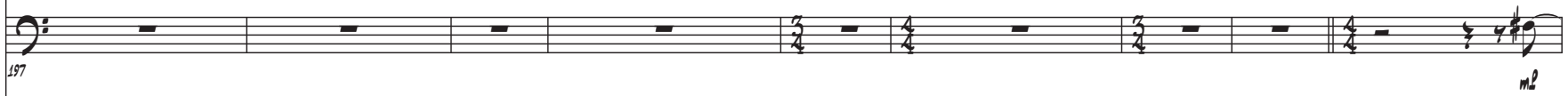

o. 5.

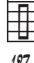


28

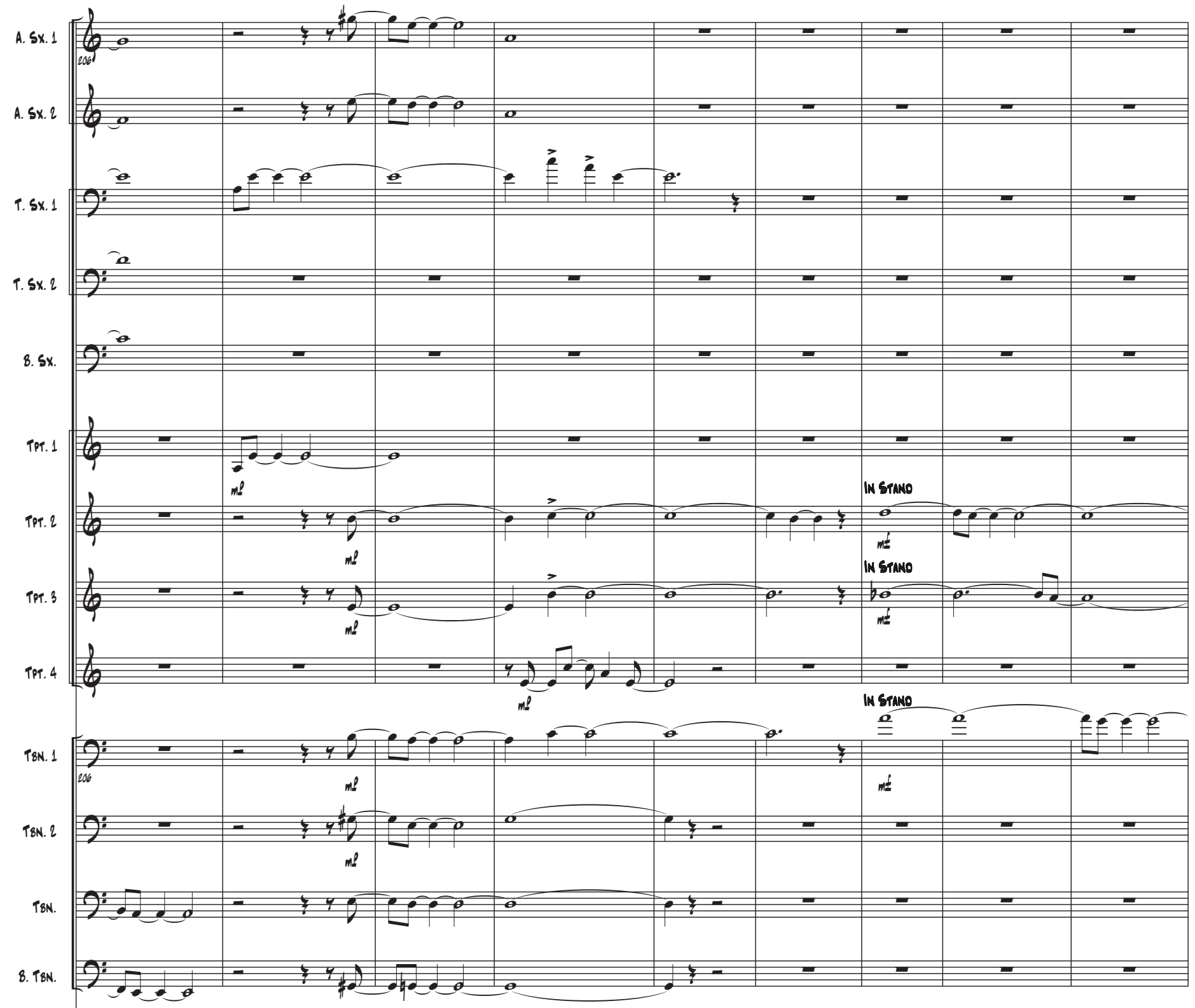

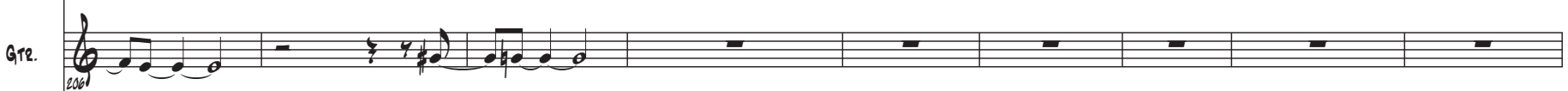

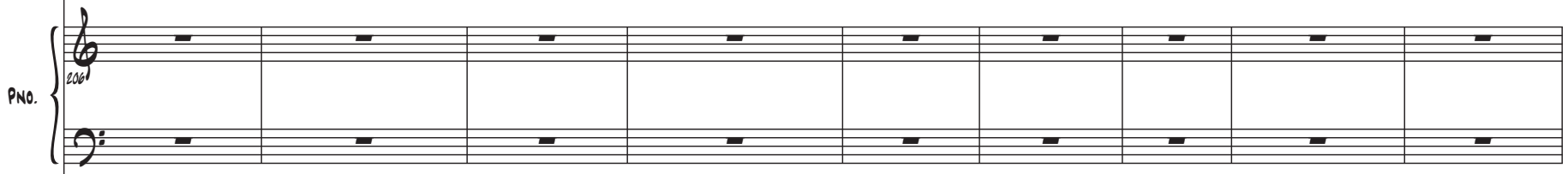

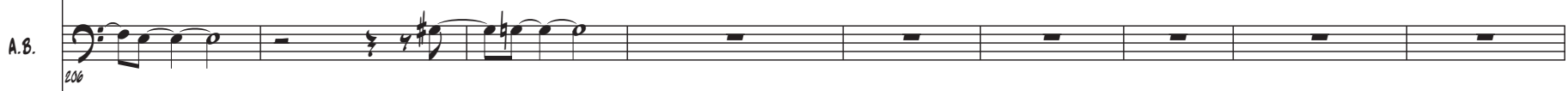

○.5.

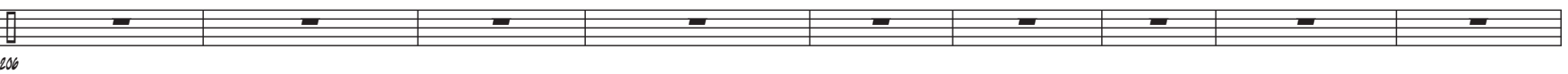



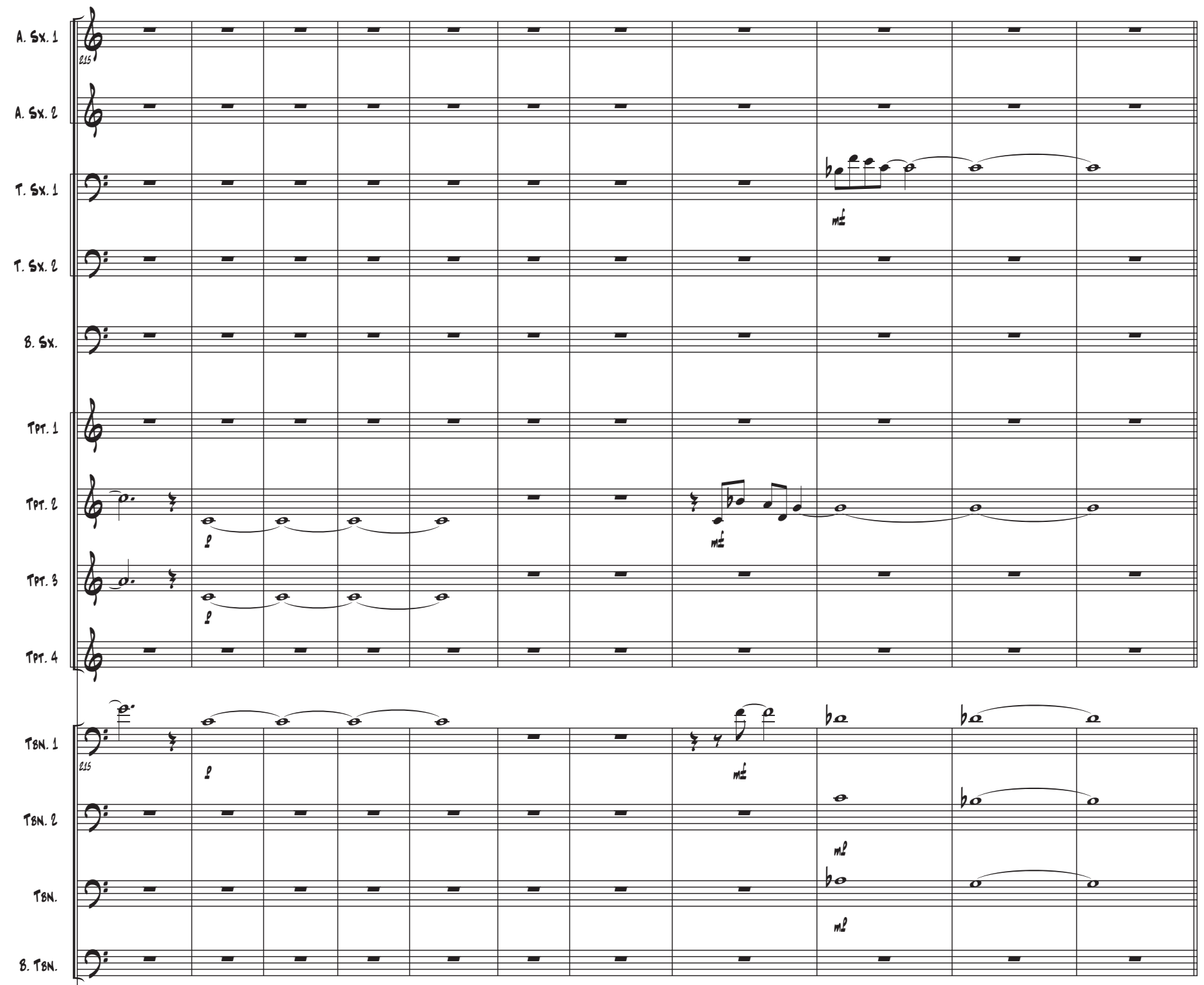

$$
A b / C \quad B b-7 \quad F / A \quad C 7 s u s / F
$$

Gre. $\int_{\text {Ulis }}$

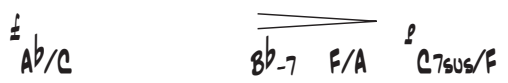
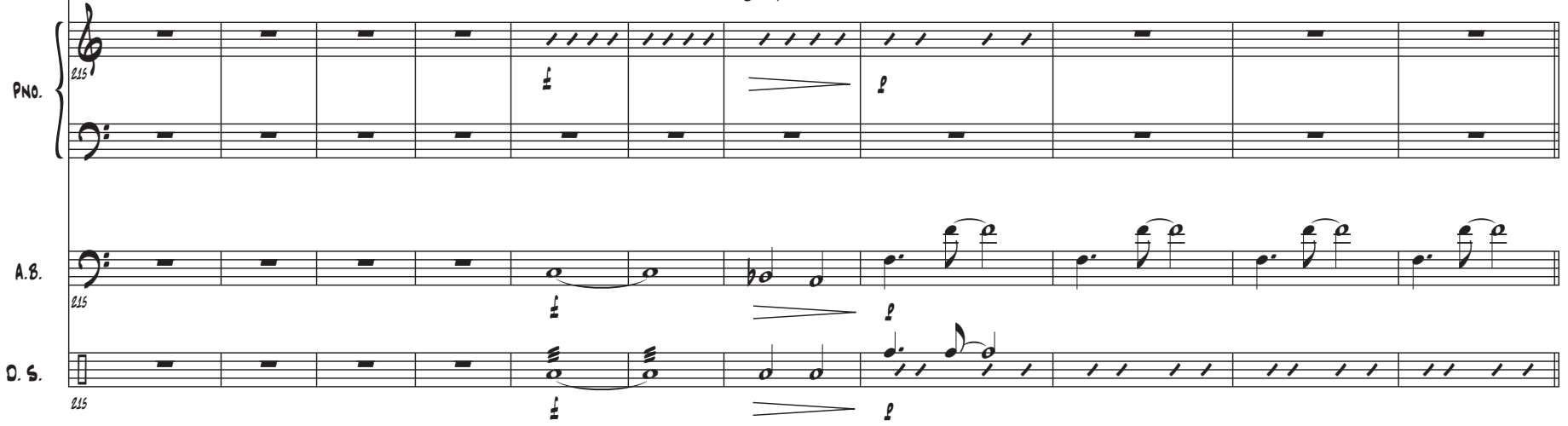
30
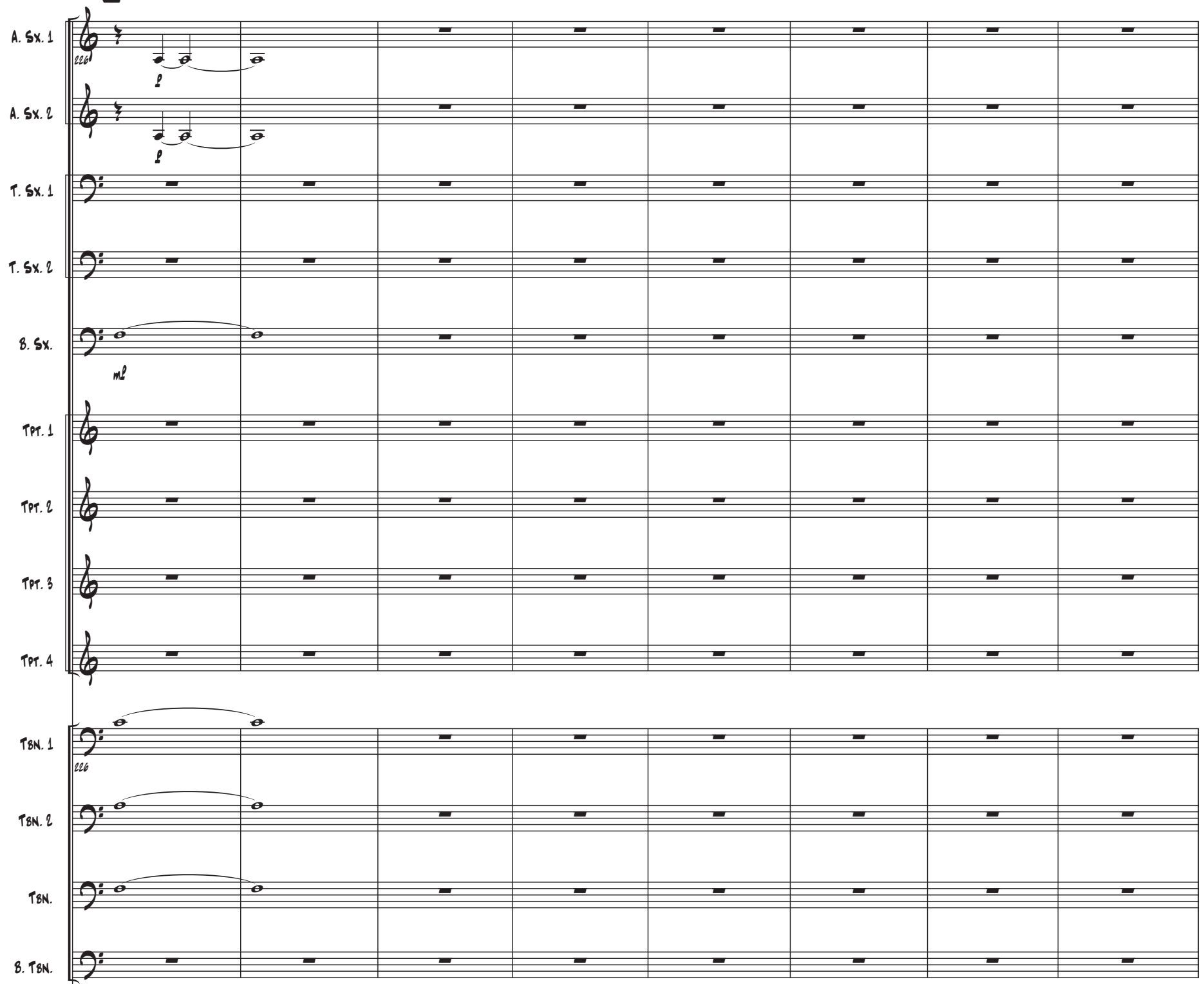

Gre.

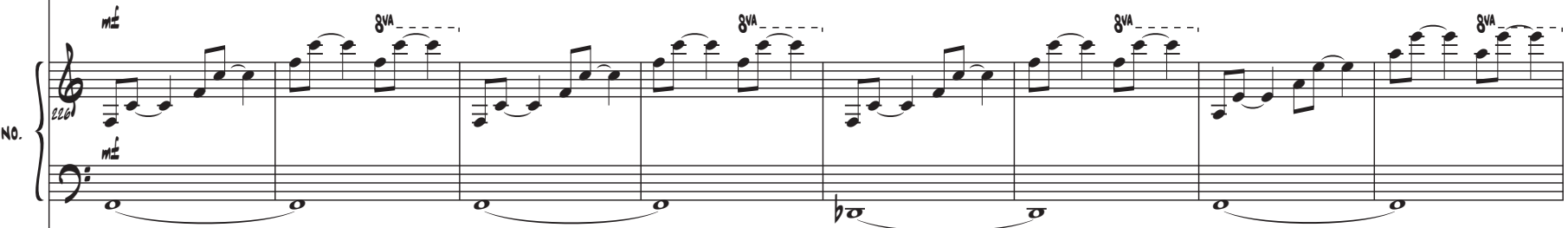

A.8.
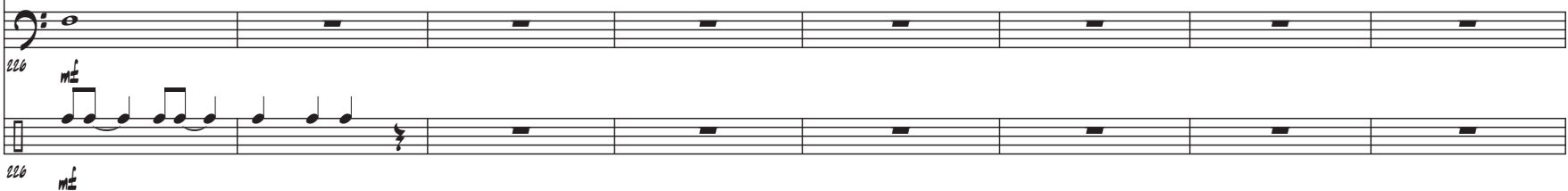

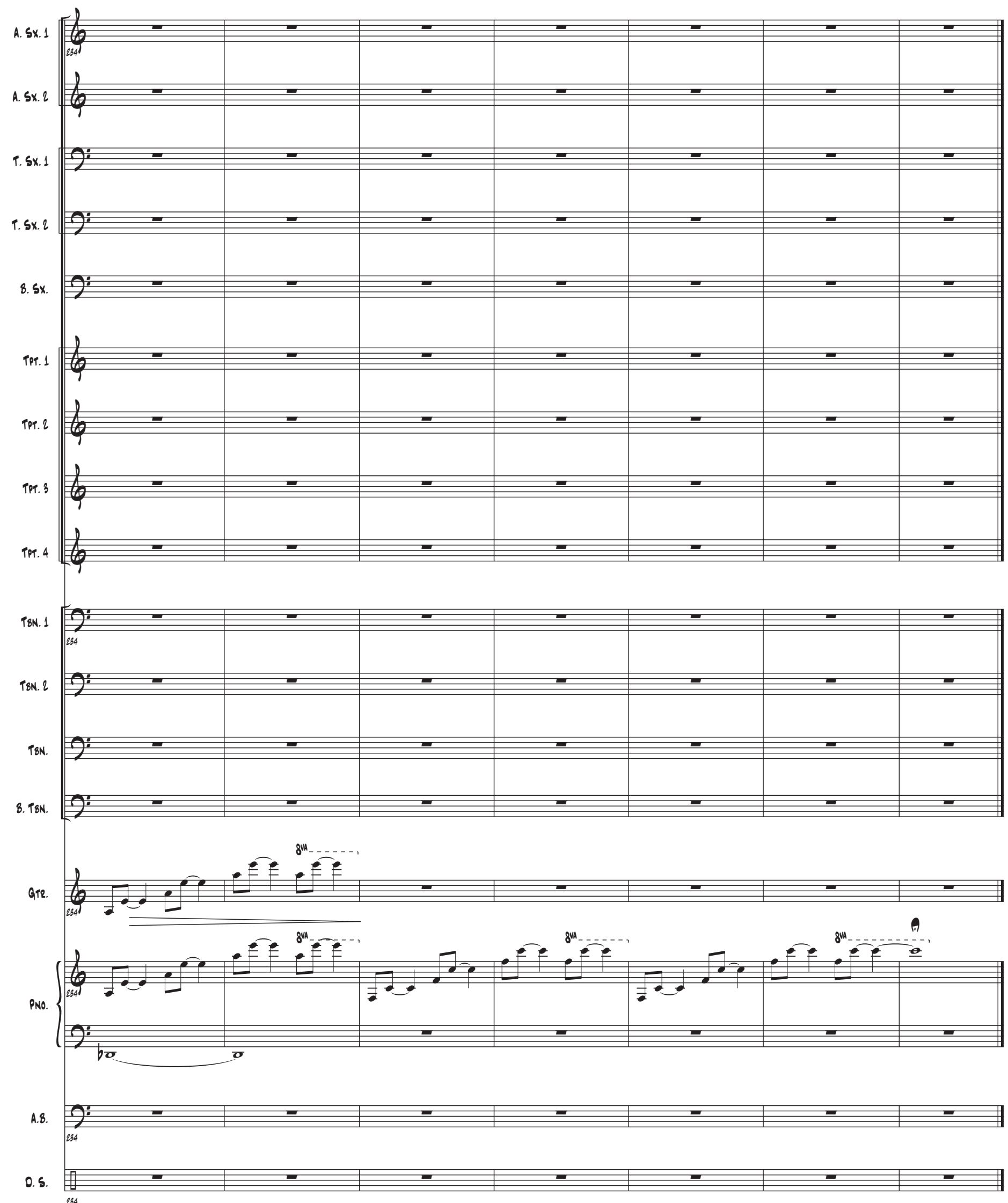



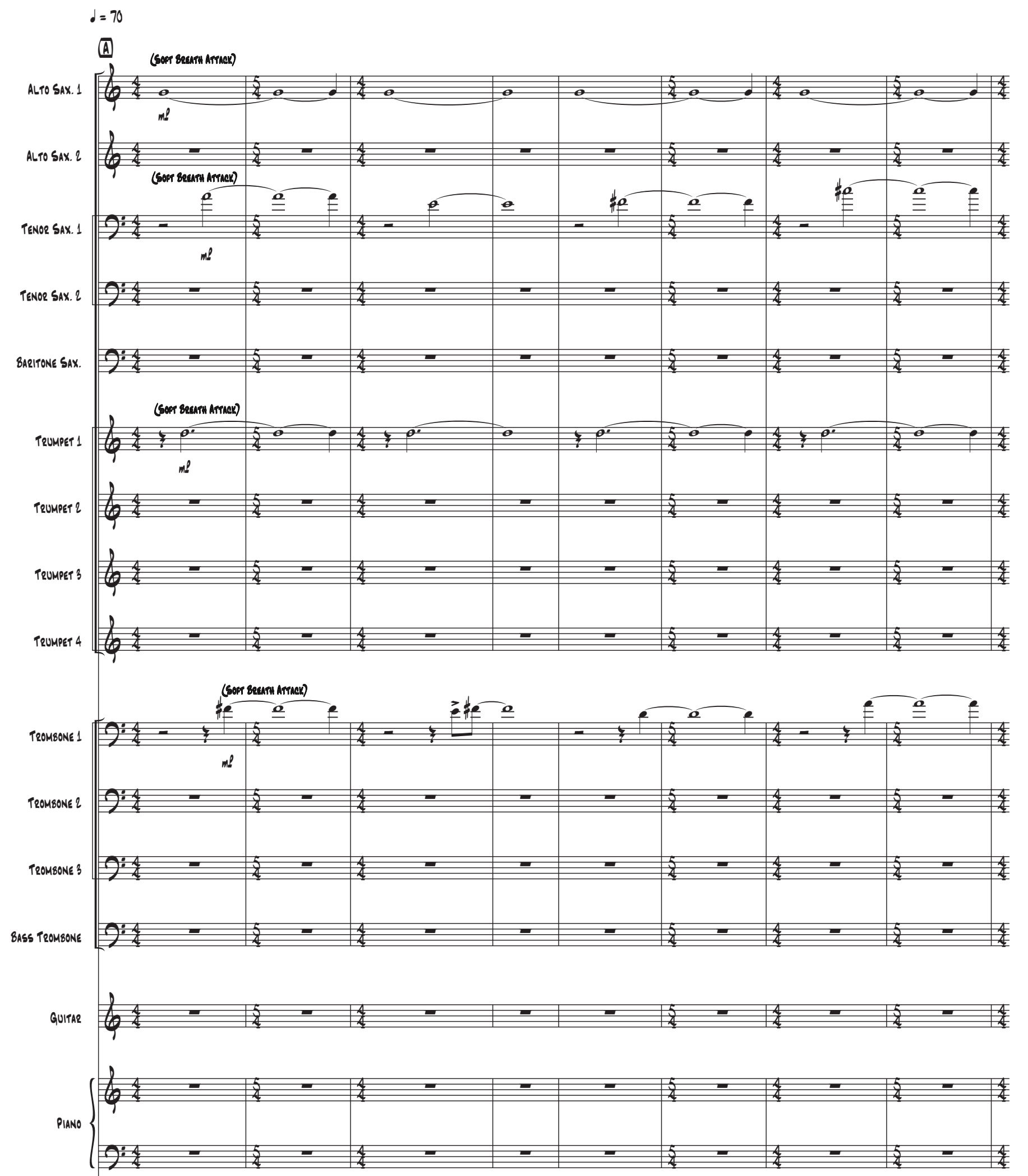

TENNOR SAX.1
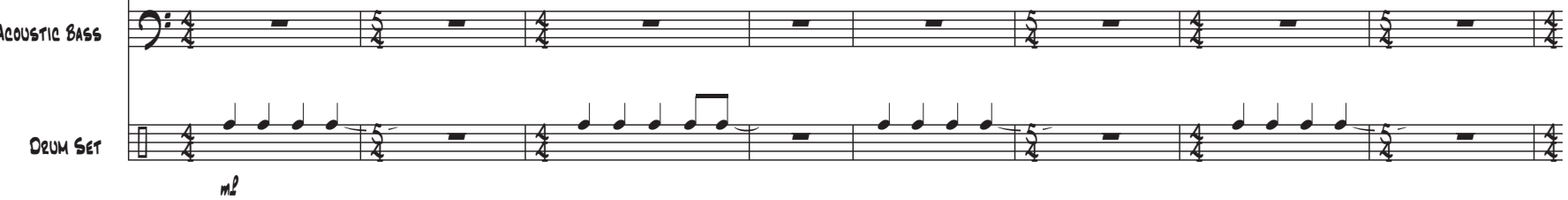

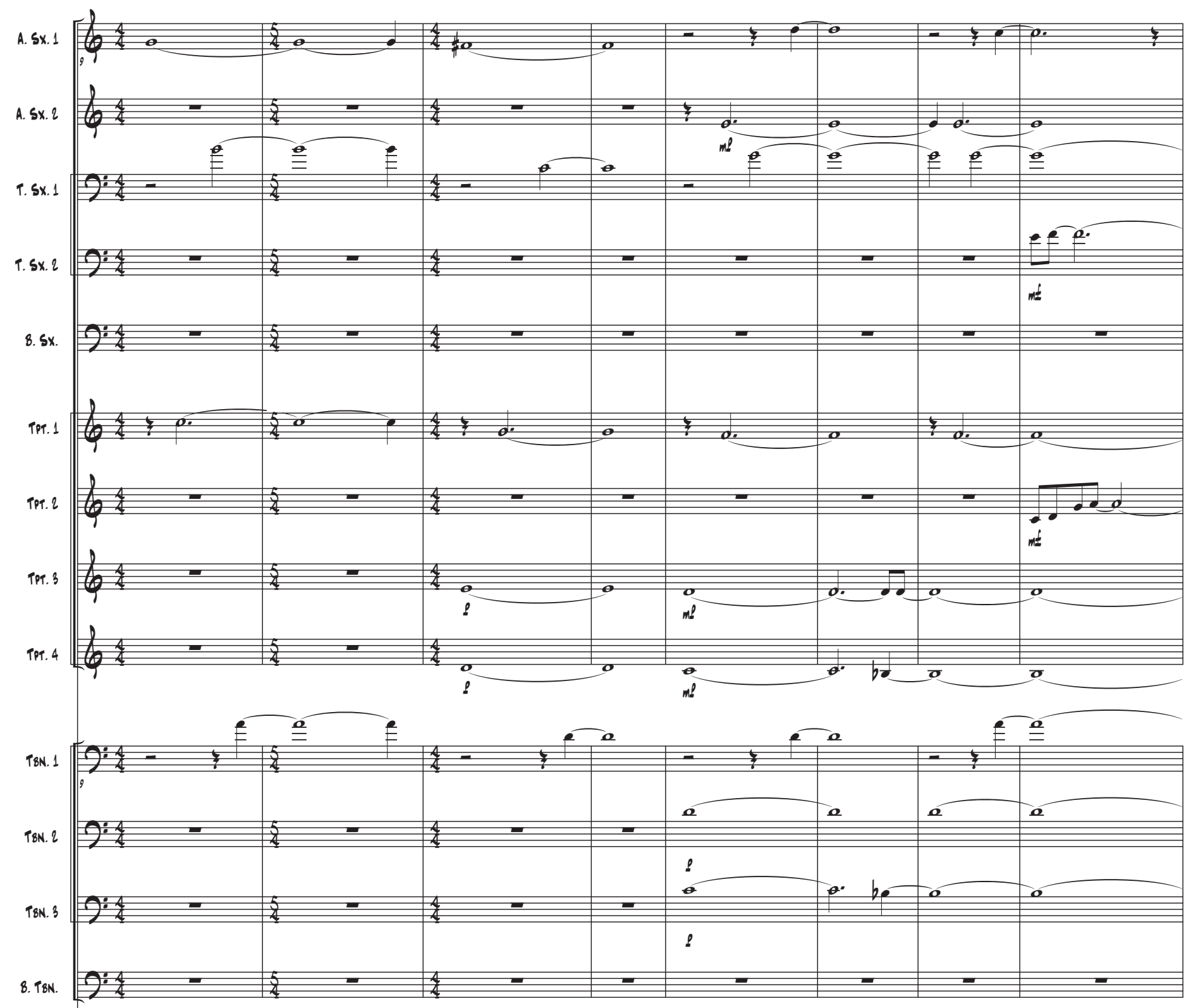

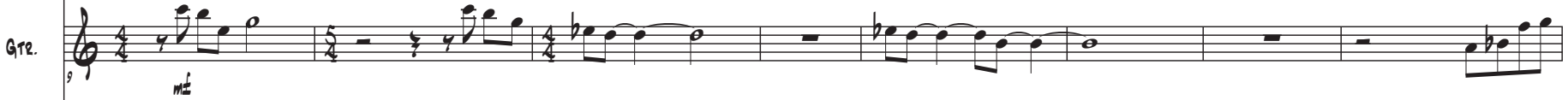

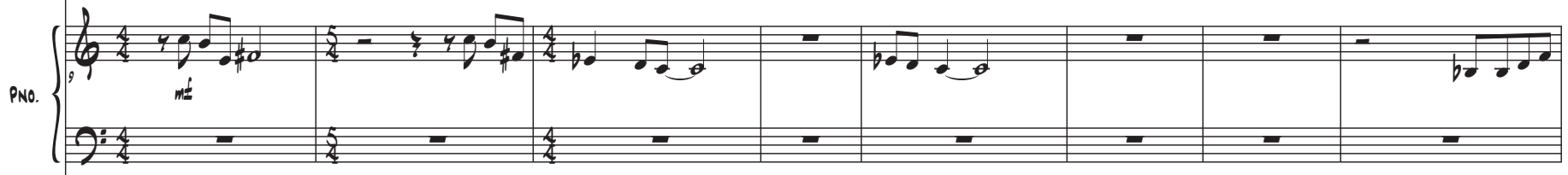

A.8. $\int_{0}^{5: 4-2-15}$

0.5. $\cdot d \cdot d \cdot d \cdot$ 

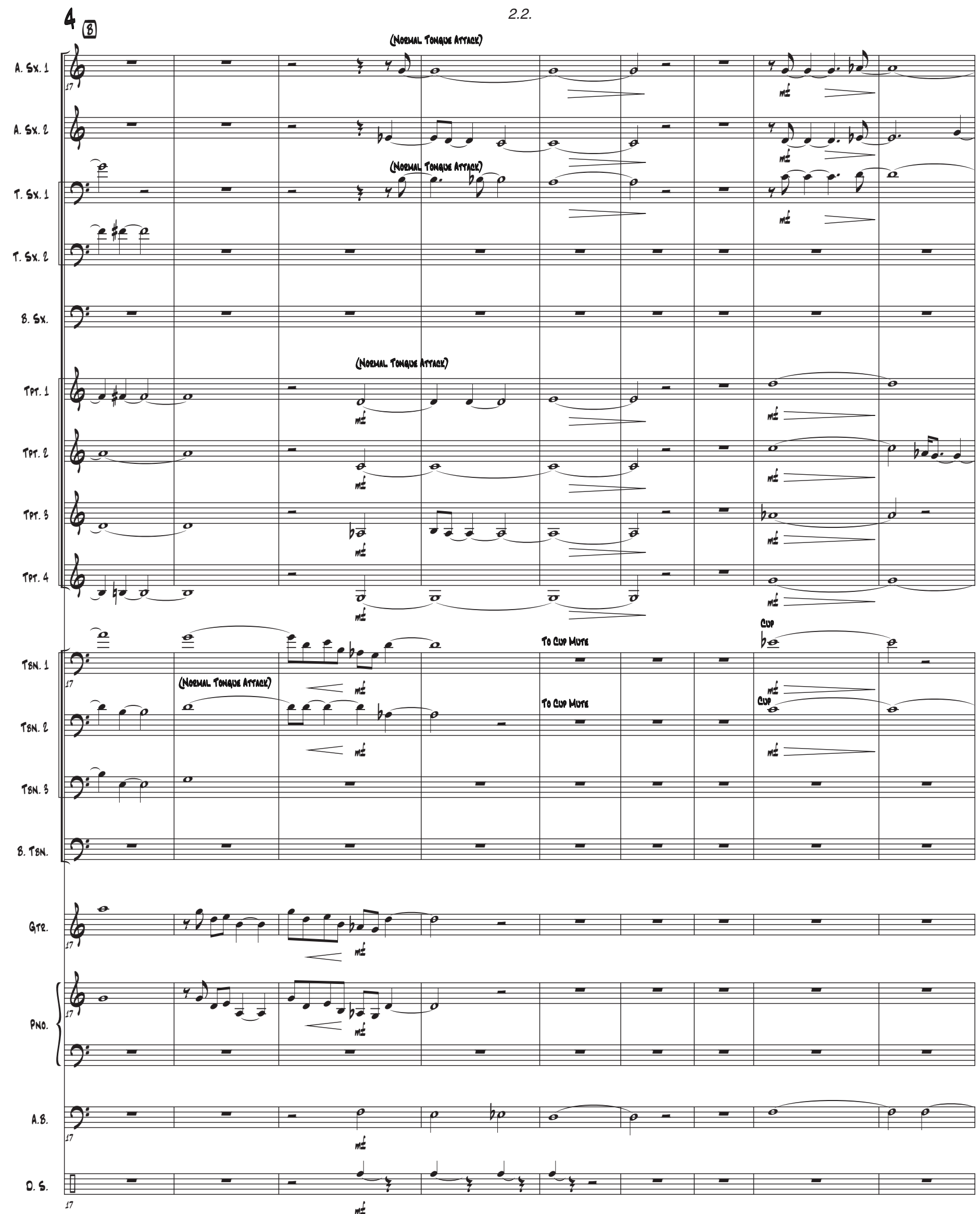

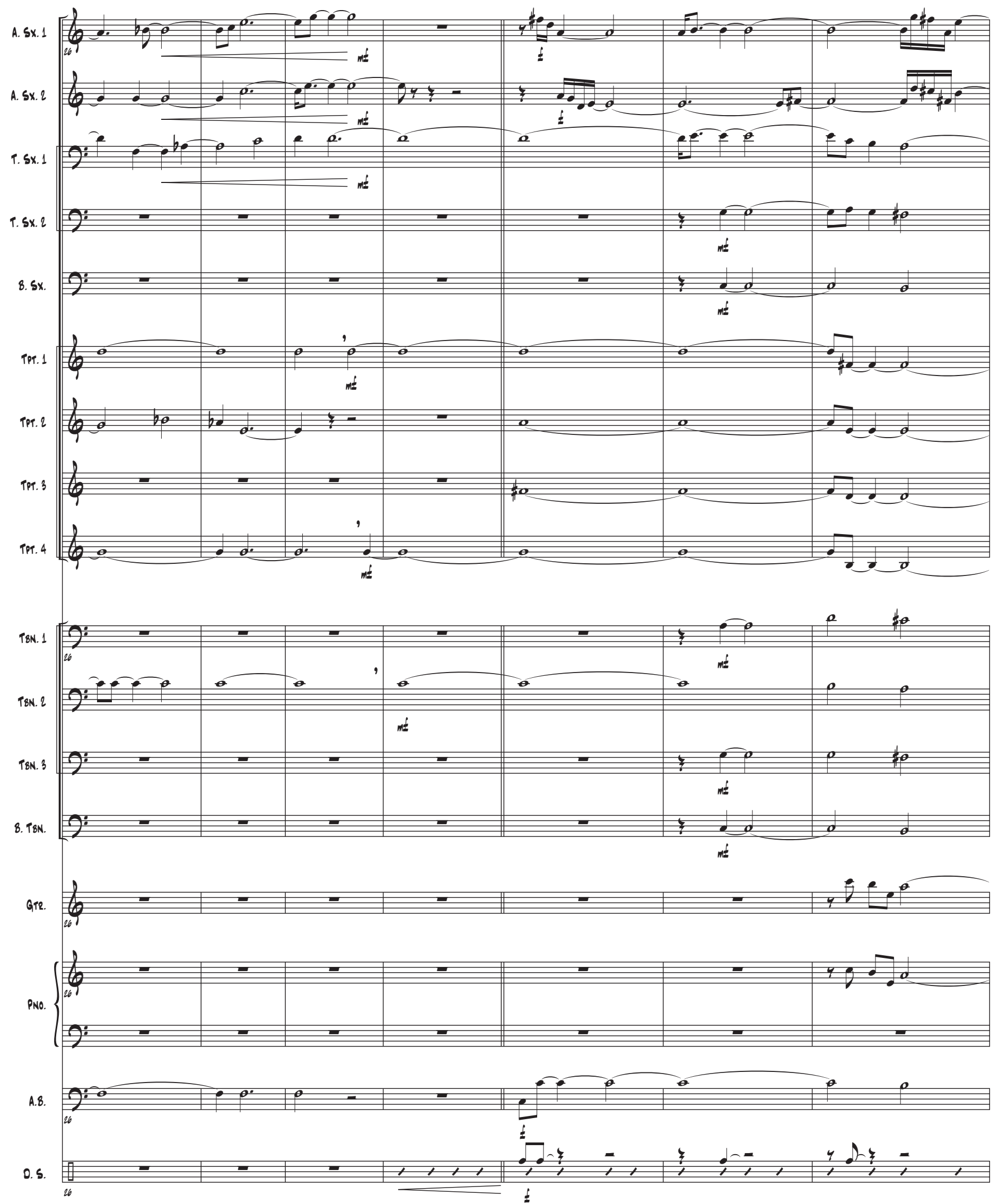

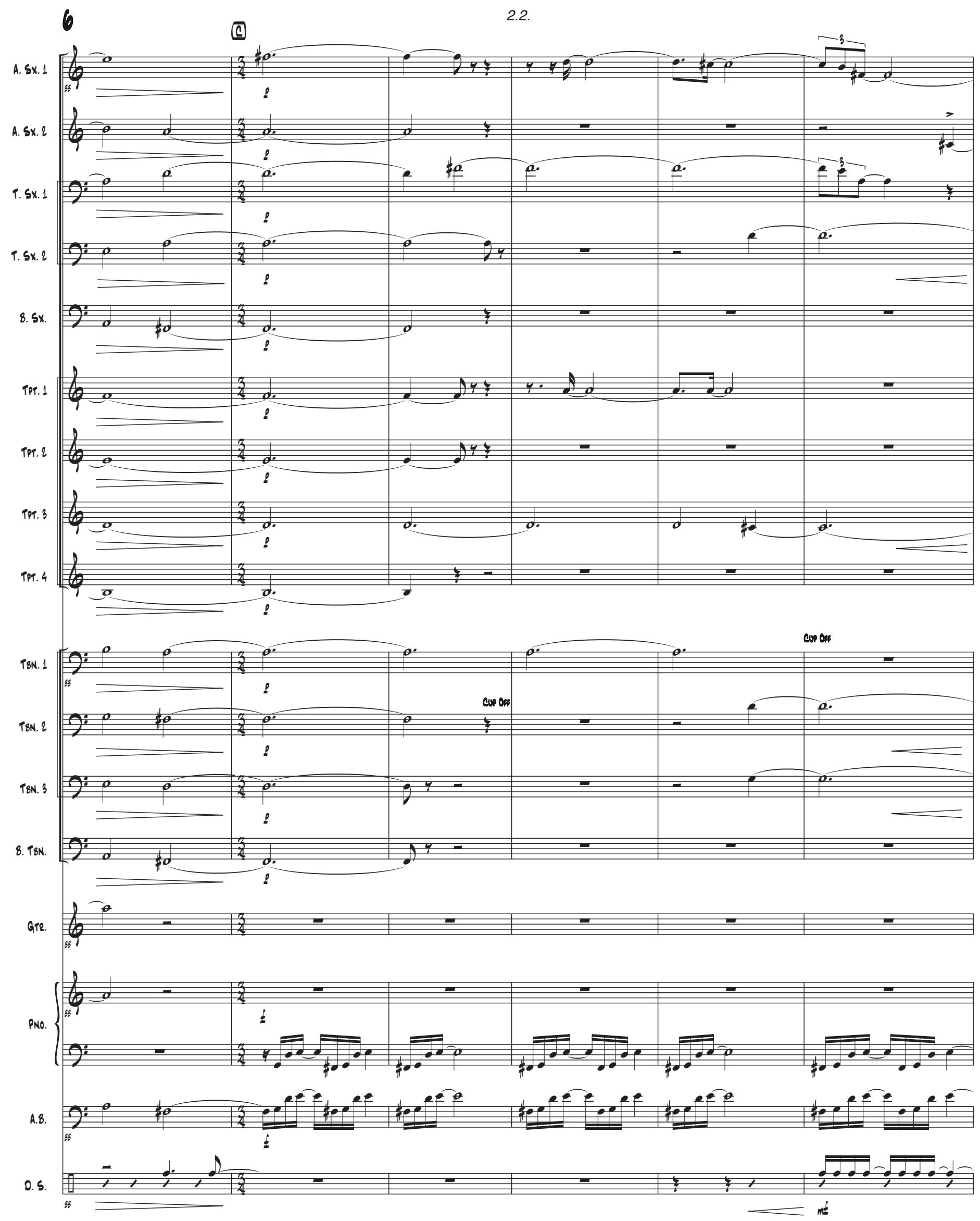

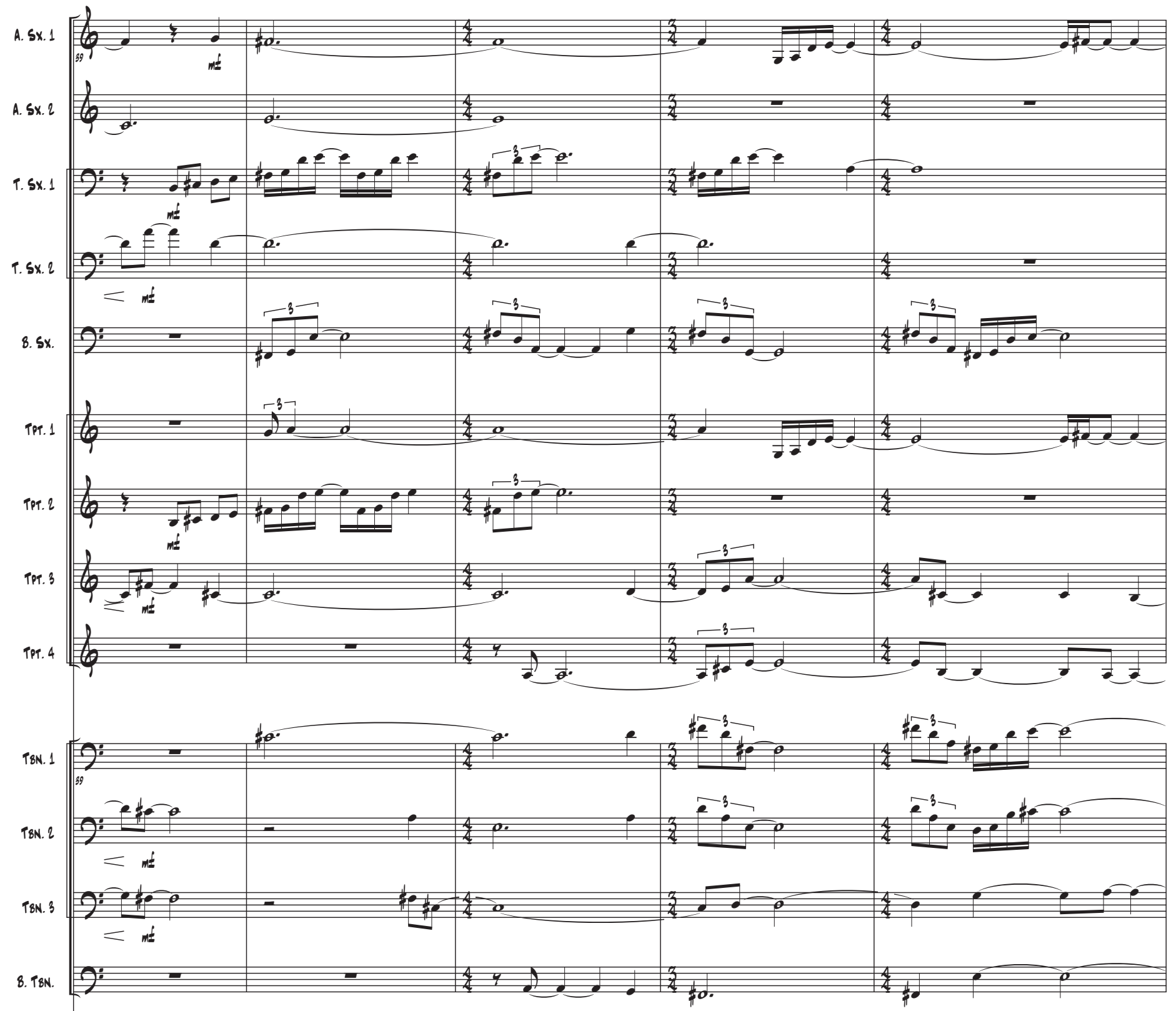

Gie. ${ }_{39}$

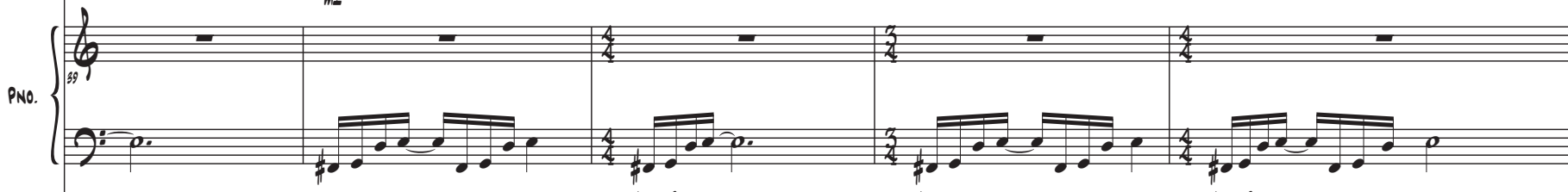
A.8.

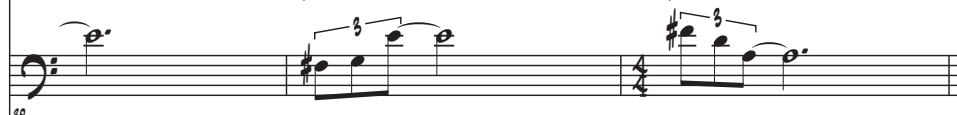
精?
怔?

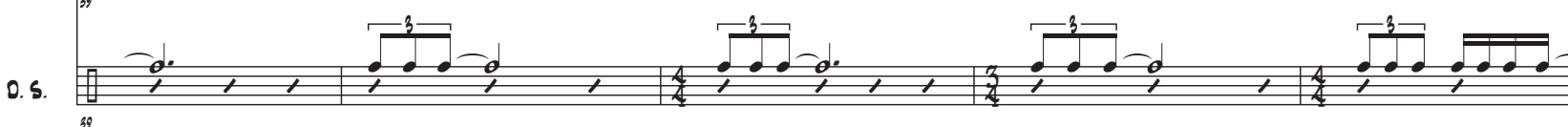



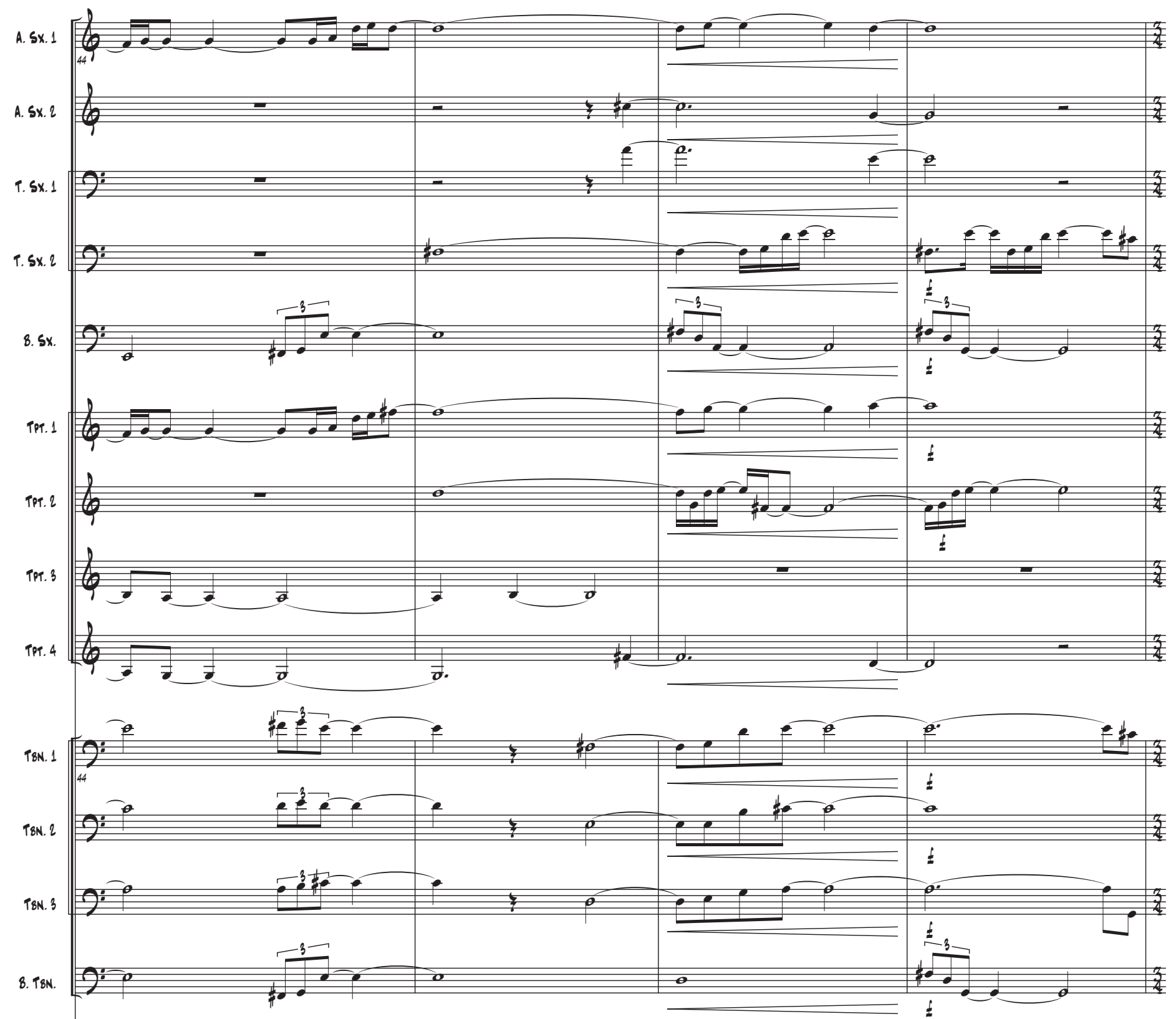
Gie. $b_{44}$
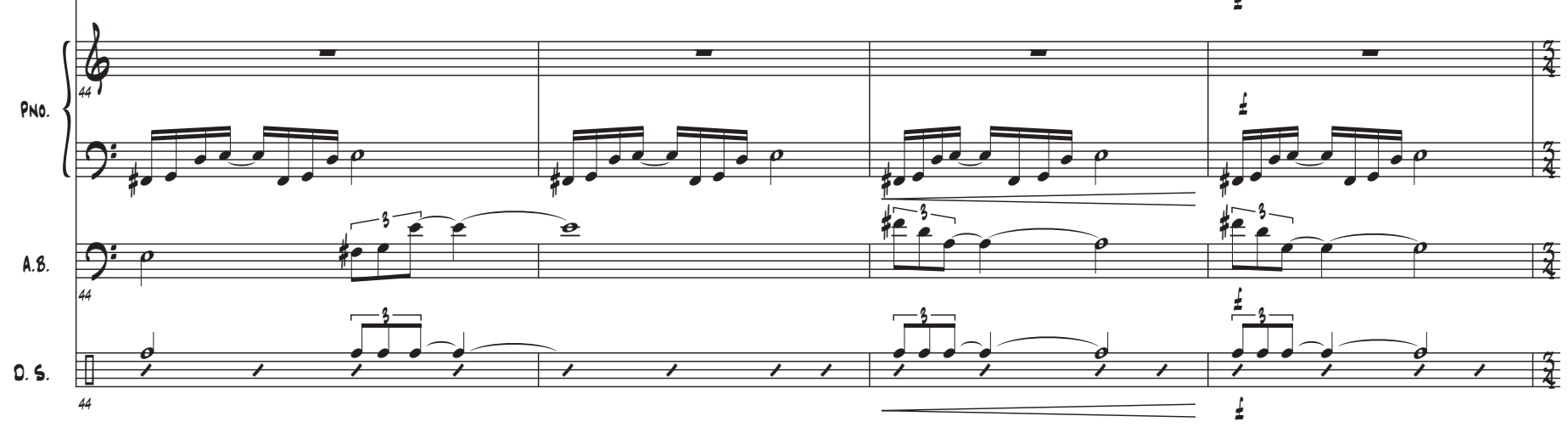

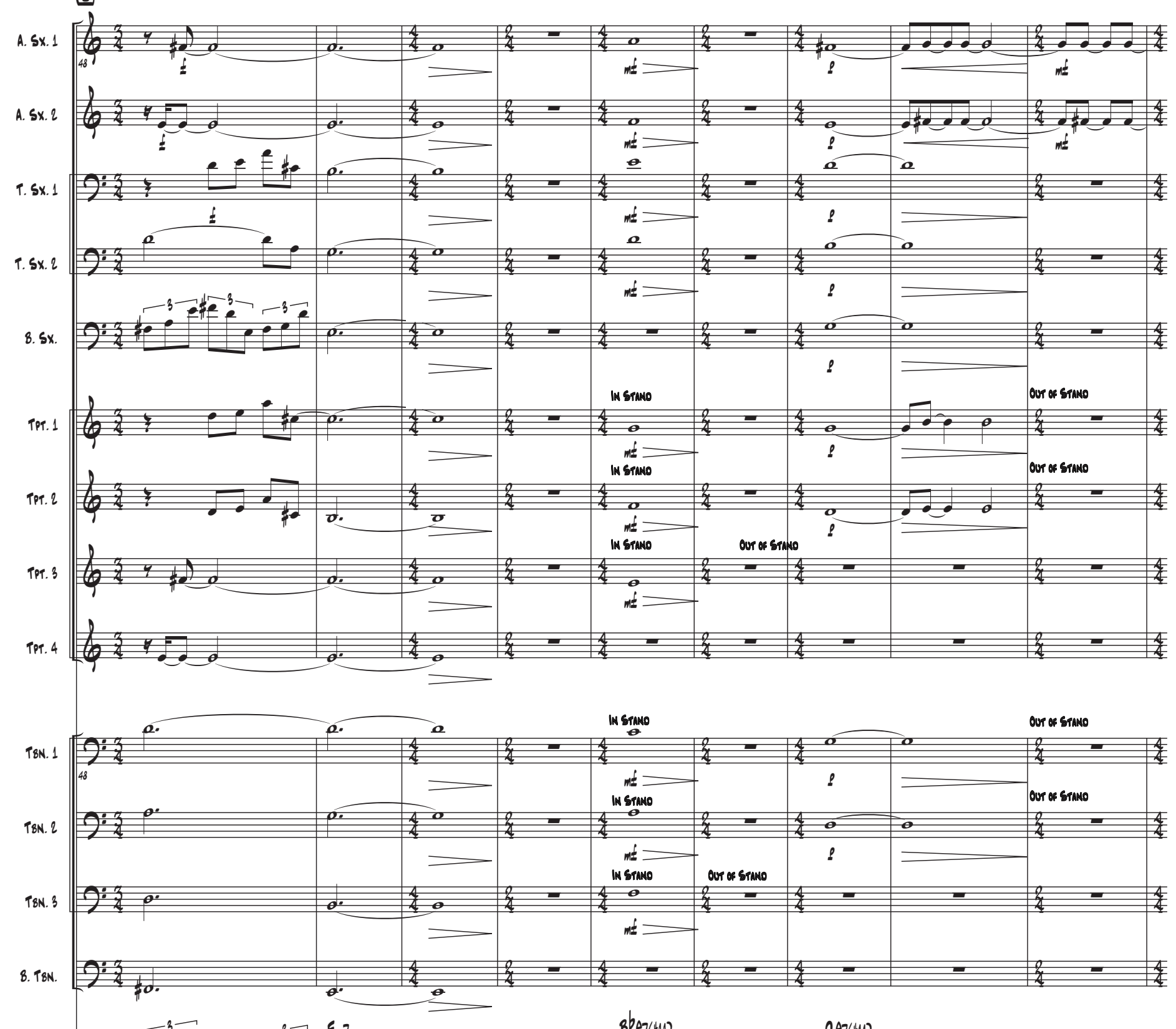

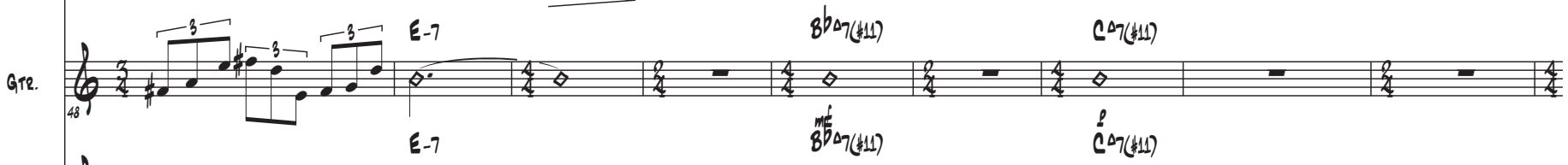

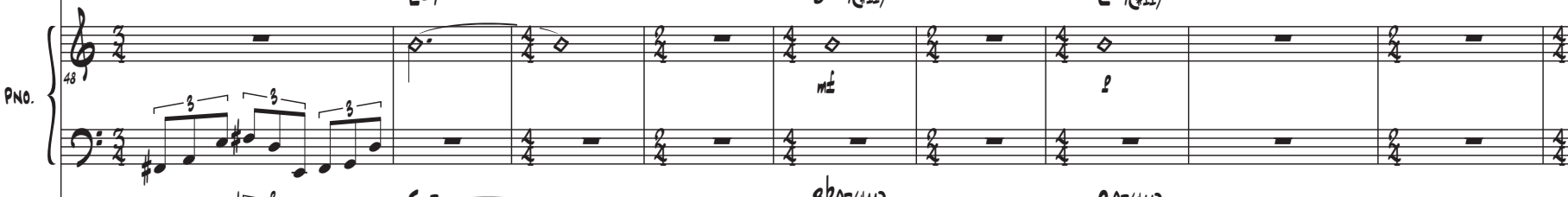
A.8. 0.5. 

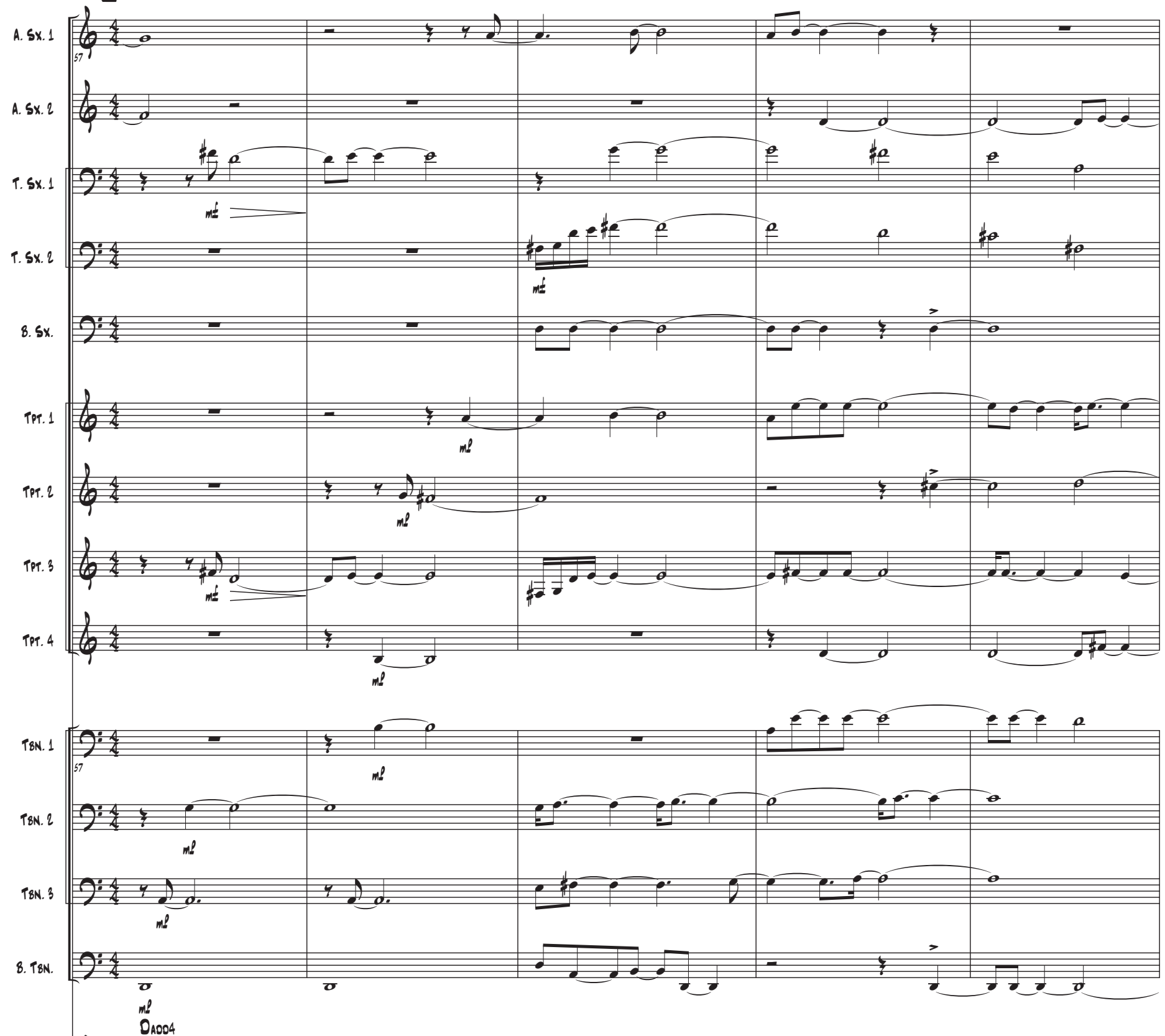

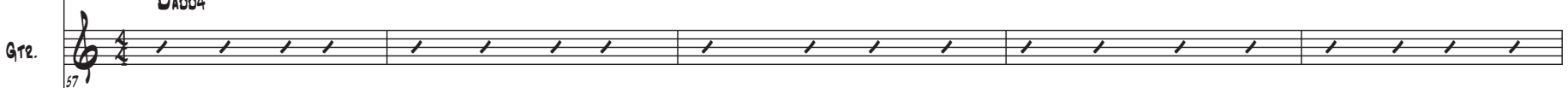

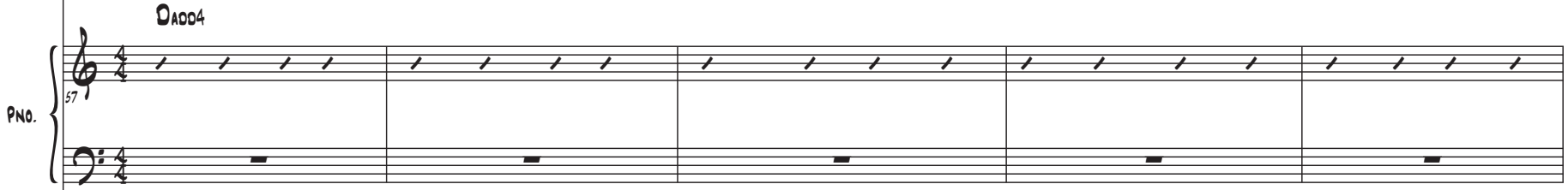
Ondoo 4

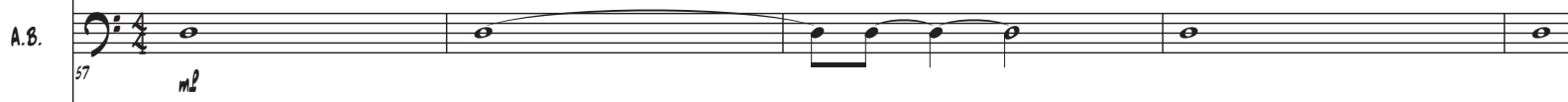

0. 5. 

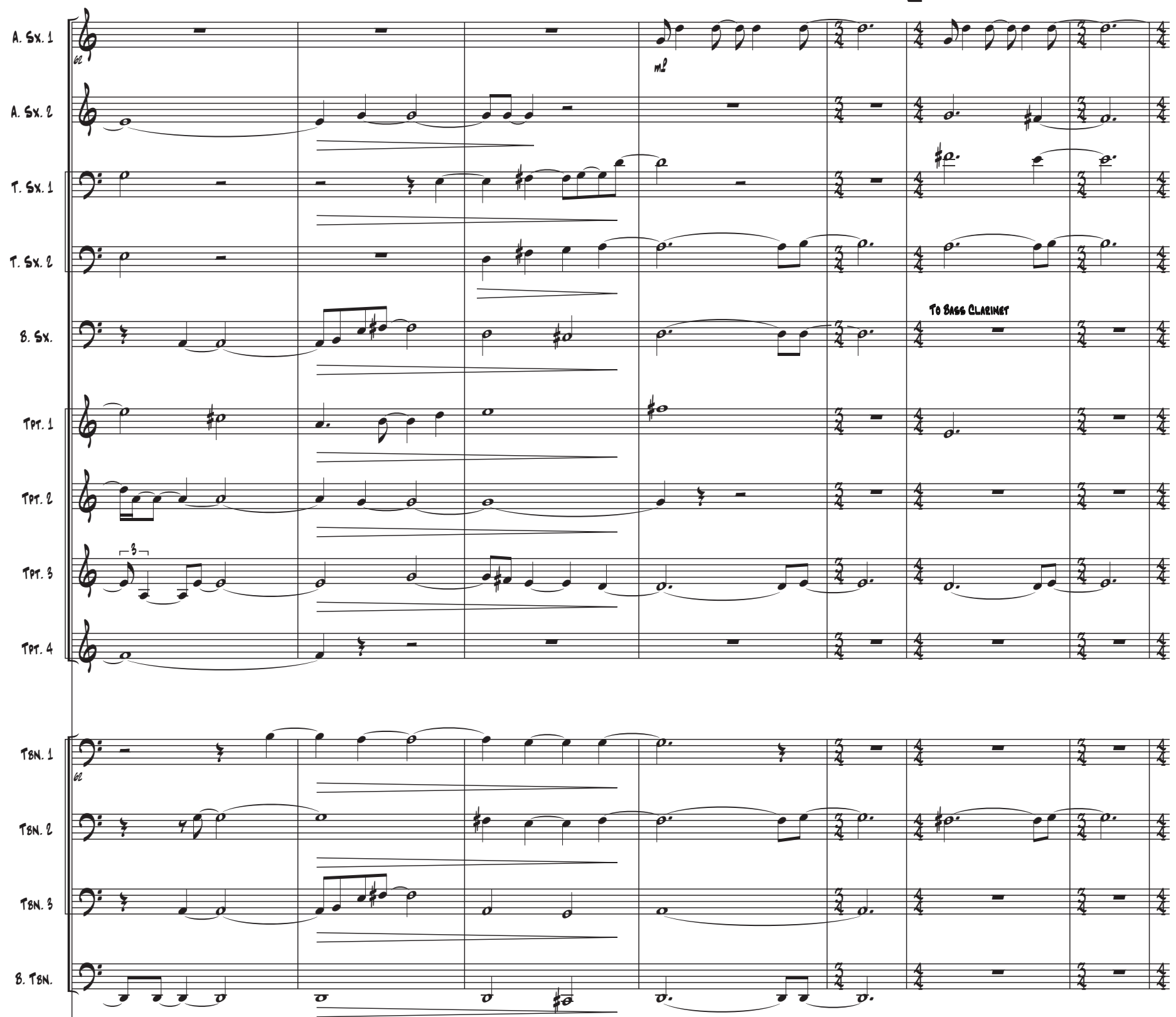

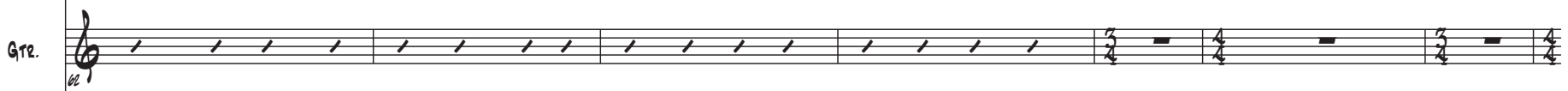

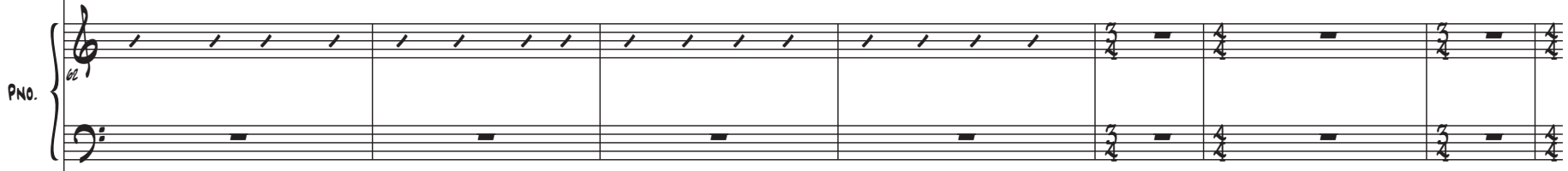

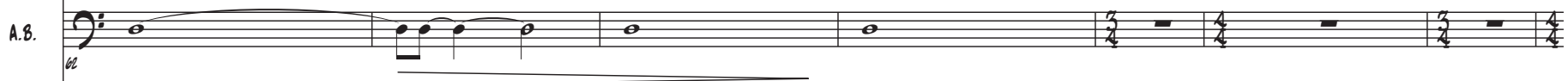
o.5. 

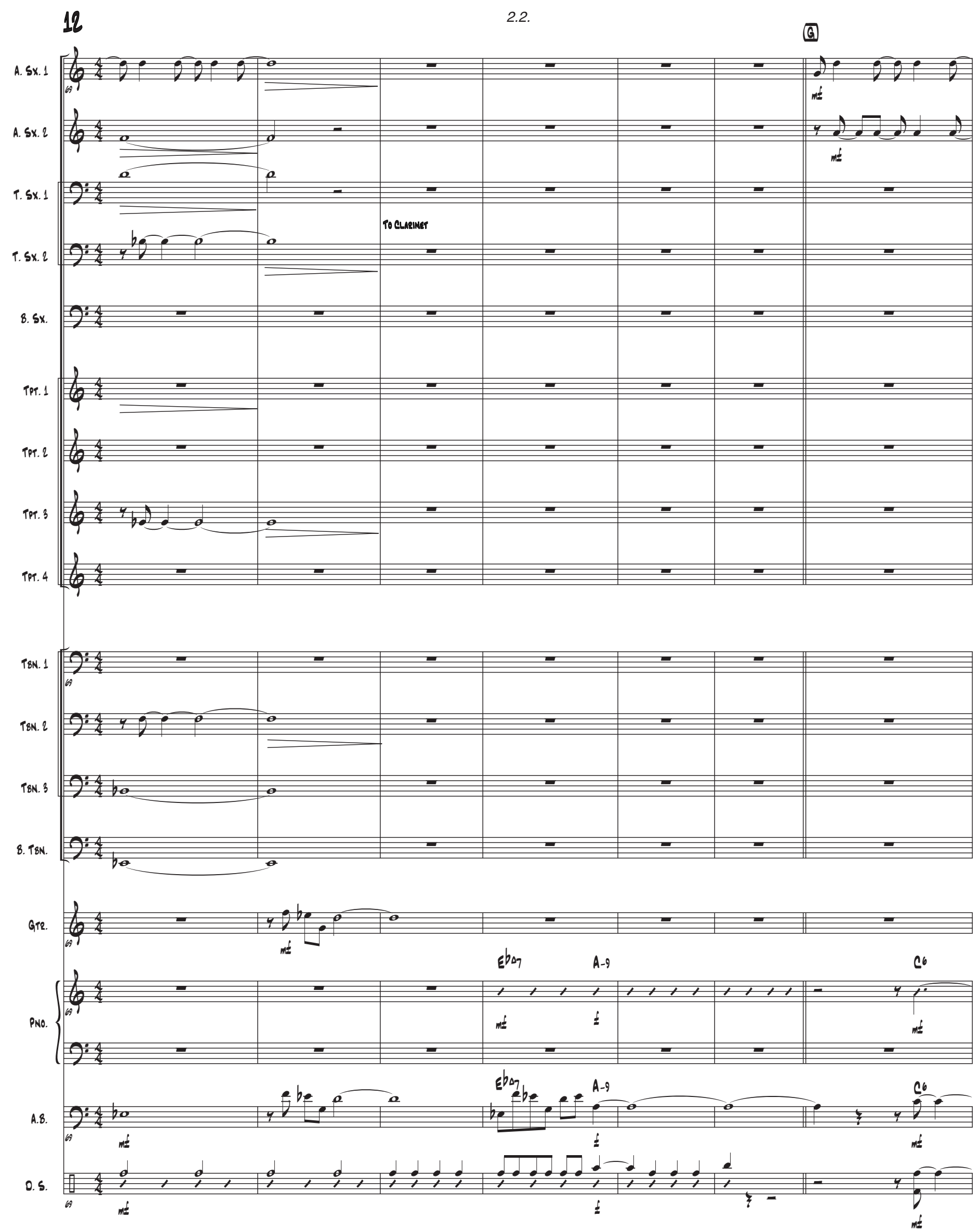


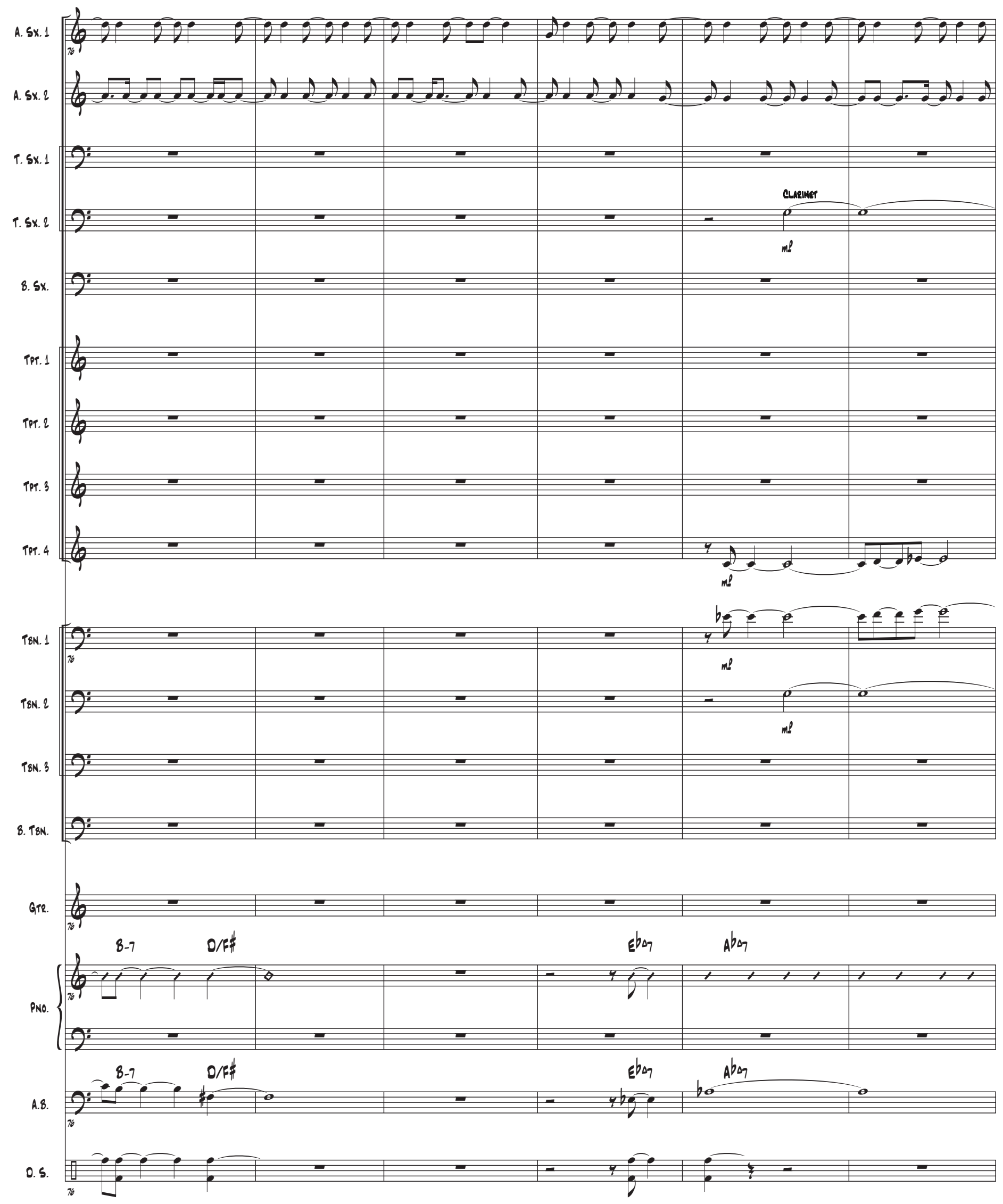


14

()
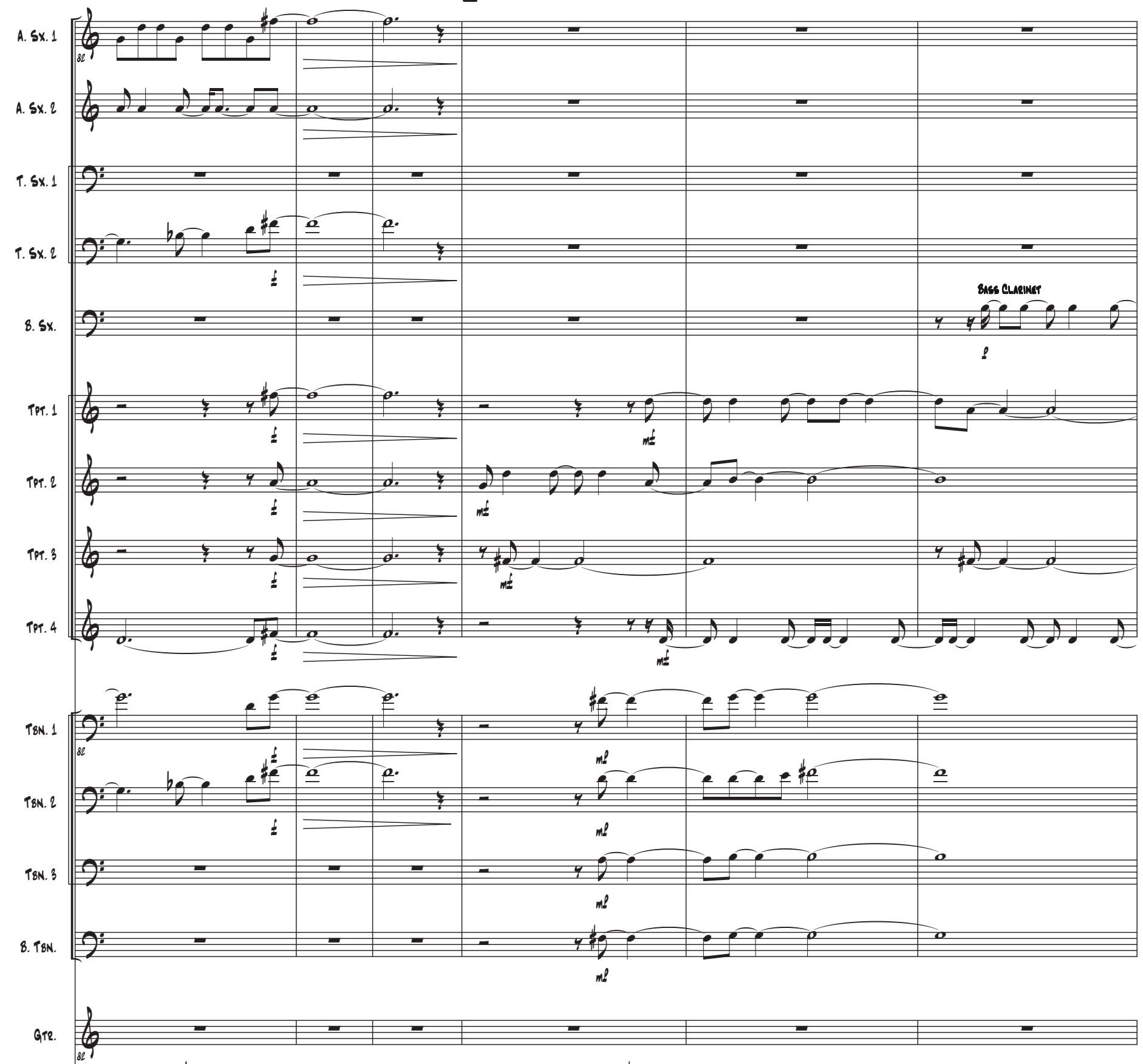

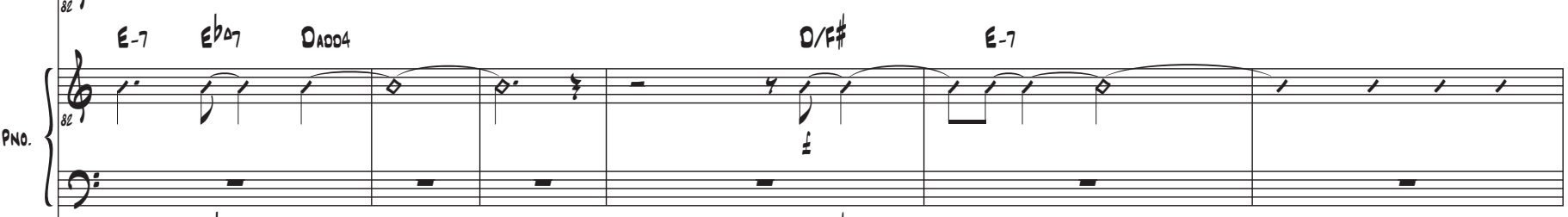

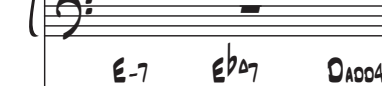

A.8.

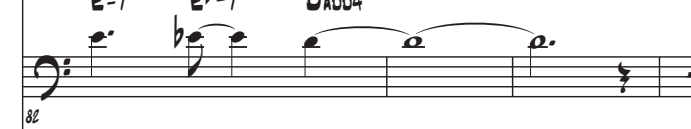

O/F E-7

0.5. 

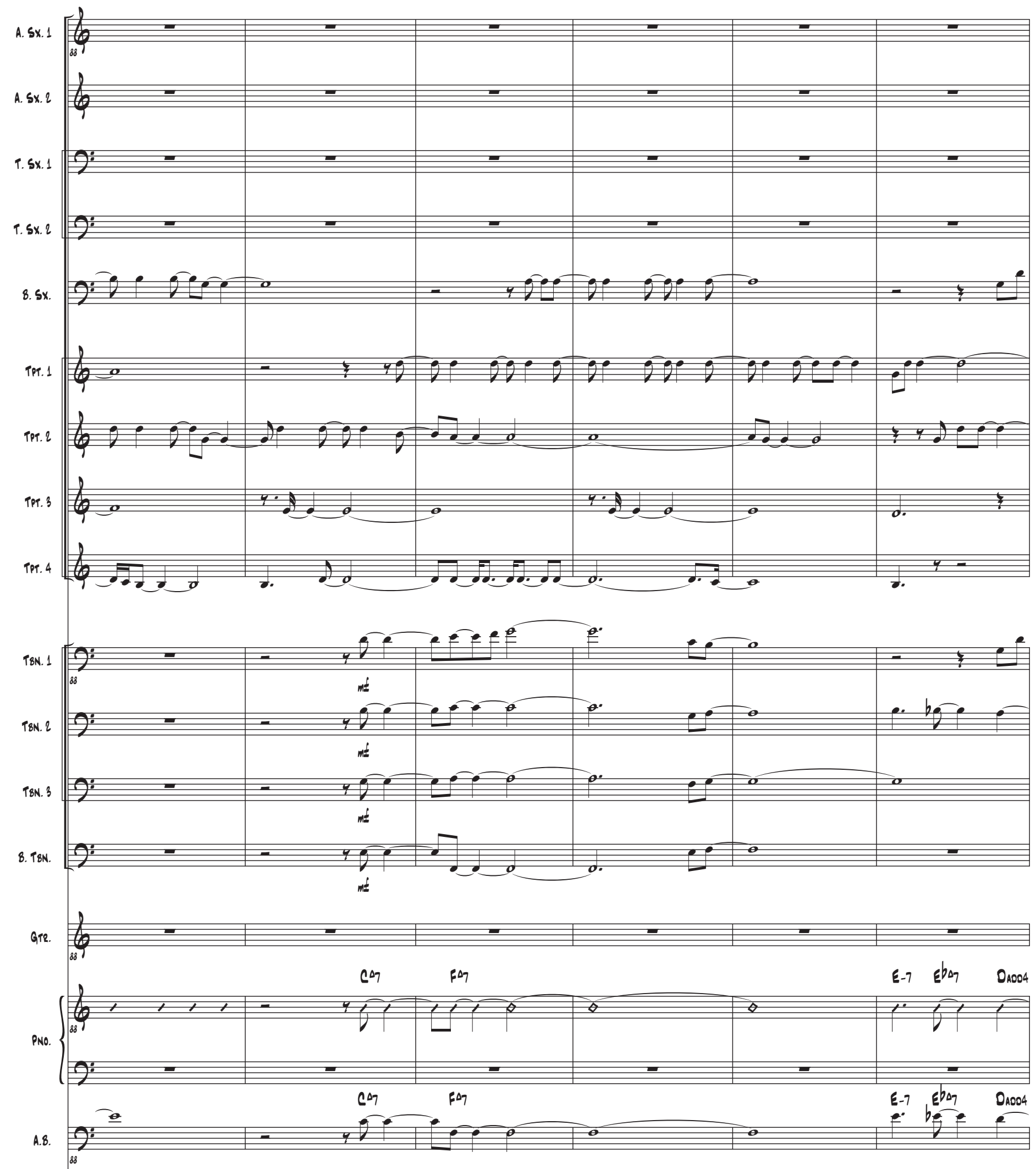

o. 5. 
16

2.2.
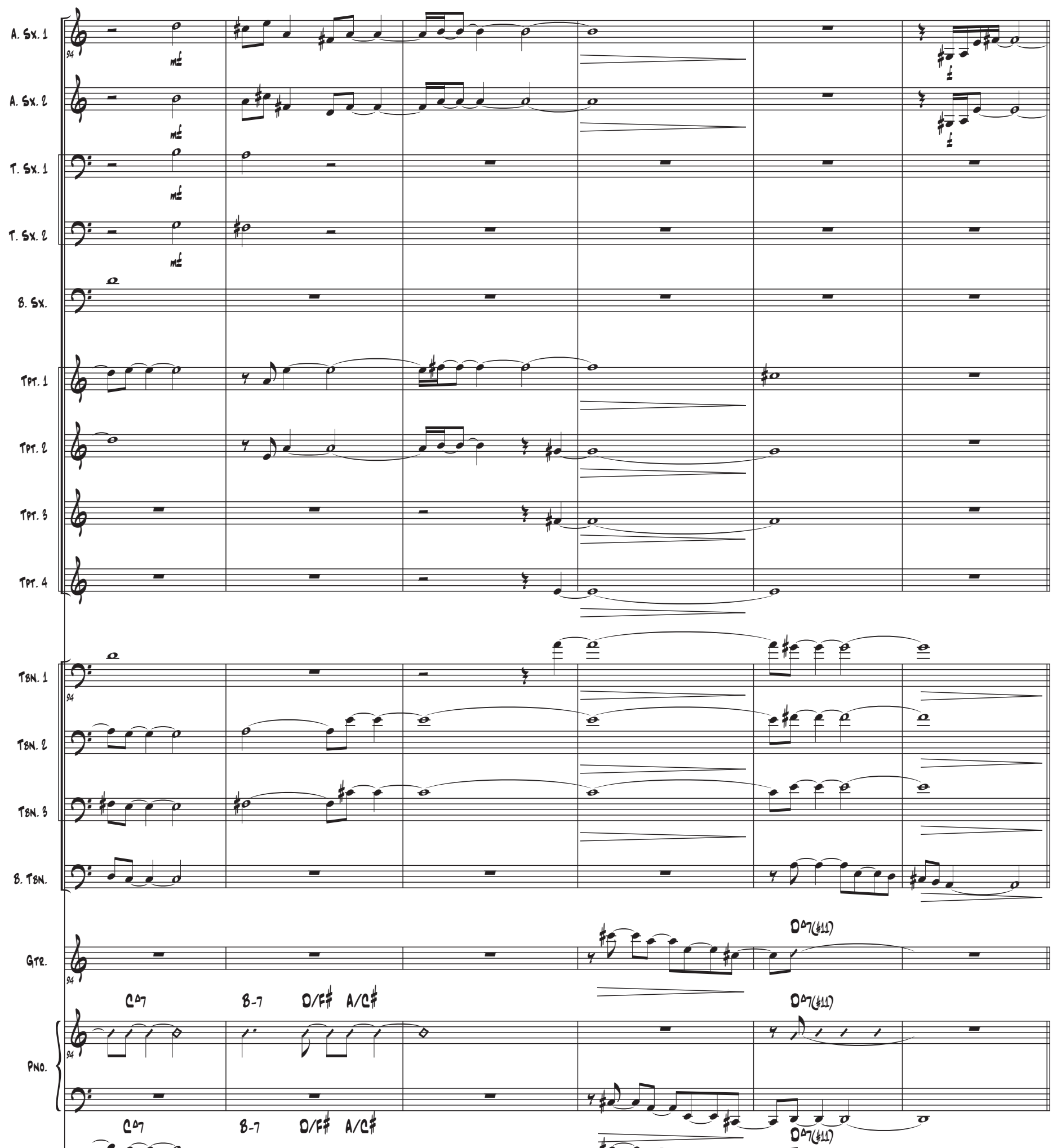

A.8.

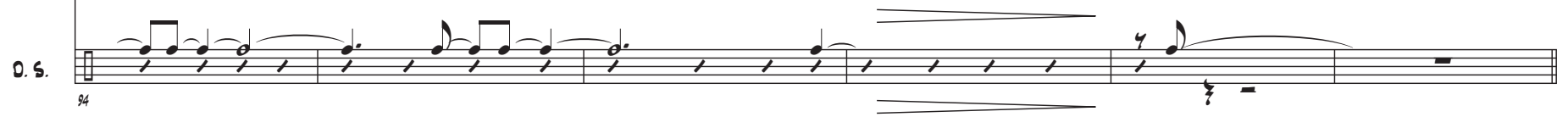



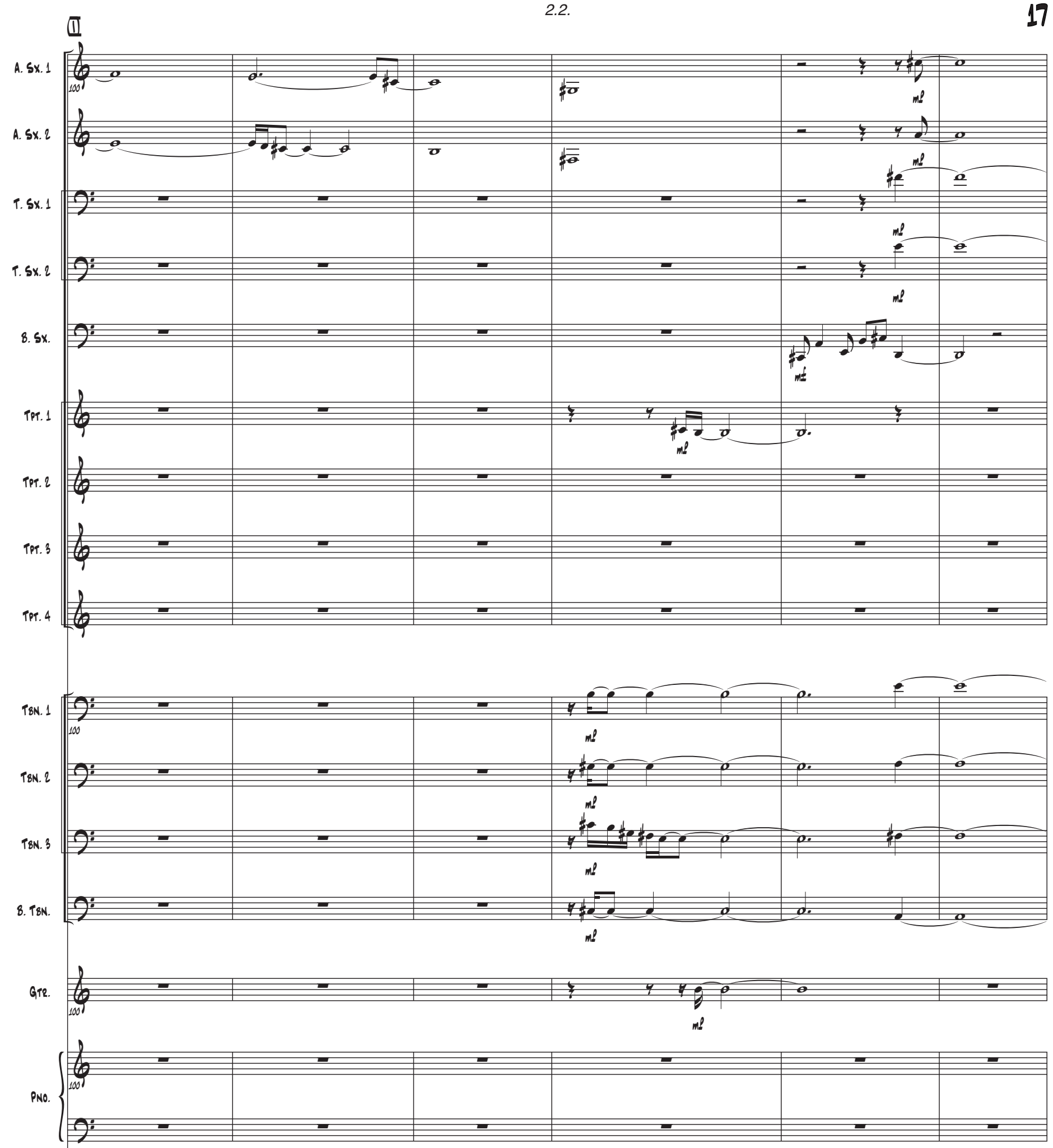

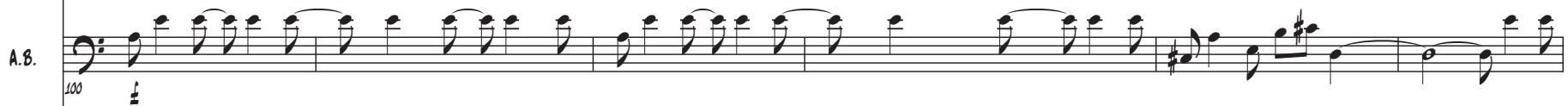



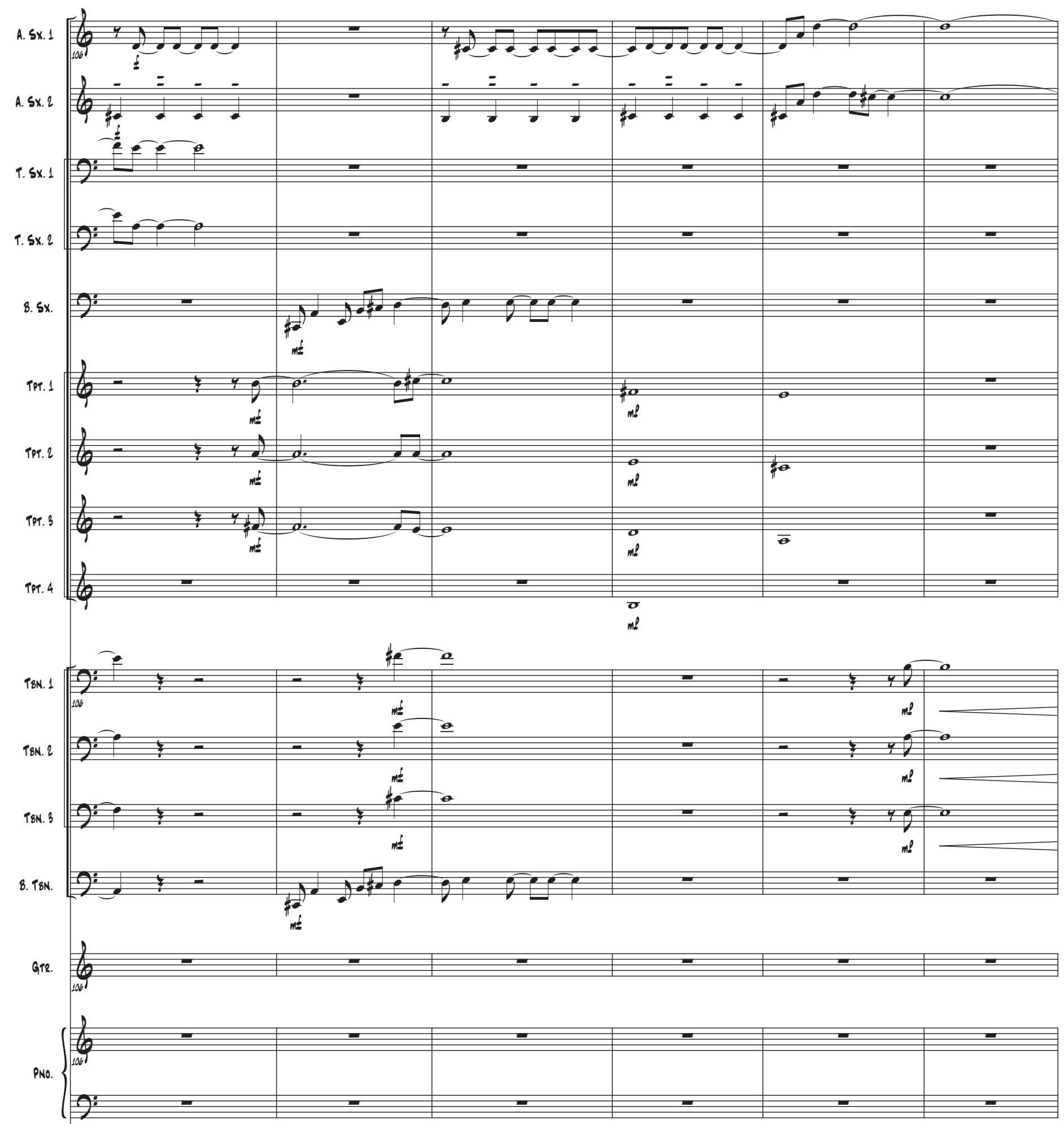

a.8.

0.5.

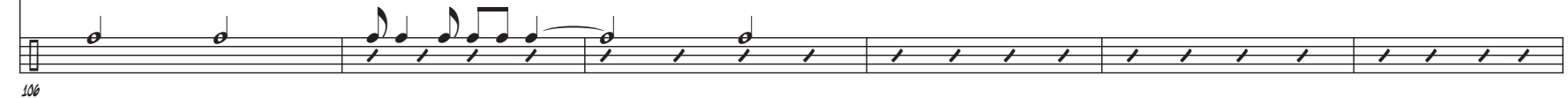



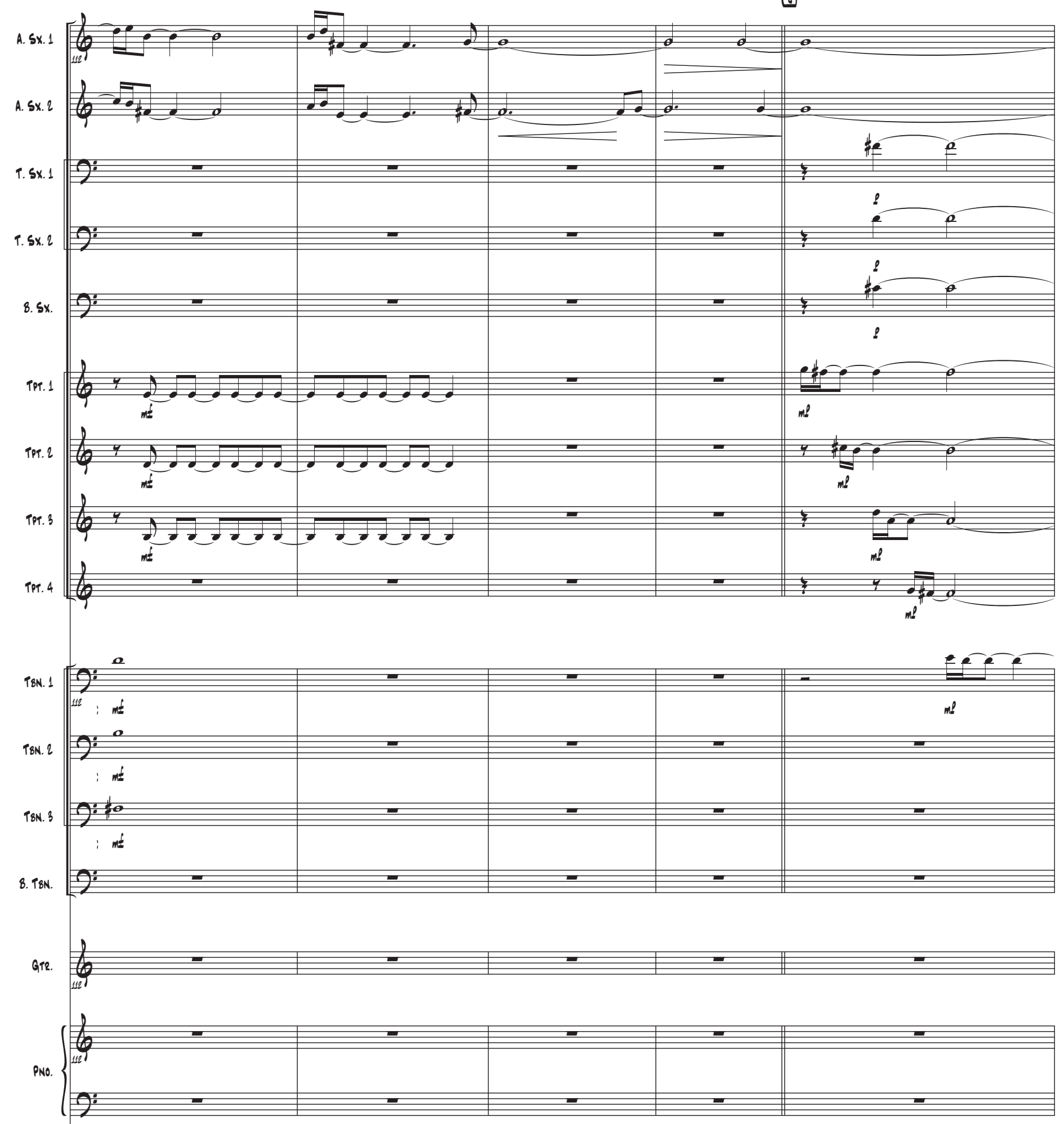

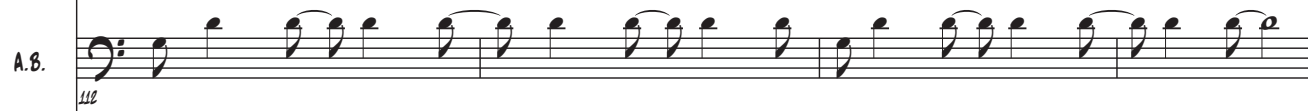

o.s. 

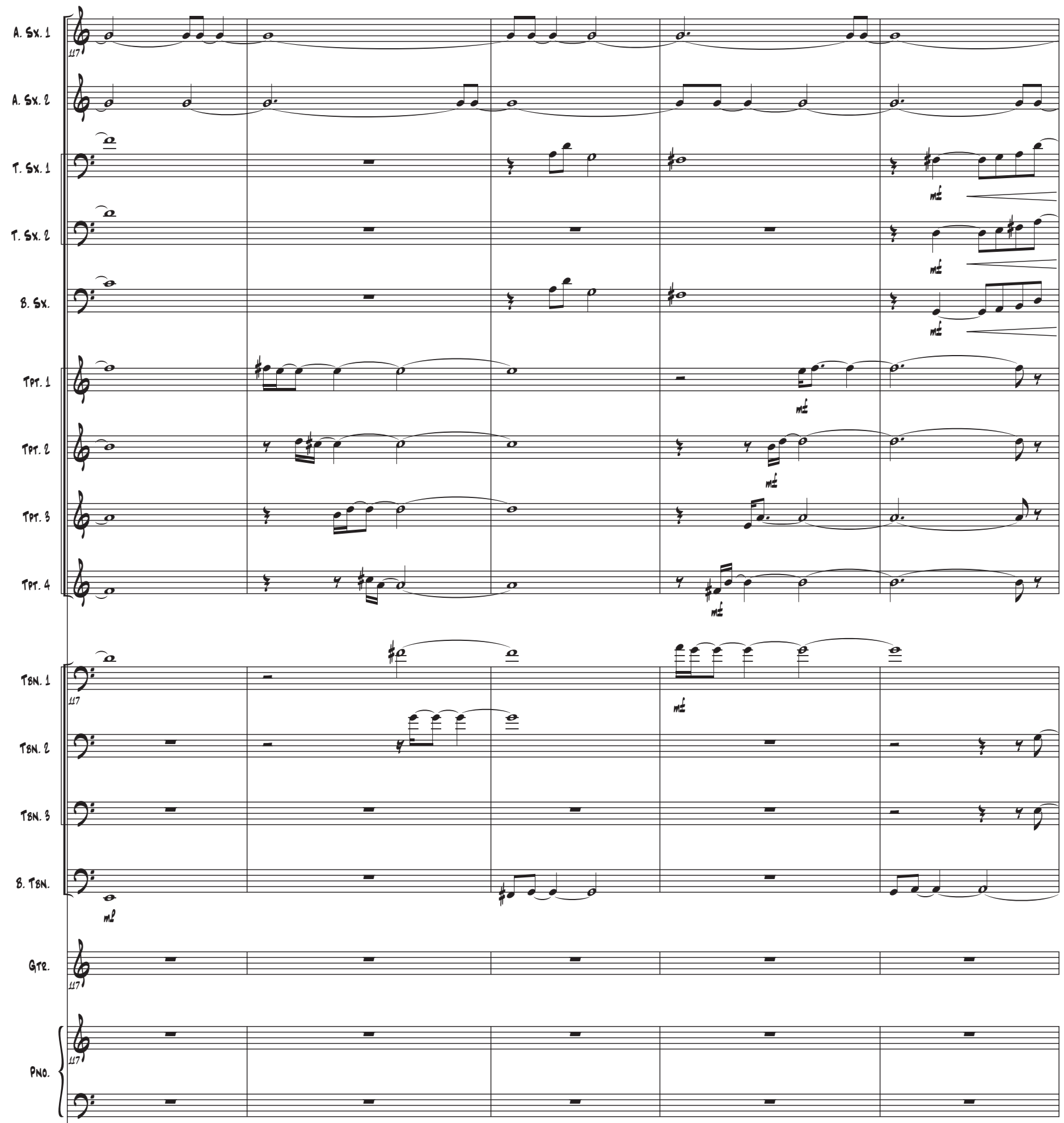

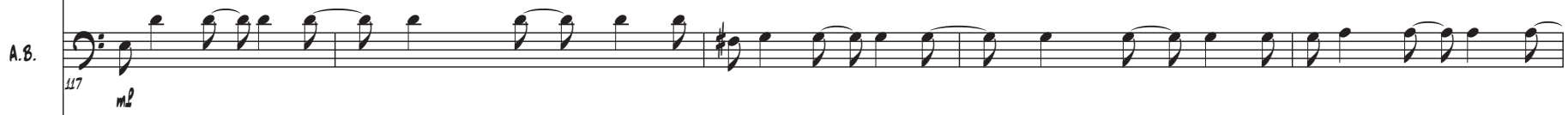

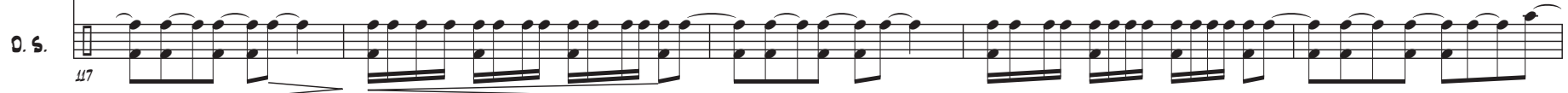



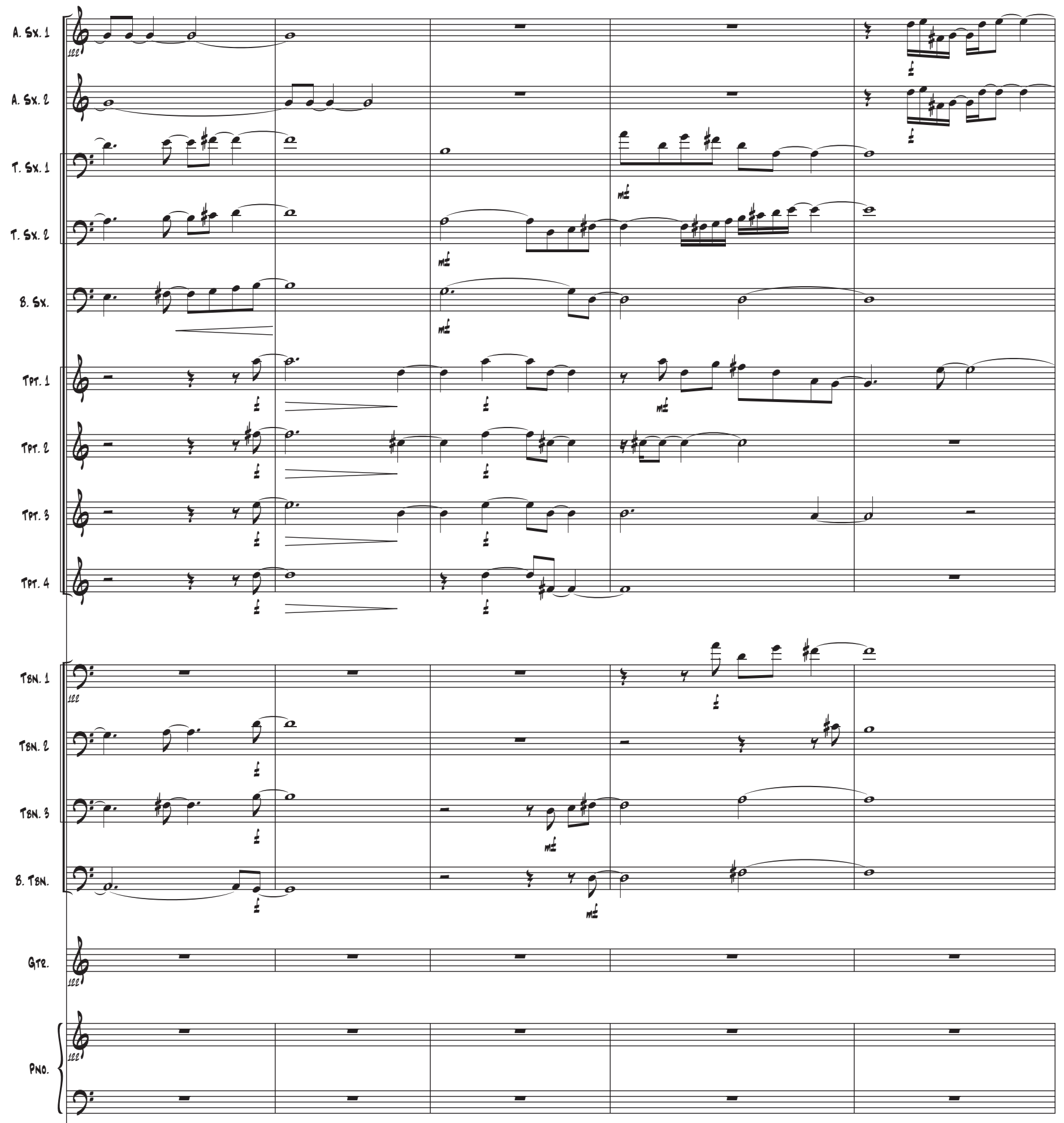

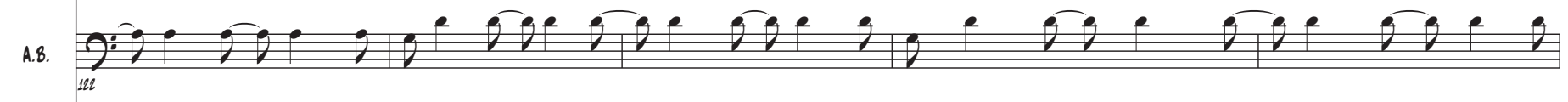

0.5. 

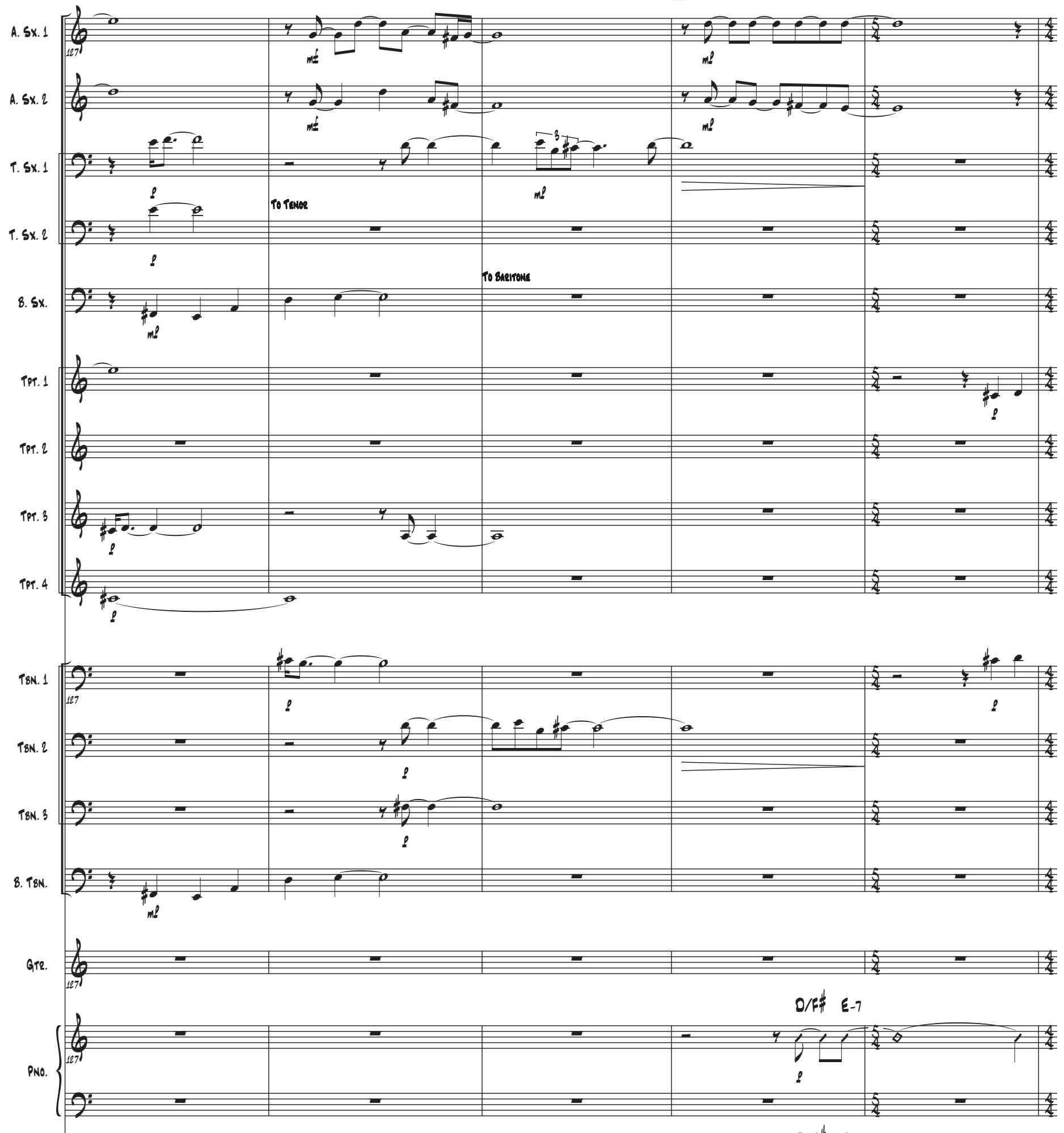

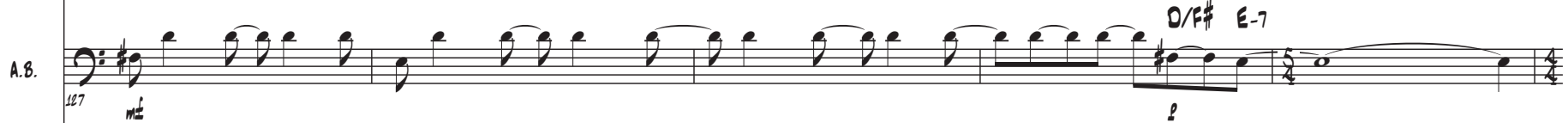

0.5. 

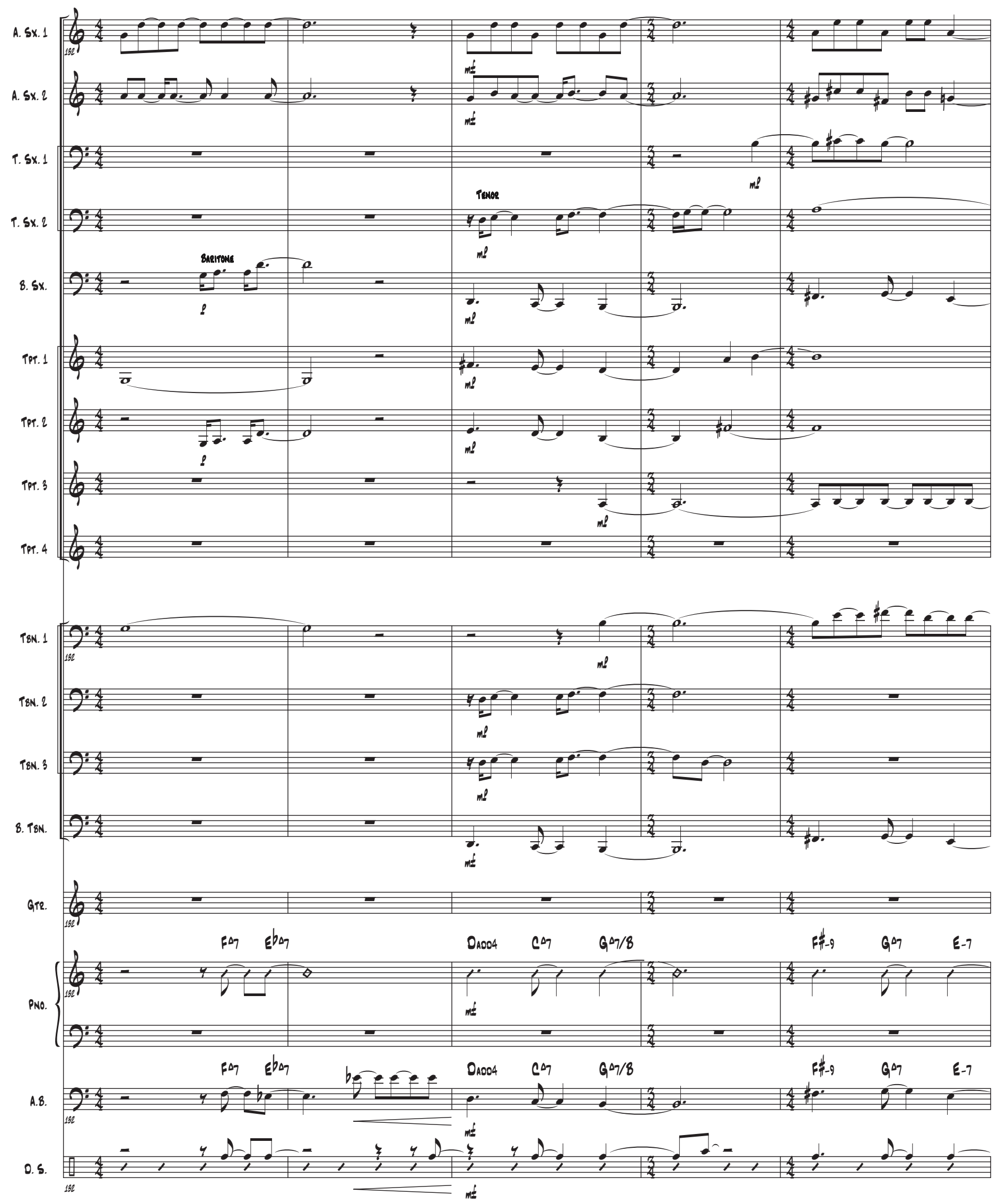
24

(1)
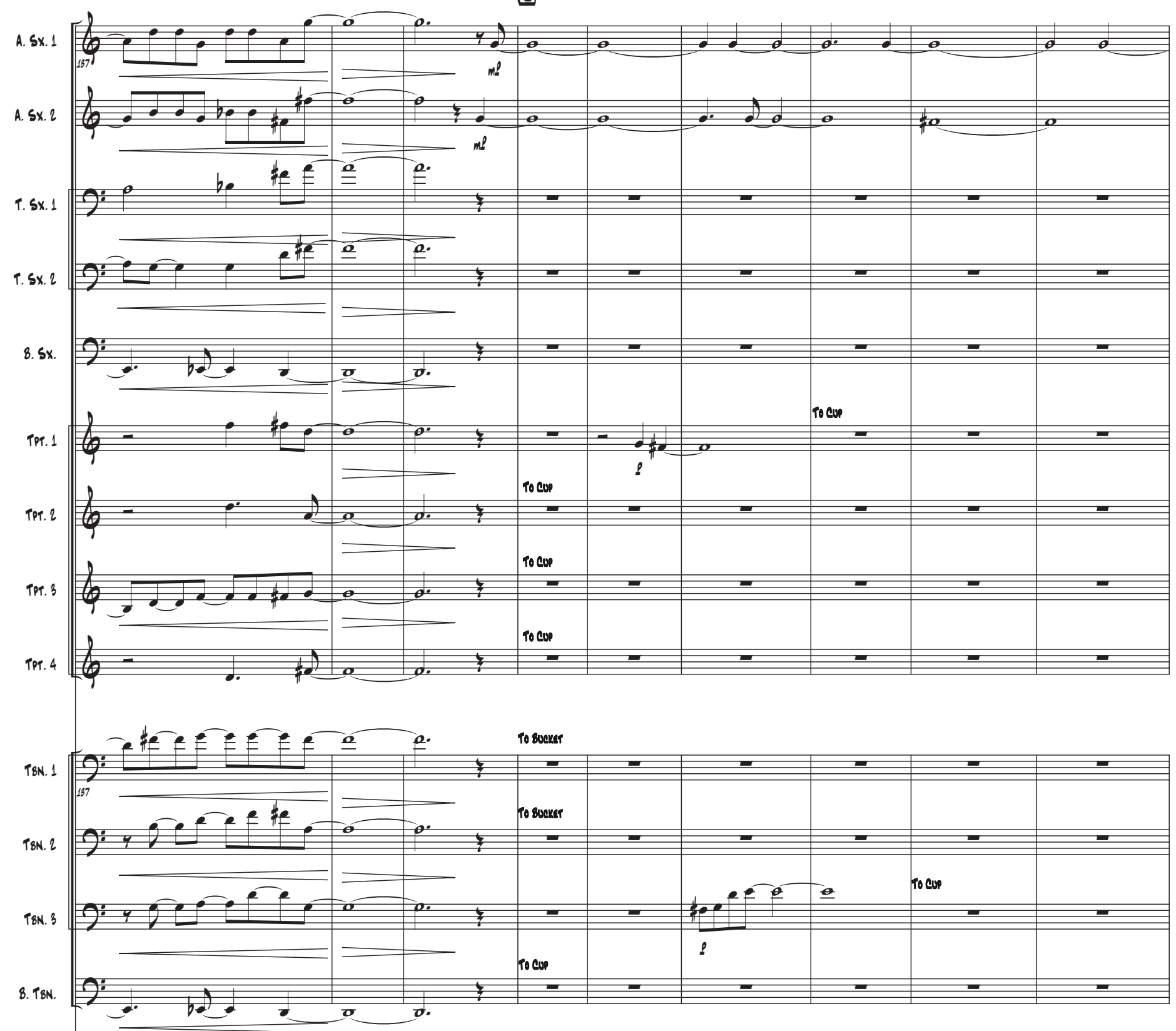

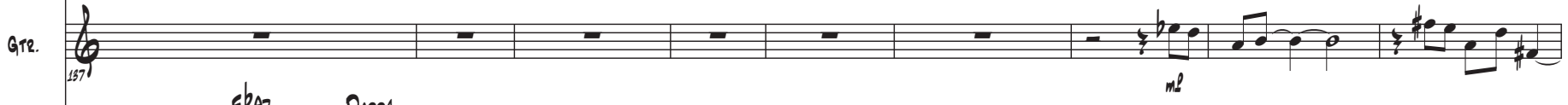

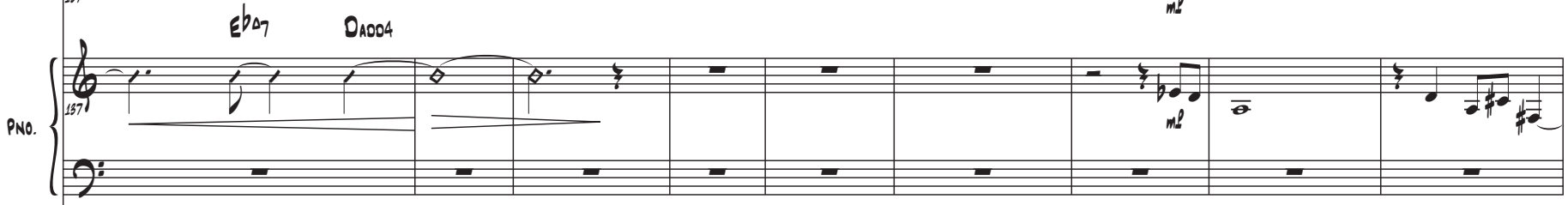
A.8.

o.s. 

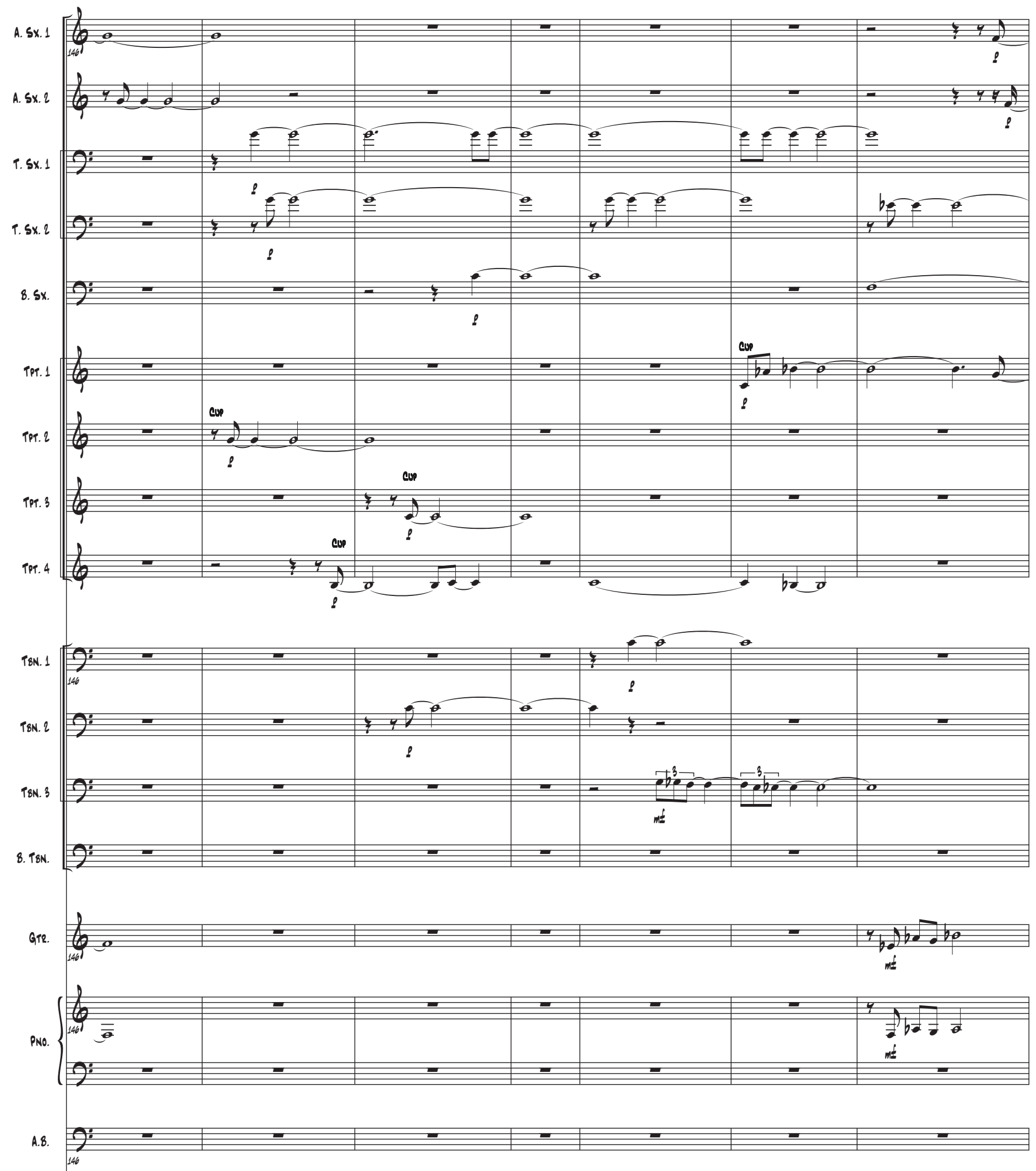

o.s. 

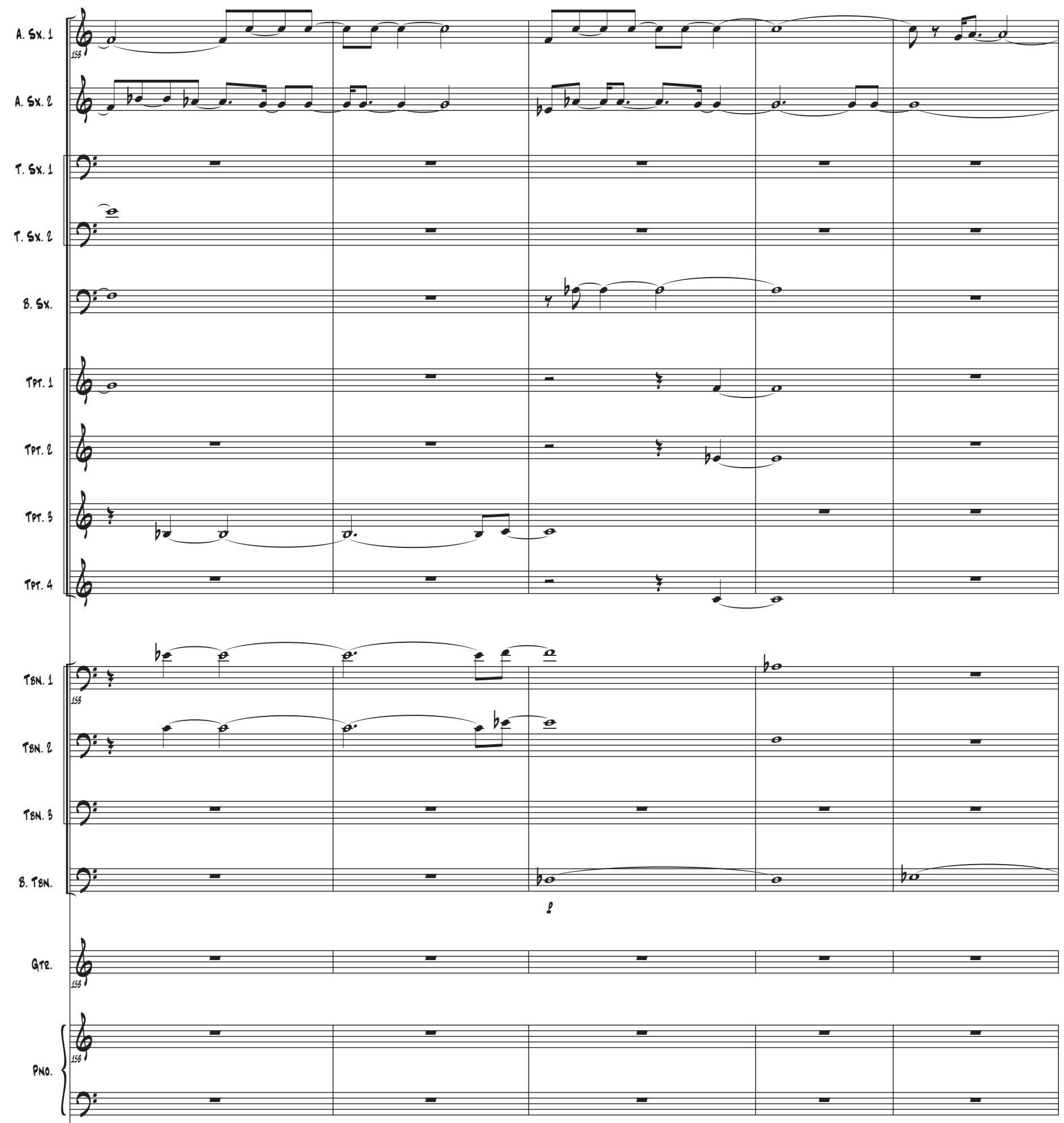

A.8.

0.5. 建 

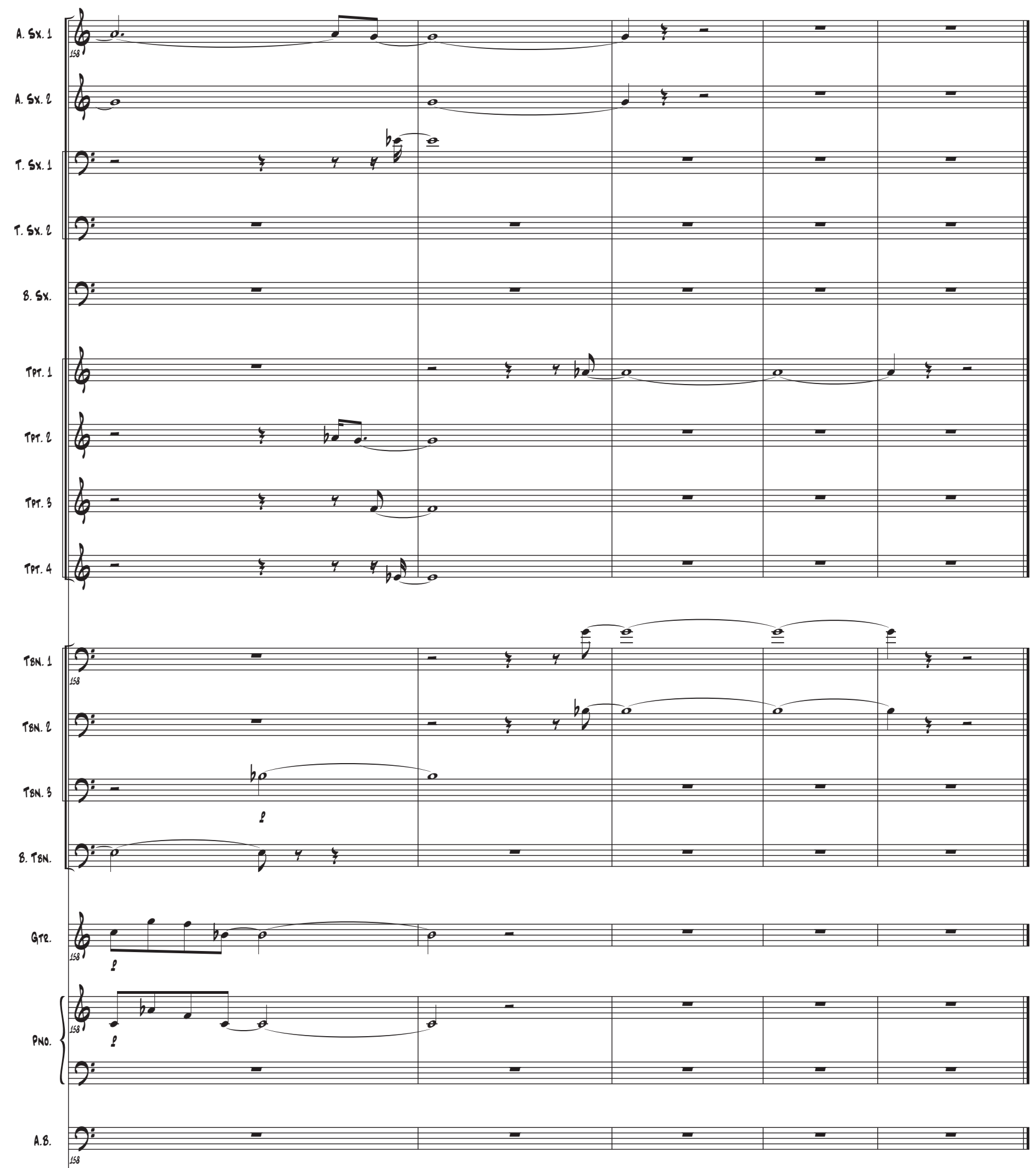

0.5. $\stackrel{.}{.} \cdot d$ 



\section{(Dream within a Dream)}
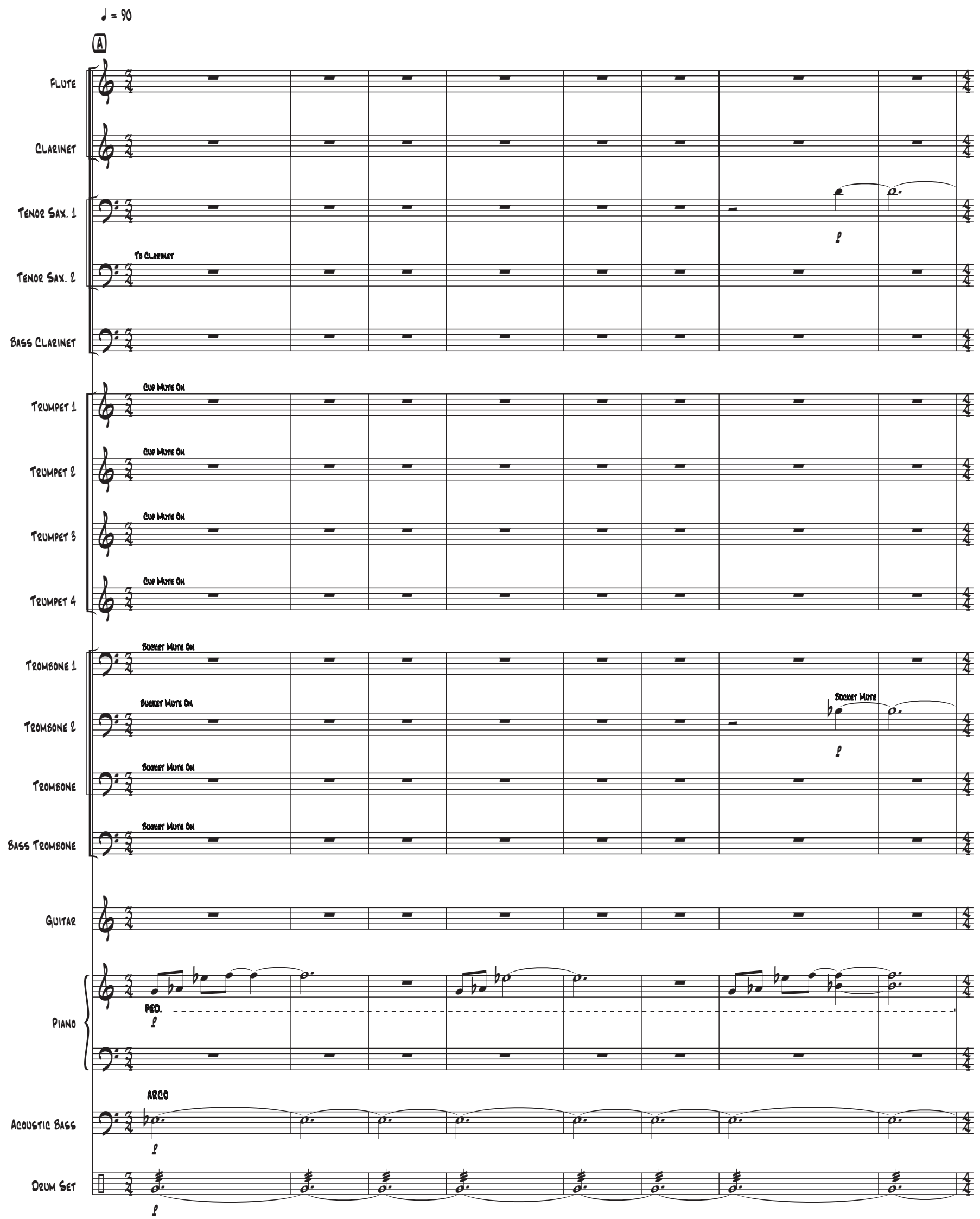

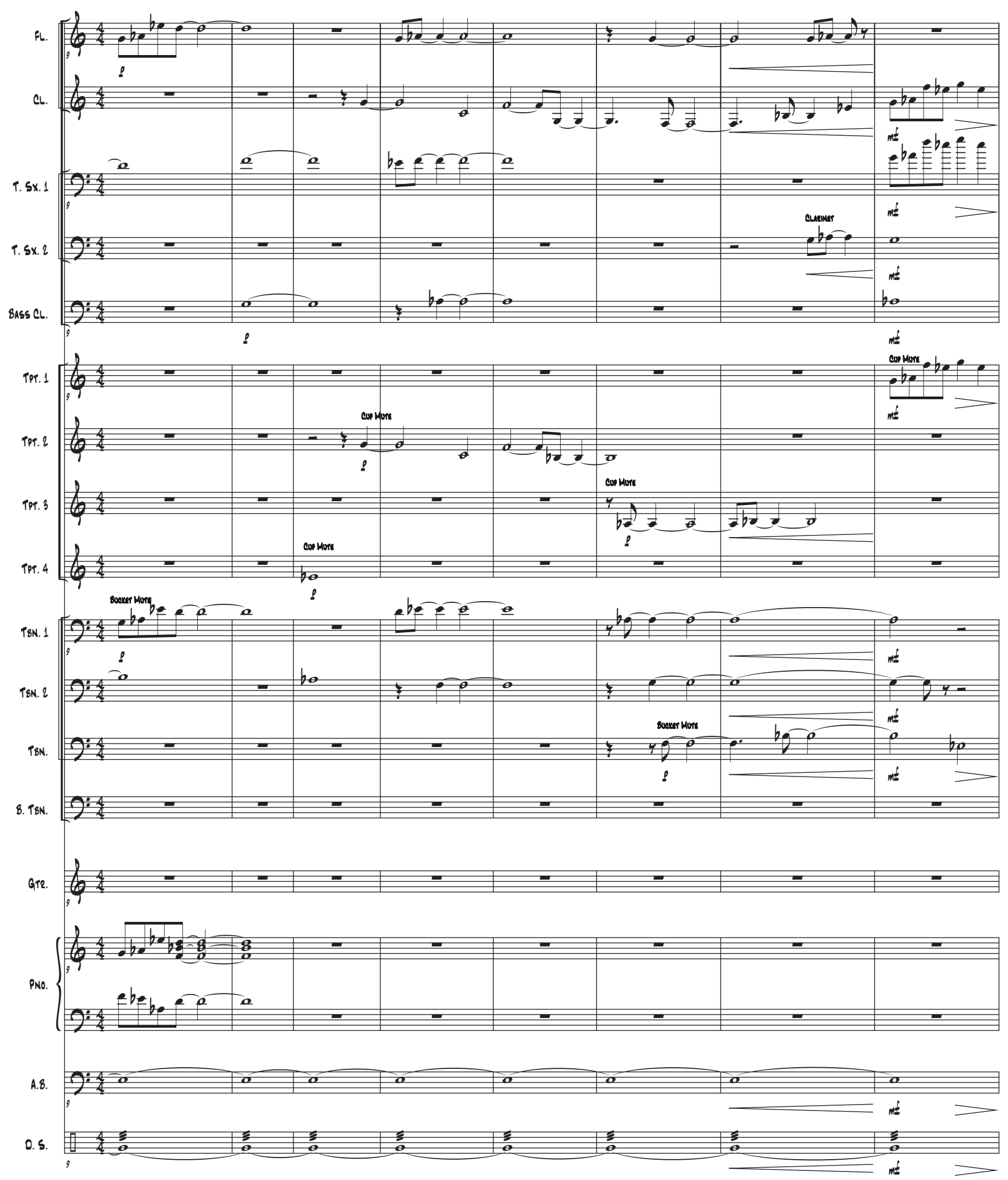


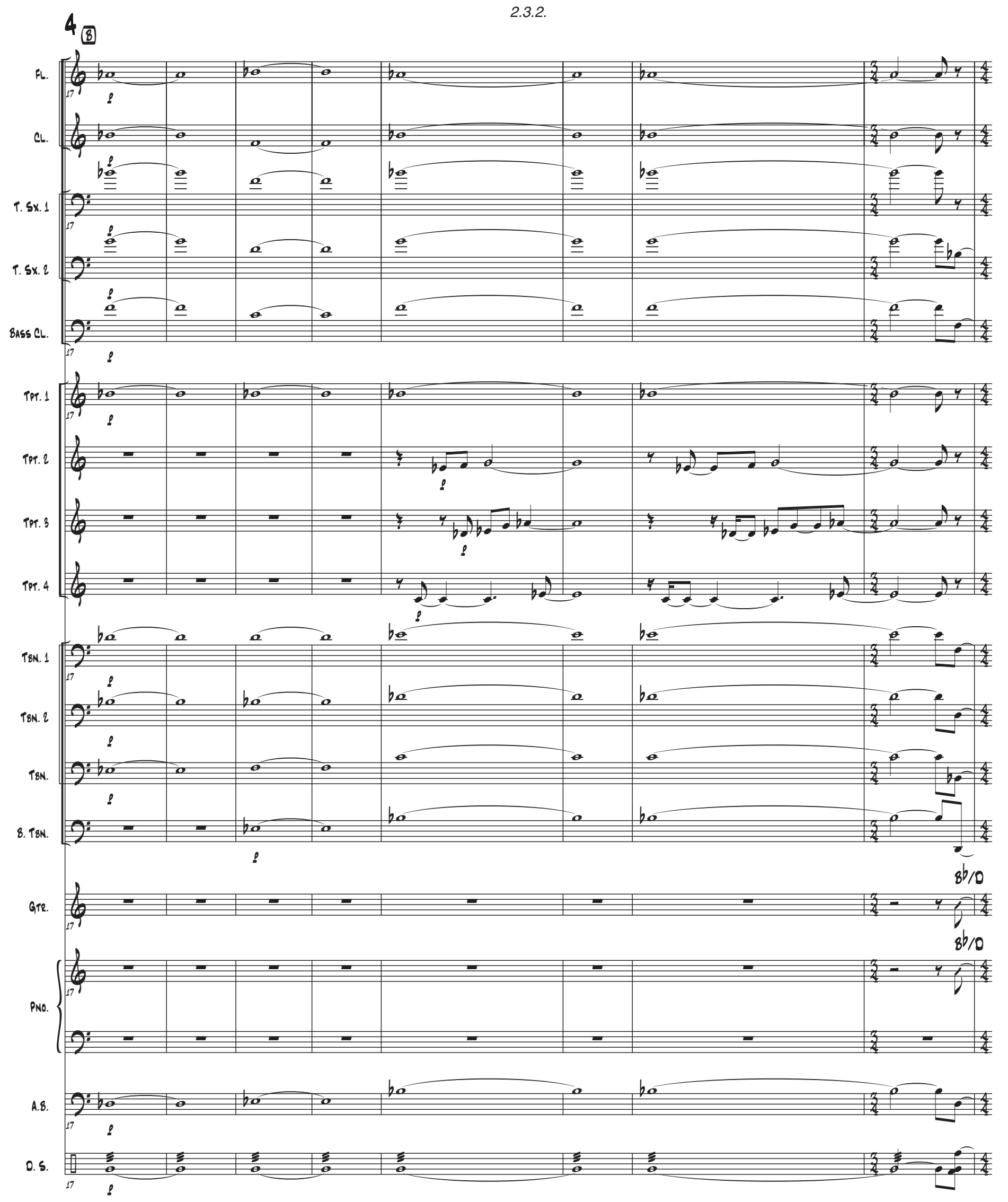


2.3.2.

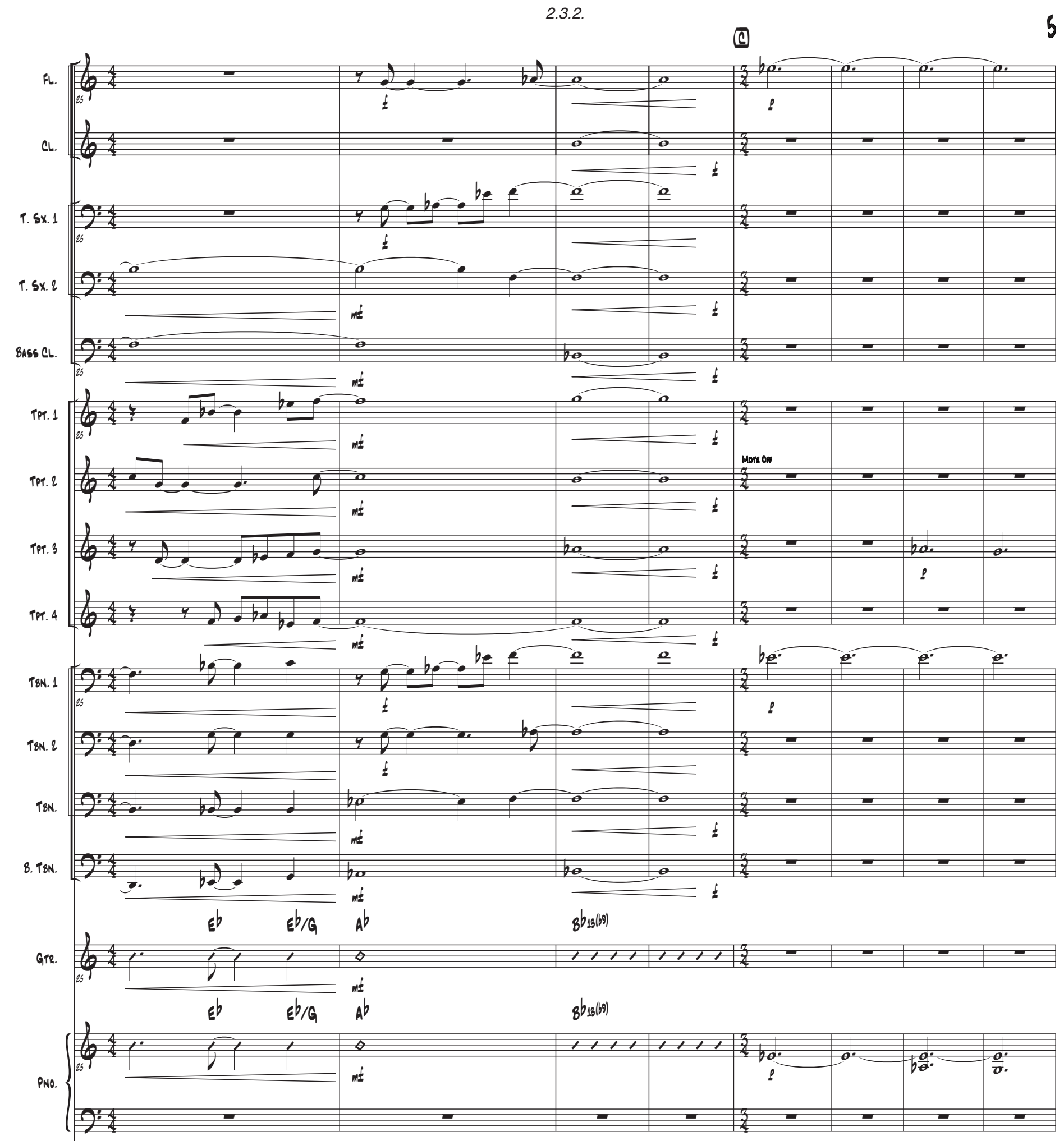

6

A.8.

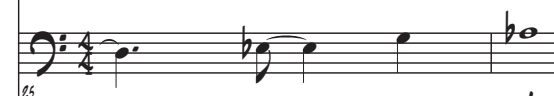

bo

o. 5 . $m$ ! 


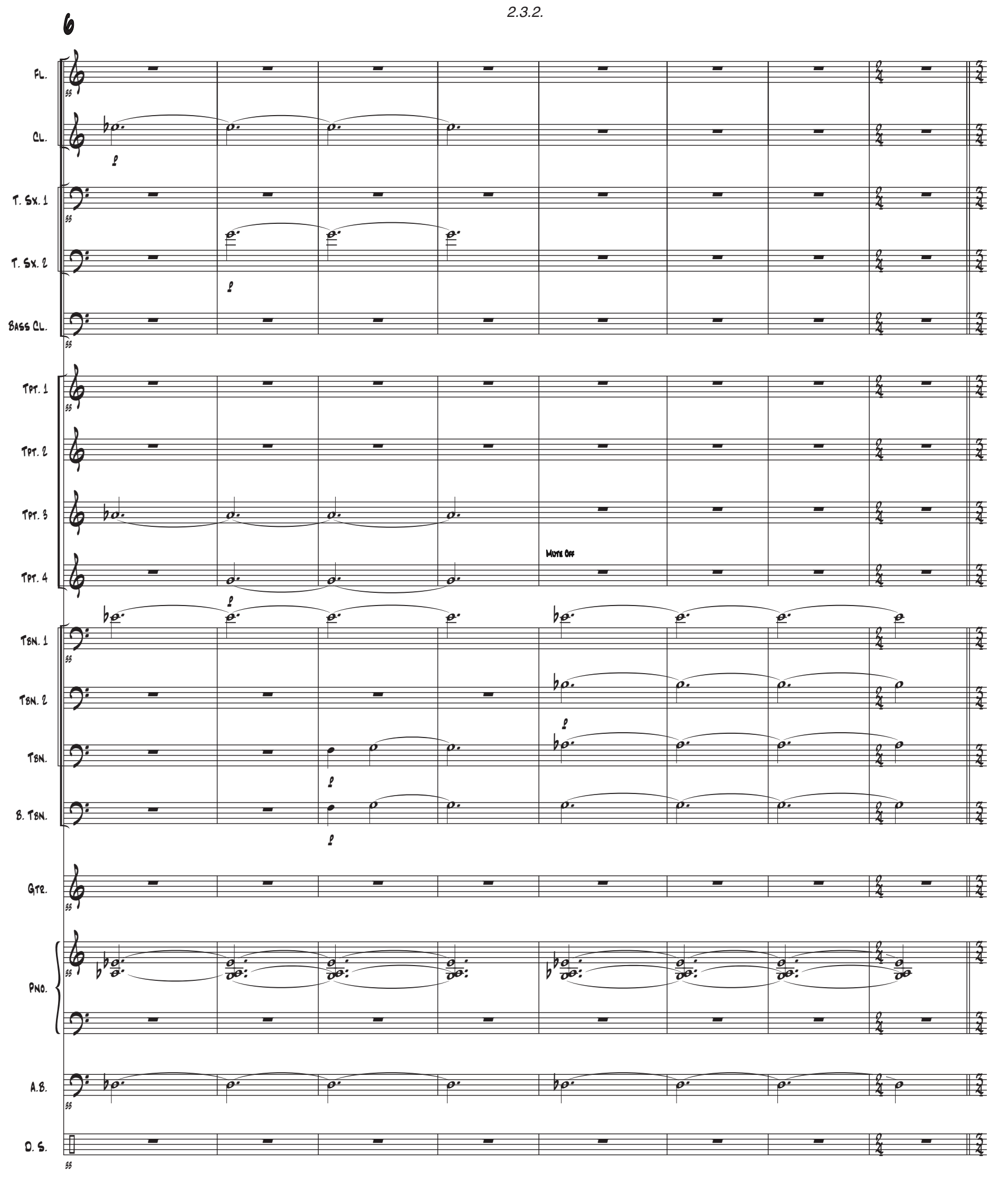



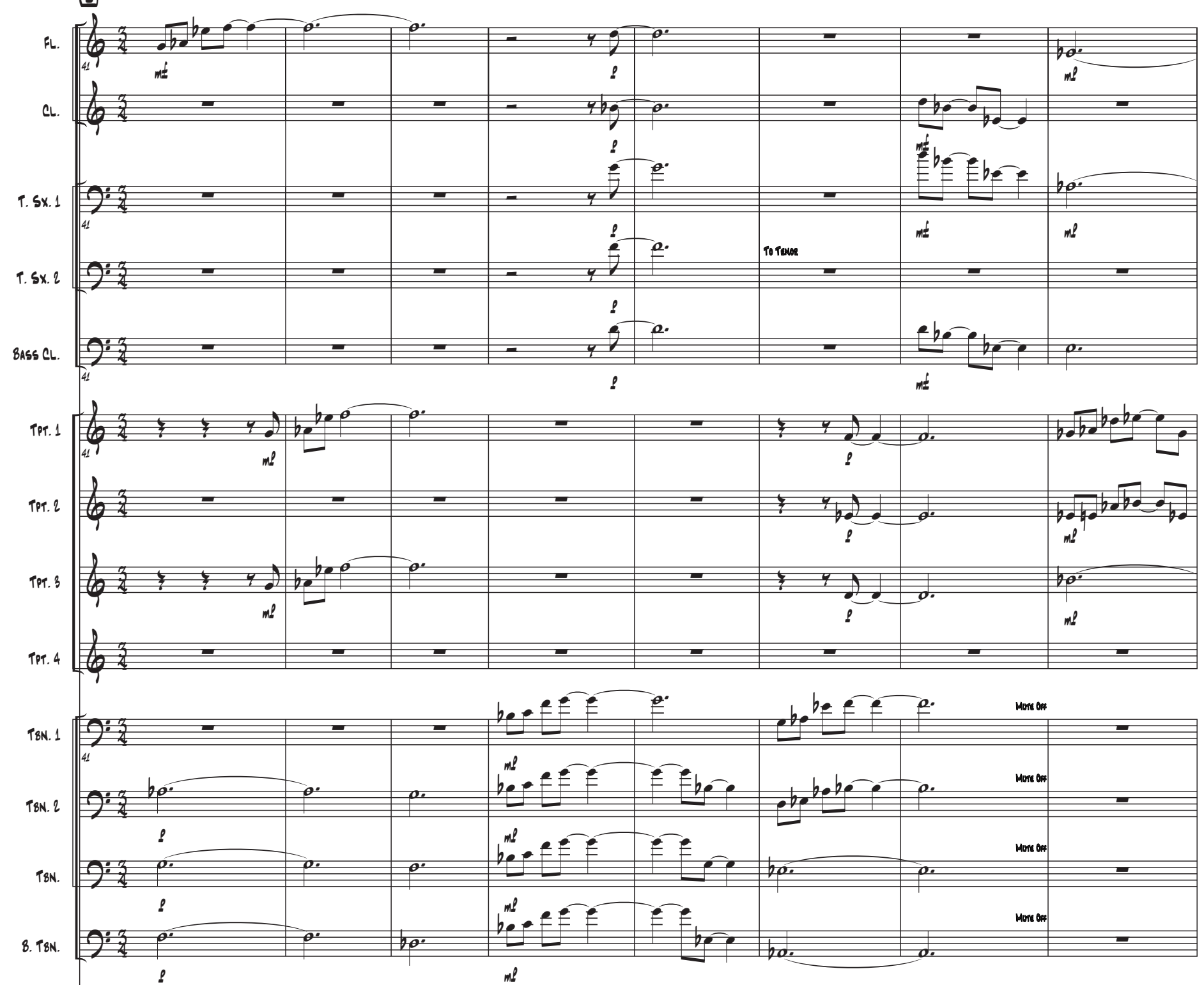

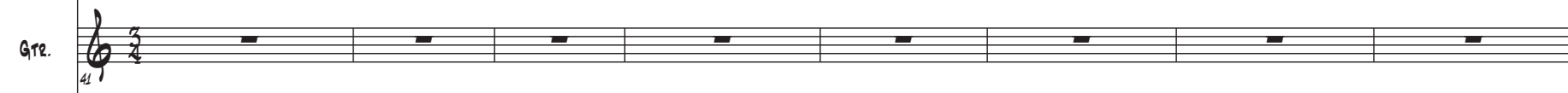

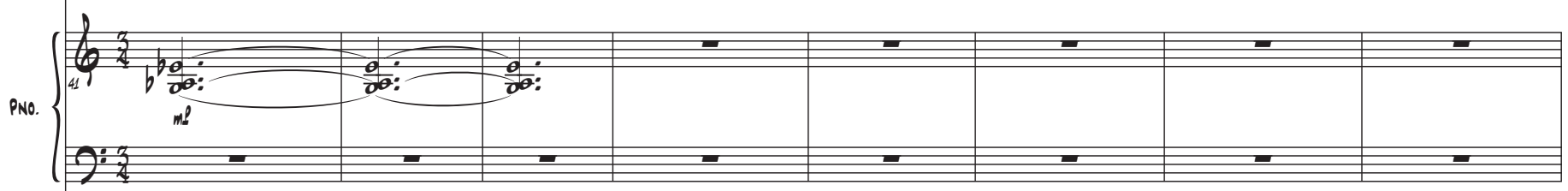

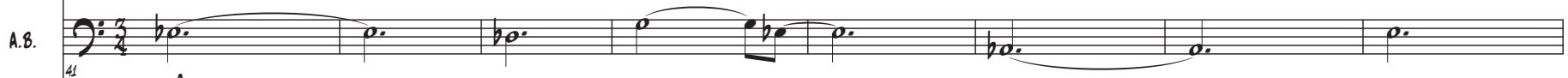
o. . 

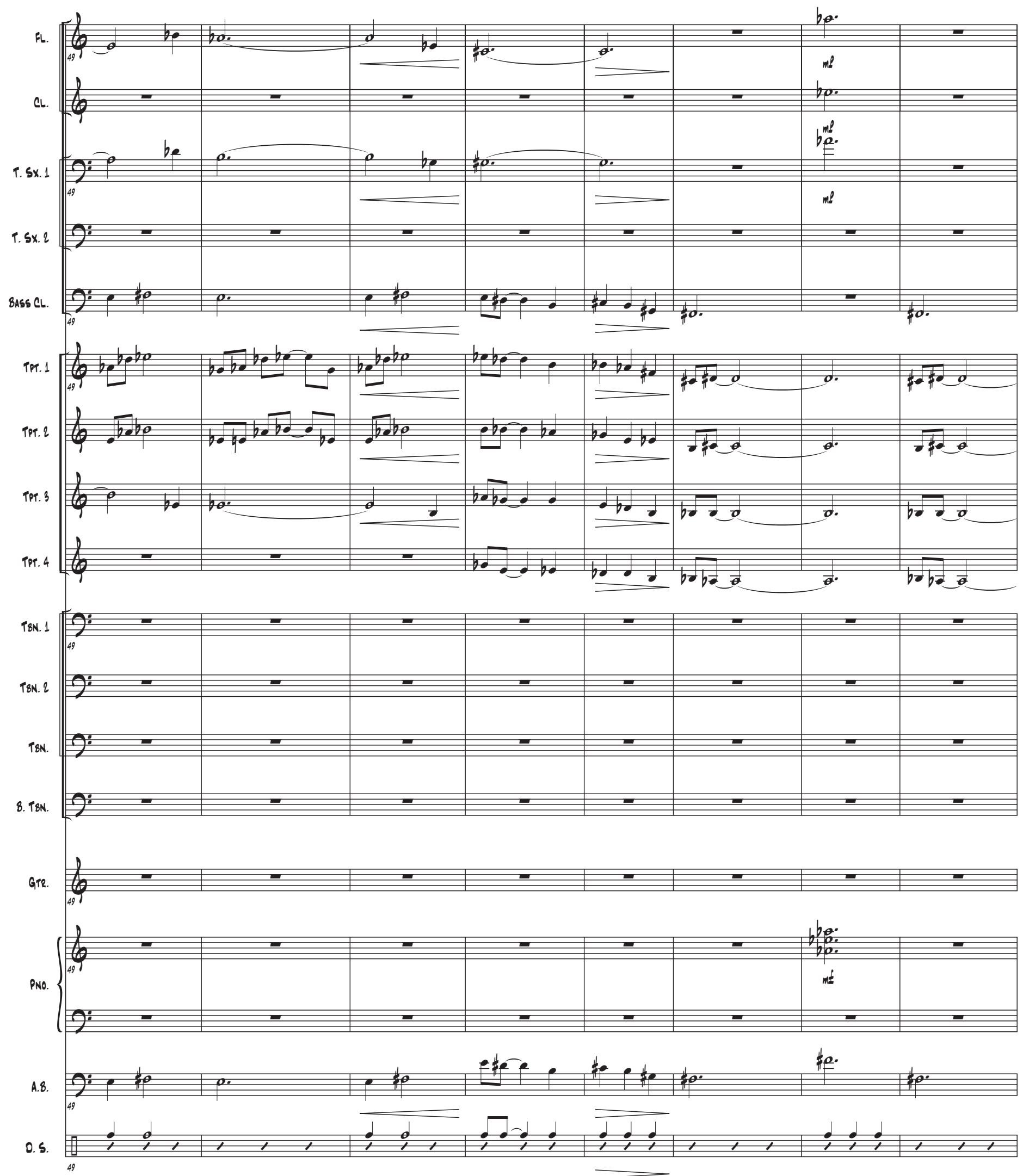
2.3.2.

9
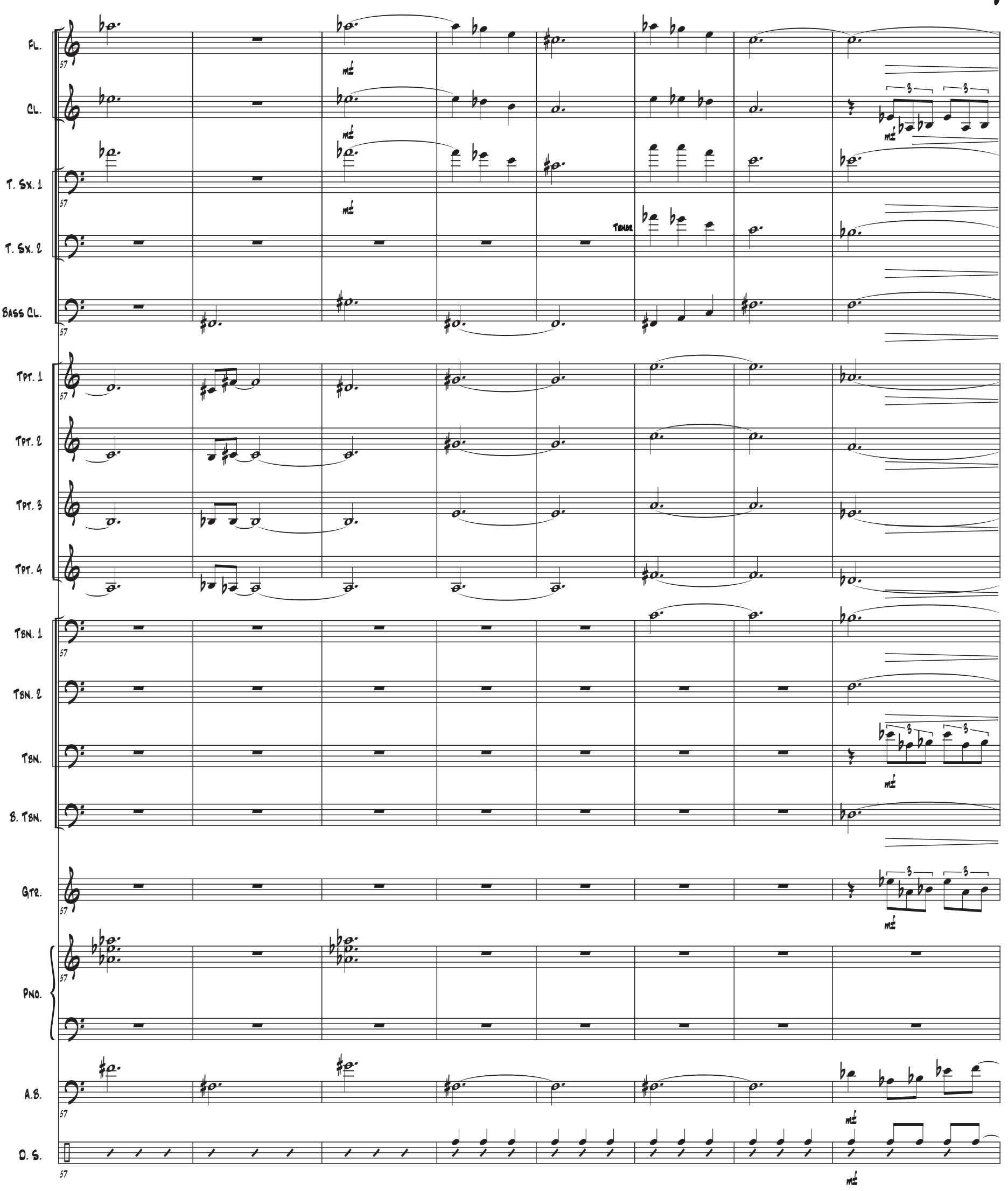

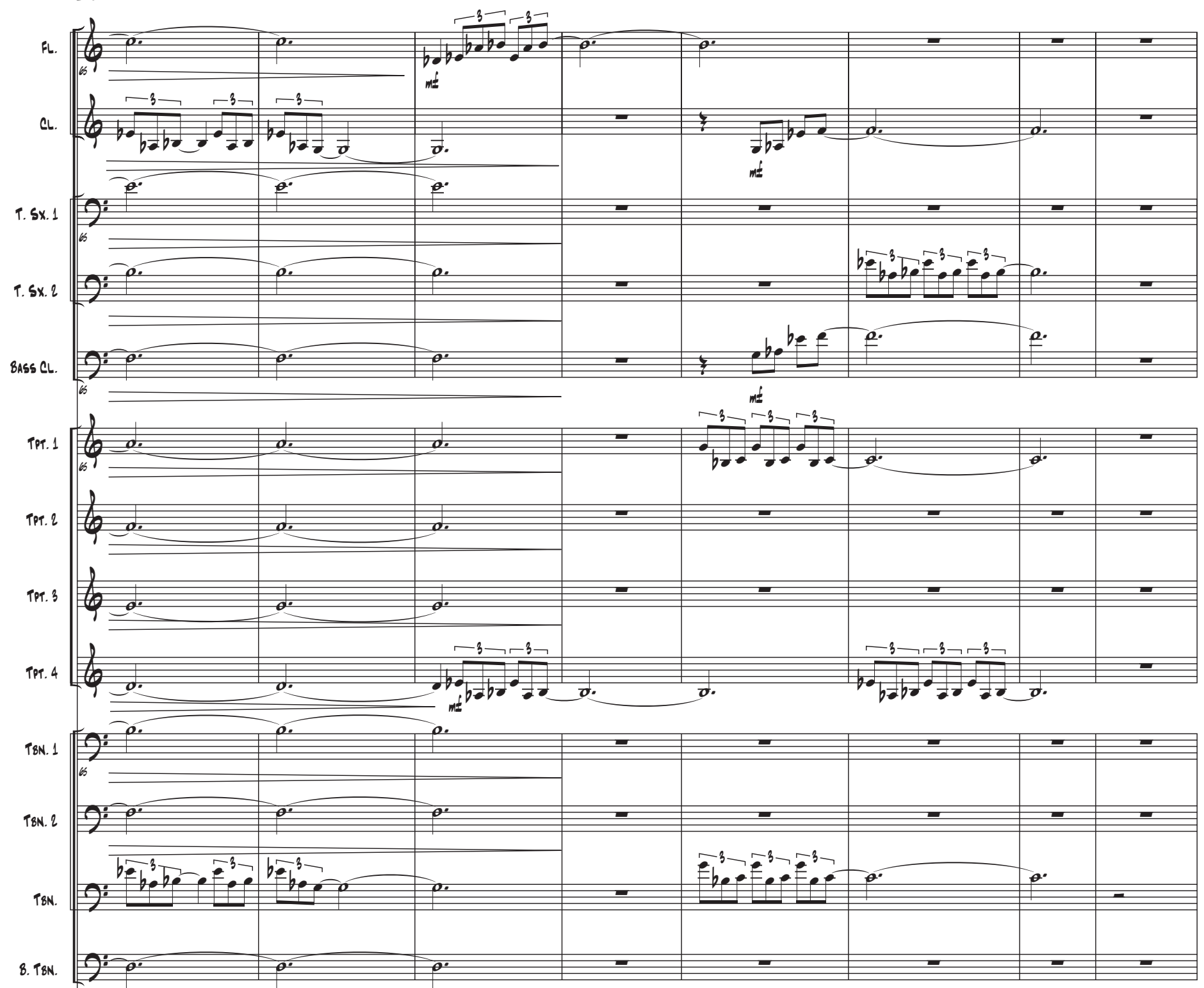

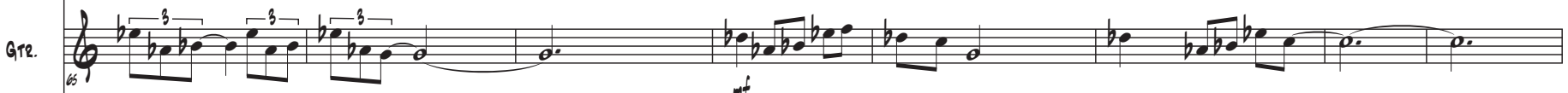

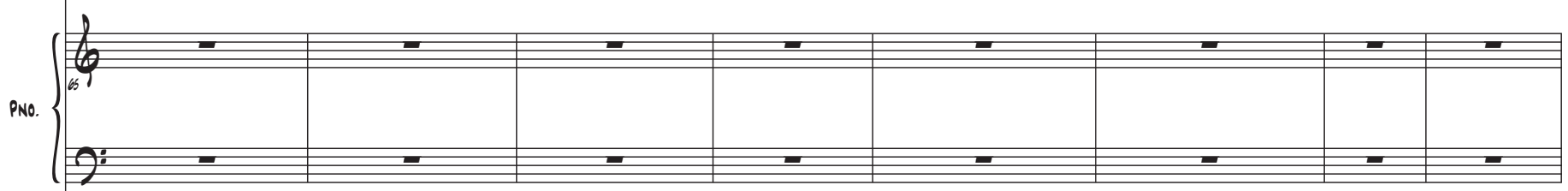

A.8.

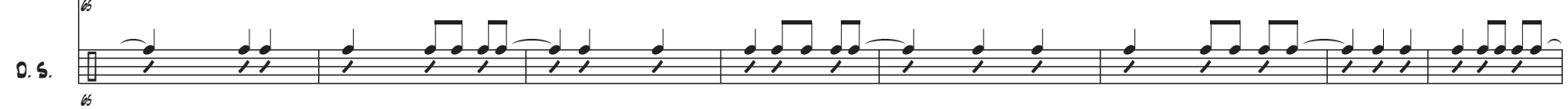


(6)
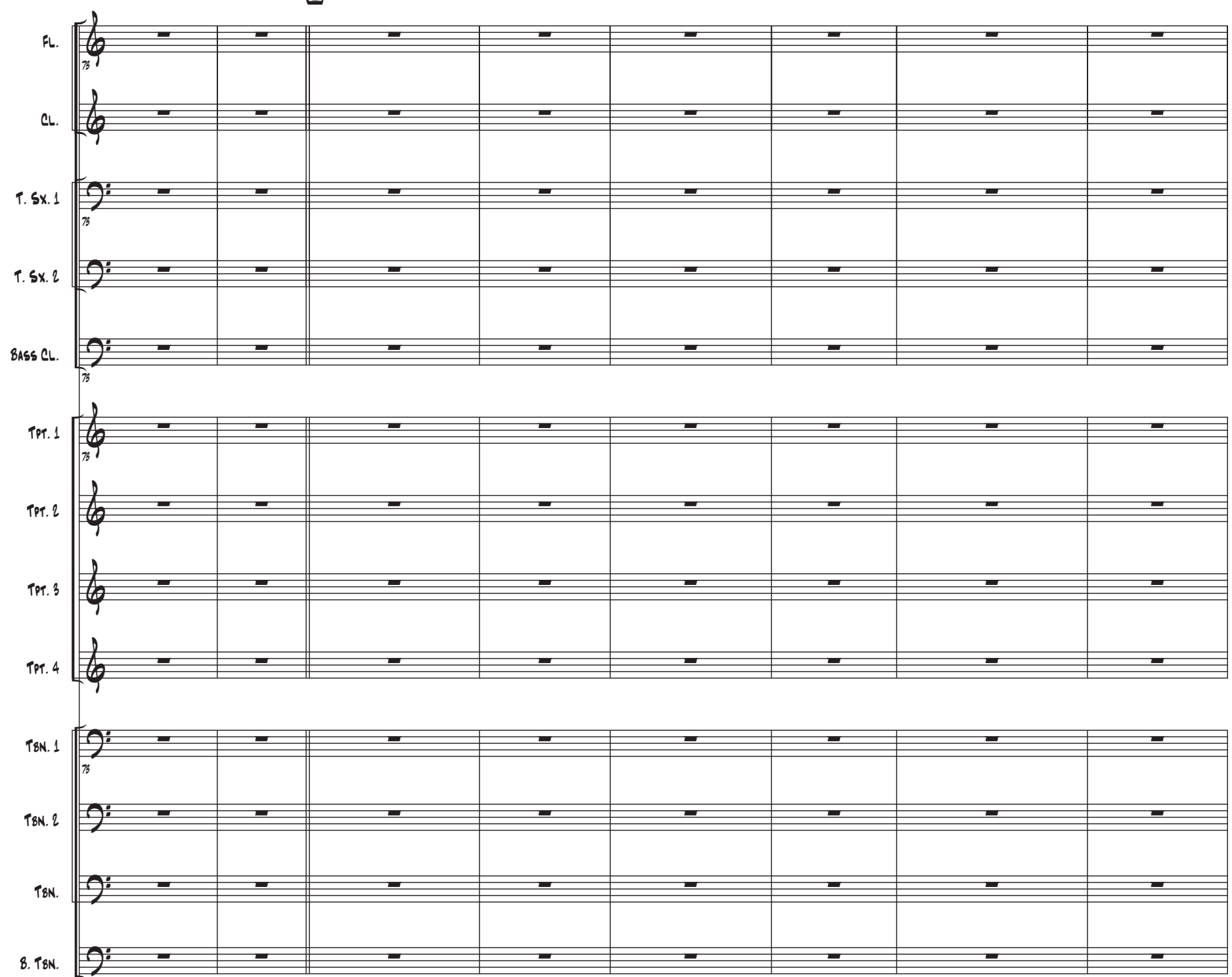

arre

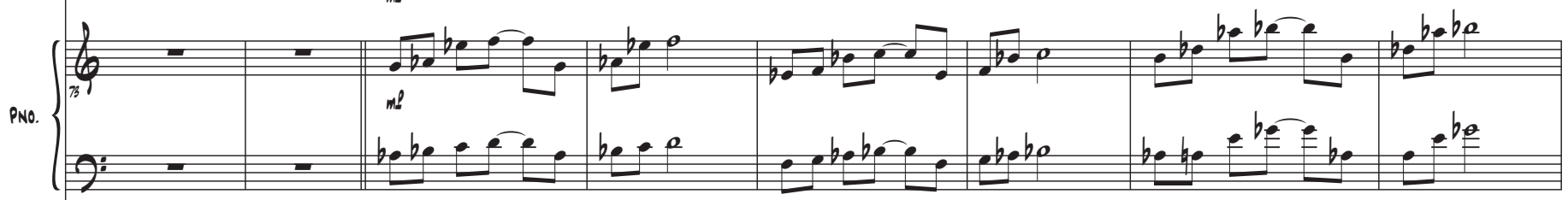

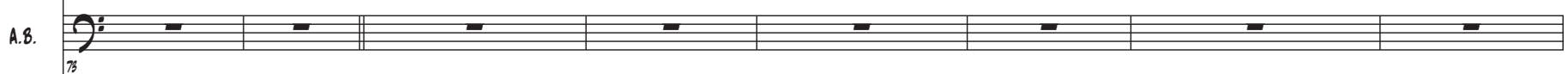

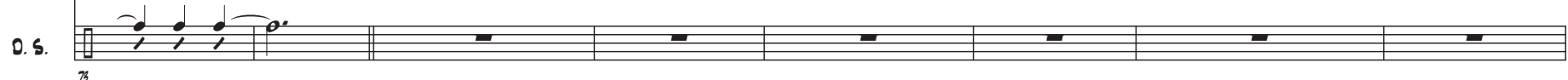



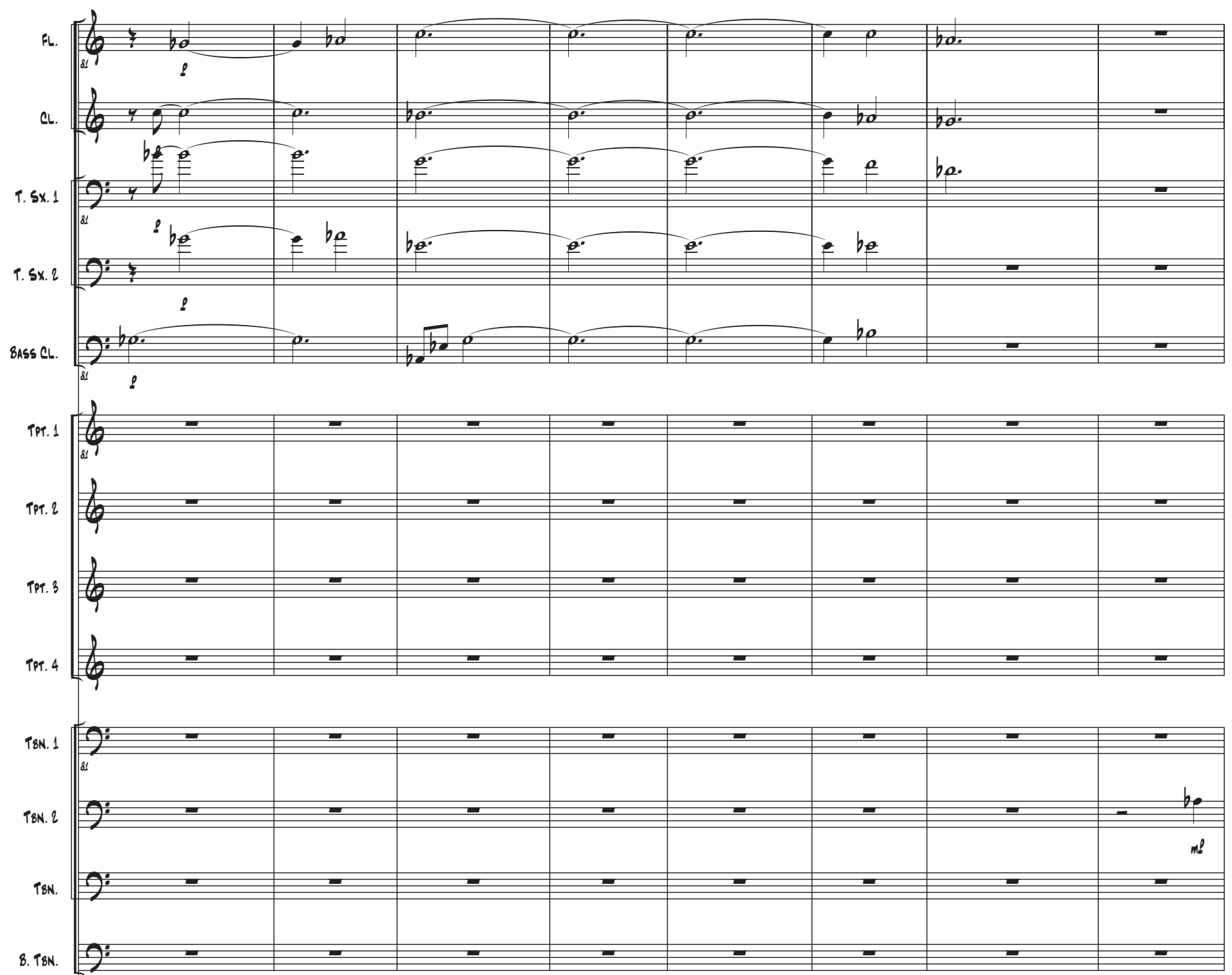

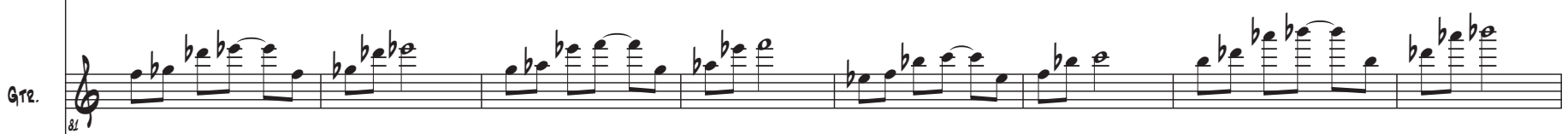

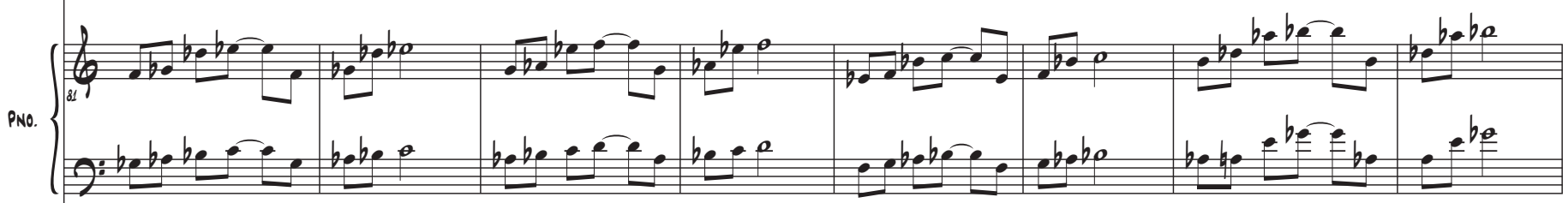

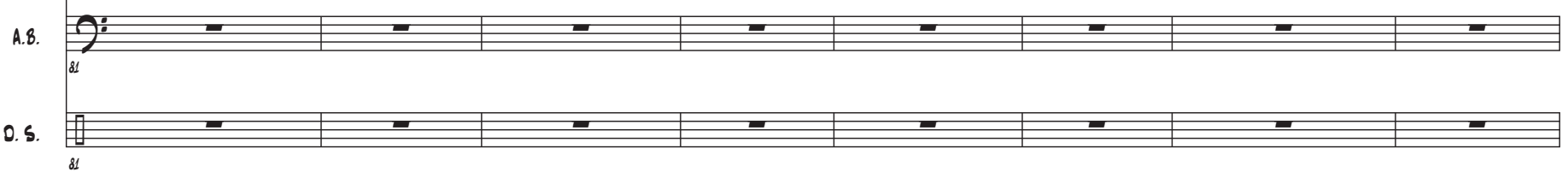



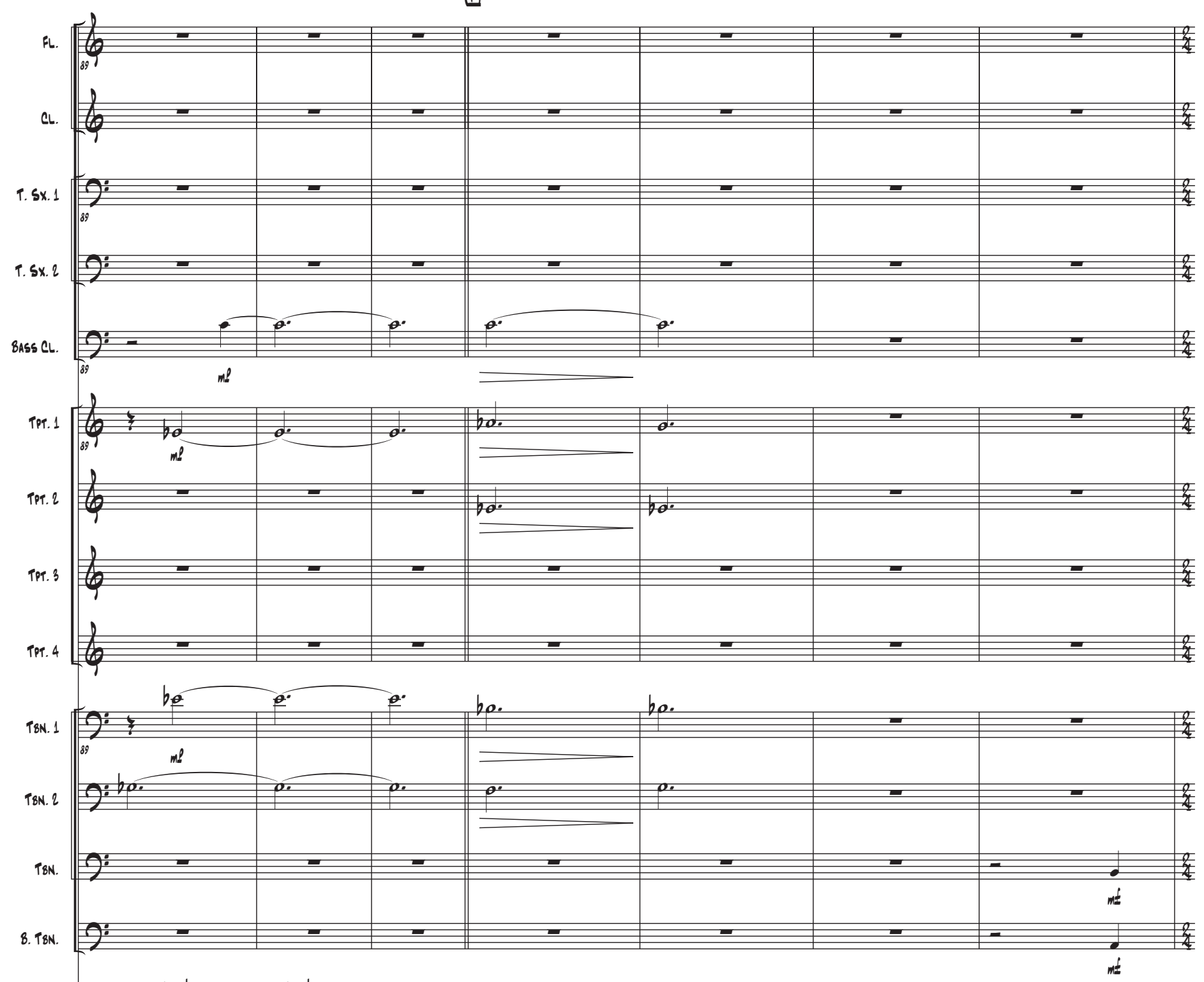
are. $b_{a 9}=b_{e} b_{e}=b_{e}^{b e}$

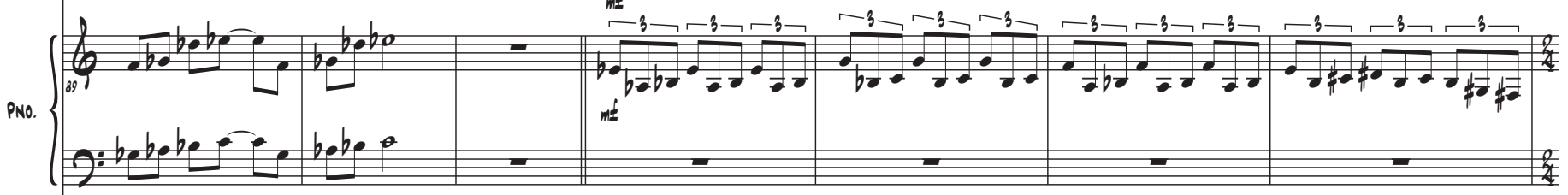

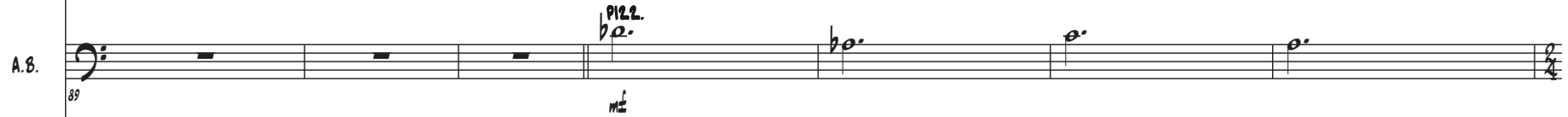

0.5. ${ }_{89}$ 

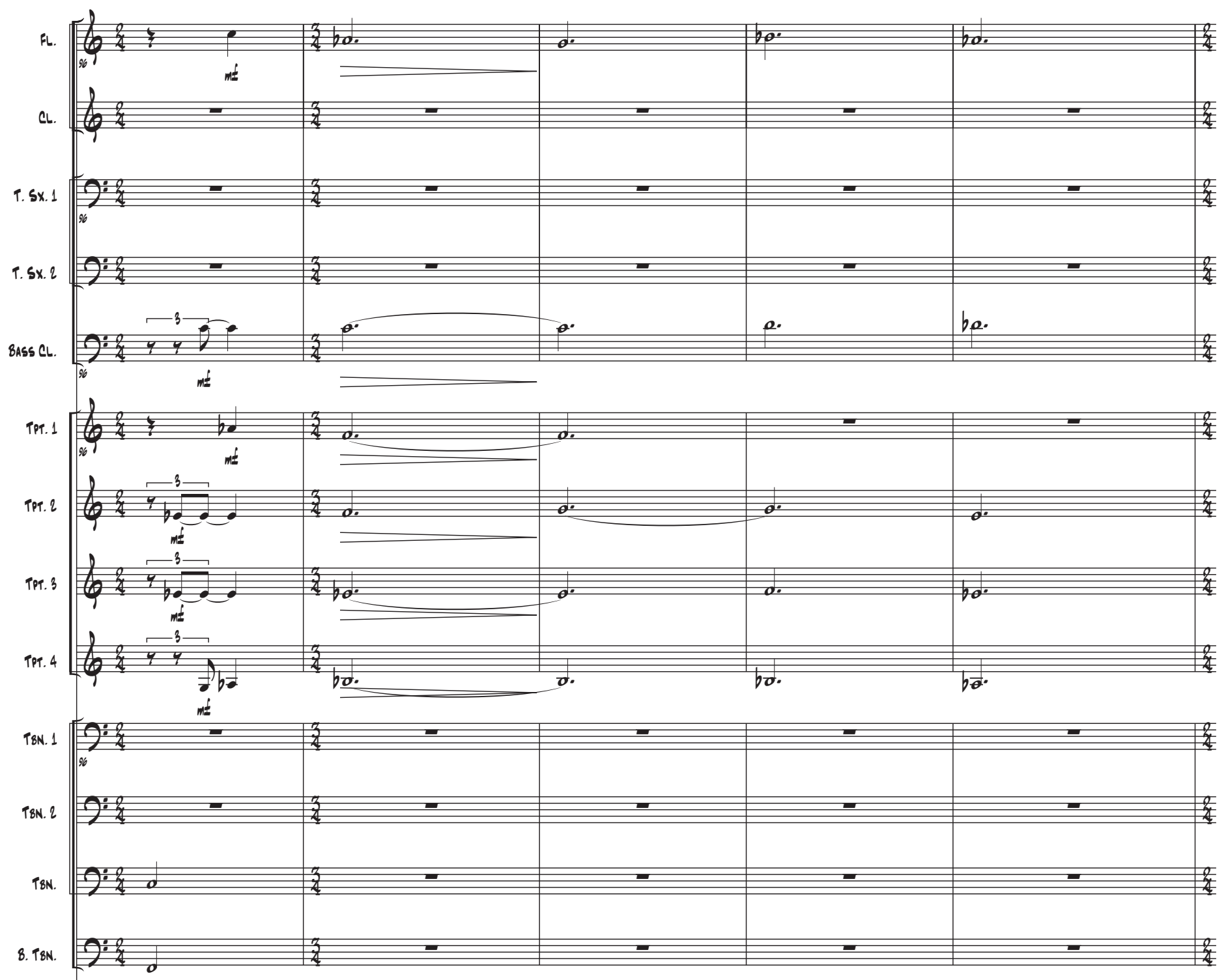

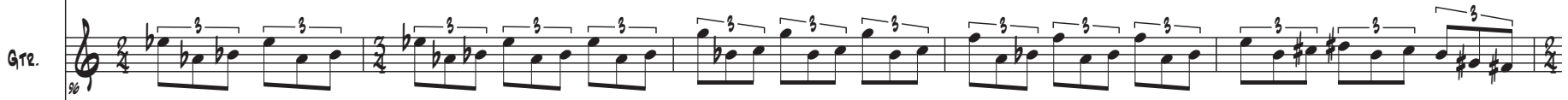

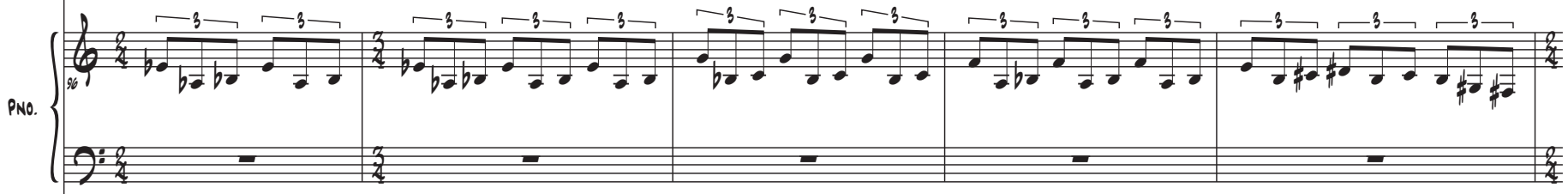

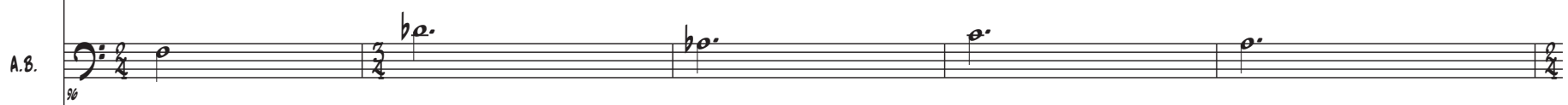
0.5. 

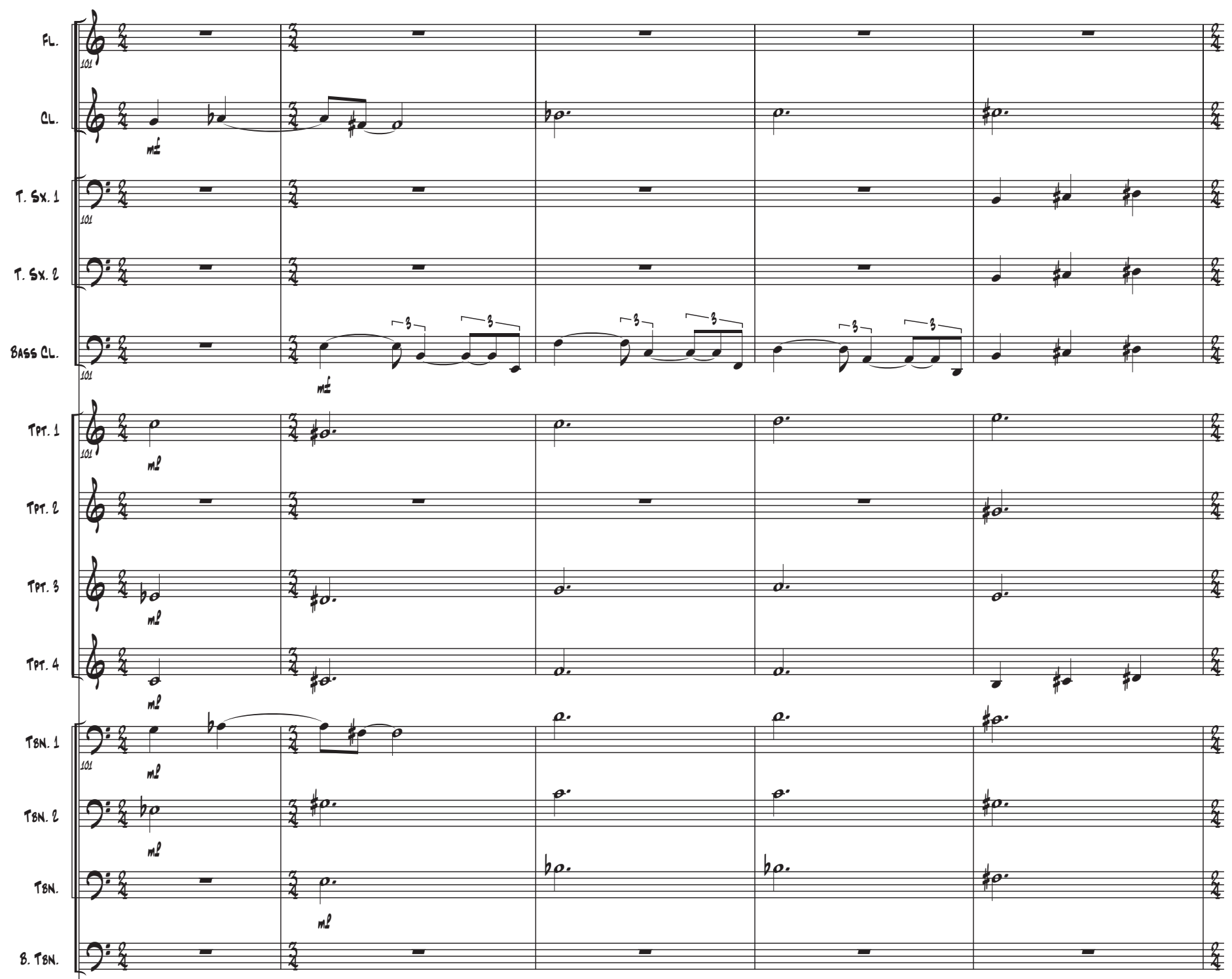

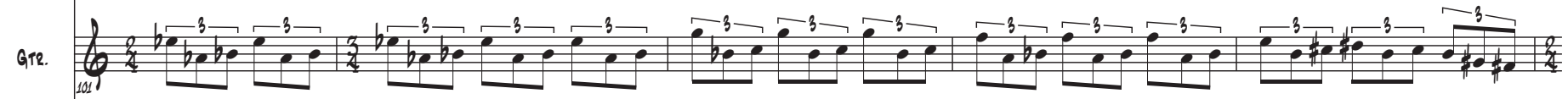

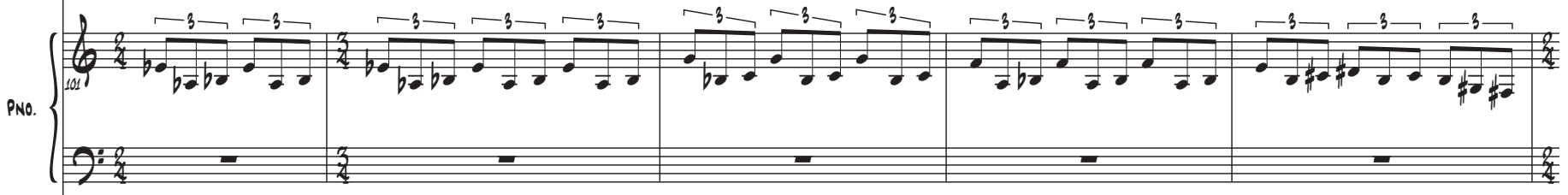
A.8. $\int_{104}^{0}: 20$ O.5. 

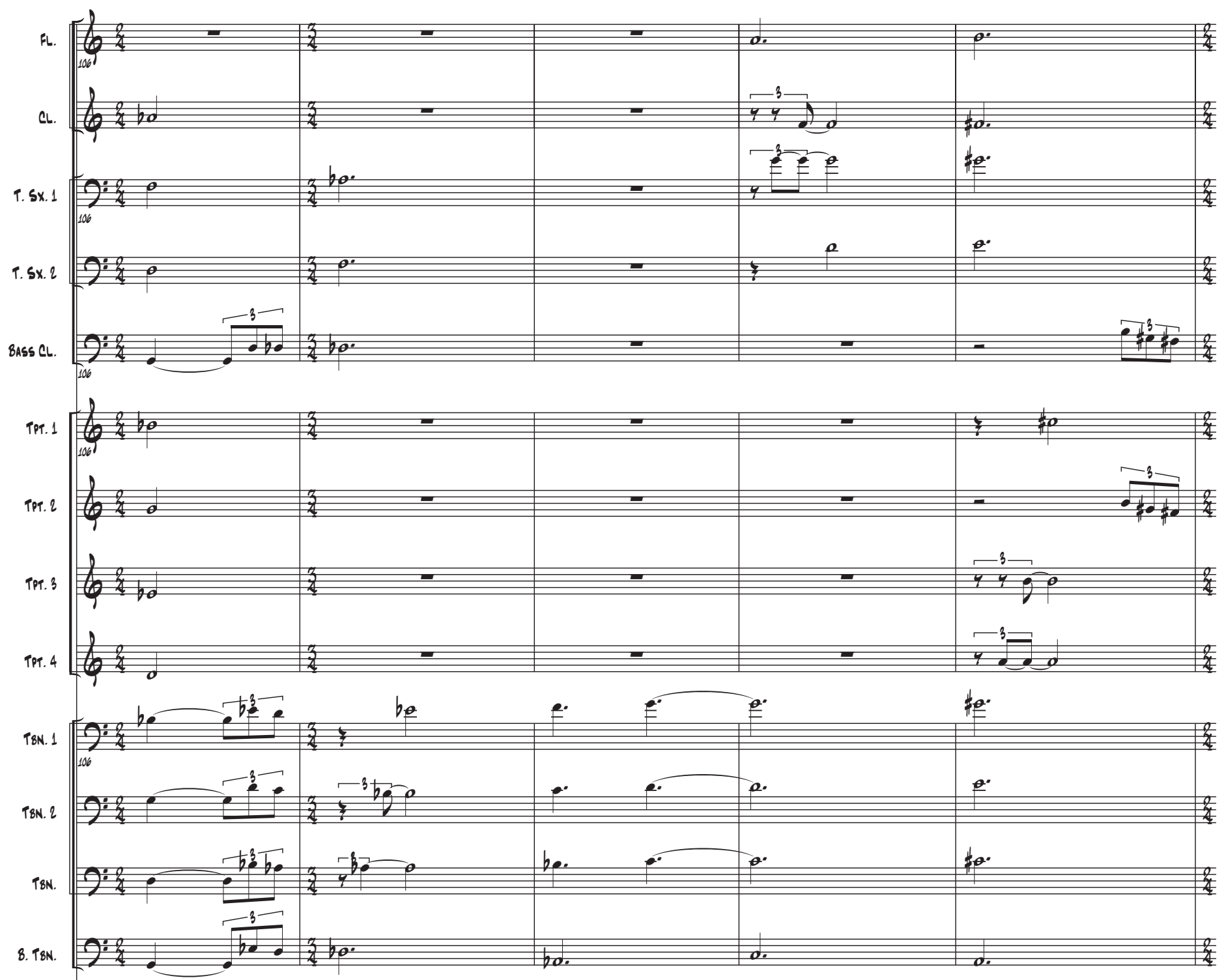

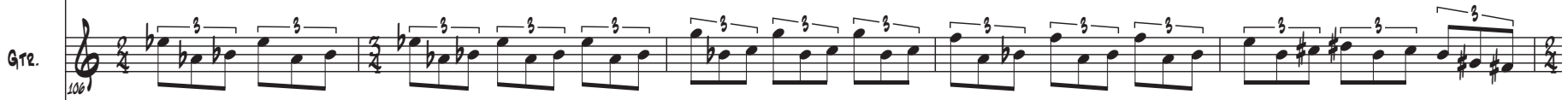

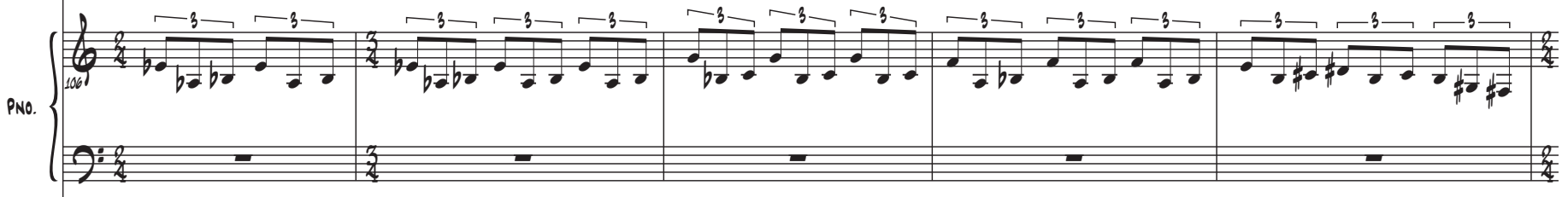
A.8. $\int_{100}^{0: 2}$ 0.5. (2) 
(G)
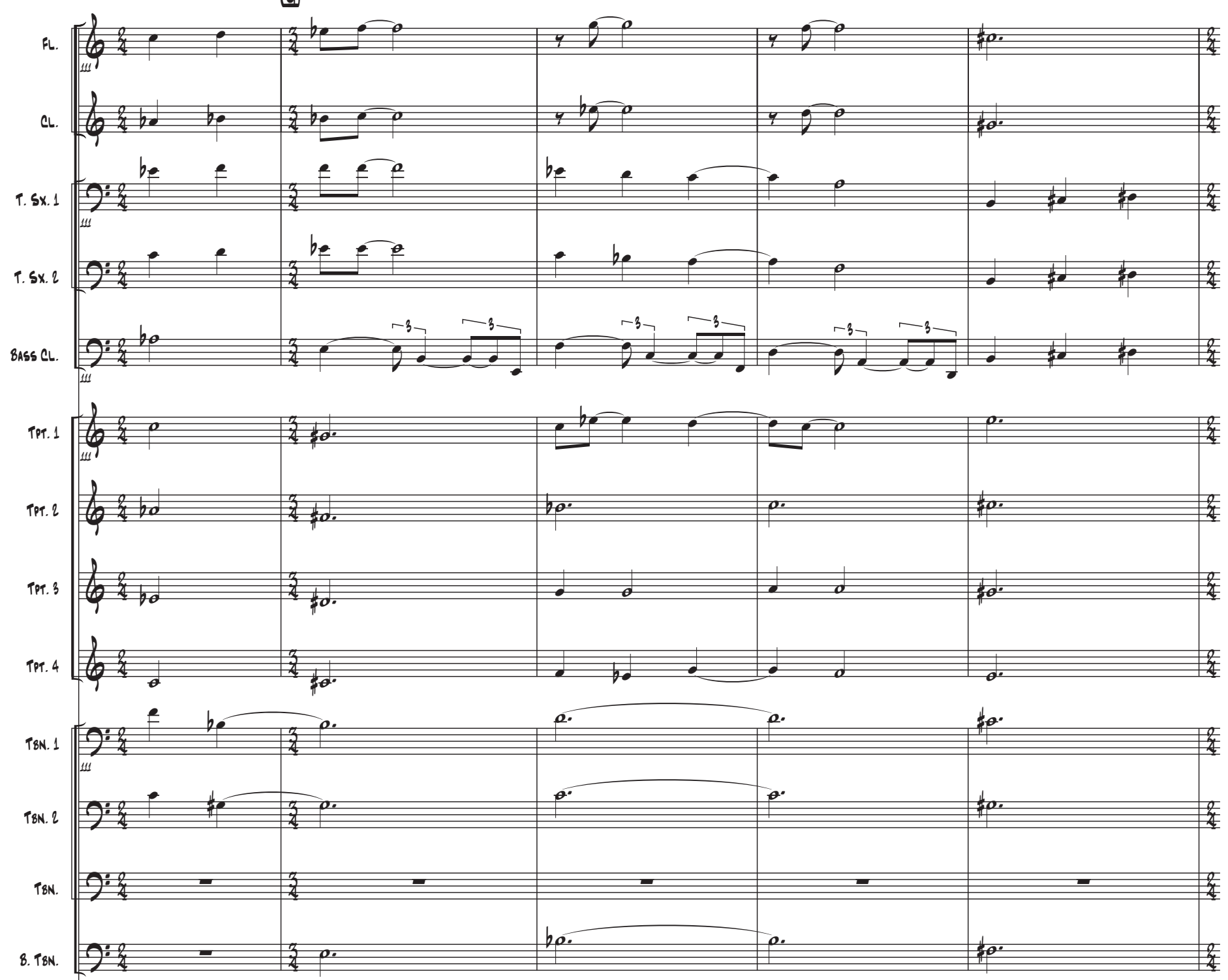

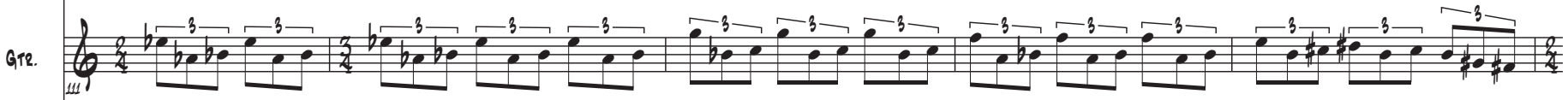

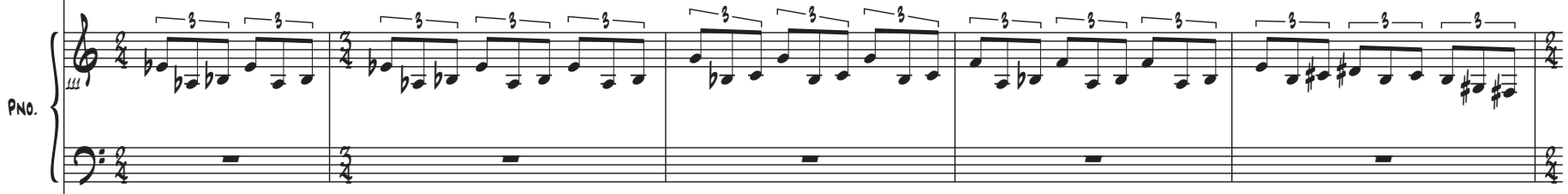
A.8. $\int_{11}^{2: 2} 0$

0.5. 

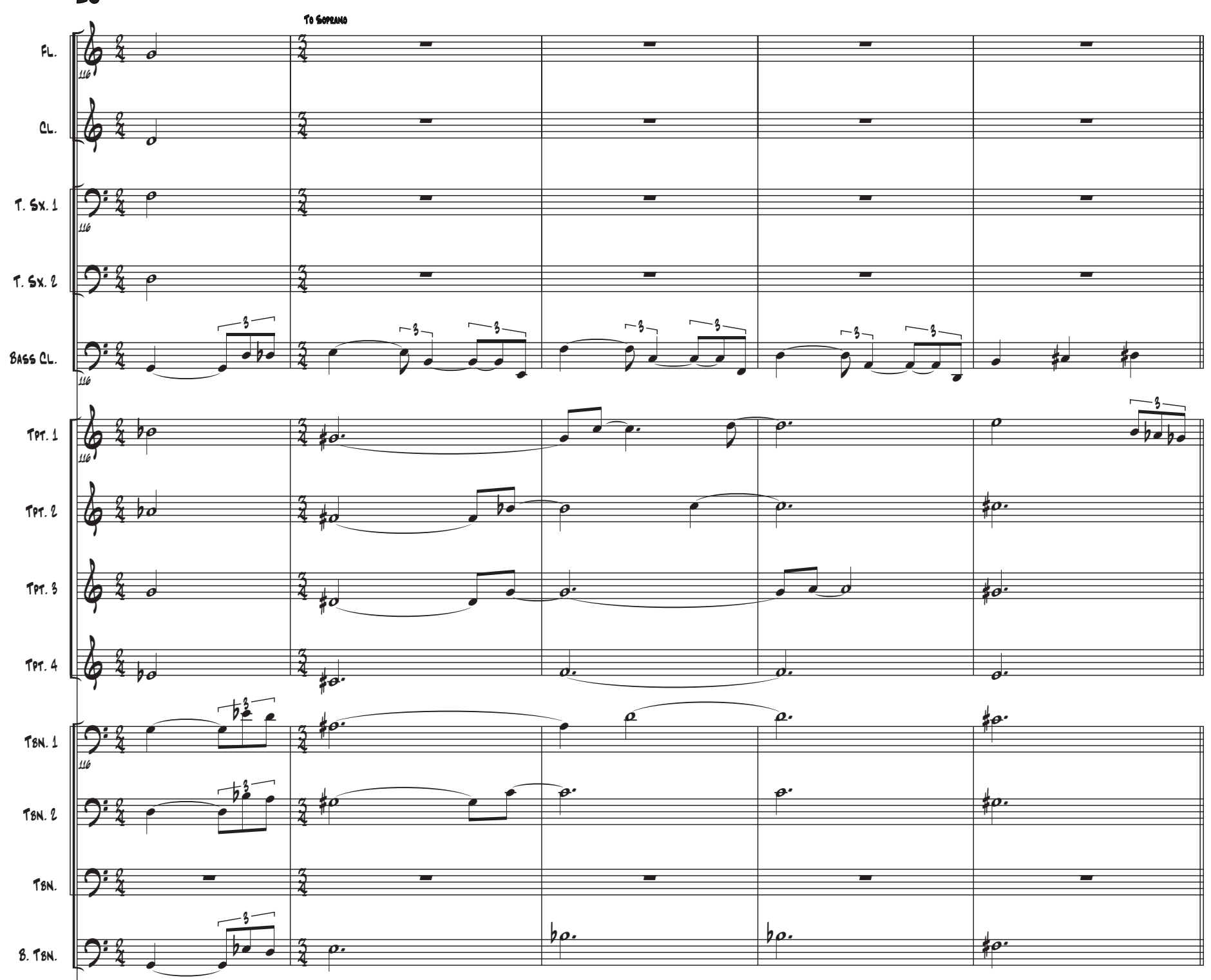

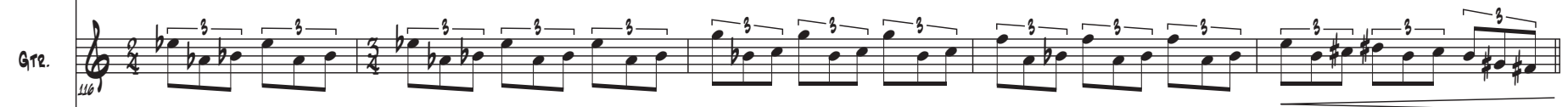

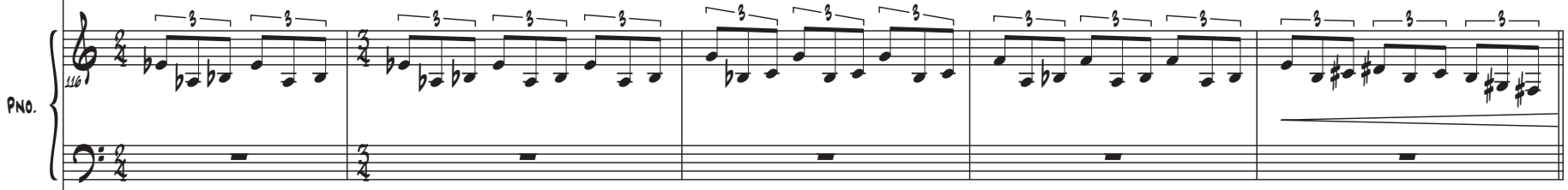
A.8. $\int_{40}^{: 2}$ 0.5. 

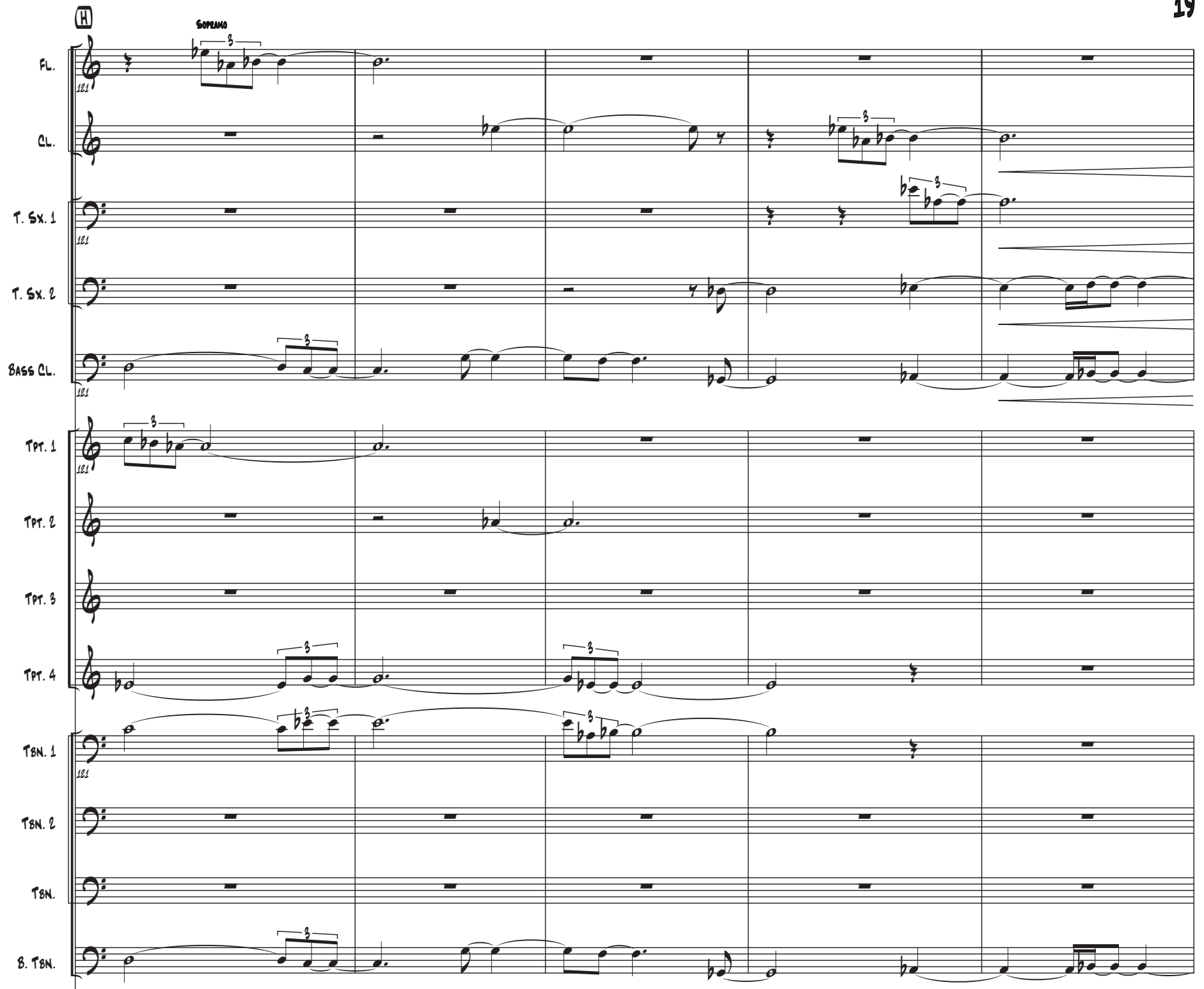

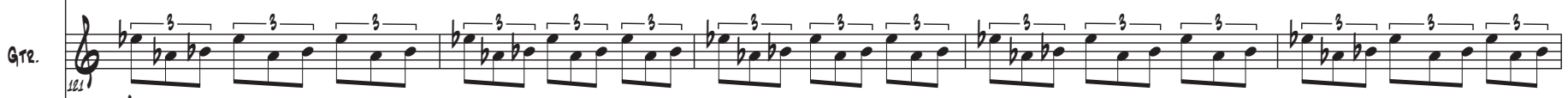

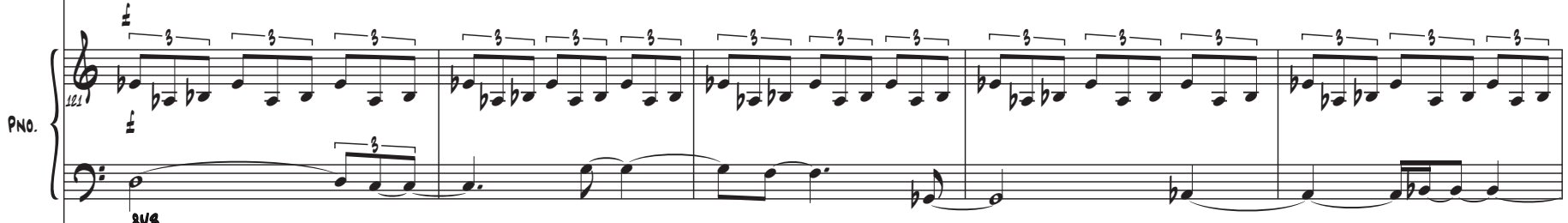

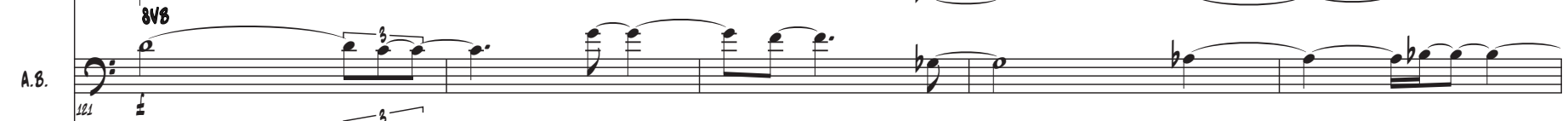

o. 5 .

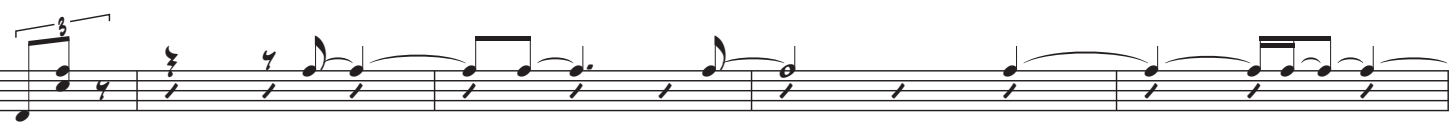



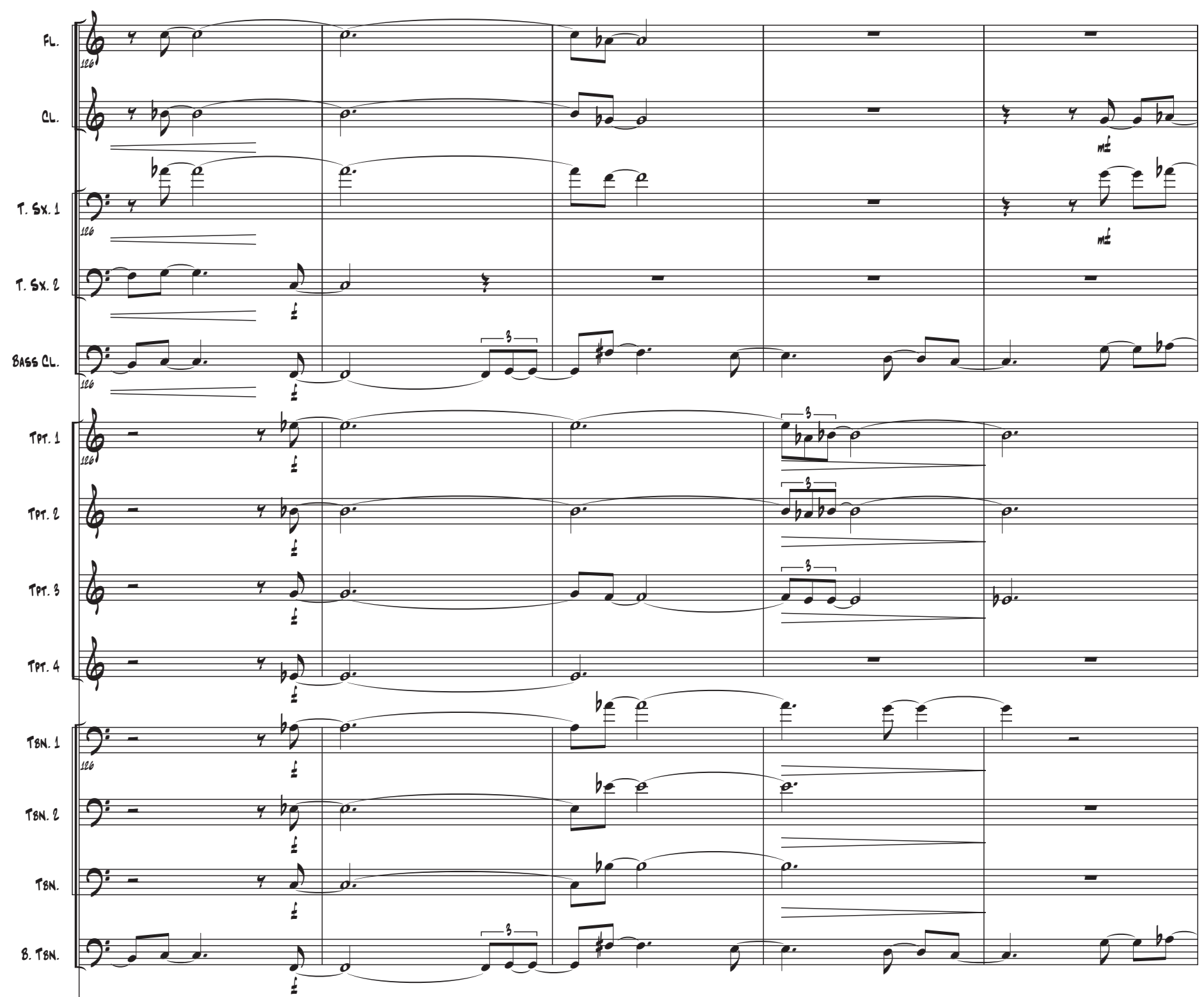

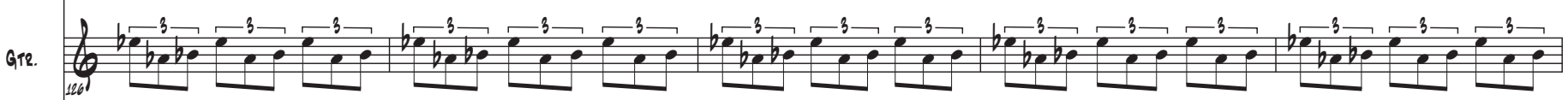

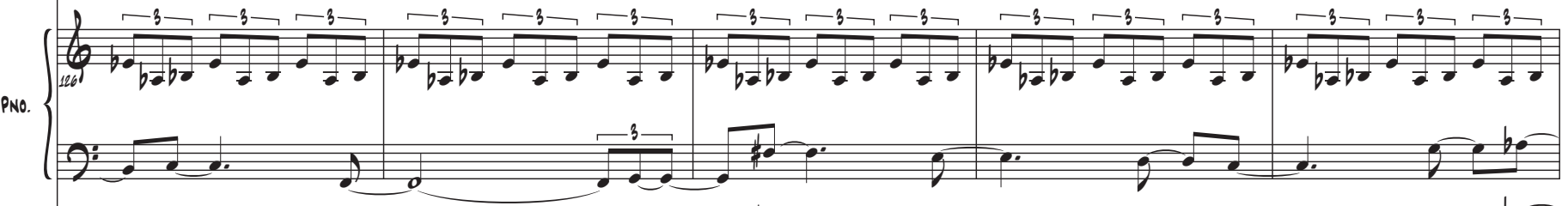

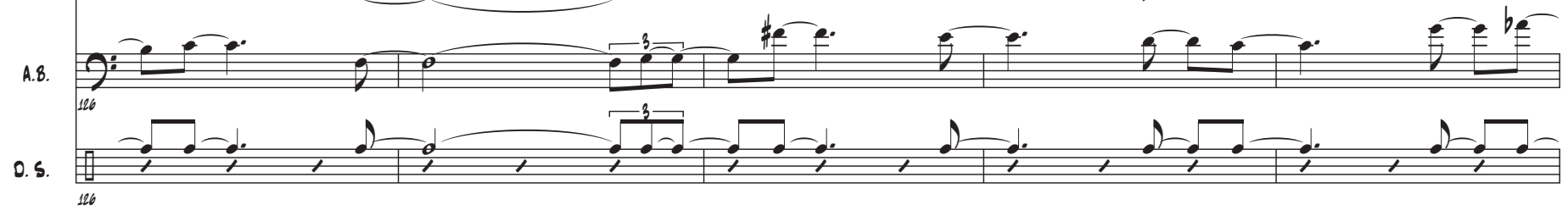



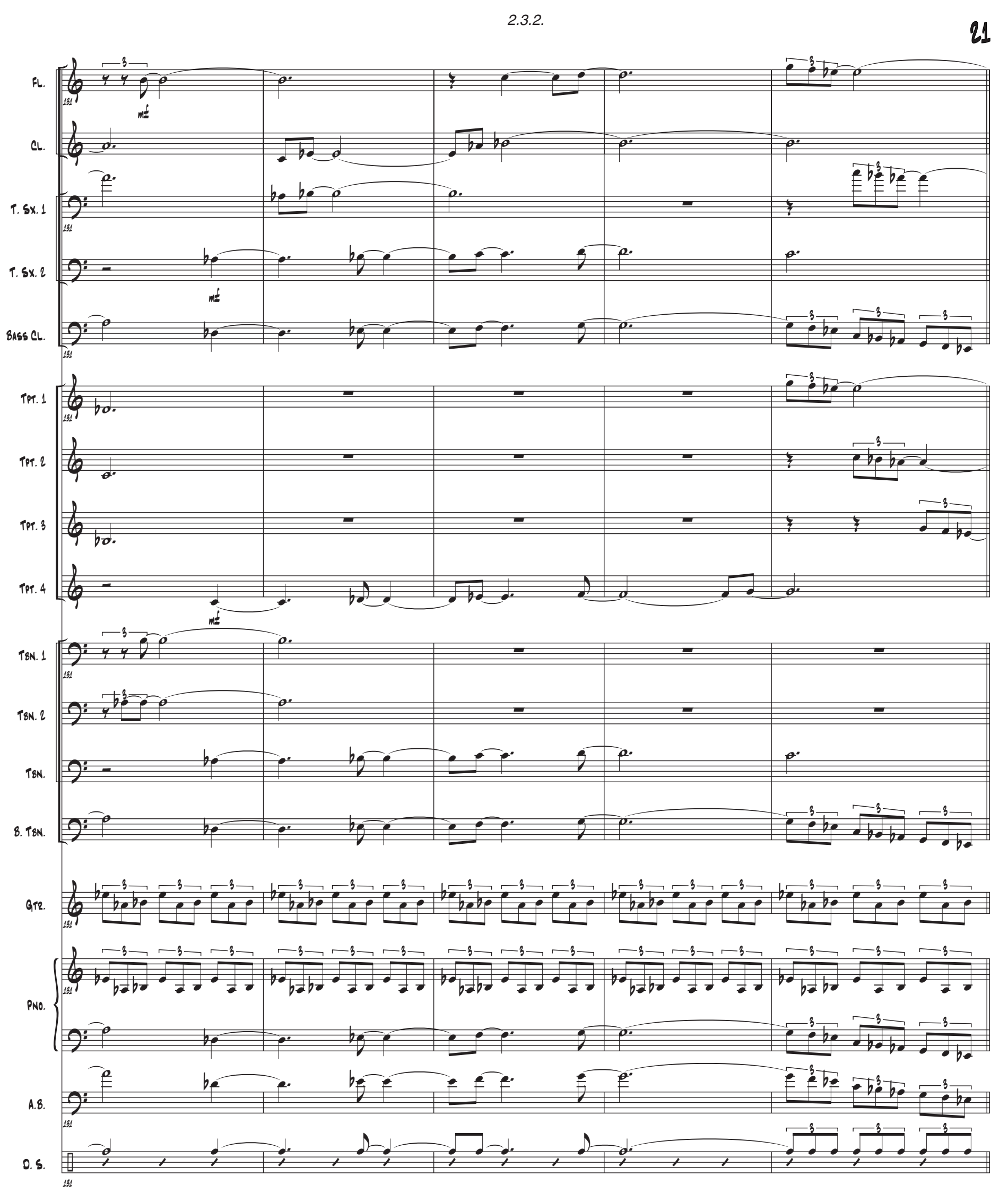

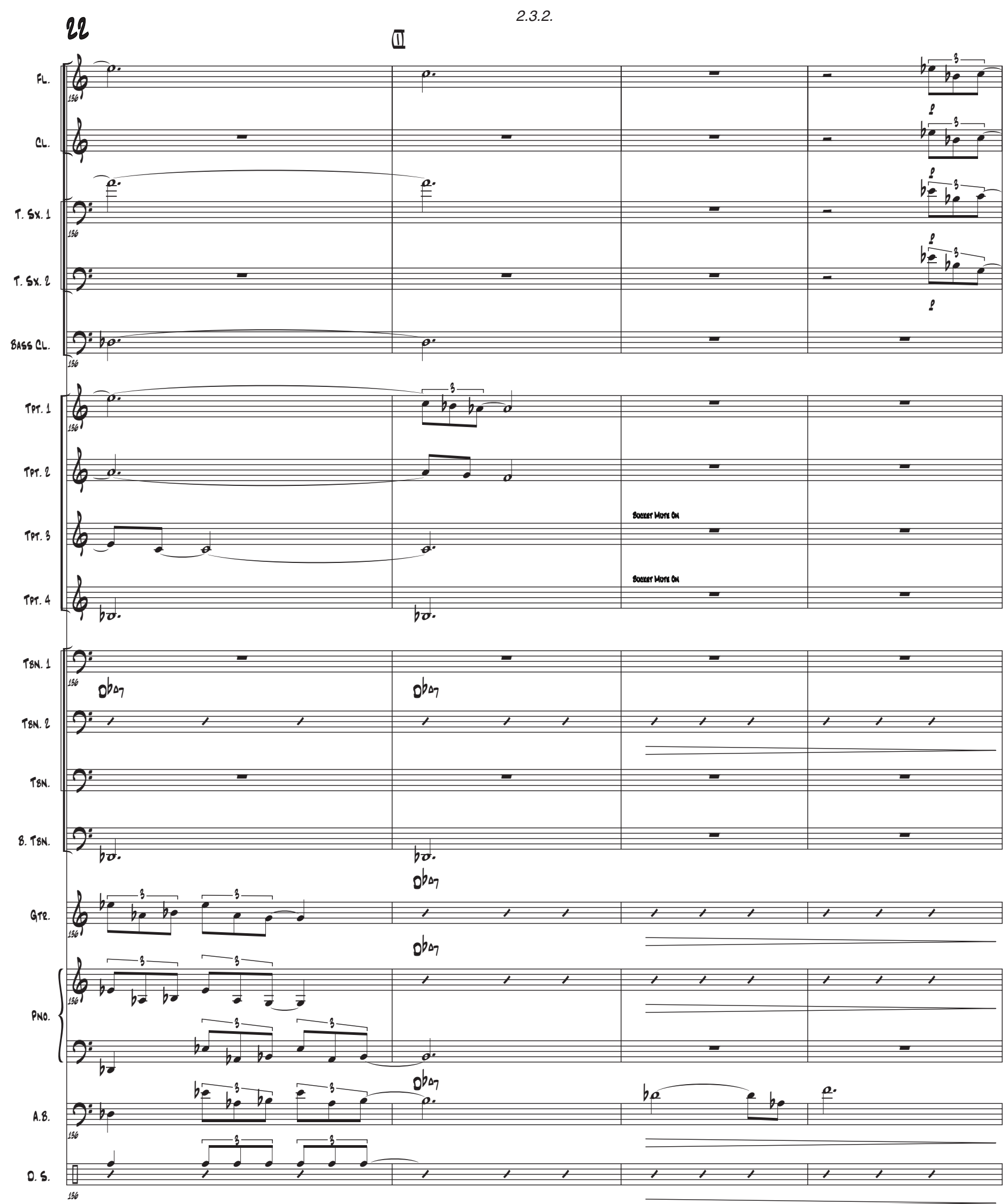

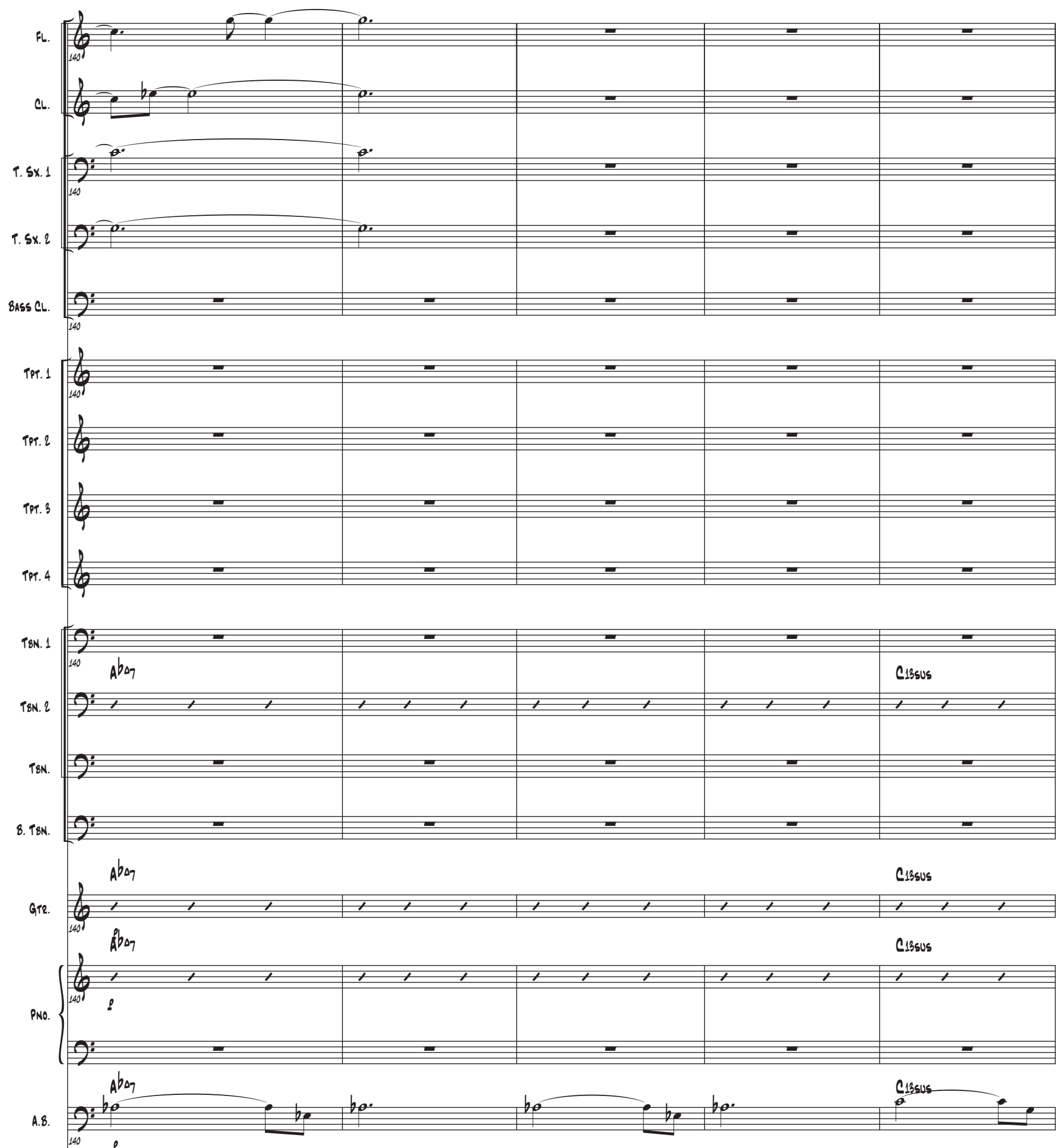

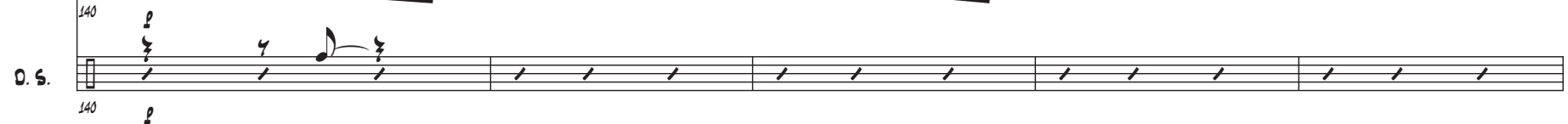



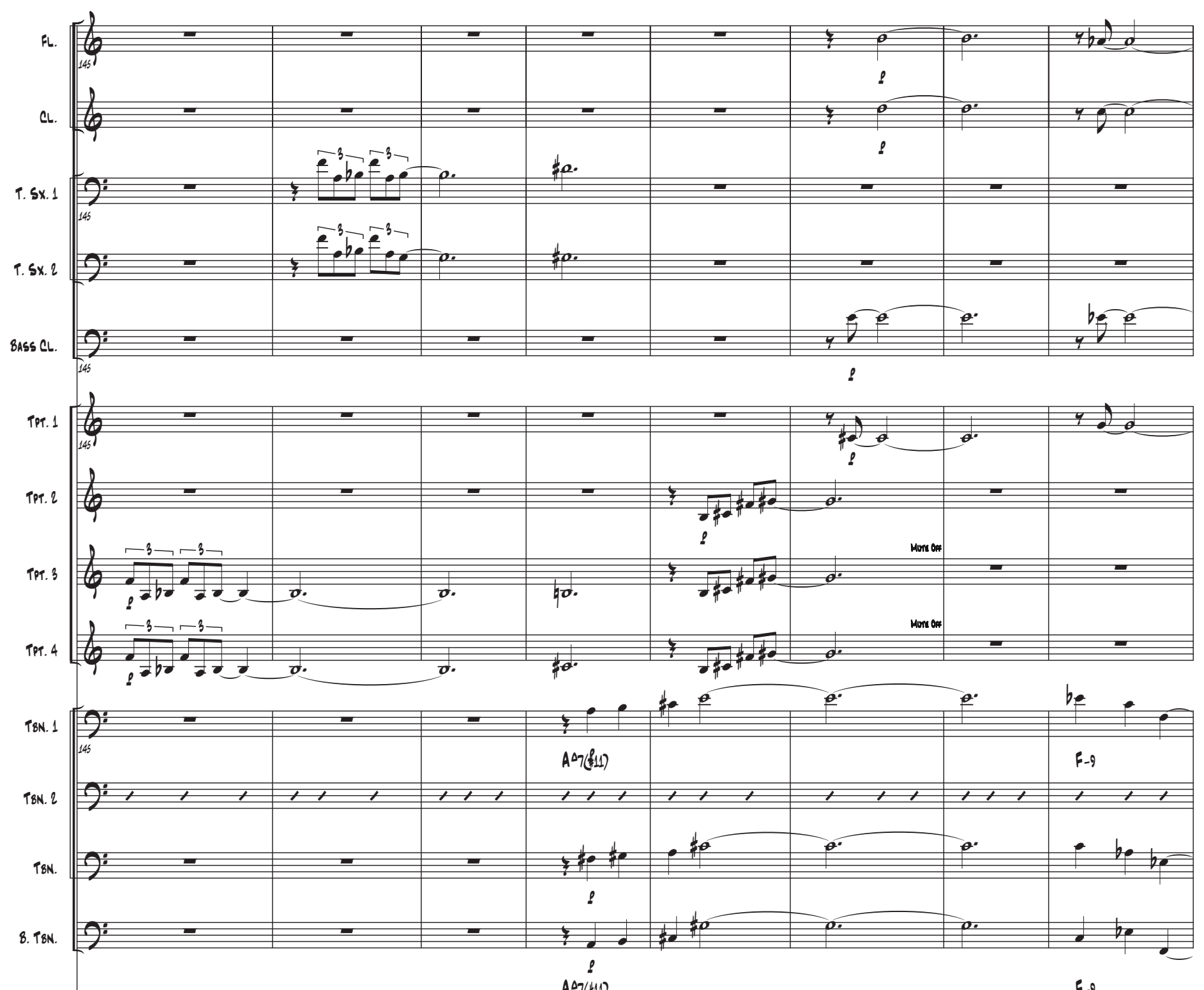

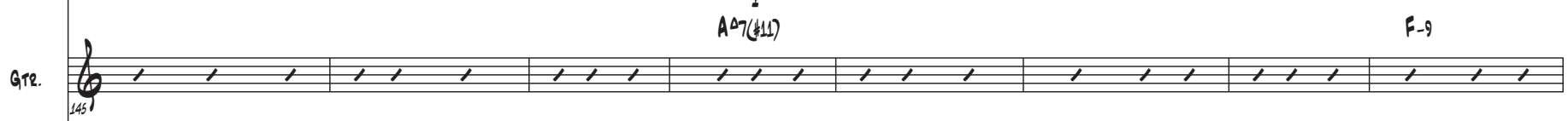

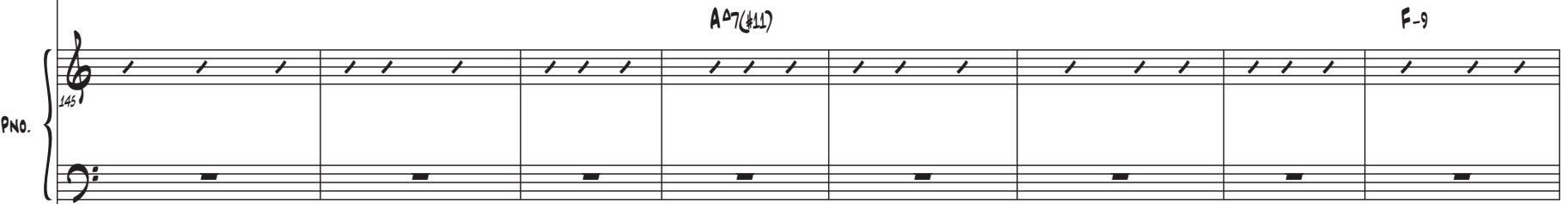
A.8. $\int_{445}^{0}$

o. s. 
2.3.2.

25
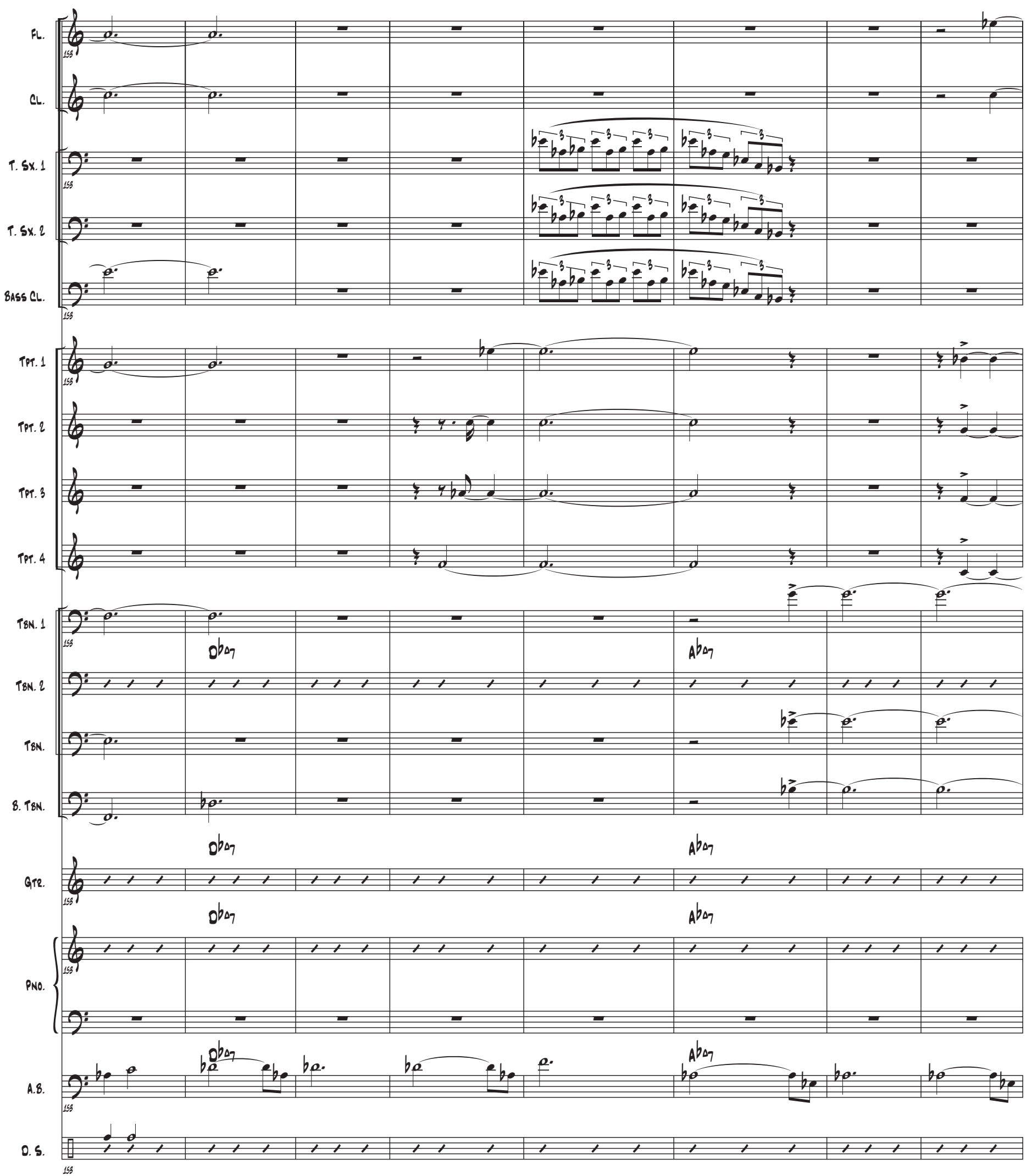

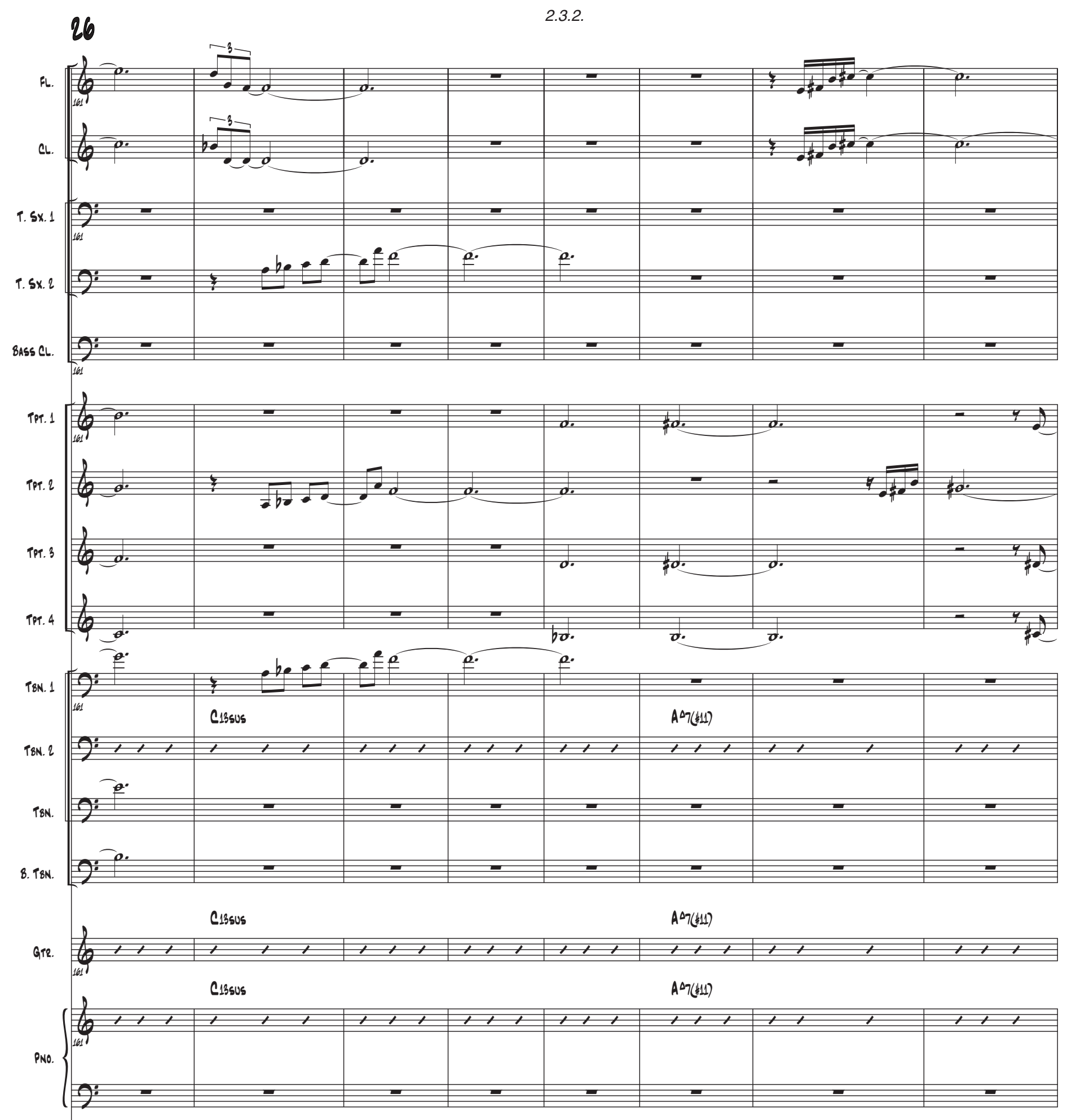

a.8.

o.5. 
(1)
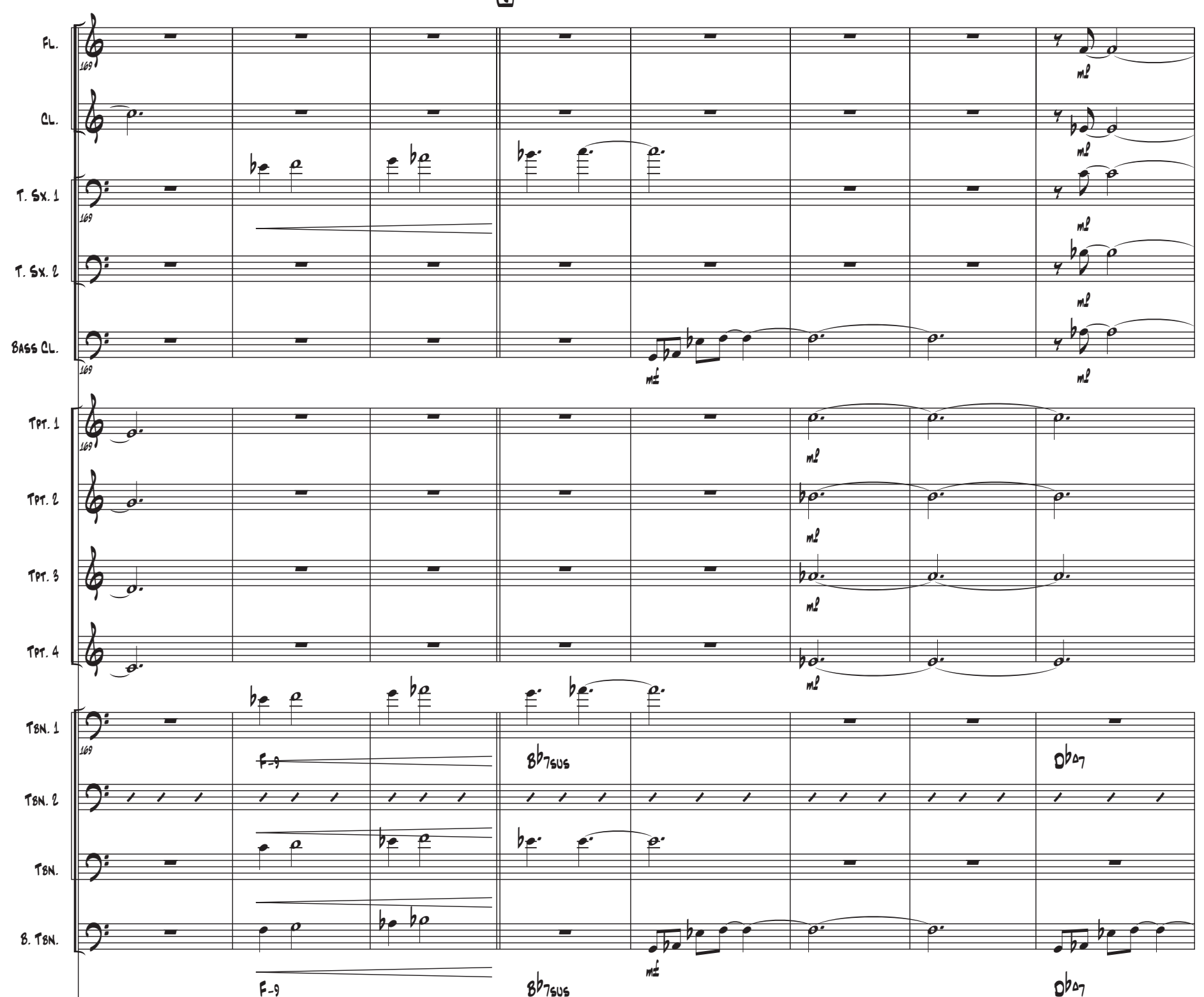

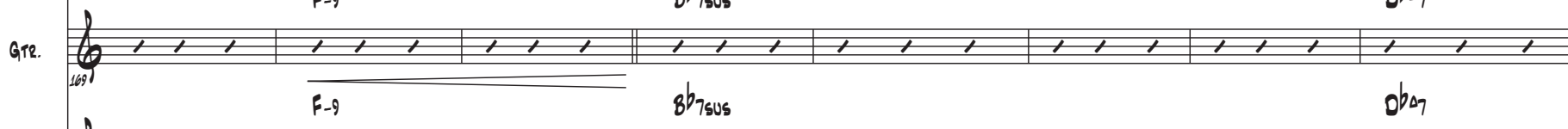

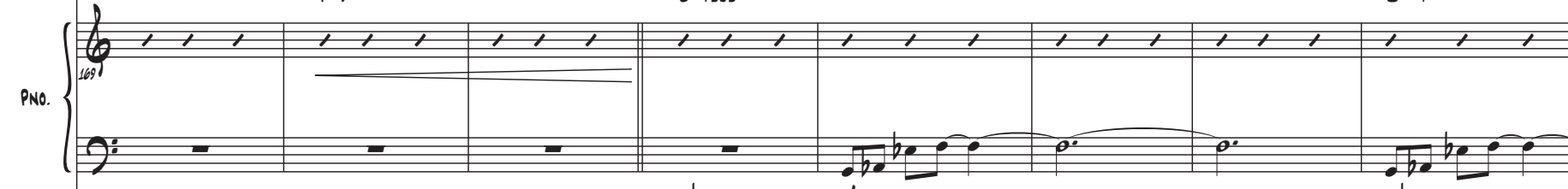

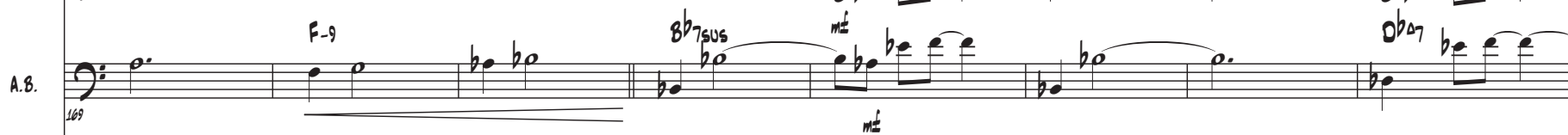
0.5. 
28

2.3.2.
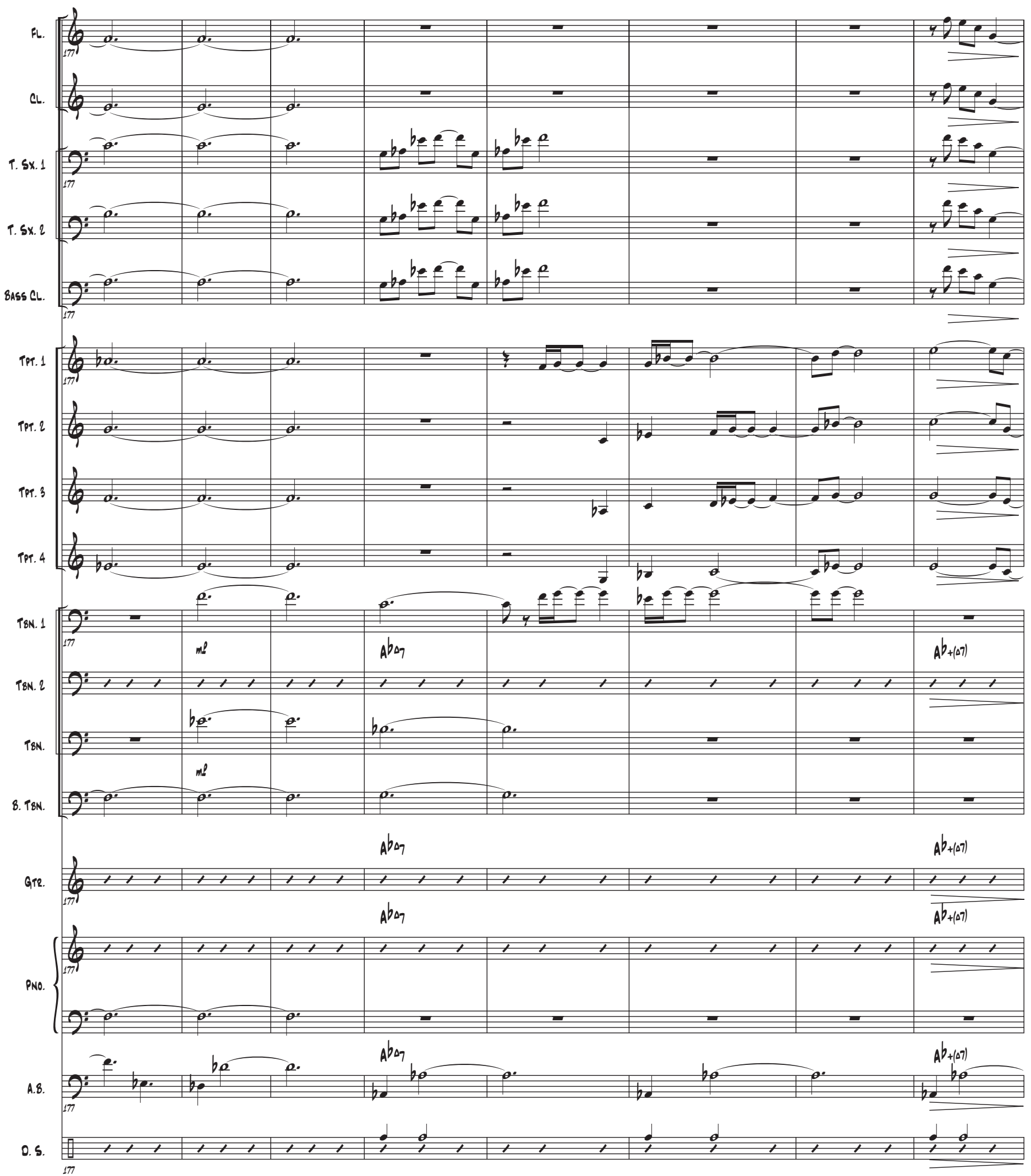

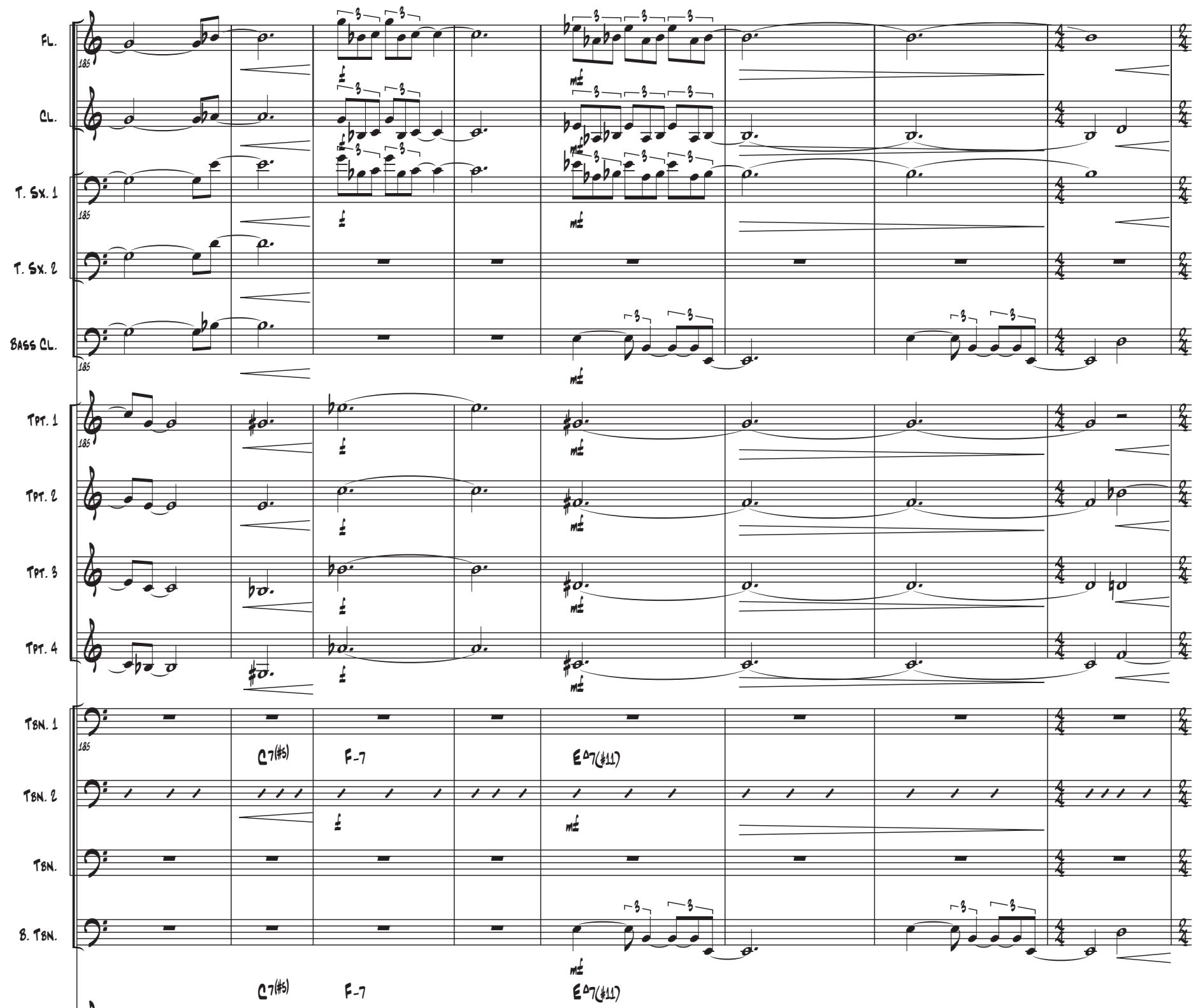

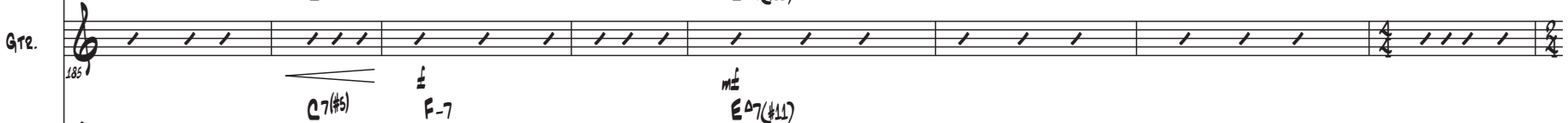

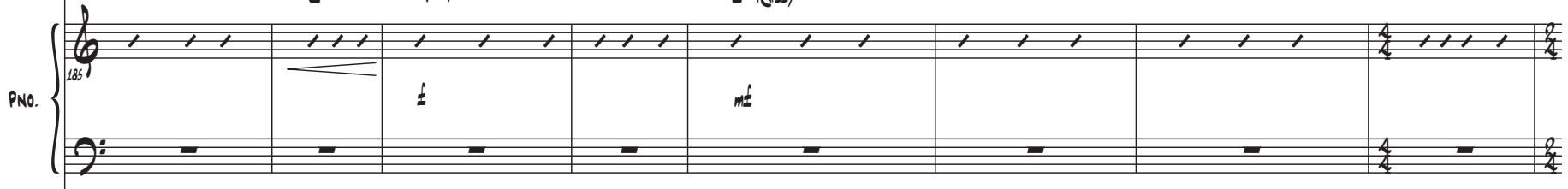

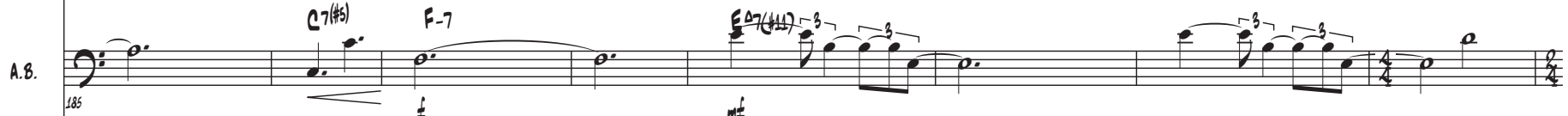

o. . 

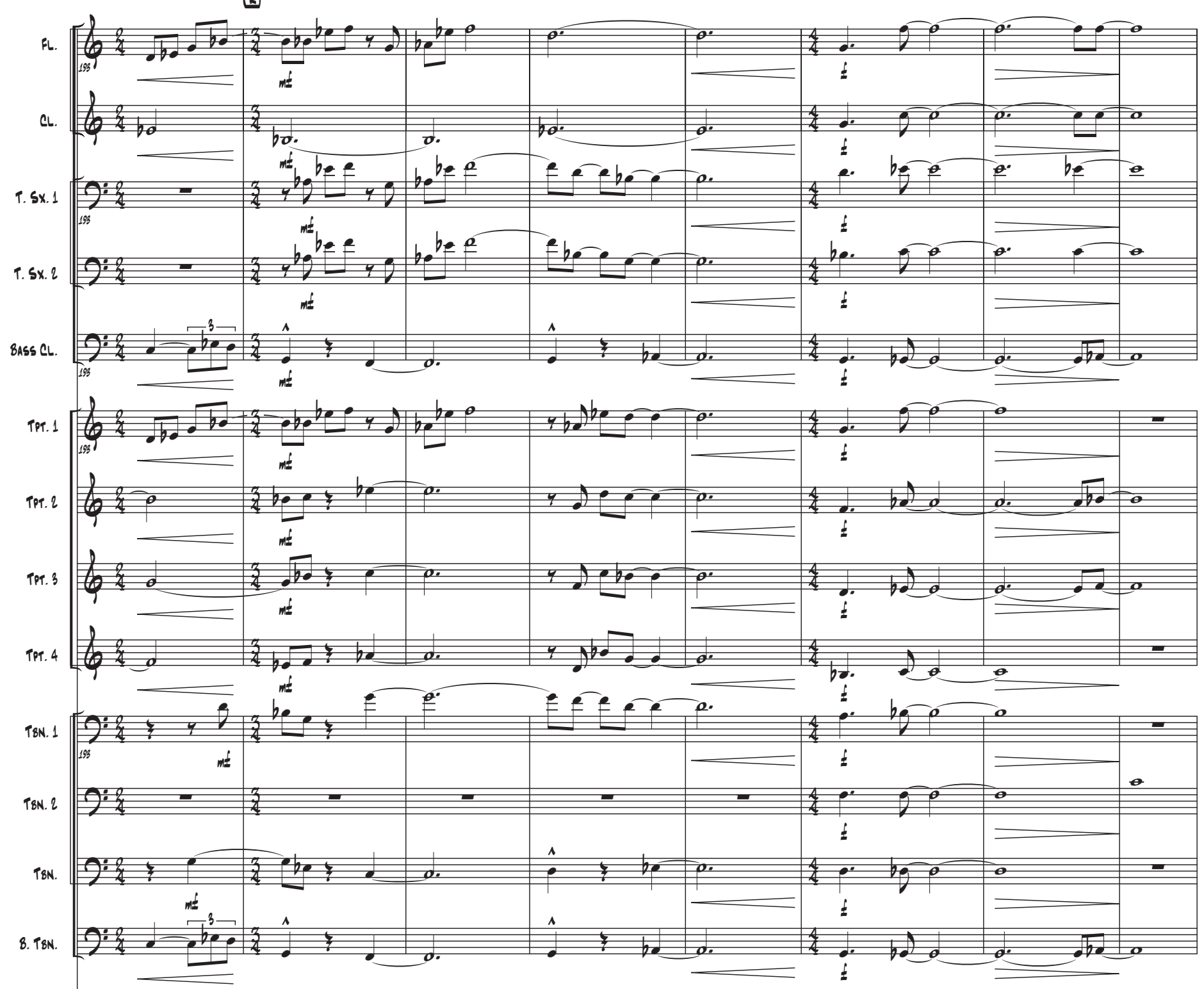

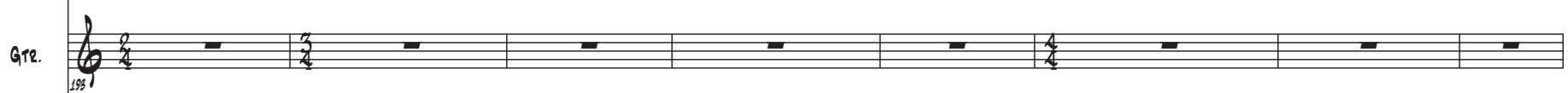

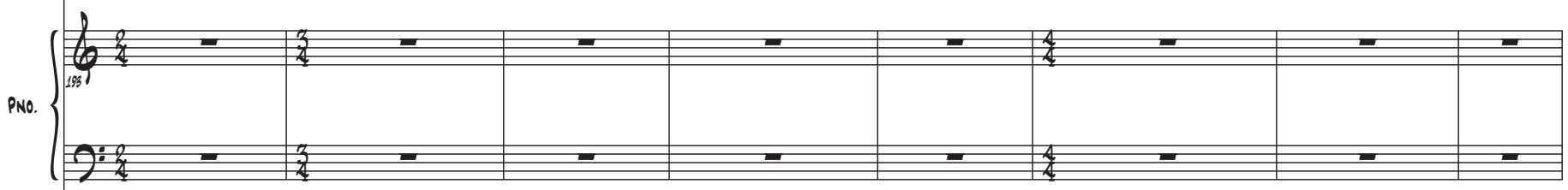

A.8. $\int_{108}^{0: 2}$

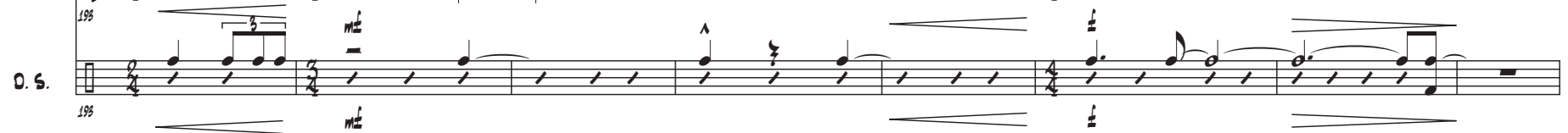



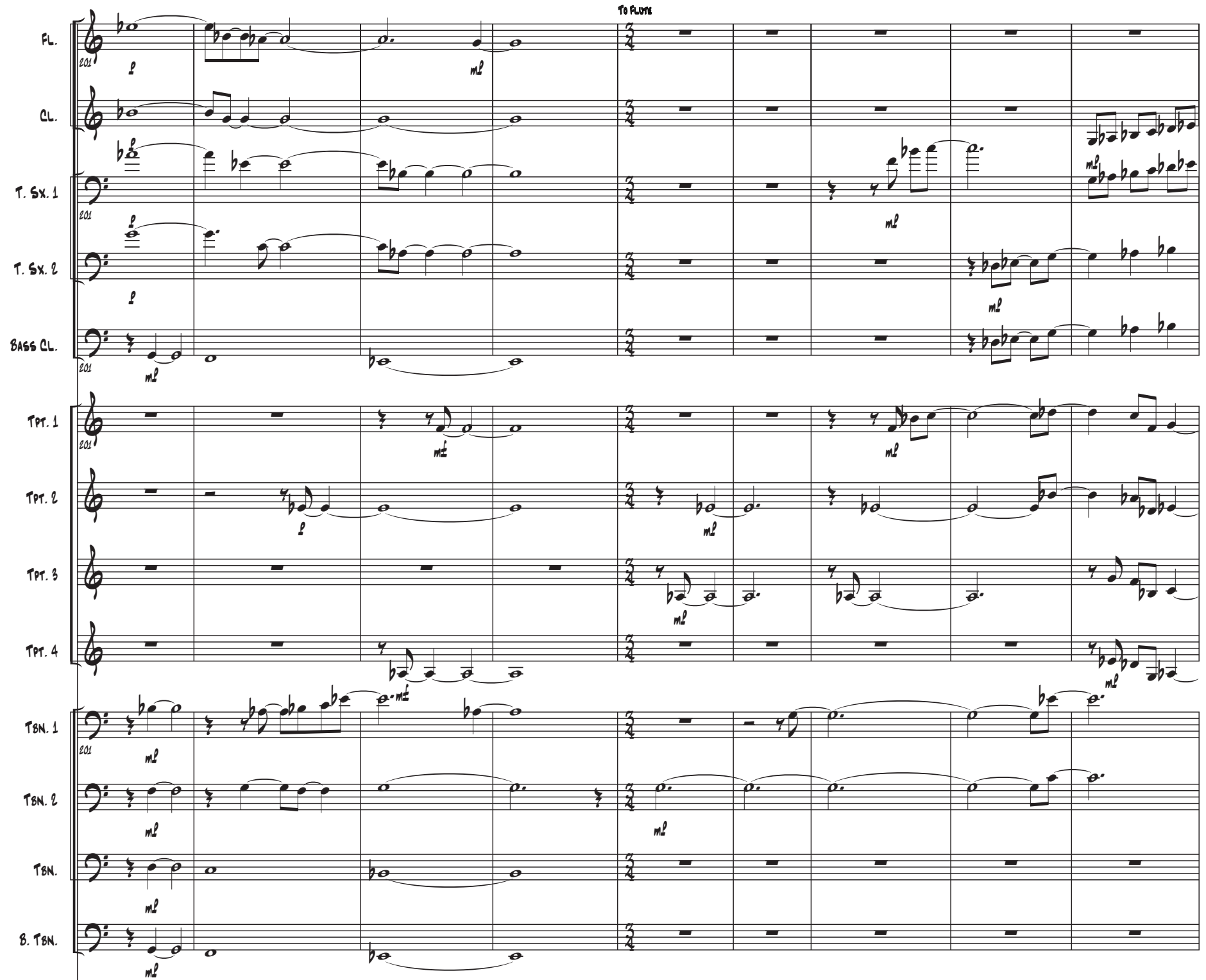

Gie. $\mathrm{l}_{\text {end }}$
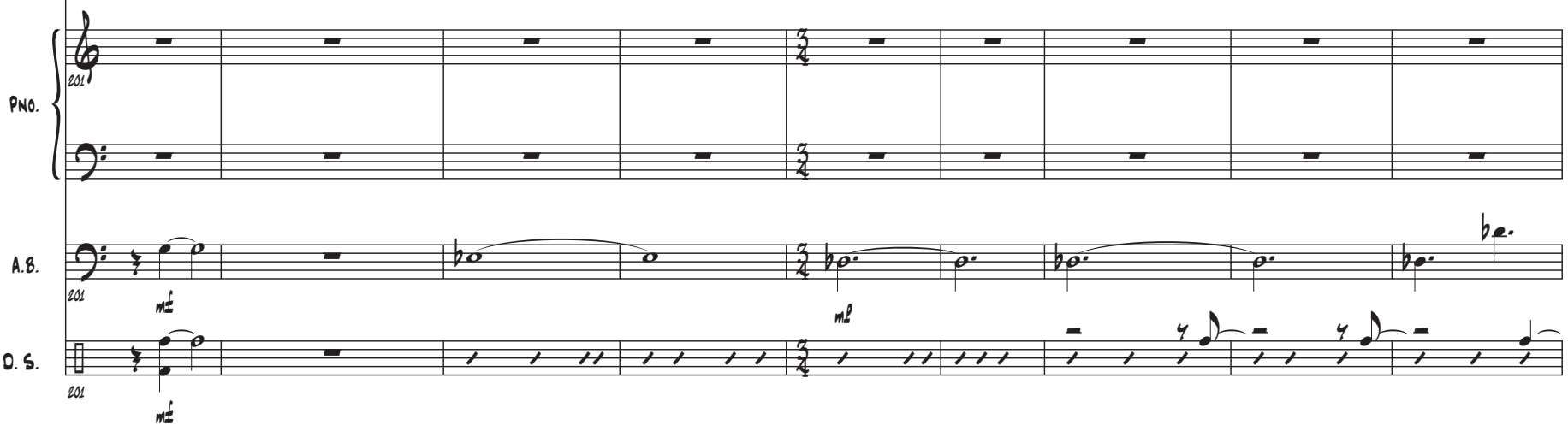

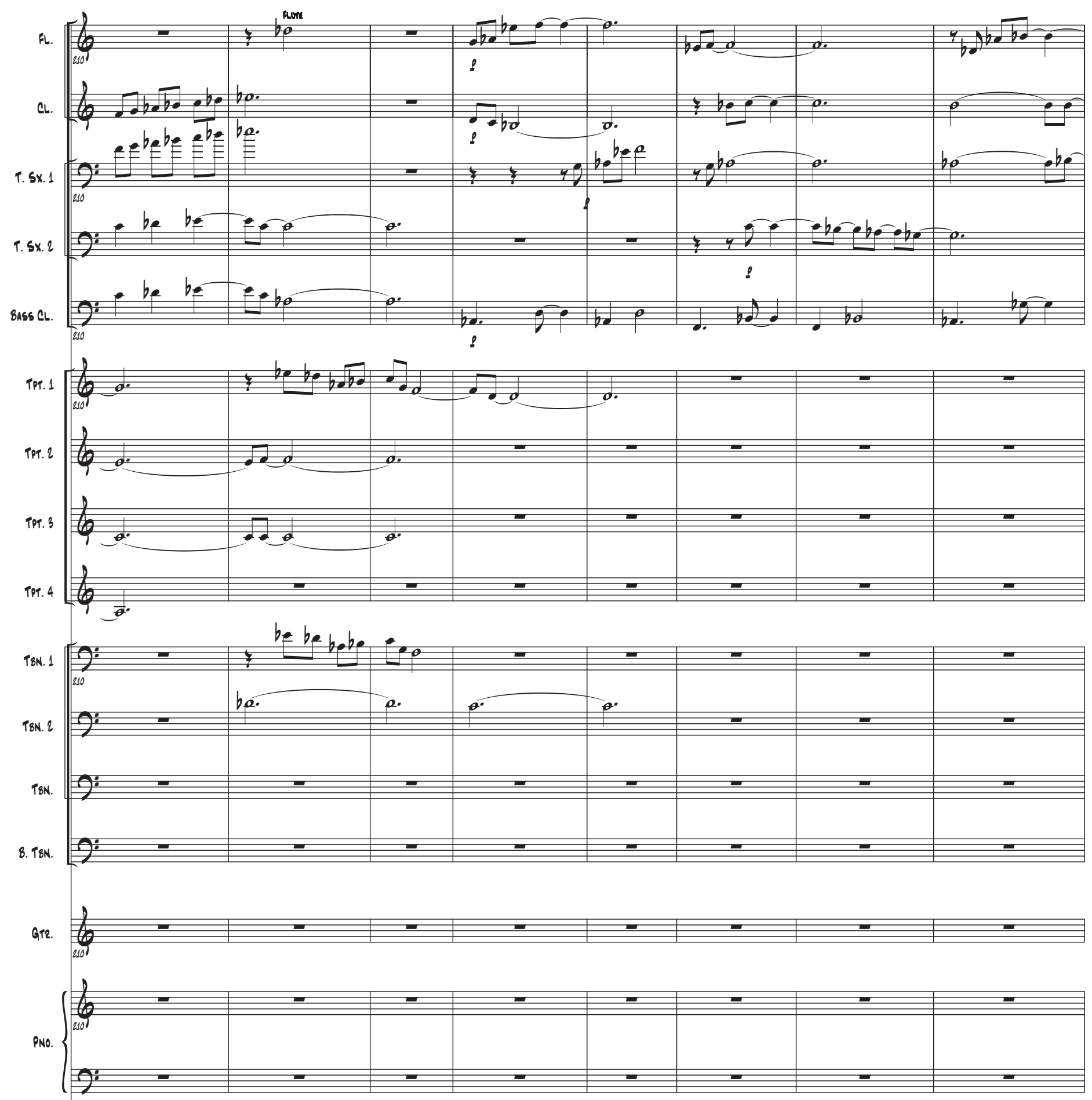

A.8. $\int_{20}^{20} b_{0}$ be. O. S. 
2.3.2.

33
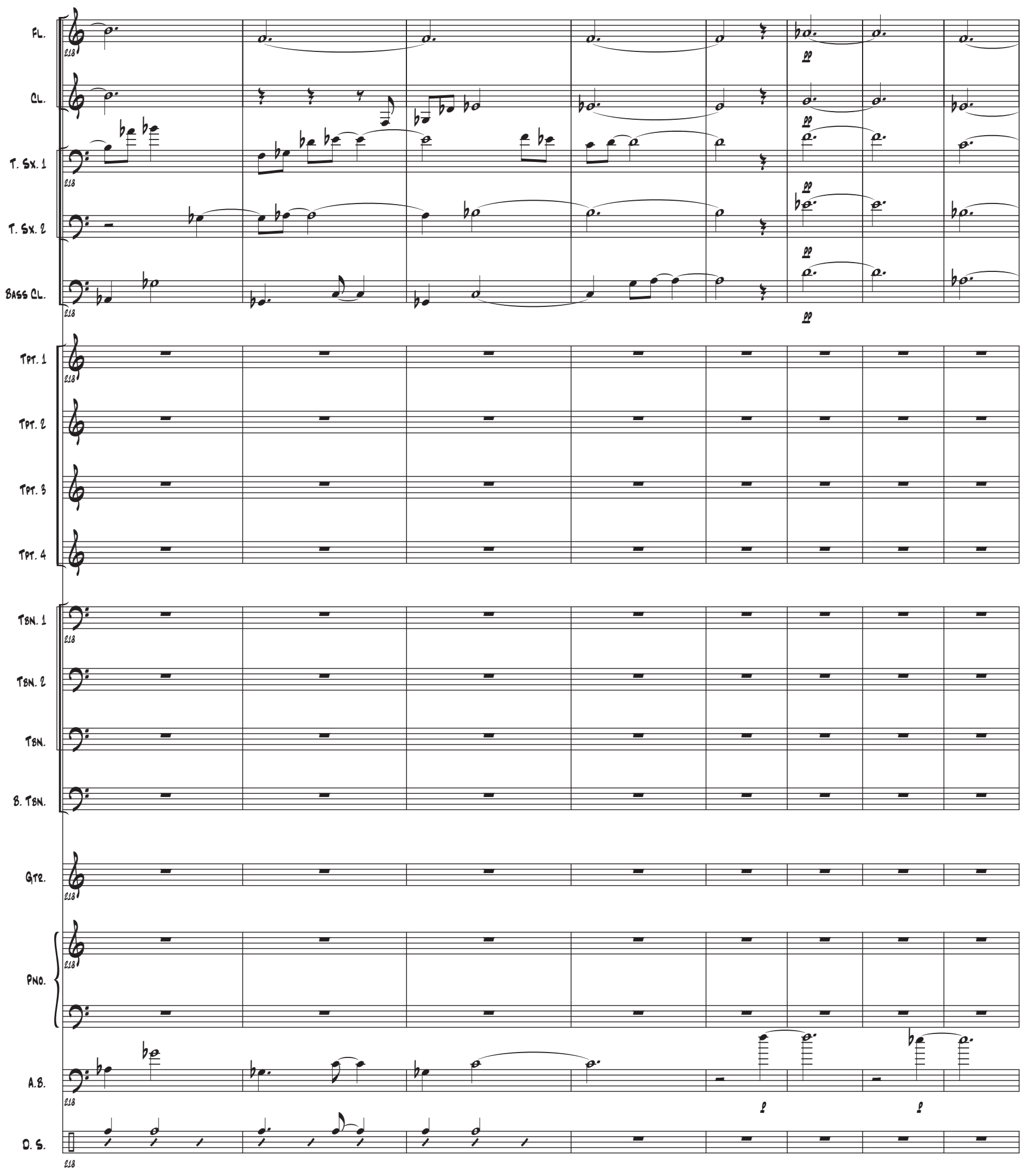

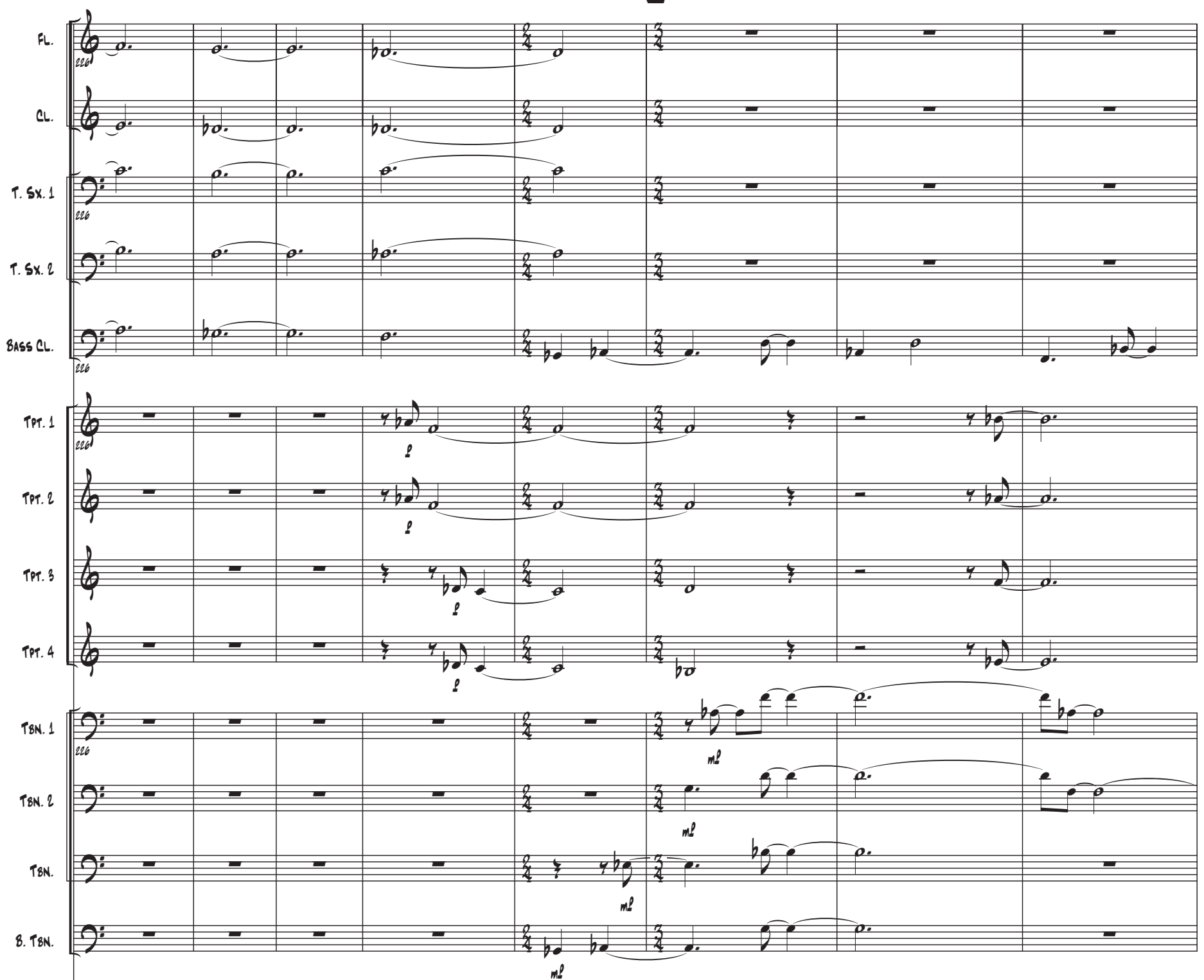

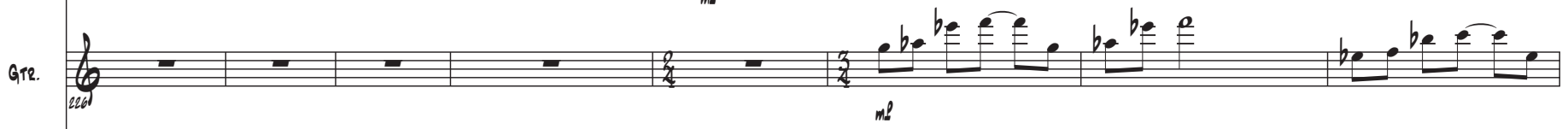

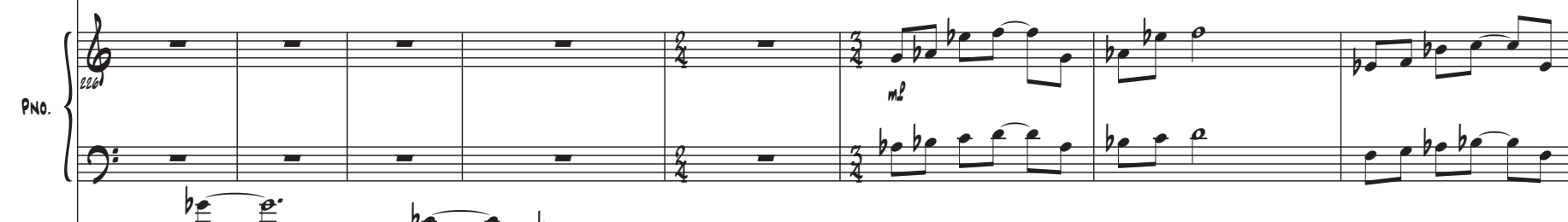

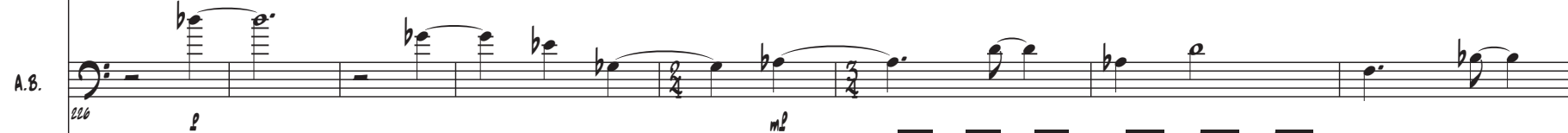
o. . 
2.3.2.

35
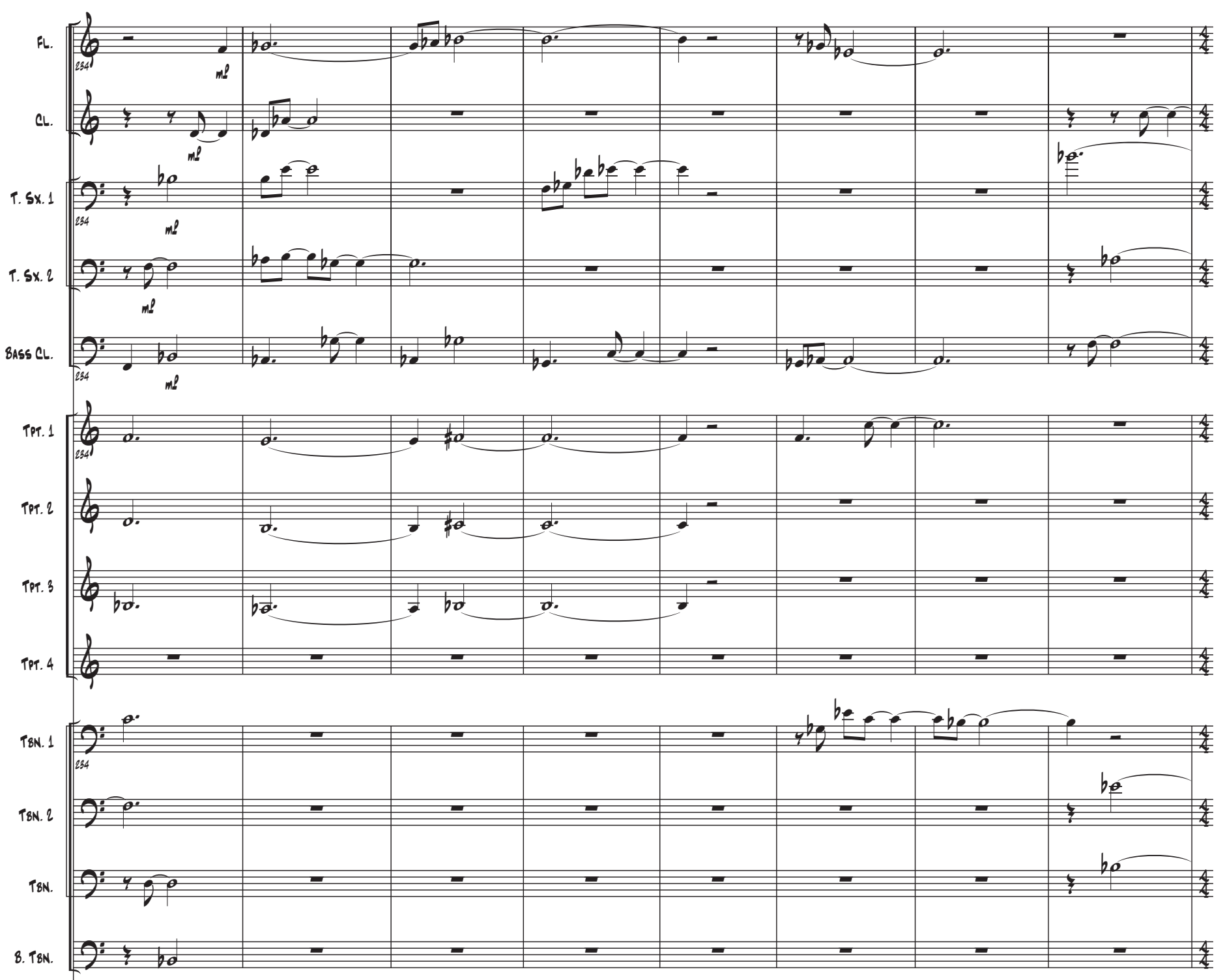

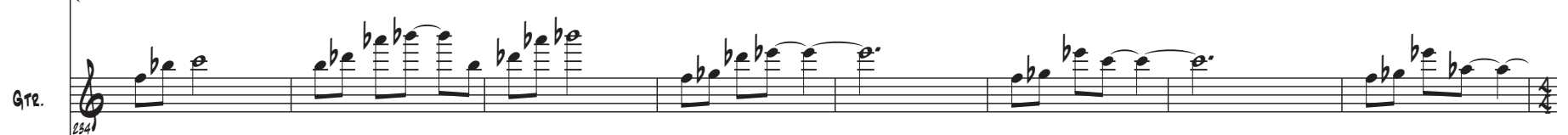

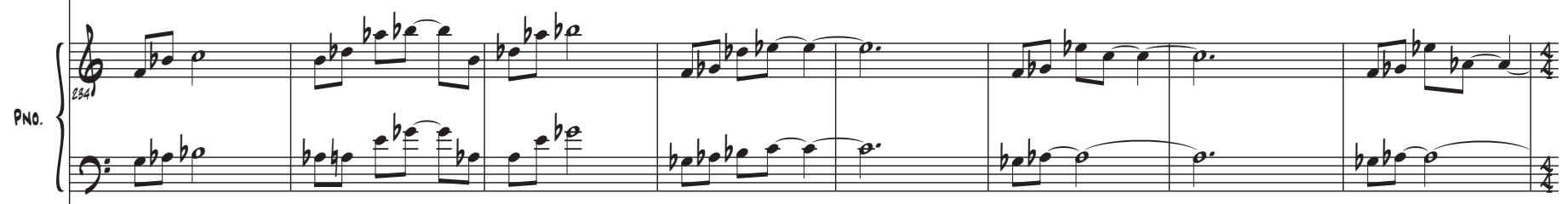

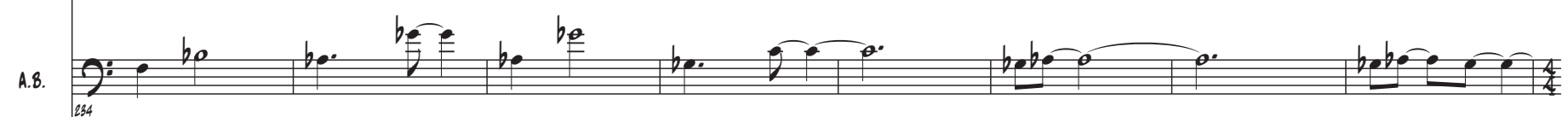

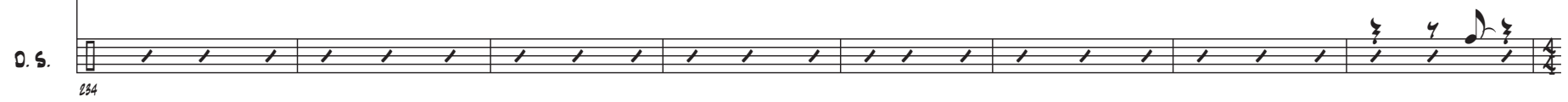



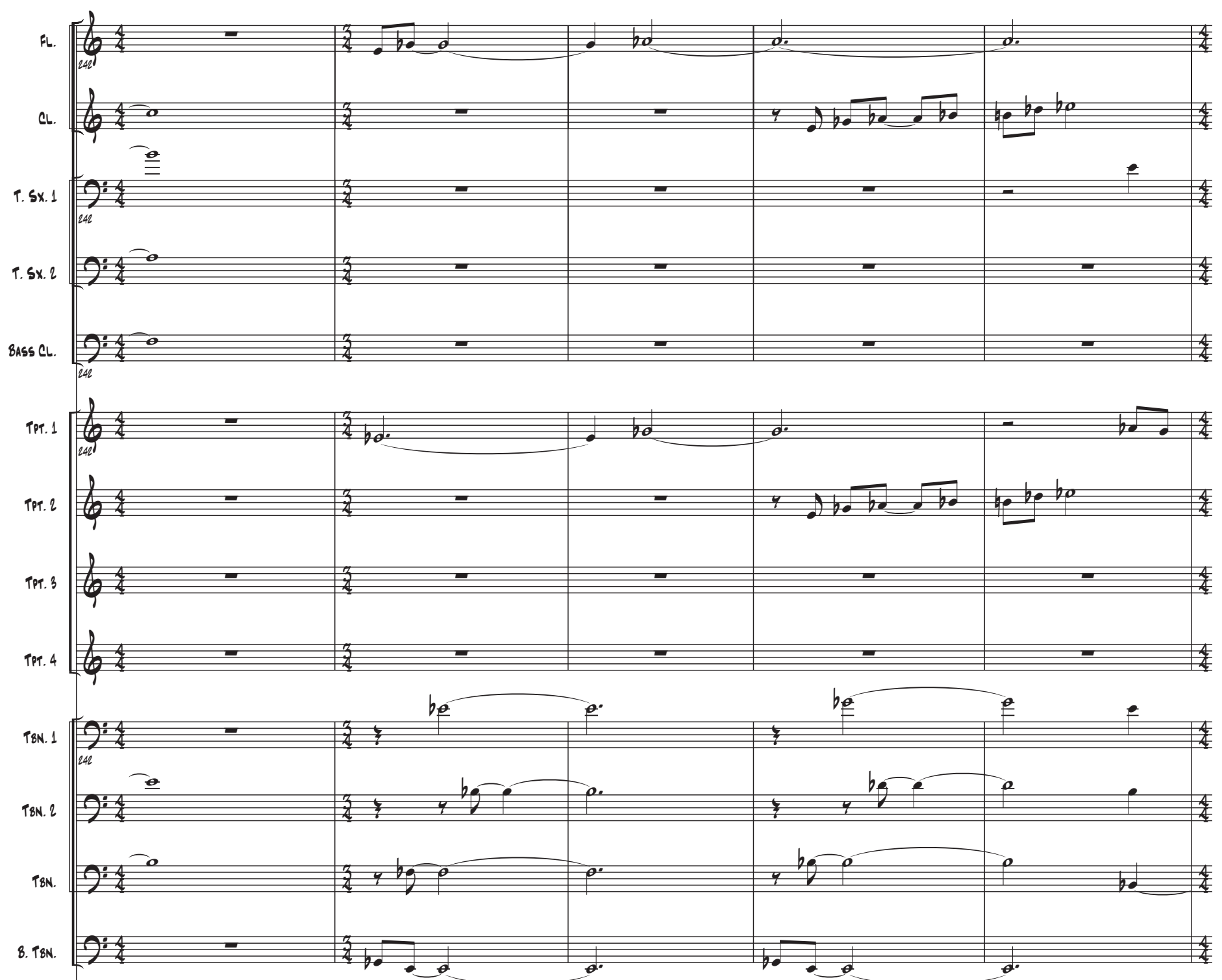

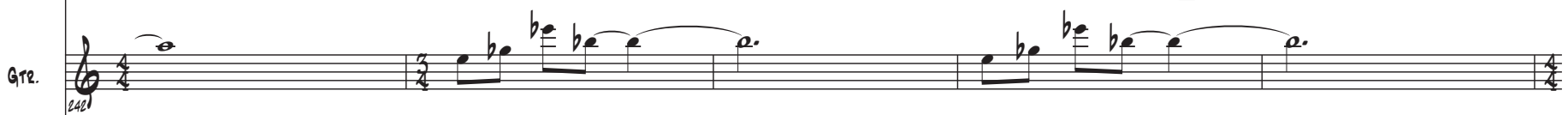
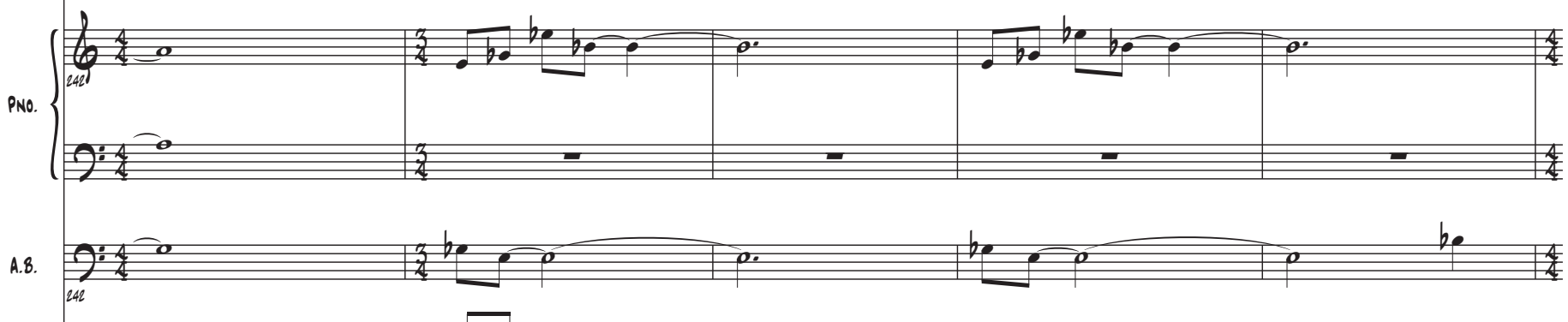

o.5. 

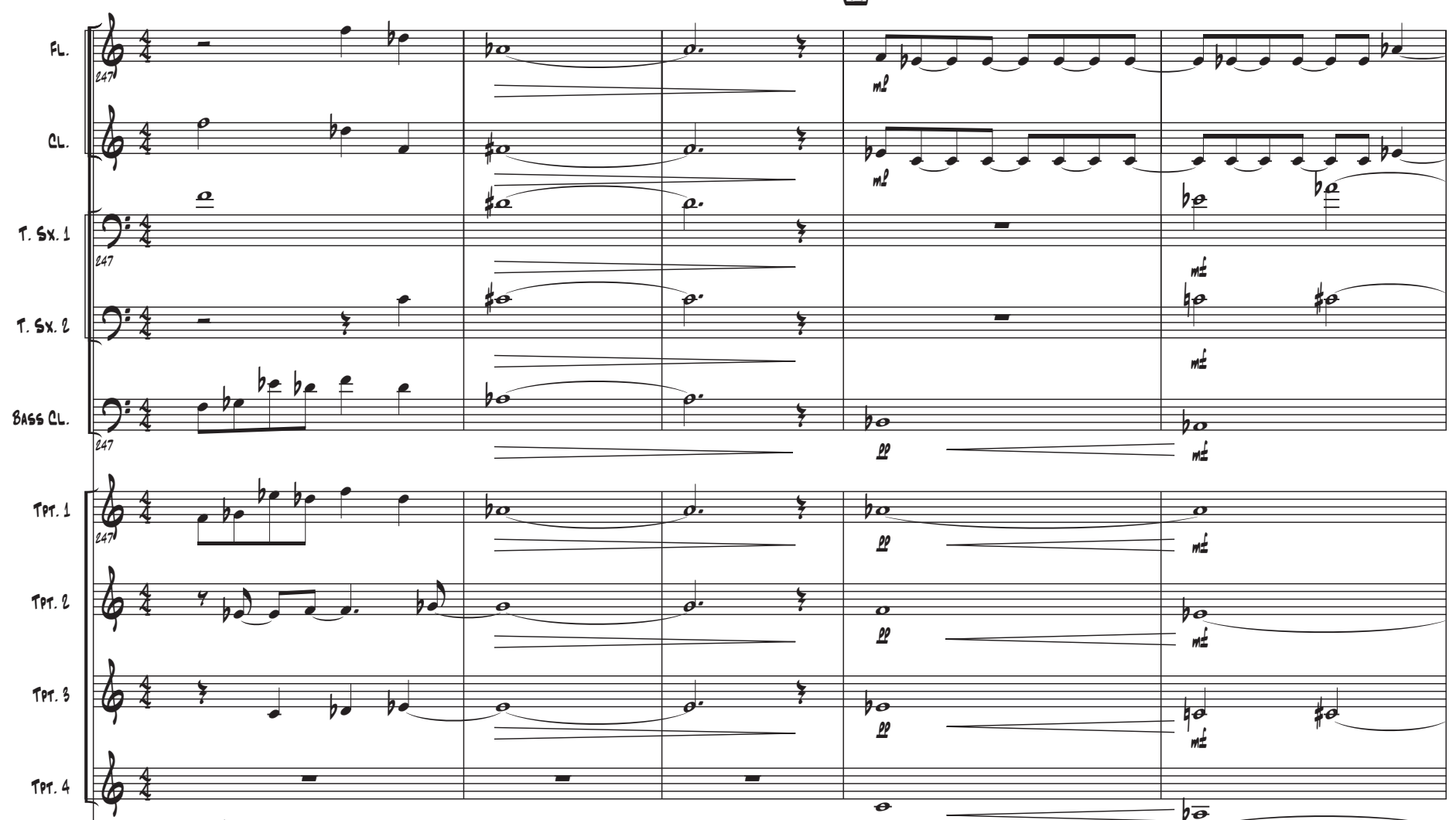

8. TSN.

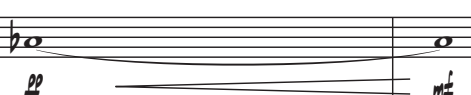



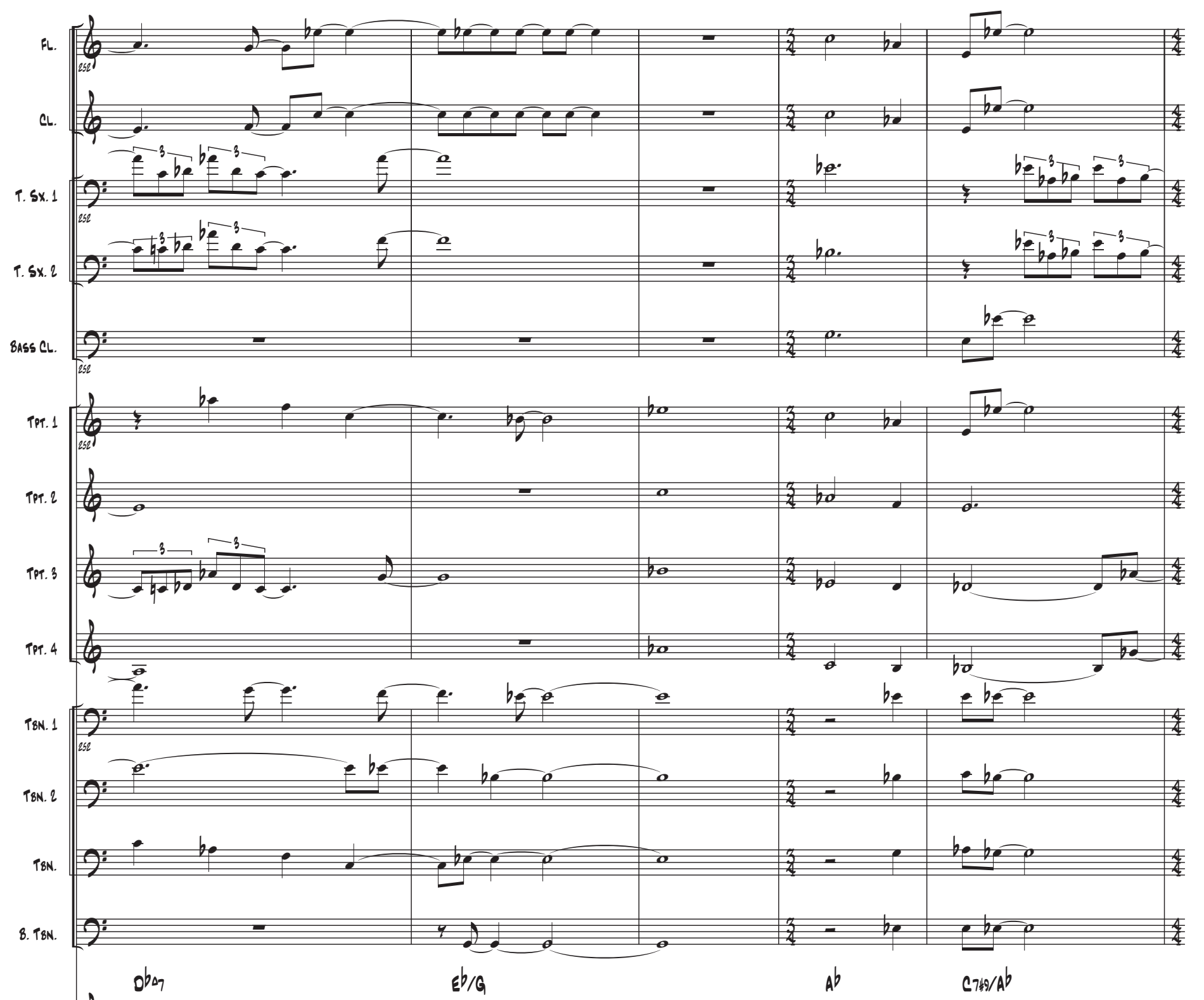

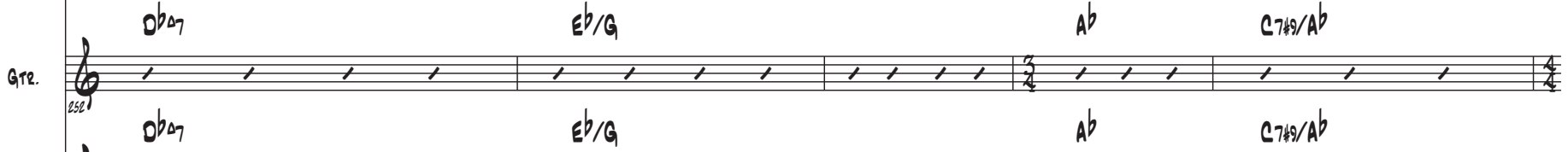

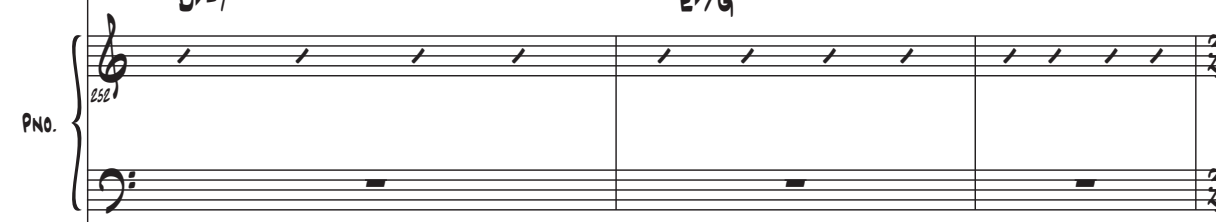
$g b_{\Delta \rightarrow} \quad \varepsilon b / G$

A.8. $\mathrm{S}_{2.2}: \mathrm{boc}_{0}$

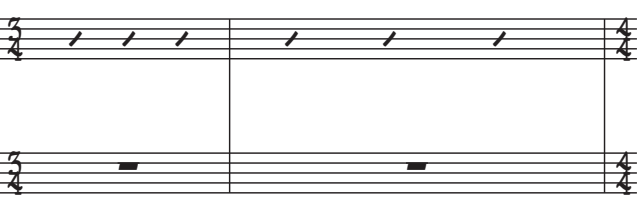

o.5. 

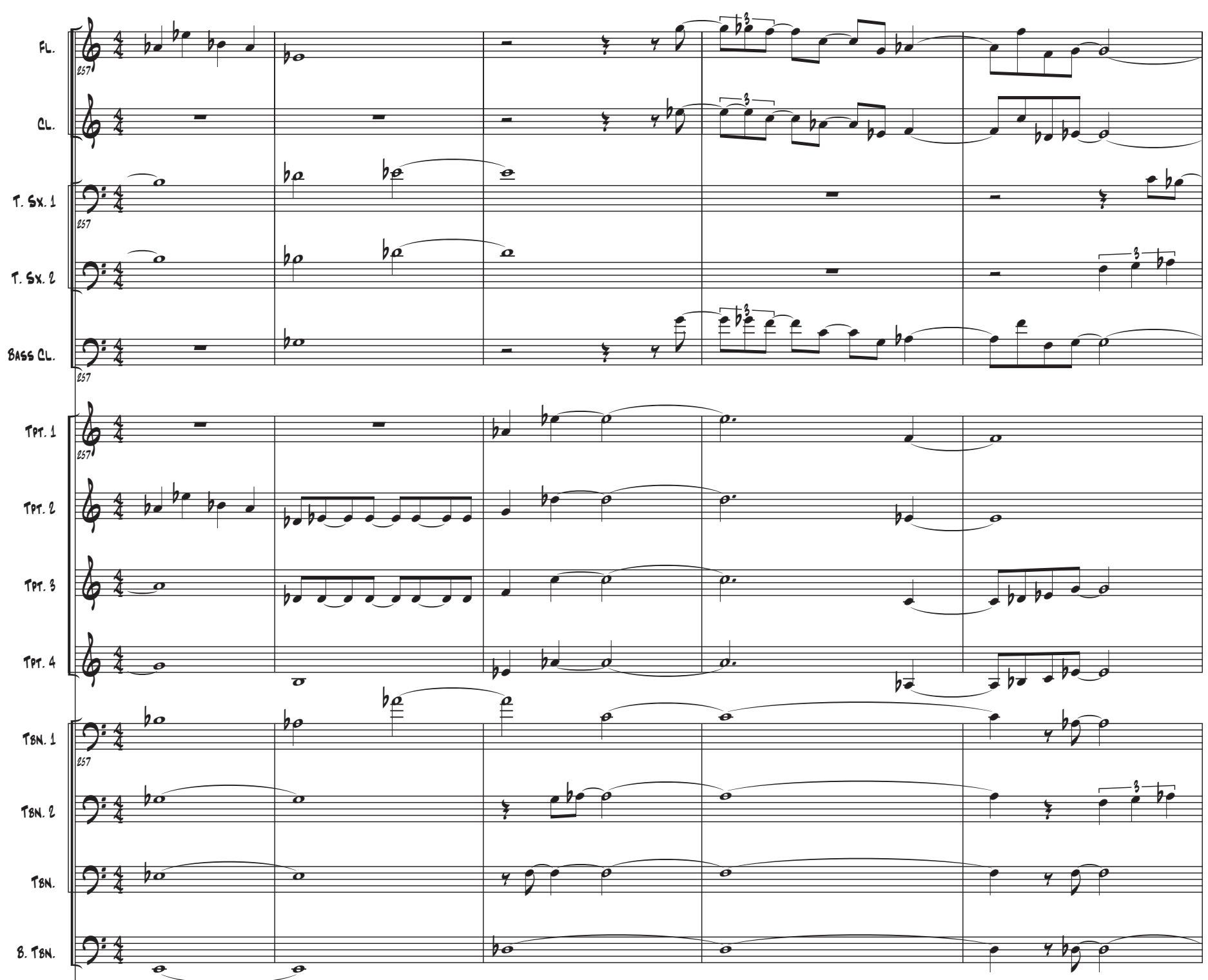

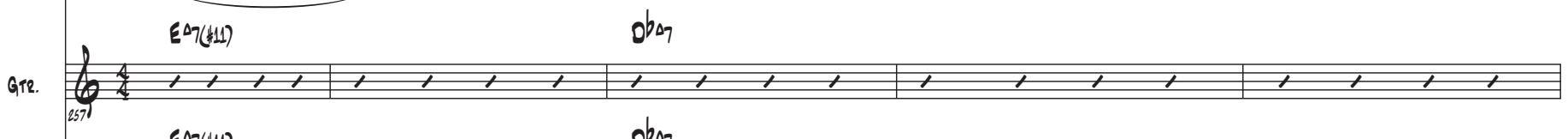

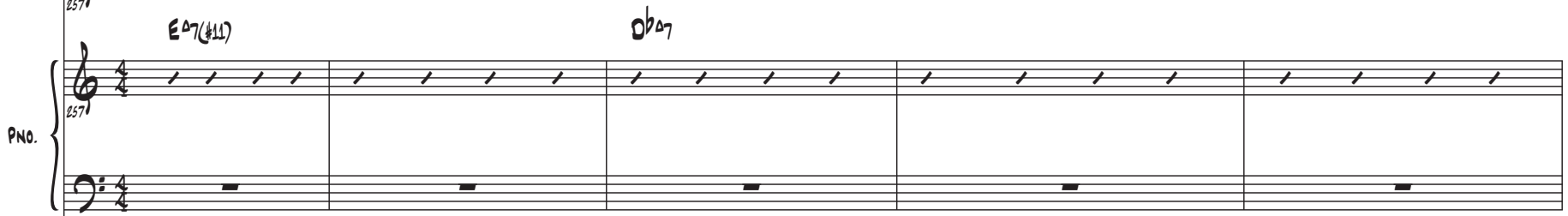
$E_{0} \rightarrow(411) \quad 0 b_{07}$

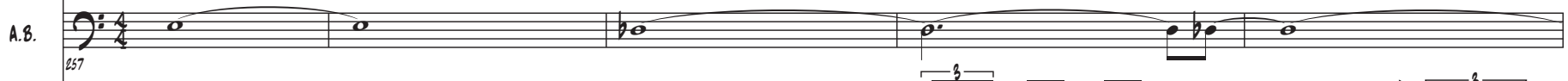

0.5. 

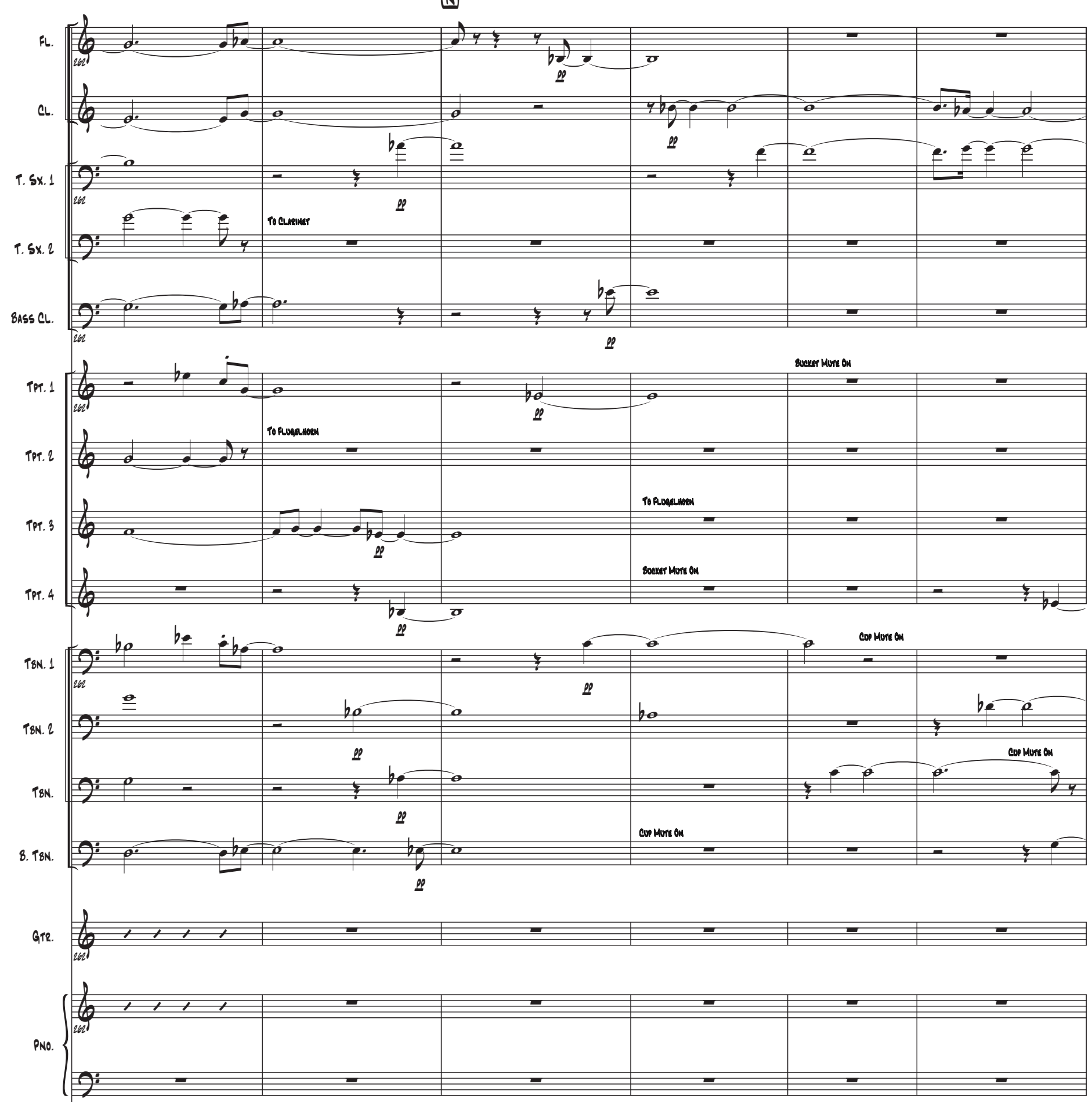

A.8. $\int_{200}^{0.0}$

bo.

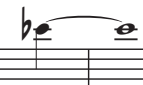

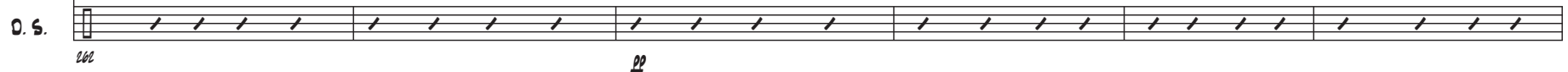


2.3.2.

41
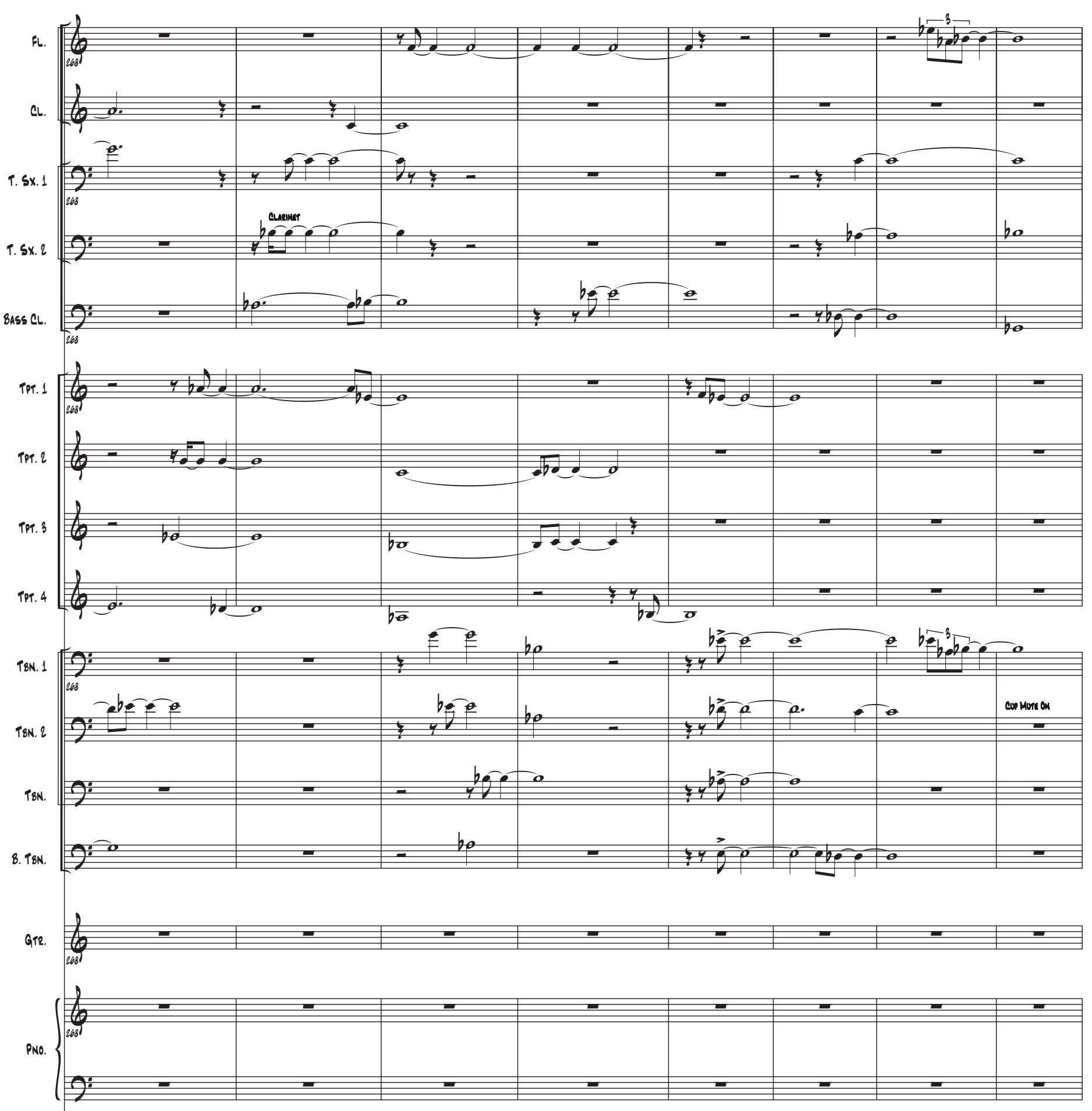

A.8. $2^{2}$

0.5. 

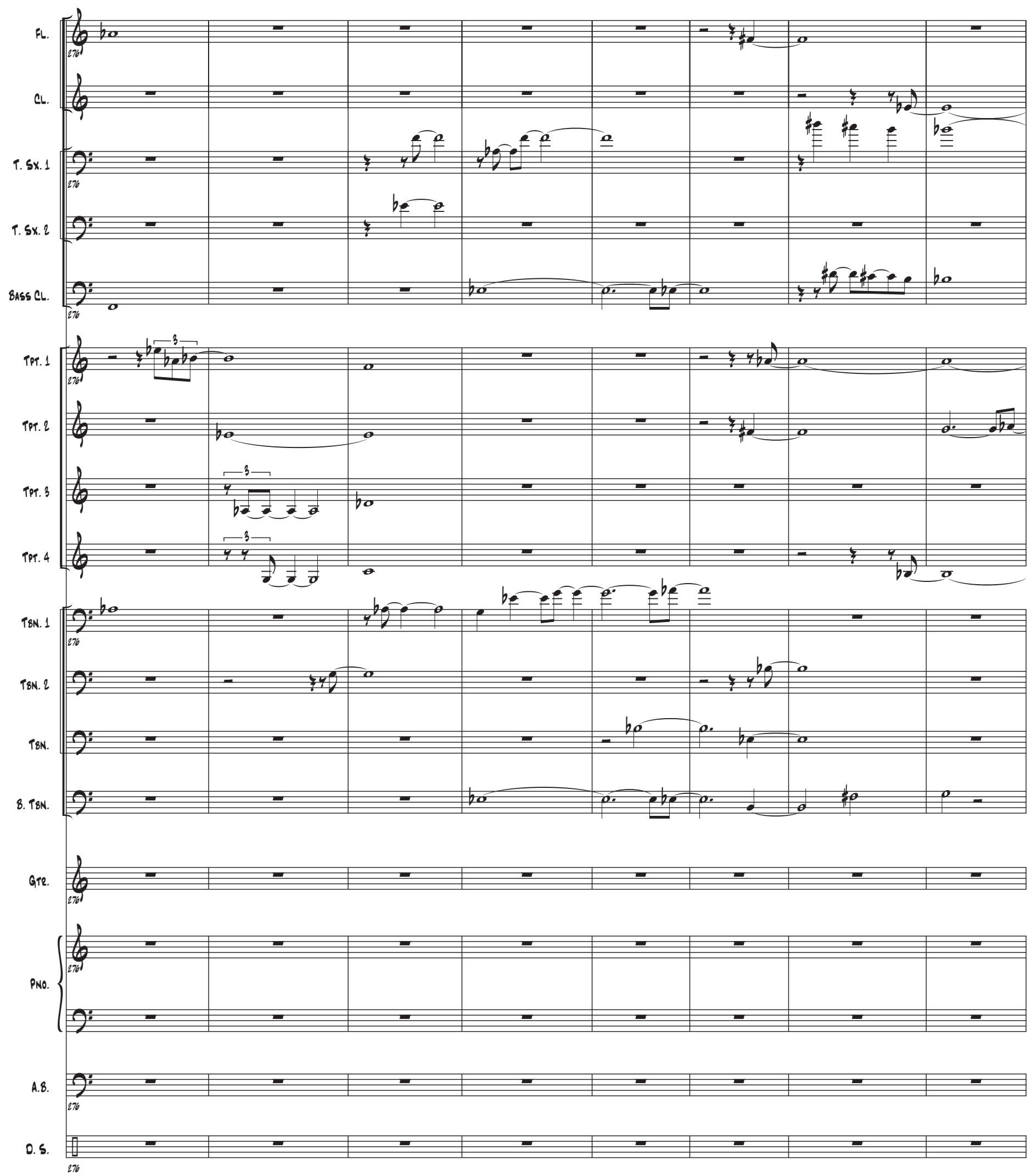

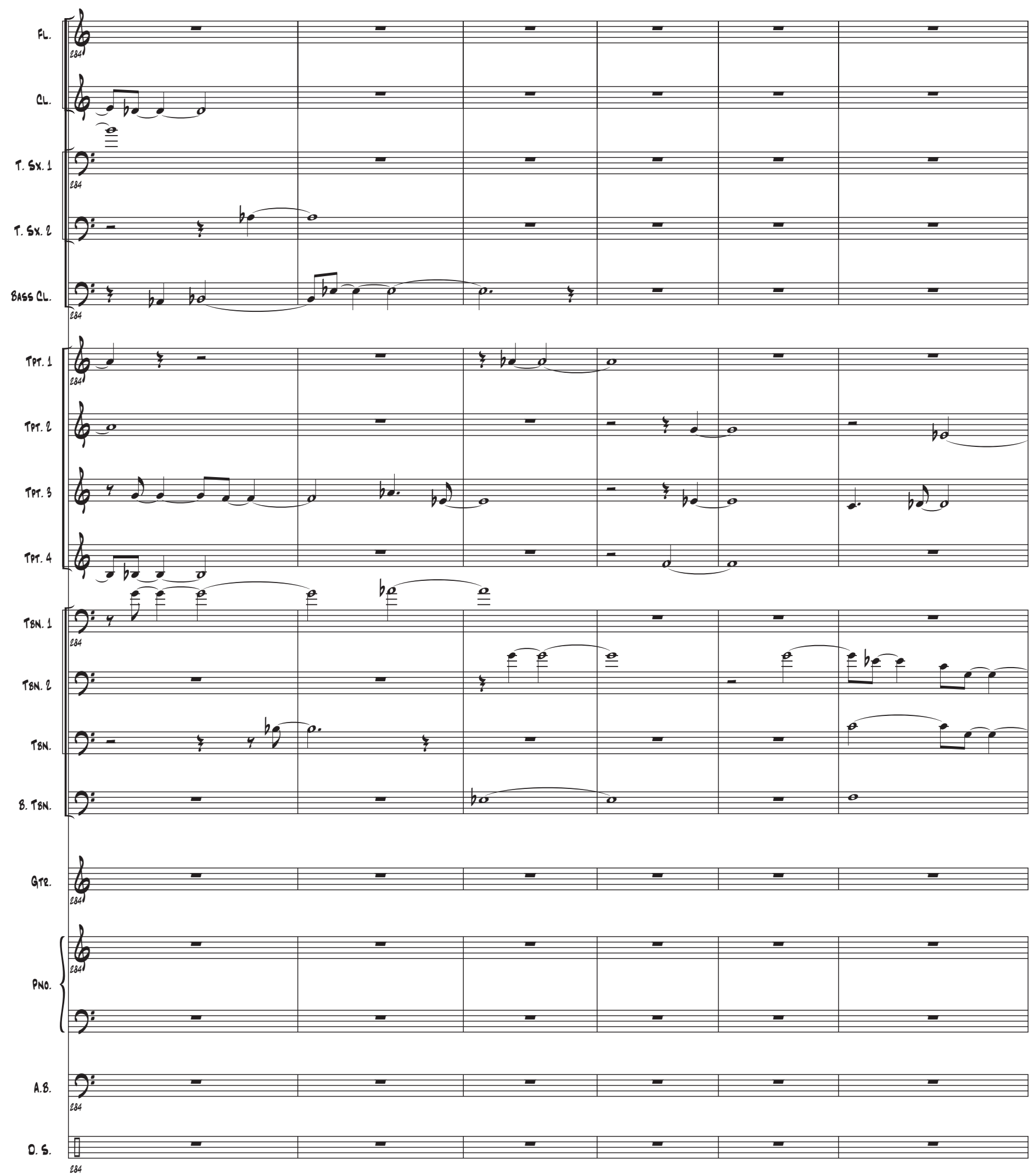

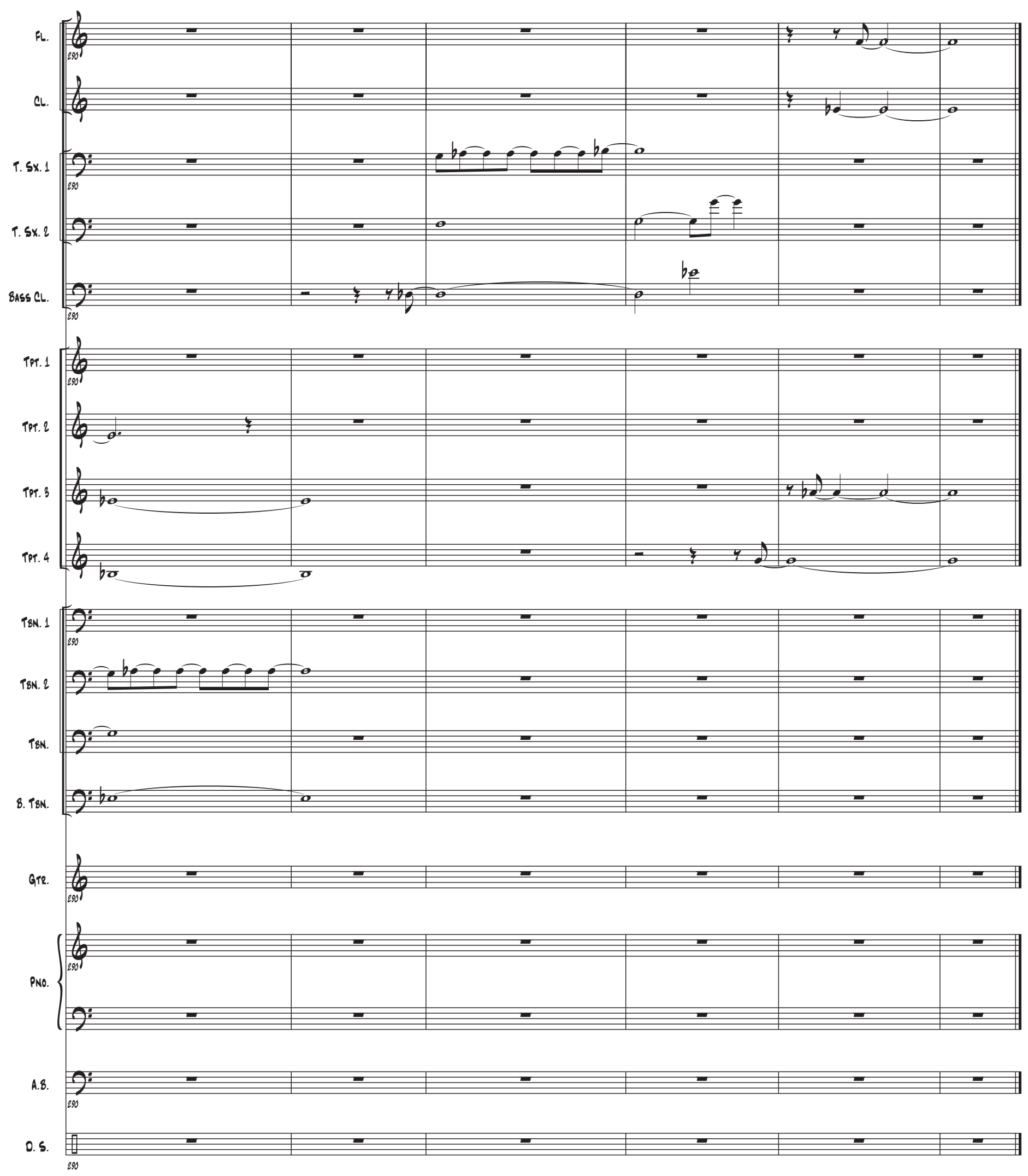



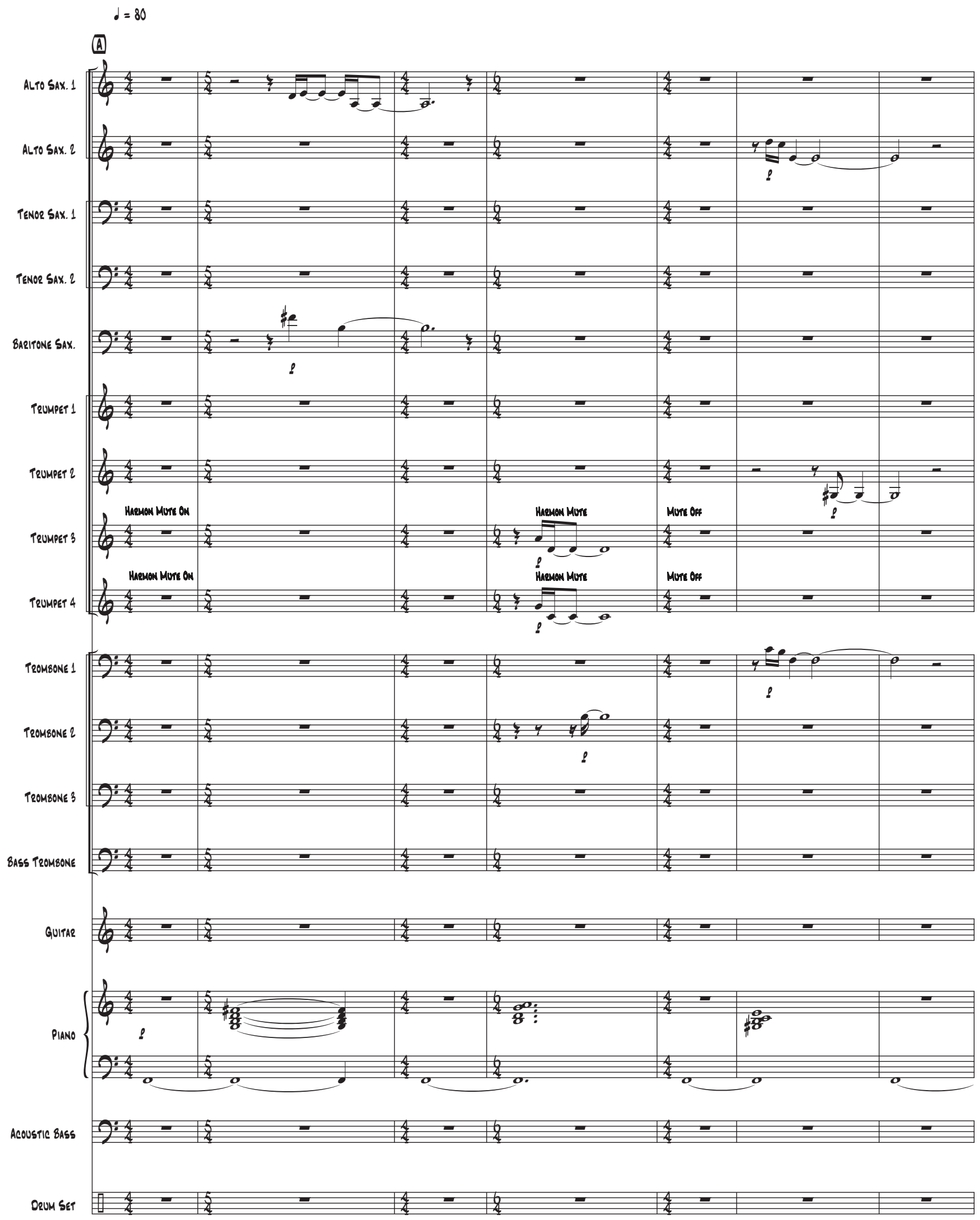



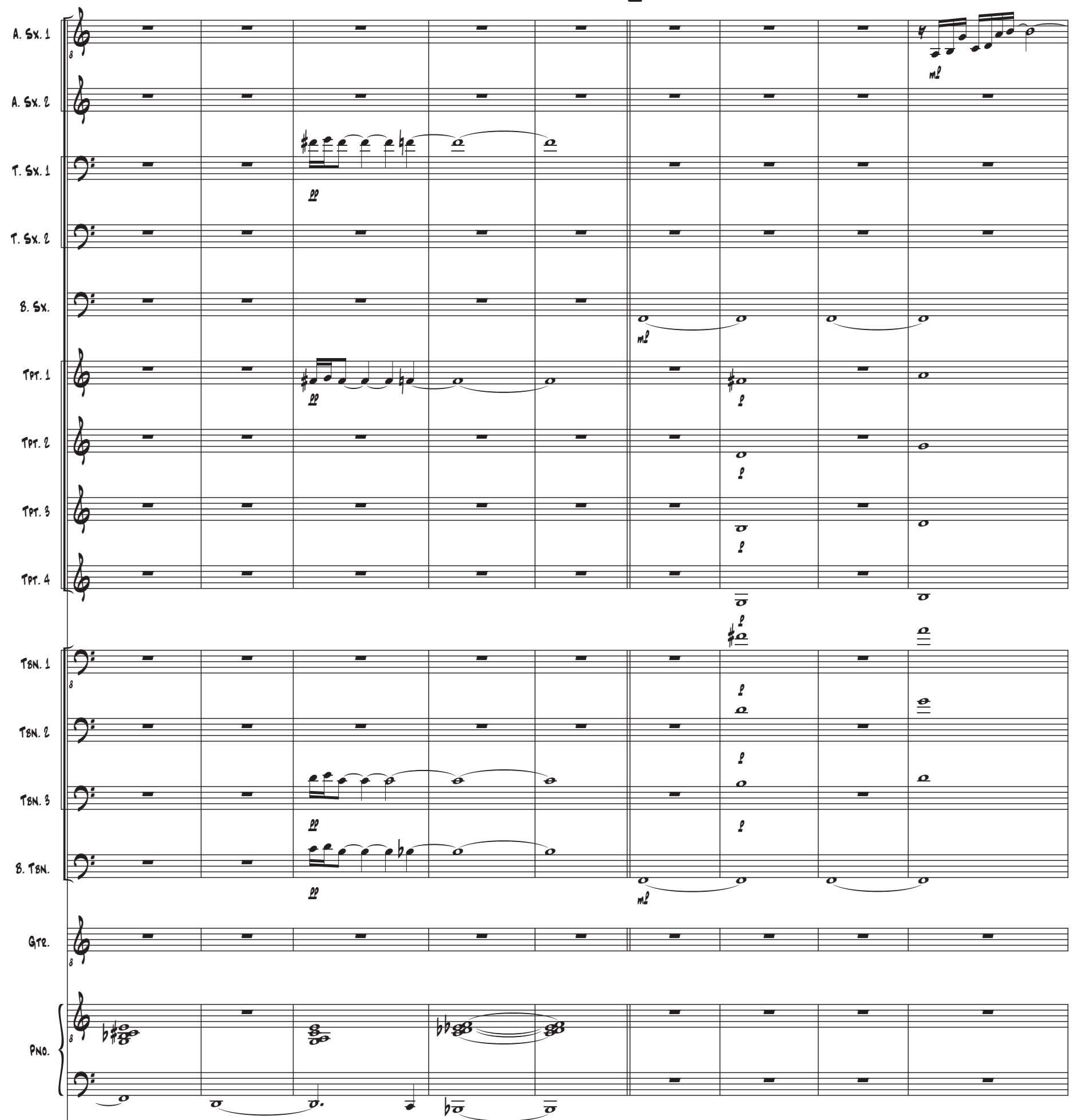

A.8.

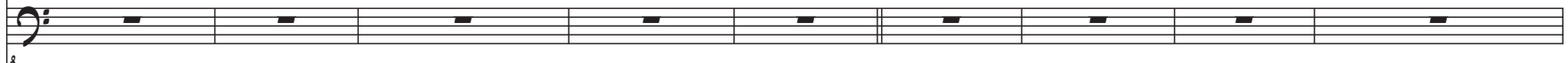

0.5. 


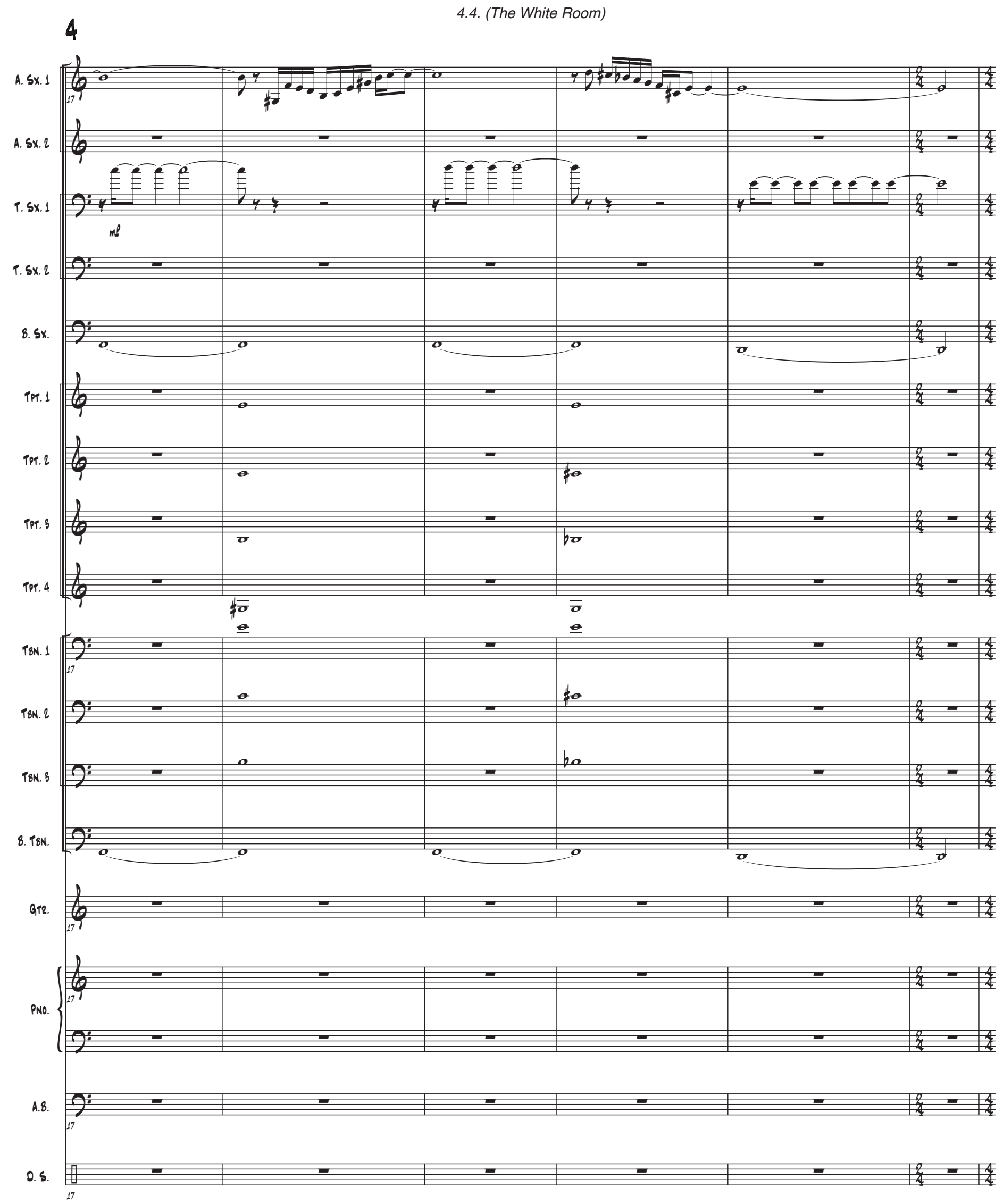




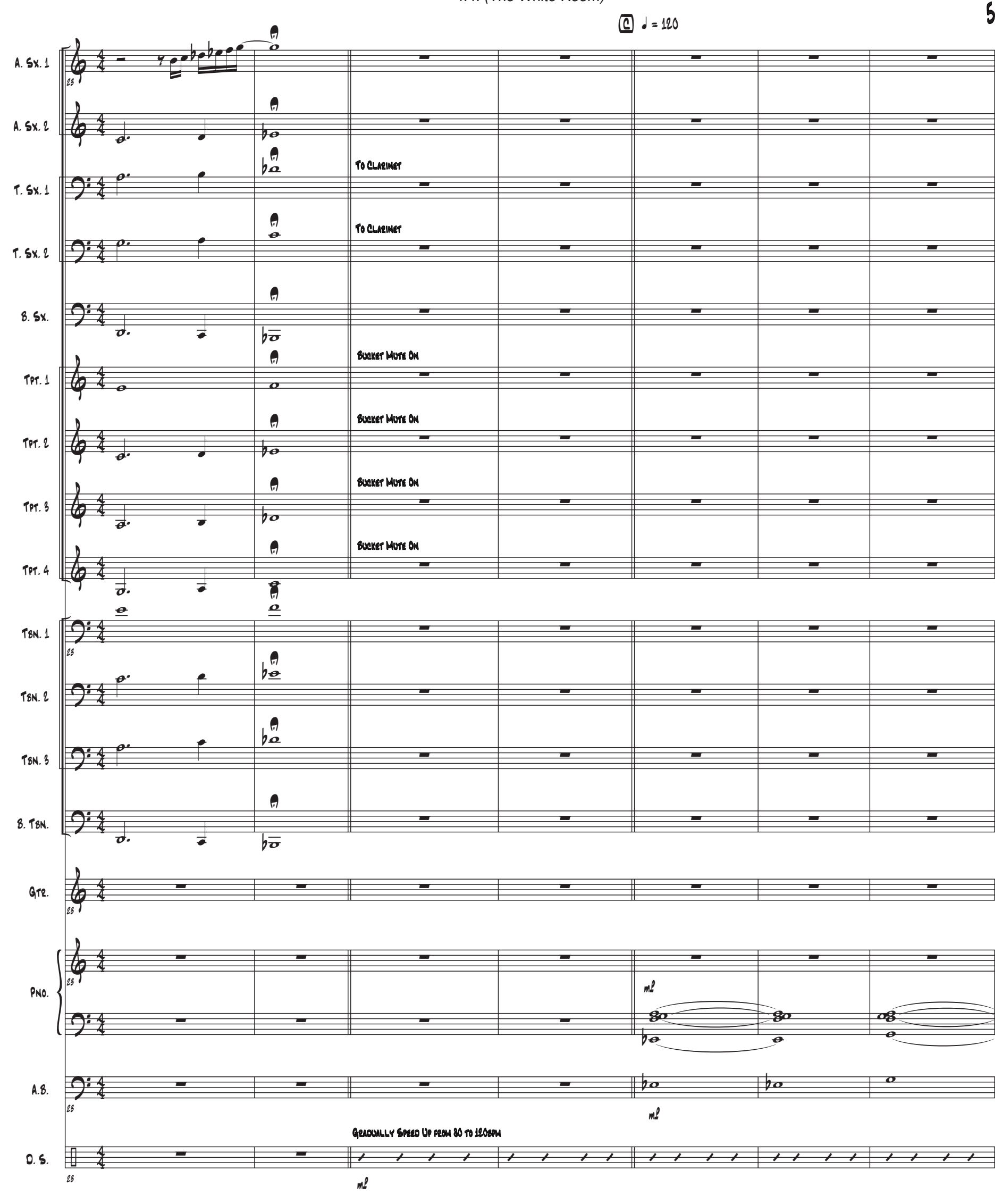



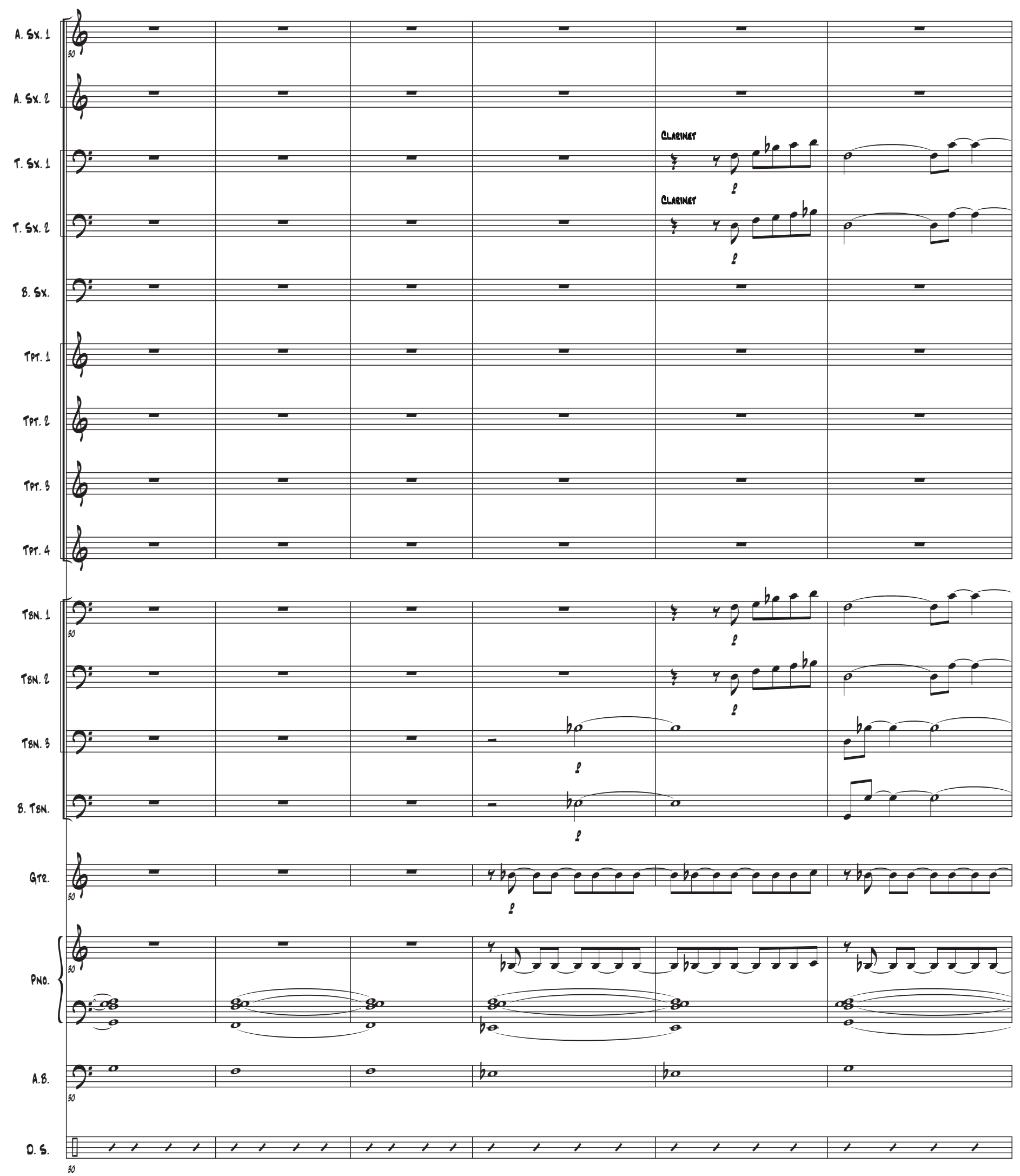


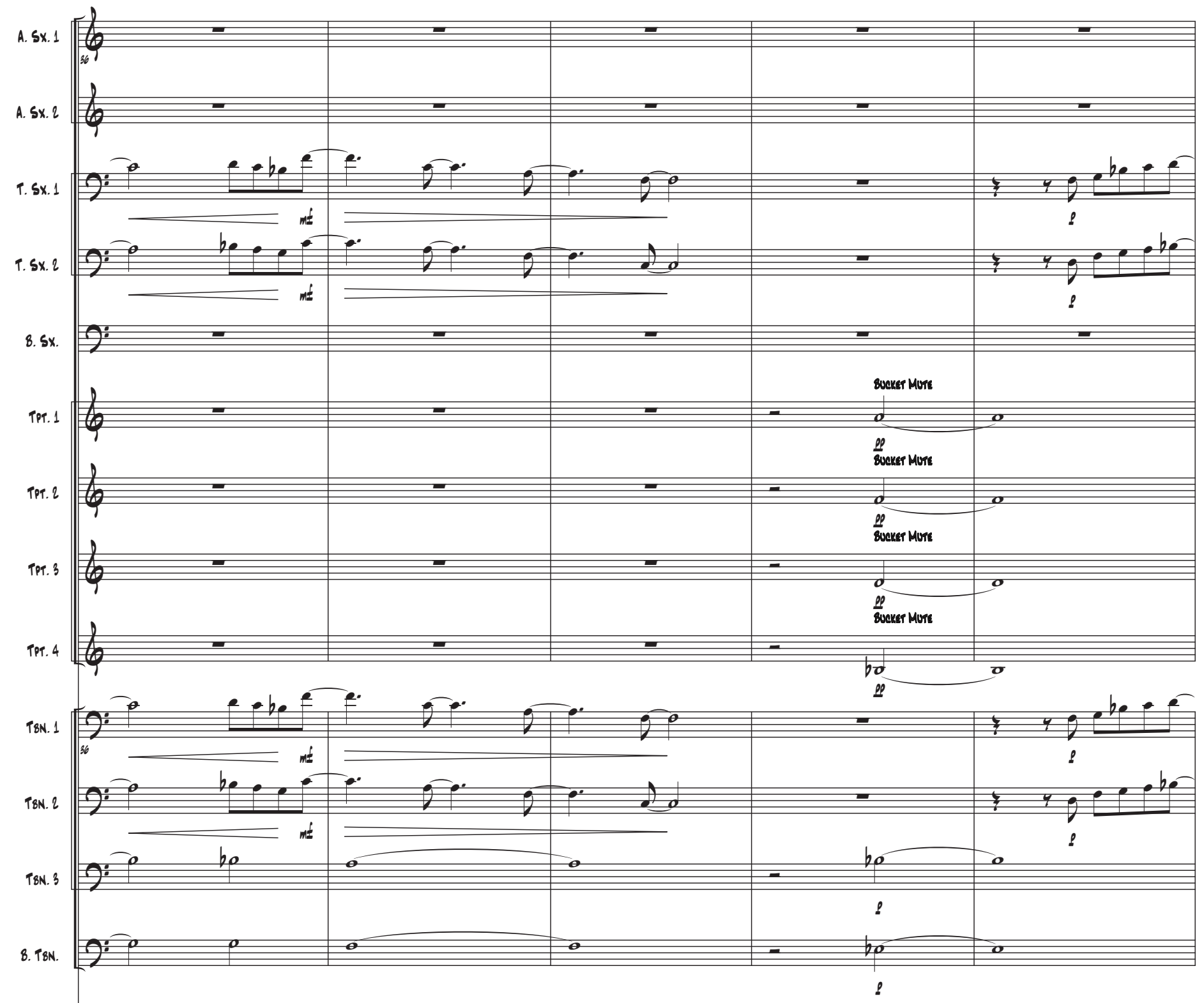

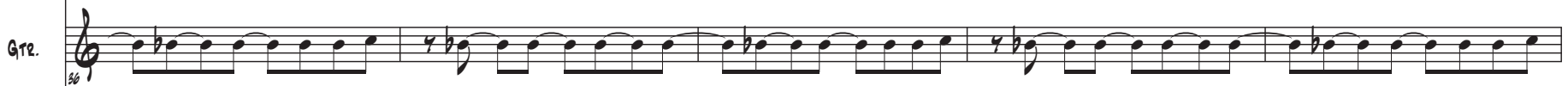

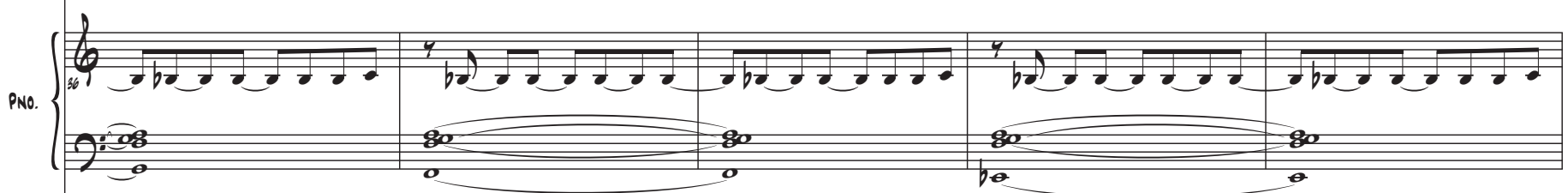

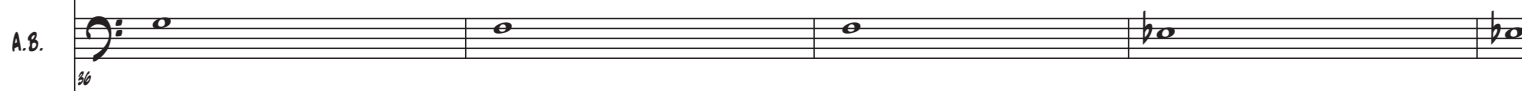

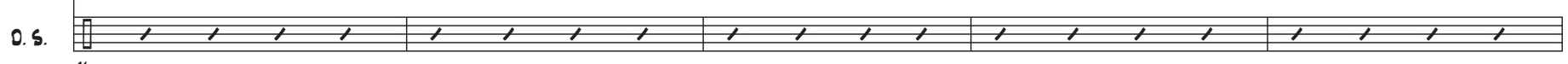



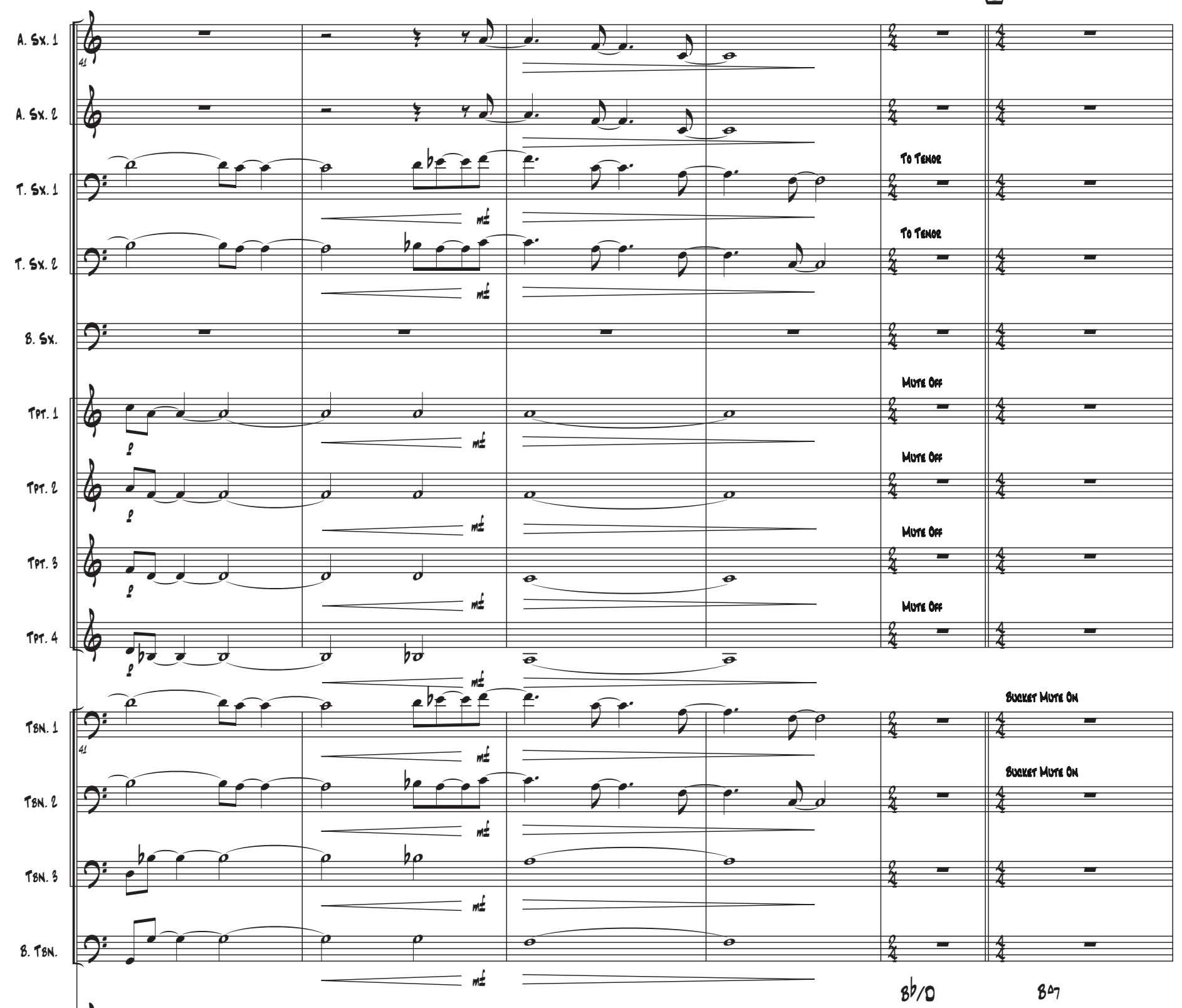

Giq. $\log _{41}$

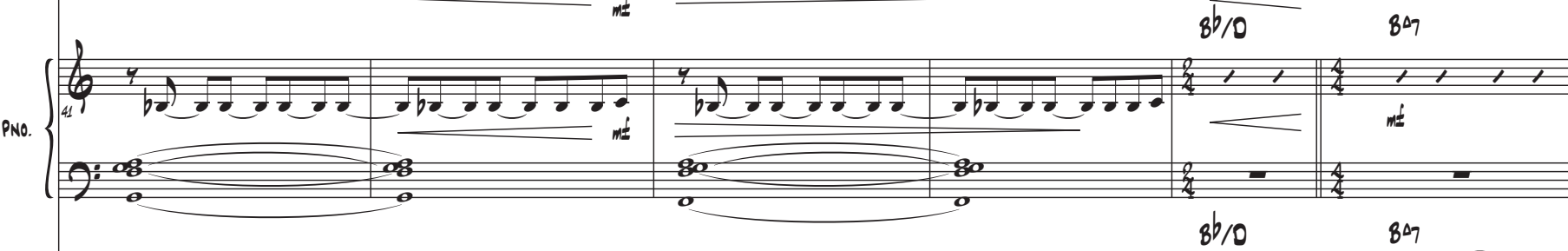

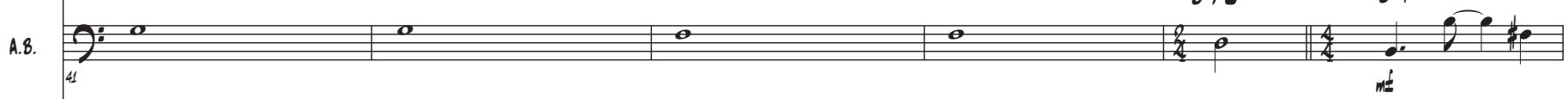

o. s. 

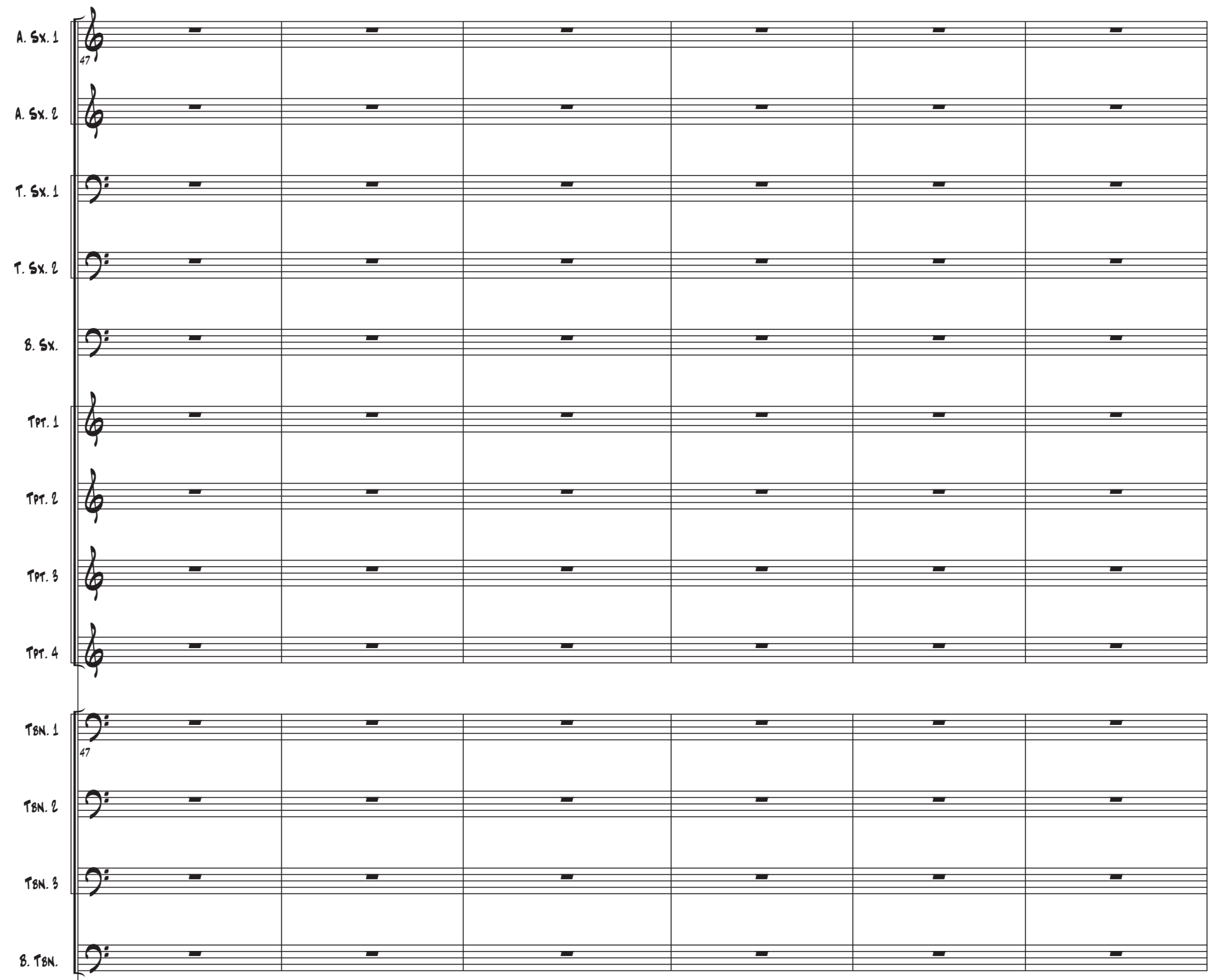

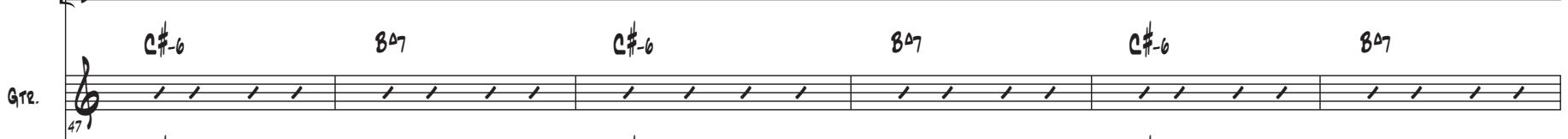

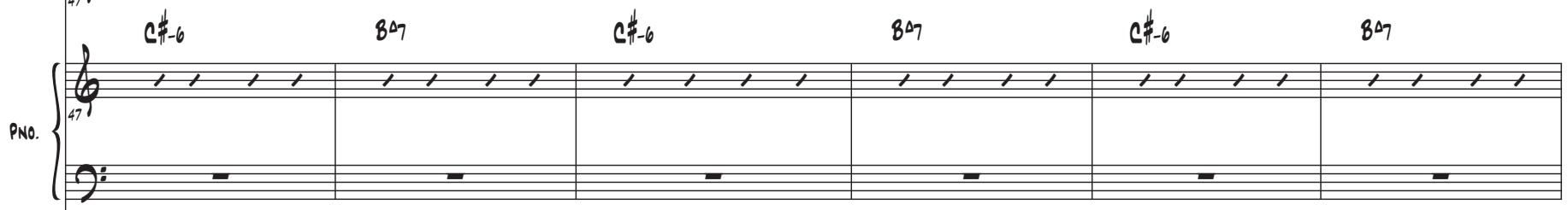
A.8.

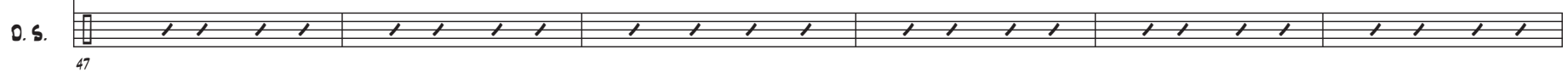




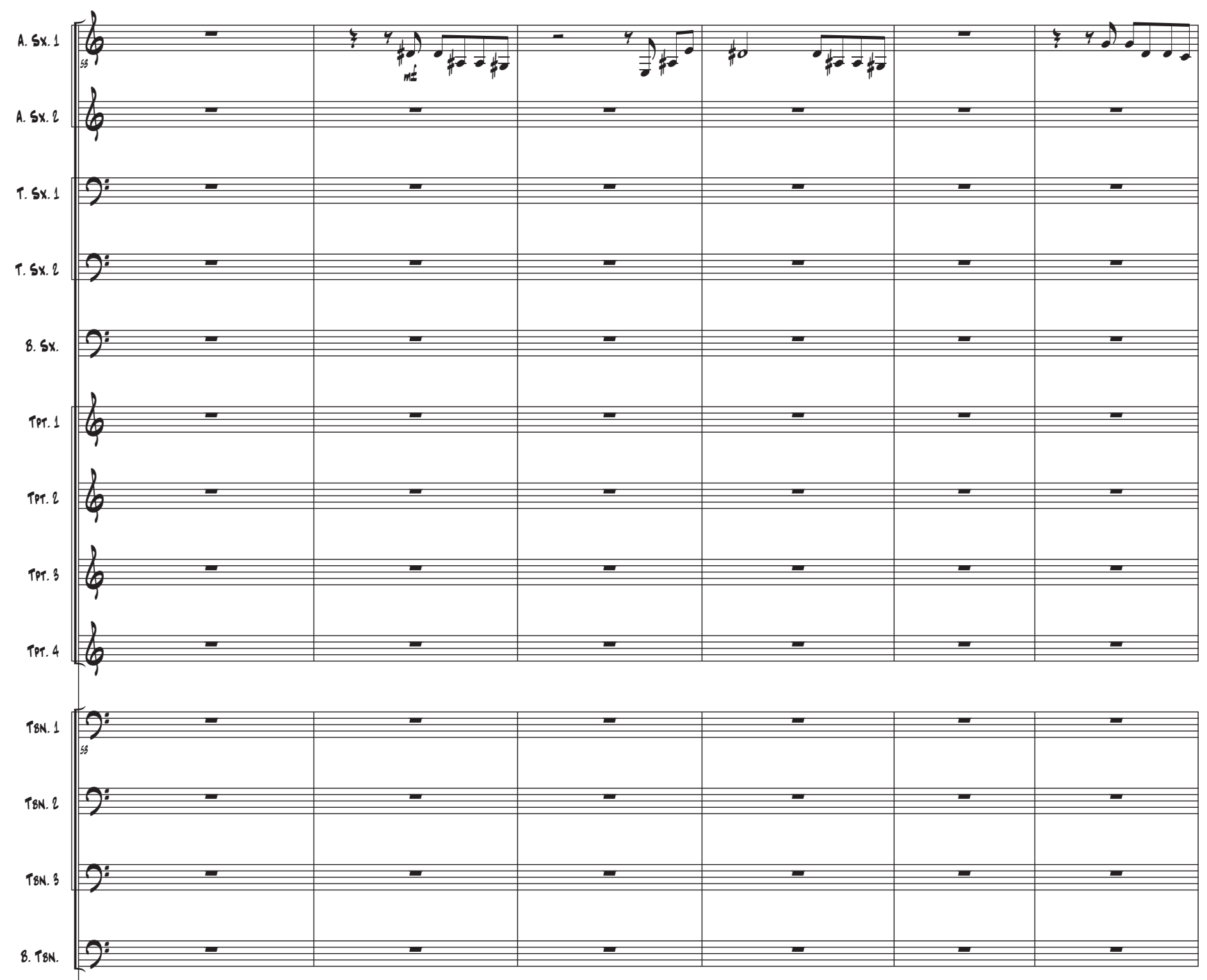

Gre. $b_{39}^{c 1,1,1}$

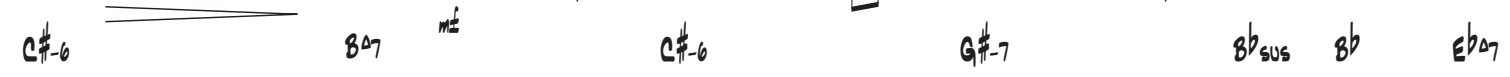

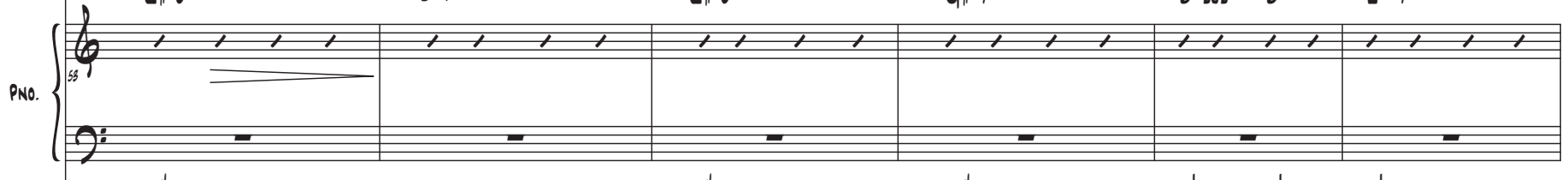
A.8. o. s. 


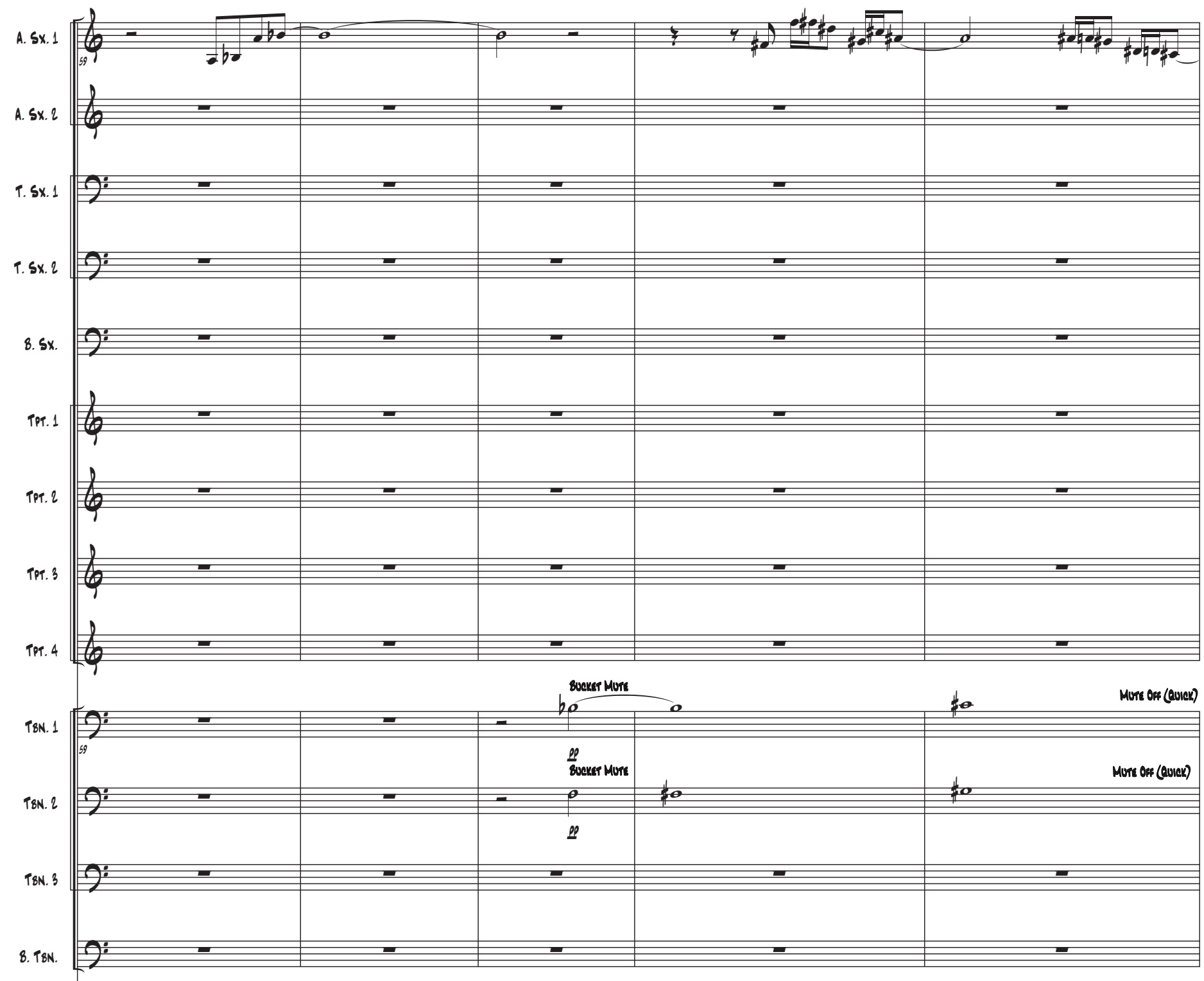

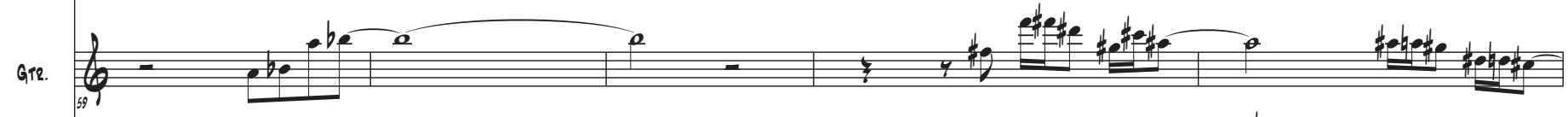

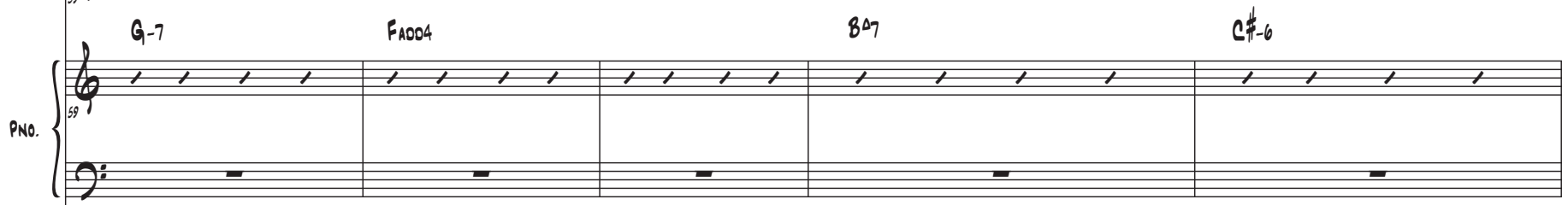

A.8.

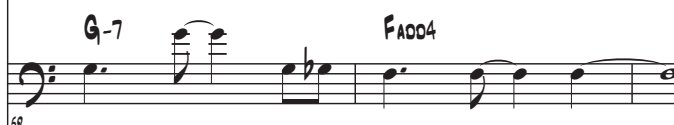

847

o. 5 .

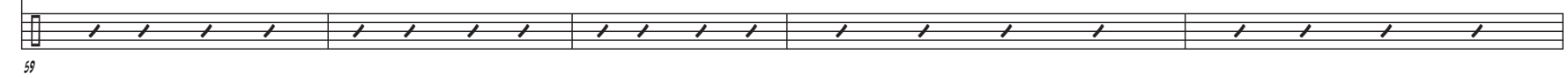




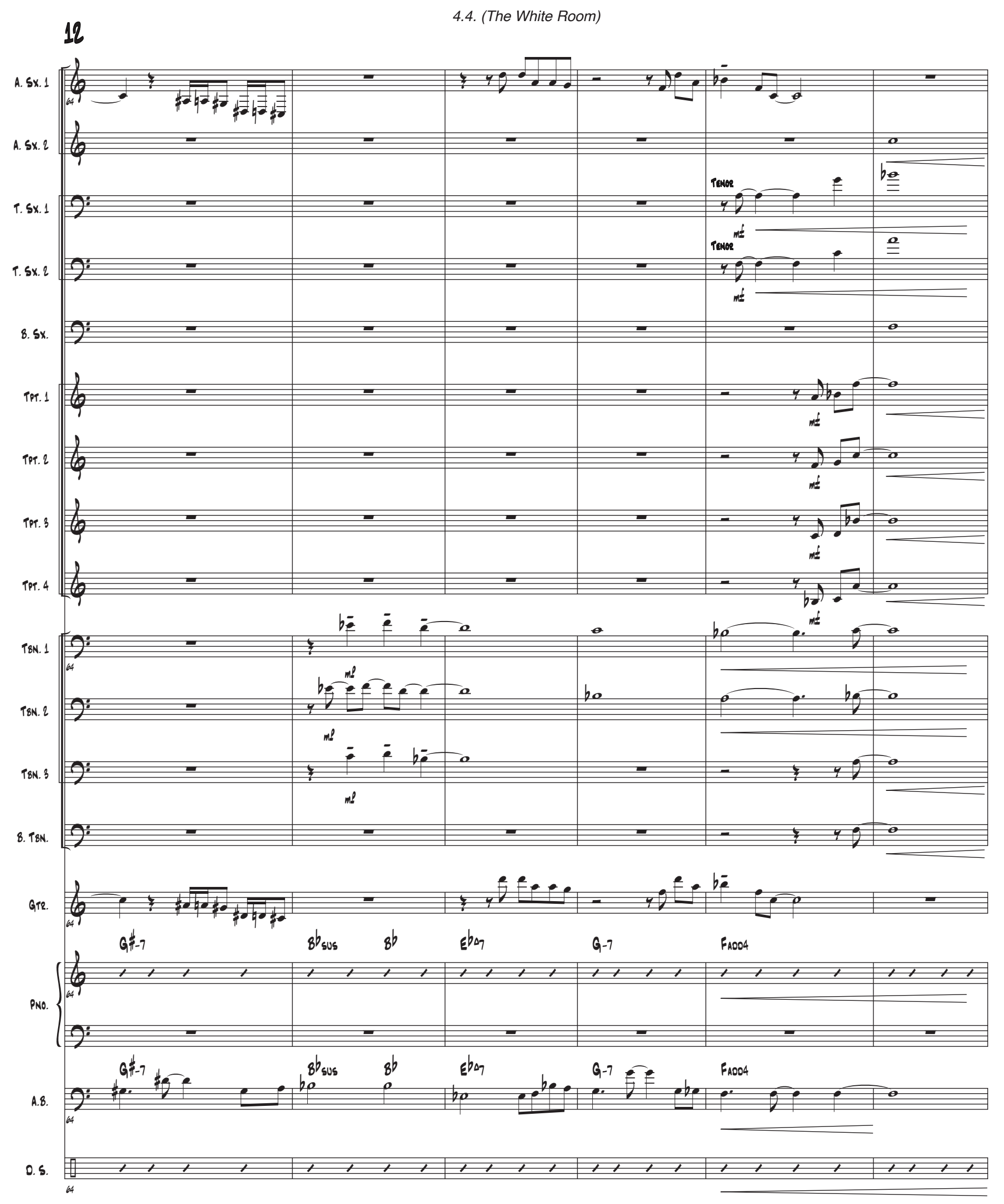




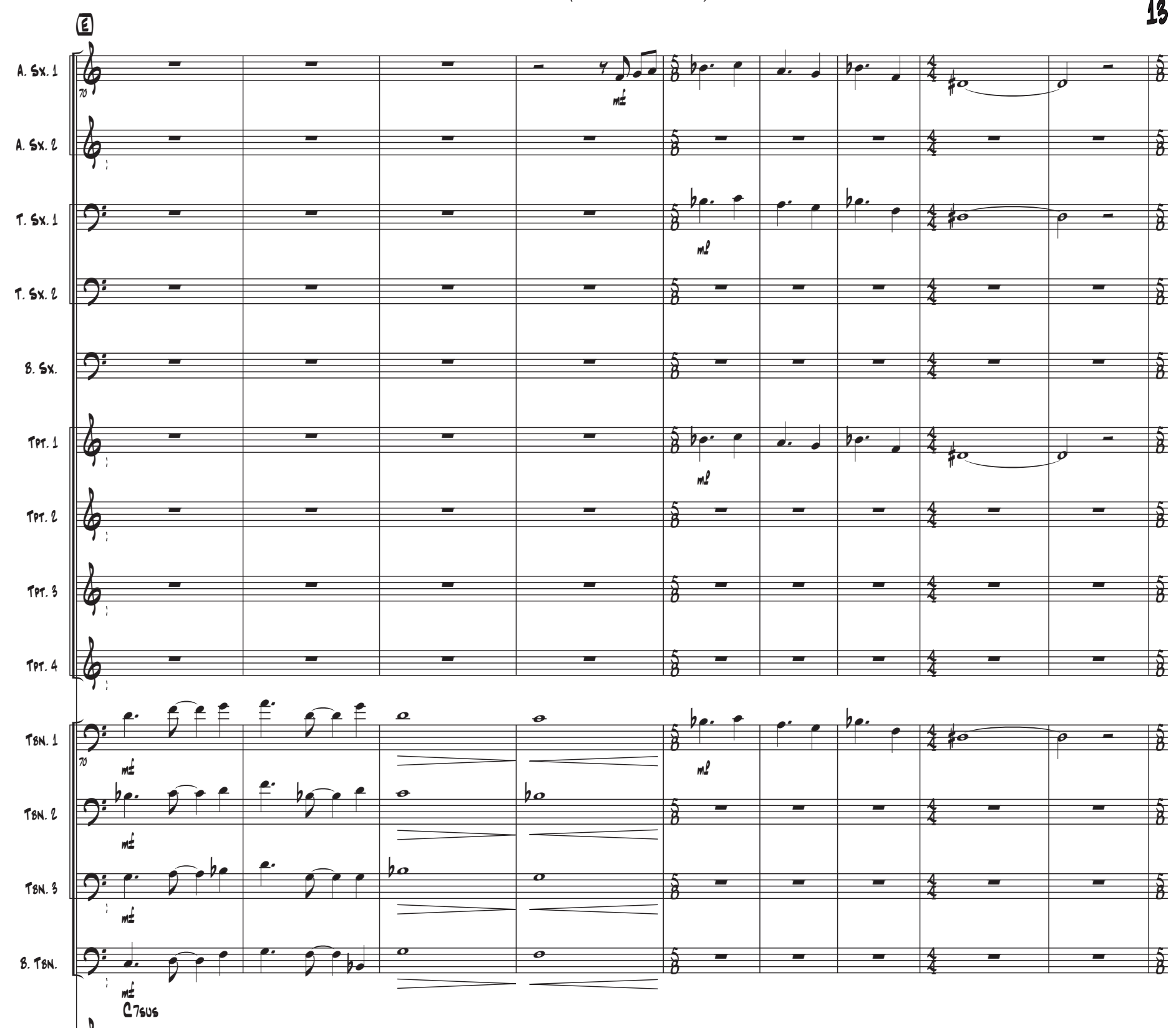

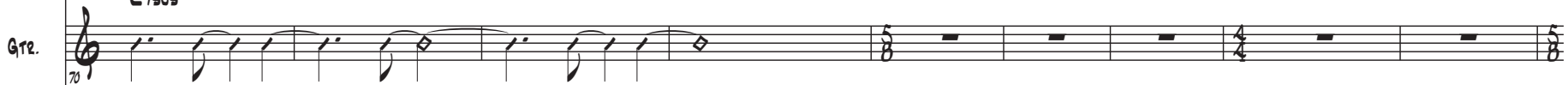
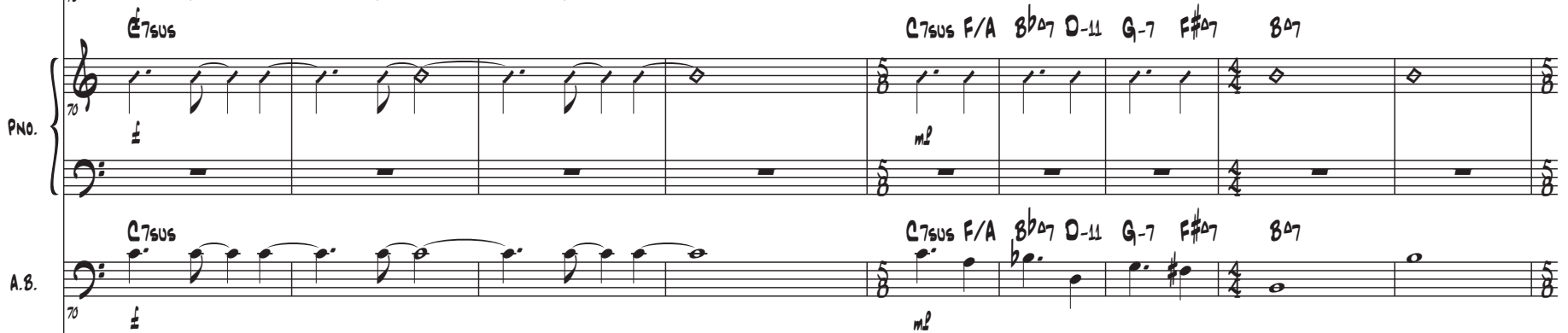

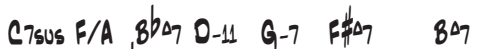

o. s. 


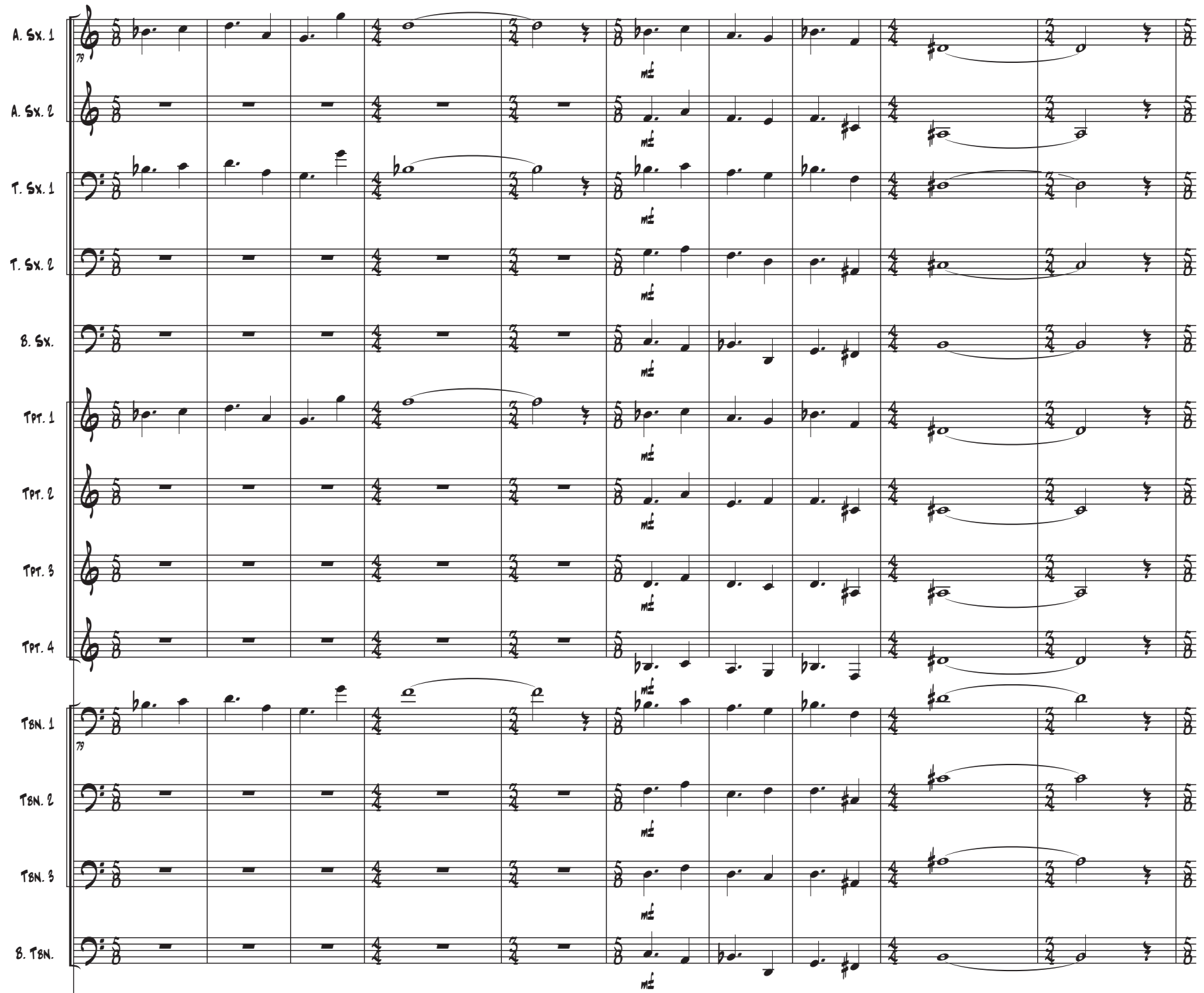

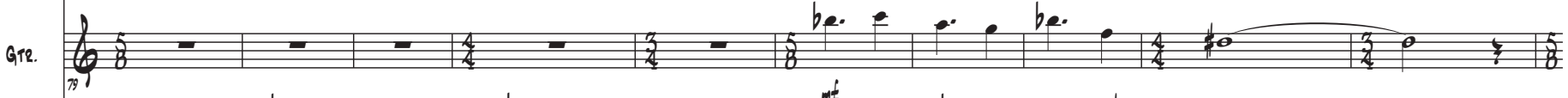

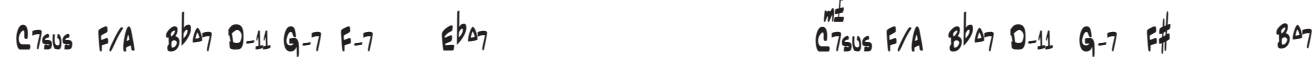

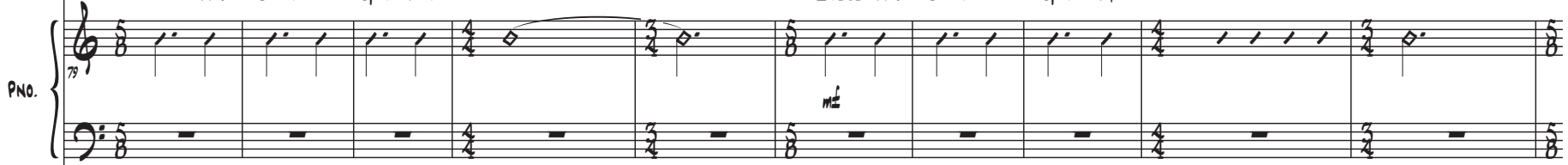

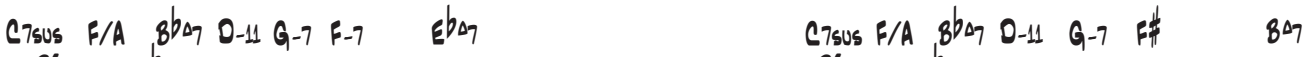
A.8. $\int_{70}$

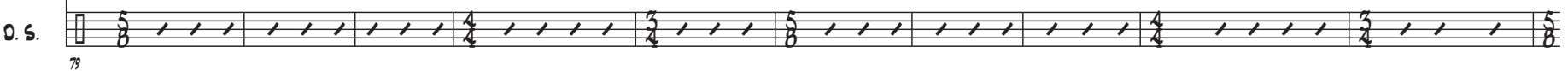



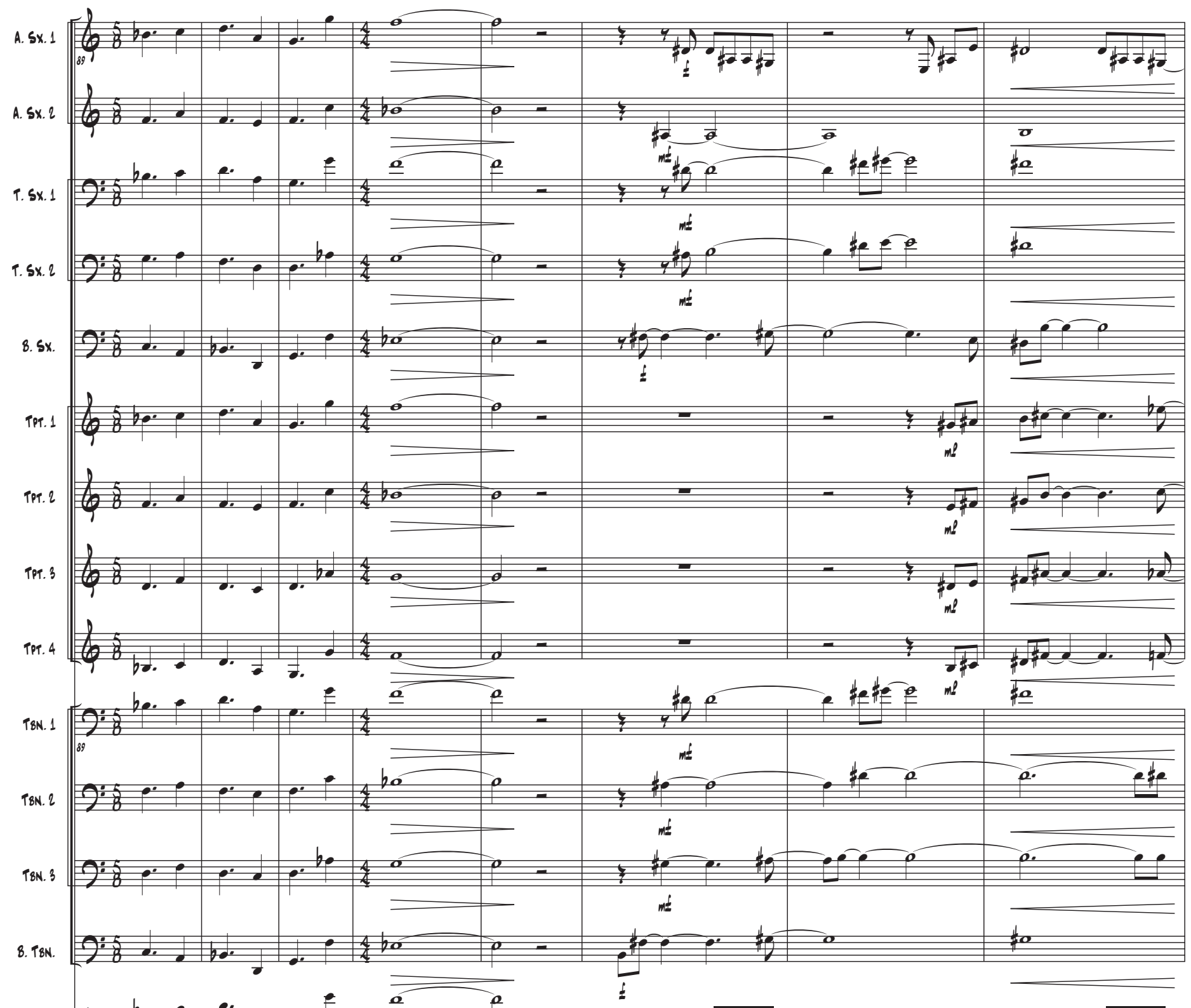

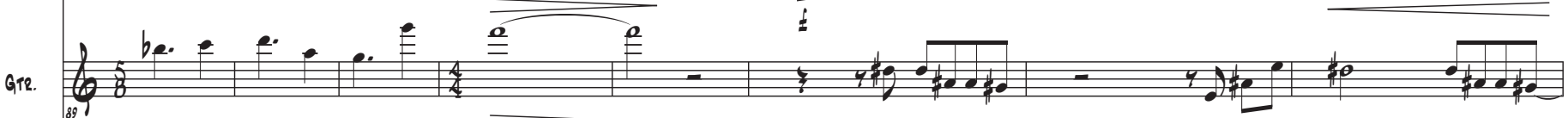

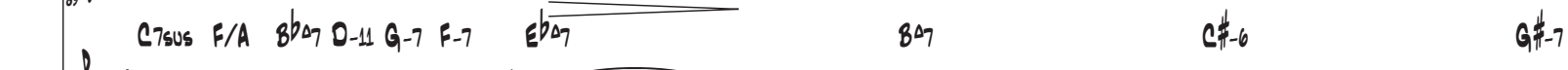

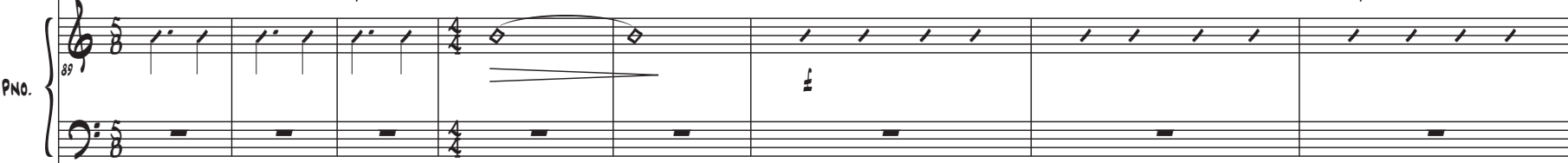

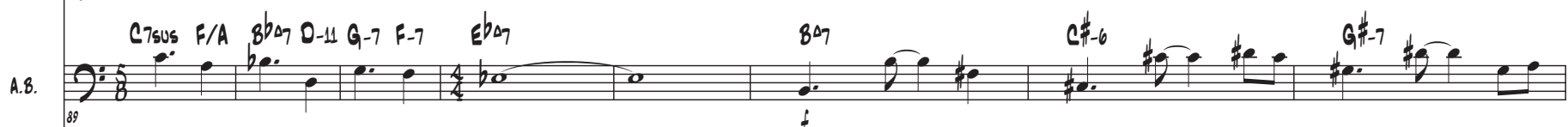

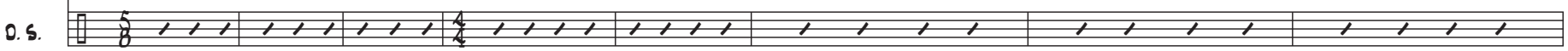




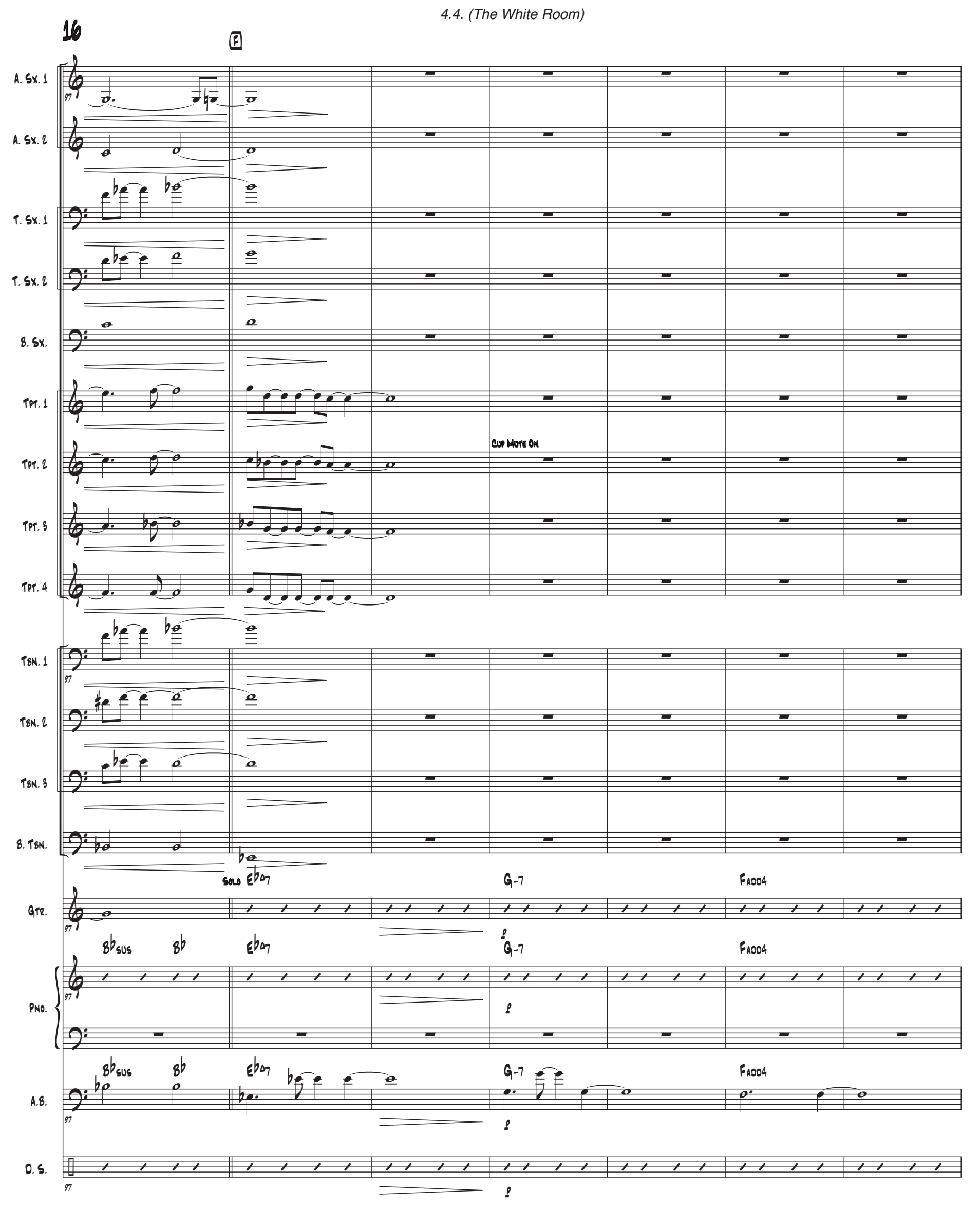



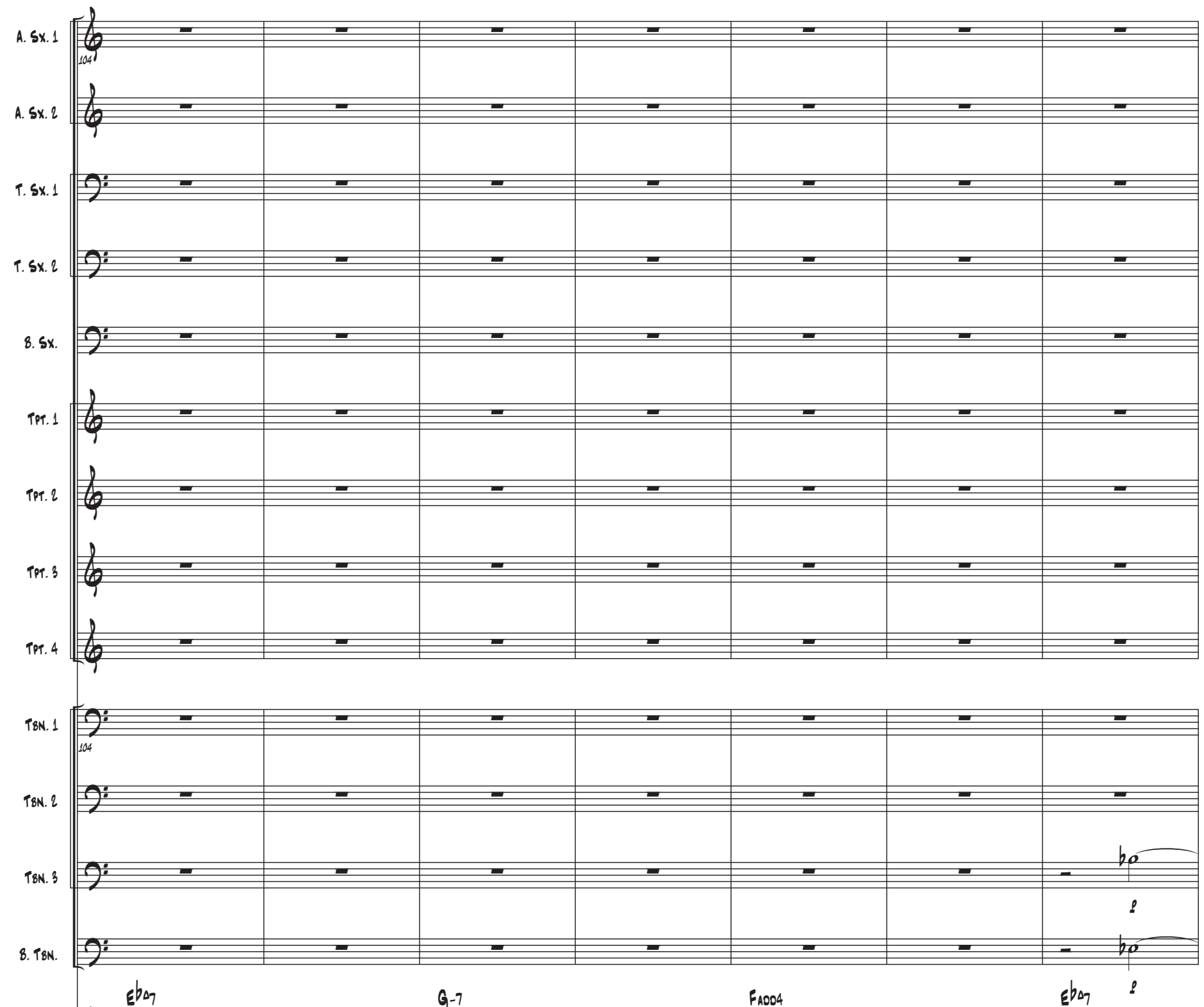

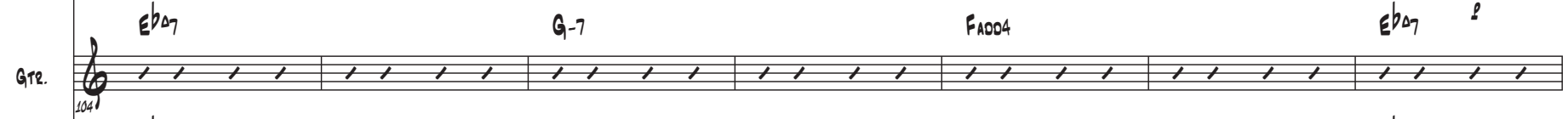

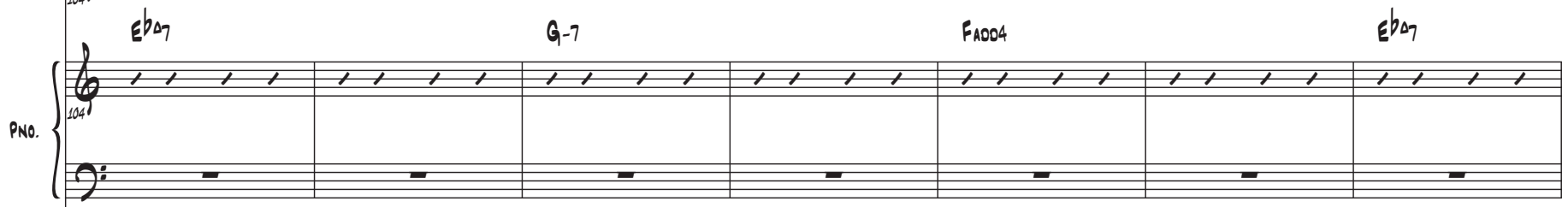

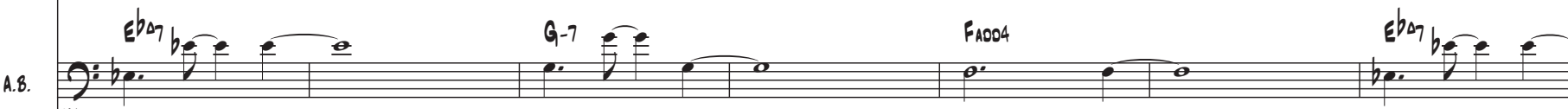

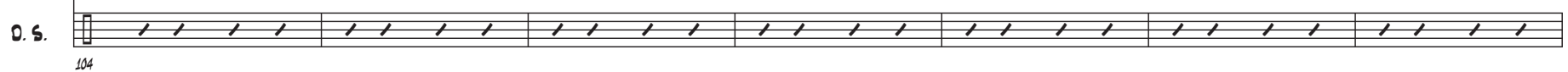




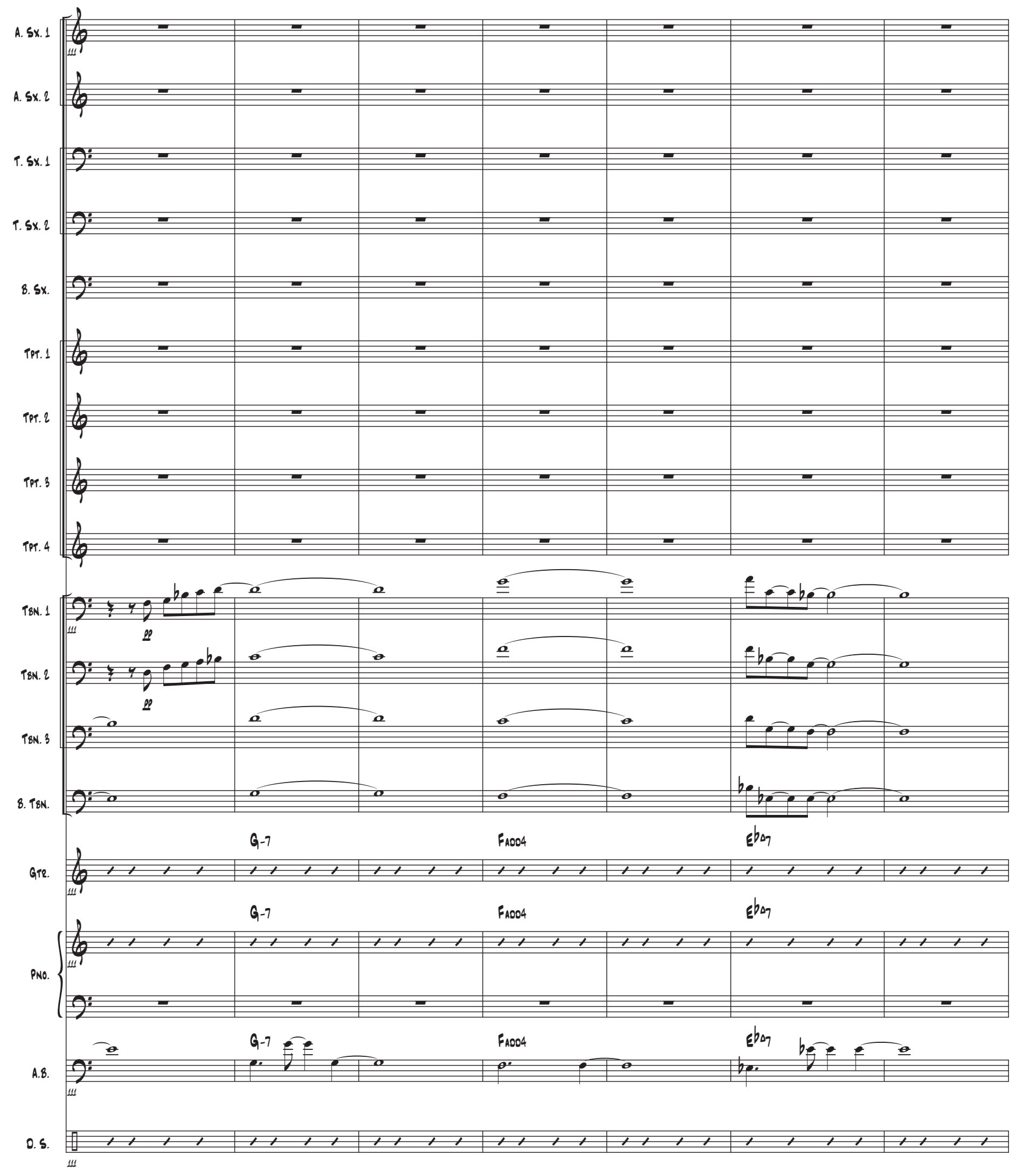



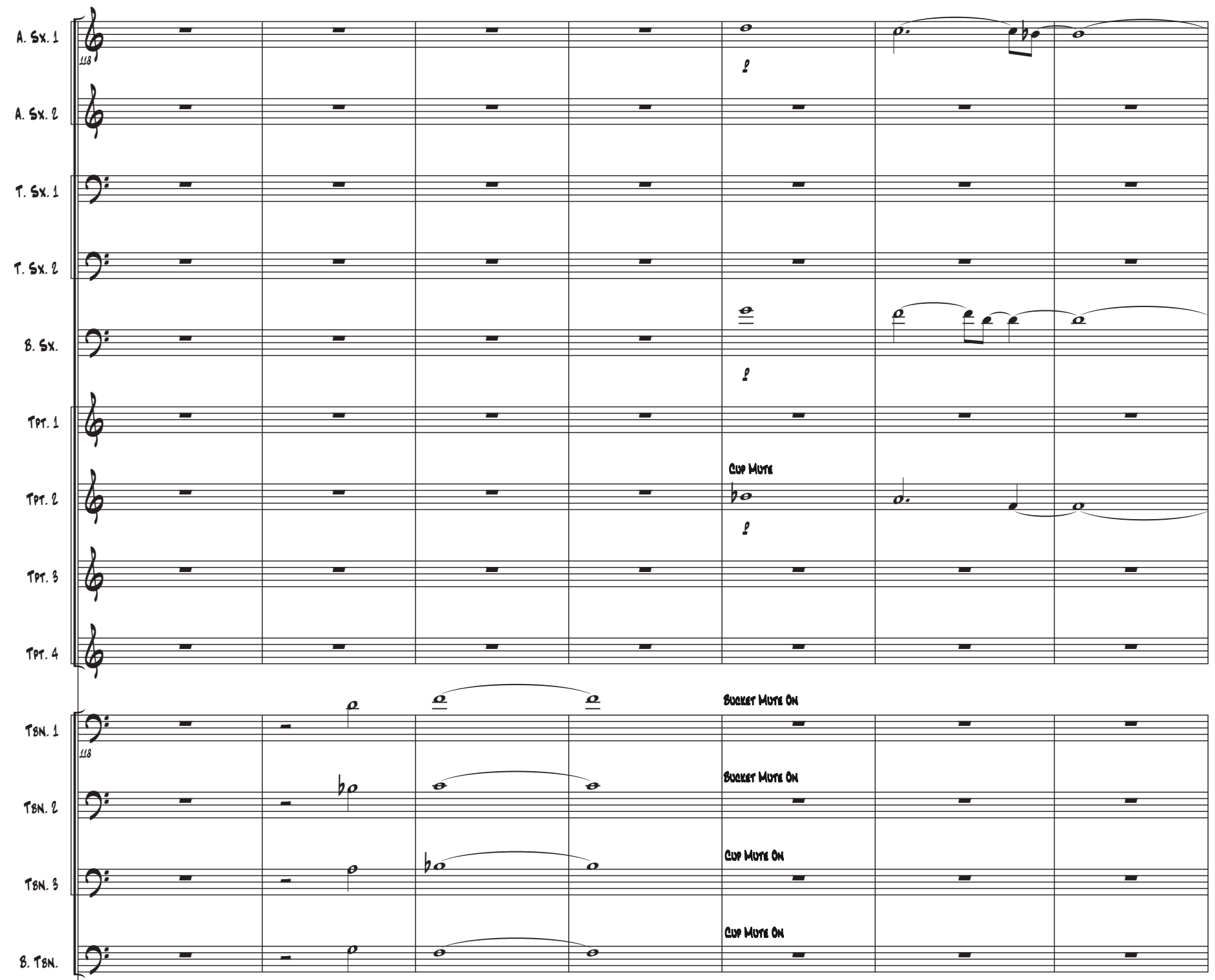

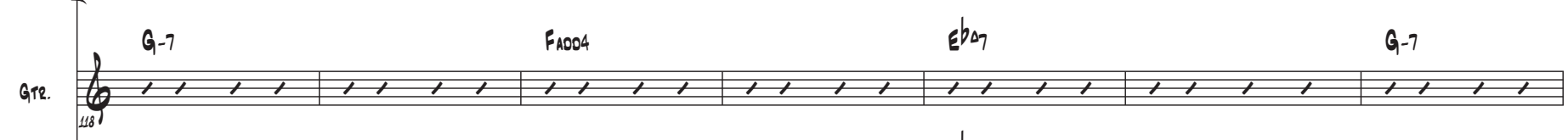

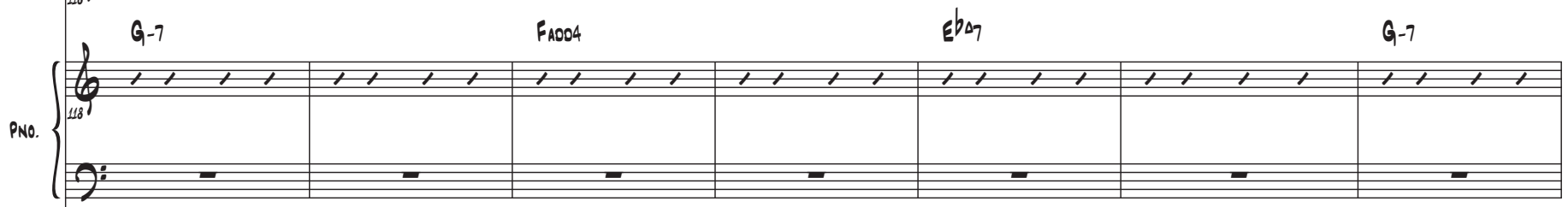

A.8.

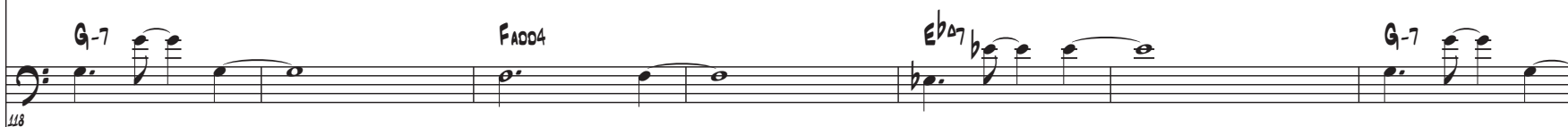

o. s. 

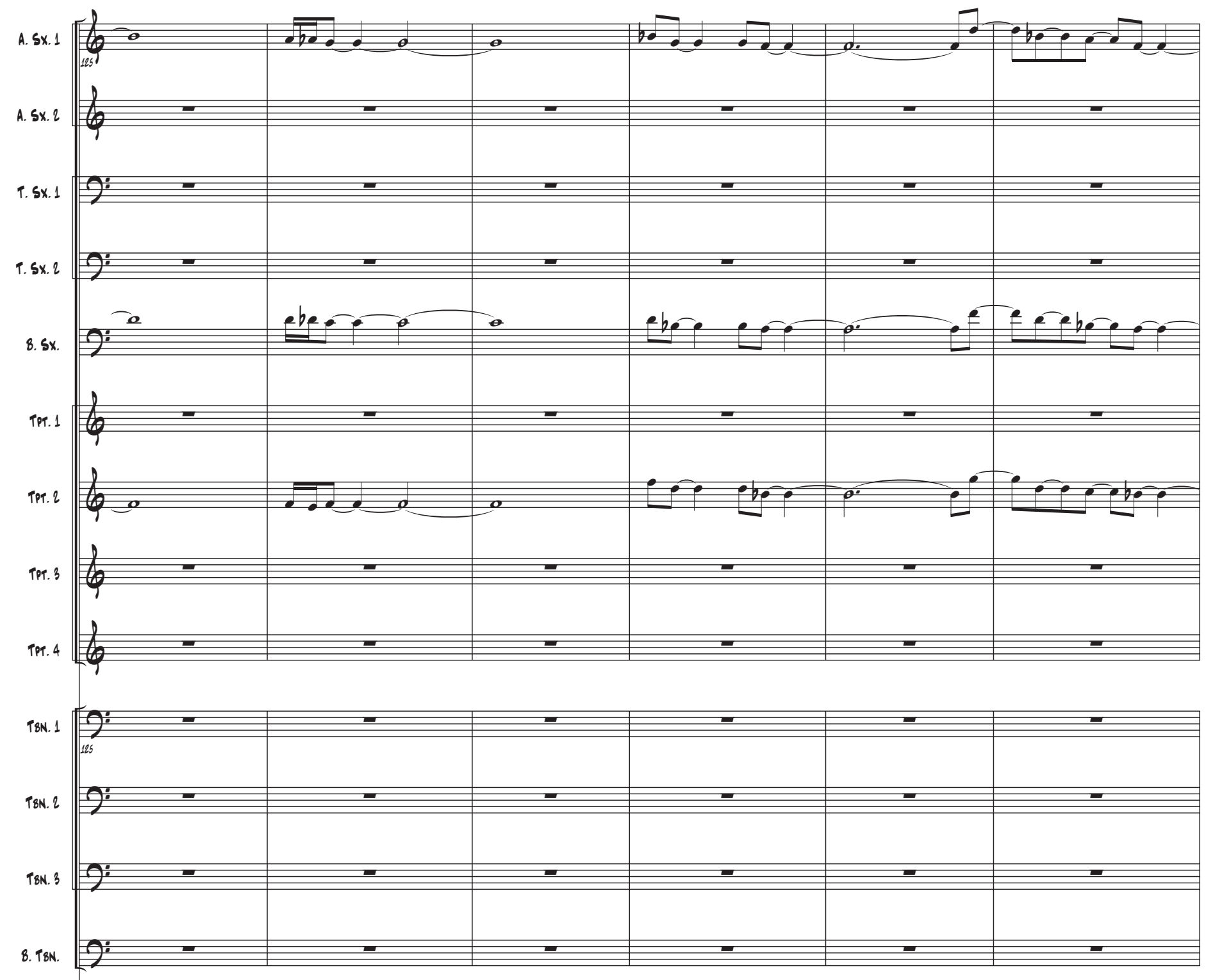

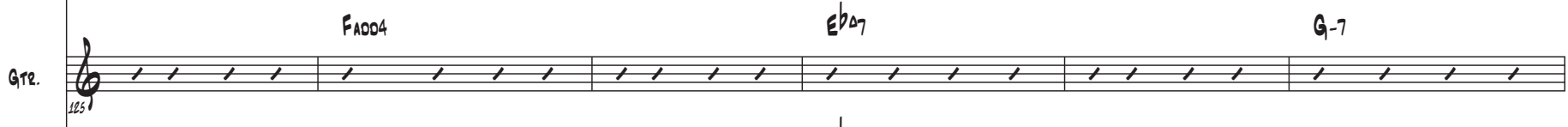
Fa004 $\quad \xi b_{\Delta 7} \quad G-7$

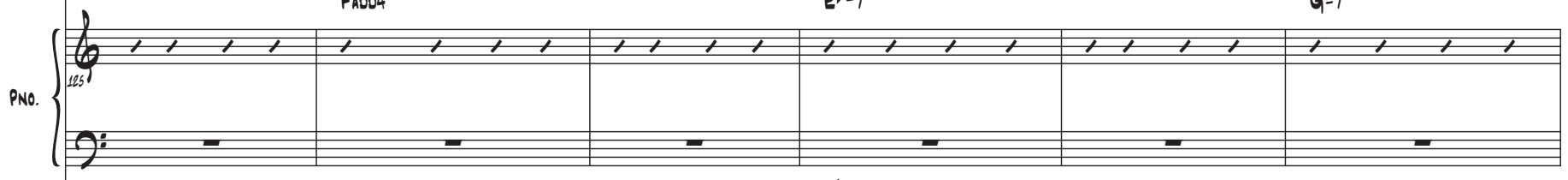

A.8. 20 125

o.s. 


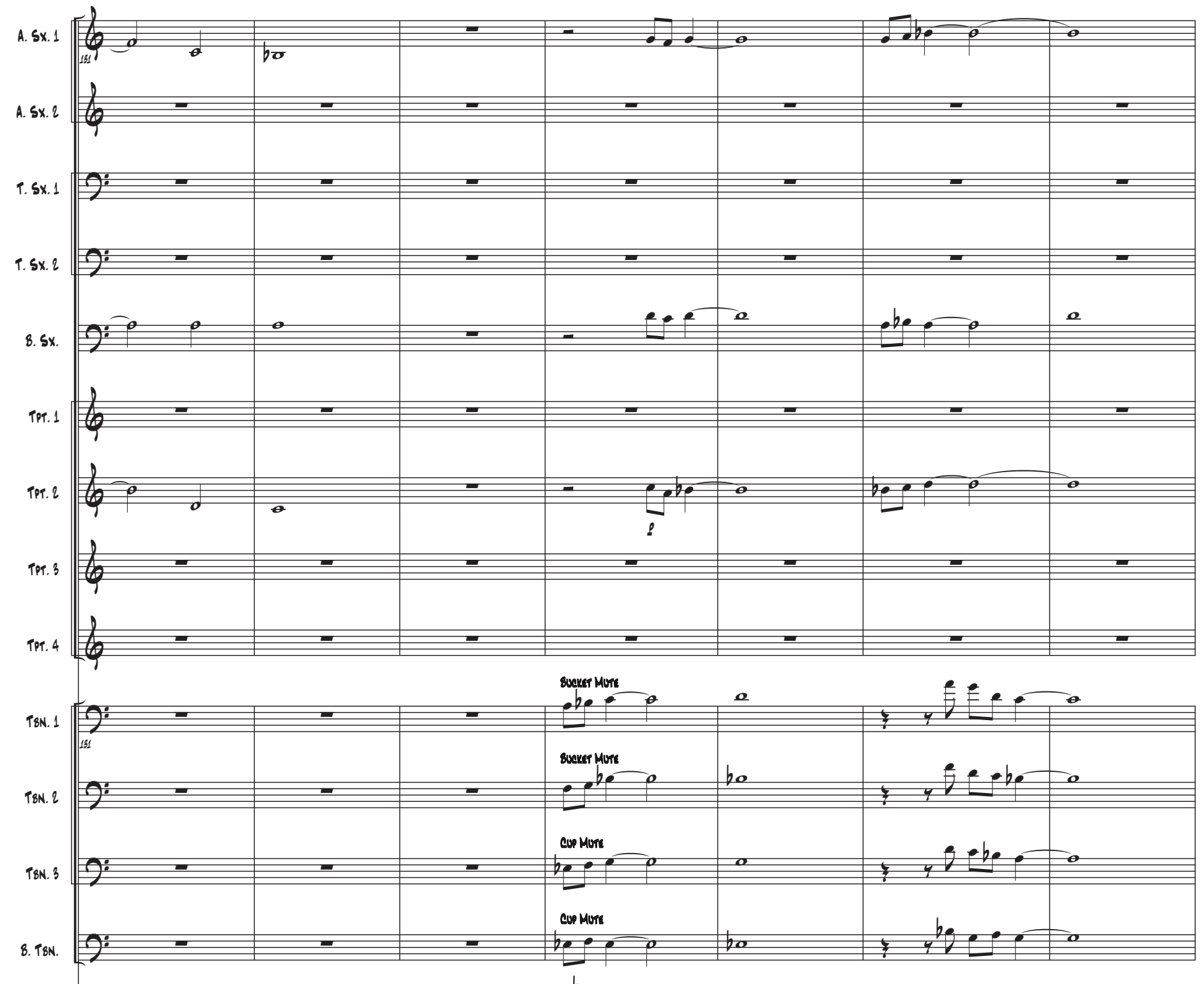

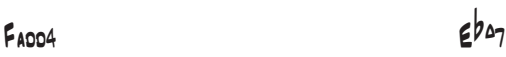

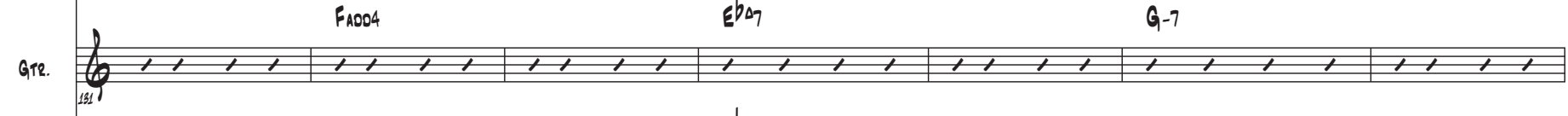

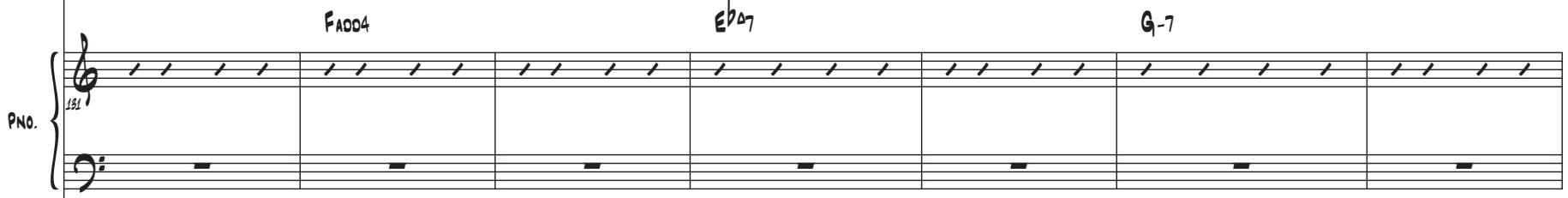

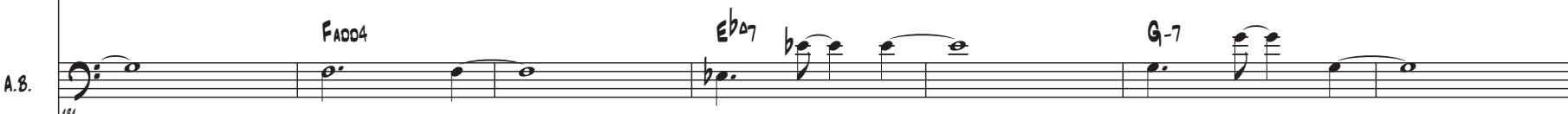

o. s. 

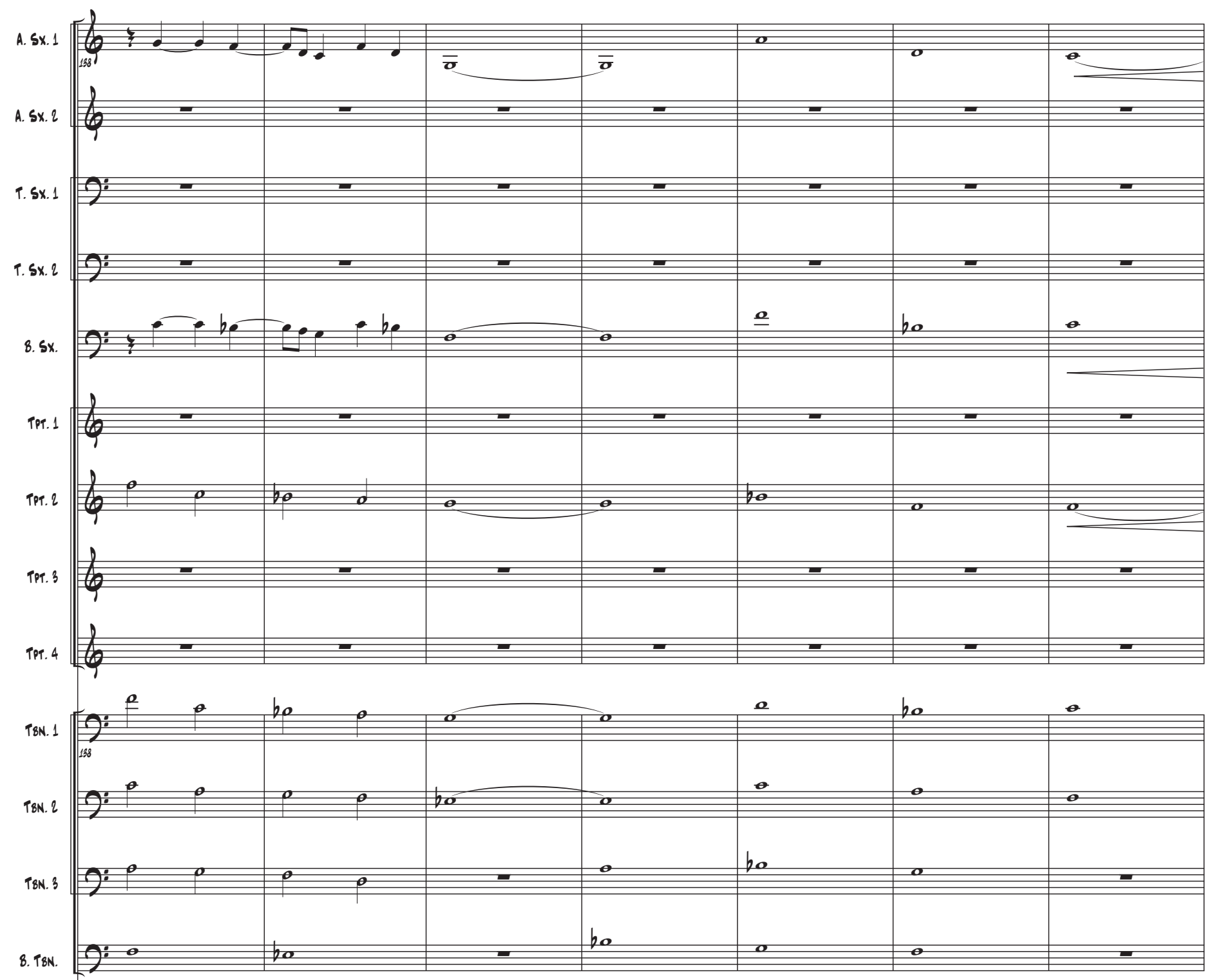

GTe. $\int_{1189}^{F_{A 004}}$

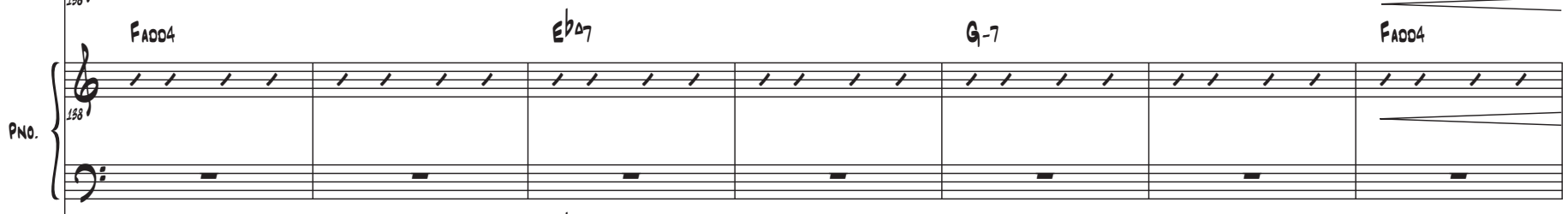

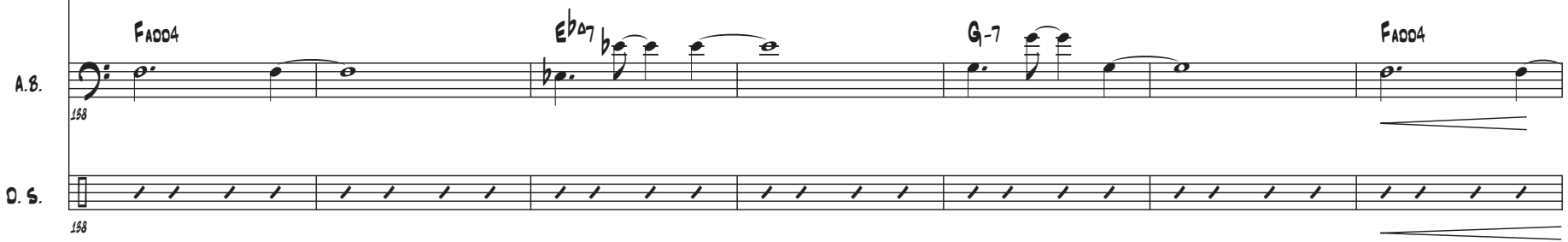


(6)
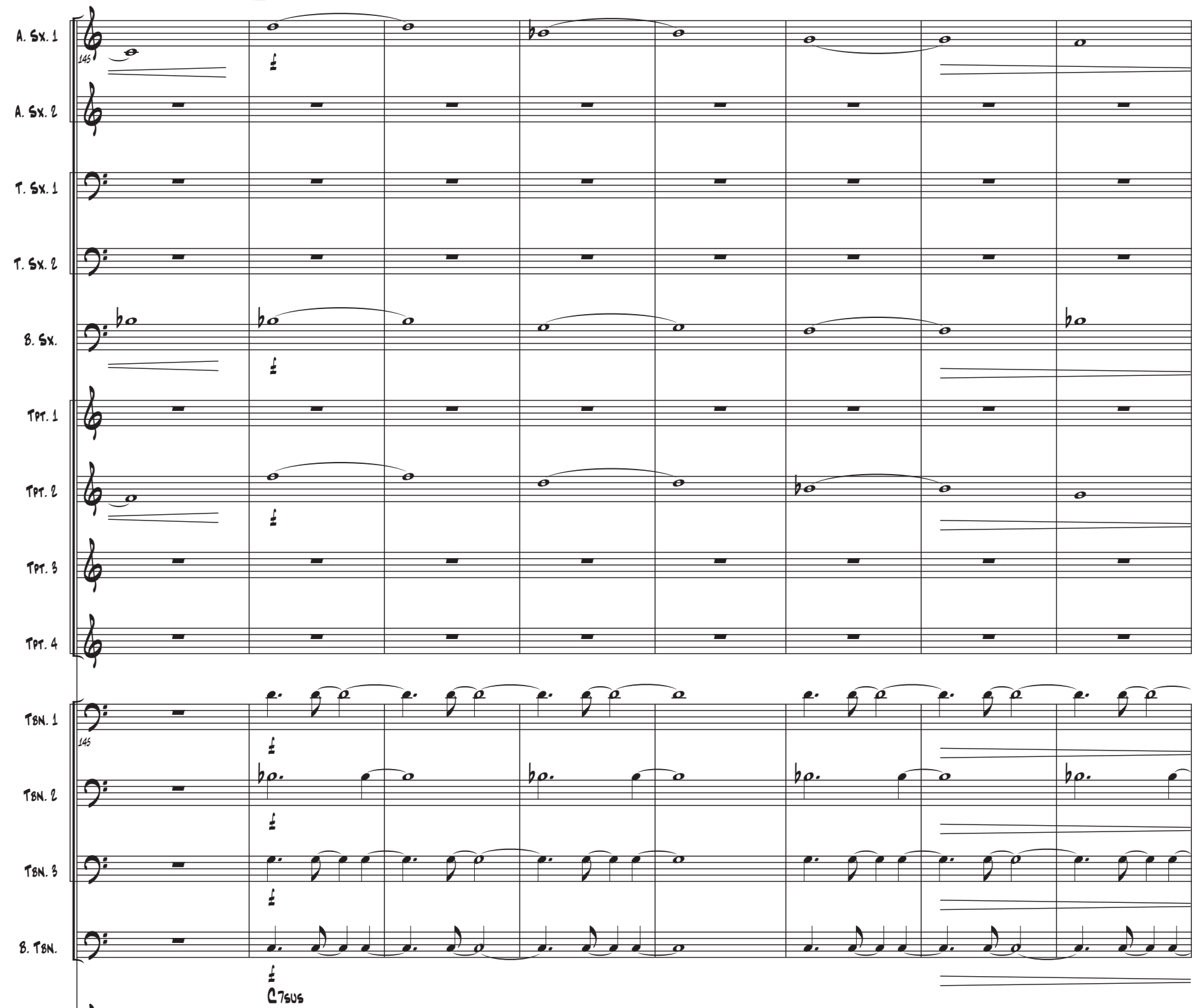

Gie. $\int_{\text {lus }}$ Cisus

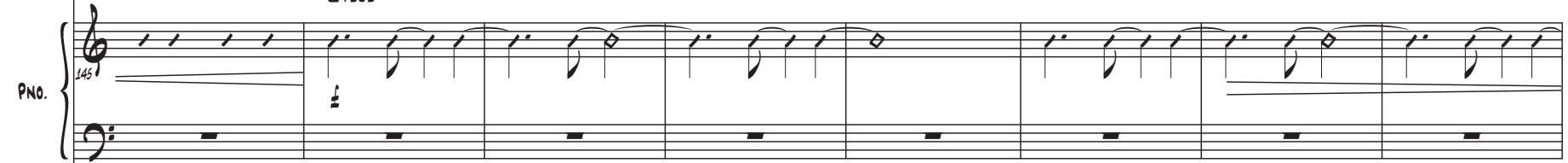

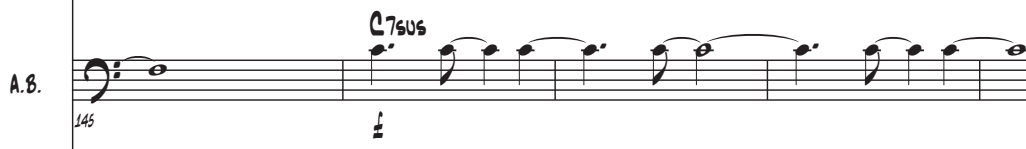

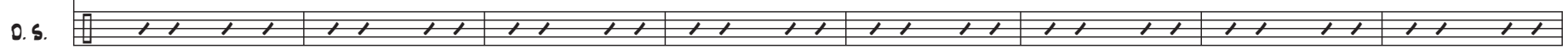
${ }_{145} \rightleftharpoons$ ! 

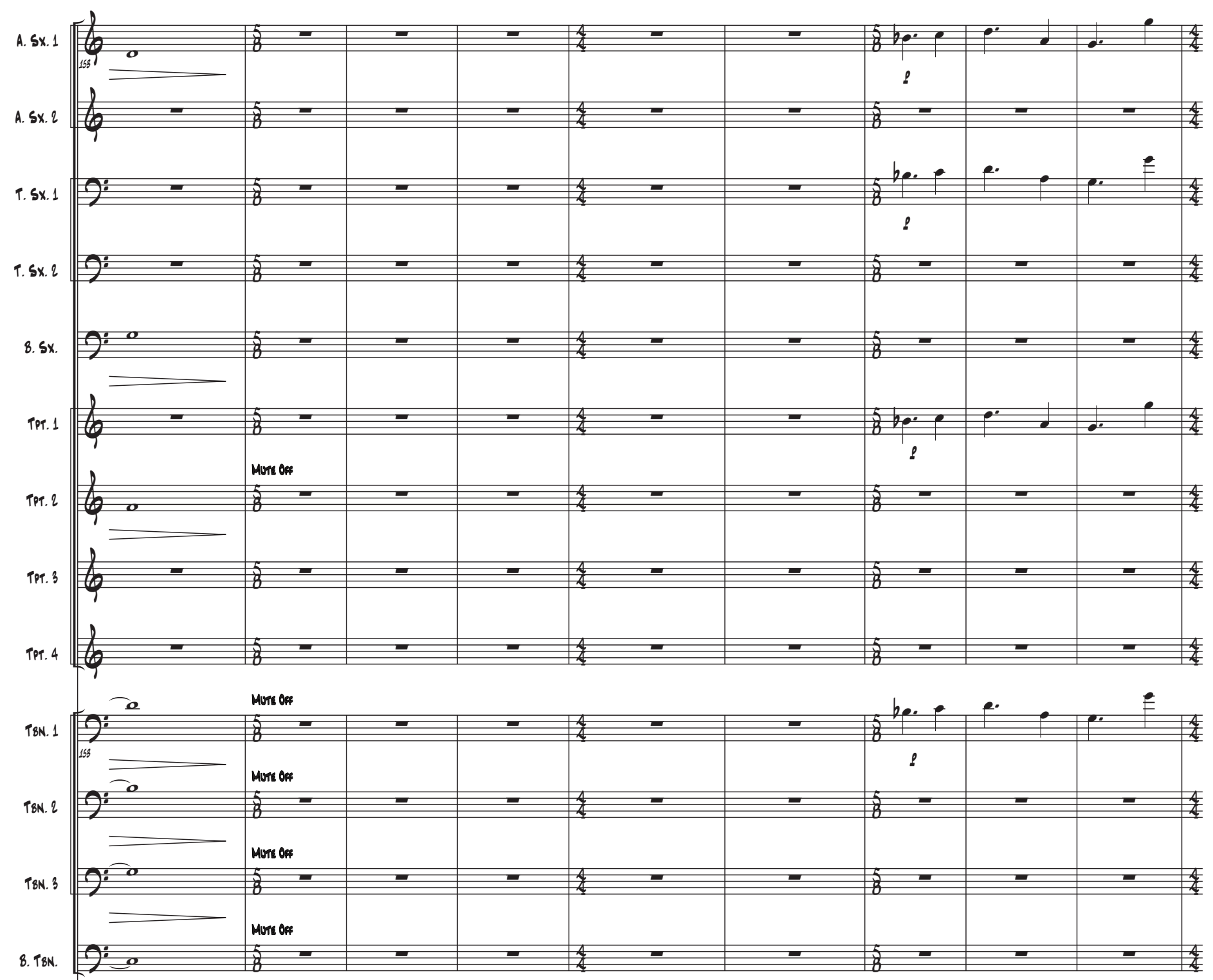

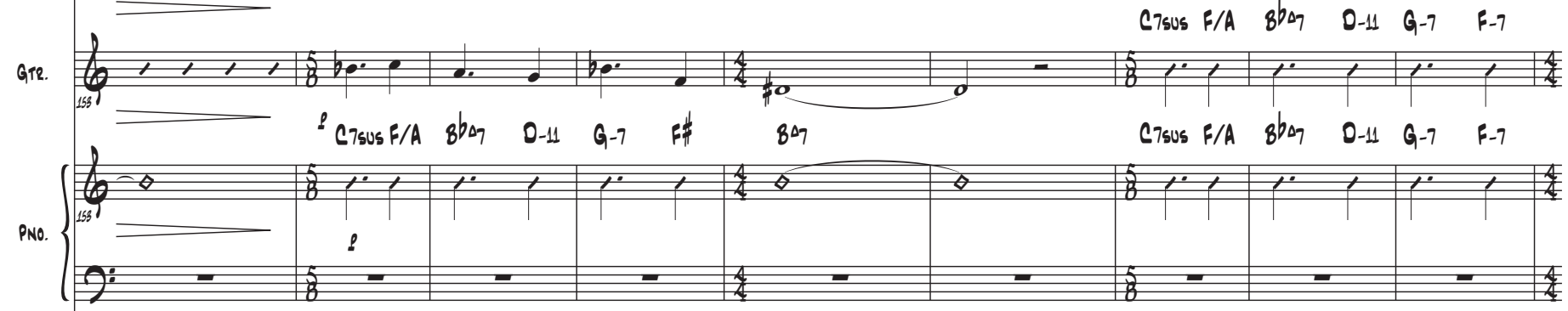

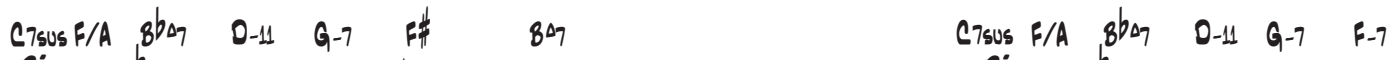

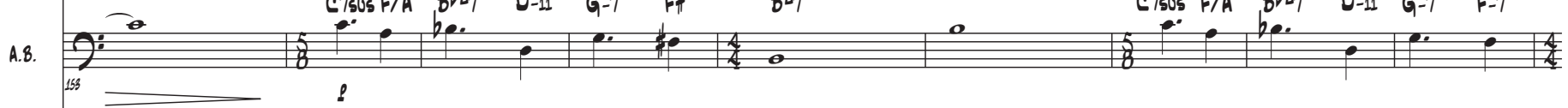

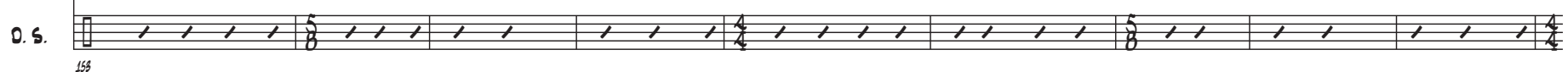



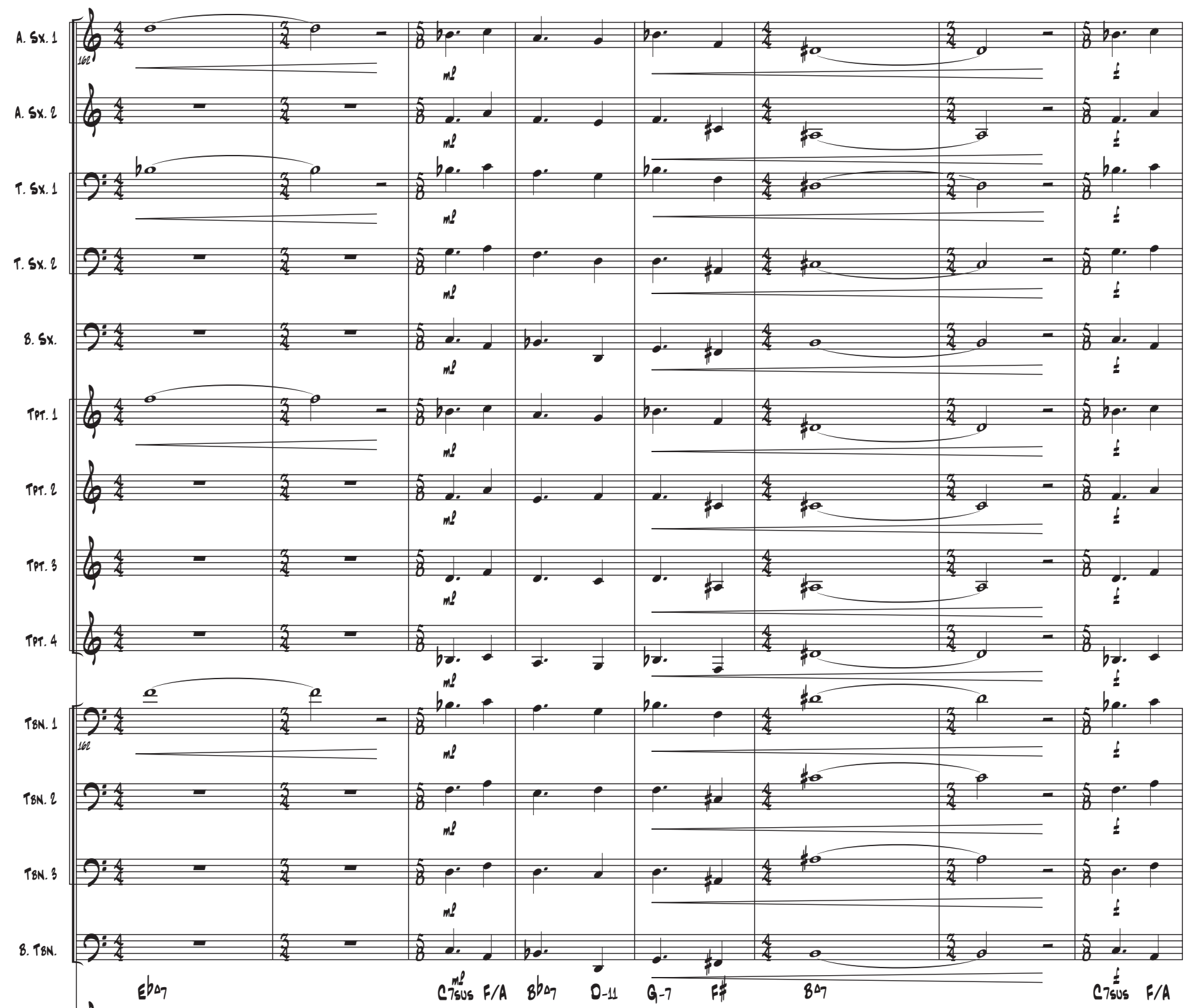

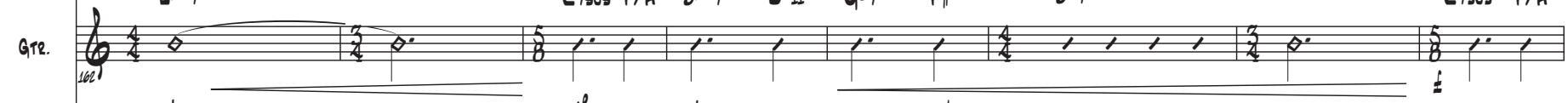

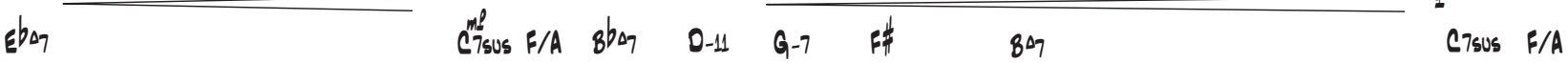

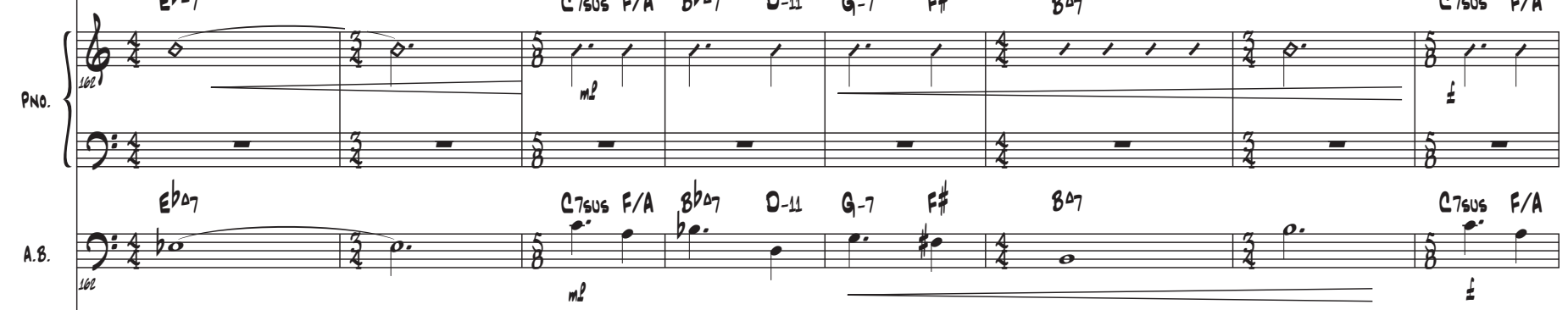

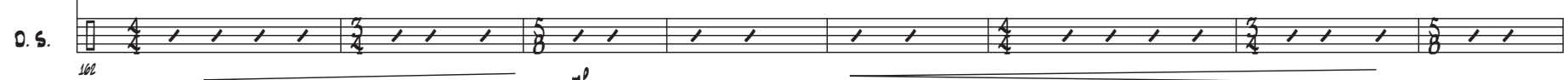



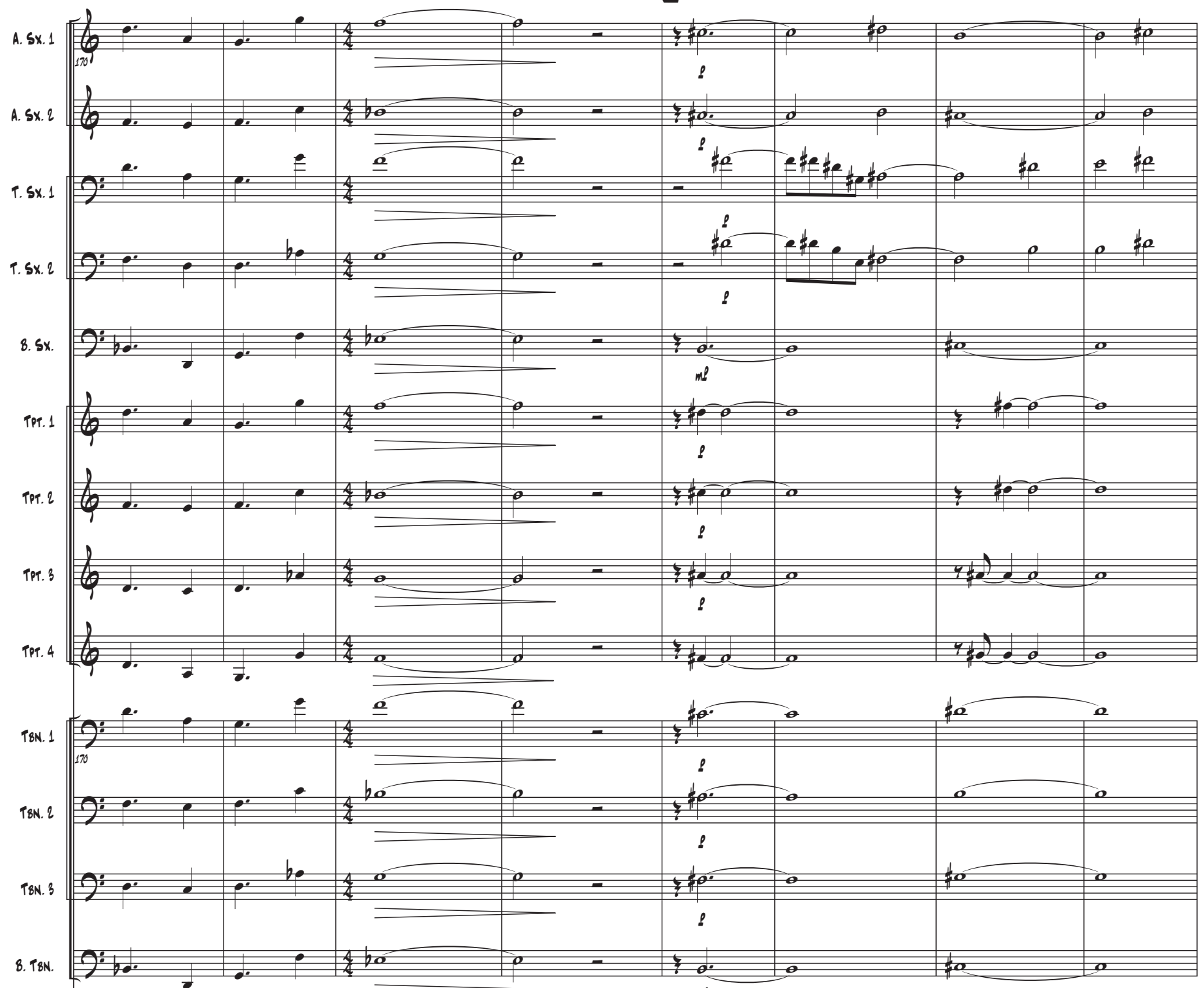

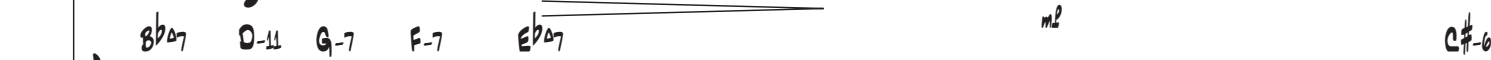

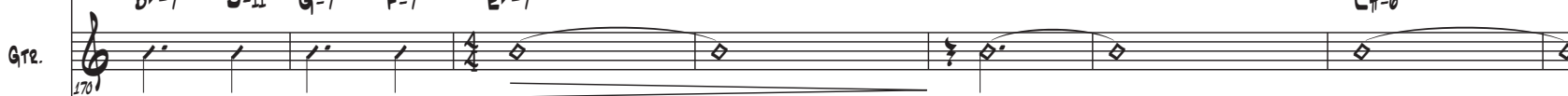

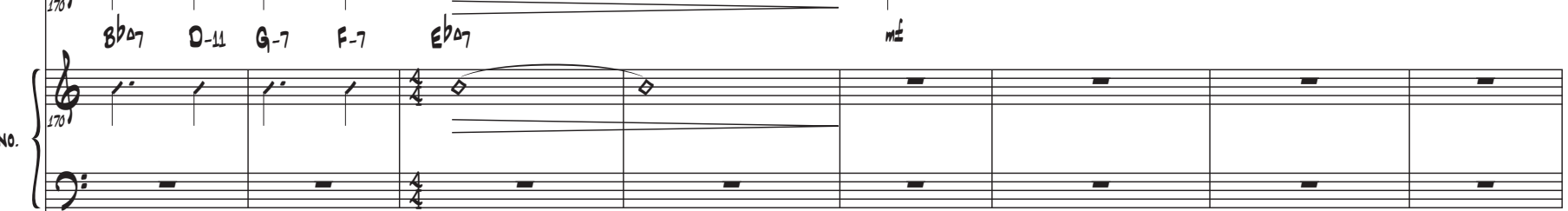

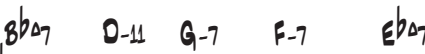

A.8.

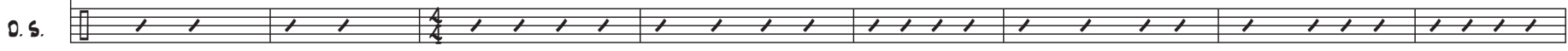
170 

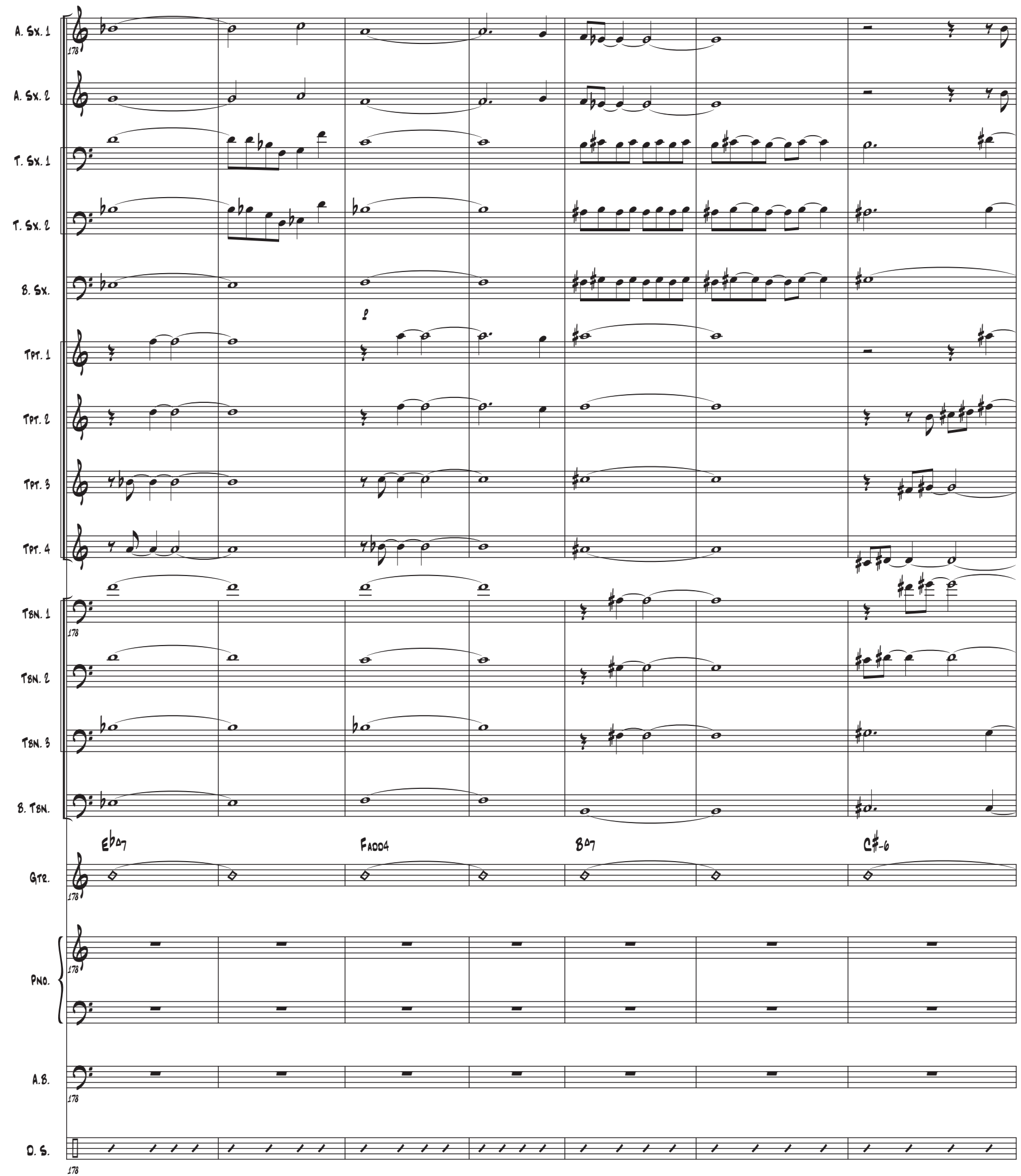
28

4.4. (The White Room)

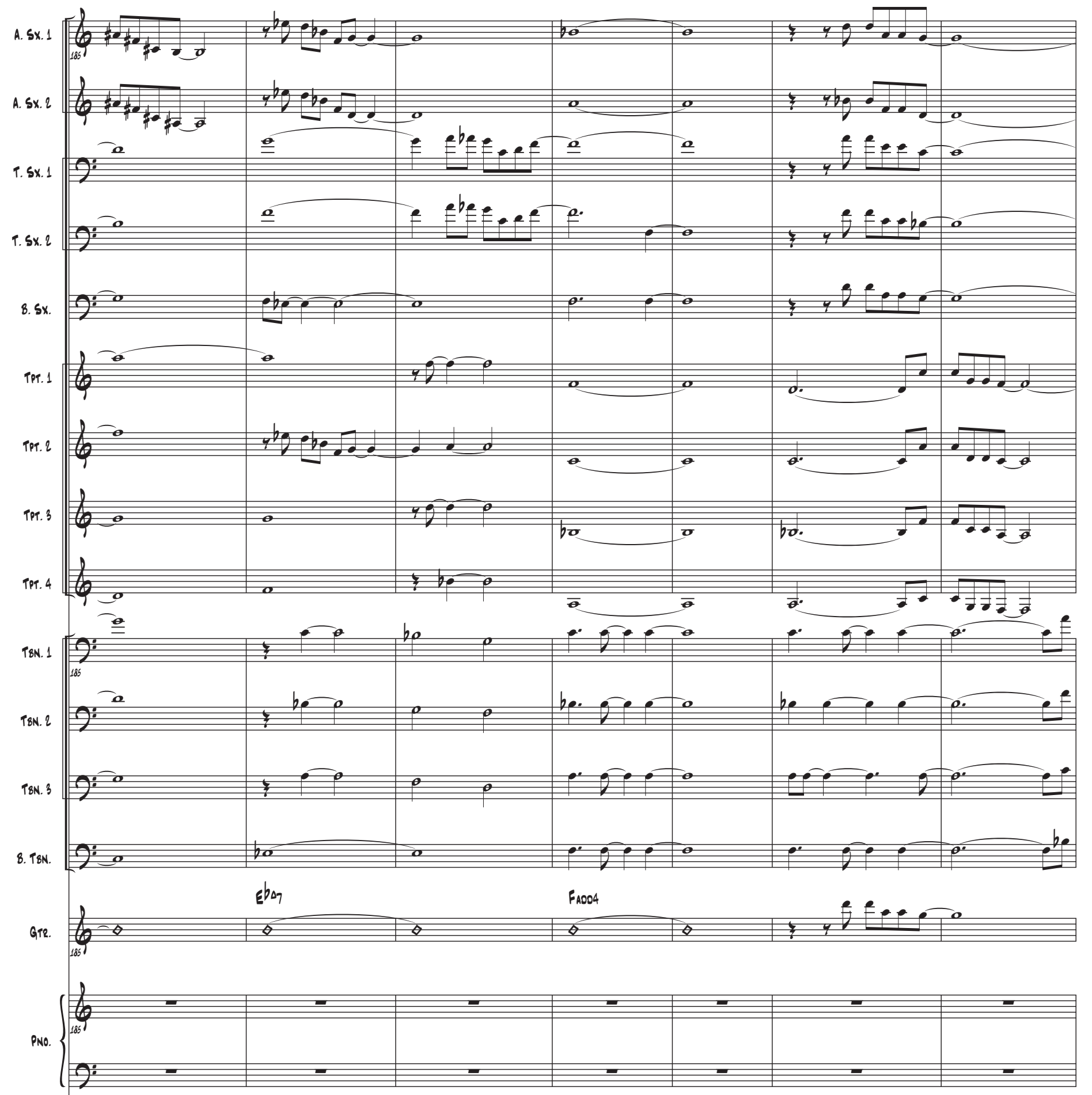

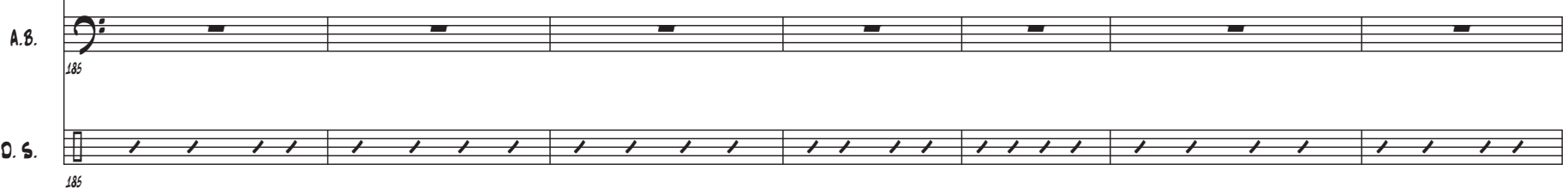



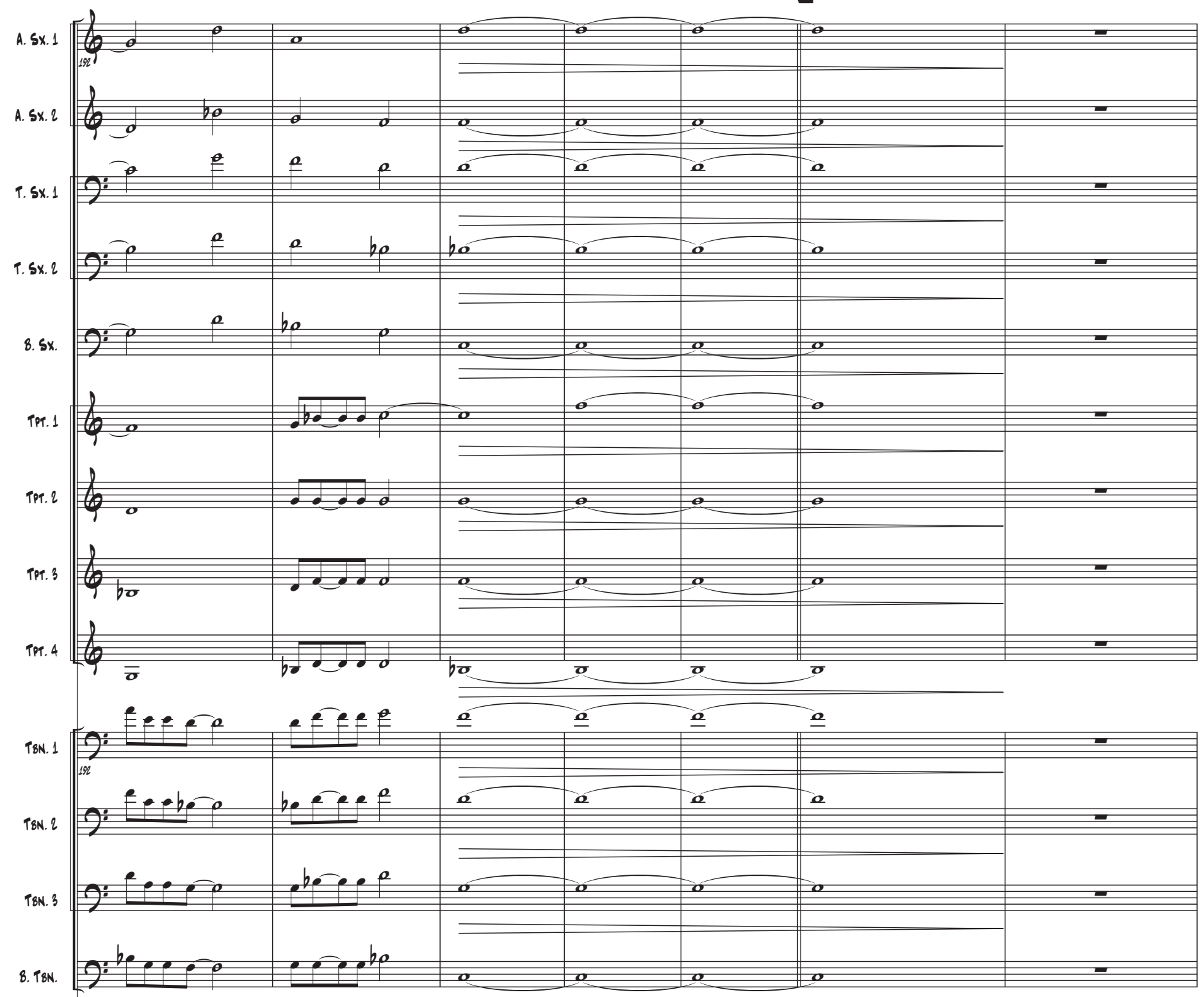

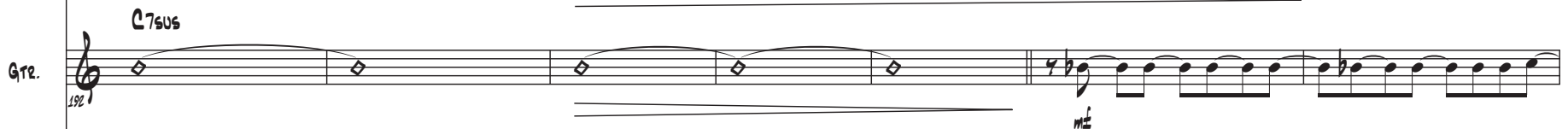
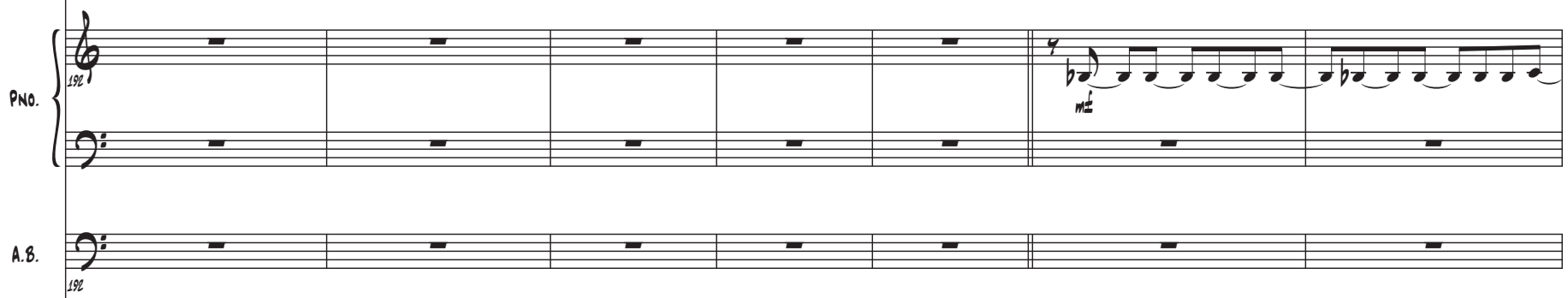

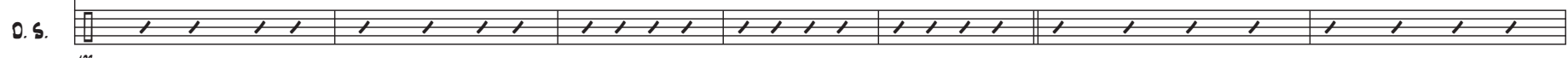




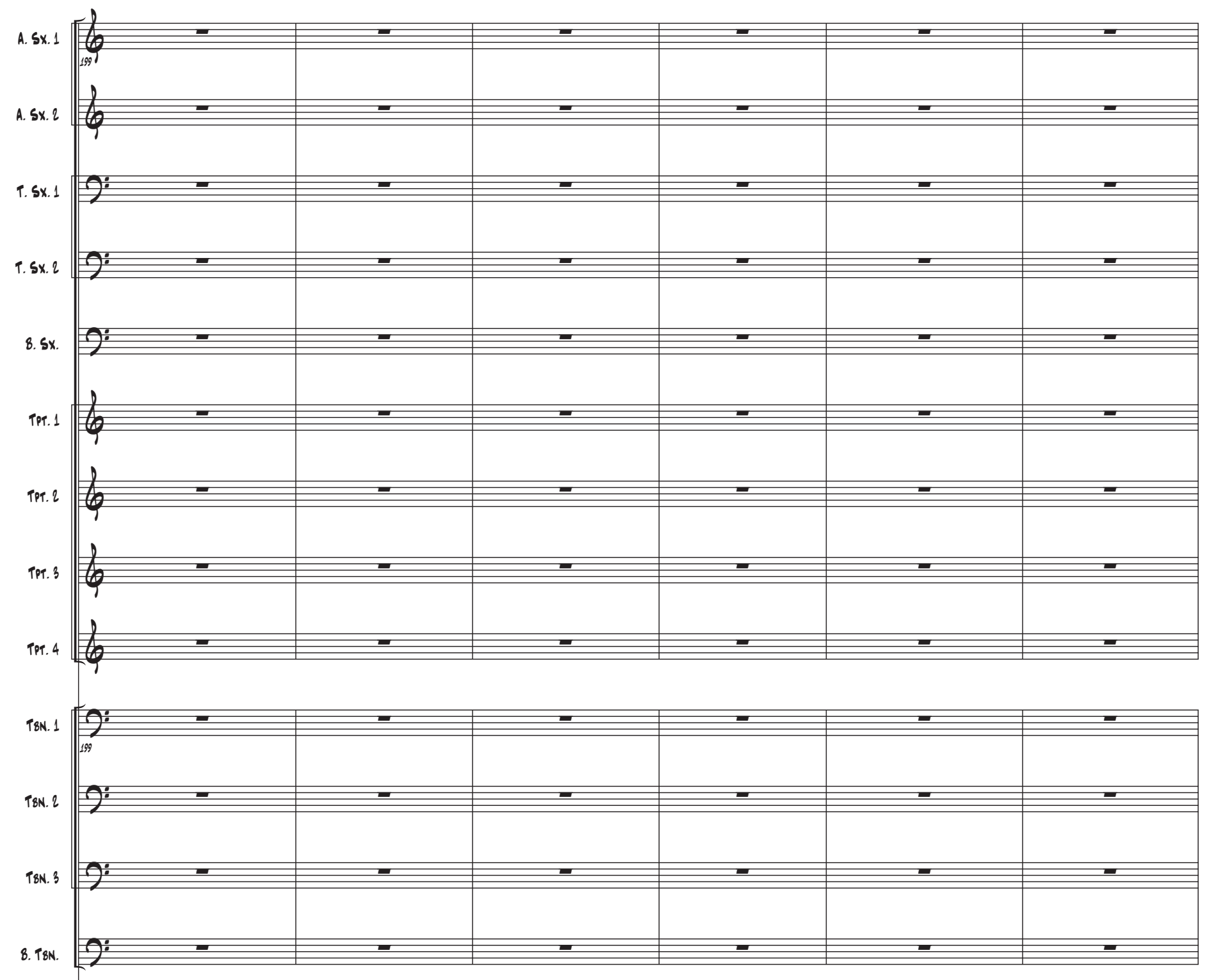

are. $b_{\text {sq }}$

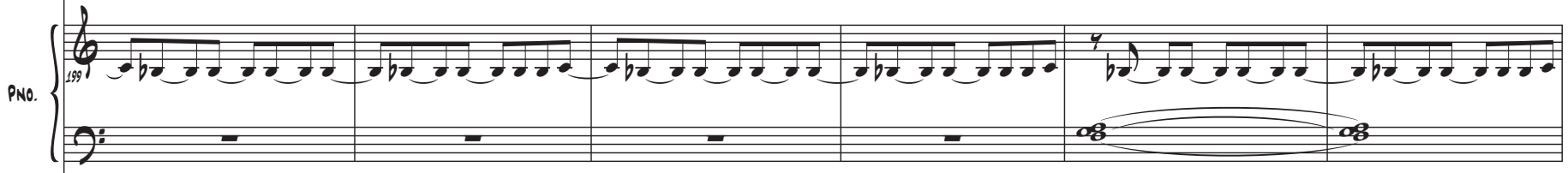

A.8.

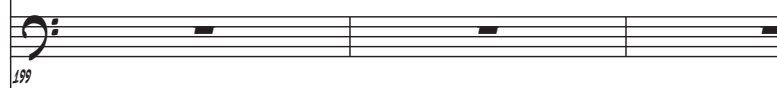

o. s. 


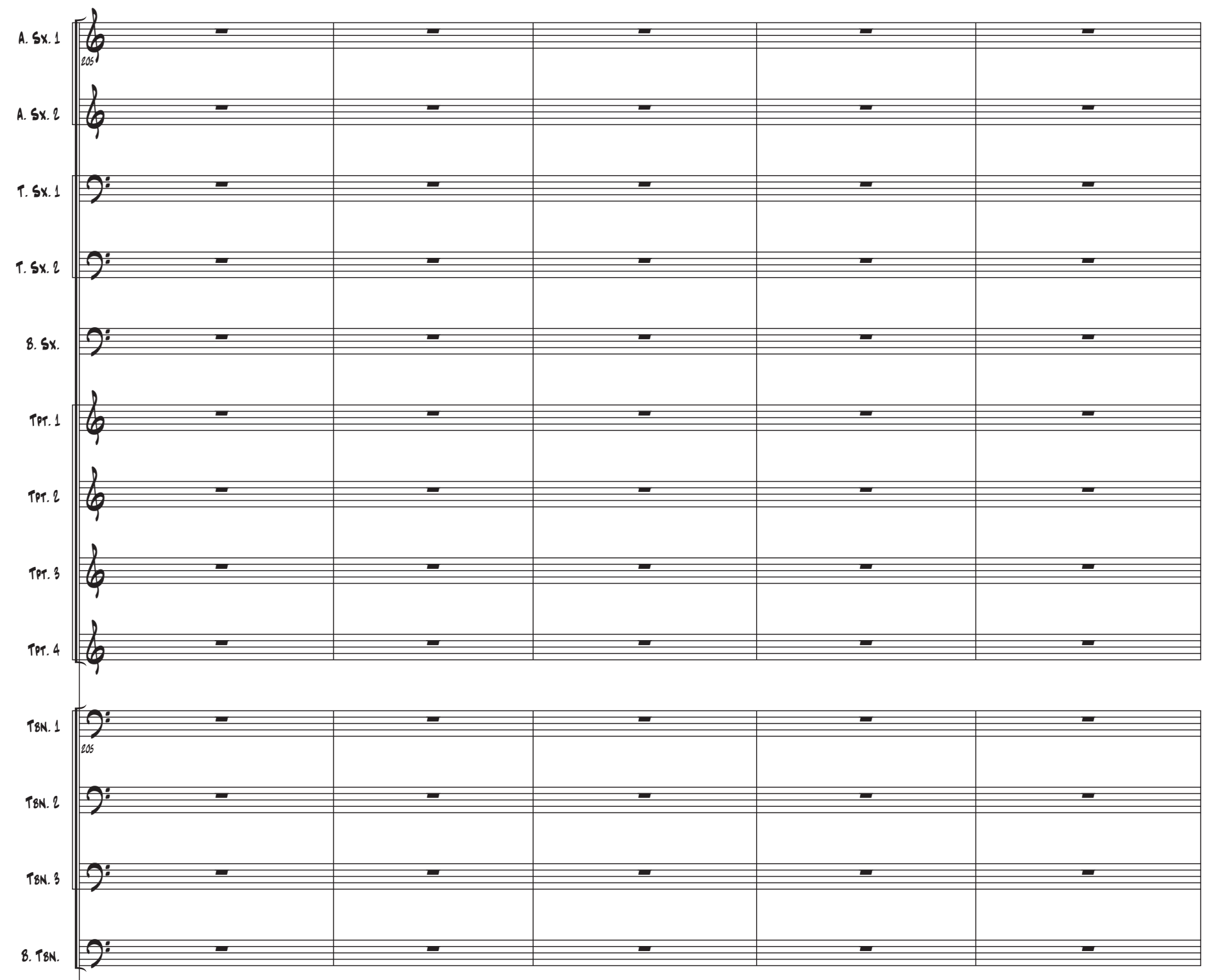

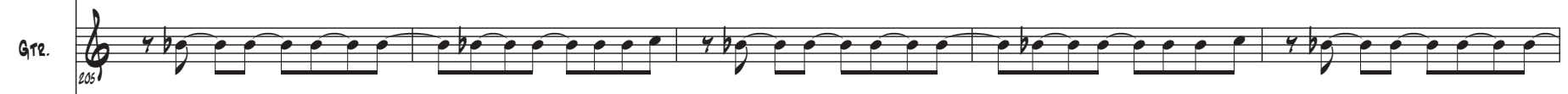

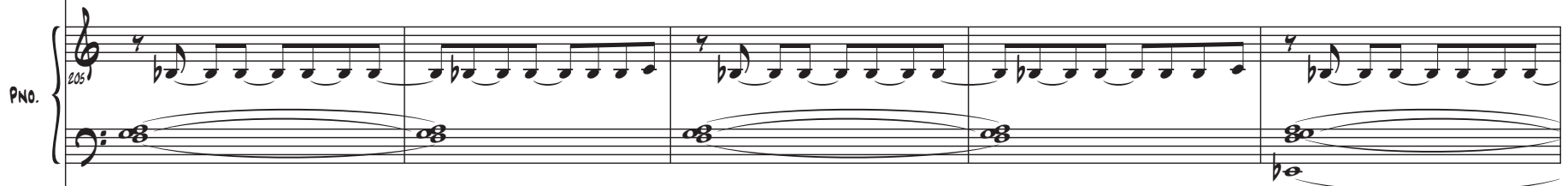

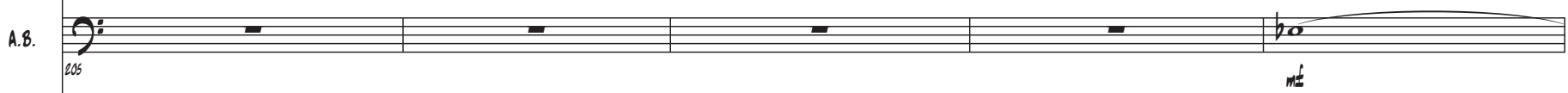

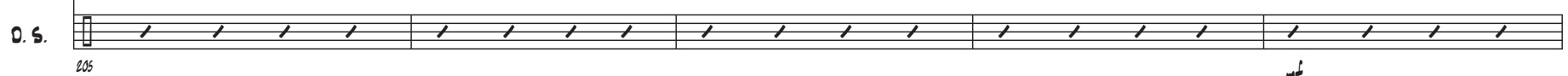



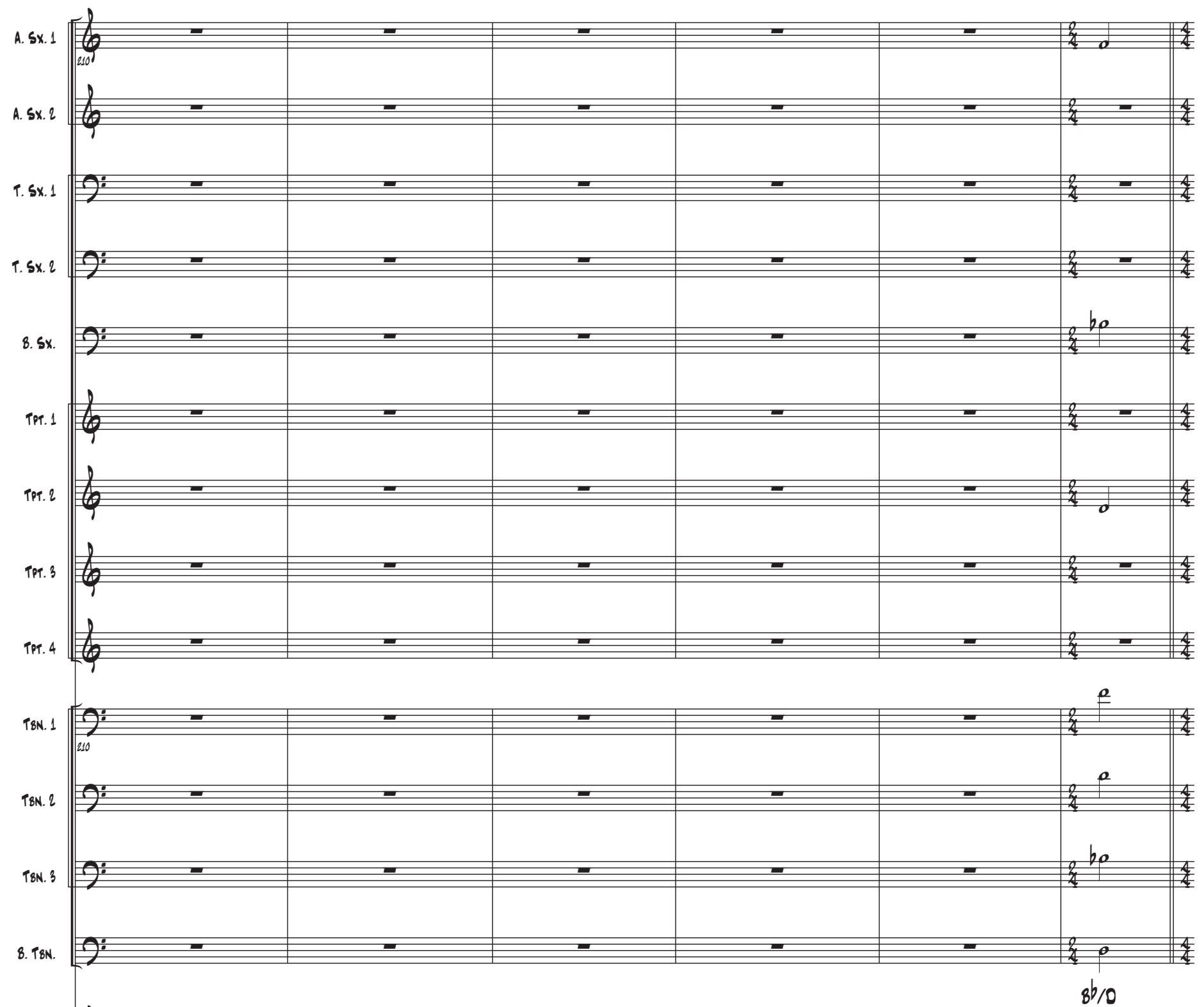

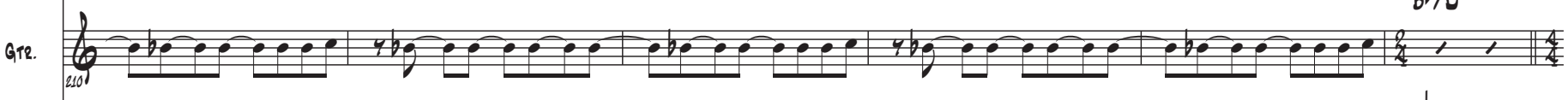
$8 b / 0$
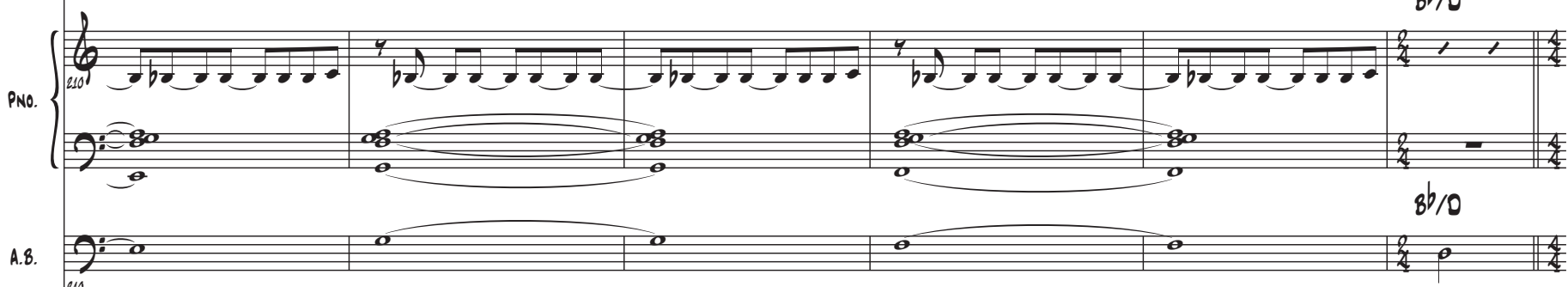

0.5. 

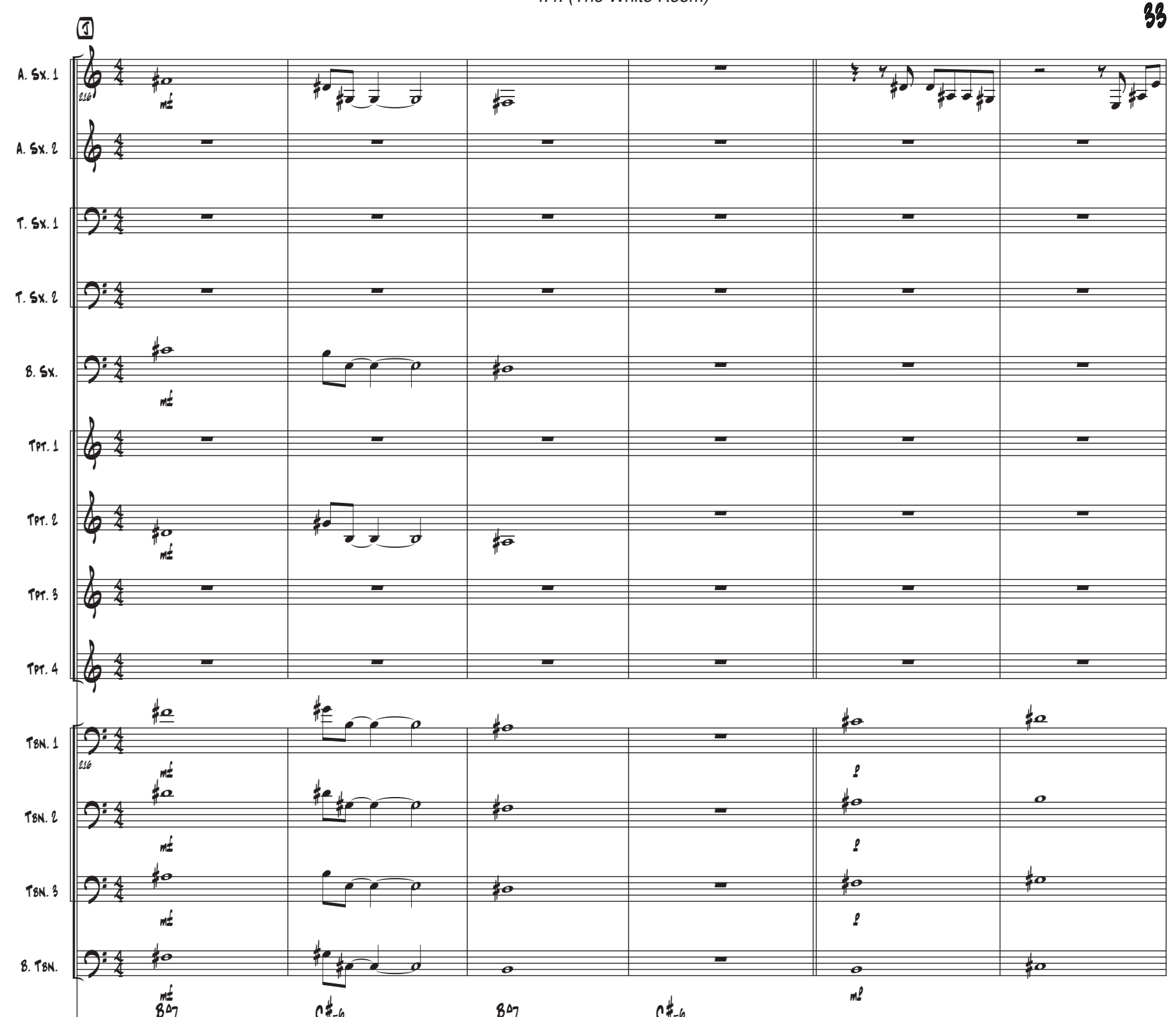

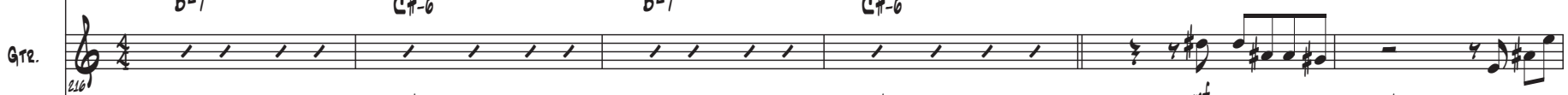

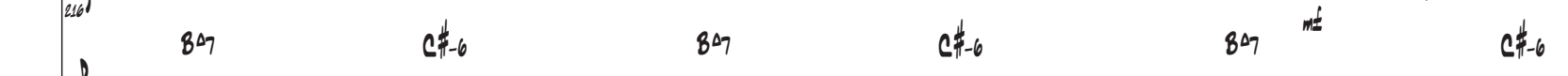

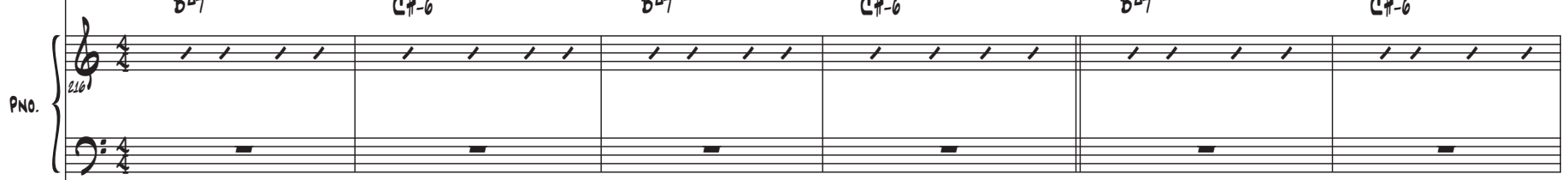
A.8. $2: 400 \%$

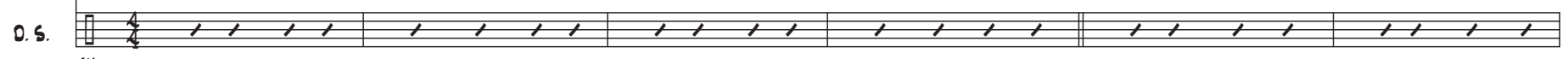




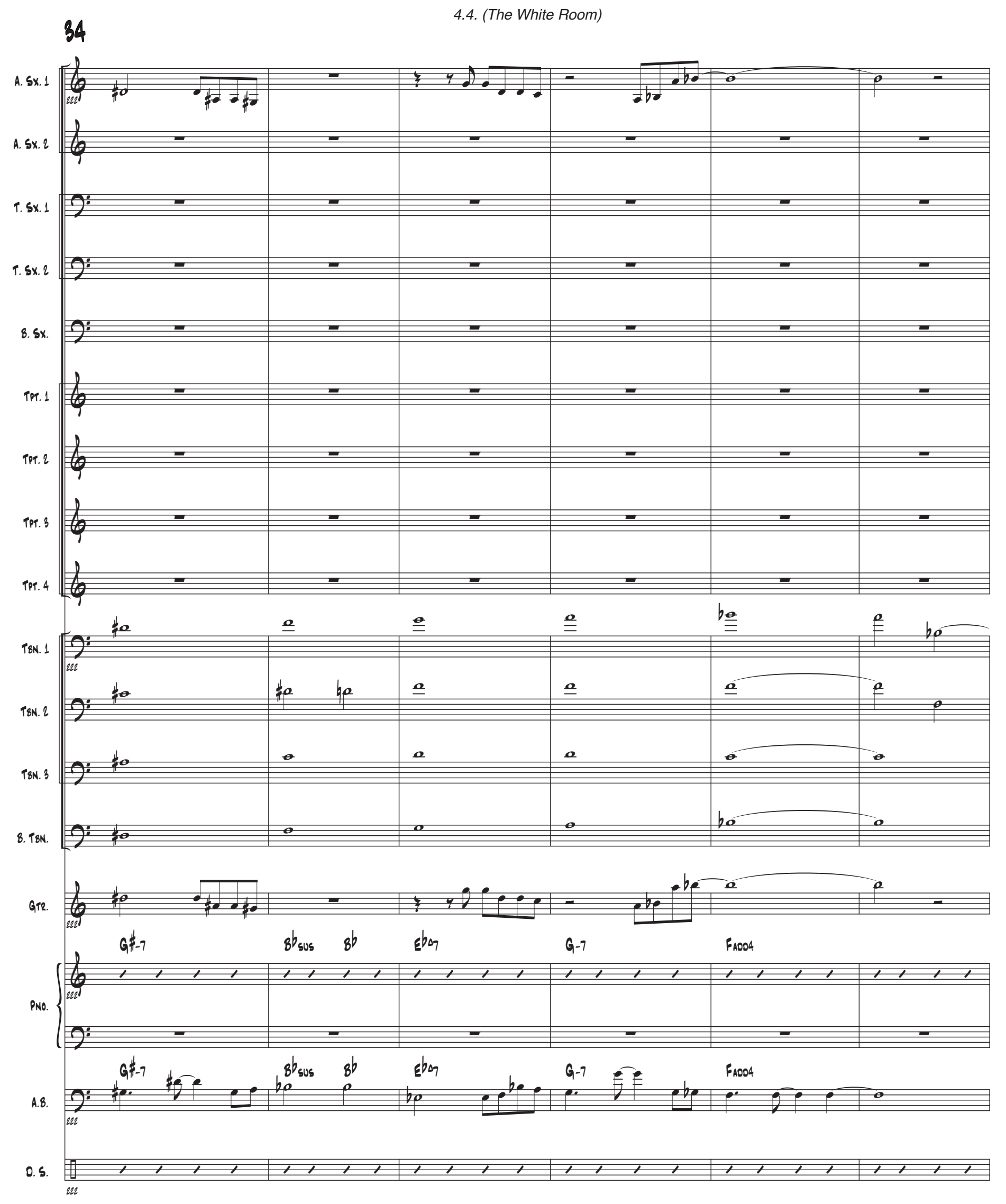


4.4. (The White Room)

35
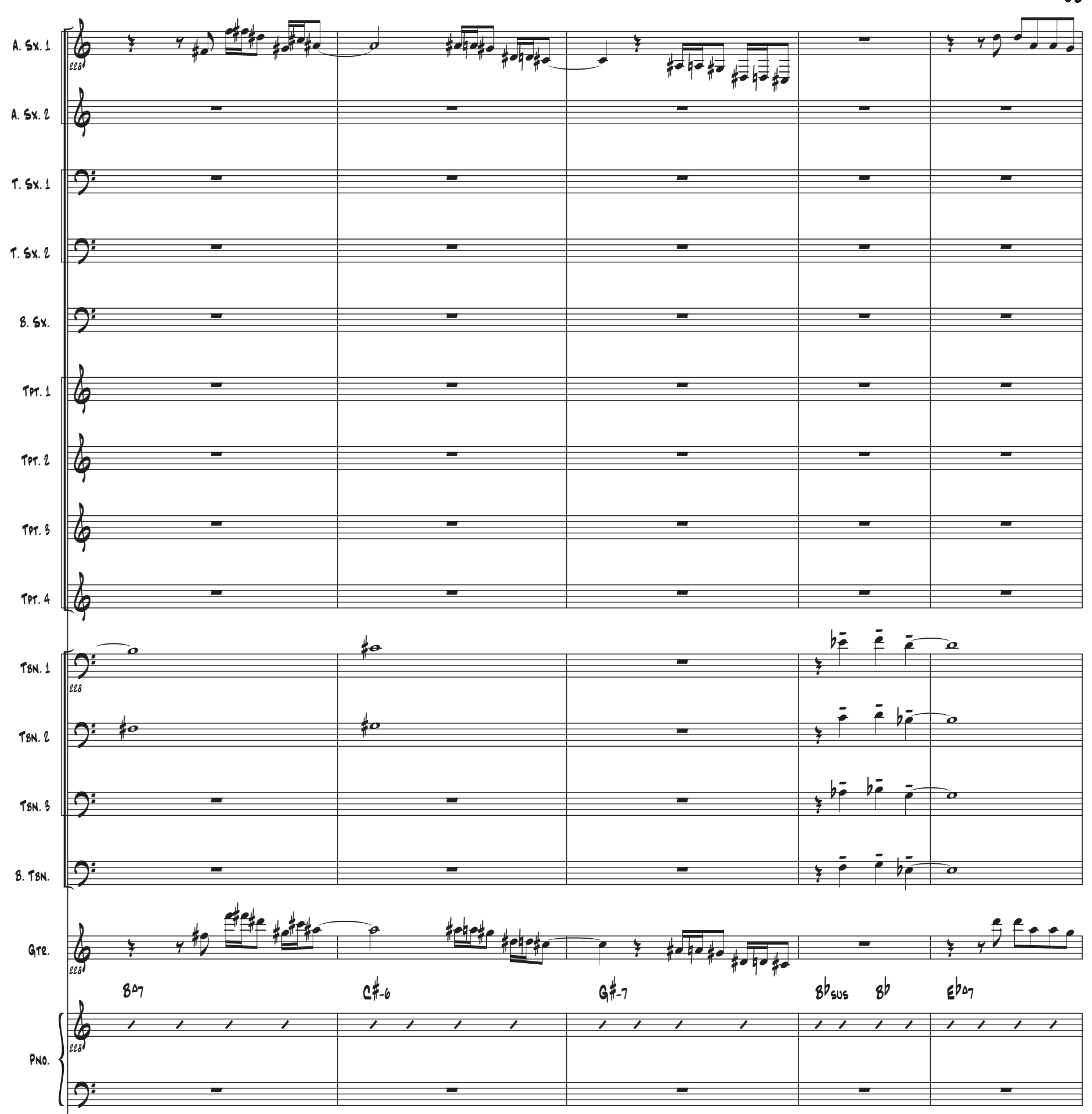

A.8.

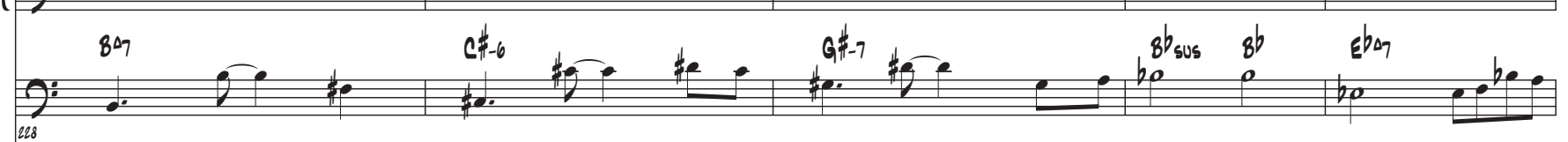

o.s.

228 
(B)
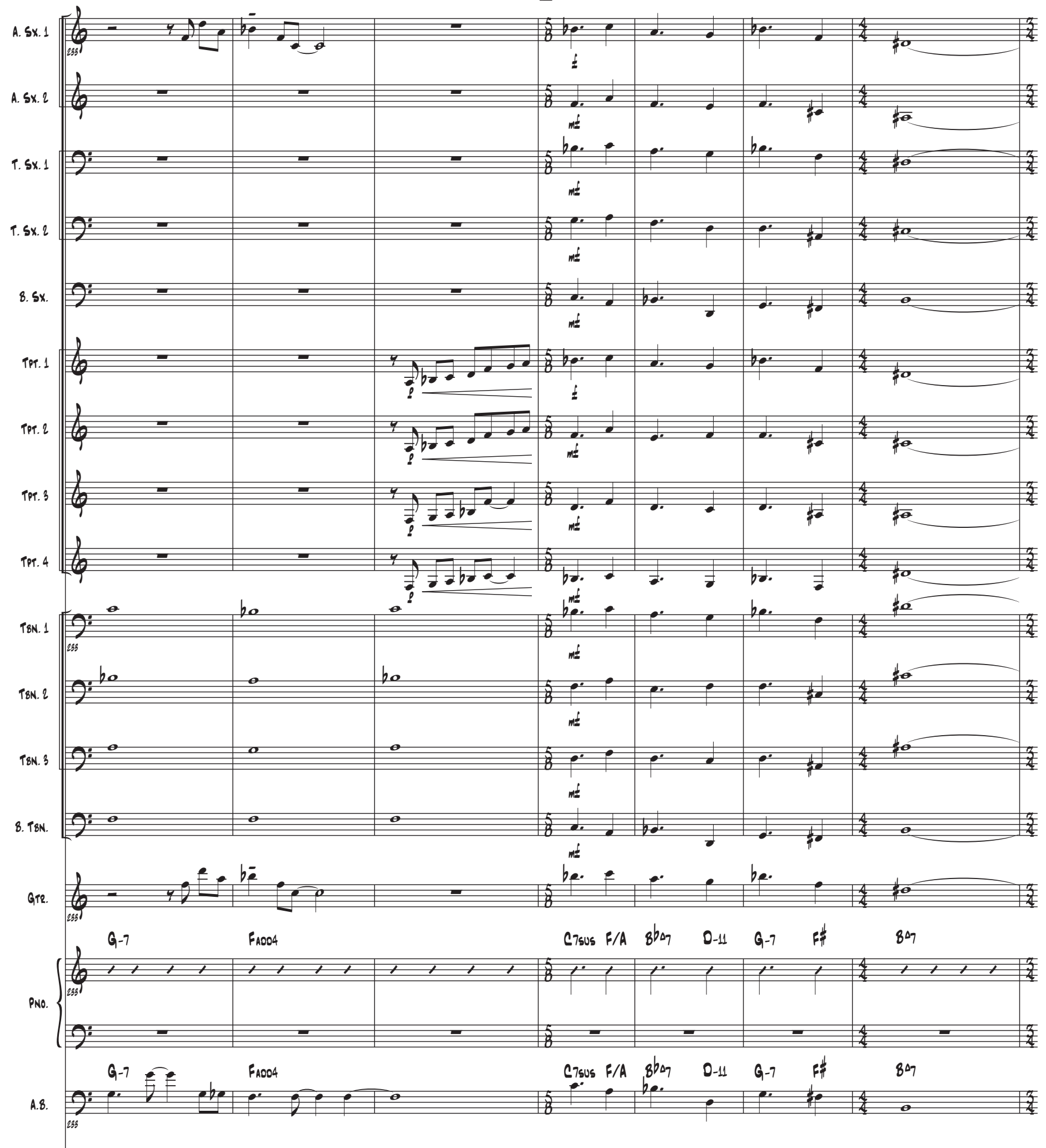

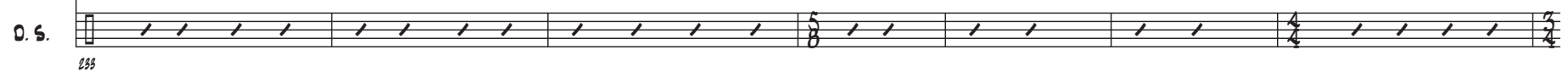



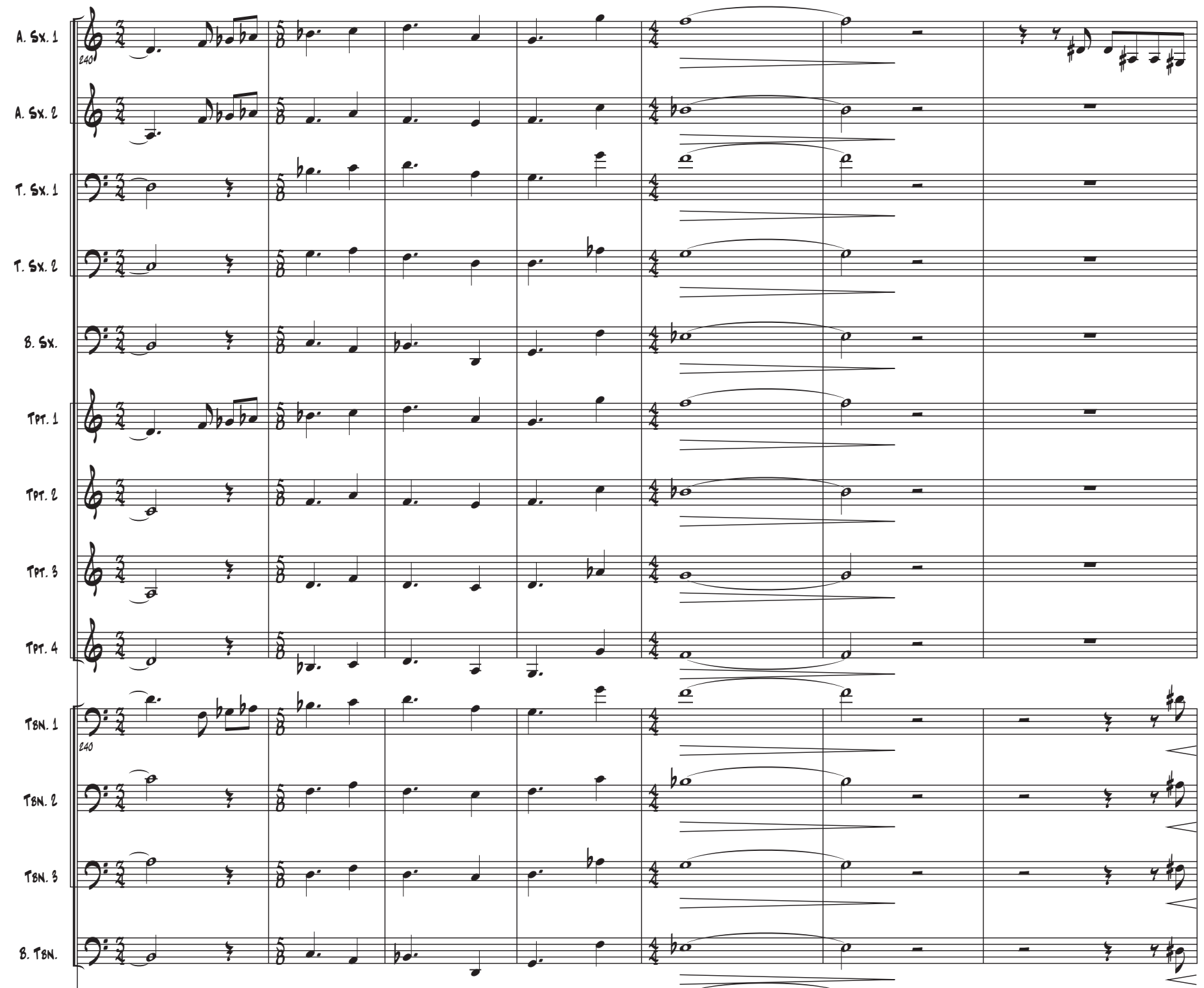
Gie. $\int_{240}^{30}$

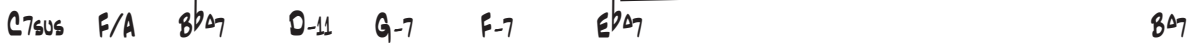

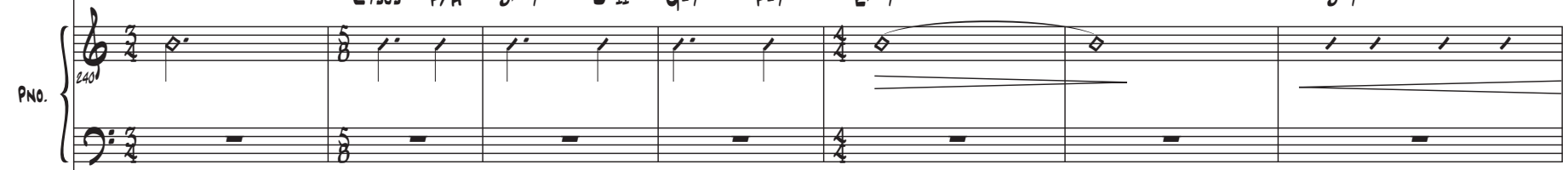
C7sus $F / A \quad B b_{\Delta 7} \quad 0_{-11} \quad G-7 \quad F-7 \quad E b_{\Delta 7}$

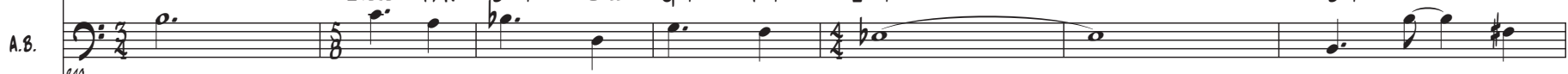

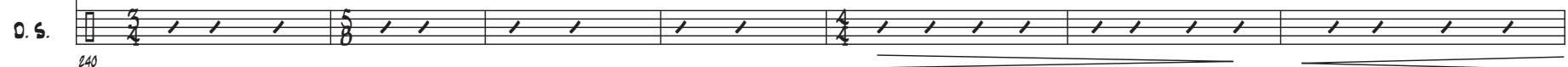




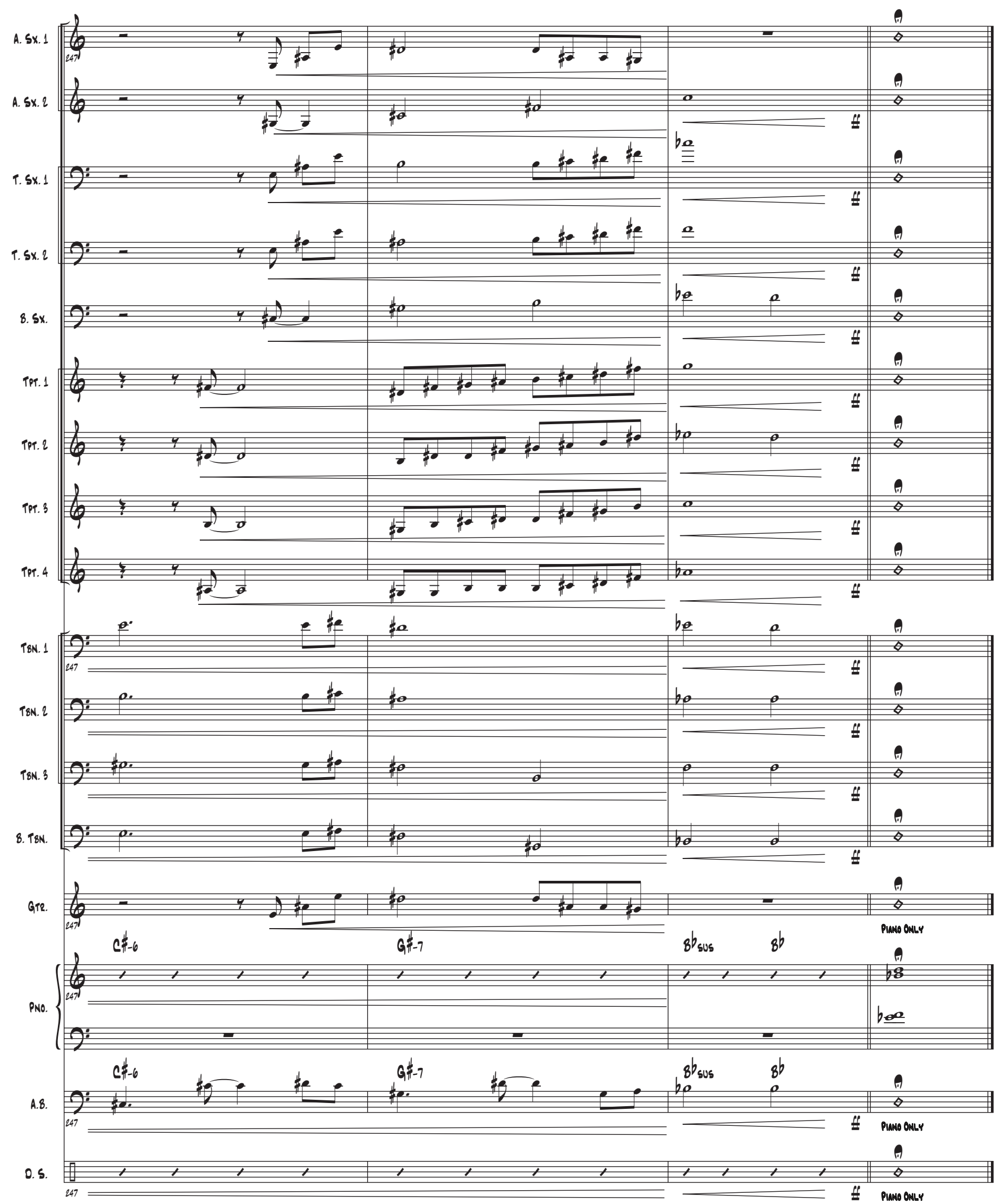





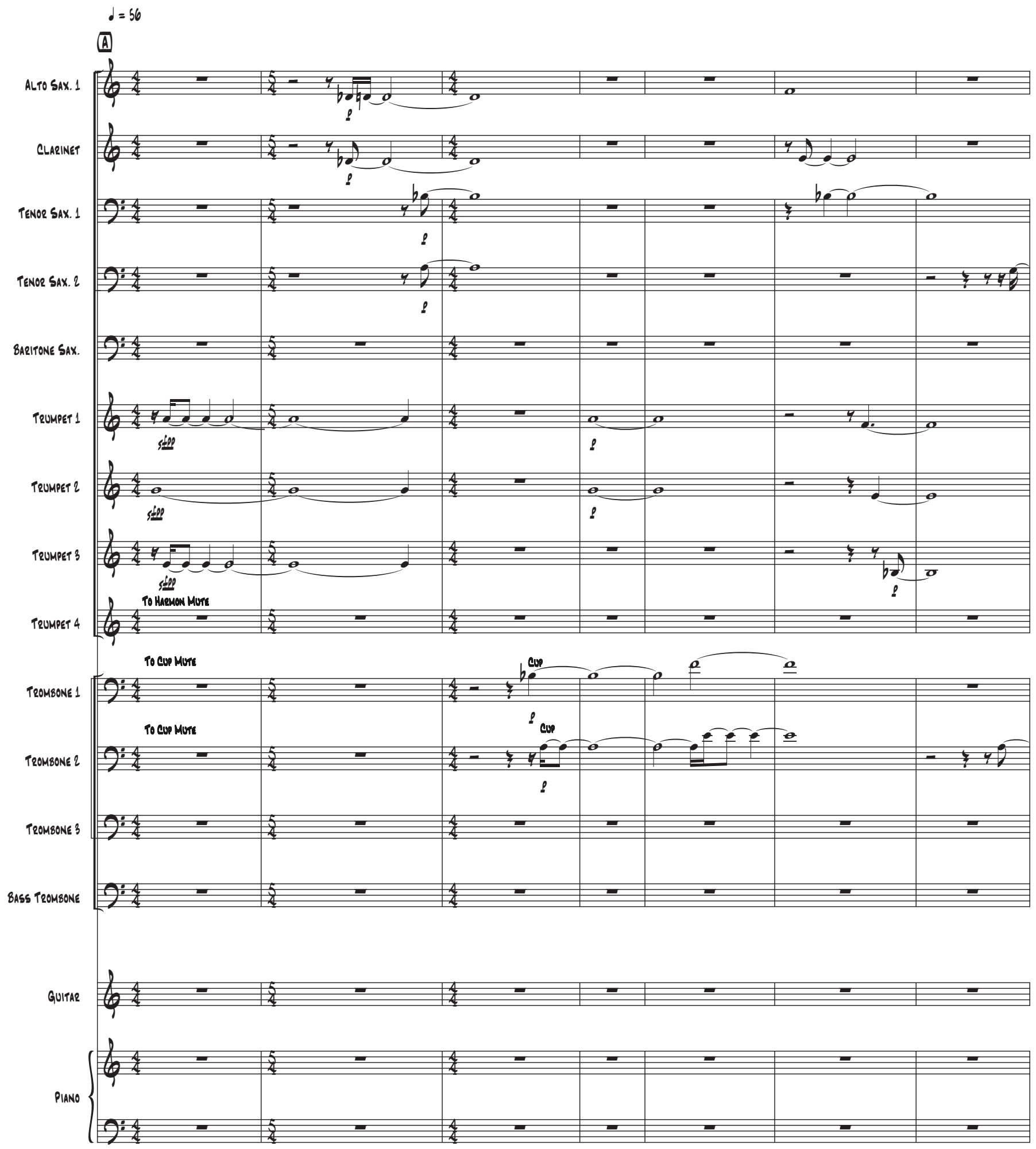



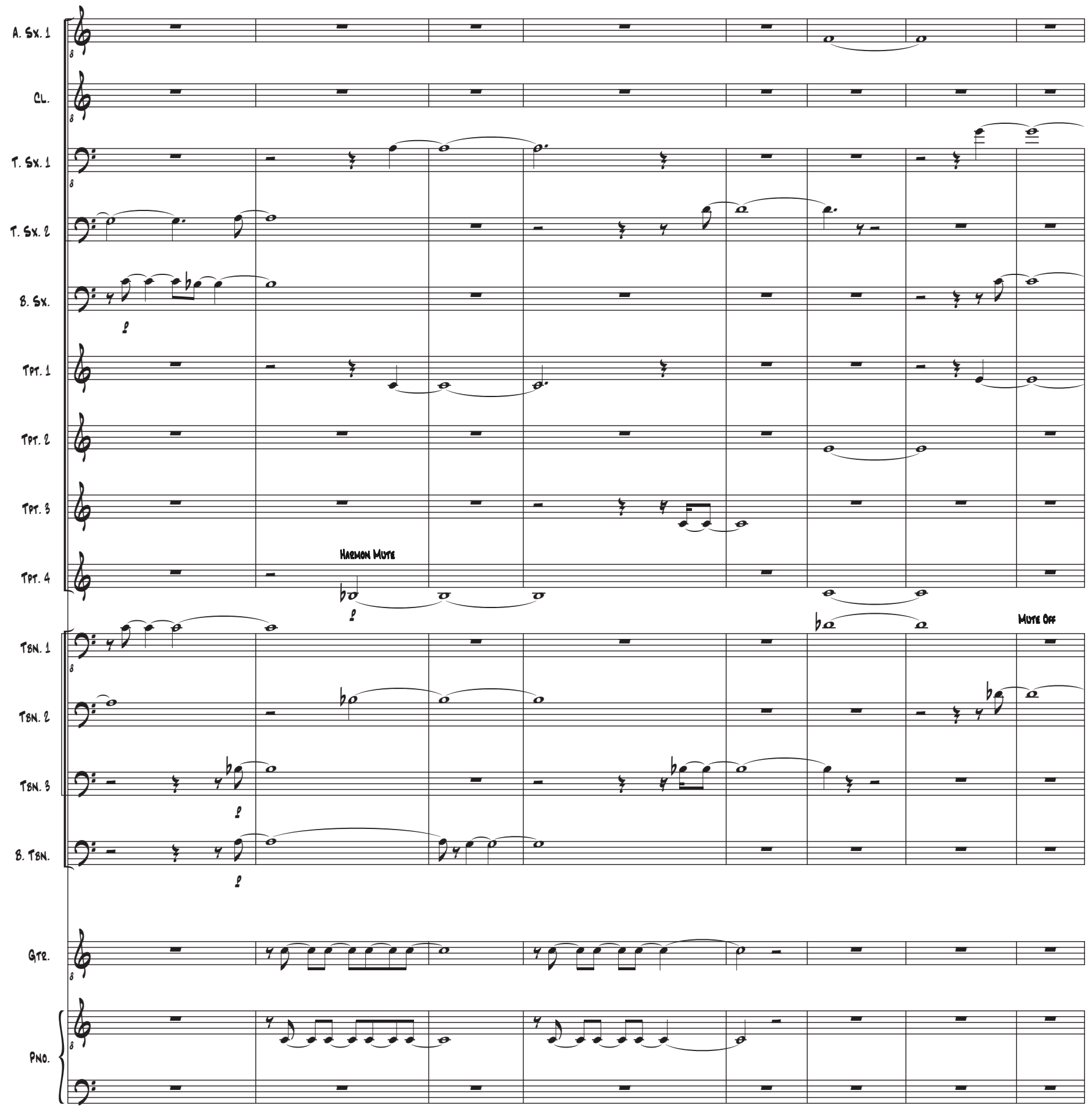


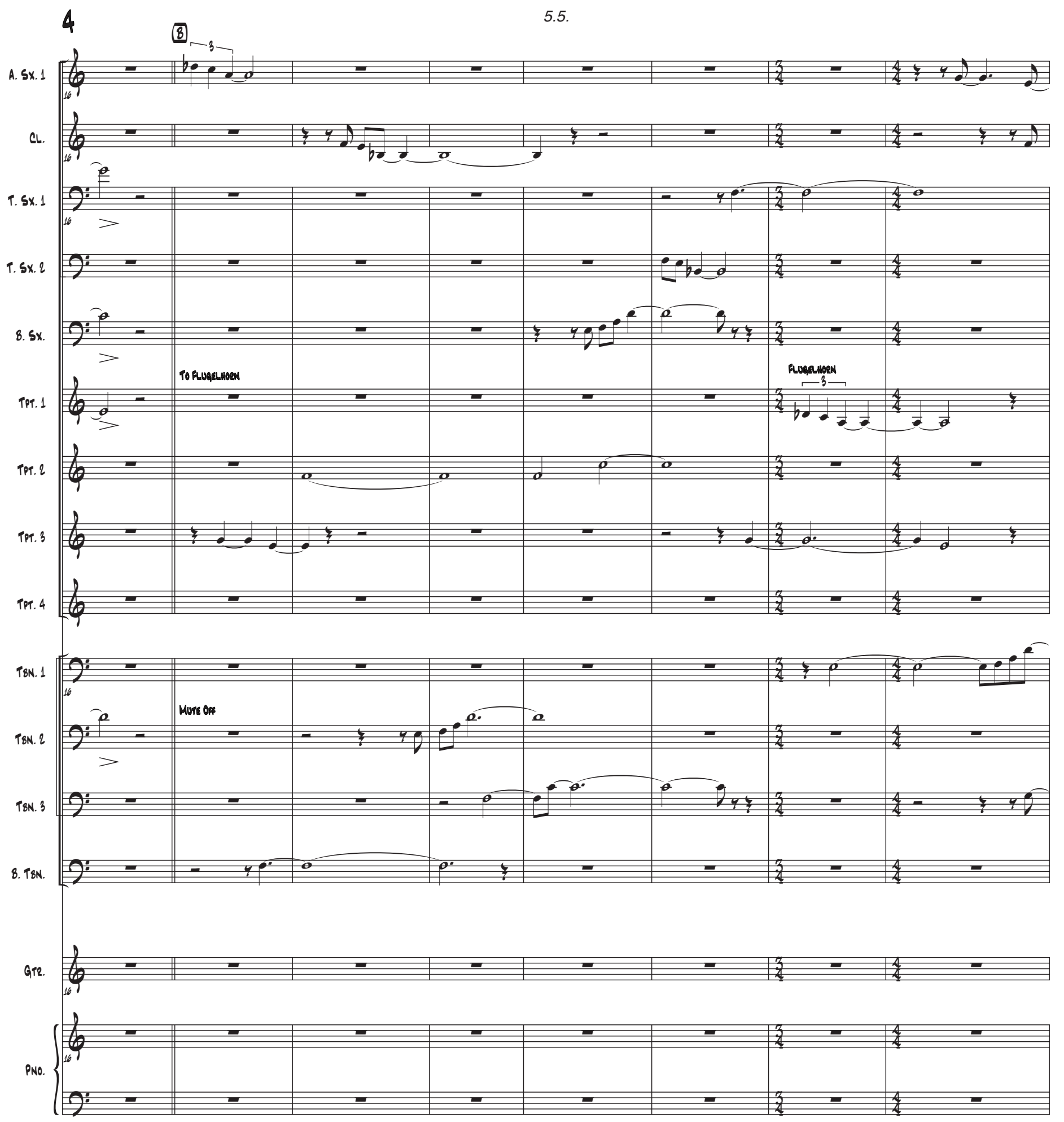



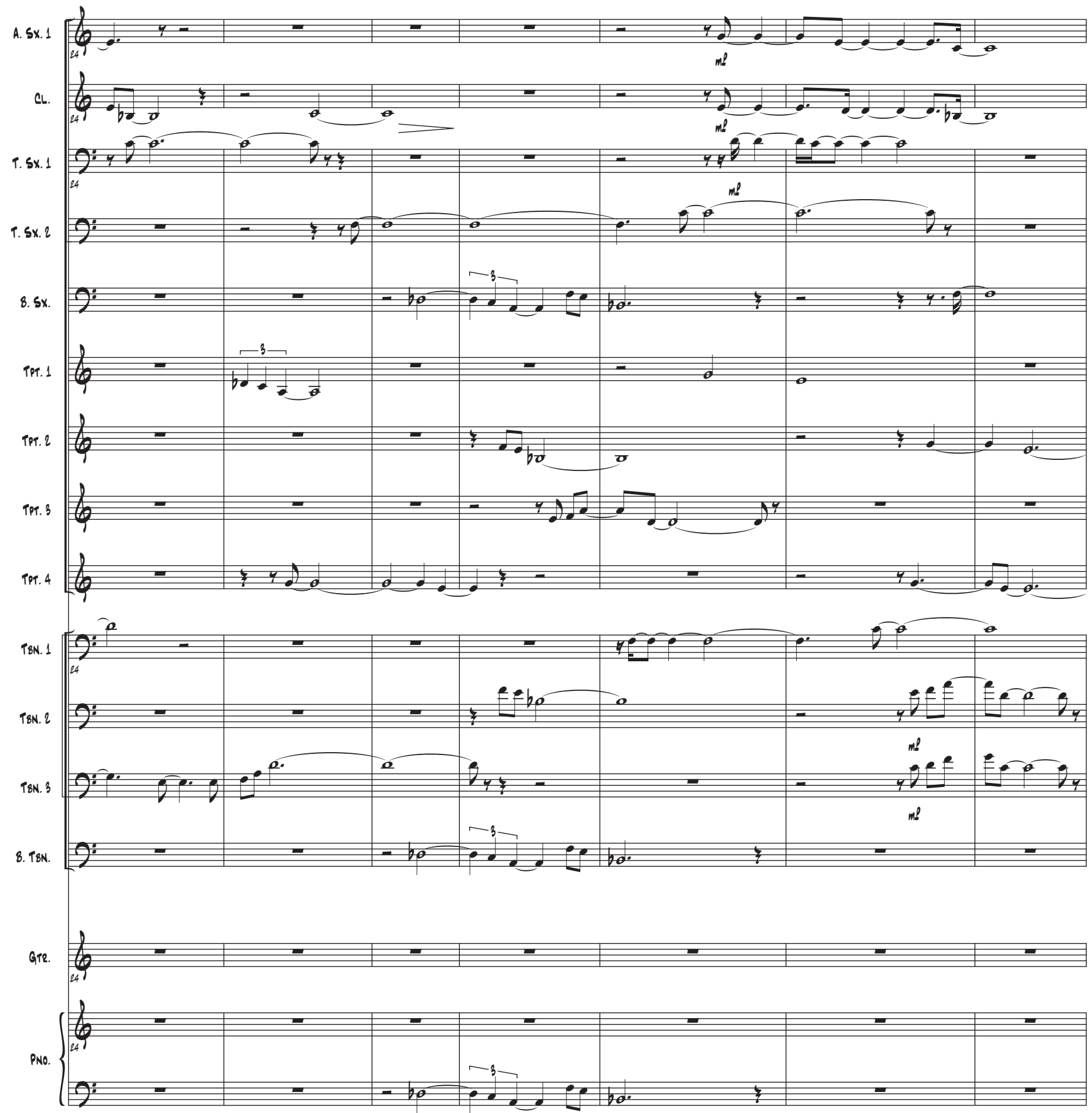


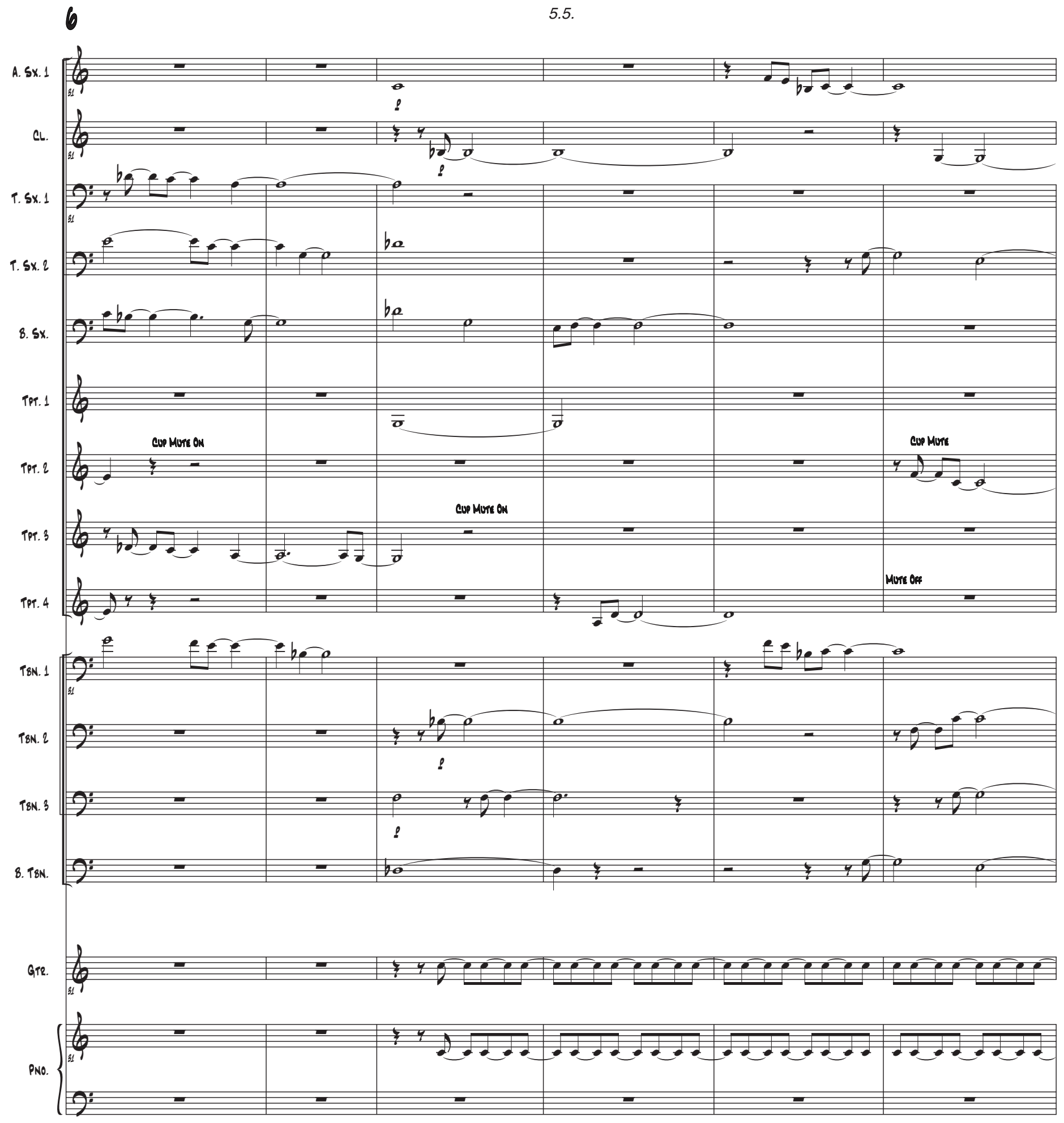


(c)
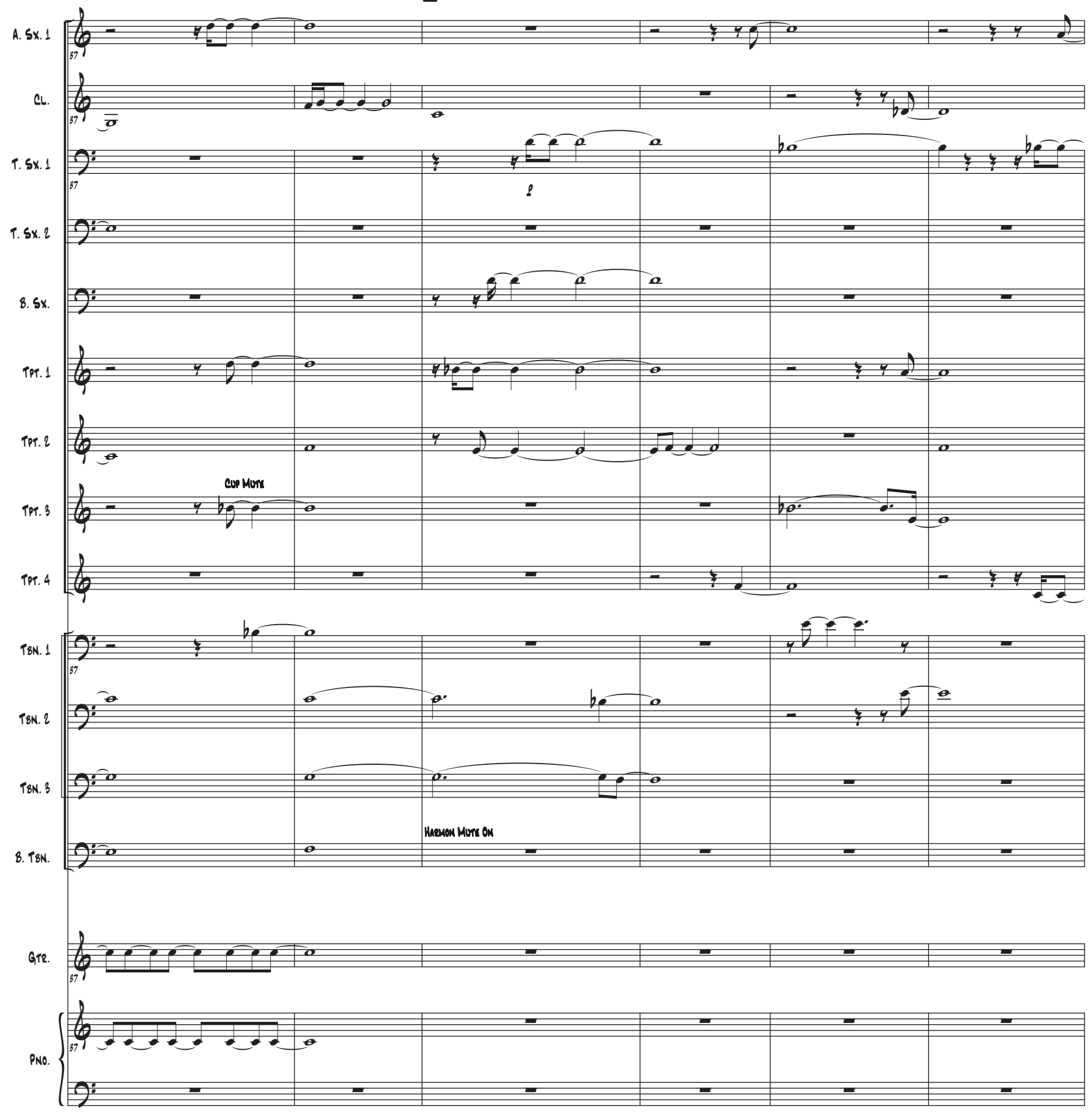

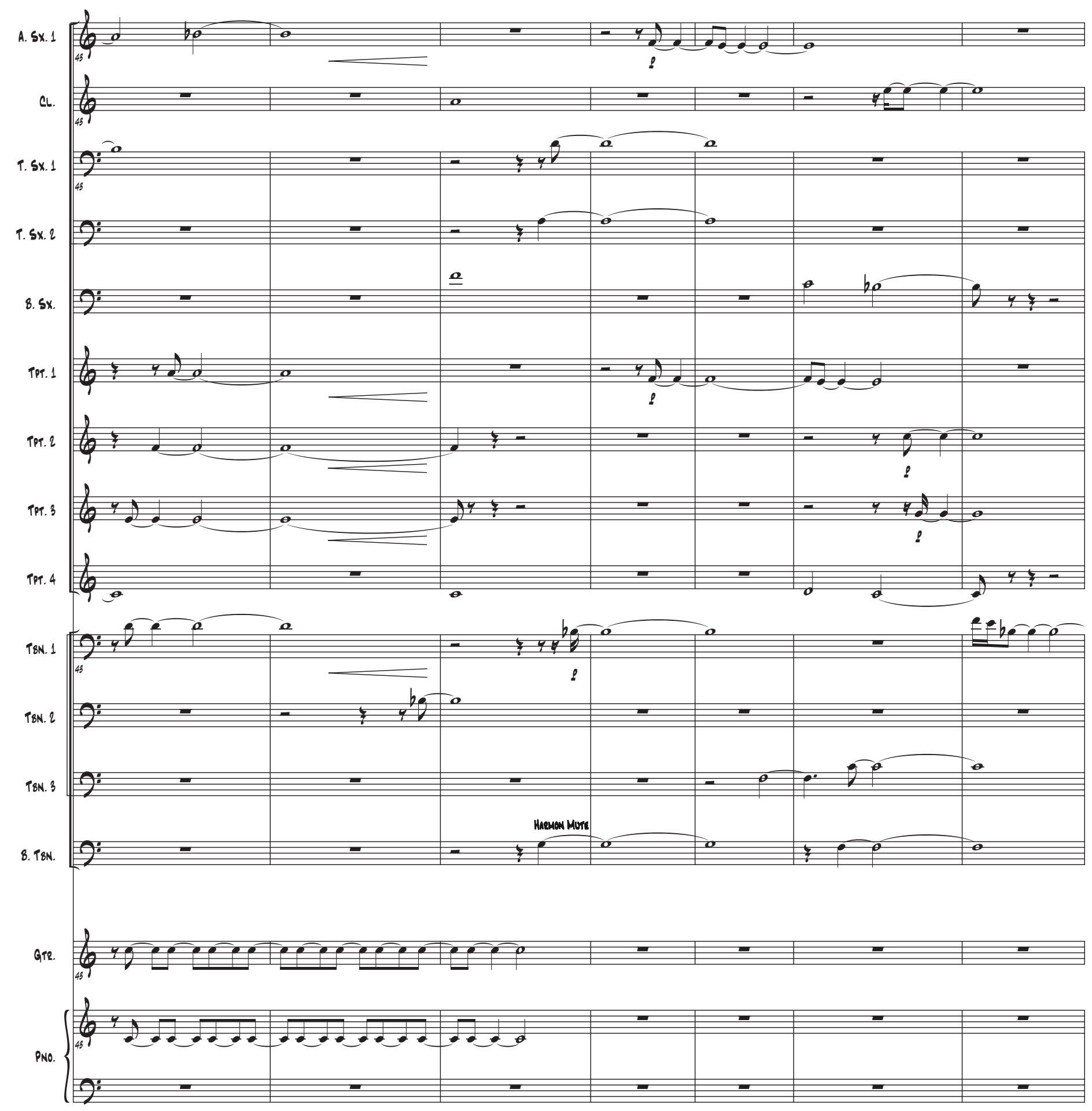

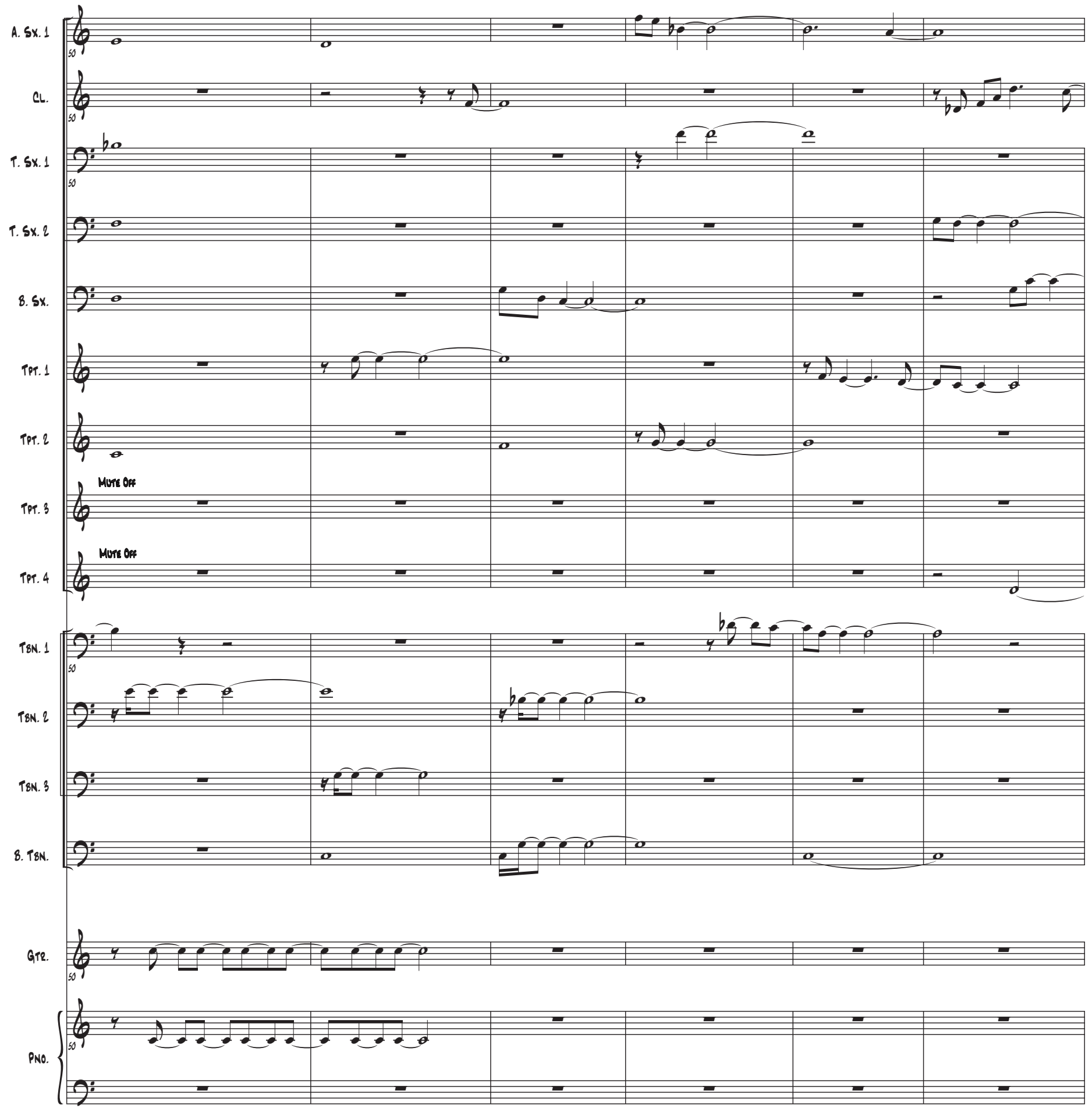


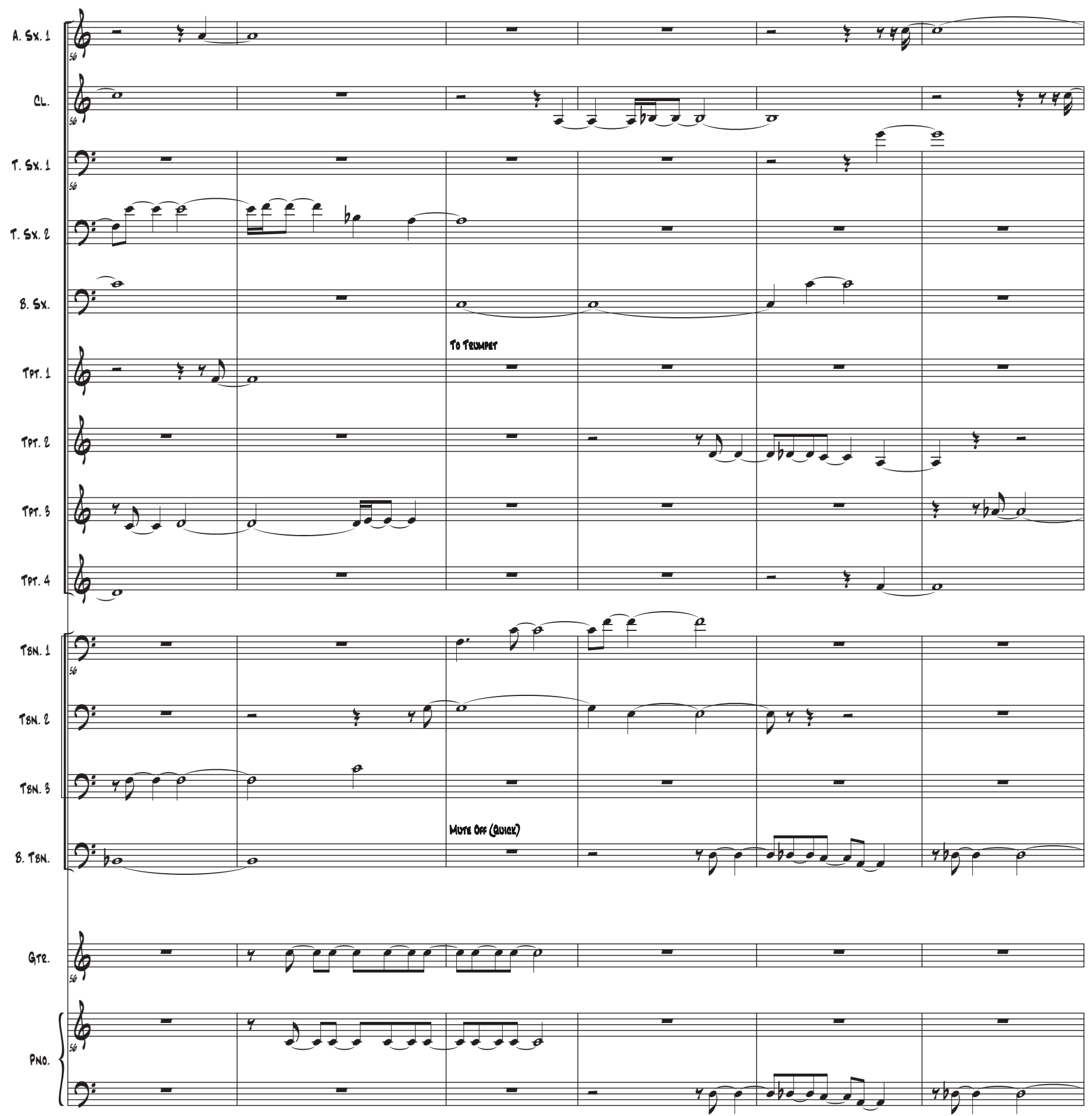



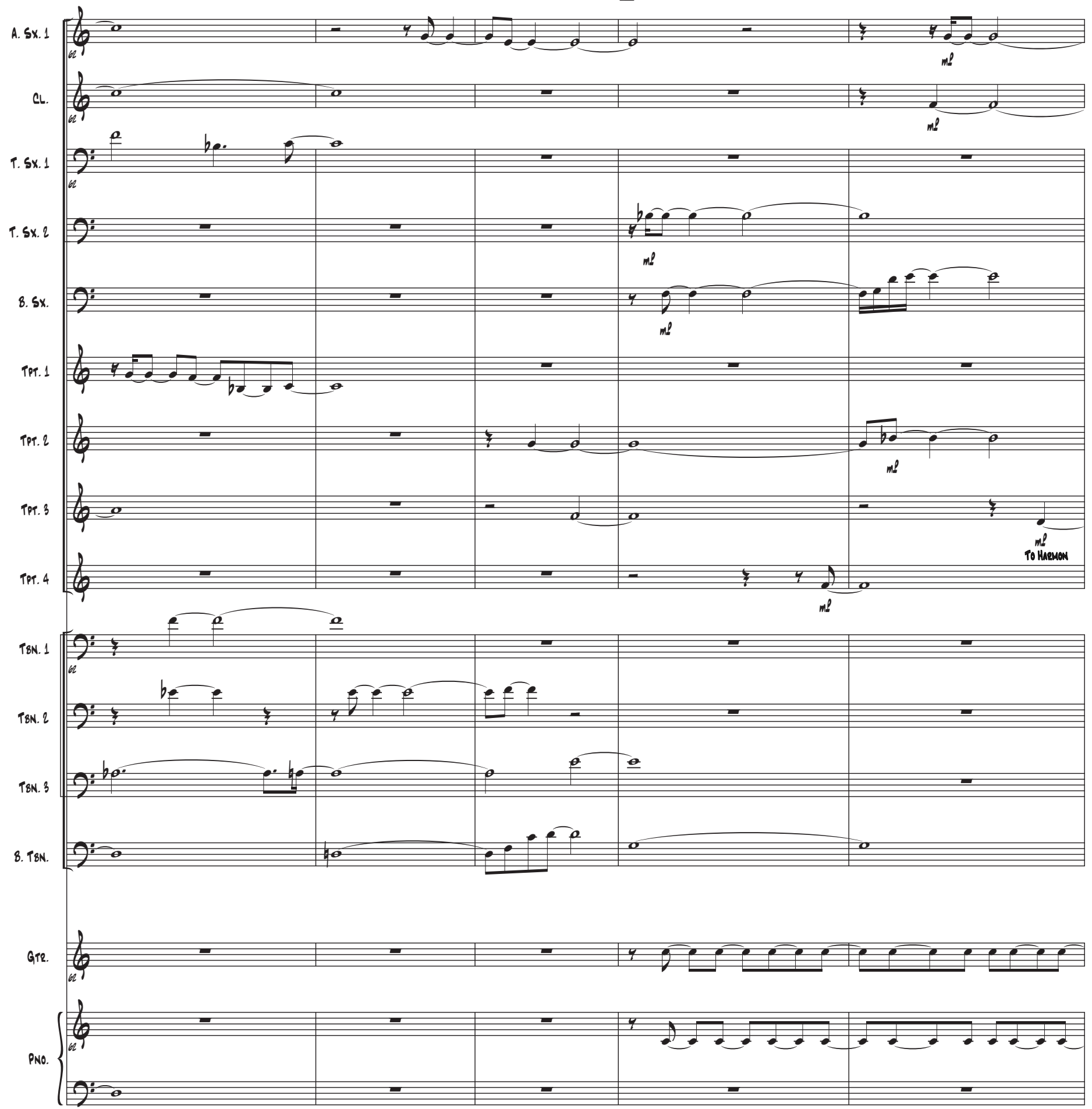


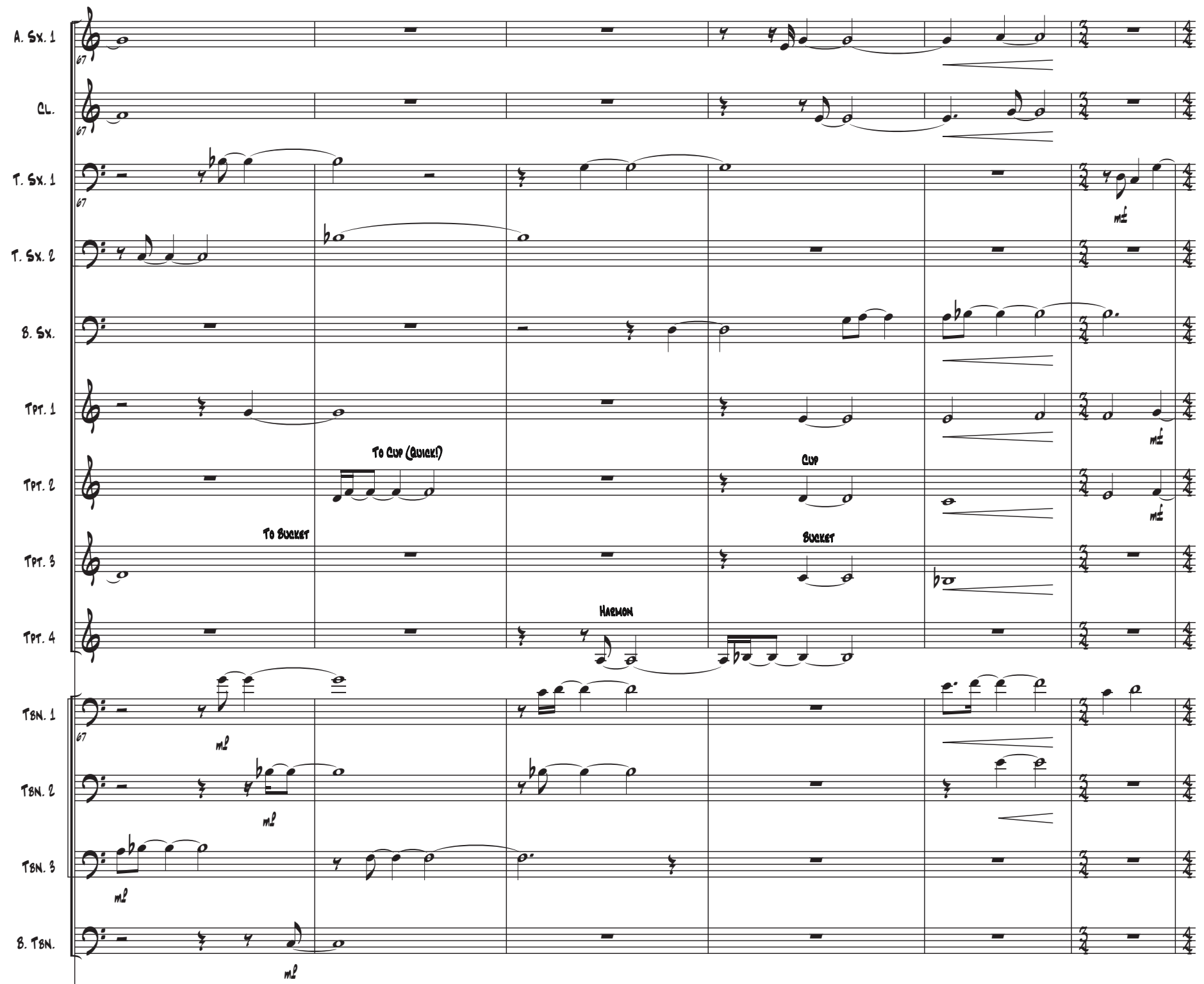

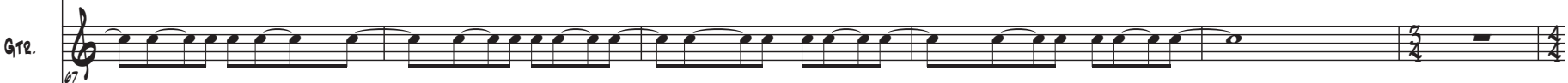

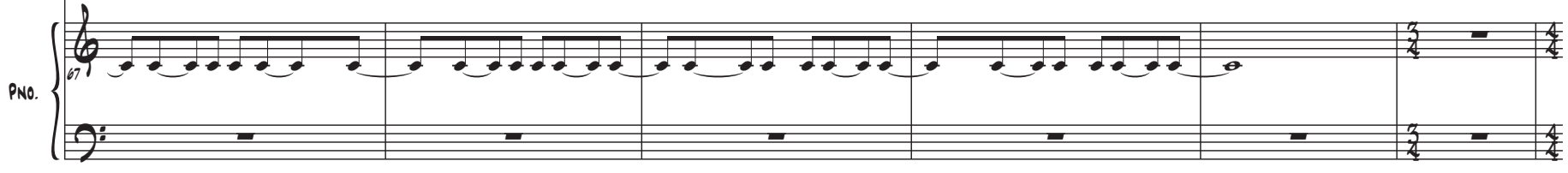




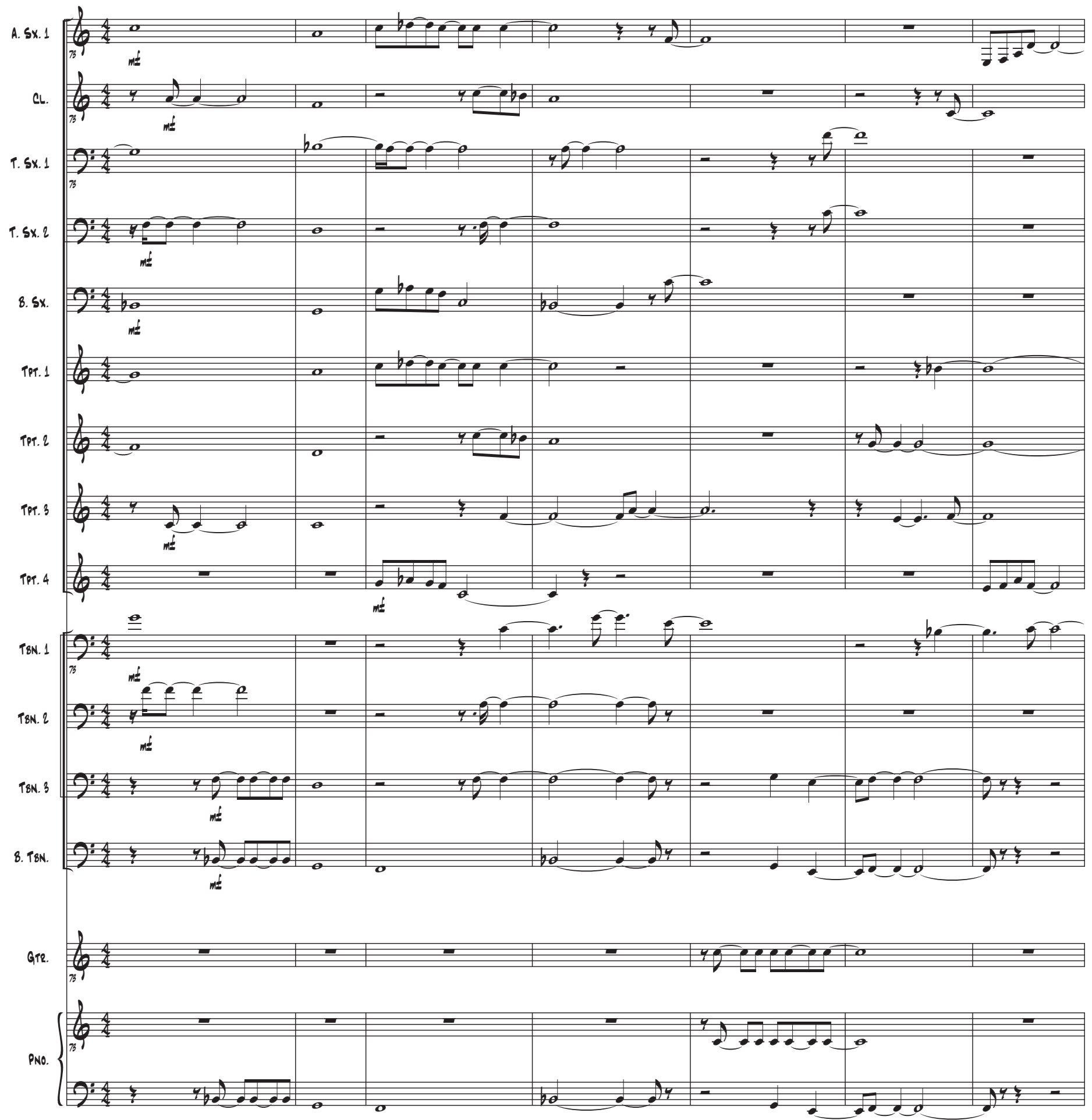




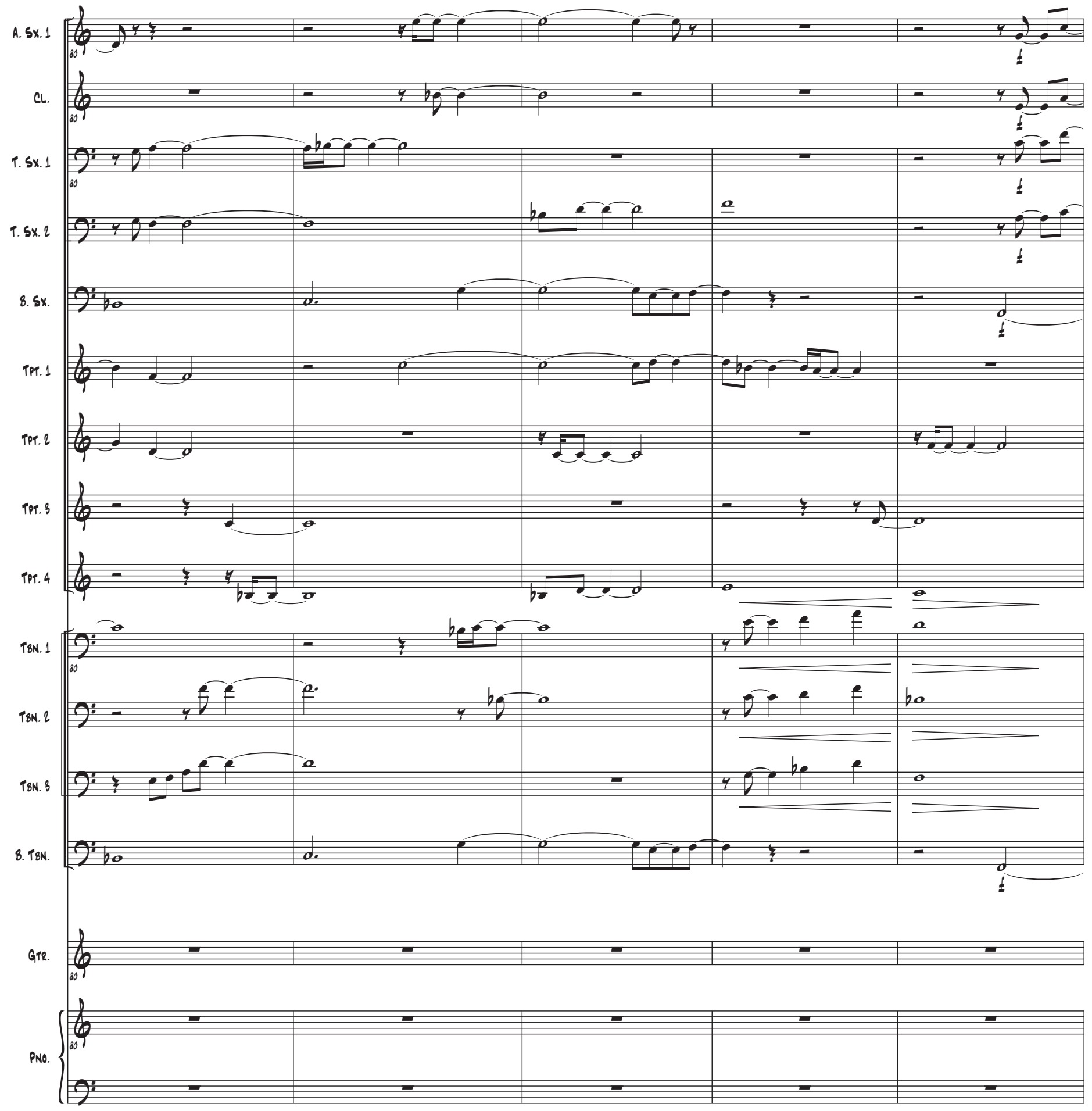



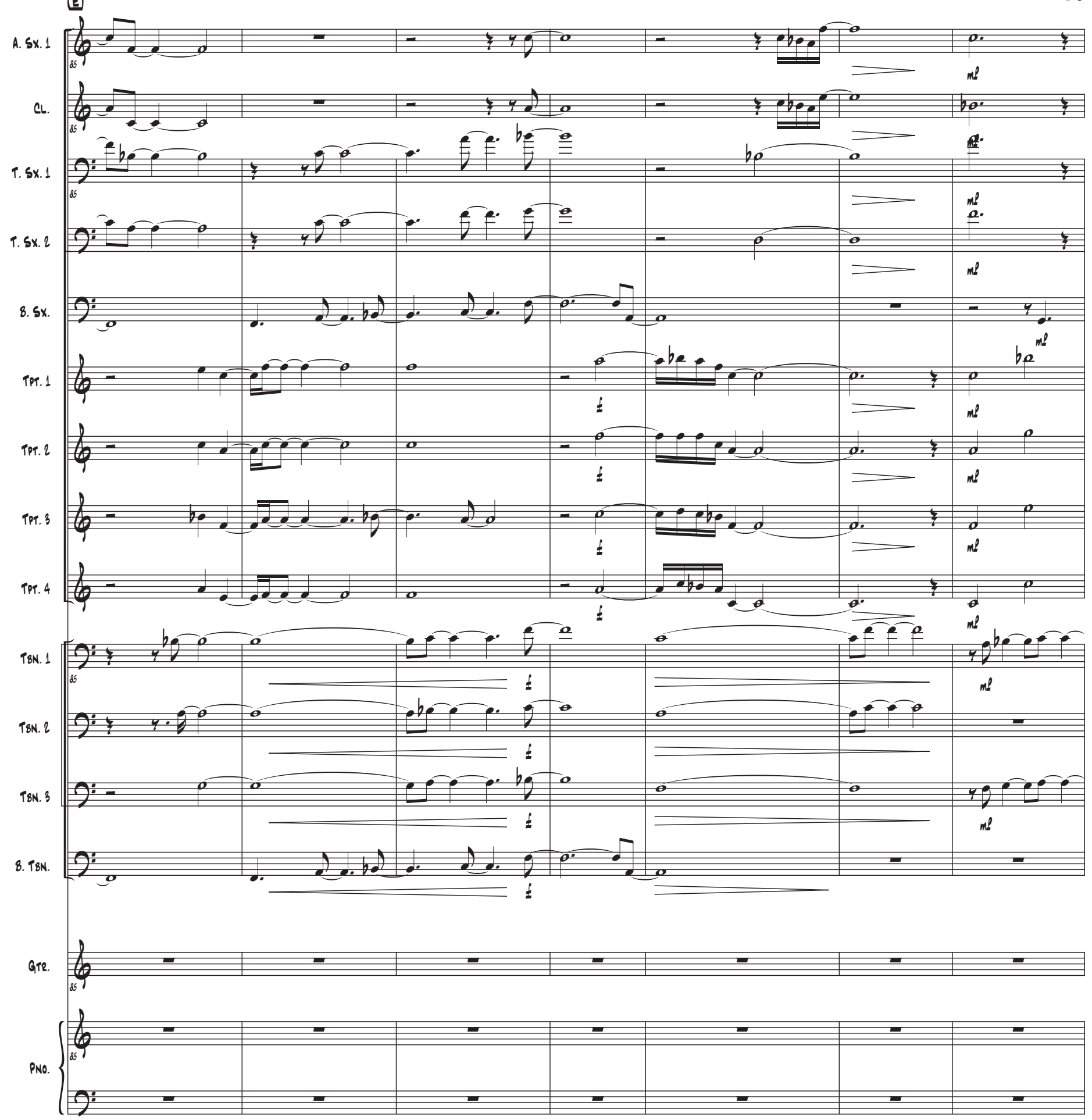


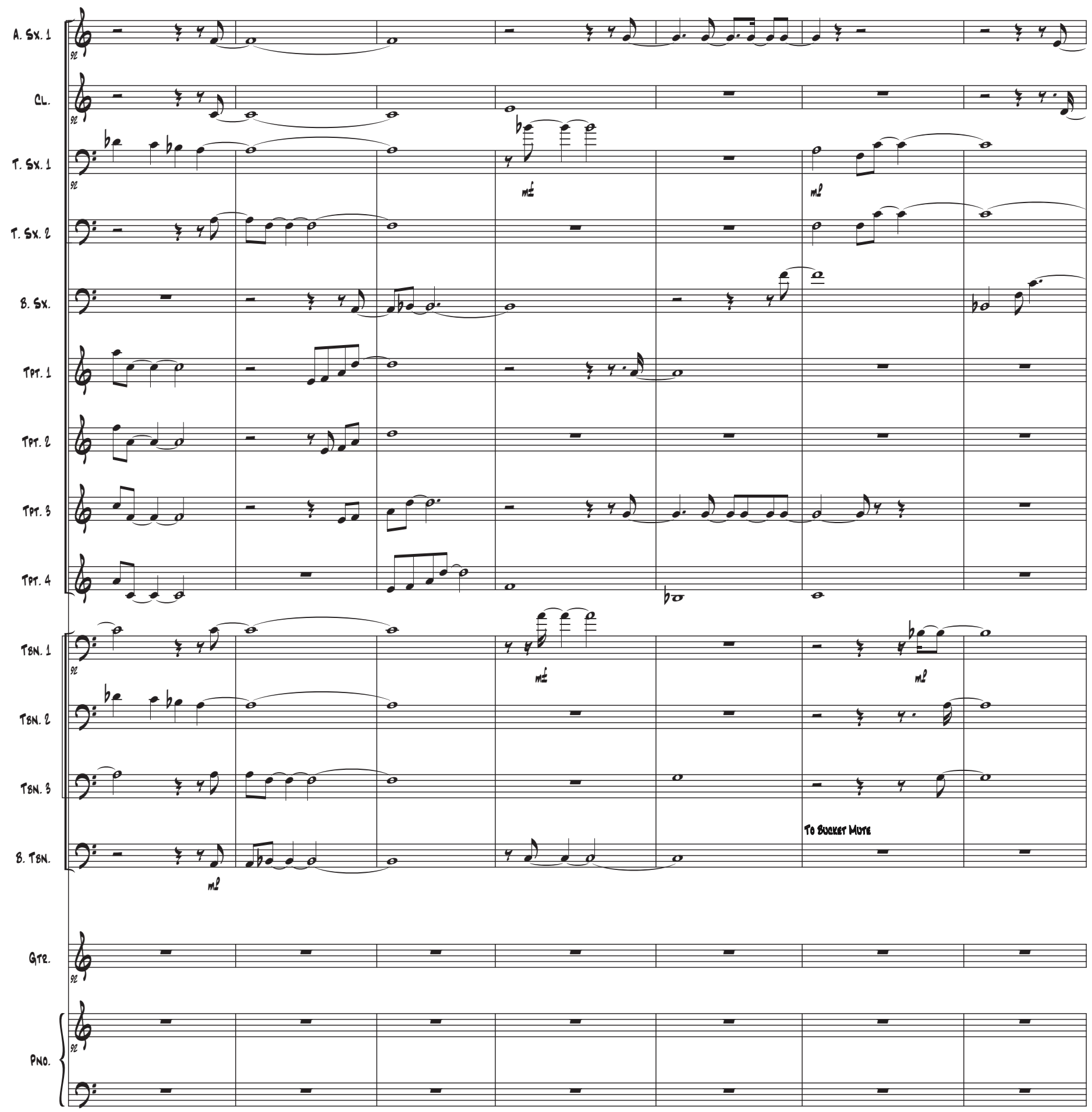




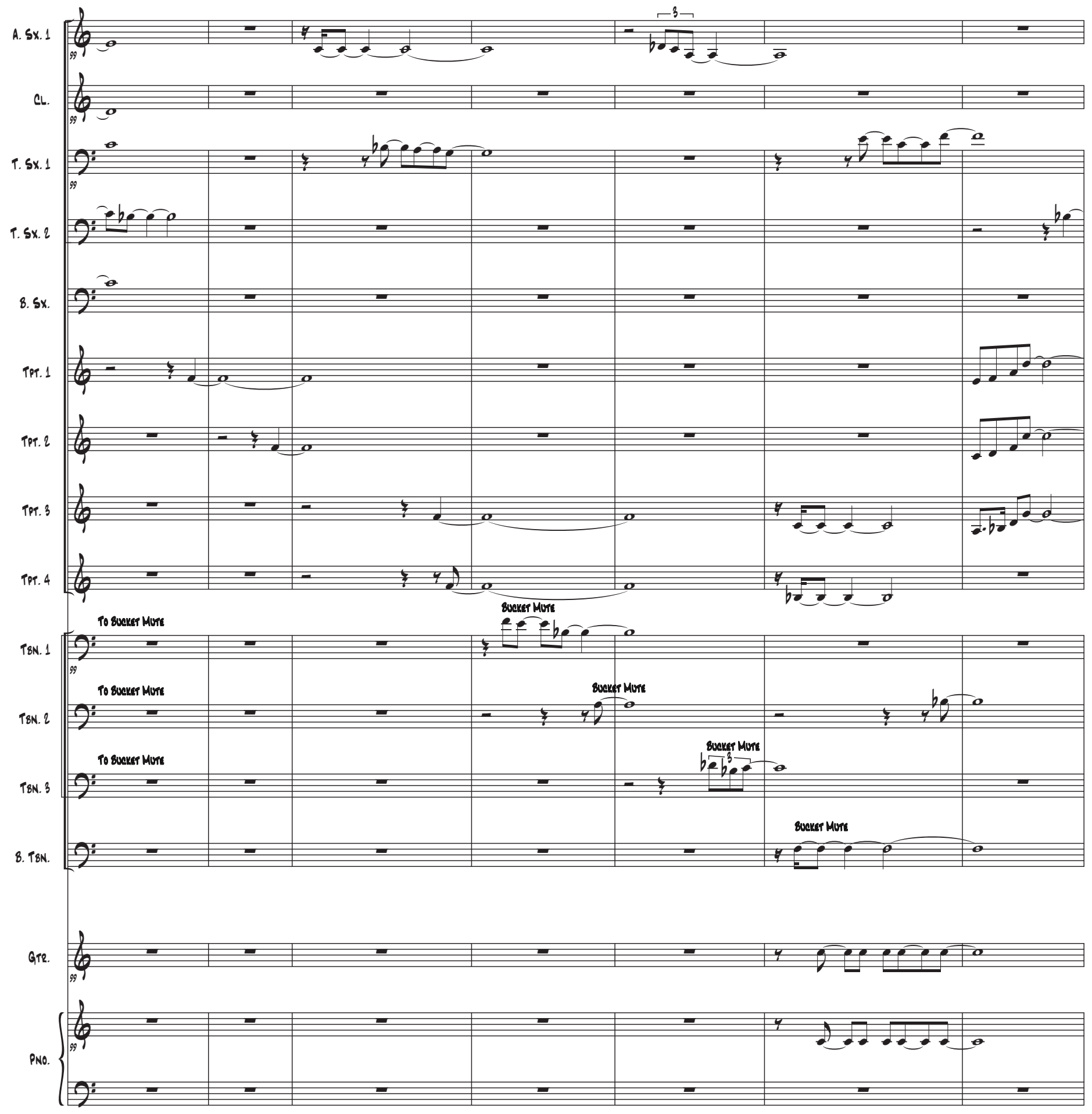


18
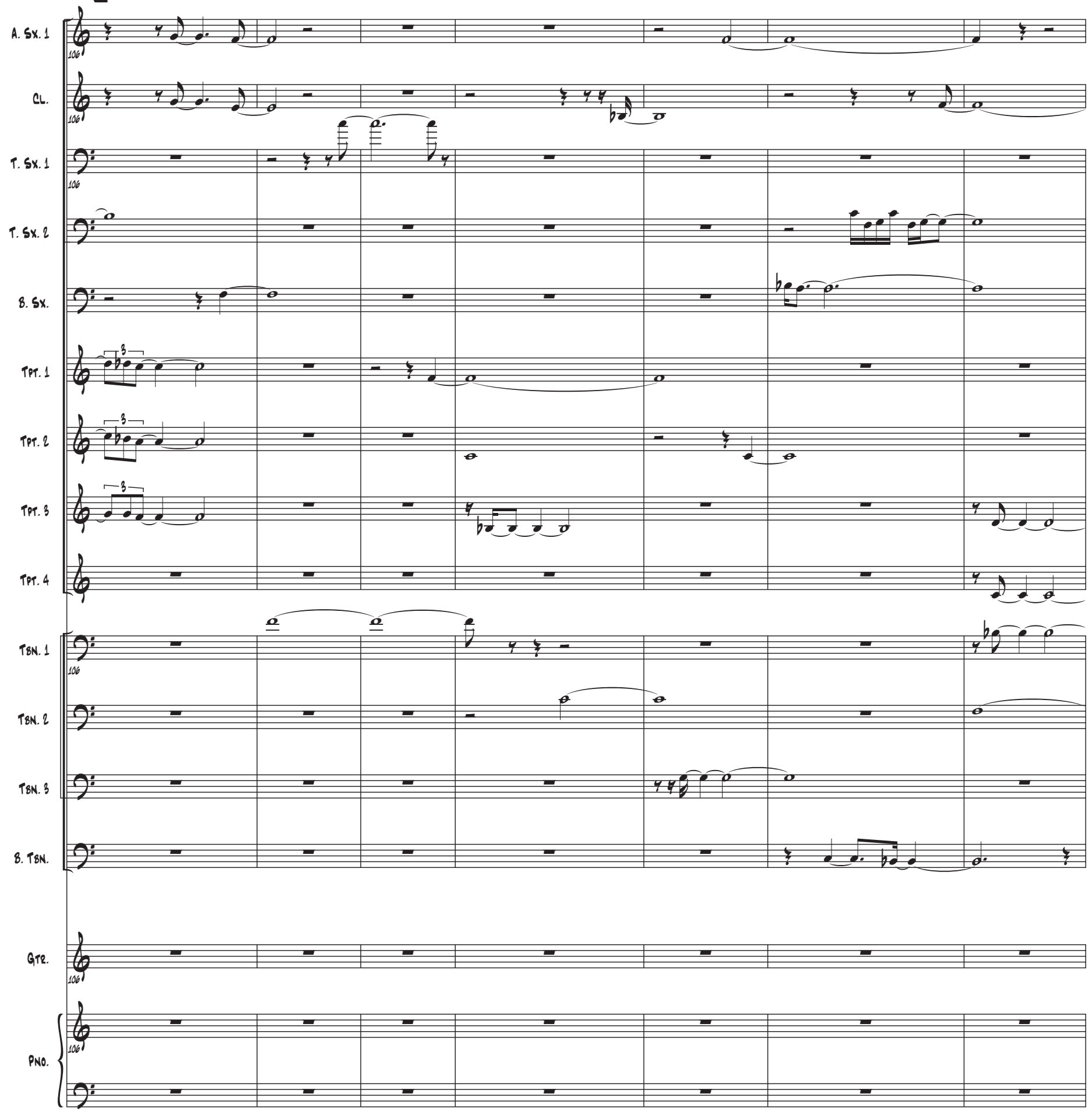


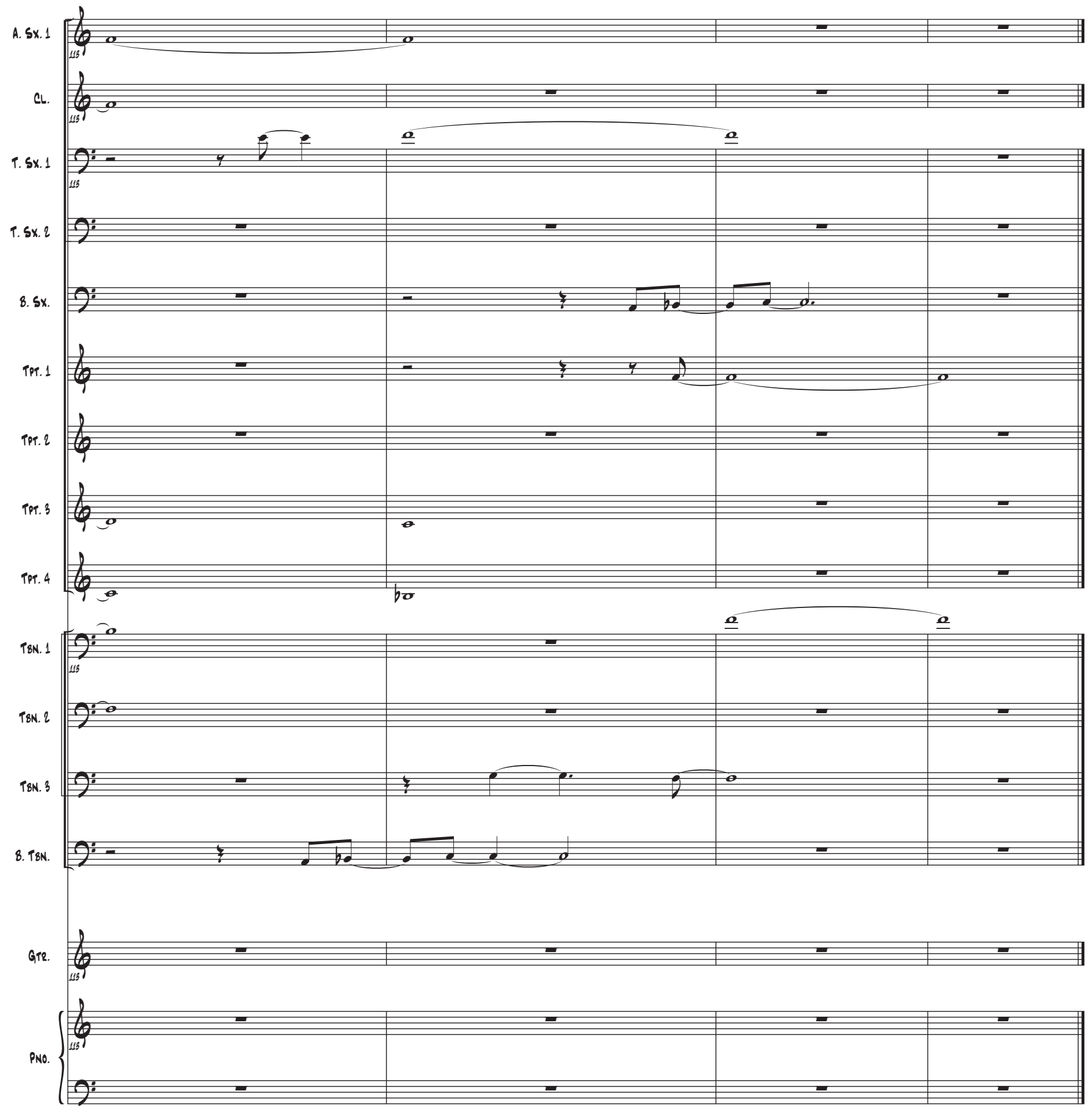

